\title{
The Temporal Ultraviolet Limit for Complex Bosonic Many-body Models.
}

\author{
Tadeusz Balaban \\ Department of Mathematics \\ Rutgers, The State University of New Jersey \\ 110 Frelinghuysen $\mathrm{Rd}$ \\ Piscataway, NJ 08854-8019 \\ tbalaban@math.rutgers.edu \\ Joel Feldman* \\ Department of Mathematics \\ University of British Columbia \\ Vancouver, B.C. \\ CANADA V6T 122 \\ feldman@math.ubc.ca \\ http://www.math.ubc.ca/ feldman/ \\ Horst Knörrer, Eugene Trubowitz \\ Mathematik \\ ETH-Zentrum \\ CH-8092 Zürich \\ SWITZERLAND \\ knoerrer@math.ethz.ch, trub@math.ethz.ch \\ http://www.math.ethz.ch/ knoerrer/
}

* Research supported in part by the Natural Sciences and Engineering Research Council of Canada and the Forschungsinstitut für Mathematik, ETH Zürich. 


\section{Introduction}

We are developing a set of tools and techniques for analyzing the large distance/infrared behaviour of a system of identical bosons, as the temperature tends to zero. In this paper we retain an infrared cutoff. That is, we consider bosons moving in the discrete torus $X=\mathbb{Z}^{\mathrm{D}} / L \mathbb{Z}^{\mathrm{D}}$, endowed with the standard Euclidean metric $d(\mathbf{x}, \mathbf{y})$. Our long term goal is to rigorously treat the infrared limit $L \rightarrow \infty$. See the introduction of [BFKT1].

The total energy of our many boson systems has two sources. First, each particle in the system has a kinetic energy. We shall denote the corresponding quantum mechanical observable by $\mathrm{h}$. The most common is $-\frac{1}{2 m} \Delta$, but, in this paper, more general operators are allowed. We assume that $\mathrm{h}=\nabla^{*} \mathcal{H} \nabla$ where $\mathcal{H}: L^{2}\left(X^{*}\right) \rightarrow L^{2}\left(X^{*}\right)$ is a translation invariant, real, strictly positive, operator, $X^{*}$ is the set of all bonds (ordered pairs of nearest neighbour points of $X$, but with the pair $(\mathbf{y}, \mathbf{x})$ viewed as $-(\mathbf{x}, \mathbf{y}))$ and the gradient $(\nabla f)((\mathbf{x}, \mathbf{y}))=f(\mathbf{y})-f(\mathbf{x})$. Second, the particles interact with each other through a twobody potential, $2 v(\mathbf{x}, \mathbf{y})$, which is assumed to be real, symmetric, translation invariant and exponentially decaying. For stability, $v$ is also required to be repulsive, in the sense that, viewed as the kernel of a convolution operator, it is strictly positive.

We assume that the system is in thermodynamic equilibrium and that expectations of observables are given by the grand canonical ensemble at temperature $T=\frac{1}{k \beta}>0$ and chemical potential $\mu$. We concentrate on the partition function $\operatorname{Tr} e^{-\frac{1}{k T}(H-\mu N)}$. Here, $H$ is the Hamiltonian and $N$ is the number operator. The techniques developed here can also be applied to correlations functions. See (I.8).

In [BFKT2], we developed a functional integral representation for the partition function. See (I.2) below. The integration variable of this functional integral is a complex field $\alpha_{\tau}(\mathbf{x})$ depending on position $\mathbf{x} \in X$ and time/temperature $\tau \in\left(0, \frac{1}{k T}\right]$. Here, we have periodic boundary conditions, that is $\alpha_{0}=\alpha_{\frac{1}{k T}}$. The representation of [BFKT2] can be viewed as a rigorous version of the formal representation

$$
\operatorname{Tr} e^{-\frac{1}{k T}(H-\mu N)}=\int \cdots \int \prod_{\substack{\mathbf{x} \in X \\ 0 \leq \tau \leq \frac{1}{k T}}} \frac{d \alpha_{\tau}^{*}(\mathbf{x}) d \alpha_{\tau}(\mathbf{x})}{2 \pi i} e^{\mathcal{A}_{X}\left(\alpha^{*}, \alpha\right)}
$$

where 


$$
\begin{gathered}
\mathcal{A}_{X}\left(\alpha^{*}, \alpha\right)=\int_{0}^{\frac{1}{k T}} d \tau \int_{X} d \mathbf{x}\left\{\alpha_{\tau}(\mathbf{x})^{*} \frac{\partial}{\partial \tau} \alpha_{\tau}(\mathbf{x})-\alpha_{\tau}(\mathbf{x})^{*}\left(h \alpha_{\tau}\right)(\mathbf{x})+\mu \alpha_{\tau}(\mathbf{x})^{*} \alpha_{\tau}(\mathbf{x})\right\} \\
-\int_{0}^{\frac{1}{k T}} d \tau \iint_{X^{2}} d \mathbf{x} d \mathbf{y} \alpha_{\tau}(\mathbf{x})^{*} \alpha_{\tau}(\mathbf{y})^{*} v(\mathbf{x}, \mathbf{y}) \alpha_{\tau}(\mathbf{x}) \alpha_{\tau}(\mathbf{y})
\end{gathered}
$$

This formal representations is frequently used in the physics literature. See [NO, (2.66)]. The ultraviolet problem is to integrate out, in this representation, all variables $\alpha_{\tau}(\mathbf{x})$ except for those having $\tau$ in a lattice with spacing of order one. In this paper, we treat the ultraviolet problem for our rigorous version of the formal representation above.

As pointed out in the introduction to [BFKT1], it is not possible to give rigorous mathematical meaning to the functional integral above in a straightforward way. For this reason, we derived (in Theorem 2.2 of [BFKT2]) the representation

$$
\begin{aligned}
& \operatorname{Tr} e^{-\frac{1}{k T}(H-\mu N)} \\
& =\lim _{\varepsilon \rightarrow 0} \int \prod_{\tau \in \varepsilon \mathbb{Z} \cap\left(0, \frac{1}{k T}\right]}\left[d \mu_{\mathrm{R}(\varepsilon)}\left(\alpha_{\tau}^{*}, \alpha_{\tau}\right) \zeta_{\varepsilon}\left(\alpha_{\tau-\varepsilon}, \alpha_{\tau}\right) e^{\left\langle\alpha_{\tau-\varepsilon}^{*}, j(\varepsilon) \alpha_{\tau}\right\rangle-\varepsilon\left\langle\alpha_{\tau-\varepsilon}^{*} \alpha_{\tau} v \alpha_{\tau-\varepsilon}^{*} \alpha_{\tau}\right\rangle}\right]
\end{aligned}
$$

Here, for any $r>0$,

$$
d \mu_{r}\left(\alpha^{*}, \alpha\right)=\prod_{\mathbf{x} \in X} \frac{d \alpha^{*}(\mathbf{x}) \wedge d \alpha(\mathbf{x})}{2 \pi \imath} e^{-\alpha^{*}(\mathbf{x}) \alpha(\mathbf{x})} \chi(|\alpha(\mathbf{x})|<r)
$$

denotes the unnormalized Gaussian measure, cut off at radius $r$, and $\zeta_{\varepsilon}(\alpha, \beta)$ is the characteristic function of

$$
\left\{\alpha, \beta: \mathbb{C}^{X} \rightarrow \mathbb{C} \mid\|\alpha-\beta\|_{\infty}<p_{0}(\varepsilon)\right\}
$$

The cutoffs $\mathrm{R}(\varepsilon)>0$ and $p_{0}(\varepsilon) \geq \ln \frac{1}{\varepsilon}$ are decreasing functions of $\varepsilon$ defined for all $0<\varepsilon \leq 1$ that obey

$$
\mathrm{R}(\varepsilon) \geq \frac{1}{\sqrt[4]{\varepsilon}} p_{0}(\varepsilon) \quad \text { and } \quad \lim _{\varepsilon \rightarrow 0} \sqrt{\varepsilon} \mathrm{R}(\varepsilon)=0
$$

Furthermore, for any $t>0$, the operator $j(t)=e^{-t(\mathrm{~h}-\mu)}$. We write the $(\mathbb{R}$-style) scalar product, $\langle f, g\rangle=\sum_{\mathbf{x} \in X} f(\mathbf{x}) g(\mathbf{x})$ for any two fields $f, g: X \rightarrow \mathbb{C} .^{(1)}$

In this paper, we treat the ultraviolet problem in the representation (I.2). The final result is to write the partition function as a functional integral which involves $\alpha_{\tau}$ for only finitely many values of $\tau$, independent of $\varepsilon$. To achieve this, we have to integrate out all

(1) Thus the usual scalar product over $\mathbb{C}^{|X|}$ is $\left\langle f^{*}, g\right\rangle$. 
but a fixed number of fields $\alpha_{\tau}$ in the representation (I.2). For $n \geq 1$ and $\varepsilon>0$, set

$$
\begin{aligned}
& I_{n}\left(\varepsilon ; \alpha^{*}, \beta\right) \\
& \quad=\int_{\tau \in \varepsilon \mathbb{Z} \cap\left(0,2^{n} \varepsilon\right)} d \mu_{\mathrm{R}(\varepsilon)}\left(\alpha_{\tau}^{*}, \alpha_{\tau}\right) \prod_{\tau \in \varepsilon \mathbb{Z} \cap\left(0,2^{n} \varepsilon\right]}\left[\zeta_{\varepsilon}\left(\alpha_{\tau-\varepsilon}, \alpha_{\tau}\right) e^{\left\langle\alpha_{\tau-\varepsilon}^{*}, j(\varepsilon) \alpha_{\tau}\right\rangle-\varepsilon\left\langle\alpha_{\tau-\varepsilon}^{*} \alpha_{\tau} v \alpha_{\tau-\varepsilon}^{*} \alpha_{\tau}\right\rangle}\right]
\end{aligned}
$$

with $\alpha_{0}=\alpha$ and $\alpha_{2^{n} \varepsilon}=\beta$. If $\varepsilon=\frac{1}{2^{m}} \frac{1}{p k T}$, for $m, p \in \mathbb{N}$, then

$$
\begin{array}{r}
\int \prod_{\tau \in \varepsilon \mathbb{Z} \cap\left(0, \frac{1}{k T}\right]}\left[d \mu_{\mathrm{R}(\varepsilon)}\left(\alpha_{\tau}^{*}, \alpha_{\tau}\right) \zeta_{\varepsilon}\left(\alpha_{\tau-\varepsilon}, \alpha_{\tau}\right) e^{\left.\left\langle\alpha_{\tau-\varepsilon}^{*}, j(\varepsilon) \alpha_{\tau}\right\rangle-\varepsilon\left\langle\alpha_{\tau-\varepsilon}^{*} \alpha_{\tau} v \alpha_{\tau-\varepsilon}^{*} \alpha_{\tau}\right\rangle\right]}\right. \\
=\int \prod_{n=1}^{p}\left[d \mu_{\mathrm{R}(\varepsilon)}\left(\phi_{n}^{*}, \phi_{n}\right) I_{m}\left(\varepsilon ; \phi_{n-1}^{*}, \phi_{n}\right)\right]
\end{array}
$$

with the convention $\phi_{0}=\phi_{p}$. Combining (I.2) and (I.4) we get

$$
\operatorname{Tr} e^{-\frac{1}{k T}(H-\mu N)}=\lim _{m \rightarrow \infty} \int \prod_{n=1}^{p}\left[d \mu_{\mathrm{R}\left(\frac{1}{2^{m} p k T}\right)}\left(\phi_{n}^{*}, \phi_{n}\right) I_{m}\left(\frac{1}{2^{m} p k T} ; \phi_{n-1}^{*}, \phi_{n}\right)\right]
$$

In this paper we show that, that for all sufficiently small ${ }^{(2)} \theta>0$,

$$
I_{\theta}\left(\alpha^{*}, \beta\right)=\lim _{m \rightarrow \infty} I_{m}\left(2^{-m} \theta ; \alpha^{*}, \beta\right)
$$

exists and we also exhibit properties of $I_{\theta}$ that we deem useful for a potential infrared analysis. If $\theta$ was chosen sufficiently small, we will write $I_{\theta}$ as the sum of a dominant part (which is shown to have a logarithm) and terms indexed by proper subsets of $X$ and which are exponentially small in the size of the subsets.

The partition function can be written as

$$
\operatorname{Tr} e^{-\frac{1}{k T}(H-\mu N)}=\int \prod_{n=1}^{p}\left[\prod_{\mathbf{x} \in X} \frac{d \phi_{n}(\mathbf{x})^{*} \phi_{n}(\mathbf{x})}{2 \pi \imath} e^{-\phi_{n}(\mathbf{x})^{*} \phi_{n}(\mathbf{x})}\right] I_{\theta}\left(\phi_{n-1}^{*}, \phi_{n}\right)
$$

More generally, one also gets a representation for the Green's functions ${ }^{(3)}$

$$
\frac{\operatorname{Tr} e^{-\frac{1}{k T}(H-\mu N)} \mathbb{T} \prod_{j=1}^{\ell} \psi^{(\dagger)}\left(\beta_{j}, \mathbf{x}_{j}\right)}{\operatorname{Tr} e^{-\frac{1}{k T}(H-\mu N)}}
$$

(2) The smallness condition on $\theta$ does not depend on the interaction $v$.

(3) Here $\psi\left(\beta_{j}, \mathbf{x}_{j}\right)$ and $\psi^{\dagger}\left(\beta_{j}, \mathbf{x}_{j}\right)$ are annihilation and creation operators, conjugated by $e^{\beta_{j}(H-\mu N)}$, and $\mathbb{T}$ denotes time ordering. 
with $\mathbf{x}_{1}, \cdots, \mathbf{x}_{\ell} \in X$ and $0 \leq \beta_{1}, \cdots, \beta_{\ell} \leq \frac{1}{k T}$. To this end, choose a sufficiently fine partition, $0=\tau_{0}<\tau_{1}<\cdots<\tau_{p}=\frac{1}{k T}$ that contains $\beta_{1}, \cdots, \beta_{\ell}$. It follows from [BFKT2, Theorem 3.7] that the numerator of (I.7) is equal to

$$
\int \prod_{n=1}^{p}\left\{\left[\prod_{\mathbf{x} \in X} \frac{d \phi_{\tau_{n}}(\mathbf{x})^{*} \phi_{\tau_{n}}(\mathbf{x})}{2 \pi \imath} e^{-\phi_{\tau_{n}}(\mathbf{x})^{*} \phi_{\tau_{n}}(\mathbf{x})}\right] I_{\tau_{n}-\tau_{n-1}}\left(\phi_{\tau_{n-1}}^{*}, \phi_{\tau_{n}}\right)\right\} \prod_{j=1}^{\ell} \phi_{\beta_{j}}\left(\mathbf{x}_{j}\right)^{(*)}
$$

with $\phi_{0}=\phi_{\frac{1}{k T}}$.

The functions $I_{n}\left(\varepsilon ; \alpha^{*}, \beta\right)$ can also be defined recursively by

$$
\begin{aligned}
& I_{1}\left(\varepsilon ; \alpha^{*}, \beta\right)=\int d \mu_{\mathrm{R}(\varepsilon)}\left(\phi^{*}, \phi\right) \zeta_{\varepsilon}\left(\alpha^{*}, \phi\right) e^{\left\langle\alpha^{*}, j(\varepsilon) \phi\right\rangle+\left\langle\phi^{*}, j(\varepsilon) \beta\right\rangle} \\
& e^{-\varepsilon\left(\left\langle\alpha^{*} \phi, v \alpha^{*} \phi\right\rangle+\left\langle\phi^{*} \beta, v \phi^{*} \beta\right\rangle\right)} \zeta_{\varepsilon}\left(\phi^{*}, \beta\right)
\end{aligned}
$$

and

$$
I_{n+1}\left(\varepsilon ; \alpha^{*}, \beta\right)=\int d \mu_{\mathrm{R}(\varepsilon)}\left(\phi^{*}, \phi\right) I_{n}\left(\varepsilon ; \alpha^{*}, \phi\right) I_{n}\left(\varepsilon ; \phi^{*}, \beta\right)
$$

This recursive definition is called decimation, because we successively integrate out every second field. The description of and estimates on $I_{n}$ will be obtained inductively.

The integrals (I.9) and (I.10) are oscillatory. Their dominant contributions are extracted by stationary phase. In [BFKT5], we describe the construction and estimates that one obtains if, in (I.9) and (I.10), one always ignores contributions far away from the critical point of the ("free part") of the exponent. We call this the "stationary phase approximation". To be somewhat more precise, fix a suitable (see [BFKT5, Hypothesis I.1]) non negative decreasing function $r(t)$, and assume that one keeps in (I.9) and (I.10) only the integral over fields that are within distance $\mathrm{r}\left(2^{n} \varepsilon\right)$ from the critical point of the exponent, evaluated at $v \equiv 0$. Then the dominant contribution to $I_{n}\left(\varepsilon ; \alpha^{*}, \beta\right)$ is

$$
I_{n}^{(\mathrm{SP})}\left(\varepsilon ; \alpha^{*}, \beta\right)=\mathcal{Z}_{2^{n} \varepsilon}(\varepsilon)^{|X|} e^{\left\langle\alpha^{*}, j\left(2^{n} \varepsilon\right) \beta\right\rangle+V_{2^{n}}\left(\varepsilon ; \alpha^{*}, \beta\right)+E_{2^{n} \varepsilon}\left(\varepsilon ; \alpha^{*}, \beta\right)}
$$

where, for every $\delta$ that is an integer multiple of $\varepsilon$,

$$
V_{\delta}\left(\varepsilon ; \alpha^{*}, \beta\right)=-\varepsilon \sum_{\tau \in \varepsilon \mathbb{Z} \cap[0, \delta)}\left\langle\left[j(\tau) \alpha^{*}\right][j(\delta-\tau-\varepsilon) \beta], v\left[j(\tau) \alpha^{*}\right][j(\delta-\tau-\varepsilon) \beta]\right\rangle
$$

and the functions $E_{\delta}$ are recursively defined by

$$
\begin{aligned}
E_{\varepsilon}\left(\varepsilon ; \alpha^{*}, \beta\right) & =0 \\
E_{2 \delta}\left(\varepsilon ; \alpha^{*}, \beta\right) & =E_{\delta}\left(\varepsilon ; \alpha^{*}, j(\delta) \beta\right)+E_{\delta}\left(\varepsilon ; j(\delta) \alpha^{*}, \beta\right)+\log \frac{\int d \mu_{r(\delta)}\left(z^{*}, z\right) e^{\partial \mathcal{A}_{\delta}\left(\varepsilon ; \alpha^{*}, \beta ; z^{*}, z\right)}}{\int d \mu_{r(\delta)}\left(z^{*}, z\right)}
\end{aligned}
$$


with

$$
\begin{aligned}
\partial \mathcal{A}_{\delta}\left(\varepsilon ; \alpha^{*}, \beta ; z_{*}, z\right)= & {\left[V_{\delta}\left(\varepsilon ; \alpha^{*}, j(\delta) \beta+z\right)-V_{\delta}\left(\varepsilon ; \alpha^{*}, j(\delta) \beta\right)\right] } \\
& +\left[V_{\delta}\left(\varepsilon ; j(\delta) \alpha^{*}+z_{*}, \beta\right)-V_{\delta}\left(\varepsilon ; j(\delta) \alpha^{*}, \beta\right)\right] \\
& +\left[E_{\delta}\left(\varepsilon ; \alpha^{*}, j(\delta) \beta+z\right)-E_{\delta}\left(\varepsilon ; \alpha^{*}, j(\delta) \beta\right)\right] \\
& +\left[E_{\delta}\left(\varepsilon ; j(\delta) \alpha^{*}+z_{*}, \beta\right)-E_{\delta}\left(\varepsilon ; j(\delta) \alpha^{*}, \beta\right)\right]
\end{aligned}
$$

The normalization constant $\mathcal{Z}_{\delta}(\varepsilon)$, which is extremely close to one, is chosen so that $E_{\delta}(\varepsilon ; 0,0)=0$. See (I.7), (I.8) and (I.9) in [BFKT5]. The motivation for this recursion relation comes from a stationary phase construction and is given below and also in the section $\S$ II of [BFKT5]. Theorem I.4 of [BFKT5] shows that, under suitable assumptions on the function $\mathrm{r}(t)$ and the number $\theta$, the logarithm in (I.11) always exists as an analytic function of the fields, and that one can get a good estimate on

$$
E_{\theta}\left(\alpha^{*}, \beta\right)=\lim _{m \rightarrow \infty} E_{\theta}\left(2^{-m} \theta ; \alpha^{*}, \beta\right)
$$

To motivate the recursive definition (I.11) of $E_{\delta}\left(\varepsilon ; \alpha^{*}, \beta\right)$ we replace $I_{n}$ by

$$
I_{n}^{(\mathrm{SP})}\left(\varepsilon ; \alpha^{*}, \beta\right)=\mathcal{Z}_{\varepsilon_{n}}(\varepsilon)^{|X|} e^{\left\langle\alpha^{*}, j\left(\varepsilon_{n}\right) \beta\right\rangle+V_{\varepsilon_{n}}\left(\varepsilon ; \alpha^{*}, \beta\right)+E_{\varepsilon_{n}}\left(\varepsilon ; \alpha^{*}, \beta\right)}
$$

in the recursion relation (I.10). Here, $\varepsilon_{n}=2^{n} \varepsilon$. The resulting integral

$$
\begin{aligned}
& \int d \mu_{\mathrm{R}(\varepsilon)}\left(\phi^{*}, \phi\right) I_{n}^{(\mathrm{SP})}\left(\varepsilon ; \alpha^{*}, \phi\right) I_{n}^{(\mathrm{SP})}\left(\varepsilon ; \phi^{*}, \beta\right) \\
& =\mathcal{Z}_{\varepsilon_{n}}(\varepsilon)^{2|X|} \int d \mu_{\mathrm{R}(\varepsilon)}\left(\phi^{*}, \phi\right) e^{\left\langle\alpha^{*}, j\left(\varepsilon_{n}\right) \phi\right\rangle+\left\langle\phi^{*}, j\left(\varepsilon_{n}\right) \beta\right\rangle} e^{V_{\varepsilon_{n}}\left(\varepsilon ; \alpha^{*}, \phi\right)+V_{\varepsilon_{n}}\left(\varepsilon ; \phi^{*}, \beta\right)} \\
& \quad=\mathcal{Z}_{\varepsilon_{n}}(\varepsilon)^{2|X|} \int\left[\prod_{\mathbf{x} \in X} \frac{d \phi^{*}(\mathbf{x}) d \phi(\mathbf{x})}{2 \pi \imath} \chi(|\phi(\mathbf{x})|<\mathrm{R}(\varepsilon))\right] e^{\mathcal{A}\left(\alpha^{*}, \beta ; \phi^{*}, \phi^{*}, \phi\right)}
\end{aligned}
$$

with

$$
\begin{aligned}
\mathcal{A}\left(\alpha^{*}, \beta ; \phi_{*}, \phi\right)= & -\left\langle\phi_{*}, \phi\right\rangle+\left\langle\alpha^{*}, j\left(\varepsilon_{n}\right) \phi\right\rangle+\left\langle\phi_{*}, j\left(\varepsilon_{n}\right) \beta\right\rangle \\
& +V_{\varepsilon_{n}}\left(\varepsilon ; \alpha^{*}, \phi\right)+V_{\varepsilon_{n}}\left(\varepsilon ; \phi_{*}, \beta\right)+E_{\varepsilon_{n}}\left(\varepsilon ; \alpha^{*}, \phi\right)+E_{\varepsilon_{n}}\left(\varepsilon ; \phi_{*}, \beta\right)
\end{aligned}
$$

Here we have written $\mathcal{A}$ as a function of four independent complex fields $\alpha^{*}, \beta, \phi_{*}$ and $\phi$. The activity in (I.12) is obtained by evaluating $\mathcal{A}\left(\alpha^{*}, \beta ; \phi_{*}, \phi\right)$ with $\phi_{*}=\phi^{*}$, the complex conjugate of $\phi$. The reason for introducing independent complex fields $\phi_{*}$ and $\phi$ lies in the fact that the critical point (with respect to the variables $\phi_{*}, \phi$ ) of the quadratic part

$$
-\left\langle\phi_{*}, \phi\right\rangle+\left\langle j\left(\varepsilon_{n}\right) \alpha^{*}, \phi\right\rangle+\left\langle\phi_{*}, j\left(\varepsilon_{n}\right) \beta\right\rangle=-\left\langle\phi_{*}-j\left(\varepsilon_{n}\right) \alpha^{*}, \phi-j\left(\varepsilon_{n}\right) \beta\right\rangle+\left\langle\alpha^{*}, j\left(\varepsilon_{n+1}\right) \beta\right\rangle
$$


of $\mathcal{A}$ is "not real". Precisely, the critical point is

$$
\phi_{*}^{\text {crit }}=j\left(\varepsilon_{n}\right) \alpha^{*} \quad \phi^{\text {crit }}=j\left(\varepsilon_{n}\right) \beta
$$

and in general $\left(\phi_{*}^{\text {crit }}\right)^{*} \neq \phi^{\text {crit }}$.

It is reasonable to expect that the dominant contribution to the integral in (I.12) comes from the fields $\phi(\mathbf{x})$ in a neighbourhood of the critical point. We now sketch, approximately, the strategy that we use to verify that this is indeed the case. We decompose, for each $\mathbf{x} \in X$, the domain of integration $\{|\phi(\mathbf{x})|<\mathrm{R}(\varepsilon)\}$ into the "small field region", where $\phi^{*}(\mathbf{x})$ is close to $\phi_{*}^{\text {crit }}(\mathbf{x})$ and $\phi(\mathbf{x})$ is close to $\phi^{\text {crit }}(\mathbf{x})$, and the "large field region" where this is not the case. Precisely, write

$$
\chi(|\phi(\mathbf{x})|<\mathrm{R}(\varepsilon))=\chi_{\mathbf{x}, \text { small }}\left(\phi(\mathbf{x}), \phi^{*}(\mathbf{x})\right)+\chi_{\mathbf{x}, \text { large }}(\phi(\mathbf{x}))
$$

where

$$
\chi_{\mathbf{x}, \text { small }}\left(\phi, \phi_{*}\right)=\left\{\begin{array}{cc}
1 & \text { if } \phi_{*}=\phi^{*},|\phi|<\mathrm{R}(\varepsilon) \\
\quad \text { and }\left|\phi_{*}-\phi_{*}^{\text {crit }}(\mathbf{x})\right|<\mathrm{r}\left(\varepsilon_{n}\right),\left|\phi-\phi^{\text {crit }}(\mathbf{x})\right|<\mathrm{r}\left(\varepsilon_{n}\right) \\
0 \quad \text { otherwise }
\end{array}\right.
$$

and

$$
\chi_{\mathbf{x}, \operatorname{large}}(\phi)=\chi(|\phi|<\mathrm{R}(\varepsilon))\left(1-\chi_{\mathbf{x}, \text { small }}\left(\phi, \phi^{*}\right)\right)
$$

We multiply out the products of sums of characteristic functions and get that (I.12) is equal to

$$
\begin{aligned}
\sum_{\Lambda \subset X} \mathcal{Z}_{\varepsilon_{n}}(\varepsilon)^{2|X|}\left[\prod_{\mathbf{x} \in \Lambda} \int \frac{d \phi_{*}(\mathbf{x}) \wedge d \phi(\mathbf{x})}{2 \pi \imath} \chi_{\mathbf{x}, \text { small }}\left(\phi(\mathbf{x}), \phi_{*}(\mathbf{x})\right)\right] \\
\left.\qquad \prod_{\mathbf{x} \in X \backslash \Lambda} \int \frac{d \phi^{*}(\mathbf{x}) \wedge d \phi(\mathbf{x})}{2 \pi \imath} \chi_{\mathbf{x}, \operatorname{large}}(\phi(\mathbf{x}))\right]\left.e^{\mathcal{A}\left(\alpha^{*}, \beta ; \phi_{*}, \phi\right)}\right|_{\substack{\phi_{*}(\mathbf{x})=\phi^{*}(\mathbf{x}) \\
\text { for } \mathbf{x} \in X \backslash \Lambda}}
\end{aligned}
$$

Select a term of (I.14), that is, a subset $\Lambda$ of $X$. For points $\mathbf{x} \in \Lambda$, we introduce the "fluctuation variables" $z_{*}(\mathbf{x}), z(\mathbf{x})$ by the change of variables

$$
\phi_{*}(\mathbf{x})=\phi_{*}^{\mathrm{crit}}(\mathbf{x})+z_{*}(\mathbf{x}), \quad \phi(\mathbf{x})=\phi^{\mathrm{crit}}(\mathbf{x})+z(\mathbf{x})
$$

For points outside $\Lambda$, we do not perform any change of variables. So the fluctuation fields $z_{*}, z$ are supported on $\Lambda$, and the change of variables is

$$
\begin{gathered}
\phi_{*}=\Lambda \phi_{*}^{\mathrm{crit}}+z_{*}+\Lambda^{c} \phi_{*}=\Lambda j\left(\varepsilon_{n}\right) \alpha^{*}+z_{*}+\Lambda^{c} \phi_{*} \\
\phi=\Lambda \phi^{\mathrm{crit}}+z+\Lambda^{c} \phi=\Lambda j\left(\varepsilon_{n}\right) \beta+z+\Lambda^{c} \phi
\end{gathered}
$$


Here, we also denote by $\Lambda$ the operator "multiplication by the characteristic function of the set $\Lambda "$. Under this change of variables the domain of integration

$$
\left\{\left(\phi(\mathbf{x}), \phi_{*}(\mathbf{x})\right) \mid \chi_{\mathbf{x}, \operatorname{small}}\left(\phi(\mathbf{x}), \phi_{*}(\mathbf{x})\right)=1\right\}
$$

is transformed into

$$
\begin{array}{r}
D(\mathbf{x})=\left\{\left(z_{*}(\mathbf{x}), z(\mathbf{x})\right)\left|\left(\phi_{*}^{\text {crit }}(\mathbf{x})+z_{*}(\mathbf{x})\right)^{*}=\phi^{\text {crit }}(\mathbf{x})+z(\mathbf{x}), \quad\right| \phi^{\text {crit }}(\mathbf{x})+z(\mathbf{x}) \mid<\mathrm{R}(\varepsilon)\right. \\
\text { and } \left.\left|z_{*}(\mathbf{x})\right| \leq \mathrm{r}\left(\varepsilon_{n}\right),|z(\mathbf{x})| \leq \mathrm{r}\left(\varepsilon_{n}\right)\right\}
\end{array}
$$

Observe that for $\left(z_{*}(\mathbf{x}), z(\mathbf{x})\right) \in D(\mathbf{x})$, in general $z_{*}(\mathbf{x}) \neq z^{*}(\mathbf{x})$.

The quadratic part of the effective action

$$
\mathcal{A}\left(\alpha^{*}, \beta ; \Lambda \phi_{*}^{\text {crit }}+z_{*}+\Lambda^{c} \phi_{*}, \Lambda \phi^{\text {crit }}+z+\Lambda^{c} \phi\right)
$$

in the new variables is

$$
\begin{aligned}
&-\left\langle\Lambda j\left(\varepsilon_{n}\right) \alpha^{*}+z_{*}+\Lambda^{c} \phi_{*}, \Lambda j\left(\varepsilon_{n}\right) \beta+z+\Lambda^{c} \phi\right\rangle \\
&+\left\langle\alpha^{*}, j\left(\varepsilon_{n}\right)\left(\Lambda j\left(\varepsilon_{n}\right) \beta+z+\Lambda^{c} \phi\right)\right\rangle+\left\langle j\left(\varepsilon_{n}\right)\left(\Lambda j\left(\varepsilon_{n}\right) \alpha^{*}+z_{*}+\Lambda^{c} \phi_{*}\right), \beta\right\rangle \\
&=-\left\langle z_{*}, z\right\rangle-\left\langle\Lambda^{c} \phi_{*}, \Lambda^{c} \phi\right\rangle+Q_{\Lambda}\left(\alpha^{*}, \beta ; \Lambda^{c} \phi_{*}, \Lambda^{c} \phi\right)
\end{aligned}
$$

where

$$
Q_{\Lambda}\left(\alpha^{*}, \beta ; \Lambda^{c} \phi_{*}, \Lambda^{c} \phi\right)=\left\langle\Lambda j\left(\varepsilon_{n}\right) \alpha^{*}, \Lambda j\left(\varepsilon_{n}\right) \beta\right\rangle+\left\langle\alpha^{*}, j\left(\varepsilon_{n}\right) \Lambda^{c} \phi\right\rangle+\left\langle j\left(\varepsilon_{n}\right) \Lambda^{c} \phi, \beta\right\rangle
$$

Observe that the terms linear in $z_{*}, z$ cancelled, because we centered the change of variables at the critical point. Inserting this change of variables in (I.14), we see that (I.12) is equal to

$$
\begin{aligned}
& \mathcal{Z}_{\varepsilon_{n}}(\varepsilon)^{2|X|} \sum_{\Lambda \subset X}\left[\prod_{\mathbf{x} \in \Lambda} \int_{D(\mathbf{x})} \frac{d z_{*}(\mathbf{x}) \wedge d z(\mathbf{x})}{2 \pi \imath} e^{-z_{*}(\mathbf{x}) z(\mathbf{x})}\right] \\
& {\left[\prod_{\mathbf{x} \in \Lambda^{c}} \int \frac{d \phi^{*}(\mathbf{x}) d \phi(\mathbf{x})}{2 \pi \imath} e^{-\phi^{*}(\mathbf{x}) \phi(\mathbf{x})} \chi_{\mathbf{x}, \operatorname{large}}(\phi(\mathbf{x}))\right] e^{\tilde{\mathcal{A}}_{\Lambda}\left(\alpha^{*}, \beta ; \phi, z_{*}, z\right)} }
\end{aligned}
$$

where

$$
\begin{aligned}
\tilde{\mathcal{A}}_{\Lambda}\left(\alpha^{*}, \beta ; \phi, z_{*}, z\right)= & Q_{\Lambda}\left(\alpha^{*}, \beta ; \Lambda^{c} \phi^{*}, \Lambda^{c} \phi\right) \\
& +V_{\varepsilon_{n}}\left(\varepsilon ; \alpha^{*}, \Lambda \phi^{\text {crit }}+z+\Lambda^{c} \phi\right)+V_{\varepsilon_{n}}\left(\varepsilon ; \Lambda \phi_{*}^{\text {crit }}+z_{*}+\Lambda^{c} \phi^{*}, \beta\right) \\
& +E_{\varepsilon_{n}}\left(\varepsilon ; \alpha^{*}, \Lambda \phi^{\text {crit }}+z+\Lambda^{c} \phi\right)+E_{\varepsilon_{n}}\left(\varepsilon ; \Lambda \phi_{*}^{\text {crit }}+z_{*}+\Lambda^{c} \phi^{*}, \beta\right)
\end{aligned}
$$


If we apply Stokes' Theorem (Lemma A.1 of [BFKT5]) with $X$ replaced by $\Lambda, r=\mathrm{r}\left(\varepsilon_{n}\right)$, $\sigma=\sigma_{*}=0$ and $\rho=\left(\phi_{*}^{\text {crit }}\right)^{*}-\phi^{\text {crit }}$ to (I.18) we see that (I.12) is equal to

$$
\begin{aligned}
\mathcal{Z}_{\varepsilon_{n}}(\varepsilon)^{2|X|} \sum_{\Omega \subset \Lambda \subset X} & {\left[\prod_{\mathbf{x} \in \Omega} \int_{|z(\mathbf{x})| \leq \mathrm{r}\left(\varepsilon_{n}\right)} \frac{d z^{*}(\mathbf{x}) d z(\mathbf{x})}{2 \pi \imath} e^{-|z(\mathbf{x})|^{2}}\right]\left[\prod_{\mathbf{x} \in \Lambda \backslash \Omega} \int_{C(\mathbf{x})} \frac{d z_{*}(\mathbf{x}) \wedge d z(\mathbf{x})}{2 \pi \imath} e^{-z_{*}(\mathbf{x}) z(\mathbf{x})}\right] } \\
& {\left.\left[\prod_{\mathbf{x} \in \Lambda^{c}} \int \frac{d \phi^{*}(\mathbf{x}) d \phi(\mathbf{x})}{2 \pi \imath} e^{-|\phi(\mathbf{x})|^{2}} \chi_{\mathbf{x}, \operatorname{large}}(\phi(\mathbf{x}))\right] e^{\tilde{\mathcal{A}}_{\Lambda}\left(\alpha^{*}, \beta ; \phi, z_{*}, z\right)}\right|_{\substack{z_{*}(\mathbf{x})=z^{*}(\mathbf{x}) \\
\text { for } \mathbf{x} \in \Omega}}(\mathrm{I}, 20) }
\end{aligned}
$$

where, for each $\mathbf{x} \in X, C(\mathbf{x})$ is a two real dimensional submanifold of $\mathbb{C}^{2}$ whose boundary is the union of "circles" $\partial D(\mathbf{x})$ and $\left\{\left(z_{*}(\mathbf{x}), z(\mathbf{x})\right) \in \mathbb{C}^{2}\left|z_{*}^{*}(\mathbf{x})=z(\mathbf{x}),\right| z(\mathbf{x}) \mid=\mathrm{r}\left(\varepsilon_{n}\right)\right\}$. For points $\mathbf{x} \in \Omega$, the domain of integration has been moved "back to the reals".

Whenever $\chi_{\mathbf{x} \text {, large }}(\phi(\mathbf{x}))=1$ or $\left(z_{*}(\mathbf{x}), z(\mathbf{x})\right) \in C(\mathbf{x})$ for some $\mathbf{x} \in X$, then $\tilde{\mathcal{A}}_{\Lambda}\left(\alpha^{*}, \beta ; \phi, z_{*}, z\right)$ or $-z_{*}(\mathbf{x}) z(\mathbf{x})$ has extremely large negative real part and the contribution to the integral is very small. (See the discussion following Theorem III.35.) For this reason we kept only the term of (I.20) with $\Omega=\Lambda=X$ for the "stationary phase approximation" in [BFKT5]. In this case, $Q_{X}\left(\alpha^{*}, \beta ; \Lambda^{c} \phi^{*}, \Lambda^{c} \phi\right)=\left\langle\alpha^{*}, j\left(\varepsilon_{n+1}\right) \beta\right\rangle$. Thus, the stationary phase approximation to

$$
\int d \mu_{\mathrm{R}(\varepsilon)}\left(\phi^{*}, \phi\right) I_{n}^{(\mathrm{SP})}\left(\varepsilon ; \alpha^{*}, \phi\right) I_{n}^{(\mathrm{SP})}\left(\varepsilon ; \phi^{*}, \beta\right)
$$

is

$$
\begin{array}{r}
\mathcal{Z}_{\varepsilon_{n}}(\varepsilon)^{2|X|} e^{\left\langle\alpha^{*}, j\left(\varepsilon_{n+1}\right) \beta\right\rangle}\left[\prod_{\mathbf{x} \in X} \int_{|z(\mathbf{x})|<\mathrm{r}\left(\varepsilon_{n}\right)} \frac{d z^{*}(\mathbf{x}) \wedge d z(\mathbf{x})}{2 \pi \imath} e^{-|z(\mathbf{x})|^{2}}\right] e^{\tilde{\mathcal{A}}\left(\alpha^{*}, \beta ; z^{*}, z\right)} \\
=\mathcal{Z}_{n}^{2|X|} e^{\left\langle\alpha^{*}, j\left(\varepsilon_{n+1}\right) \beta\right\rangle} \int d \mu_{r\left(\varepsilon_{n}\right)}\left(z^{*}, z\right) e^{\tilde{\mathcal{A}}_{X}\left(\alpha^{*}, \beta ; z^{*}, z\right)}
\end{array}
$$

where

$$
\begin{aligned}
\tilde{\mathcal{A}}_{X}\left(\alpha^{*}, \beta ; z_{*}, z\right)= & V_{\varepsilon_{n}}\left(\varepsilon ; \alpha^{*}, \phi^{\text {crit }}+z\right)+V_{\varepsilon_{n}}\left(\varepsilon ; \phi_{*}^{\text {crit }}+z_{*}, \beta\right) \\
& +E_{\varepsilon_{n}}\left(\varepsilon ; \alpha^{*}, \phi^{\text {crit }}+z\right)+E_{\varepsilon_{n}}\left(\varepsilon ; \phi_{*}^{\text {crit }}+z_{*}, \beta\right)
\end{aligned}
$$

By construction

$$
\begin{aligned}
V_{\varepsilon_{n}}\left(\varepsilon ; \alpha^{*}, \phi^{\text {crit }}\right)+V_{\varepsilon_{n}}\left(\varepsilon ; \phi_{*}^{\text {crit }}, \beta\right) & =V_{\varepsilon_{n}}\left(\varepsilon ; \alpha^{*}, j\left(\varepsilon_{n}\right) \beta\right)+V_{\varepsilon_{n}}\left(\varepsilon ; j\left(\varepsilon_{n}\right) \alpha^{*}, \beta\right) \\
& =V_{\varepsilon_{n+1}}\left(\varepsilon ; \alpha^{*}, \beta\right)
\end{aligned}
$$

so that

$$
\begin{aligned}
V_{\varepsilon_{n}}\left(\varepsilon ; \alpha^{*}, \phi^{\text {crit }}+z\right)+ & V_{\varepsilon_{n}}\left(\varepsilon ; \phi_{*}^{\text {crit }}+z^{*}, \beta\right) \\
= & V_{\varepsilon_{n+1}}\left(\varepsilon ; \alpha^{*}, \beta\right)+\left[V_{\varepsilon_{n}}\left(\varepsilon ; \alpha^{*}, j\left(\varepsilon_{n}\right) \beta+z\right)-V_{\varepsilon_{n}}\left(\varepsilon ; \alpha^{*}, j\left(\varepsilon_{n}\right) \beta\right)\right] \\
& +\left[V_{\varepsilon_{n}}\left(\varepsilon ; j\left(\varepsilon_{n}\right) \alpha^{*}+z^{*}, \beta\right)-V_{\varepsilon_{n}}\left(\varepsilon ; j\left(\varepsilon_{n}\right) \alpha^{*}, \beta\right)\right]
\end{aligned}
$$


Consequently, the stationary phase approximation to

$$
\int d \mu_{\mathrm{R}(\varepsilon)}\left(\phi^{*}, \phi\right) I_{n}^{(\mathrm{SP})}\left(\varepsilon ; \alpha^{*}, \phi\right) I_{n}^{(\mathrm{SP})}\left(\varepsilon ; \phi^{*}, \beta\right)
$$

can also be written as

$$
\begin{aligned}
& \mathcal{Z}_{\varepsilon_{n}}(\varepsilon)^{2|X|} e^{\left\langle\alpha^{*}, j\left(\varepsilon_{n+1}\right) \beta\right\rangle+V_{\varepsilon_{n+1}}\left(\varepsilon ; \alpha^{*}, \beta\right)} \\
& e^{E_{\varepsilon_{n}}\left(\varepsilon ; \alpha^{*}, j\left(\varepsilon_{n}\right) \beta\right)+E_{\varepsilon_{n}}\left(\varepsilon ; j\left(\varepsilon_{n}\right) \alpha^{*}, \beta\right)} \int d \mu_{r\left(\varepsilon_{n}\right)}\left(z^{*}, z\right) e^{\partial \mathcal{A}_{\varepsilon_{n}}\left(\varepsilon ; \alpha^{*}, \beta ; z^{*}, z\right)}
\end{aligned}
$$

This is compatible with (I.11) if we take

$$
\mathcal{Z}_{\varepsilon_{n+1}}(\varepsilon)=\mathcal{Z}_{\varepsilon_{n}}(\varepsilon)^{2} \int_{|z|<\mathrm{r}\left(\varepsilon_{n}\right)} \frac{d z^{*} \wedge d z}{2 \pi i} e^{-|z|^{2}}
$$

It turns out that, for each fixed $\Omega \subset X$, the sum over all sets $\Lambda$ with $\Omega \subset \Lambda \subset X$ in (I.20) can be written in the form

$$
\mathcal{Z}_{\varepsilon_{n+1}}(\varepsilon)^{|X|} e^{\left\langle\alpha^{*}, j\left(\varepsilon_{n+1}\right) \beta\right\rangle_{\Omega}} e^{V_{\Omega ; \varepsilon_{n+1}}\left(\varepsilon ; \alpha^{*}, \beta\right)+E_{\Omega ; \varepsilon_{n+1}}\left(\varepsilon ; \alpha^{*}, \beta\right)} \varphi_{\Omega ; \varepsilon_{n+1}}\left(\varepsilon ; \alpha^{*}, \beta\right)
$$

where $V_{\Omega ; \varepsilon_{n+1}}$ and $E_{\Omega ; \varepsilon_{n+1}}$ depend only on $\alpha^{*}(\mathbf{x})$ and $\beta(\mathbf{x})$ with $\mathbf{x} \in \Omega$, and are given by the same formulae as $V_{\varepsilon_{n+1}}$ and $E_{\varepsilon_{n+1}}$ but with the total space $X$ replaced by $\Omega$ everywhere, and where $\varphi_{\Omega ; \varepsilon_{n+1}}$ is a very small function that encapsulates the sum over $\Lambda$ and various integral operators.

The sets $\Lambda$, resp. $\Omega$, introduced above are called "small field sets" of the first, resp. second, kind. The discussion of the previous paragraph shows that (I.20) is equal to

$$
\int d \mu_{\mathrm{R}(\varepsilon)}\left(\phi^{*}, \phi\right) I_{n}^{(\mathrm{SP})}\left(\varepsilon ; \alpha^{*}, \phi\right) I_{n}^{(\mathrm{SP})}\left(\varepsilon ; \phi^{*}, \beta\right)
$$

and it indicates that $I_{n+1}^{(\mathrm{SP})}\left(\varepsilon ; \alpha^{*}, \phi\right)$ is its dominant contribution. The next decimation step would be to consider

$$
\begin{aligned}
\int d \mu_{\mathrm{R}(\varepsilon)}\left(\phi^{*}, \phi\right)\left[\int d \mu_{\mathrm{R}(\varepsilon)}\left(\phi_{1}^{*}, \phi_{1}\right) I_{n}^{(\mathrm{SP})}\left(\varepsilon ; \alpha^{*}, \phi_{1}\right) I_{n}^{(\mathrm{SP})}\left(\varepsilon ; \phi_{1}^{*}, \phi\right)\right] \\
{\left[\int d \mu_{\mathrm{R}(\varepsilon)}\left(\phi_{1}^{*}, \phi_{2}\right) I_{n}^{(\mathrm{SP})}\left(\varepsilon ; \phi^{*}, \phi_{2}\right) I_{n}^{(\mathrm{SP})}\left(\varepsilon ; \phi_{2}^{*}, \beta\right)\right] }
\end{aligned}
$$

Inserting (I.20) and (I.22) for the two integrals in brackets gives a normalization constant times a sum over small field sets $\Omega_{1}$ and $\Omega_{2}$ of integrals

$$
\left.\int\left[\prod_{\mathbf{x} \in X} \frac{d \phi^{*}(\mathbf{x}) d \phi(\mathbf{x})}{2 \pi \imath} \chi(\mid \phi(\mathbf{x}))<\mathrm{R}(\varepsilon)\right)\right] e^{\mathcal{A}^{\prime}\left(\alpha^{*}, \beta ; \phi^{*}, \phi\right)} \varphi_{\Omega_{1} ; \varepsilon_{n+1}}\left(\varepsilon ; \alpha^{*}, \phi\right) \varphi_{\Omega_{2} ; \varepsilon_{n+1}}\left(\varepsilon ; \phi^{*}, \beta\right)
$$


with

$$
\begin{aligned}
\mathcal{A}^{\prime}\left(\alpha^{*}, \beta ; \phi_{*}, \phi\right)=-\left\langle\phi_{*}\right. & , \phi\rangle+\left\langle\alpha^{*}, j\left(\varepsilon_{n+1}\right) \phi\right\rangle_{\Omega_{1}}+\left\langle\phi_{*}, j\left(\varepsilon_{n+1}\right) \beta\right\rangle_{\Omega_{2}} \\
& +V_{\Omega_{1} ; \varepsilon_{n+1}}\left(\varepsilon ; \alpha^{*}, \phi\right)+V_{\Omega_{2} ; \varepsilon_{n+1}}\left(\varepsilon ; \phi_{*}, \beta\right) \\
& +E_{\Omega_{1} ; \varepsilon_{n+1}}\left(\varepsilon ; \alpha^{*}, \phi\right)+E_{\Omega_{2} ; \varepsilon_{n+1}}\left(\varepsilon ; \phi_{*}, \beta\right)
\end{aligned}
$$

This oscillatory integral is similar to (I.12). The small factors for points $\mathbf{x} \in\left(X \backslash \Omega_{1}\right) \cup$ $\left(X \backslash \Omega_{2}\right)$ mentioned above are so strong that we only have to perform a stationary phase argument for points inside $\Omega_{1} \cap \Omega_{2}$. That is, we would, as in (I.18), write (I.23) as

$$
\begin{aligned}
\sum_{\Lambda^{\prime} \subset \Omega_{1} \cap \Omega_{2}}\left[\prod_{\mathbf{x} \in \Lambda^{\prime}} \int_{D^{\prime}(\mathbf{x})} \frac{d z_{*}(\mathbf{x}) \wedge d z(\mathbf{x})}{2 \pi \imath} e^{-z_{*}(\mathbf{x}) z(\mathbf{x})}\right] \\
{\left[\prod_{\mathbf{x} \in X \backslash \Lambda^{\prime}} \int \frac{d \phi^{*}(\mathbf{x}) d \phi(\mathbf{x})}{2 \pi \imath} e^{-|\phi(\mathbf{x})|^{2}} \chi_{\mathbf{x}, \text { large }}^{\prime}(\phi(\mathbf{x}))\right] e^{\tilde{\mathcal{A}}^{\prime}\left(\alpha^{*}, \beta ; z_{*}, z\right)} f^{\prime}\left(\alpha^{*}, \beta ; z_{*}, z\right) }
\end{aligned}
$$

where $\left.D^{\prime} \mathbf{x}\right)$ and $\chi_{\mathbf{x} \text {, large }}^{\prime}$ are defined as were $D(\mathbf{x})$ and $\chi_{\mathbf{x}}$, large, but with $\varepsilon_{n}$ replaced by $\varepsilon_{n+1}$, and where $\mathcal{A}^{\prime}\left(\alpha^{*}, \beta ; \phi, z_{*}, z\right)$ and $f^{\prime}\left(\alpha^{*}, \beta ; \phi, z_{*}, z\right)$ are obtained using the change of variables around the critical point of the quadratic form.

The next step would be to again apply Stokes' Theorem for the variables $z_{*}(\mathbf{x}), z(\mathbf{x})$ with $\mathbf{x} \in \Lambda$. Here, a small technical difficulty arises. Namely, the factor $f^{\prime}\left(\alpha^{*}, \beta ; z_{*}, z\right)$ in the integrand need not be analytic in $z_{*}, z$ and the version of Stokes' Theorem presented in Appendix A of [BFKT5] cannot be applied directly. To circumvent this difficulty, we introduce a constant $\mathfrak{c}>0$ and define the cut-off propagator

$$
j_{\mathfrak{c}}(\tau)(\mathbf{x}, \mathbf{y})=j(\tau)(\mathbf{x}, \mathbf{y}) \cdot \begin{cases}1 & \text { if } d(\mathbf{x}, \mathbf{y}) \leq \mathfrak{c} \\ 0 & \text { if } d(\mathbf{x}, \mathbf{y})>\mathfrak{c}\end{cases}
$$

where $d(\mathbf{x}, \mathbf{y})$ is the distance on the torus $X$, replace $j(t)$ by $j_{\mathfrak{c}}(t)$ in the formulae above and control the error terms. We apply Stokes' theorem only for points $\mathbf{x} \in \Lambda^{\prime}$ that have distance at least $\mathfrak{c}$ from $\left(X \backslash \Omega_{1}\right) \cup\left(X \backslash \Omega_{2}\right)$. That is, we apply Stokes' theorem only in

$$
\Lambda=\left\{\mathbf{x} \in \Lambda^{\prime} \mid d(\mathbf{x}, \mathbf{y}) \geq \mathfrak{c} \text { for all } \mathbf{y} \in\left(X \backslash \Omega_{1}\right) \cup\left(X \backslash \Omega_{2}\right)\right\}
$$

With this construction, the analogue of $f^{\prime}\left(\alpha^{*}, \beta ; z_{*}, z\right)$ will not depend on the variables $z_{*}(\mathbf{x}), z(\mathbf{x})$ with $\mathbf{x} \in \Lambda$. The integrals for points in the "corridor"

$$
\left\{\mathbf{x} \in \Lambda^{\prime} \mid d(\mathbf{x}, \mathbf{y}) \leq \mathfrak{c} \text { for some } \mathbf{y} \in\left(X \backslash \Omega_{1}\right) \cup\left(X \backslash \Omega_{2}\right)\right\}
$$

can again be controlled by the small factors from the points $\mathbf{y} \in X \backslash \Lambda^{\prime}$. This modification leads to the somewhat more involved formulae described in $\S I I$ below.

Obviously we want to iterate the procedure described above, starting with $n=1$. In this way one creates a "hierarchy" of small field sets of the first and second kind. In 
Definition II.4, this is made more precise, and enriched with more sets that are used to describe the various "large field conditions".

The main results of this paper are estimates on all of the functions appearing in the functional integral representation of the partition function. For these estimates we use the norms developed in [BFKT3, BFKT4]. One of the simplest versions of such a norm is defined as follows. Let $\kappa, \mathrm{m}>0$. We define the norm of the power series

$$
f\left(\alpha^{*}, \beta\right)=\sum_{k, \ell \geq 0} \sum_{\substack{\mathbf{x}_{1}, \cdots, \mathbf{x}_{k} \in X \\ \mathbf{y}_{1}, \cdots, \mathbf{y}_{\ell} \in X}} a\left(\mathbf{x}_{1}, \cdots, \mathbf{x}_{k} ; \mathbf{y}_{1}, \cdots, \mathbf{y}_{\ell}\right) \alpha\left(\mathbf{x}_{1}\right)^{*} \cdots \alpha\left(\mathbf{x}_{k}\right)^{*} \beta\left(\mathbf{y}_{1}\right) \cdots \beta\left(\mathbf{y}_{\ell}\right)
$$

(with the coefficients $a\left(\mathbf{x}_{1}, \cdots, \mathbf{x}_{k} ; \mathbf{y}_{1}, \cdots, \mathbf{y}_{\ell}\right)$ invariant under permutations of $\mathbf{x}_{1}, \cdots, \mathbf{x}_{k}$ and of $\left.\mathbf{y}_{1}, \cdots, \mathbf{y}_{\ell}\right)$ to be

$$
\left.\left\|f\left(\alpha^{*}, \beta\right)\right\|_{\kappa, \mathrm{m}}=\sum_{k, \ell \geq 0} \max _{\mathbf{x} \in X} \max _{1 \leq i \leq k+\ell} \sum_{\substack{(\overrightarrow{\mathbf{x}}, \overrightarrow{\mathbf{y}}) \in X^{k} \times X^{\ell} \\(\overrightarrow{\mathbf{x}}, \overrightarrow{\mathbf{y}})_{i}=\mathbf{x}}} \kappa^{k+\ell} e^{\mathrm{m} \tau(\overrightarrow{\mathbf{x}}, \overrightarrow{\mathbf{y}})}\right)|a(\overrightarrow{\mathbf{x}} ; \overrightarrow{\mathbf{y}})|
$$

where $\tau(\overrightarrow{\mathbf{x}}, \overrightarrow{\mathbf{y}})$ is the minimal length of a tree which contains vertices at the points of the set $\left\{\mathbf{x}_{1}, \cdots, \mathbf{x}_{k}, \mathbf{y}_{1}, \cdots, \mathbf{y}_{\ell}\right\}$.

Our main results, the description of and bounds on $I_{\theta}$, are stated in Section II.6 (Theorems II.16 and II.18). The other sections of Chapter II introduce the notation used in these Theorems. Chapter III gives an outline of the proof and contains discussions which might illuminate the concepts introduced in Chapter II. The proof of Theorem II.16 is split over Chapters III-V. The proof of Theorem II.18 is split over Chapters III and VI. 


\section{Formulation of the Main Theorem}

Our main result is a representation of the "effective density" $I_{\theta}$ of (I.6) as a sum over subsets $\Omega$ of $X$. For each $\Omega \subset X$ the corresponding summand is the product of

- the exponential of a function that is analytic in the fields and that depends only on $\Omega$.

- A function that involves all possible "hierarchies" (collections of large and small field sets - see Definition II.4) that lead to $\Omega$, which is not necessarily analytic, but can be proven to be very small (unless $\Omega=X$ ). Indeed, if $\Omega^{c} \neq \emptyset$, this function is $O\left(\|v\| \|^{n}\right.$ ) (this norm will be defined in (II.5)) for all $n \in \mathbb{N}$ and also decreases exponentially quickly with $\left|\Omega^{c}\right|$.

The first factor will be called the "small field part". It is described in Section II.1 below.

\section{II.1 The Small Field Parts}

Let $\Omega$ be a subset of $X$. For any kernel $w(\mathbf{x}, \mathbf{y})$ on $X$ denote by

$$
w_{\Omega}(\mathbf{x}, \mathbf{y})= \begin{cases}w(\mathbf{x}, \mathbf{y}) & \text { if } \mathbf{x}, \mathbf{y} \in \Omega \\ 0 & \text { otherwise }\end{cases}
$$

its truncation to $\Omega$. For any $t>0$, set

$$
\begin{aligned}
j_{(\Omega)}(t) & =\mathbb{1}_{\Omega}+\sum_{\ell=1}^{\infty} \frac{1}{\ell !}\left(-t(\mathrm{~h}-\mu)_{\Omega}\right)^{\ell} \\
& =e^{-t(\mathrm{~h}-\mu)_{\Omega}}-\mathbb{1}_{X \backslash \Omega}
\end{aligned}
$$

and

$$
j_{(\Omega), \mathfrak{c}}(t)(\mathbf{x}, \mathbf{y})= \begin{cases}j_{(\Omega)}(t)(\mathbf{x}, \mathbf{y}) & \text { if } d(\mathbf{x}, \mathbf{y}) \leq \mathfrak{c} \\ 0 & \text { if } d(\mathbf{x}, \mathbf{y})>\mathfrak{c}\end{cases}
$$

For each $0<t \leq \frac{1}{2 k T}$ and each analytic function $V\left(\alpha_{*}, \beta\right)$, that depends only on the variables $\alpha_{*}(\mathbf{x}), \beta(\mathbf{x})$ with $\mathbf{x} \in \Omega$, consider the formal renormalization group operator $\mathfrak{R}_{\Omega ; t}(V ; \cdot, \cdot)$ at scale $t$ with "principal interaction $V$ " that is defined as the following generalization of (I.11). It associates to any two analytic functions ${ }^{(1)} f_{1}\left(\alpha_{*}, \beta\right), f_{2}\left(\alpha_{*}, \beta\right)$

(1) We introduce the complex field $\alpha_{*}$ in order to clarify the analyticity properties of the functions $f_{1}, f_{2}$. We shall usually evaluate $\alpha_{*}$ at $\alpha^{*}$. 
that depend only on the variables $\alpha_{*}(\mathbf{x}), \beta(\mathbf{x})$ with $\mathbf{x} \in \Omega$ the function

$$
\begin{aligned}
& \mathfrak{R}_{\Omega ; t}(V ;\left.f_{1}, f_{2}\right)\left(\alpha_{*}, \beta\right) \\
&=f_{1}\left(\alpha_{*}, j_{(\Omega), \mathfrak{c}}(t) \beta\right)+f_{2}\left(j_{(\Omega), \mathfrak{c}}(t) \alpha_{*}, \beta\right)+\log \frac{\int d \mu_{\Omega, \mathrm{r}(t)}\left(z^{*}, z\right) e^{\mathcal{A}\left(\alpha_{*}, \beta ; z^{*}, z\right)}}{\int d \mu_{\Omega, \mathrm{r}(t)}\left(z^{*}, z\right)} \\
& \quad-\left\langle\left[j_{(\Omega)}(t)-j_{(\Omega), \mathfrak{c}}(t)\right] \alpha_{*},\left[j_{(\Omega)}(t)-j_{(\Omega), \mathfrak{c}}(t)\right] \beta\right\rangle \\
& \quad+V\left(\alpha_{*}, j_{(\Omega), \mathfrak{c}}(t) \beta\right)-V\left(\alpha_{*}, j_{(\Omega)}(t) \beta\right)+V\left(j_{(\Omega), \mathfrak{c}}(t) \alpha_{*}, \beta\right)-V\left(j_{(\Omega)}(t) \alpha_{*}, \beta\right)
\end{aligned}
$$

where $\mathcal{A}\left(\alpha_{*}, \beta ; z_{*}, z\right)$ is

$$
\begin{aligned}
{\left[f _ { 1 } \left(\alpha_{*}, z\right.\right.} & \left.\left.+j_{(\Omega), \mathfrak{c}}(t) \beta\right)-f_{1}\left(\alpha_{*}, j_{(\Omega), \mathfrak{c}}(t) \beta\right)\right]+\left[f_{2}\left(z_{*}+j_{(\Omega), \mathfrak{c}}(t) \alpha_{*}, \beta\right)-f_{2}\left(j_{(\Omega), \mathfrak{c}}(t) \alpha_{*}, \beta\right)\right] \\
& +\left\langle\left[j_{(\Omega)}(t)-j_{(\Omega), \mathfrak{c}}(t)\right] \alpha_{*}, z\right\rangle+\left\langle z_{*},\left[j_{(\Omega)}(t)-j_{(\Omega), \mathfrak{c}}(t)\right] \beta\right\rangle \\
& +\left[V\left(\alpha_{*}, z+j_{(\Omega), \mathfrak{c}}(t) \beta\right)-V\left(\alpha_{*}, j_{(\Omega), \mathfrak{c}}(t) \beta\right)\right] \\
& +\left[V\left(z_{*}+j_{(\Omega), \mathfrak{c}}(t) \alpha_{*}, \beta\right)-V\left(j_{(\Omega), \mathfrak{c}}(t) \alpha_{*}, \beta\right)\right]
\end{aligned}
$$

and

$$
d \mu_{\Omega, \mathrm{r}}\left(z^{*}, z\right)=\prod_{\mathbf{x} \in \Omega} \frac{d z(\mathbf{x})^{*} \wedge d z(\mathbf{x})}{2 \pi \imath} e^{-z(\mathbf{x})^{*} z(\mathbf{x})} \chi(|z(\mathbf{x})|<r)
$$

As "principal interaction" $V$ in the renormalization group map, we use the dominant part of the interaction at the corresponding scale. Precisely, for every $\delta$ that is an integer multiple of $\varepsilon$, define

$$
V_{\Omega, \delta}\left(\varepsilon ; \alpha_{*}, \beta\right)=-\varepsilon \sum_{\tau \in \varepsilon \mathbb{Z} \cap[0, \delta)}\left\langle\left[j_{(\Omega)}(\tau) \alpha_{*}\right]\left[j_{(\Omega)}(\delta-\tau-\varepsilon) \beta\right], v_{\Omega}\left[j_{(\Omega)}(\tau) \alpha_{*}\right]\left[j_{(\Omega)}(\delta-\tau-\varepsilon) \beta\right]\right\rangle
$$

Define recursively

$$
\begin{aligned}
\mathcal{D}_{\Omega ; 0}\left(\varepsilon ; \alpha_{*}, \beta\right) & =0 \\
\mathcal{D}_{\Omega ; n+1}\left(\varepsilon ; \alpha_{*}, \beta\right) & =\Re_{\Omega ; 2^{n} \varepsilon}\left(V_{\Omega ; 2^{n} \varepsilon}(\varepsilon ; \cdot, \cdot) ; \mathcal{D}_{\Omega ; n}(\varepsilon ; \cdot, \cdot), \mathcal{D}_{\Omega ; n}(\varepsilon ; \cdot, \cdot)\right)
\end{aligned}
$$

If $\theta$ is small enough, this recursion defines analytic functions $\mathcal{D}_{\Omega ; n}\left(\varepsilon ; \alpha_{*}, \beta\right)$, when $2^{n} \varepsilon \leq \theta$, and furthermore

$$
\mathcal{D}_{\Omega ; \theta}\left(\alpha_{*}, \beta\right)=\lim _{m \rightarrow \infty} \mathcal{D}_{\Omega ; m}\left(2^{-m} \theta ; \alpha_{*}, \beta\right)
$$

exists and fulfills the estimates of Proposition II.1 and Theorem II.16 below. Our representation of the effective density will be of the form

$$
I_{\theta}\left(\alpha^{*}, \beta\right)=\sum_{\Omega \subset X} \mathcal{Z}_{\theta}^{|\Omega|} e^{\left\langle\alpha^{*}, j_{(\Omega)}(\theta) \beta\right\rangle+V_{\Omega ; \theta}\left(\alpha^{*}, \beta\right)+\mathcal{D}_{\Omega ; \theta}\left(\alpha^{*}, \beta\right)} \chi_{\theta}(\Omega ; \alpha, \beta) \varphi_{\Omega ; \theta}\left(\alpha^{*}, \beta\right)
$$


where the normalization constant $\mathcal{Z}_{\theta}$, which will be defined in Lemma II.7, is very close to one,

$$
\begin{aligned}
V_{\Omega ; \theta}\left(\alpha_{*}, \beta\right) & =\lim _{m \rightarrow \infty} V_{\Omega ; \theta}\left(2^{-m} \theta ; \alpha_{*}, \beta\right) \\
& =-\int_{0}^{\theta} d t\left\langle\left[j_{(\Omega)}(t) \alpha_{*}\right]\left[j_{(\Omega)}(\theta-t) \beta\right], v_{\Omega}\left[j_{(\Omega)}(t) \alpha_{*}\right]\left[j_{(\Omega)}(\theta-t) \beta\right]\right\rangle
\end{aligned}
$$

and $\chi_{\theta}(\Omega ; \alpha, \beta)$ implements the small field conditions for the set $\Omega$. (See Theorem II.16.)

For our construction to work, we need exponential decay of the interaction $v$. Precisely, we assume that there is a "mass" $\mathrm{m}>0$ such that

$$
\|v\| \|=\sup _{\mathbf{x} \in X} \sum_{\mathbf{y} \in X} e^{5 \mathrm{~m} d(\mathbf{x}, \mathbf{y})}|v(\mathbf{x}, \mathbf{y})|
$$

is finite.

Proposition II.1 There are constants const, $\kappa>0$ such that for all sufficiently small $\theta$ and interactions $v$, and all $\Omega \subset X$, the function $\mathcal{D}_{\Omega ; \theta}\left(\alpha_{*}, \beta\right)$ of (II.2) is well defined ${ }^{(2)}$, and obeys

$$
\left\|\mathcal{D}_{\Omega ; \theta}\right\|_{\kappa, \mathrm{m}} \leq \mathrm{const}\|v\| \|
$$

Here, we use the norm (I.25).

The proof of this Proposition is much the same as the proof of [BFKT5, Theorem I.4]. It is also a consequence of our complete analysis of $I_{\theta}$ in Theorem II.16. There, we shall also state more properties of $\mathcal{D}_{\Omega ; \theta}$.

\section{II.2 Decomposition of Space into Large and Small Field Subsets}

As indicated in the introduction, the functions $\varphi_{\Omega}$ of (II.3) are expressed in terms of "hierarchies" of large and small field subsets of $X$. Recall that we have chosen a function $r(t)$ that measures the size of the neighbourhood of a critical point at scale $t$ in which Stokes' argument is applied to move the domain of integration "back to the reals".

We fix, in addition to the functions $\mathrm{R}(t)$ and $\mathrm{r}(t)$ of Chapter I, another decreasing positive function $\mathrm{R}^{\prime}(t)$. If we are at scale $t$ and, for some point $\mathbf{x}$, one of the fields $\alpha^{*}(\mathbf{x})$ or $\beta(\mathbf{x})$ is larger than $\mathrm{R}(t)$ we shall get a controllable small factor. This leads us to introduce a further decomposition of $X$ where the corresponding large field sets will be denoted by $P_{\alpha}$ and $P_{\beta}$, respectively. Also large values of the spatial gradients of the

(2) That is, it is possible to take the logarithms involved and the limit. 
fields give rise to small factors. Spatial gradients are controlled in terms of the function $\mathrm{R}^{\prime}(t)$. The corresponding large field sets will be sets $P_{\alpha}^{\prime}$ and $P_{\beta}^{\prime}$ of bonds in the lattice $X$. Similarly, large time derivatives cause small factors. The corresponding large field sets will be denoted by the letter $Q$. The sets where, in the application of Stokes' theorem, the "side" $C(\mathbf{x})$ of the cylinder is chosen as the domain of integration, will be labelled by $R$.

All the sets in our construction should be separated by corridors. The width of these corridors is scale dependent and will be measured by a function $\mathfrak{c}(t)$. Later, in (II.18), we will make specific choices of all these functions. Their properties will be proven in Appendix F. Here, we concentrate on the purely set theoretic picture.

As indicated above, we will have to deal with bonds of the lattice $X$. We denote by $X^{*}$ the set of all bonds (i.e. pairs of neighbouring points). Furthermore, for a subset $Y$ of $X$, we set

$$
\begin{aligned}
& Y^{\star}=\{\mathrm{x} \in X \mid \mathbf{x} \text { is connected by a bond to a point of } Y\} \\
& Y^{*}=\left\{b \in X^{*} \mid b \text { has at least one end point in } Y\right\}
\end{aligned}
$$

For $P^{\prime} \subset X^{*}$, we denote by $\operatorname{supp} P^{\prime}$ the set of all end points of all bonds in $P^{\prime}$.

The large and small field sets will be conveniently indexed by intervals whose length is related to the scale at which they were created.

\section{Notation II.2}

(i) A decimation point for the interval $[0, \delta]$ is a point $\tau=\frac{p}{2^{k}} \delta$ with integers $k \geq 0$ and $0 \leq p \leq 2^{k}$.

(ii) A decimation interval in $[0, \delta]$ is an interval of the form $\mathcal{J}=\left[\frac{p}{2^{k}} \delta, \frac{p+1}{2^{k}} \delta\right]$ with $k \geq 0$ and $0 \leq p \leq 2^{k}-1$.

(iii) For a decimation point $\tau \neq 0, \delta$, there is a unique $k \geq 1$ such that $\tau \in \frac{\delta}{2^{k}} \mathbb{Z} \backslash \frac{\delta}{2^{k-1}} \mathbb{Z}$. This number $k$ is called the decimation index $\mathfrak{d}(\tau)$ of $\tau$ in $[0, \delta]$. We also set $\mathfrak{d}(0)=$ $\mathfrak{d}(\delta)=0$. We call $s=2^{-\mathfrak{d}(\tau)} \delta$ the scale of $\tau$.

The unique decimation interval that has $\tau$ as its midpoint is

$$
\mathcal{J}_{\tau}=[\tau-s, \tau+s]
$$

Its left and right halves

$$
\mathcal{J}_{\tau}^{-}=[\tau-s, \tau] \quad, \quad \mathcal{J}_{\tau}^{+}=[\tau, \tau+s]
$$

are also decimation intervals. 
Example II.3 For example, if $\tau=\frac{3}{16} \delta$, then $\tau \in \frac{\delta}{2^{4}} \mathbb{Z} \backslash \frac{\delta}{2^{3}} \mathbb{Z}$ so that $\mathfrak{d}(\tau)=4, s=\frac{\delta}{16}$, $\mathcal{J}_{\tau}=\left[\frac{2}{16} \delta, \frac{4}{16} \delta\right], \mathcal{J}_{\tau}^{-}=\left[\frac{2}{16} \delta, \frac{3}{16} \delta\right]$ and $\mathcal{J}_{\tau}^{+}=\left[\frac{3}{16} \delta, \frac{4}{16} \delta\right]$.

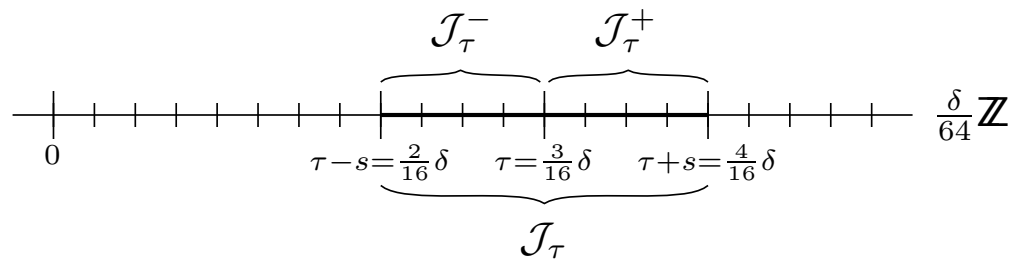

If $\tau$ is a decimation point, then the field $\alpha_{\tau}$ appears as an integration variable in the construction of $I_{m}\left(2^{-m} \delta ; \alpha^{*}, \beta\right)$ for all $m \geq \mathfrak{d}(\tau)$. When this variable is integrated, large and small field sets are introduced. We choose to label them by $\mathcal{J}_{\tau}$, because $\mathcal{J}_{\tau}$ carries the information about $\tau$, and through its length, also about the scale $2^{-\mathfrak{d}(\tau)} \delta$.

Definition II.4 (Hierarchy) A hierarchy, $\mathfrak{S}$, for scale $0<\delta \leq \frac{1}{k T}$, of large and small field sets is a collection

- $P_{\alpha}(\mathcal{J}), P_{\beta}(\mathcal{J}), Q(\mathcal{J})$ of subsets of $X$, called large field sets of the first kind

- $P_{\alpha}^{\prime}(\mathcal{J}), P_{\beta}^{\prime}(\mathcal{J})$ of subsets of $X^{*}$, also called large field sets of the first kind

- $R(\mathcal{J})$ of subsets of $X$, called large field sets of the second kind

$\circ \Lambda(\mathcal{J}), \Omega(\mathcal{J})$ of subsets of $X$, called the small field sets of the first and second kind respectively.

These sets

- are indexed by all decimation intervals $\mathcal{J}$ in $[0, \delta]$,

$\circ$ and obey the following "large/small field set" compatibility conditions. Let $\mathcal{J}$ be a decimation interval in $[0, \delta]$, and $\mathcal{J}^{-}$and $\mathcal{J}^{+}$be its left and right halves. Let $t=\operatorname{length}\left(\mathcal{J}^{+}\right)=\operatorname{length}\left(\mathcal{J}^{-}\right)=\frac{1}{2} \operatorname{length}(\mathcal{J})$. Then

- $P_{\alpha}(\mathcal{J}), P_{\beta}(\mathcal{J}) \subset \Omega\left(\mathcal{J}^{-}\right) \cap \Omega\left(\mathcal{J}^{+}\right)$and $P_{\alpha}^{\prime}(\mathcal{J}), P_{\beta}^{\prime}(\mathcal{J}) \subset\left(\Omega\left(\mathcal{J}^{-}\right) \cap \Omega\left(\mathcal{J}^{+}\right)\right)^{*}$ and $Q(\mathcal{J}) \subset\left(\Omega\left(\mathcal{J}^{-}\right) \cap \Omega\left(\mathcal{J}^{+}\right)\right)^{\star}$

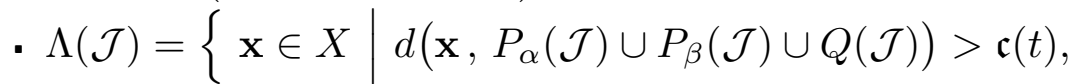

$d\left(\mathbf{x}, \operatorname{supp} P_{\alpha}^{\prime}(\mathcal{J}) \cup \operatorname{supp} P_{\beta}^{\prime}(\mathcal{J})\right)>\mathfrak{c}(t)$,

$d\left(\mathbf{x}, \Omega\left(\mathcal{J}^{-}\right)^{c} \cup \Omega\left(\mathcal{J}^{+}\right)^{c}\right)>\mathfrak{c}(t)$

- $R(\mathcal{J}) \subset \Lambda(\mathcal{J})$

. $\Omega(\mathcal{J})=\{\mathbf{x} \in \Lambda(\mathcal{J}) \mid d(\mathbf{x}, R(\mathcal{J}))>\mathfrak{c}(t)\}$

- There is a non negative integer $k_{0}$ such that $\Lambda(\mathcal{J})=\Omega(\mathcal{J})=X$, and consequently $P_{\alpha}(\mathcal{J})=P_{\beta}(\mathcal{J})=P_{\alpha}^{\prime}(\mathcal{J})=P_{\beta}^{\prime}(\mathcal{J})=Q(\mathcal{J})=R(\mathcal{J})=\emptyset$, for all decimation intervals of lengths $2^{-k} \delta$ with $k \geq k_{0}$. The smallest such $k_{0}$ is called $\operatorname{depth}(\mathfrak{S})$.

The following figure schematically illustrates the set relations amongst the various large 
and small field sets at a single scale, but is not metrically accurate.

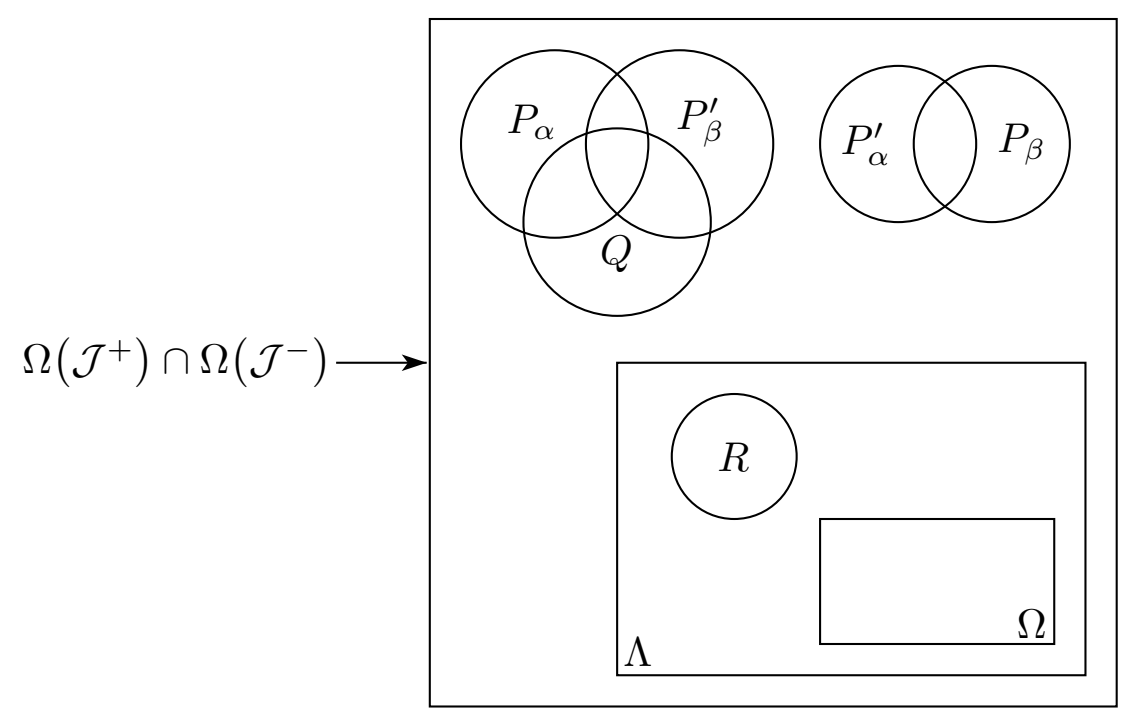

Notation II.5 Let $\mathfrak{S}$ be a hierarchy of scale $\delta$.

(i) We also denote, for example, $\Lambda(\mathcal{J})$ by $\Lambda_{\mathfrak{S}}(\mathcal{J})$, when we wish to emphasize its dependence on the hierarchy $\mathfrak{S}$.

(ii) The "summits" $\Omega_{\mathfrak{S}}([0, \delta])$ and $\Lambda_{\mathfrak{S}}([0, \delta])$ are also denoted $\Omega_{\mathfrak{S}}$ and $\Lambda_{\mathfrak{S}}$, respectively.

(iii) The decimation points, resp. intervals, in $[0, \delta]$ are called the decimation points, resp. intervals, for the hierarchy $\mathfrak{S}$.

(iv) For a decimation point $\tau$, we set

$$
\Lambda_{\tau}= \begin{cases}\Lambda\left(\mathcal{J}_{\tau}\right) & \text { if } \tau \neq 0, \delta \\ \emptyset & \text { if } \tau=0, \delta\end{cases}
$$

Here, $\mathcal{J}_{\tau}$ is the unique decimation interval centered on $\tau$. See Notation II.2. Observe that

$$
\Lambda_{\tau}=X \quad \text { if } \mathfrak{d}(\tau)>\operatorname{depth}(\mathfrak{S})
$$

Remark II.6 It follows from the definition that, for decimation intervals $\mathcal{J}^{\prime} \varsubsetneqq \mathcal{J}$

$\circ \Lambda\left(\mathcal{J}^{\prime}\right) \supset \Omega\left(\mathcal{J}^{\prime}\right) \supset \Lambda(\mathcal{J}) \supset \Omega(\mathcal{J})$

○ $\Omega\left(\mathcal{J}^{\prime}\right)^{c} \cup P_{\alpha}(\mathcal{J}) \cup P_{\beta}(\mathcal{J}) \cup \operatorname{supp} P_{\alpha}^{\prime}(\mathcal{J}) \cup \operatorname{supp} P_{\beta}^{\prime}(\mathcal{J}) \cup Q(\mathcal{J}) \subset \Lambda(\mathcal{J})^{c}$ and $\Lambda(\mathcal{J})^{c} \cup \mathcal{R}(\mathcal{J}) \subset \Omega(\mathcal{J})^{c}$

and indeed if, $\emptyset \neq \Omega\left(\mathcal{J}^{\prime}\right) \neq X$, then $d\left(\Omega\left(\mathcal{J}^{\prime}\right)^{c}, \Omega(\mathcal{J})\right) \geq d\left(\Omega\left(\mathcal{J}^{\prime}\right)^{c}, \Lambda(\mathcal{J})\right)>\mathfrak{c}\left(\left|\mathcal{J}^{\prime}\right|\right)$. Since $X$ is a finite set and $\mathfrak{c}(t) \geq 1$ for all $t$, it then follows that there is a natural number $k_{X}$ with the property that 
○ if $\mathcal{J}^{\prime}$ is a decimation interval with $\Omega\left(\mathcal{J}^{\prime}\right) \neq X$, then $\Omega(\mathcal{J})=\Lambda(\mathcal{J})=P_{\alpha}(\mathcal{J})=$ $P_{\beta}(\mathcal{J})=P_{\alpha}^{\prime}(\mathcal{J})=P_{\beta}^{\prime}(\mathcal{J})=Q(\mathcal{J})=R(\mathcal{J})=\emptyset$ for all decimation intervals $\mathcal{J} \supsetneqq \mathcal{J}^{\prime}$ with $\frac{|\mathcal{J}|}{\left|\mathcal{J}^{\prime}\right|} \geq 2^{k_{X}}$

In particular, for each $\delta>0$, there are only finitely many hierarchies $\mathfrak{S}$ for scale $\delta$ that have $\Omega_{\mathfrak{S}}([0, \delta]) \neq \emptyset$. Each such hierarchy has depth at most $k_{X}$.

\section{II.3 The Large Field Integral Operator}

The functions $\varphi_{\Omega ; \theta}\left(\alpha^{*}, \beta\right)$ of (II.3) will be written as a sum

$$
\varphi_{\Omega ; \theta}=\sum_{\substack{\mathfrak{S} \text { hierarchy for scale } \theta \\ \Omega_{\mathfrak{S}}([0, \theta])=\Omega}} \varphi_{\mathfrak{S} ; \theta}
$$

Each of the functions $\varphi_{\mathfrak{S} ; \theta}\left(\alpha^{*}, \beta\right)$ will be an integral over variables in the large field regions determined by the hierarchy $\mathfrak{S}$.

As we saw in the discussion of the stationary phase approximation in $\S I$, we shall need normalization constants in the representation (II.3) of the effective densities. They should obey the recursion relation (I.21). We make a particular choice of normalization constants $\mathcal{Z}_{\delta}$, by prescribing their asymptotic behaviour as $\delta \rightarrow 0$. This choice is made to simplify the proof that $\lim _{m \rightarrow \infty} I_{m}\left(2^{-m} \theta ; \cdots\right)$ exists.

Lemma II.7 There is a unique function $\delta \in(0,1) \rightarrow \mathcal{Z}_{\delta} \in(0,1)$ that obeys

$$
\mathcal{Z}_{2 \delta}=\mathcal{Z}_{\delta}^{2} \int_{|z| \leq \mathrm{r}(\delta)} \frac{d z^{*} \wedge d z}{2 \pi i} e^{-|z|^{2}} \quad \lim _{\varepsilon \rightarrow 0+} \frac{1}{\varepsilon} \log \mathcal{Z}_{\varepsilon}=0
$$

Furthermore,

$$
\left|\ln \mathcal{Z}_{\delta}\right| \leq e^{-\mathrm{r}(\delta)^{2}}
$$

This lemma is proven in Appendix C.

The large field integral operator arises from the "left over" fields in the decimation procedure outlined in and after (I.12). The decimation steps are indexed by decimation points $\tau \in(0, \delta)$. When the field $\phi$ is being integrated out in such a step, one gets, as in (I.20), a sum over pairs of small field sets $\Omega\left(\mathcal{J}_{\tau}\right) \subset \Lambda\left(\mathcal{J}_{\tau}\right)$ and

(i) the fluctuation integral with variables $|z(\mathbf{x})| \leq \mathrm{r}\left(\frac{1}{2}\left|\mathcal{J}_{\tau}\right|\right)$ for $\mathbf{x} \in \Omega\left(\mathcal{J}_{\tau}\right)$

(ii) the integral over the Stokes' cylinders $C(\mathbf{x}), \mathbf{x} \in \Lambda\left(\mathcal{J}_{\tau}\right) \backslash \Omega\left(\mathcal{J}_{\tau}\right)$, with the variables $z_{*}(\mathbf{x}), z(\mathbf{x})$

(iii) the "large field integral of the first kind" for points $x \in \Lambda\left(\mathcal{J}_{\tau}\right)^{c}$, with variables $\phi(\mathbf{x})$ which violated at least one of the small field conditions of the first kind 
In the decimation step, the fluctuation integral (i) is performed, while the integrals (ii) and (iii) are are not performed explicitly and form part of the large field integral operator. For labelling purposes, the integration variables of (ii) are renamed $z_{* \tau}(\mathbf{x})$ and $z_{\tau}(\mathbf{x})$, and the integration variables of (iii) are renamed $\alpha_{\tau}(\mathbf{x})$. We call the fields $\alpha_{\tau}(\mathbf{x}), \mathbf{x} \in \Lambda\left(\mathcal{J}_{\tau}\right)^{c}$, and $z_{* \tau}(\mathbf{x}), z_{\tau}(\mathbf{x}), \mathbf{x} \in \Lambda\left(\mathcal{J}_{\tau}\right) \backslash \Omega\left(\mathcal{J}_{\tau}\right)$, the residual fields. The integral operator associated to a full hierarchy, $\mathfrak{S}$, is the concatenation of all integral operators associated to all decimation points $\tau$ for the hierarchy with decimation index $\mathfrak{d}(\tau)<\operatorname{depth}(\mathfrak{S})$.

The definition of the integral operators involves the constant $\mathfrak{c}$ and the cut-off propagator $j_{\mathfrak{c}}(\tau)$ of $(\mathrm{I} .24)$.

Definition II.8 (Large Field Integral Operator) Let $\mathfrak{S}$ be a hierarchy for scale $\delta>0$.

(i) Let $\tau$ be a decimation point for $\mathfrak{S}$ with $\mathfrak{d}(\tau) \leq \operatorname{depth}(\mathfrak{S})$. The scale of $\tau$ is $s=2^{-\mathfrak{d}(\tau)} \delta$, and its corresponding decimation interval is $\mathcal{J}=\mathcal{J}_{\tau}=\left[\tau_{\ell}, \tau_{r}\right]$ with $\tau_{\ell}=\tau-s$ and $\tau_{r}=\tau+s$. The integral operator associated to the decimation point $\tau$ is

$$
\begin{aligned}
& \mathcal{I}_{\left(\mathcal{J} ; \alpha^{*}, \beta\right)}=\mathcal{I}_{\left(\mathcal{J}, \mathfrak{S} ; \alpha^{*}, \beta\right)}=\left(\prod_{\mathbf{x} \in \Lambda(\mathcal{J}) \backslash(R(\mathcal{J}) \cup \Omega(\mathcal{J}))} \int_{\left|z_{\tau}(\mathbf{x})\right| \leq \mathrm{r}(s)} \frac{d z_{\tau}(\mathbf{x})^{*} \wedge d z_{\tau}(\mathbf{x})}{2 \pi i} e^{-z_{\tau}(\mathbf{x})^{*} z_{\tau}(\mathbf{x})}\right) \\
& \left(\prod_{\mathbf{x} \in R(\mathcal{J})} \int_{C_{s}\left(\mathbf{x} ; \alpha^{*}, \beta\right)} \frac{d z_{* \tau}(\mathbf{x}) \wedge d z_{\tau}(\mathbf{x})}{2 \pi i} e^{-z_{* \tau}(\mathbf{x}) z_{\tau}(\mathbf{x})}\right) \\
& \left(\prod_{\mathbf{x} \in X \backslash \Lambda(\mathcal{J})} \int \frac{d \alpha_{\tau}(\mathbf{x})^{*} \wedge d \alpha_{\tau}(\mathbf{x})}{2 \pi i}\right) \chi_{\mathcal{J}}\left(\alpha, \alpha_{\tau}, \beta\right) \\
& \mathcal{Z}_{s}^{\left|\Omega\left(\mathcal{J}^{-}\right) \backslash \Omega(\mathcal{J})\right|} \mathcal{Z}_{s}^{\left|\Omega\left(\mathcal{J}^{+}\right) \backslash \Omega(\mathcal{J})\right|}
\end{aligned}
$$

Here, for each $\mathbf{x} \in R(\mathcal{J}), C_{s}\left(\mathbf{x} ; \alpha^{*}, \beta\right)$ is a two real dimensional surface in

$$
\left\{\left(z_{*}, z\right) \in \mathbb{C}^{2}|| z_{*}|,| z \mid<\mathrm{R}(s)\right\}
$$

whose boundary is the union of the circle $\left\{\left(z_{*}, z\right) \in \mathbb{C}^{2}\left|z_{*}^{*}=z,\right| z \mid=\mathrm{r}(s)\right\}$ and the curve bounding $(3)$

$$
\begin{array}{r}
\left\{\left(z_{*}, z\right) \in \mathbb{C}^{2}|| z_{*}-\left(\left[1-j_{\mathfrak{c}}(s)\right] \alpha^{*}\right)(\mathbf{x})|\leq \mathrm{r}(s),| z-\left(\left[1-j_{\mathfrak{c}}(s)\right] \beta\right)(\mathbf{x}) \mid \leq \mathrm{r}(s)\right. \\
\left.z_{*}^{*}-z=\left(j_{\mathfrak{c}}(s)[\beta-\alpha]\right)(\mathbf{x})\right\}
\end{array}
$$

Analyticity and Stokes' theorem ensures the action of the integral operator is independent of the choice of the surfaces $C_{s}\left(\mathbf{x} ; \alpha^{*}, \beta\right)$. See [BFKT5, $\S I I$ and Lemma A.1]. We choose

(3) The set (II.7) is a technically precise variant of (I.16). 
$C_{s}\left(\mathbf{x} ; \alpha^{*}, \beta\right)$ to depend only on the values of the fields $\alpha$ and $\beta$ at points $\mathbf{y} \in X$ with $d(\mathbf{x}, \mathbf{y}) \leq \mathfrak{c}$. This is possible because the boundary curves have the same property, since $j_{\mathfrak{c}}(s)$ has range $\mathfrak{c}$.

The characteristic function $\chi_{\mathcal{J}}\left(\alpha, \alpha_{\tau}, \beta\right)$ implementing the large and small field conditions of the first kind is given in Appendix A.

If $\Omega(\mathcal{J})=X$, we set $\mathcal{I}_{\left(\mathcal{J} ; \alpha^{*}, \beta\right)}=11$.

(ii) The integral operator associated to the hierarchy $\mathfrak{S}$ is

$$
\mathcal{I}_{\left(\mathfrak{S} ; \alpha^{*}, \beta\right)} \prod_{n=0, \cdots, \operatorname{depth}(\mathfrak{S})} \prod_{\substack{\text { decimation intervals } \\ \mathcal{J}=\left[\tau_{l}, \tau_{r}\right] \subset[0, \delta] \\ \text { of length } 2-n_{\delta}}} \mathcal{I}_{\left(\mathcal{J} ; \alpha_{\tau_{\ell}}^{*}, \alpha_{\tau_{r}}\right)}
$$

Observe that the arguments $\alpha_{\tau_{l}}^{*}, \alpha_{\tau_{r}}$ in each $\mathcal{I}_{\left(\mathcal{J} ; \alpha_{\tau_{\ell}}^{*}, \alpha_{\tau_{r}}\right)}$ are the integration variables for an integral appearing to its left. This is the reason for ordering the product $\prod_{n=0, \cdots, \operatorname{depth}(\mathfrak{S})}$ with larger values of $n$ to the right.

(iii) We will bound, in Theorem II.18, the "absolute value"

$$
\left|\mathcal{I}_{\left(\mathfrak{S} ; \alpha^{*}, \beta\right)}\right|=\prod_{n=0, \cdots, \operatorname{depth}(\mathfrak{S})} \prod_{\substack{\text { time intervals } \\ \mathcal{J}=\left[\tau_{l}, \tau_{r}\right] \subset[0, \delta] \\ \text { of length } 2^{-n} \delta}}\left|\mathcal{I}_{\left(\mathcal{J} ; \alpha_{\tau_{\ell}}^{*}, \alpha_{\tau_{r}}\right)}\right|
$$

of the integral operator. Here $\left|\mathcal{I}_{\left(\mathcal{J} ; \alpha^{*}, \beta\right)}\right|$ is constructed by replacing

$$
\int_{C_{s}\left(\mathbf{x} ; \alpha^{*}, \beta\right)} \frac{d z_{* \tau}(\mathbf{x}) \wedge d z_{\tau}(\mathbf{x})}{2 \pi i} e^{-z_{* \tau}(\mathbf{x}) z_{\tau}(\mathbf{x})} \quad \text { by } \quad \int_{C_{s}\left(\mathbf{x} ; \alpha^{*}, \beta\right)}\left|\frac{d z_{* \tau}(\mathbf{x}) \wedge d z_{\tau}(\mathbf{x})}{2 \pi i}\right| e^{-\operatorname{Re} z_{* \tau}(\mathbf{x}) z_{\tau}(\mathbf{x})}
$$

in the formula for $\mathcal{I}_{\left(\mathcal{J} ; \alpha^{*}, \beta\right)}$ of part (i).

The integral operator $\mathcal{I}_{\mathfrak{S}}$ integrates over the fields $\alpha_{\tau}, z_{* \tau}, z_{\tau}$ with $\tau \in \varepsilon \mathbb{Z} \cap(0, \delta)$, where $\varepsilon=2^{-\operatorname{depth}(\mathfrak{S})} \delta$. We introduce the shorthand notation

$$
\vec{\alpha}=\left(\alpha_{\tau}\right)_{\tau \in \varepsilon \mathbb{Z} \cap(0, \delta)} \quad, \quad \vec{z}=\left(z_{\tau}\right)_{\tau \in \varepsilon \mathbb{Z} \cap(0, \delta)} \quad, \quad \vec{z}_{*}=\left(z_{* \tau}\right)_{\tau \in \varepsilon \mathbb{Z} \cap(0, \delta)}
$$

for these "residual" fields.

In Theorem II.18, we give an estimate on the integral operators $\mathcal{I}_{\mathfrak{S}}$.

\section{II.4 The Background Field}

In (I.17), we described the change of the quadratic part of the effective interaction after one decimation step. We iterate this procedure and are led to explicit, but relatively 
complicated expressions for the quadratic part of the effective action at a given scale. To organize the description of the quadratic part and also of the dominant quartic part, we introduce "background fields". The effective action depends on the fields $\alpha_{\tau}$ both directly and through their complex conjugates, but is an analytic function if we treat the complex conjugates as independent variables. Consequently we introduce new complex fields $\alpha_{* \tau}$ that will often be evaluated at $\alpha_{\tau}^{*}$.

Definition II.9 (The Background Field) Let $\mathfrak{S}$ be a hierarchy for scale $\delta$. Set $\varepsilon=2^{-n} \delta$ with the integer $n \geq \operatorname{depth}(\mathfrak{S})$. Given fields $\alpha_{*}, \beta, \vec{\alpha}_{*}=\left(\alpha_{* \tau}(\mathbf{x})\right)_{\substack{\tau \in \varepsilon \mathbb{Z} \cap(0, \delta) \\ \mathbf{x} \in X}}$ and $\vec{\alpha}=\left(\alpha_{\tau}(\mathbf{x})\right)_{\substack{\tau \in \varepsilon \mathbb{Z} \cap(0, \delta) \\ \mathbf{x} \in X}}$, we define the background fields ${ }^{(4)}$ for $\mathfrak{S}$ by

$$
\begin{aligned}
\Gamma_{* \mathfrak{S}}\left(\tau ; \alpha_{*}, \vec{\alpha}_{*}\right) & =\Gamma_{* \tau}^{0}(\mathfrak{S}) \alpha_{*}+\sum_{\tau^{\prime} \in \varepsilon \mathbb{Z} \cap(0, \delta)} \Gamma_{* \tau}^{\tau^{\prime}}(\mathfrak{S}) \alpha_{* \tau^{\prime}} \\
\Gamma_{\mathfrak{S}}(\tau ; \vec{\alpha}, \beta) & =\sum_{\tau^{\prime} \in \varepsilon \mathbb{Z} \cap(0, \delta)} \Gamma_{\tau}^{\tau^{\prime}}(\mathfrak{S}) \alpha_{\tau^{\prime}}+\Gamma_{\tau}^{\delta}(\mathfrak{S}) \beta
\end{aligned}
$$

For $\tau \in(0, \delta)$ and decimation points $\tau^{\prime} \in[0, \delta]$, the coefficients $\Gamma_{* \tau}^{\tau^{\prime}}(\mathfrak{S})=\Gamma_{* \tau}^{\tau^{\prime}}$, with $\tau^{\prime} \neq \delta$, and $\Gamma_{\tau}^{\tau^{\prime}}(\mathfrak{S})=\Gamma_{\tau}^{\tau^{\prime}}$, with $\tau^{\prime} \neq 0$, are defined as follows:

$\circ$ For $\tau=\tau^{\prime} \in(0, \delta)$,

$$
\Gamma_{* \tau}^{\tau}=\Gamma_{\tau}^{\tau}=\Lambda_{\tau}^{c}
$$

Here, we use the following notation. If $Y$ is a subset of $X$, the operator "multiplication by the characteristic function of $Y$ " is also denoted by $Y$.

$\circ$ For $\tau \neq \tau^{\prime}, \Gamma_{* \tau}^{\tau^{\prime}}=0$ unless $\tau>\tau^{\prime}$ and $\left[\tau^{\prime}, \tau\right]$ is strictly contained in a decimation interval with $\tau^{\prime}$ as its left endpoint ${ }^{(5)}$. If $\mathcal{J}$ is the smallest such decimation interval and $\delta^{\prime}$ its length, then

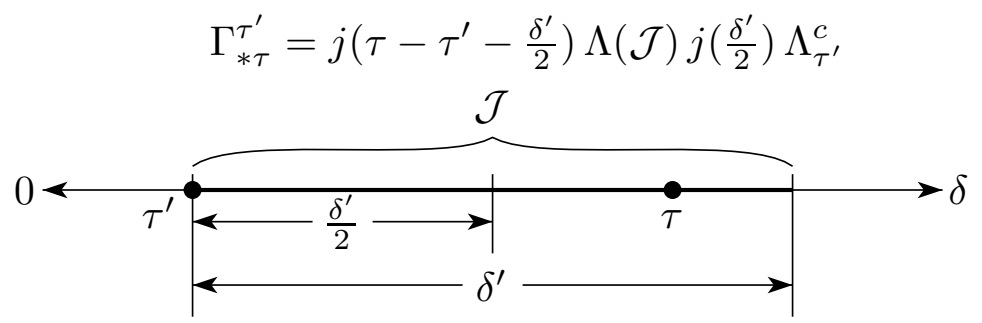

○ Similarly for $\tau \neq \tau^{\prime}, \Gamma_{\tau}^{\tau^{\prime}}=0$ unless $\tau<\tau^{\prime}$ and $\left[\tau, \tau^{\prime}\right]$ is strictly contained in a decimation interval with $\tau^{\prime}$ as its right endpoint. If $\mathcal{J}$ is the smallest such interval and $\delta^{\prime}$ its length, then

$$
\Gamma_{\tau}^{\tau^{\prime}}=j\left(\tau^{\prime}-\tau-\frac{\delta^{\prime}}{2}\right) \Lambda(\mathcal{J}) j\left(\frac{\delta^{\prime}}{2}\right) \Lambda_{\tau^{\prime}}^{c}
$$

(4) For each fixed $\tau \in(0, \delta), \alpha_{*}$ and $\vec{\alpha}_{*}$, the background field $\Gamma_{* \mathfrak{S}}\left(\tau ; \alpha_{*}, \vec{\alpha}_{*}\right)$ is a function of $\mathbf{x} \in X$.

(5) This implies that $\mathfrak{d}(\tau)>\mathfrak{d}\left(\tau^{\prime}\right)$ whenever $\tau$ is a decimation point. Observe that there is a maximal decimation interval with $\tau^{\prime}$ as its left endpoint. If $\tau^{\prime} \neq 0$, it is $\left[\tau^{\prime}, \tau^{\prime}+2^{-\mathfrak{d}\left(\tau^{\prime}\right)} \delta\right]$. If $\tau^{\prime}=0$ it is $[0, \delta]$. 
Remark II.10 The Definition II.9, of the background field, is independent of the choice of integer $n \geq \operatorname{depth}(\mathfrak{S})$. To see this, let $\varepsilon_{\mathfrak{S}}=2^{-\operatorname{depth}(\mathfrak{S})} \delta$. The only place in the definition where $\varepsilon$ appears is in the range of summation $\sum_{\tau^{\prime} \in \varepsilon \mathbb{Z} \cap(0, \delta)}$. If $\tau^{\prime} \in\left(\varepsilon \mathbb{Z} \backslash \varepsilon_{\mathfrak{S}} \mathbb{Z}\right) \cap(0, \delta)$, then $\mathfrak{d}\left(\tau^{\prime}\right)>\operatorname{depth}(\mathfrak{S})$ so that $\Lambda_{\tau^{\prime}}^{c}=\emptyset$ and $\Gamma_{\tau}^{\tau^{\prime}}(\mathfrak{S})=\Gamma_{* \tau}^{\tau^{\prime}}(\mathfrak{S})=0$.

The dominant contributions to the quadratic part of the effective action associated to the hierarchy $\mathfrak{S}$ for scale $\delta$ will be

$$
Q_{\mathfrak{S}}\left(\alpha_{*}, \beta ; \vec{\alpha}_{*}, \vec{\alpha}\right)=Q_{\varepsilon, \delta}\left(\alpha_{*}, \beta ; \Gamma_{* \mathfrak{S}}\left(\cdot ; \alpha_{*}, \vec{\alpha}_{*}\right), \Gamma_{\mathfrak{S}}(\cdot ; \vec{\alpha}, \beta)\right)
$$

with $\varepsilon=2^{-\operatorname{depth}(\mathfrak{S})} \delta$, where

$$
\begin{aligned}
& Q_{\varepsilon, \delta}\left(\alpha_{*}, \beta ; \vec{\gamma}_{*}, \vec{\gamma}\right) \\
& \quad=\sum_{\tau \in \varepsilon \mathbb{Z} \cap(0, \delta)}\left\langle\gamma_{* \tau}, \gamma_{\tau}\right\rangle-\left\langle\alpha_{*}, j(\varepsilon) \gamma_{\varepsilon}\right\rangle-\sum_{\tau \in \varepsilon \mathbb{Z} \cap(0, \delta-\varepsilon)}\left\langle\gamma_{* \tau}, j(\varepsilon) \gamma_{\tau+\varepsilon}\right\rangle-\left\langle\gamma_{* \delta-\varepsilon}, j(\varepsilon) \beta\right\rangle \\
& \quad=-\left\langle\alpha_{*}, j(\varepsilon) \gamma_{\varepsilon}\right\rangle+\sum_{\tau \in \varepsilon \mathbb{Z} \cap(0, \delta-\varepsilon)}\left\langle\gamma_{* \tau}, \gamma_{\tau}-j(\varepsilon) \gamma_{\tau+\varepsilon}\right\rangle+\left\langle\gamma_{* \delta-\varepsilon}, \gamma_{\delta-\varepsilon}-j(\varepsilon) \beta\right\rangle \\
& \quad=\left\langle\gamma_{* \varepsilon}-j(\varepsilon) \alpha_{*}, \gamma_{\varepsilon}\right\rangle+\sum_{\tau \in \varepsilon \mathbb{Z} \cap(\varepsilon, \delta)}\left\langle\gamma_{* \tau}-j(\varepsilon) \gamma_{* \tau-\varepsilon}, \gamma_{\tau}\right\rangle-\left\langle\gamma_{* \delta-\varepsilon}, j(\varepsilon) \beta\right\rangle
\end{aligned}
$$

The dominant part to the quartic part of the effective action will be

$$
\mathcal{V}_{\mathfrak{S}}\left(\alpha_{*}, \beta ; \vec{\alpha}_{*}, \vec{\alpha}\right)=-\int_{0}^{\delta} d \tau\left\langle\Gamma_{* \mathfrak{S}}\left(\tau ; \alpha_{*}, \vec{\alpha}_{*}\right) \Gamma_{\mathfrak{S}}(\tau ; \vec{\alpha}, \beta), v \Gamma_{* \mathfrak{S}}\left(\tau ; \alpha_{*}, \vec{\alpha}_{*}\right) \Gamma_{\mathfrak{S}}(\tau ; \vec{\alpha}, \beta)\right\rangle
$$

The contributions characteristic of the small field set $\Omega_{\mathfrak{S}}([0, \delta])$ are not being integrated over. Therefore we set

$$
\begin{aligned}
& Q_{\mathfrak{S}}^{r e s}\left(\alpha_{*}, \beta ; \vec{\alpha}_{*}, \vec{\alpha}\right)=Q_{\mathfrak{S}}\left(\alpha_{*}, \beta ; \vec{\alpha}_{*}, \vec{\alpha}\right)-\left\langle\alpha_{*}, j_{(\Omega)}(\delta) \beta\right\rangle \\
& \mathcal{V}_{\mathfrak{S}}^{r e s}\left(\alpha_{*}, \beta ; \vec{\alpha}_{*}, \vec{\alpha}\right)=\mathcal{V}_{\mathfrak{S}}\left(\alpha_{*}, \beta ; \vec{\alpha}_{*}, \vec{\alpha}\right)-V_{\Omega ; \delta}\left(\alpha_{*}, \beta\right)
\end{aligned}
$$

\section{II.5 Norms}

Our main result will be, that for sufficiently small $0<\theta \leq \frac{1}{k T}$, the effective density can be represented in the form

$$
\begin{aligned}
I_{\theta}\left(\alpha^{*}, \beta\right)= & \sum_{\Omega \subset X} \mathcal{Z}_{\theta}^{|\Omega|} e^{\left\langle\alpha^{*}, j_{(\Omega)}(\theta) \beta\right\rangle+V_{\Omega ; \theta}\left(\alpha^{*}, \beta\right)+\mathcal{D}_{\Omega ; \theta}\left(\alpha^{*}, \beta\right)} \chi_{\theta}(\Omega ; \alpha, \beta) \\
& \sum_{\substack{\mathcal{S} \text { hierarchy for scale } \theta \\
\Omega_{\mathfrak{S}}=\Omega}} \mathcal{I}_{\left(\mathcal{S}_{\mathfrak{s}}, \beta\right)}\left(e^{-Q_{\mathfrak{S}}^{\text {res }}\left(\alpha^{*}, \beta ; \vec{\alpha}^{*}, \vec{\alpha}\right)+\mathcal{V}_{\mathfrak{S}}^{\text {res }}\left(\alpha^{*}, \beta ; \vec{\alpha}^{*}, \vec{\alpha}\right)} e^{\mathcal{B}_{\mathfrak{S}}\left(\alpha^{*}, \beta ; \overrightarrow{p_{\uparrow}}\right)+\mathcal{L}_{\mathfrak{S}}\left(\alpha^{*}, \beta ; \vec{p}\right)}\right)
\end{aligned}
$$


In this formula, $V_{\Omega ; \theta}, Q_{\mathfrak{S}}^{r e s}$ and $\mathcal{V}_{\mathfrak{S}}^{\text {res }}$ are explicit functions; their definitions have been given in (II.4), (II.10), (II.11) and (II.12). Observe that they are evaluated with $\alpha_{*}=\alpha^{*}$. The pure small field part $\mathcal{D}_{\Omega ; \theta}$ has been constructed in (II.2). The functions $\mathcal{L}_{\mathfrak{S}}$ and $\mathcal{B}_{\mathfrak{S}}$ depend on the "residual fields" $\vec{\alpha}, \vec{z}_{*}, \vec{z}$ that are the integration variables of $\mathcal{I}_{\mathfrak{S}}$. Again we choose to write them as analytic functions of

$$
\vec{\rho}=\left(\vec{\alpha}_{*}, \vec{\alpha}, \vec{z}_{*}, \vec{z}\right)
$$

as well as $\alpha^{*}$ and $\beta$. When they appear inside the integral operator we evaluate them at

$$
\overrightarrow{\rho_{\uparrow}}=\left.\left(\vec{\alpha}_{*}, \vec{\alpha}, \vec{z}_{*}, \vec{z}\right)\right|_{\substack{\vec{\alpha}_{*}=\vec{\alpha}^{*} \\ z_{* \tau}(\mathbf{x})=z_{\mathcal{T}}(\mathbf{x})^{*} \text { for } \mathbf{x} \in \Lambda\left(\mathcal{J}_{\tau}\right) \backslash\left(R\left(\mathcal{J}_{\mathcal{T}}\right) \cap \Omega\left(\mathcal{J}_{\mathcal{T}}\right)\right)}}
$$

The function $\mathcal{L}_{\mathfrak{S}}\left(\alpha_{*}, \beta ; \vec{\rho}\right)$ will be analytic in the fields and depends only on the values of the fields $\alpha_{* \tau}(\mathbf{x}), \alpha_{\tau}(\mathbf{x})$ for points $\mathbf{x} \in X \backslash \Omega$. It is called the "pure large field contribution". The function $\mathcal{B}_{\mathfrak{S}}\left(\alpha_{*}, \beta ; \vec{\rho}\right)$ depends on the fields at points $\mathbf{x}$ both inside and outside $\Omega$ and is called the "boundary contribution".

In Proposition II.1, we gave estimates on $\mathcal{D}_{\Omega ; \theta}$, expressed in terms of the norms (I.25). The norms that we use to measure $\mathcal{L}_{\mathfrak{S}}$ and $\mathcal{B}_{\mathfrak{S}}$ are similar to the ones introduced in (I.25), but are more sophisticated. They weight the variables $\alpha_{* \tau}(\mathbf{x}), \alpha_{\tau}(\mathbf{x})$ so as to take into account their maximum possible magnitudes on $\mathcal{I}_{\mathfrak{S}}$ 's domain of integration. The abstract framework for these norms was developed in [BFKT4, §II]. For the convenience of the reader, we review it. In Definition II.13, we introduce the concrete weight factors used in this paper.

\section{Definition II.11}

(i) A weight factor on $X$ is a function $\kappa: X \rightarrow(0, \infty]$.

(ii) Let $n_{1}, \cdots, n_{s}$ be nonnegative integers and $\overrightarrow{\mathbf{x}}_{1} \in X^{n_{1}}, \cdots, \overrightarrow{\mathbf{x}}_{s} \in X^{n_{s}}$. If $\delta$ is any metric on $X$, we define the tree size $\tau_{\delta}\left(\overrightarrow{\mathbf{x}}_{1}, \cdots, \overrightarrow{\mathbf{x}}_{s}\right)$ as the length (with respect to the metric $\delta$ ) of the shortest tree in $X$ whose set of vertices contains $\mathbf{x}_{1,1}, \mathbf{x}_{1,2}, \cdots, \mathbf{x}_{1, n_{1}}, \cdots, \mathbf{x}_{s, n_{s}}$.

(iii) For any subset $\Omega$ of X we construct a metric $d_{\Omega}$ on $X$ as follows: Denote by $\bar{\Omega}$ the union of closed unit cubes centered at the points of $\Omega$. For a curve $\gamma$ in $\mathbb{R}^{n}$ we set

$$
\operatorname{length}_{\Omega}(\gamma)=2 \cdot \operatorname{length}(\gamma \cap \bar{\Omega})+\operatorname{length}\left(\gamma \cap\left(\mathbb{R}^{n} \backslash \bar{\Omega}\right)\right)
$$

where length is the ordinary length in $X$.

For any two points $\mathbf{x}, \mathbf{y} \in X$ define

$$
d_{\Omega}(\mathbf{x}, \mathbf{y})=\inf \left\{\operatorname{mlength}_{\Omega}(\gamma) \mid \gamma \text { a curve joining } \mathbf{x} \text { to } \mathbf{y}\right\}
$$


where $\mathrm{m}$ is the "mass" introduced just before (I.25). Clearly,

$$
\mathrm{m} d \leq d_{\Omega} \leq 2 \mathrm{~m} d
$$

Recall that $d$ is the standard metric on $X$.

If $\Omega \subset \Omega^{\prime} \subset X$ and the set $S=\left\{\mathbf{x}_{1,1}, \mathbf{x}_{1,2}, \cdots, \mathbf{x}_{1, n_{1}}, \cdots, \mathbf{x}_{s, n_{s}}\right\}$ contains both a point of $\Omega$ and of $X \backslash \Omega^{\prime}$ then

$$
\tau_{d_{\Omega}}\left(\overrightarrow{\mathbf{x}}_{1}, \cdots, \overrightarrow{\mathbf{x}}_{s}\right) \leq \tau_{d_{\Omega^{\prime}}}\left(\overrightarrow{\mathbf{x}}_{1}, \cdots, \overrightarrow{\mathbf{x}}_{s}\right)-m \operatorname{dist}\left(\bar{\Omega}, \mathbb{R}^{n} \backslash \bar{\Omega}^{\prime}\right)
$$

where, for subsets $U, V$ of $\mathbb{R}^{n}$

$$
\operatorname{dist}(U, V)=\inf \{\operatorname{length}(\gamma) \mid \gamma \text { a curve joining a point of } U \text { to a point of } V\}
$$

Definition II.12 Let $\phi_{1}, \cdots, \phi_{s}$ be a collection of fields on $X$.

(i) Let $f\left(\phi_{1}, \cdots, \phi_{s}\right)$ be a function which is defined and analytic on a neighbourhood of the origin in $\mathbb{C}^{s|X|}$. Then $f$ has a unique expansion of the form

$$
f\left(\phi_{1}, \cdots, \phi_{s}\right)=\sum_{n_{1}, \cdots, n_{s} \geq 0} \sum_{\left(\overrightarrow{\mathbf{x}}_{1}, \cdots, \overrightarrow{\mathbf{x}}_{s}\right) \in X^{n_{1}} \times \cdots \times X^{n_{s}}} a\left(\overrightarrow{\mathbf{x}}_{1}, \cdots, \overrightarrow{\mathbf{x}}_{s}\right) \phi_{1}\left(\overrightarrow{\mathbf{x}}_{1}\right) \cdots \phi_{s}\left(\overrightarrow{\mathbf{x}}_{s}\right)
$$

with the coefficients $a\left(\overrightarrow{\mathbf{x}}_{1}, \cdots, \overrightarrow{\mathbf{x}}_{s}\right)$ invariant under permutations of the components of each vector $\overrightarrow{\mathbf{x}}_{j}$. The functions $a\left(\overrightarrow{\mathbf{x}}_{1}, \cdots, \overrightarrow{\mathbf{x}}_{s}\right)$ are called the (symmetric) coefficient system for $f$.

(ii) For any $n_{1}, \cdots, n_{s} \geq 0$ and any function $b\left(\overrightarrow{\mathbf{x}}_{1}, \cdots, \overrightarrow{\mathbf{x}}_{s}\right)$ on $X^{n_{1}} \times \cdots \times X^{n_{s}}$, we define the norm $\|b\|_{n_{1}, \cdots, n_{s}}$ as follows:

○If there is at least one field, that is if $\sum_{j=1}^{s} n_{j} \neq 0$, then

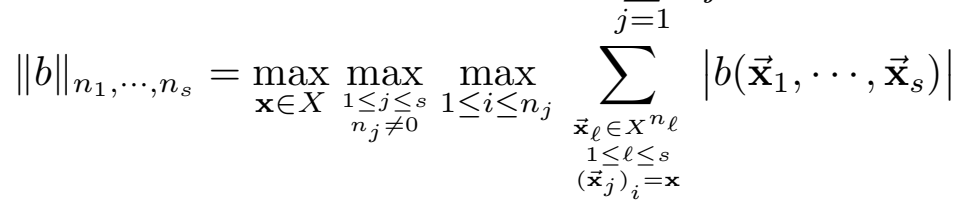

- For the constant term, that is if $\sum_{j=1}^{s} n_{j}=0$,

$$
\|b\|_{n_{1}, \cdots, n_{s}}=|b(-, \cdots,-)|
$$

(ii) Given weight factors $\kappa_{1}, \cdots, \kappa_{s}$, and a metric $\delta$ on $X$, the weight system with metric $\delta$ that associates the weight factor $\kappa_{j}$ to the field $\phi_{j}$ is defined by

$$
w_{\delta}\left(\overrightarrow{\mathbf{x}}_{1}, \cdots, \overrightarrow{\mathbf{x}}_{s}\right)=e^{\tau_{\delta}\left(\overrightarrow{\mathbf{x}}_{1}, \cdots, \overrightarrow{\mathbf{x}}_{s}\right)} \prod_{j=1}^{s} \prod_{\ell=1}^{n_{j}} \kappa_{j}\left(\mathbf{x}_{j, \ell}\right)
$$


for all $\left(\overrightarrow{\mathbf{x}}_{1}, \cdots, \overrightarrow{\mathbf{x}}_{s}\right) \in X^{n_{1}} \times \cdots \times X^{n_{s}}$ and all nonnegative integers $n_{1}, \cdots, n_{s}$.

If $\Omega$ is a subset of $X$, the weight system with core $\Omega$ that associates the weight factor $\kappa_{j}$ to the field $\phi_{j}$ (and the weight factor one to the history field) is $w_{d_{\Omega}}$.

(iv) Let $f\left(\phi_{1}, \cdots, \phi_{s}\right)$ be a function which is defined and analytic on a neighbourhood of the origin in $\mathbb{C}^{s|X|}$ and $a$ the symmetric coefficient system of $f$. We define the norm, with weight $w$, of $f$ to be

$$
\|f\|_{w}=\sum_{n_{1}, \cdots, n_{s} \geq 0}\left\|w\left(\overrightarrow{\mathbf{x}}_{1}, \cdots, \overrightarrow{\mathbf{x}}_{s}\right) a\left(\overrightarrow{\mathbf{x}}_{1}, \cdots, \overrightarrow{\mathbf{x}}_{s}\right)\right\|_{n_{1}, \cdots, n_{s}}
$$

The functions $\mathcal{B}_{\mathfrak{S}}\left(\alpha_{*}, \beta ; \vec{\rho}\right)$ and $\mathcal{L}_{\mathfrak{S}}\left(\alpha_{*}, \beta ; \vec{\rho}\right)$ in (II.13) depend on the fields $\alpha_{*}, \beta$ and, in addition, on the residual fields $\vec{\rho}=\left(\vec{\alpha}_{*}, \vec{\alpha}, \vec{z}_{*}, \vec{z}\right)$ that are integrated over in the large field integral operator $\mathcal{I}_{\mathfrak{S}}$. The weight factors that we associate to these variables depend on the functions $\mathrm{r}(t)$ and $\mathrm{R}(t)$ introduced before. Recall that $r(t)$ measures the size of the region close to a critical point where the stationary phase construction at scale $t$ is performed (see the Introduction just after (I.10) ). $R(t)$ is the threshold between "large" and "small" fields for scale $t$, see the beginning of Section II.2.

Definition II.13 (Weight Factors) Let $\mathfrak{S}$ be a hierarchy for scale $\delta$.

(i) We define the weight factor $\kappa_{* \mathfrak{S}, 0}$ for the field $\alpha_{*}$ by

$$
\kappa_{* \mathfrak{S}, 0}(\mathbf{x})=\min \{2 \mathrm{R}(t) \mid \mathbf{x} \in \Lambda([0, t]) \text { such that }[0, t] \text { is a decimation interval }\}
$$

and, for $\tau$ a decimation point in $(0, \delta)$, the weight factor $\kappa_{* \mathfrak{S}, \tau}$ for the field $\alpha_{* \tau}$ by

$$
\kappa_{* \mathfrak{S}, \tau}(\mathbf{x})= \begin{cases}\infty & \text { if } \mathbf{x} \in \Lambda_{\tau} \\ \min \{\mathrm{R}(t) \mid \mathbf{x} \in \Lambda([\tau, \tau+t]),[\tau, \tau+t] \text { a decimation interval }\} & \text { otherwise }\end{cases}
$$

Similarly we define the weight factor $\kappa_{\mathfrak{S}, \delta}$ for the field $\beta$ by

$$
\kappa_{\mathfrak{S}, \delta}(\mathbf{x})=\min \{2 \mathrm{R}(t) \mid \mathbf{x} \in \Lambda([\delta-t, \delta]),[\delta-t, \delta] \text { a decimation interval }\}
$$

and, for $\tau$ a decimation point in $(0, \delta)$, the weight factor $\kappa_{\mathfrak{S}, \tau}$ for the field $\alpha_{\tau}$ by

$$
\kappa_{\mathfrak{S}, \tau}(\mathbf{x})= \begin{cases}\infty & \text { if } \mathbf{x} \in \Lambda_{\tau} \\ \min \{\mathrm{R}(t) \mid \mathbf{x} \in \Lambda([\tau-t, \tau]),[\tau-t, \tau] \text { a decimation interval }\} & \text { otherwise }\end{cases}
$$


The significance of the " $\infty$ " lines is the following. If $\mathfrak{d}(\tau) \leq \operatorname{depth}(\mathfrak{S})$ and $\mathbf{x} \in \Lambda_{\tau}$, then the integration variables $\alpha_{* \tau}(\mathbf{x}), \alpha_{\tau}(\mathbf{x})$ have been replaced by the integration variable $z_{* \tau}(\mathbf{x}), z_{\tau}(\mathbf{x})$ during the decimation step for $\tau$. Thus $\alpha_{* \tau}(\mathbf{x}), \alpha_{\tau}(\mathbf{x})$ no longer appear as arguments. If $\mathfrak{d}(\tau)>\operatorname{depth}(\mathfrak{S})$, that is if $\alpha_{* \tau}$ and $\alpha_{\tau}$ do not appear as integration variables in $\mathcal{I}_{\mathfrak{S}}$ at all, then $\Lambda_{\tau}=X$ and $\kappa_{*, \tau}(\mathbf{x})=\kappa_{\tau}(\mathbf{x})=\infty$ for all $\mathbf{x}$.

The spatial decay of these weight factors is discussed in Appendix B.

(ii) We define weight factors $\lambda_{\tau}$ for the "residual" fields $z_{* \tau}, z_{\tau}, \tau$ a decimation point in $(0, \delta)$ by

$$
\lambda_{\mathfrak{S}, \tau}(\mathbf{x})= \begin{cases}32 \mathrm{r}\left(2^{-\mathfrak{d}(\tau)} \delta\right) & \text { if } \mathbf{x} \in \Lambda_{\tau} \backslash \Omega\left(\mathcal{J}_{\tau}\right) \\ \infty & \text { otherwise }\end{cases}
$$

(iii) We denote by $w_{\mathfrak{S}}$ the weight system with core $\Omega_{\mathfrak{S}}$ that associates the weight factor $\kappa_{* \mathfrak{S}, 0}$ to the field $\alpha_{*}$, the weight factor $\kappa_{\mathfrak{S}, \delta}$ to the field $\beta$, and, for $\tau \in(0, \delta)$, the weight factors $\kappa_{* \mathfrak{S}, \tau}, \kappa_{\mathfrak{S}, \tau}, \lambda_{\mathfrak{S}, \tau}$ and $\lambda_{\mathfrak{S}, \tau}$ to the fields $\alpha_{* \tau}, \alpha_{\tau}, z_{* \tau}$ and $z_{\tau}$, respectively. We will generally write $\|\cdot\|_{\mathfrak{S}}$ in place of $\|\cdot\|_{w_{\mathfrak{S}}}$.

Our main result (Theorem II.16 below) states, that, under suitable assumptions on the functions $R(t)$ and $\mathrm{r}(t)$ the decomposition (II.13) of $I_{\theta}$ exists, and gives bounds on $\left\|\mathcal{B}_{\mathfrak{S}}\right\|_{\mathfrak{S}}$ and $\left\|\mathcal{L}_{\mathfrak{S}}\right\|_{\mathfrak{S}}$.

\section{II.6 Summary and Statement of the Main Theorems}

We are studying many particle systems of Bosons on the finite lattice $X$ whose single particle Hamiltonian $\mathrm{h}$ is of the form $\mathrm{h}=\nabla^{*} \mathcal{H} \nabla$ with a translation invariant, strictly positive operator $\mathcal{H}: L^{2}\left(X^{*}\right) \rightarrow L^{2}\left(X^{*}\right)$. For our construction, we assume that there are constants $0<c_{\mathcal{H}}<C_{\mathcal{H}}$ such that all of its eigenvalues lie between $c_{\mathcal{H}}$ and $C_{\mathcal{H}}$. Also we assume that

$$
D_{\mathcal{H}}=\sum_{\substack{\mathbf{x} \in X \\ 1 \leq i, j \leq d}} e^{6 \mathrm{~m} d(\mathbf{x}, 0)}\left|\mathcal{H}\left(b_{i}(0), b_{j}(\mathbf{x})\right)\right|<\infty
$$

where $\mathrm{m}$ is the mass used in (II.5). Here, for each $1 \leq i \leq \mathrm{D}$ and $\mathbf{x} \in X, b_{i}(\mathbf{x})=\left(\mathbf{x}, \mathbf{x}+e_{i}\right)$ denotes the bond with base point $\mathbf{x}$ and direction $e_{i}$. The interactions $v(\mathbf{x}, \mathbf{y})$ we allow are assumed to be translation invariant, repulsive and exponentially decaying in that the norm

$$
\left\|v\left|\|=\sup _{\mathbf{x} \in X} \sum_{\mathbf{y} \in X} e^{5 \mathrm{~m} d(\mathbf{x}, \mathbf{y})}\right| v(\mathbf{x}, \mathbf{y}) \mid\right.
$$

introduced in (II.5), is sufficiently small. We discuss the system at temperature $T=\frac{1}{k \beta}>0$ and chemical potential $\mu$. 
The representation (II.13) of the effective density that we want to achieve depends on

- the functions $R(t)$ and $\mathrm{R}^{\prime}(t)$ that implement the large field conditions at scale $t$.

- the function $r(t)$ that gives the size of the region near the critical point at scale $t$ where stationary phase is applied.

- the function $\mathfrak{c}(t)$ that measures the size of the "corridors" in the hierarchies.

- the constant $\mathfrak{c}$ that measures the size of the cut off of the single particle operator that is needed for the analyticity in Stokes' argument (see (I.24)).

- a constant $\mathfrak{v}>0$ that measures, roughly speaking, the size of the interaction $v$. The precise conditions relating $v$ and $\mathfrak{v}$ are given in Hypothesis II.14, below.

- a constant $c_{v}>0$ that, roughly speaking, imposes a lower bound on the smallest eigenvalue, $v_{1}$, of $v$, viewed as the kernel of a convolution operator acting on $L^{2}(X)$. Again, see Hypothesis II.14, below. Clearly $v_{1} \leq\|v\| \leq\|v\| \|$.

- the chemical potential $\mu$

Hypothesis II.14 The two-body potential $v(\mathbf{x}, \mathbf{y})$ is a real, symmetric, translation invariant function on $X \times X$ that obeys

$$
\frac{1}{4} \mathfrak{v} \leq\|\| v \mid \| \leq \frac{1}{2} \mathfrak{v} \quad \text { and } \quad v_{1} \geq c_{v}\|v\| \|
$$

For our construction, we fix strictly positive exponents $e_{\mathrm{r}}, e_{\mathrm{R}}, e_{\mathrm{R}^{\prime}}$ and $e_{\mu}$ that obey

$$
\begin{array}{rlrl}
3 e_{\mathrm{R}}+4 e_{\mathrm{r}}<1 & 1 \leq 4 e_{\mathrm{R}}+2 e_{\mathrm{r}} & 2\left(e_{\mathrm{R}}+e_{\mathrm{r}}\right)<e_{\mu} \leq 1 \\
e_{\mathrm{R}^{\prime}}+e_{\mathrm{r}}<1 & \frac{1}{2} \leq e_{\mathrm{R}^{\prime}} &
\end{array}
$$

and a constant $K_{\mu}>0$. We make the particular, $\mathfrak{v}$-dependent, choices

$$
\begin{aligned}
& \mathrm{r}(t)=\left(\frac{1}{t \mathfrak{v}}\right)^{e_{\mathrm{r}}} \quad \mathrm{R}(t)=\left(\frac{1}{t \mathfrak{v}}\right)^{e_{\mathrm{R}}} \mathrm{r}(t) \quad \mathrm{R}^{\prime}(t)=\left(\frac{1}{t}\right)^{e_{\mathrm{R}^{\prime}}} \mathrm{r}(t) \\
& \mathfrak{c}=\log ^{2} \frac{1}{\mathfrak{v}} \quad \mathfrak{c}(t)=\log ^{2} \frac{1}{t \mathfrak{v}}
\end{aligned}
$$

and assume that

$$
|\mu| \leq K_{\mu} \mathfrak{v}^{e_{\mu}}
$$

Example II.15 Natural choices for $e_{\mathrm{R}}$ and $e_{\mathrm{R}^{\prime}}$ are $e_{\mathrm{R}}=\frac{1}{4}, e_{\mathrm{R}^{\prime}}=\frac{1}{2}$. It is also natural to choose $e_{\mu}$ bigger than, but close to $\frac{1}{2}$.

We are working with a Riemann sum approximation to the quartic term in (I.1) which is, roughly speaking, (-2 times) a sum over $\tau \in \varepsilon \mathbb{Z} \cap\left[0, \frac{1}{k T}\right]$ of

$$
\varepsilon\left\langle\alpha_{\tau}^{*} \alpha_{\tau}, v \alpha_{\tau}^{*} \alpha_{\tau}\right\rangle \geq \varepsilon v_{1} \sum_{\mathbf{x} \in X}\left|\alpha_{\tau}(\mathbf{x})\right|^{4} \geq \varepsilon v_{1} \mathrm{R}(\varepsilon)^{4}\left|\left\{\mathbf{x} \in X|| \alpha_{\tau}(\mathbf{x}) \mid>\mathrm{R}(\varepsilon)\right\}\right|
$$


The coefficient $\varepsilon v_{1} \mathrm{R}(\varepsilon)^{4}=\frac{v_{1}}{\mathfrak{v}} \varepsilon \mathfrak{v} \mathrm{R}(\varepsilon)^{4}$ would be exactly $\frac{v_{1}}{\mathfrak{v}}$, which is of order one, for the choice $e_{R}=\frac{1}{4}$, if $e_{\mathrm{r}}$ were zero. We think of $e_{r}$ as being small.

Similarly, the h term in (I.1) is, roughly speaking, a sum over $\tau \in \varepsilon \mathbb{Z} \cap\left[0, \frac{1}{k T}\right]$ of (minus)

$$
\varepsilon\left\|\nabla \alpha_{\tau}\right\|_{L^{2}\left(X^{*}\right)} \geq \varepsilon \mathrm{R}^{\prime}(\varepsilon)^{2}\left|\left\{b \in X^{*}|| \nabla \alpha_{\tau}(b) \mid>\mathrm{R}^{\prime}(\varepsilon)\right\}\right|
$$

The coefficient $\varepsilon \mathrm{R}^{\prime}(\varepsilon)^{2}$ would be exactly one, for the choice $e_{\mathrm{R}^{\prime}}=\frac{1}{2}$, if $e_{\mathrm{r}}$ were zero. The combined $v$ and $\mu$ terms in (I.1) are, roughly speaking, a sum over $\tau \in \varepsilon \mathbb{Z} \cap\left[0, \frac{1}{k T}\right]$ of $(-\varepsilon$ times $)$

$$
\begin{aligned}
\frac{1}{2}\left\langle\alpha_{\tau}^{*} \alpha_{\tau}, v \alpha_{\tau}^{*} \alpha_{\tau}\right\rangle-\mu\left\|\alpha_{\tau}\right\|^{2} & \geq \sum_{\mathbf{x} \in X}\left[\frac{1}{2} v_{1}\left|\alpha_{\tau}(\mathbf{x})\right|^{4}-\mu\left|\alpha_{\tau}(\mathbf{x})\right|^{2} \mid\right. \\
& =\sum_{\mathbf{x} \in X}\left[\frac{1}{2} v_{1}\left(\left|\alpha_{\tau}(\mathbf{x})\right|^{2}-\frac{\mu}{v_{1}}\right)^{2}-\frac{\mu^{2}}{2 v_{1}} \mid\right. \\
& \geq-\sum_{\mathbf{x} \in X} \frac{\mu^{2}}{2 v_{1}}
\end{aligned}
$$

With this choice of $e_{\mu}$, the right hand side is small. Making $e_{\mu}$ larger would make the right hand side smaller, but would also make the critical value, $\frac{\mu}{v_{1}}$, of $\left|\alpha_{\tau}(\mathbf{x})\right|$ smaller too. This is not desirable for generating symmetry breaking in the infrared regime.

With these choices, (II.17) is satisfied if $e_{\mathrm{r}}>0$ is small enough.

Our main theorems are Theorems II.16 and II.18, below. They are proven in $\S$ III.8.

Theorem II.16 There is a constant $K$, such that for all sufficiently ${ }^{(6)}$ small $\mathfrak{v}, \theta>0$, the limit $I_{\theta}\left(\alpha^{*}, \beta\right)=\lim _{m \rightarrow \infty} I_{m}\left(2^{-m} \theta ; \alpha^{*}, \beta\right)$ exists and has the representation

$$
\begin{aligned}
I_{\theta}\left(\alpha^{*}, \beta\right)= & \sum_{\Omega \subset X} \mathcal{Z}_{\theta}^{|\Omega|} e^{\left\langle\alpha^{*}, j_{(\Omega)}(\theta) \beta\right\rangle+V_{\Omega ; \theta}\left(\alpha^{*}, \beta\right)+\mathcal{D}_{\Omega ; \theta}\left(\alpha^{*}, \beta\right)} \chi_{\theta}(\Omega ; \alpha, \beta) \\
& \sum_{\substack{\mathfrak{S} \text { hierarchy for scale } \theta \\
\Omega_{\mathfrak{S}}=\Omega}} \mathcal{I}_{\left(\mathfrak{S} ; \alpha^{*}, \beta\right)}\left(e^{-Q_{\mathfrak{S}}^{r e s}\left(\alpha^{*}, \beta ; \vec{\alpha}^{*}, \vec{\alpha}\right)+\mathcal{V}_{\mathfrak{S}}^{r e s}\left(\alpha^{*}, \beta ; \vec{\alpha}^{*}, \vec{\alpha}\right)} e^{\mathcal{B}_{\mathfrak{S}}\left(\alpha^{*}, \beta ; \overrightarrow{\rho_{\uparrow}}\right)+\mathcal{L}_{\mathfrak{S}}\left(\alpha^{*}, \beta ; \overrightarrow{\rho_{\uparrow}}\right)}\right)
\end{aligned}
$$

with the following properties.

- $V_{\Omega ; \theta}$ and $\mathcal{D}_{\Omega ; \theta}$ are the functions defined in (II.4) and (II.2). Furthermore, $\mathcal{D}_{\Omega ; \theta}$ can be decomposed in the form

$$
\mathcal{D}_{\Omega ; \theta}\left(\alpha_{*}, \beta\right)=\mathcal{R}_{\Omega ; \theta}\left(\alpha_{*}, \beta\right)+\mathcal{E}_{\Omega ; \theta}\left(\alpha_{*}, \beta\right)
$$

(6) See Hypothesis F.7. 
with a function $\mathcal{R}_{\Omega ; \theta}\left(\alpha_{*}, \beta\right)$ that is bilinear in $\alpha_{*}$ and $\beta$, is independent ${ }^{(7)}$ of the interaction $v$, and fulfills the estimate

$$
\left\|\mathcal{R}_{\Omega ; \theta}\right\|_{2 \mathrm{R}(\theta), 2 \mathrm{~m}} \leq K e^{-2 \mathrm{mc}} \theta^{2} \mathrm{r}(\theta)^{2} \mathrm{R}(\theta)^{2} \leq K \theta \mathfrak{v}^{\mathrm{m} \log \frac{1}{\mathfrak{v}}}
$$

and a function $\mathcal{E}_{\Omega ; \theta}\left(\alpha_{*}, \beta\right)$ that has degree at least two ${ }^{(8)}$ both in $\alpha_{*}$ and in $\beta$ and fulfills the estimate

$$
\left\|\mathcal{E}_{\Omega ; \theta}\right\|_{2 \mathrm{R}(\theta), 2 \mathrm{~m}} \leq K \theta^{2}\|v\|^{2} \mathrm{r}(\theta)^{2} \mathrm{R}(\theta)^{6} \leq K\left(\frac{\|v\|}{\mathfrak{v}}\right)^{2}
$$

- $\chi_{\theta}(\Omega ; \alpha, \beta)$ is the characteristic function imposing the small field conditions. It is one if

- $|\alpha(\mathbf{x})|,|\beta(\mathbf{x})| \leq \mathrm{R}(\theta)$ for all $\mathbf{x} \in \Omega$ and

- $|\nabla \alpha(b)|,|\nabla \beta(b)| \leq \mathrm{R}^{\prime}(\theta)$ for all bonds $b$ on $X$ that have at least one end in $\Omega$ and - $|\alpha(\mathbf{x})-\beta(\mathbf{x})| \leq \mathrm{r}(\theta)$ for all $\mathbf{x}$ within a distance one from $\Omega$

and it is zero otherwise

- For each hierarchy $\mathfrak{S}$ for scale $\theta, \mathcal{B}_{\mathfrak{S}}\left(\alpha_{*}, \beta ; \vec{\rho}\right)$ is an analytic function of its arguments and fulfills the estimate

$$
\left\|\mathcal{B}_{\mathfrak{S}}\right\|_{\mathfrak{S}} \leq K \theta\|v\| \| \mathrm{r}(\theta) \mathrm{R}(\theta)^{3} \leq K \frac{\|v\|}{\mathfrak{v}}
$$

- For each hierarchy $\mathfrak{S}$ for scale $\theta, \mathcal{L}_{\mathfrak{S}}$ has the decomposition

$$
\mathcal{L}_{\mathfrak{S}}\left(\alpha_{*}, \beta ; \vec{\rho}\right)=\sum_{\substack{\text { decimation } \\ \text { intervals } \\ \mathcal{J} \subset[0, \theta]}} \mathcal{L}_{\mathfrak{S}}\left(\mathcal{J} ; \alpha_{*}, \beta ; \vec{\rho}\right)
$$

where, for each decimation interval $\mathcal{J} \subset[0, \theta]$, the function $\mathcal{L}_{\mathfrak{S}}\left(\mathcal{J} ; \alpha_{*}, \beta ; \vec{\rho}\right)$ is an analytic function of its arguments that

- is "large field with respect to the interval $\mathcal{J}$ " (that is, it depends only on values of the fields at points $\mathbf{x} \in X \backslash \Omega_{\mathfrak{S}}(\mathcal{J})$ and depends only on the variables $\alpha_{* \tau}, \alpha_{\tau}, z_{* \tau}, z_{\tau}$ with $\tau \in \mathcal{J}$. (If $\tau=0 \in \mathcal{J}$, then replace $\alpha_{* 0}(\mathbf{x})$ by $\alpha_{*}(\mathbf{x})$. If $\tau=\theta \in \mathcal{J}$, then replace $\alpha_{\theta}(\mathbf{x})$ by $\beta(\mathbf{x})$ )

- and fulfills the estimate

$$
\left\|\mathcal{L}_{\mathfrak{S}}(\mathcal{J} ; \cdot)\right\|_{\mathfrak{S}} \leq K \delta\|\| v \| \mathrm{r}(\delta) \mathrm{R}(\delta)^{3}=K \frac{\|v\| \|}{\mathfrak{v}}(\mathfrak{v} \delta)^{1-3 e_{\mathrm{R}}-4 e_{\mathrm{r}}}
$$

where $\delta$ is the length of the time interval $\mathcal{J}$.

- The functions $\mathcal{R}_{\Omega ; \theta}, \mathcal{E}_{\Omega ; \theta}, \mathcal{B}_{\mathfrak{S}}$ and $\mathcal{L}_{\mathfrak{S}}(\mathcal{J})$ are all invariant under $\alpha_{*} \rightarrow e^{-i \theta} \alpha_{*}$, $\beta \rightarrow e^{i \theta} \beta, \vec{\rho}=\left(\vec{\alpha}_{*}, \vec{\alpha}, \vec{z}_{*}, \vec{z}\right) \rightarrow\left(e^{-i \theta} \vec{\alpha}_{*}, e^{i \theta} \vec{\alpha}, e^{-i \theta} \vec{z}_{*}, e^{i \theta} \vec{z}\right)$.

- For each hierarchy $\mathfrak{S}$ for scale $\theta, \mathcal{I}_{\mathfrak{S}}$ is the integral operator of Definition II.8. Its properties are described in Theorem II.18, below.

(7) $\mathcal{R}_{\Omega ; \theta}$ is constructed like $\mathcal{D}_{\Omega ; \theta}$, but with $v=0$.

(8) By this we mean that every monomial appearing in the power series expansion of these functions contains a factor of the form $\alpha_{*}\left(\mathbf{x}_{1}\right) \alpha_{*}\left(\mathbf{x}_{2}\right) \beta\left(\mathbf{x}_{3}\right) \beta\left(\mathbf{x}_{4}\right)$. 
Definition II.17 Let $\Omega \subset X$ and $\theta, \mathfrak{v}>0$. We define the technical small field regulator

$$
\operatorname{Reg}_{\mathcal{S F}}(\Omega ; \alpha, \beta)=\operatorname{Reg}_{\mathcal{S F}}^{(2)}(\Omega ; \alpha, \beta)+\operatorname{Reg}_{\mathcal{S F}}^{(4)}(\Omega ; \alpha, \beta)
$$

with

$$
\begin{aligned}
\operatorname{Reg}_{\mathcal{S F}}^{(2)}(\Omega ; \alpha, \beta)= & K_{\text {reg }} \theta|\mu|\left[\|\alpha\|_{\Omega}^{2}+\|\beta\|_{\Omega}^{2}\right] \\
\operatorname{Reg}_{\mathcal{S} \mathcal{F}}^{(4)}(\Omega ; \alpha, \beta)= & K_{\text {reg }} \theta \mathfrak{v}\left\{\|\alpha\|_{L^{4}(\Omega)}^{3}+\|\beta\|_{L^{4}(\Omega)}^{3}\right\}\left\{\|\alpha-\beta\|_{L^{4}(\tilde{\Omega})}\right. \\
& \left.+\theta\left[\mu+e^{-5 \operatorname{mc}(\theta)}\right]\left[\|\alpha\|_{L^{4}(\tilde{\Omega})}+\|\beta\|_{L^{4}(\tilde{\Omega})}\right]+\theta\left[\|\nabla \alpha\|_{L^{4}\left(\tilde{\Omega}^{*}\right)}+\|\nabla \beta\|_{L^{4}\left(\tilde{\Omega}^{*}\right)}\right]\right\}
\end{aligned}
$$

Here $\tilde{\Omega}$ is the set of points of $X$ that are within a distance $\mathfrak{c}(\theta)$ of $\Omega$, and $K_{\text {reg }}=$ $2^{9} \exp \left\{20 e^{12 \mathrm{~m}} \mathrm{D} D_{\mathcal{H}}\right\}$.

In addition to the constants of (II.17), we choose $0<e_{\ell}<2 e_{\mathrm{r}}$ and set

$$
\ell(t)=\left(\frac{1}{t \mathfrak{v}}\right)^{e_{\ell}}
$$

Theorem II.18 Let $\Omega \subset X$ and assume that $\alpha$ and $\beta$ obey the small field conditions $\chi_{\theta}(\Omega ; \alpha, \beta)=1$. Then,

$$
\begin{aligned}
& e^{-\frac{1}{2}\|\alpha\|^{2}-\frac{1}{2}\|\beta\|^{2}} e^{\operatorname{Re}\left(\left\langle\alpha^{*}, j_{(\Omega)}(\theta) \beta\right\rangle+V_{\Omega ; \theta}\left(\alpha_{*}, \beta\right)\right)} e^{-\operatorname{Reg}_{\mathcal{S} \mathcal{F}}(\Omega ; \alpha, \beta)}
\end{aligned}
$$

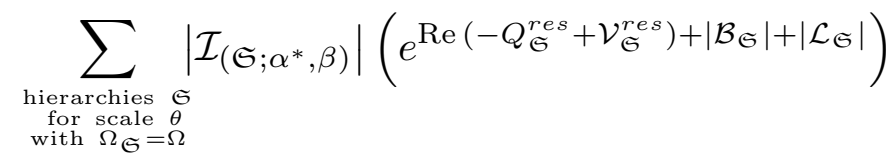

$$
\begin{aligned}
& \leq e^{-\frac{1}{4} \ell(\theta)\left|\Omega^{c}\right|}\left[\prod_{\mathbf{x} \in \Omega^{c}} \frac{1}{1+|\alpha(\mathbf{x})|^{3}} \frac{1}{1+|\beta(\mathbf{x})|^{3}}\right]
\end{aligned}
$$

In the above theorem,

○ the factor $\prod_{\mathbf{x} \in \Omega^{c}} \frac{1}{1+|\alpha(\mathbf{x})|^{3}} \frac{1}{1+|\beta(\mathbf{x})|^{3}}$ on the right hand side ensures the left hand side, which is a function of $\{\alpha(\mathbf{x}), \beta(\mathbf{x})\}_{\mathbf{x} \in X}$ is integrable,

○ the factor $e^{-\ell(\theta)\left|\Omega^{c}\right|}$ on the right hand side tells us that when the large field region $\Omega^{c}$ is large, then the left hand side is small and

A stronger bound than that of Theorems II.18 is given in (III.22). 


\section{Strategy of the Proof}

Our proof of Theorem II.16 goes roughly as follows. In a first step, we fix $\varepsilon=2^{-k} \theta$ for some $k \in \mathbb{N}$ and show that there is a representation for $I_{k}\left(\varepsilon ; \alpha^{*}, \beta\right)$ similar to (II.13), but with a sum over hierarchies for scale $\theta$ which have depth at most $k$. In a second step we compare the resulting representations for $I_{k}\left(2^{-k} \theta ; \alpha^{*}, \beta\right)$ and $I_{k+1}\left(2^{-(k+1)} \theta ; \alpha^{*}, \beta\right)$ in order to take the limit $k \rightarrow \infty$.

For the first step, we use the decimation strategy sketched in the introduction. We construct, for $n=1,2, \cdots, k$ a representation of $I_{n}\left(\varepsilon ; \alpha^{*}, \beta\right)$ similar to (II.13), but with a sum over hierarchies for scale $2^{n} \varepsilon=2^{n-k} \theta$. In the step (I.10) from $n$ to $n+1$,

$$
I_{n+1}\left(\varepsilon ; \alpha^{*}, \beta\right)=\int d \mu_{\mathrm{R}(\varepsilon)}\left(\phi^{*}, \phi\right) I_{n}\left(\varepsilon ; \alpha^{*}, \phi\right) I_{n}\left(\varepsilon ; \phi^{*}, \beta\right)
$$

write the representation of $I_{n}\left(\varepsilon ; \alpha^{*}, \phi\right)$ resp. $I_{n}\left(\varepsilon ; \phi^{*}, \beta\right)$ as a sum over such hierarchies $\mathfrak{S}_{1}$ resp. $\mathfrak{S}_{2}$ and write the integral as a sum of integrals, indexed by $\left(\mathfrak{S}_{1}, \mathfrak{S}_{2}\right)$. One such integral leads to the sum of terms in the representation of $I_{n+1}$ that are associated to the hierarchies for scale $2^{n+1} \varepsilon=2^{n-k+1} \theta$ that are preceded by $\left(\mathfrak{S}_{1}, \mathfrak{S}_{2}\right)$ in the following sense.

Definition III.1 A pair $\left(\mathfrak{S}_{1}, \mathfrak{S}_{2}\right)$ of hierarchies for scale $\delta$ is said to precede the hierarchy $\mathfrak{S}$ for scale $2 \delta$ if

$$
S_{\mathfrak{S}}(\mathcal{J})=S_{\mathfrak{S}_{1}}(\mathcal{J}) \quad \text { and } \quad S_{\mathfrak{S}}(\delta+\mathcal{J})=S_{\mathfrak{S}_{2}}(\mathcal{J})
$$

for all $S=\Lambda, \Omega, P_{\alpha}, P_{\beta}, P_{\alpha}^{\prime}, P_{\beta}^{\prime}, Q, R$ and all decimation intervals $\mathcal{J}$ in $[0, \delta]$. In this case, we write

$$
\left(\mathfrak{S}_{1}, \mathfrak{S}_{2}\right) \prec \mathfrak{S}
$$

We also denote, for any field $\vec{\alpha}=\left(\alpha_{\tau}(\mathbf{x})\right)_{\substack{\tau \in \varepsilon \mathbb{Z} \cap(0,2 \delta) \\ \mathbf{x} \in X}}$, the left and right half fields to be

$$
\vec{\alpha}_{l}=\left(\alpha_{\tau}(\mathbf{x})\right) \underset{\substack{\tau \in \varepsilon \mathbb{Z} \cap(0, \delta) \\ \mathbf{x} \in X}}{ } \quad \vec{\alpha}_{r}=\left(\alpha_{\tau+\delta}(\mathbf{x})\right)_{\substack{\tau \in \varepsilon \mathbb{Z} \cap(0, \delta) \\ \mathbf{x} \in X}}
$$

Given hierarchies $\mathfrak{S}_{1}, \mathfrak{S}_{2}$ for scale $\delta$, the choice of a hierarchy $\mathfrak{S}$ with $\left(\mathfrak{S}_{1}, \mathfrak{S}_{2}\right) \prec \mathfrak{S}$ amounts to the choice of

(i) the small/large field sets of the first kind

$$
\begin{aligned}
& P_{\alpha}([0,2 \delta]), P_{\beta}([0,2 \delta]) \subset \Omega_{\mathfrak{S}_{1}} \cap \Omega_{\mathfrak{S}_{2}} \\
& P_{\alpha}^{\prime}([0,2 \delta]), P_{\beta}^{\prime}([0,2 \delta]) \subset\left(\Omega_{\mathfrak{S}_{1}} \cap \Omega_{\mathfrak{S}_{2}}\right)^{*} \\
& Q([0,2 \delta]) \subset\left(\Omega_{\mathfrak{S}_{1}} \cap \Omega_{\mathfrak{S}_{2}}\right)^{\star}
\end{aligned}
$$


By Definition II.4, these sets determine $\Lambda([0,2 \delta])$.

(ii) the large field set of the second kind $R([0,2 \delta]) \subset \Lambda([0,2 \delta])$. Again, by Definition II.4, this set determines the new small field set of the second kind $\Omega([0,2 \delta])$.

In a decimation step as outlined above, we start with two hierarchies $\mathfrak{S}_{1}, \mathfrak{S}_{2}$ for scale $\delta$. Then we first decompose $\Omega_{\mathfrak{S}_{1}} \cap \Omega_{\mathfrak{S}_{2}}$ into large/small field sets of the first kind, and afterwards decompose the resulting small field sets $\Lambda$ according to the choice of the regions where "stationary phase" is applied. To formalize the first step, we use

Definition III.2 Let $\Omega_{0} \subset X$ and $\delta>0$. We denote by $F_{\delta}\left(\Omega_{0}\right)$ the set of all choices of "small/large field sets of the first kind"

$$
\mathfrak{A}=\left(\Lambda, P_{\alpha}, P_{\beta}, P_{\alpha}^{\prime}, P_{\beta}^{\prime}, Q\right)
$$

with

$$
\begin{aligned}
& \circ \Lambda, P_{\alpha}, P_{\beta} \subset \Omega_{0}, P_{\alpha}^{\prime}, P_{\beta}^{\prime} \subset \Omega_{0}^{*} \text { and } Q \subset \Omega_{0}^{\star} \\
& \circ \Lambda=\left\{\mathbf{x} \in X \mid d\left(\mathbf{x}, P_{\alpha} \cup P_{\beta} \cup Q \cup \operatorname{supp} P_{\alpha}^{\prime} \cup \operatorname{supp} P_{\beta}^{\prime} \cup \Omega_{0}^{c}\right)>\mathfrak{c}(\delta)\right\}
\end{aligned}
$$

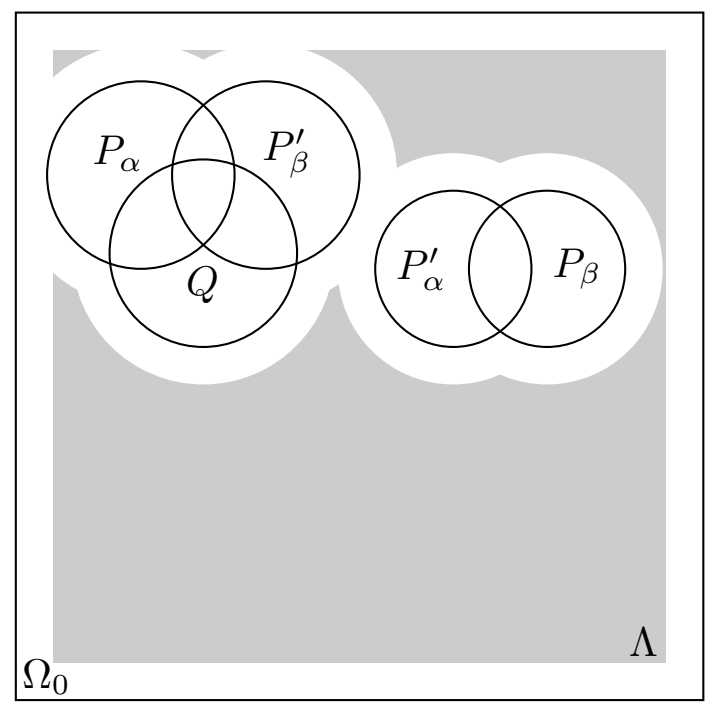

\section{III.1 History Fields}

As described in the previous section, the decimation step from scale $\delta$ to scale $2 \delta$ involves integrals of products of pairs of terms like in (II.13), indexed by two hierarchies of $\mathfrak{S}_{1}, \mathfrak{S}_{2}$ for scale $\delta$. The result of this integral will be represented as a sum over all hierarchies $\mathfrak{S}$ of scale $2 \delta$ that are preceded by $\left(\mathfrak{S}_{1}, \mathfrak{S}_{2}\right)$. Each such term should contain 
a factor

$$
e^{\left\langle\alpha^{*}, j_{\left(\Omega_{\mathfrak{S}}\right)}(2 \delta) \beta\right\rangle+V_{\Omega_{\mathfrak{S}} ; 2 \delta}\left(\varepsilon ; \alpha^{*}, \beta\right)+\mathcal{D}_{\Omega_{\mathfrak{S}} ; 2 \delta}\left(\varepsilon ; \alpha^{*}, \beta\right)}
$$

For the construction of $\mathcal{D}_{\Omega_{\mathfrak{S}} ; 2 \delta}(\varepsilon ; \cdot, \cdot)$ out of $\mathcal{D}_{\Omega_{\mathfrak{S}_{1}} ; \delta}(\varepsilon ; \cdot, \cdot)$ and $\mathcal{D}_{\Omega_{\mathfrak{S}_{2}} ; \delta}(\varepsilon ; \cdot, \cdot)$ we need to know which contributions to $\mathcal{D}_{\Omega_{\mathfrak{S}_{i}} ; \delta}(\varepsilon ; \cdot, \cdot)$ involved points outside the new small field region $\Omega_{\mathfrak{S}}$.

To keep track of precisely which points were involved in the construction of each contribution, we introduce the concept of a history field. This is a field on $X$ that takes only the values 0 and 1 . In particular

$$
\mathfrak{h}^{2}=\mathfrak{h}
$$

The history field is never integrated over. We put in a history field at each point where some construction is performed. That is, we shall always work with "history complete" functions in the following sense.

\section{Definition III.3}

(i) A function $f\left(\phi_{1}, \cdots, \phi_{s} ; \mathfrak{h}\right)$ in the fields $\phi_{1}, \cdots, \phi_{s}, \mathfrak{h}$ is called history complete if it is in fact a function of $\phi_{1} \mathfrak{h}, \cdots, \phi_{s} \mathfrak{h}, \mathfrak{h}$. If $f$ is a history complete analytic function, any non trivial monomial in its power series expansion that contains a factor $\phi_{i}(\mathbf{x})$ automatically also contains a factor $\mathfrak{h}(\mathbf{x})$.

(ii) Given a power series $f\left(\phi_{1}, \cdots, \phi_{s} ; \mathfrak{h}\right)$ and a subset $\Omega$ of $X$ we set

$$
\left.f\right|_{\Omega}=\left.f\left(\phi_{1}, \cdots, \phi_{s} ; \mathfrak{h}\right)\right|_{\mathfrak{h}(\mathbf{x})=0 \text { for } \mathbf{x} \in X \backslash \Omega}
$$

If $f$ is history complete, $\left.f\right|_{\Omega}$ depends only on the fields $\phi_{1}(\mathbf{x}), \cdots, \phi_{s}(\mathbf{x}), \mathfrak{h}(\mathbf{x})$ with $\mathbf{x} \in \Omega$.

The starting points of our construction are the single particle Hamiltonian $\mathrm{h}=\nabla^{*} \mathcal{H} \nabla$ and the interaction $v$. Already at this point, we have to monitor the points in space involved in the construction, for example in the exponential $j(t)=e^{t(\mathrm{~h}-\mu)}$. This is the motivation for the notion of $\mathfrak{h}$-operator introduced in [BFKT4, Definition IV.1]. For the convenience of the reader, we repeat some of the concepts introduced in [BFKT4].

\section{Definition III.4}

(i) An $\mathfrak{h}$-operator or $\mathfrak{h}$-linear map $A$ on $\mathbb{C}^{X}$ is a linear operator on $\mathbb{C}^{X}$ whose kernel is of the form

$$
A(\mathbf{x}, \mathbf{y})=\sum_{\ell=0}^{\infty} \sum_{\left(\mathbf{x}_{1}, \cdots, \mathbf{x}_{\ell}\right) \in X^{\ell}} A\left(\mathbf{x} ; \mathbf{x}_{1}, \cdots, \mathbf{x}_{\ell} ; \mathbf{y}\right) \mathfrak{h}(\mathbf{x}) \mathfrak{h}\left(\mathbf{x}_{1}\right) \cdots \mathfrak{h}\left(\mathbf{x}_{\ell}\right) \mathfrak{h}(\mathbf{y})
$$


(ii) The composition $A \circ B$ of two $\mathfrak{h}$-operators $A, B$ on $\mathbb{C}^{X}$ is by definition the $\mathfrak{h}$-operator with kernel

$$
\begin{aligned}
(A \circ B)(\mathbf{x}, \mathbf{y}) & =\sum_{\mathbf{z} \in X} A(\mathbf{x}, \mathbf{z}) B(\mathbf{z}, \mathbf{y}) \\
& =\sum_{\mathbf{z} \in X} \sum_{\substack{\ell, \ell^{\prime} \geq 0 \\
\mathbf{x}_{1}, \cdots, \mathbf{x}_{\ell} \\
\mathbf{y}_{1}, \cdots \mathbf{y}_{\ell^{\prime}}}} A\left(\mathbf{x} ; \mathbf{x}_{1}, \cdots, \mathbf{x}_{\ell} ; \mathbf{z}\right) B\left(\mathbf{z} ; \mathbf{y}_{1}, \cdots, \mathbf{y}_{\ell^{\prime}} ; \mathbf{y}\right) \\
& \mathfrak{h}(\mathbf{x}) \mathfrak{h}\left(\mathbf{x}_{1}\right) \cdots \mathfrak{h}\left(\mathbf{x}_{\ell}\right) \mathfrak{h}(\mathbf{z}) \mathfrak{h}\left(\mathbf{y}_{1}\right) \cdots \mathfrak{h}\left(\mathbf{y} \ell_{\ell^{\prime}}\right) \mathfrak{h}(\mathbf{y})
\end{aligned}
$$

Here we used that $\mathfrak{h}^{2}=\mathfrak{h}$.

(iii) For an "ordinary" linear operator $J$ on $\mathbb{C}^{X}$ with kernel $J(\mathbf{x}, \mathbf{y})$, we define the associated $\mathfrak{h}$-operator by

$$
\bar{J}(\mathbf{x}, \mathbf{y})=\mathfrak{h}(\mathbf{x}) J(\mathbf{x}, \mathbf{y}) \mathfrak{h}(\mathbf{y})
$$

and the associated $\mathfrak{h}$-exponential as

$$
\operatorname{exph}(J)=\mathfrak{h}+\sum_{\ell=1}^{\infty} \frac{1}{\ell !} \bar{J}^{\ell}=\mathfrak{h} e^{\mathfrak{h} J \mathfrak{h}}=e^{\mathfrak{h} J \mathfrak{h}} \mathfrak{h}
$$

The $\mathfrak{h}$-exponential obeys the product rule exph $\left(J_{1}\right) \operatorname{exph}\left(J_{2}\right)=\operatorname{exph}\left(J_{1}+J_{2}\right)$, provided the operators $\bar{J}_{1}$ and $\bar{J}_{2}$ commute.

(iv) If $\phi$ is any field on $X$ and $A$ an $\mathfrak{h}$-operator, we set

$$
\begin{aligned}
(A \phi)(\mathbf{x}) & =\sum_{\mathbf{y} \in X} A(\mathbf{x}, \mathbf{y}) \phi(\mathbf{y}) \\
& =\sum_{\ell=0}^{\infty} \sum_{\mathbf{x}_{1}, \cdots, \mathbf{x}_{\ell}, \mathbf{y}} A\left(\mathbf{x} ; \mathbf{x}_{1}, \cdots, \mathbf{x}_{\ell} ; \mathbf{y}\right) \mathfrak{h}(\mathbf{x}) \mathfrak{h}\left(\mathbf{x}_{1}\right) \cdots \mathfrak{h}\left(\mathbf{x}_{\ell}\right) \mathfrak{h}(\mathbf{y}) \phi(\mathbf{y})
\end{aligned}
$$

To keep the notation simple, we shall frequently use the same symbol for a history complete function or $\mathfrak{h}$-operator as we used in Chapter II for the function or operator one gets if one sets $\mathfrak{h}(\mathbf{x})=1$ for all $\mathbf{x} \in X$. So we shall again write $v$ for the operator $\bar{v}$, and set

$$
j(t)=\operatorname{exph}(-t(\mathrm{~h}-\mu))
$$

Observe that

$$
\left.j(t)\right|_{\substack{\mathfrak{h}(\mathbf{x})=0 \text { for } \mathbf{x} \in X \backslash \Omega \\ \mathfrak{h}(\mathbf{x})=1 \text { for } \mathbf{x} \in \Omega}}=j_{(\Omega)}(t)
$$

with the operator $j_{(\Omega)}(t)$ introduced at the beginning of Section II.1. Again we define

$$
j_{\mathfrak{c}}(\tau)(\mathbf{x}, \mathbf{y})=j(\tau)(\mathbf{x}, \mathbf{y}) \cdot \begin{cases}1 & \text { if } d(\mathbf{x}, \mathbf{y}) \leq \mathfrak{c} \\ 0 & \text { if } d(\mathbf{x}, \mathbf{y})>\mathfrak{c}\end{cases}
$$




\section{III.2 Properties of the Background Fields}

Here, and through the rest of the paper, use the same definition as before for background fields (that is Definition II.9), just with $j(t)$ being interpreted as the $\mathfrak{h}$-operator of (III.1). Here, we want to study some of their properties. In particular, we develop a recursion relation (Proposition III.6).

\section{Remark III.5 (The structure of the background fields)}

Let $\mathfrak{S}$ be a hierarchy for scale $\delta$.

(i) When the history field is identically zero, the background field becomes

$$
\left.\Gamma_{* \mathfrak{S}}\left(\tau ; \alpha_{*}, \vec{\alpha}_{*}\right)\right|_{\mathfrak{h}=0}=\left.\Lambda_{\tau}^{c} \alpha_{* \tau} \quad \Gamma_{\mathfrak{S}}(\tau ; \vec{\alpha}, \beta)\right|_{\mathfrak{h}=0}=\Lambda_{\tau}^{c} \alpha_{\tau}
$$

The differences $\Gamma_{* \mathfrak{S}}\left(\tau ; \alpha_{*}, \vec{\alpha}_{*}\right)-\Lambda_{\tau}^{c} \alpha_{* \tau}$ and $\Gamma_{\mathfrak{S}}(\tau ; \vec{\alpha}, \beta)-\Lambda_{\tau}^{c} \alpha_{\tau}$ are history complete. Their restrictions to the small field region $\Omega_{\mathfrak{S}}$ are

$$
\left.\Gamma_{* \mathfrak{S}}\left(\tau ; \alpha_{*}, \vec{\alpha}_{*}\right)\right|_{\Omega_{\mathfrak{S}}}-\Lambda_{\tau}^{c} \alpha_{* \tau}=\left.j(\tau) \alpha_{*}\right|_{\Omega_{\mathfrak{S}}},\left.\quad \Gamma_{\mathfrak{S}}(\tau ; \vec{\alpha}, \beta)\right|_{\Omega_{\mathfrak{S}}}-\Lambda_{\tau}^{c} \alpha_{\tau}=\left.j(\delta-\tau) \beta\right|_{\Omega_{\mathfrak{S}}}
$$

(ii) Let $\tau=\delta \sum_{k=1}^{\mathfrak{d}(\tau)} \frac{a_{k}}{2^{k}}, a_{k} \in\{0,1\}$, be the "binary expansion" of the decimation point $\tau \in(0, \delta)$, and let $\tau^{\prime} \in(0, \delta)$ be another decimation point different from $\tau$. Then $\Gamma_{* \tau}^{\tau^{\prime}}=0$ unless $\tau^{\prime}$ is one of the "binary approximations" $\delta \sum_{k=1}^{d} \frac{a_{k}}{2^{k}}\left(1 \leq d<\mathfrak{d}(\tau), \quad a_{d}=1\right)$ of $\tau$. In this case, let $d^{\prime}=\min \left\{k>d \mid a_{k}=1\right\}$. Then

$$
\Gamma_{* \tau}^{\tau^{\prime}}=j\left(\tau-\tau^{\prime}-\frac{\delta}{2^{d^{\prime}}}\right) \Lambda\left(\left[\tau^{\prime}, \tau^{\prime}+\frac{\delta}{2^{d^{\prime}}-1}\right]\right) j\left(\frac{\delta}{2^{d^{\prime}}}\right) \Lambda_{\tau^{\prime}}^{c}
$$

In particular, $\Gamma_{* \tau}^{\tau^{\prime}}=0$ whenever $\tau^{\prime}>\tau$ or $\mathfrak{d}\left(\tau^{\prime}\right)>\mathfrak{d}(\tau)$.

Analogous statements hold for $\Gamma_{\tau}^{\tau^{\prime}}$, in terms of the binary expansions of $\delta-\tau$ and $\delta-\tau^{\prime}$. In particular, $\Gamma_{\tau}^{\tau^{\prime}}=0$ whenever $\tau^{\prime}<\tau$ or $\mathfrak{d}\left(\tau^{\prime}\right)>\mathfrak{d}(\tau)$.

(iii) If $\tau \neq \tau^{\prime}$, then $\Gamma_{* \tau}^{\tau^{\prime}}$ and $\Gamma_{\tau}^{\tau^{\prime}}$ are always of the form $j\left(\tau_{1}\right) \Lambda_{1} j\left(\tau_{2}\right) \Lambda_{2}^{c}$ with $\tau_{1}, \tau_{2} \geq 0$, $\tau_{1}+\tau_{2}=\left|\tau-\tau^{\prime}\right|$ and $\Lambda_{1}$ and $\Lambda_{2}^{c}$ being (possibly trivial) characteristic functions.

(iv) Let $\tau \in(0, \delta)$ and $\tau^{\prime} \in[0, \delta]$ be decimation points with $\mathfrak{d}(\tau)>\mathfrak{d}\left(\tau^{\prime}\right)$. (For $\tau^{\prime}=0, \delta$, set $\mathfrak{d}\left(\tau^{\prime}\right)=0$.) Furthermore let $0<t \leq \frac{\delta}{2^{\mathfrak{d}(\tau)}}$. If $\tau$ is not of the form $\tau^{\prime}+\frac{\delta}{2^{d}}$ for any $\mathfrak{d}\left(\tau^{\prime}\right) \leq d \leq \operatorname{depth}(\mathfrak{S})$, then $\Gamma_{* \tau}^{\tau^{\prime}}=j(t) \Gamma_{* \tau-t}^{\tau^{\prime}}$. If $\tau$ is not of the form $\tau^{\prime}-\frac{\delta}{2^{d}}$ for any $\mathfrak{d}\left(\tau^{\prime}\right) \leq d \leq \operatorname{depth}(\mathfrak{S})$, then $\Gamma_{\tau}^{\tau^{\prime}}=j(t) \Gamma_{\tau+t}^{\tau^{\prime}}$. For more information, see Lemma E.16.

(v) If $\mathfrak{d}\left(\tau^{\prime}\right)>\operatorname{depth}(\mathfrak{S})$ then $\Gamma_{* \tau}^{\tau^{\prime}}=\Gamma_{\tau}^{\tau^{\prime}}=0$ for all $\tau \in(0, \delta)$. 
Proof: (i) Since $j(t)$ vanishes when $\mathfrak{h}$ is identically zero, $\left.\Gamma_{* \tau}^{\tau^{\prime}}\right|_{\mathfrak{h}=0}=\Gamma_{\tau}^{\tau^{\prime}}=\left.\right|_{\mathfrak{h}=0}=0$ for all $\tau^{\prime} \neq \tau$. For all $\tau^{\prime} \in(0, \delta), \Omega_{\mathfrak{S}} \cap \Lambda_{\tau^{\prime}}^{c}=\emptyset$ and hence $\left.\Gamma_{* \tau}^{\tau^{\prime}}\right|_{\Omega_{\mathfrak{S}}}=\left.\Gamma_{\tau}^{\tau^{\prime}}\right|_{\Omega_{\mathfrak{S}}}=0$ whenever $\tau^{\prime} \neq \tau$. Finally,

$$
\left.\Gamma_{* \tau}^{0}\right|_{\Omega_{\mathfrak{S}}}=\left.j\left(\tau-\frac{\delta^{\prime}}{2}\right) \Omega_{\mathfrak{S}} j\left(\frac{\delta^{\prime}}{2}\right)\right|_{\Omega_{\mathfrak{S}}}=\left.j\left(\tau-\frac{\delta^{\prime}}{2}\right) j\left(\frac{\delta^{\prime}}{2}\right)\right|_{\Omega_{\mathfrak{S}}}=\left.j(\tau)\right|_{\Omega_{\mathfrak{S}}}
$$

and $\left.\Gamma_{\tau}^{\delta}\right|_{\Omega_{\mathfrak{S}}}=\left.j(\delta-\tau)\right|_{\Omega_{\mathfrak{S}}}$

(ii) Assume that $\Gamma_{* \tau}^{\tau^{\prime}} \neq 0$. Denote by $\mathcal{J}$ the smallest decimation interval with $\tau^{\prime}$ as its left endpoint that strictly contains $\left[\tau^{\prime}, \tau\right]$. As $\mathcal{J}$ is a decimation interval, there is $d^{\prime} \geq \mathfrak{d}\left(\tau^{\prime}\right)+1$ such that $\mathcal{J}=\left[\tau^{\prime}, \tau^{\prime}+\frac{\delta}{2^{d^{\prime}-1}}\right]$. Since $\left[\tau^{\prime}, \tau^{\prime}+\frac{\delta}{2^{d^{\prime}}}\right]$ is also a decimation interval, but does not contain $\tau$ in its interior, we have $\tau^{\prime}+\frac{\delta}{2^{d^{\prime}}} \leq \tau<\tau^{\prime}+\frac{\delta}{2^{d^{\prime}-1}}$.

Set $d=\mathfrak{d}\left(\tau^{\prime}\right)<\mathfrak{d}(\tau)$ and let $\tau^{\prime}=\delta \sum_{k=1}^{d} \frac{b_{k}}{2^{k}}, \quad b_{k} \in\{0,1\}$ be the "binary expansion" of $\tau^{\prime}$. As $\frac{\delta}{2^{d^{\prime}}} \leq \tau-\tau^{\prime}<\frac{\delta}{2^{d^{\prime}-1}}$ and $d^{\prime}>d$ we have

$$
a_{k}=b_{k} \text { for } k=1, \cdots, d, \quad a_{d}=1, \quad a_{d+1}=\cdots=a_{d^{\prime}-1}=0, \quad a_{d^{\prime}}=1
$$

So $\tau^{\prime}$ is a binary approximation of $\tau$. The remaining claims follow directly from the Definition II.9 of the background fields.

(iii) follows by inspection. Recall that $j(0)=\mathfrak{h}$.

(iv) We consider $\Gamma_{* \tau}^{\tau^{\prime}}$. By part (ii) we may assume that $\tau>\tau^{\prime}$. Observe that $\left[\tau^{\prime}, \tau^{\prime}+\frac{\delta}{2^{\mathfrak{o}\left(\tau^{\prime}\right)}}\right]$ is the maximal decimation interval with $\tau^{\prime}$ as left endpoint. If $\tau$ does not lie in this interval, then $\tau-t \geq \tau-\frac{\delta}{2^{\mathfrak{o}(\tau)}} \geq \tau^{\prime}+\frac{\delta}{2^{\mathfrak{o}\left(\tau^{\prime}\right)}}$, and consequently $\Gamma_{* \tau}^{\tau^{\prime}}=\Gamma_{* \tau-t}^{\tau^{\prime}}=0$. So we may assume that $\tau \in\left(\tau^{\prime}, \tau^{\prime}+\frac{\delta}{2^{\mathfrak{D}\left(\tau^{\prime}\right)}}\right)$.

Let $\mathcal{J}=\left[\tau^{\prime}, \tau^{\prime}+\frac{\delta}{2^{d-1}}\right], d>\mathfrak{d}\left(\tau^{\prime}\right)$ be the smallest decimation interval with $\tau^{\prime}$ as left endpoint such that $\left[\tau^{\prime}, \tau\right] \varsubsetneqq \mathcal{J}$. Then $\tau^{\prime}+\frac{\delta}{2^{d}} \leq \tau<\tau^{\prime}+\frac{\delta}{2^{d-1}}$. If $\tau \neq \tau^{\prime}+\frac{\delta}{2^{d}}$ then, again, $\tau-t \geq \tau-\frac{\delta}{2^{\mathfrak{d}(\tau)}} \geq \tau^{\prime}+\frac{\delta}{2^{d}}$ and consequently

$$
\Gamma_{* \tau}^{\tau^{\prime}}-j(t) \Gamma_{* \tau-t}^{\tau^{\prime}}=\left[j\left(\tau-\tau^{\prime}-\frac{\delta}{2^{d}}\right)-j(t) j\left(\tau-t-\tau^{\prime}-\frac{\delta}{2^{d}}\right)\right] \Lambda(\mathcal{J}) j\left(\frac{\delta}{2^{d}}\right) \Lambda_{\tau^{\prime}}^{c}=0
$$

If $\tau=\tau^{\prime}+\frac{\delta}{2^{d}}$, but $d>\operatorname{depth}(\mathfrak{S})$, then $\Lambda(\mathcal{J})=X$ and

$$
\Gamma_{* \tau}^{\tau^{\prime}}=\Lambda(\mathcal{J}) j\left(\frac{\delta}{2^{d}}\right) \Lambda_{\tau^{\prime}}^{c}=j\left(\tau-\tau^{\prime}\right) \Lambda_{\tau^{\prime}}^{c} \quad, \quad \Gamma_{* \tau-t}^{\tau^{\prime}}=j\left(\tau-t-\tau^{\prime}\right) \Lambda_{\tau^{\prime}}^{c}
$$

(v) In this case $\Lambda_{\tau^{\prime}}^{c}=\emptyset$. 
In the decimation step from scale $\delta$ to scale $2 \delta$, we are passing from the product of the terms indexed by hierarchies $\mathfrak{S}_{1}, \mathfrak{S}_{2}$ for scale $\delta$ to a sum of terms indexed by hierarchies $\mathfrak{S}$ that are preceded by $\left(\mathfrak{S}_{1}, \mathfrak{S}_{2}\right)$ in the sense of Definition III.1. For this reason, we need

Proposition III.6 (Recursion relation for the background fields) Let $\left(\mathfrak{S}_{1}, \mathfrak{S}_{2}\right)$ be a pair of hierarchies for scale $\delta$ that precede the hierarchy $\mathfrak{S}$ for scale $2 \delta$. Let $\tau \in(0,2 \delta)$ be a decimation point. Then

$$
\Gamma_{* \mathfrak{S}}\left(\tau ; \alpha_{*}, \vec{\alpha}_{*}\right)= \begin{cases}\Gamma_{* \mathfrak{S}_{1}}\left(\tau ; \alpha_{*}, \vec{\alpha}_{* l}\right) & \text { if } \tau \in(0, \delta) \\ \Lambda_{\mathfrak{S}} j(\delta) \alpha_{*}+\Lambda_{\mathfrak{S}}^{c} \alpha_{* \delta} & \text { if } \tau=\delta \\ \Gamma_{* \mathfrak{S}_{2}}\left(\tau-\delta ; \Lambda_{\mathfrak{S}} j(\delta) \alpha_{*}+\Lambda_{\mathfrak{S}}^{c} \alpha_{* \delta}, \vec{\alpha}_{* r}\right)+\partial \Gamma_{* \tau} \alpha_{*} & \text { if } \tau \in(\delta, 2 \delta)\end{cases}
$$

Here, for $\tau \in(\delta, 2 \delta)$, the difference operator $\partial \Gamma_{* \tau}$ is defined as follows. Let $\mathcal{J}$ be the smallest decimation interval for $\mathfrak{S}$ with left endpoint $\delta$ such that $[\delta, \tau]$ is strictly contained in $\mathcal{J}$, and let $\delta^{\prime}$ be the length of $\mathcal{J}$. Then

$$
\partial \Gamma_{* \tau}=j\left(\tau-\delta-\frac{\delta^{\prime}}{2}\right) \Lambda_{\mathfrak{S}}(\mathcal{J})^{c} j\left(\frac{\delta^{\prime}}{2}\right) \Lambda_{\mathfrak{S}} j(\delta)
$$

Similarly,

$$
\Gamma_{\mathfrak{S}}(\tau ; \vec{\alpha}, \beta)= \begin{cases}\Gamma_{\mathfrak{S}_{1}}\left(\tau ;, \vec{\alpha}_{l}, \Lambda_{\mathfrak{S}} j(\delta) \beta+\Lambda_{\mathfrak{S}}^{c} \alpha_{\delta}\right)+\partial \Gamma_{\tau} \beta & \text { if } \tau \in(0, \delta) \\ \Lambda_{\mathfrak{S}} j(\delta) \beta+\Lambda_{\mathfrak{S}}^{c} \alpha_{\delta} & \text { if } \tau=\delta \\ \Gamma_{\mathfrak{S}_{2}}\left(\tau-\delta ; \vec{\alpha}_{r}, \beta\right) & \text { if } \tau \in(\delta, 2 \delta)\end{cases}
$$

Here, for $\tau \in(0, \delta)$, the difference operator $\partial \Gamma_{\tau}$ is defined as follows. Let $\mathcal{J}$ be the smallest decimation interval for $\mathfrak{S}$ with right endpoint $\delta$ such that $[\tau, \delta]$ is strictly contained in $\mathcal{J}$, and let $\delta^{\prime}$ be the length of $\mathcal{J}$. Then

$$
\partial \Gamma_{\tau}=j\left(\delta-\tau-\frac{\delta^{\prime}}{2}\right) \Lambda_{\mathfrak{S}}(\mathcal{J})^{c} j\left(\frac{\delta^{\prime}}{2}\right) \Lambda_{\mathfrak{S}} j(\delta)
$$

Proof: If $\tau \in(0, \delta)$ and $0 \leq \tau^{\prime} \leq \tau$ is a decimation point, then $\Gamma_{* \tau}^{\tau^{\prime}}(\mathfrak{S})=\Gamma_{* \tau}^{\tau^{\prime}}\left(\mathfrak{S}_{1}\right)$ by Definition II.9. By Remark III.5.ii, $\Gamma_{* \tau}^{\tau^{\prime}}(\mathfrak{S})=0$ and $\Gamma_{* \tau}^{\tau^{\prime}}\left(\mathfrak{S}_{1}\right)=0$ whenever $\tau^{\prime}>\tau$. If $\tau=\delta$ then $\Gamma_{* \mathfrak{S}}\left(\tau ; \alpha_{*}, \vec{\alpha}_{*}\right)=\Lambda_{\mathfrak{S}} j(\delta) \alpha_{*}+\Lambda_{\mathfrak{S}}^{c} \alpha_{* \delta}$ directly by Definition II.9. Now let $\tau \in(\delta, 2 \delta)$. Directly from Definition II.9,

$$
\Gamma_{* \tau}^{\tau^{\prime}}(\mathfrak{S})= \begin{cases}\Gamma_{* \tau-\delta}^{0}\left(\mathfrak{S}_{2}\right) \Lambda_{\mathfrak{S}}^{c} & \text { if } \tau^{\prime}=\delta \\ \Gamma_{* \tau-\delta}^{\tau^{\prime}-\delta}\left(\mathfrak{S}_{2}\right) & \text { if } \delta<\tau^{\prime}<2 \delta \\ 0 & \text { if } 0<\tau^{\prime}<\delta\end{cases}
$$


so that

$$
\begin{aligned}
\Gamma_{* \mathfrak{S}}\left(\tau ; \alpha_{*}, \vec{\alpha}_{*}\right)-\Gamma_{* \mathfrak{S}_{2}}\left(\tau-\delta ; \Lambda_{\mathfrak{S}} j(\delta) \alpha_{*}+\Lambda_{\mathfrak{S}}^{c} \alpha_{* \delta}, \vec{\alpha}_{* r}\right) \\
=\Gamma_{* \tau}^{0}(\mathfrak{S}) \alpha_{*}-\Gamma_{* \tau-\delta}^{0}\left(\mathfrak{S}_{2}\right) \Lambda_{\mathfrak{S}} j(\delta) \alpha_{*}
\end{aligned}
$$

Let $\mathcal{J}$ be the interval defined in the Proposition, and set $\mathcal{J}^{\prime}=\{t-\delta \mid t \in \mathcal{J}\}$. Then $\Lambda_{\mathfrak{S}}(\mathcal{J})=\Lambda_{\mathfrak{S}_{2}}\left(\mathcal{J}^{\prime}\right)$ and

$$
\begin{aligned}
\Gamma_{* \tau}^{0}(\mathfrak{S})-\Gamma_{* \tau-\delta}^{0}\left(\mathfrak{S}_{2}\right) \Lambda_{\mathfrak{S}} j(\delta) & =j(\tau-\delta) \Lambda_{\mathfrak{S}} j(\delta)-j\left(\tau-\delta-\frac{\delta^{\prime}}{2}\right) \Lambda_{\mathfrak{S}_{2}}\left(\mathcal{J}^{\prime}\right) j\left(\frac{\delta^{\prime}}{2}\right) \Lambda_{\mathfrak{S}} j(\delta) \\
& =j\left(\tau-\delta-\frac{\delta^{\prime}}{2}\right) \Lambda_{\mathfrak{S}_{2}}\left(\mathcal{J}^{\prime}\right)^{c} j\left(\frac{\delta^{\prime}}{2}\right) \Lambda_{\mathfrak{S}} j(\delta)=\partial \Gamma_{* \tau}
\end{aligned}
$$

Here, we used $j(\tau-\delta)=j\left(\tau-\delta-\frac{\delta^{\prime}}{2}\right)\left(\Lambda_{\mathfrak{S}_{2}}\left(\mathcal{J}^{\prime}\right)^{c}+\Lambda_{\mathfrak{S}_{2}}\left(\mathcal{J}^{\prime}\right)\right) j\left(\frac{\delta^{\prime}}{2}\right)$.

The recursion representation for $\Gamma_{\mathfrak{S}}$ is proven similarly.

Further properties and alternative definitions of the background fields are given in Appendix E.

\section{III.3 The Explicit Quadratic and Quartic Terms in the Effective Action}

In (II.9), we defined the prospective dominant contribution to the quadratic part of the effective action associated to a hierarchy $\mathfrak{S}$ for scale $\delta$ to be

$$
Q_{\mathfrak{S}}\left(\alpha_{*}, \beta ; \vec{\alpha}_{*}, \vec{\alpha}\right)=Q_{\varepsilon, \delta}\left(\alpha_{*}, \beta ; \Gamma_{* \mathfrak{S}}\left(\cdot ; \alpha_{*}, \vec{\alpha}_{*}\right), \Gamma_{\mathfrak{S}}(\cdot ; \vec{\alpha}, \beta)\right)
$$

where $Q_{\varepsilon, \delta}$ was defined in (II.10) and $\varepsilon$ was chosen to be $2^{-\operatorname{depth}(\mathfrak{S})} \delta$. Here, and through the rest of the paper, we use the same definition but with $j(t)$ being interpreted as the $\mathfrak{h}$-operator of (III.1) and with the background fields $\Gamma_{* \mathfrak{S}}, \Gamma_{\mathfrak{S}}$ of $\S$ III.2.

Part (i) of the following Lemma shows that, in (III.3), we could have chosen $\varepsilon=$ $2^{-n} \delta$ for any $n \geq \operatorname{depth}(\mathfrak{S})$. Part (ii) isolates the pure small field part and the historyindependent part. Part (iii) gives a recursion relation.

\section{Lemma III.7}

(i) Let $\mathfrak{S}$ be a hierarchy at scale $\delta$. For all $k, n \geq \operatorname{depth}(\mathfrak{S})$

$$
Q_{\frac{\delta}{2^{k}}, \delta}\left(\alpha_{*}, \beta ; \Gamma_{* \mathfrak{S}}\left(\cdot ; \alpha_{*}, \vec{\alpha}_{*}\right), \Gamma_{\mathfrak{S}}(\cdot ; \vec{\alpha}, \beta)\right)=Q_{\frac{\delta}{2^{n}}, \delta}\left(\alpha_{*}, \beta ; \Gamma_{* \mathfrak{S}}\left(\cdot ; \alpha_{*}, \vec{\alpha}_{*}\right), \Gamma_{\mathfrak{S}}(\cdot ; \vec{\alpha}, \beta)\right)
$$

(ii) Let $\mathfrak{S}$ be a hierarchy at scale $\delta$. When the history field is identically zero,

$$
\left.Q_{\mathfrak{S}}\left(\alpha_{*}, \beta ; \vec{\alpha}_{*}, \vec{\alpha}\right)\right|_{\mathfrak{h}=0}=\sum_{\tau \in \varepsilon \mathbb{Z} \cap(0, \delta)}\left\langle\Lambda_{\tau}^{c} \alpha_{* \tau}, \Lambda_{\tau}^{c} \alpha_{\tau}\right\rangle
$$


where $\varepsilon=2^{-\operatorname{depth}(\mathfrak{S})} \delta$. The difference $Q_{\mathfrak{S}}\left(\alpha_{*}, \beta ; \vec{\alpha}_{*}, \vec{\alpha}\right)-\sum_{\tau}\left\langle\Lambda_{\tau}^{c} \alpha_{* \tau}, \Lambda_{\tau}^{c} \alpha_{\tau}\right\rangle$ is history complete. Its restriction to the small field region $\Omega_{\mathfrak{S}}$ is

$$
\left.Q_{\mathfrak{S}}\left(\alpha_{*}, \beta ; \vec{\alpha}_{*}, \vec{\alpha}\right)\right|_{\Omega_{\mathfrak{S}}}-\sum_{\tau \in \varepsilon \mathbb{Z} \cap(0, \delta)}\left\langle\Lambda_{\tau}^{c} \alpha_{* \tau}, \Lambda_{\tau}^{c} \alpha_{\tau}\right\rangle=-\left.\left\langle\alpha_{*}, j(\delta) \beta\right\rangle\right|_{\Omega_{\mathfrak{S}}}
$$

(iii) Let $\mathfrak{S}_{1}, \mathfrak{S}_{2}$ be hierarchies of scale $\delta$ such that $\left(\mathfrak{S}_{1}, \mathfrak{S}_{2}\right) \prec \mathfrak{S}$, where $\mathfrak{S}$ is a hierarchy of scale $2 \delta$. Then

$$
\begin{aligned}
Q_{\mathfrak{S}}\left(\alpha_{*}, \beta ; \vec{\alpha}_{*}, \vec{\alpha}\right)=Q_{\mathfrak{S}_{1}} & \left(\alpha_{*}, \Lambda j(\delta) \beta+\Lambda^{c} \alpha_{\delta} ; \vec{\alpha}_{* l}, \vec{\alpha}_{l}\right)+Q_{\mathfrak{S}_{2}}\left(\Lambda j(\delta) \alpha_{*}+\Lambda^{c} \alpha_{* \delta}, \beta ; \vec{\alpha}_{* r}, \vec{\alpha}_{r}\right) \\
& +\left\langle\Lambda j(\delta) \alpha_{*}, \Lambda j(\delta) \beta\right\rangle+\left\langle\Lambda^{c} \alpha_{* \delta}, \Lambda^{c} \alpha_{\delta}\right\rangle \\
& +\sum_{\tau \in \varepsilon \mathbb{Z} \cap[0, \delta)}\left\langle\Gamma_{* \mathfrak{S}_{1}}\left(\tau ; \alpha_{*}, \vec{\alpha}_{* l}\right),\left(\partial \Gamma_{\tau}-j(\varepsilon) \partial \Gamma_{\tau+\varepsilon}\right) \beta\right\rangle \\
& +\sum_{\tau \in \varepsilon \mathbb{Z} \cap(0, \delta]}\left\langle\left(\partial \Gamma_{* \delta+\tau}-j(\varepsilon) \partial \Gamma_{* \delta+\tau-\varepsilon}\right) \alpha_{*}, \Gamma_{\mathfrak{S}_{2}}\left(\tau ; \vec{\alpha}_{r}, \beta\right)\right\rangle
\end{aligned}
$$

where $\Lambda=\Lambda_{\mathfrak{S}}, \varepsilon=2^{-\operatorname{depth}(\mathfrak{S})}$ and the differences $\partial \Gamma_{* \tau}, \partial \Gamma_{\tau}$ were defined in Proposition III. 6 for $\tau \in(\delta, 2 \delta)$ and $\tau \in(0, \delta)$, respectively. Here, we have also set $\partial \Gamma_{* \delta}=\partial \Gamma_{* 2 \delta}=$ $\partial \Gamma_{0}=\partial \Gamma_{\delta}=0$ and $\Gamma_{* \mathfrak{S}_{1}}(0 ; \cdot)=\alpha_{*}, \Gamma_{\mathfrak{S}_{2}}(\delta ; \cdot)=\beta$.

(iv) Let $\mathfrak{S}$ be a hierarchy of scale $\delta$. Then for any fields $\phi_{*}, \phi$

$$
\begin{aligned}
Q_{\mathfrak{S}}\left(\alpha_{*}, \Lambda j(\delta) \beta+\Lambda^{c} \phi ; \vec{\alpha}_{*}, \vec{\alpha}\right)-Q_{\mathfrak{S}}\left(\alpha_{*}, \Lambda j_{\mathfrak{c}}(\delta) \beta+\Lambda^{c} \phi ; \vec{\alpha}_{*}, \vec{\alpha}\right) \\
=\sum_{\tau \in \varepsilon \mathbb{Z} \cap[0, \delta)}\left\langle\Gamma_{* \mathfrak{S}}\left(\tau ; \alpha_{*}, \vec{\alpha}_{*}\right),\left(\Gamma_{\tau}^{\delta}-j(\varepsilon) \Gamma_{\tau+\varepsilon}^{\delta}\right) \Lambda\left(j(\delta)-j_{\mathfrak{c}}(\delta)\right) \beta\right\rangle \\
Q_{\mathfrak{S}}\left(\Lambda j(\delta) \alpha_{*}+\Lambda^{c} \phi_{*}, \beta ; \vec{\alpha}_{*}, \vec{\alpha}\right)-Q_{\mathfrak{S}}\left(\Lambda j_{\mathfrak{c}}(\delta) \alpha_{*}+\Lambda^{c} \phi_{*}, \beta ; \vec{\alpha}_{*}, \vec{\alpha}\right) \\
=\sum_{\tau \in \varepsilon \mathbb{Z} \cap(0, \delta]}\left\langle\left(\Gamma_{* \tau}^{0}-j(\varepsilon) \Gamma_{* \tau-\varepsilon}^{0}\right) \Lambda\left(j(\delta)-j_{\mathfrak{c}}(\delta)\right) \alpha_{*}, \Gamma_{\mathfrak{S}}(\tau ; \vec{\alpha}, \beta)\right\rangle
\end{aligned}
$$

where $\Lambda=\Lambda_{\mathfrak{S}}$ and, again, $\varepsilon=2^{-\operatorname{depth}(\mathfrak{S})}$. Here, we have also set $\Gamma_{* \delta}^{0}=\Gamma_{0}^{\delta}=0$ and $\Gamma_{* \mathfrak{S}}(0 ; \cdot)=\alpha_{*}, \Gamma_{\mathfrak{S}}(\delta ; \cdot)=\beta$ and, correspondingly, $\Gamma_{* 0}^{0}=\Gamma_{\delta}^{\delta}=11$.

Proof: (i) It suffices to prove this in the case that $k>\operatorname{depth}(\mathfrak{S})$ and $n=k-1$. To simplify notation, set $\varepsilon=2^{-k} \delta$,

$$
\gamma_{* \tau}=\left\{\begin{array}{ll}
\Gamma_{* \mathfrak{S}}\left(\tau ; \alpha_{*}, \vec{\alpha}_{*}\right) & \text { if } \tau \neq 0 \\
\alpha_{*} & \text { if } \tau=0
\end{array} \quad \gamma_{\tau}= \begin{cases}\Gamma_{\mathfrak{S}}(\tau ; \vec{\alpha}, \beta) & \text { if } \tau \neq \delta \\
\beta & \text { if } \tau=\delta\end{cases}\right.
$$

If $\tau, \tau^{\prime}$ are decimation points with $\mathfrak{d}(\tau)=k$ and $\mathfrak{d}\left(\tau^{\prime}\right) \leq \operatorname{depth}(\mathfrak{S})$, then by Remark III.5.iv, $\Gamma_{* \tau}^{\tau^{\prime}}=j(\varepsilon) \Gamma_{* \tau-\varepsilon}^{\tau^{\prime}}$. Combining this with Remark III.5.v, we see that

$$
\gamma_{* \tau}=j(\varepsilon) \gamma_{* \tau-\varepsilon} \quad \text { if } \mathfrak{d}(\tau)=k
$$


So by (II.10)

$$
\begin{aligned}
Q_{\varepsilon, \delta}\left(\alpha_{*}, \beta ; \vec{\gamma}_{*}, \vec{\gamma}\right) & =\sum_{\tau \in \varepsilon \mathbb{Z} \cap(0, \delta)}\left\langle\gamma_{* \tau}-j(\varepsilon) \gamma_{* \tau-\varepsilon}, \gamma_{\tau}\right\rangle-\left\langle\gamma_{* \delta-\varepsilon}, j(\varepsilon) \beta\right\rangle \\
& =\sum_{\tau \in 2 \varepsilon \mathbb{Z} \cap(0, \delta)}\left\langle\gamma_{* \tau}-j(\varepsilon) \gamma_{* \tau-\varepsilon}, \gamma_{\tau}\right\rangle-\left\langle j(\varepsilon) \gamma_{* \delta-2 \varepsilon}, j(\varepsilon) \beta\right\rangle \\
& =\sum_{\tau \in 2 \varepsilon \mathbb{Z} \cap(0, \delta)}\left\langle\gamma_{* \tau}-j(\varepsilon) j(\varepsilon) \gamma_{* \tau-2 \varepsilon}, \gamma_{\tau}\right\rangle-\left\langle\gamma_{* \delta-2 \varepsilon}, j(\varepsilon) j(\varepsilon) \beta\right\rangle \\
& =Q_{2 \varepsilon, \delta}\left(\alpha_{*}, \beta ; \vec{\gamma}_{*}, \vec{\gamma}\right)
\end{aligned}
$$

(ii) Define $\gamma_{* \tau}$ and $\gamma_{\tau}$ as above. By Remark III.5.i,

$$
\left.j(\varepsilon) \gamma_{* \tau-\varepsilon}\right|_{\mathfrak{h}=0}=\left.0 \quad j(\varepsilon) \gamma_{* \tau-\varepsilon}\right|_{\Omega_{\mathfrak{S}}}=\left.j(\tau) \alpha_{*}\right|_{\Omega_{\mathfrak{S}}}
$$

Both equations follow from the definition (II.10) and Remark III.5.i.

(iii) Let $\varepsilon=2^{-\operatorname{depth}(\mathfrak{S})}(2 \delta)=2^{-(\operatorname{depth}(\mathfrak{S})-1)} \delta$. Define $\gamma_{* \tau}$ and $\gamma_{\tau}$ as above, but with $\delta$ replaced by $2 \delta$. By Proposition III. 6

$$
\gamma_{* \tau}=\left\{\begin{array}{ll}
\gamma_{* \tau}^{(1)} & \text { if } \tau \in(0, \delta) \\
\Lambda j(\delta) \alpha_{*}+\Lambda^{c} \alpha_{* \delta} & \text { if } \tau=\delta \\
\gamma_{* \tau-\delta}^{(2)}+\partial \Gamma_{* \tau} \alpha_{*} & \text { if } \tau \in(\delta, 2 \delta)
\end{array} \quad \gamma_{\tau}= \begin{cases}\gamma_{\tau}^{(1)}+\partial \Gamma_{\tau} \beta & \text { if } \tau \in(0, \delta) \\
\Lambda j(\delta) \beta+\Lambda^{c} \alpha_{\delta} & \text { if } \tau=\delta \\
\gamma_{\tau-\delta}^{(2)} & \text { if } \tau \in(\delta, 2 \delta)\end{cases}\right.
$$

where $\Lambda=\Lambda_{\mathfrak{S}}$ and

$$
\begin{aligned}
\gamma_{* \tau}^{(1)} & =\Gamma_{* \mathfrak{S}_{1}}\left(\tau ; \alpha_{*}, \vec{\alpha}_{* l}\right) & \gamma_{\tau}^{(1)} & =\Gamma_{\mathfrak{S}_{1}}\left(\tau ; \vec{\alpha}_{l}, \Lambda j(\delta) \beta+\Lambda^{c} \alpha_{\delta}\right) \\
\gamma_{* \tau}^{(2)} & =\Gamma_{* \mathfrak{S}_{2}}\left(\tau ; \Lambda j(\delta) \alpha_{*}+\Lambda^{c} \alpha_{* \delta}, \vec{\alpha}_{* r}\right) & \gamma_{\tau}^{(2)} & =\Gamma_{\mathfrak{S}_{2}}\left(\tau ; \vec{\alpha}_{r}, \beta\right)
\end{aligned}
$$

Then

$$
\begin{aligned}
Q_{\mathfrak{S}}= & \sum_{\tau \in \varepsilon \mathbb{Z} \cap(0, \delta)}\left\langle\gamma_{* \tau}^{(1)}-j(\varepsilon) \gamma_{* \tau-\varepsilon}^{(1)}, \gamma_{\tau}^{(1)}+\partial \Gamma_{\tau} \beta\right\rangle \\
& +\left\langle\Lambda j(\delta) \alpha_{*}+\Lambda^{c} \alpha_{* \delta}-j(\varepsilon) \gamma_{* \delta-\varepsilon}^{(1)}, \Lambda j(\delta) \beta+\Lambda^{c} \alpha_{\delta}\right\rangle \\
& +\left\langle\gamma_{* \varepsilon}^{(2)}+\partial \Gamma_{* \delta+\varepsilon} \alpha_{*}-j(\varepsilon)\left(\Lambda j(\delta) \alpha_{*}+\Lambda^{c} \alpha_{* \delta}\right), \gamma_{\varepsilon}^{(2)}\right\rangle \\
& +\sum_{\tau \in \varepsilon \mathbb{Z} \cap(\varepsilon, \delta)}\left\langle\gamma_{* \tau}^{(2)}+\partial \Gamma_{* \delta+\tau} \alpha_{*}-j(\varepsilon)\left(\gamma_{* \tau-\varepsilon}^{(2)}+\partial \Gamma_{* \delta+\tau-\varepsilon} \alpha_{*}\right), \gamma_{\tau}^{(2)}\right\rangle \\
& -\left\langle\gamma_{* \delta-\varepsilon}^{(2)}+\partial \Gamma_{* 2 \delta-\varepsilon} \alpha_{*}, j(\varepsilon) \beta\right\rangle
\end{aligned}
$$




$$
\begin{aligned}
& =\sum_{\tau \in \varepsilon \mathbb{Z} \cap(0, \delta)}\left\langle\gamma_{* \tau}^{(1)}-j(\varepsilon) \gamma_{* \tau-\varepsilon}^{(1)}, \gamma_{\tau}^{(1)}\right\rangle-\left\langle j(\varepsilon) \gamma_{* \delta-\varepsilon}^{(1)}, \Lambda j(\delta) \beta+\Lambda^{c} \alpha_{\delta}\right\rangle \\
& +\left\langle\gamma_{* \varepsilon}^{(2)}-j(\varepsilon)\left(\Lambda j(\delta) \alpha_{*}+\Lambda^{c} \alpha_{* \delta}\right), \gamma_{\varepsilon}^{(2)}\right\rangle \\
& \quad+\sum_{\tau \in \varepsilon \mathbb{Z} \cap(\varepsilon, \delta)}\left\langle\gamma_{* \tau}^{(2)}-j(\varepsilon) \gamma_{* \tau-\varepsilon}^{(2)}, \gamma_{\tau}^{(2)}\right\rangle-\left\langle\gamma_{* \delta-\varepsilon}^{(2)}, j(\varepsilon) \beta\right\rangle \\
& \quad+\left\langle\Lambda j(\delta) \alpha_{*}, \Lambda j(\delta) \beta\right\rangle+\left\langle\Lambda^{c} \alpha_{* \delta}, \Lambda^{c} \alpha_{\delta}\right\rangle \\
& \quad+\sum_{\tau \in \varepsilon \mathbb{Z} \cap(0, \delta)}\left\langle\gamma_{* \tau}^{(1)}-j(\varepsilon) \gamma_{* \tau-\varepsilon}^{(1)}, \partial \Gamma_{\tau} \beta\right\rangle \\
& \quad+\left\langle\partial \Gamma_{* \delta+\varepsilon} \alpha_{*}, \gamma_{\varepsilon}^{(2)}\right\rangle+\sum_{\tau \in \varepsilon \mathbb{Z} \cap(\varepsilon, \delta)}\left\langle\partial \Gamma_{* \delta+\tau} \alpha_{*}-j(\varepsilon) \partial \Gamma_{* \delta+\tau-\varepsilon} \alpha_{*}, \gamma_{\tau}^{(2)}\right\rangle \\
& \quad-\left\langle\partial \Gamma_{* 2 \delta-\varepsilon} \alpha_{*}, j(\varepsilon) \beta\right\rangle \\
& =Q_{\mathfrak{S}_{1}}\left(\alpha_{*}, \Lambda j(\delta) \beta+\Lambda^{c} \alpha_{\delta} ; \vec{\alpha}_{* l}, \vec{\alpha}_{l}\right)+Q_{\mathfrak{S}_{2}}\left(\Lambda j(\delta) \alpha_{*}+\Lambda^{c} \alpha_{* \delta}, \beta ; \vec{\alpha}_{* r}, \vec{\alpha}_{r}\right) \\
& +\left\langle\Lambda j(\delta) \alpha_{*}, \Lambda j(\delta) \beta\right\rangle+\left\langle\Lambda^{c} \alpha_{* \delta}, \Lambda^{c} \alpha_{\delta}\right\rangle \\
& \quad+\sum_{\tau \in \varepsilon \mathbb{Z} \cap[0, \delta)}\left\langle\gamma_{* \tau}^{(1)},\left(\partial \Gamma_{\tau}-j(\varepsilon) \partial \Gamma_{\tau+\varepsilon}\right) \beta\right\rangle \\
& +\sum_{\tau \in \varepsilon \mathbb{Z} \cap(0, \delta]}\left\langle\left(\partial \Gamma_{* \delta+\tau}-j(\varepsilon) \partial \Gamma_{* \delta+\tau-\varepsilon}\right) \alpha_{*}, \gamma_{\tau}^{(2)}\right\rangle
\end{aligned}
$$

with $\partial \Gamma_{0}=\partial \Gamma_{\delta}=\partial \Gamma_{* \delta}=\partial \Gamma_{* 2 \delta}=0$.

(iv) The first equality follows from (II.10) and the fact that

$$
\Gamma_{\mathfrak{S}}\left(\tau ; \vec{\alpha}, \Lambda j(\delta) \beta+\Lambda^{c} \phi ; \vec{\alpha}\right)-\Gamma_{\mathfrak{S}}\left(\tau ; \vec{\alpha}, \Lambda j_{\mathfrak{c}}(\delta) \beta+\Lambda^{c} \phi ; \vec{\alpha}\right)=\Gamma_{\tau}^{\delta} \Lambda\left(j(\delta)-j_{\mathfrak{c}}(\delta)\right) \beta
$$

The second equality follows from (II.10) and the fact that

$$
\Gamma_{* \mathfrak{S}}\left(\tau ; \Lambda j(\delta) \alpha_{*}+\Lambda^{c} \phi_{*} ; \vec{\alpha}_{*}\right)-\Gamma_{* \mathfrak{S}}\left(\tau ; \Lambda j_{\mathfrak{c}}(\delta) \alpha_{*}+\Lambda^{c} \phi_{*} ; \vec{\alpha}_{*}\right)=\Gamma_{* \tau}^{0} \Lambda\left(j(\delta)-j_{\mathfrak{c}}(\delta)\right) \alpha_{*}
$$

The quartic part of the interaction for a given decimation step depends on the scale

at which the process has been started. To keep track of this we need the Riemann sums approximating (II.11).

\section{Definition III.8}

(i) Let $\mathfrak{S}$ be a hierarchy for scale $\delta$, and let $\varepsilon=2^{-n} \delta$ with $n \geq \operatorname{depth}(\mathfrak{S})$. We define

$$
\mathcal{V}_{\mathfrak{S}}\left(\varepsilon ; \alpha_{*}, \beta ; \vec{\alpha}_{*}, \vec{\alpha}\right)=\varepsilon \mathcal{V}_{n}\left(\alpha_{*}, \beta ; \Gamma_{* \mathfrak{S}}\left(\cdot ; \alpha_{*}, \vec{\alpha}_{*}\right), \Gamma_{\mathfrak{S}}(\cdot ; \vec{\alpha}, \beta)\right)
$$

where

$$
\begin{aligned}
\mathcal{V}_{n}\left(\alpha_{*}, \beta ; \vec{\gamma}_{*}, \vec{\gamma}\right)=-\left[\left\langle\alpha_{*} \gamma_{\varepsilon}, v \alpha_{*} \gamma_{\varepsilon}\right\rangle\right. & +\sum_{\tau \in \varepsilon \mathbb{Z} \cap(0, \delta-\varepsilon)}\left\langle\gamma_{* \tau} \gamma_{\tau+\varepsilon}, v \gamma_{* \tau} \gamma_{\tau+\varepsilon}\right\rangle \\
& \left.+\left\langle\gamma_{* \delta-\varepsilon} \beta, v \gamma_{* \delta-\varepsilon} \beta\right\rangle\right]
\end{aligned}
$$


and $\Gamma_{* \mathfrak{S}}\left(\tau ; \alpha_{*}, \vec{\alpha}_{*}\right), \Gamma_{\mathfrak{S}}(\tau ; \vec{\alpha}, \beta)$ are the background fields.

(ii) In the remark below we shall identify the small field part of $\mathcal{V}_{\mathfrak{S}}$. For that purpose, we define for any subset $\Omega$ of $X$ and every $\delta$ that is an integer multiple of $\varepsilon$

$$
V_{\Omega, \delta}\left(\varepsilon ; \alpha_{*}, \beta\right)=-\left.\varepsilon \sum_{\tau \in \varepsilon \mathbb{Z} \cap[0, \delta)}\left\langle\left[j(\tau) \alpha^{*}\right][j(\delta-\tau-\varepsilon) \beta], v\left[j(\tau) \alpha^{*}\right][j(\delta-\tau-\varepsilon) \beta]\right\rangle\right|_{\Omega}
$$

(Evaluating this at $\mathfrak{h}=1$ gives the $V_{\Omega, \delta}\left(\varepsilon ; \alpha_{*}, \beta\right)$ of (II.1).)

Clearly, $\mathcal{V}_{\mathfrak{S}}$ is history complete (in particular $\left.\mathcal{V}_{\mathfrak{S}}\right|_{\mathfrak{h}=0}=0$ ). It follows from Remark III.5.i that

\section{Remark III.9}

(i) If $\varepsilon=2^{-n} \delta$ with $n \geq \operatorname{depth}(\mathfrak{S})$ then $\left.\mathcal{V}_{\mathfrak{S}}\left(\varepsilon ; \alpha_{*}, \beta ; \vec{\alpha}_{*}, \vec{\alpha}\right)\right|_{\Omega}=V_{\Omega, \delta}\left(\varepsilon ; \alpha_{*}, \beta\right)$ for all $\Omega \subset \Omega_{\mathfrak{S}}$.

(ii) $V_{\Omega, \delta}\left(\varepsilon ; \alpha_{*}, j(\delta) \beta\right)+V_{\Omega, \delta}\left(\varepsilon ; j(\delta) \alpha_{*}, \beta\right)=V_{\Omega, 2 \delta}\left(\varepsilon ; \alpha_{*}, \beta\right)$

\section{III.4 Properties of the Large Field Integral Operator}

In our representation of $I_{n}\left(\varepsilon ; \alpha^{*}, \beta\right)$, we persist in using the integral operator $\mathcal{I}_{\left(\mathfrak{S} ; \alpha^{*}, \beta\right)}$ of Definition II.8, except that the surface $C_{s}\left(\mathbf{x} ; \alpha^{*}, \beta\right)$ is now $\mathfrak{h}$-dependent through the operators $j_{\mathfrak{c}}(s)$ of (II.7). Note that the characteristic functions $\chi_{\mathcal{J}}\left(\alpha, \alpha_{\tau}, \beta\right)$ and the cutoff Gaussian measures do not depend on $\mathfrak{h}$.

\section{Lemma III.10}

(i) Let $\left(\mathfrak{S}_{1}, \mathfrak{S}_{2}\right)$ be a pair of hierarchies for scale $\delta$ that precede the hierarchy $\mathfrak{S}$ for scale $2 \delta$. We have the recursion relation

$$
\begin{aligned}
& \mathcal{I}_{\left(\mathfrak{S} ; \alpha^{*}, \beta\right)}\left[d \mu\left(\vec{\alpha}, \vec{z}_{*}, \vec{z}\right)\right] \\
& =\mathcal{I}_{\left([0,2 \delta], \mathfrak{S} ; \alpha^{*}, \beta\right)} \mathcal{I}_{\left(\mathfrak{S}_{1} ; \alpha^{*}, \alpha_{\delta}\right)}\left[d \mu\left(\vec{\alpha}_{l}, \vec{z}_{* l}, \vec{z}_{l}\right)\right] \quad \mathcal{I}_{\left(\mathfrak{S}_{2} ; \alpha_{\delta}^{*}, \beta\right)}\left[d \mu\left(\vec{\alpha}_{r}, \vec{z}_{* r}, \vec{z}_{r}\right)\right]
\end{aligned}
$$

(ii) Let $\mathfrak{S}$ be a hierarchy for scale $\delta$ and set $\varepsilon=2^{-\operatorname{depth}(\mathfrak{S})} \delta$. The large field operator $\mathcal{I}_{\left(\mathfrak{S} ; \alpha^{*}, \beta\right)}\left[d \mu\left(\vec{\alpha}, \vec{z}_{*}, \vec{z}\right)\right]$ depends on $\alpha(\mathbf{y})$ and $\beta(\mathbf{y})$ only for $\mathbf{y} \in \Omega_{\mathfrak{S}}^{c}$.

Proof: Part (i) is trivial. To prove part (ii) by induction, it suffices to show that $\mathcal{I}_{\left([0,2 \delta], \mathfrak{S} ; \alpha^{*}, \beta\right)}$ depends on $\alpha(\mathbf{y})$ and $\beta(\mathbf{y})$ only for $\mathbf{y} \in \Omega([0,2 \delta])^{c}$. The dependence of 
$\mathcal{I}_{\left([0,2 \delta], \mathfrak{S} ; \alpha^{*}, \beta\right)}$ on $\alpha(\mathbf{y})$ and $\beta(\mathbf{y})$ arises only through two mechanisms. First, through the integration domain $C_{\delta}\left(\mathbf{x} ; \alpha^{*}, \beta\right)$ with $\mathbf{x} \in R([0,2 \delta])$. By construction, this integration domain depends only on $\alpha(\mathbf{y})$ and $\beta(\mathbf{y})$ for $\mathbf{y}$ within a distance $\mathfrak{c}$ of $R([0,2 \delta])$. Since $\Omega([0,2 \delta])$ is the set of all points of $\Lambda([0,2 \delta])$ whose distance from $R([0,2 \delta])$ is at least $\mathfrak{c}(\delta)$, any such $\mathbf{y}$ is in $\Omega([0,2 \delta])^{c}$. The second dependence is through the characteristic function $\chi_{[0,2 \delta]}\left(\alpha, \alpha_{\delta}, \beta\right)$. One sees by direct inspection of its definition in Appendix $\mathrm{A}$ that this characteristic function is independent of $\alpha(\mathbf{y})$ and $\beta(\mathbf{y})$ for all $\mathbf{y} \in \Omega([0,2 \delta])$.

Remark III.11 Let $\mathfrak{S}$ be a hierarchy for scale $\delta$ and set $\varepsilon=2^{-\operatorname{depth}(\mathfrak{S})} \delta$. The integral operator $\mathcal{I}_{\left(\mathfrak{S} ; \alpha^{*}, \beta\right)}\left[d \mu\left(\vec{\alpha}, \vec{z}_{*}, \vec{z}\right)\right]$ acts on the space of functions that are defined and measurable in the variables

$$
\begin{array}{lll}
z_{\tau}(\mathbf{x}) & \mathbf{x} \in \Lambda(\mathcal{J}) \backslash(R(\mathcal{J}) \cup \Omega(\mathcal{J})) & \text { with }\left|z_{\tau}(\mathbf{x})\right| \leq \mathrm{r}(s) \\
z_{* \tau}(\mathbf{x}), z_{\tau}(\mathbf{x}) & \mathbf{x} \in R(\mathcal{J}) & \text { with }\left|z_{* \tau}(\mathbf{x})\right|,\left|z_{\tau}(\mathbf{x})\right| \leq \mathrm{R}(s) \\
\alpha_{\tau}(\mathbf{x}) & \mathbf{x} \in \Lambda(\mathcal{J})^{c} & \text { with }\left|\alpha_{\tau}(\mathbf{x})\right| \leq \mathrm{R}(\varepsilon)
\end{array}
$$

and analytic in the variables $z_{* \tau}(\mathbf{x}), z_{\tau}(\mathbf{x}), \mathbf{x} \in R\left(\mathcal{J}_{\tau}\right)$. Here $\mathcal{J}=\mathcal{J}_{\tau}$ runs over all decimation intervals in $[0, \delta]$ of length $2 \varepsilon \leq 2 s \leq \delta$.

Proof: The first two restrictions appear explicitly in the limits of integration in Definition II.8. The restriction $\left|\alpha_{\tau}(\mathbf{x})\right| \leq \mathrm{R}(\varepsilon)$ is an immediate consequence of Lemma A.4.a.

We shall prove a bound on these large field integral operators, with $\mathfrak{h} \equiv 1$, in Theorem II.18. To obtain a good bound, we make a specific choice of $C_{s}\left(\mathbf{x} ; \alpha^{*}, \beta\right)$. Roughly speaking, each point $\mathbf{x} \in R(\mathcal{J})$ will provide a very small factor for the size of the integral over $C_{s}\left(\mathbf{x} ; \alpha^{*}, \beta\right)$ which arises from the factor $e^{-z_{* \tau}(\mathbf{x}) z_{\tau}(\mathbf{x})}$. This will be established in Proposition III.38, below.

\section{III.5 Norms and the Renormalization Group Map}

In our description of the effective density in Theorem II.16, the non-explicit quantities $\mathcal{D}_{\Omega_{\mathfrak{S}}}, \mathcal{B}_{\mathfrak{S}}$ and $\mathcal{L}_{\mathfrak{S}}$ are estimated with the help of the norms $\|\cdot\|_{\mathfrak{S}}$. These norms were defined abstractly in Definition II.12 and then made concrete by the choice of the weight factors in Definition II.13. In the construction itself, all functions involve history fields. For this reason, we extend, in Definition III.12 below, the abstract definition of norms to the case of functions that depend on a history field too. In this abstract setting, we recall the main theorem from $[\mathrm{BFKT} 4, \S \mathrm{II}]$ that will allow us to control the fluctuation 
integrals (Theorem III.14). A fluctutation integral is performed for every triple $\mathfrak{S}_{1}, \mathfrak{S}_{2}, \mathfrak{S}$ of hierarchies with $\left(\mathfrak{S}_{1}, \mathfrak{S}_{2}\right) \prec \mathfrak{S}$. (See the discussion before Definition III.1.) Therefore, we discuss, in Remark III.17, how the weight factors for $\mathfrak{S}_{1}, \mathfrak{S}_{2}$ and $\mathfrak{S}$ are related.

In each decimaation step, the fluctuation integral is introduced through coordinates that are centred on the critical point of the quadratic part of the effective interaction. This change of variables affects our norms and is controlled using the operator norm of Definition III.18, below.

Definition III.12 Let $\phi_{1}, \cdots, \phi_{s}$ be a collection of fields on $X$ and let $\mathfrak{h}$ be a history field on $X$.

(i) Let $f\left(\phi_{1}, \cdots, \phi_{s} ; \mathfrak{h}\right)$ be a function which is defined and analytic on a neighbourhood of the origin in $\mathbb{C}^{(s+1)|X|}$. Then $f$ has a unique expansion of the form

$$
f\left(\phi_{1}, \cdots, \phi_{s} ; \mathfrak{h}\right)=\sum_{n_{1}, \cdots, n_{s+1} \geq 0} \sum_{\left(\overrightarrow{\mathbf{x}}_{1}, \cdots, \overrightarrow{\mathbf{x}}_{s+1}\right) \in X^{n_{1}} \times \cdots \times X^{n_{s+1}}} a\left(\overrightarrow{\mathbf{x}}_{1}, \cdots, \overrightarrow{\mathbf{x}}_{s} ; \overrightarrow{\mathbf{x}}_{s+1}\right) \phi_{1}\left(\overrightarrow{\mathbf{x}}_{1}\right) \cdots \phi_{s}\left(\overrightarrow{\mathbf{x}}_{s}\right) \mathfrak{h}\left(\overrightarrow{\mathbf{x}}_{s+1}\right)
$$

with the coefficients $a\left(\overrightarrow{\mathbf{x}}_{1}, \cdots, \overrightarrow{\mathbf{x}}_{s} ; \overrightarrow{\mathbf{x}}_{s+1}\right)$ invariant under permutations of the components of each vector $\overrightarrow{\mathbf{x}}_{j}$. The functions $a\left(\overrightarrow{\mathbf{x}}_{1}, \cdots, \overrightarrow{\mathbf{x}}_{s} ; \overrightarrow{\mathbf{x}}_{s+1}\right)$ are called the (symmetric) coefficient system for $f$.

(ii) For any $n_{1}, \cdots, n_{s+1} \geq 0$ and any function $b\left(\overrightarrow{\mathbf{x}}_{1}, \cdots, \overrightarrow{\mathbf{x}}_{s} ; \overrightarrow{\mathbf{x}}_{s+1}\right)$ on $X^{n_{1}} \times \cdots \times X^{n_{s+1}}$, we define the norm $\|b\|_{n_{1}, \cdots, n_{s+1}}$ as follows:

$\circ$ If there is at least one nonhistory field, that is if $\sum_{j=1}^{s} n_{j} \neq 0$, then

$$
\|b\|_{n_{1}, \cdots, n_{s+1}}=\max _{\mathbf{x} \in X} \max _{\substack{1 \leq j \leq s \\ n_{j} \neq 0}} \max _{1 \leq i \leq n_{j}} \sum_{\substack{\overrightarrow{\mathbf{x}}_{\ell} \in X^{n_{\ell}} \\ 1 \leq \ell \leq s+1 \\\left(\overrightarrow{\mathbf{x}}_{j}\right)_{i}=\mathbf{x}}}\left|b\left(\overrightarrow{\mathbf{x}}_{1}, \cdots, \overrightarrow{\mathbf{x}}_{s} ; \overrightarrow{\mathbf{x}}_{s+1}\right)\right|
$$

Here $\left(\overrightarrow{\mathbf{x}}_{j}\right)_{i}$ is the $i^{\text {th }}$ component of the $n_{j}$-tuple $\overrightarrow{\mathbf{x}}_{j}$.

○ If there are only history fields, that is if $\sum_{j=1}^{s} n_{j}=0$, but $n_{s+1} \neq 0$, then we take the pure $L^{1}$ norm

$$
\|b\|_{n_{1}, \cdots, n_{s+1}}=\sum_{\overrightarrow{\mathbf{x}}_{s+1} \in X^{n_{s+1}}}\left|b\left(-, \cdots,-; \overrightarrow{\mathbf{x}}_{s+1}\right)\right|
$$

○ Finally, for the constant term, that is if $\sum_{j=1}^{s+1} n_{j}=0$,

$$
\|b\|_{n_{1}, \cdots, n_{s+1}}=|b(-, \cdots,-)|
$$

(iii) Given weight factors $\kappa_{1}, \cdots, \kappa_{s}$, and a metric $d$ on $X$, the weight system with metric $d$ that associates the weight factor $\kappa_{j}$ to the field $\phi_{j}$ (and the weight factor one to the 
history field) is defined by

$$
w_{d}\left(\overrightarrow{\mathbf{x}}_{1}, \cdots, \overrightarrow{\mathbf{x}}_{s} ; \overrightarrow{\mathbf{x}}_{s+1}\right)=e^{\tau_{d}\left(\overrightarrow{\mathbf{x}}_{1}, \cdots, \overrightarrow{\mathbf{x}}_{s+1}\right)} \prod_{j=1}^{s} \prod_{\ell=1}^{n_{j}} \kappa_{j}\left(\mathbf{x}_{j, \ell}\right)
$$

for all $\left(\overrightarrow{\mathbf{x}}_{1}, \cdots, \overrightarrow{\mathbf{x}}_{s} ; \overrightarrow{\mathbf{x}}_{s+1}\right) \in X^{n_{1}} \times \cdots \times X^{n_{s}} \times X^{n_{s+1}}$ and all nonnegative integers $n_{1}, \cdots, n_{s+1}$.

If $\Omega$ is a subset of $X$, the weight system with core $\Omega$ that associates the weight factor $\kappa_{j}$ to the field $\phi_{j}$ (and the weight factor one to the history field) is $w_{d_{\Omega}}$. The metric $d_{\Omega}$ was specified in Definition II.11.iii.

(iv) Let $f\left(\phi_{1}, \cdots, \phi_{s} ; \mathfrak{h}\right)$ be a function which is defined and analytic on a neighbourhood of the origin in $\mathbb{C}^{(s+1)|X|}$ and $a$ the symmetric coefficient system of $f$. We define the norm, with weight $w$, of $f$ to be

$$
\|f\|_{w}=\sum_{n_{1}, \cdots, n_{s+1} \geq 0}\left\|w\left(\overrightarrow{\mathbf{x}}_{1}, \cdots, \overrightarrow{\mathbf{x}}_{s}, \overrightarrow{\mathbf{x}}_{s+1}\right) a\left(\overrightarrow{\mathbf{x}}_{1}, \cdots, \overrightarrow{\mathbf{x}}_{s}, \overrightarrow{\mathbf{x}}_{s+1}\right)\right\|_{n_{1}, \cdots, n_{s+1}}
$$

Remark III.13 Let $f\left(\phi_{1}, \cdots, \phi_{s} ; \mathfrak{h}\right)$ be a power series and $\Omega$ a subset of $X$.

(i) For any weight system $w$

$$
\left\|\left.f\left(\phi_{1}, \cdots, \phi_{s} ; \mathfrak{h}\right)\right|_{\substack{\mathfrak{h}(\mathbf{x})=1 \text { for } \mathbf{x} \in \Omega \\ \mathfrak{h}(\mathbf{x})=0 \text { for } \mathbf{x} \in X \backslash \Omega}}\right\|_{w} \leq\left\|\left.f\right|_{\Omega}\right\|_{w} \leq\|f\|_{w}
$$

(ii) For any measure $d \mu\left(\phi_{s}\right)$ that is independent of $\mathfrak{h}$,

$$
\int d \mu\left(\phi_{s}\right)\left(\left.f\left(\phi_{1}, \cdots, \phi_{s} ; \mathfrak{h}\right)\right|_{\Omega}\right)=\left.\left[\int d \mu\left(\phi_{s}\right) f\left(\phi_{1}, \cdots, \phi_{s} ; \mathfrak{h}\right)\right]\right|_{\Omega}
$$

Theorem III.4 of [BFKT4, §II] uses norms as in Definitions II.12 and III.12 to control a renormalization group step. For the reader's convenience, we repeat its statement as well as the main result of [BFKT4, Corollary III.5] here.

Theorem III.14 Let $f\left(\phi_{1}, \cdots, \phi_{s} ; z_{*}, z ; \mathfrak{h}\right)$ be a function which is defined and analytic on a neighbourhood of the origin in $\mathbb{C}^{(s+3)|X|}$. Let $r \geq 0$ and denote by $d \mu\left(z^{*}, z\right)$ the measure

$$
\left.d \mu\left(z^{*}, z\right)=\prod_{\mathbf{x} \in X} \chi(|z(\mathbf{x})|) \leq r\right) e^{-z(\mathbf{x})^{*} z(\mathbf{x})} \frac{d z^{*}(\mathbf{x}) \wedge d z(\mathbf{x})}{2 \pi \imath}
$$


Furthermore let $\kappa_{1}, \cdots, \kappa_{s+2}$ be weight factors such that $\kappa_{s+1}(\mathbf{x}), \kappa_{s+2}(\mathbf{x}) \geq 4 r$ for all $\mathbf{x} \in X$, and let $\mathrm{m} \geq 0$ and $\Omega \subset X$. Denote by $w$ the weight system with core $\Omega$ that associates the weight factor $\kappa_{j}$ to the field $\phi_{j}$, and the weight factors $\kappa_{s+1}, \kappa_{s+2}$ to the fields $z^{*}$ and $z$ respectively.

If $\|f\|_{w}<\frac{1}{16}$, then there is an analytic function $g\left(\phi_{1}, \cdots, \phi_{s}\right)$ such that

$$
\frac{\int e^{f\left(\phi_{1}, \cdots, \phi_{s} ; z^{*}, z\right)} d \mu\left(z^{*}, z\right)}{\int e^{f\left(0, \cdots, 0 ; z^{*}, z\right)} d \mu\left(z^{*}, z\right)}=e^{g\left(\phi_{1}, \cdots, \phi_{s}\right)}
$$

and

$$
\|g\|_{w} \leq \frac{\|f\|_{w}}{1-16\|f\|_{w}}
$$

If, in addition, $\|f\|_{w}<\frac{1}{32}$ and the symmetric coefficient system $a\left(\overrightarrow{\mathbf{x}}_{1}, \cdots, \overrightarrow{\mathbf{x}}_{s} ; \overrightarrow{\mathbf{y}}_{*}, \overrightarrow{\mathbf{y}} ; \overrightarrow{\mathbf{x}}\right)$ of $f$ obeys $a\left(\overrightarrow{\mathbf{x}}_{1}, \cdots, \overrightarrow{\mathbf{x}}_{s} ; \overrightarrow{\mathbf{y}}_{*}, \overrightarrow{\mathbf{y}} ; \overrightarrow{\mathbf{x}}\right)=0$ whenever $\overrightarrow{\mathbf{y}}=\overrightarrow{\mathbf{y}}_{*}$, then

$$
\|g\|_{w} \leq\left(\frac{\|f\|_{w}}{\frac{1}{20}-\|f\|_{w}}\right)^{2}
$$

\section{Definition III.15}

(i) We also use the weight system $w_{\mathfrak{S}}$ of Definition II.13.iii for functions that depend on the history field, giving weight one to the history field as in Definition III.12.iii. We again write $\|\cdot\|_{\mathfrak{S}}$ for $\|\cdot\|_{w_{\mathfrak{S}}}$.

(ii) For any $\kappa, m>0$, we use $w_{\kappa, m}$ to denote the weight system with metric $m d$ the associates the constant weight factor $\kappa$ to the fields $\alpha^{*}$ and $\beta$ and the weight factor 1 to the history field. The norm $\left\|f\left(\alpha^{*}, \beta ; \mathfrak{h}\right)\right\|_{\kappa, m}=\|f\|_{w_{\kappa, m}}$.

Remark III.16 For a function $f\left(\alpha_{*}, \beta\right)$ that depends only on $\alpha_{*}(\mathbf{x})$ and $\beta(\mathbf{x})$ with $\mathrm{x} \in \Omega_{\mathfrak{S}}$,

$$
\|f\|_{2 \mathrm{R}(\delta), \mathrm{m}} \leq\|f\|_{\mathfrak{S}} \leq\|f\|_{2 \mathrm{R}(\delta), 2 \mathrm{~m}}
$$

For the decimation step, we need

Remark III.17 (Weight Factor Recursion Relation) Let $\left(\mathfrak{S}_{1}, \mathfrak{S}_{2}\right)$ be a pair of hierarchies for scale $\delta$ that precede the hierarchy $\mathfrak{S}$ for scale $2 \delta$. Then weight factors of 
Definition II.13 obey

$$
\kappa_{* \mathfrak{S}, \tau}(\mathbf{x})= \begin{cases}2 \mathrm{R}(2 \delta)=\frac{\mathrm{R}(2 \delta)}{\mathrm{R}(\delta)} \kappa_{* \mathfrak{S}_{1}, 0}(\mathbf{x}) & \text { if } \tau=0, \mathbf{x} \in \Lambda_{\mathfrak{S}} \\ \kappa_{* \mathfrak{S}_{1}, 0}(\mathbf{x}) & \text { if } \tau=0, \mathbf{x} \notin \Lambda_{\mathfrak{S}} \\ \kappa_{* \mathfrak{S}_{1}, \tau}(\mathbf{x}) & \text { if } 0<\tau<\delta \\ \infty & \text { if } \tau=\delta, \mathbf{x} \in \Lambda_{\mathfrak{S}} \\ \frac{1}{2} \kappa_{* \mathfrak{S}_{2}, 0}(\mathbf{x}) & \text { if } \tau=\delta, \mathbf{x} \notin \Lambda_{\mathfrak{S}} \\ \kappa_{* \mathfrak{S}_{2}, \tau-\delta}(\mathbf{x}) & \text { if } \delta<\tau<2 \delta\end{cases}
$$

and

$$
\kappa_{\mathfrak{S}, \tau}(\mathbf{x})= \begin{cases}2 \mathrm{R}(2 \delta)=\frac{\mathrm{R}(2 \delta)}{\mathrm{R}(\delta)} \kappa_{\mathfrak{S}_{2}, \delta}(\mathbf{x}) & \text { if } \tau=2 \delta, \mathbf{x} \in \Lambda_{\mathfrak{S}} \\ \kappa_{\mathfrak{S}_{2}, \delta}(\mathbf{x}) & \text { if } \tau=2 \delta, \mathbf{x} \notin \Lambda_{\mathfrak{S}} \\ \kappa_{\mathfrak{S}_{2}, \tau-\delta}(\mathbf{x}) & \text { if } \delta<\tau<2 \delta \\ \infty & \text { if } \tau=\delta, \mathbf{x} \in \Lambda_{\mathfrak{S}} \\ \frac{1}{2} \kappa_{\mathfrak{S}_{1}, \delta}(\mathbf{x}) & \text { if } \tau=\delta, \mathbf{x} \notin \Lambda_{\mathfrak{S}} \\ \kappa_{\mathfrak{S}_{1}, \tau}(\mathbf{x}) & \text { if } 0<\tau<\delta\end{cases}
$$

and

$$
\lambda_{\mathfrak{S}, \tau}(\mathbf{x})= \begin{cases}\lambda_{\mathfrak{S}_{1}, \tau}(\mathbf{x}) & \text { if } 0<\tau<\delta \\ 32 \mathrm{r}(\delta) & \text { if } \tau=\delta, \mathbf{x} \in \Lambda_{\mathfrak{S}} \backslash \Omega_{\mathfrak{S}} \\ \infty & \text { if } \tau=\delta, \mathbf{x} \notin \Lambda_{\mathfrak{S}} \backslash \Omega_{\mathfrak{S}} \\ \lambda_{\mathfrak{S}_{2}, \tau-\delta}(\mathbf{x}) & \text { if } \delta<\tau<2 \delta\end{cases}
$$

We will deal with linear changes in the $\phi$-fields which may be compositions of several such changes of variables. For the convenience of the reader we repeat the [BFKT4, Definition IV.2] of the operator norms we use.

Definition III.18 (Weighted $L^{1}-L^{\infty}$ operator norms) Let $\kappa, \kappa^{\prime}: X \rightarrow(0, \infty]$ be weight factors and $\delta$ an arbitrary metric and let $A$ be an $\mathfrak{h}$-linear map on $\mathbb{C}^{X}$. We define the operator norm

$$
N_{\delta}\left(A ; \kappa, \kappa^{\prime}\right)=\left\|\sum_{\substack{\mathbf{x}, \mathbf{y} \in X \\ \overrightarrow{\mathbf{x}} \in X^{(1)}}} A(\mathbf{x} ; \overrightarrow{\mathbf{x}} ; \mathbf{y}) \mathfrak{h}(\mathbf{x}) \beta_{l}(\mathbf{x}) \mathfrak{h}(\overrightarrow{\mathbf{x}}) \mathfrak{h}(\mathbf{y}) \beta_{r}(\mathbf{y})\right\|_{\omega}
$$

where $\omega$ is the weight system with metric $\delta$ that associates the weight $\frac{1}{\kappa}$ to $\beta_{l}$, the weight $\kappa^{\prime}$ to $\beta_{r}$ and the weight 1 to $\mathfrak{h}$.

For an ordinary operator $J$ on $\mathbb{C}^{X}$, we set $N_{\delta}\left(J ; \kappa, \kappa^{\prime}\right)=N_{\delta}\left(\bar{J} ; \kappa, \kappa^{\prime}\right)$. Observe that if $J$ is multiplication by the characteristic function of a set $Y$, then

$$
N_{\delta}\left(J ; \kappa, \kappa^{\prime}\right)=\sup _{\mathbf{x} \in Y} \frac{\kappa^{\prime}(\mathbf{x})}{\kappa(\mathbf{x})}
$$


In [BFKT4, Remark IV.3], we gave a more explicit reformulation of the definition of $N_{\delta}\left(A ; \kappa, \kappa^{\prime}\right)$.

The main change of variables formula [BFKT4, Proposition IV.4] is

Proposition III.19 Let $A_{j}, 1 \leq j \leq s$, be $\mathfrak{h}$-operators on $\mathbb{C}^{X}$, and let $f\left(\phi_{1}, \cdots, \phi_{s} ; \mathfrak{h}\right)$ be an analytic function on a neighbourhood of the origin in $\mathbb{C}^{(s+1)|X|}$. Define $\tilde{f}$ by

$$
\tilde{f}\left(\phi_{1}, \cdots, \phi_{s} ; \mathfrak{h}\right)=f\left(A_{1} \phi_{1}, \cdots, A_{s} \phi_{s} ; \mathfrak{h}\right)
$$

Let $\kappa_{1}, \cdots \kappa_{s}, \tilde{\kappa}_{1}, \cdots \tilde{\kappa}_{s}$ be weight factors. Denote by $w$ and $\tilde{w}$ the weight systems with metric $\delta$ that associate to the field $\phi_{j}$ the weight factor $\kappa_{j}$ and $\tilde{\kappa}_{j}$ respectively. If $N_{\delta}\left(A_{j} ; \kappa_{j}, \tilde{\kappa}_{j}\right) \leq 1$ for $1 \leq j \leq s$, then

$$
\|\tilde{f}\|_{\tilde{w}} \leq\|f\|_{w}
$$

In [BFKT4] and Appendix G, we also state variants and corollaries of this Proposition.

Most of the times, the metric $\delta$ used in Definition III.18 will be a multiple of the standard metric $d$. Therefore we introduce the notation

$$
\mathcal{N}_{\mu}\left(A ; \kappa, \kappa^{\prime}\right)=N_{\mu d}\left(A ; \kappa, \kappa^{\prime}\right)
$$

for any $\mu \geq 0$, any $\mathfrak{h}$-operator $A$ and any weight factors $\kappa, \kappa^{\prime}$. By (II.14), for any $\Omega \subset X$

$$
N_{d_{\Omega}}\left(A ; \kappa, \kappa^{\prime}\right) \leq \mathcal{N}_{2 \mathrm{~m}}\left(A ; \kappa, \kappa^{\prime}\right)
$$

Specifically, we shall use

\section{Definition III.20}

$$
\|A\|=\mathcal{N}_{5 \mathrm{~m}}(A ; 1,1)
$$

This is consistent with the definition of $\|v\| \|$ of (II.5).

The most important operators for our considerations are the propagator

$$
j(t)=\operatorname{exph}(-t(\mathrm{~h}-\mu))
$$

introduced in (III.1), and the cutoff propagator

$$
j_{\mathfrak{c}}(t)(\mathbf{x}, \mathbf{y})=j(t)(\mathbf{x}, \mathbf{y}) \cdot \begin{cases}1 & \text { if } d(\mathbf{x}, \mathbf{y}) \leq \mathfrak{c} \\ 0 & \text { if } d(\mathbf{x}, \mathbf{y})>\mathfrak{c}\end{cases}
$$

introduced in (III.2). They fulfill the estimates 
Lemma III.21 Set $K_{j}=\mathcal{N}_{6 \mathrm{~m}}(\mathrm{~h}-\mu ; 1,1)$ For all $t \geq 0$,

$$
\begin{aligned}
& \|\| j(t) \| \leq \mathcal{N}_{6 \mathrm{~m}}(j(t) ; 1,1) \leq e^{K_{j} t} \\
& \|j(t)-\mathfrak{h}\| \leq \leq \mathcal{N}_{6 \mathrm{~m}}(j(t)-\mathfrak{h} ; 1,1) \leq t K_{j} e^{K_{j} t} \\
& \left\|j_{\mathfrak{c}}(t)-j(t)\right\| \leq t K_{j} e^{K_{j} t} e^{-\mathrm{m} \mathfrak{c}} .
\end{aligned}
$$

Proof: These estimates follow directly from parts (ii), with $A=j(t)-\mathfrak{h}$, and (iv) of Remark G.4.

Further estimates on $j(t)$ are given in Appendix D.

\section{III.6 Decimation}

The first step in our proof of Theorem II.16 is the construction of the "large field/ small field" decomposition of $I_{n}\left(\varepsilon ; \alpha^{*}, \beta\right)\left(n=0,1, \cdots,\left[\log _{2} \theta / \varepsilon\right]\right)$ for fixed $\varepsilon>0$. The recursion step for this construction is Theorem III.26, below. Recall that we are assuming that the two-body potential $v$ satisfies Hypothesis II.14, and in particular that $\|v\| \leq \frac{1}{2} \mathfrak{v}$.

As in $\S I I .1$, the small field parts of the term associated to a hierarchy will depend only on the small field set, not on the hierarchy. Its description is similar to that in §II.1. First, we introduce the analogue of the renormalization group map $\mathfrak{R}_{\Omega, \delta}$ for history complete functions:

Definition III.22 Let $\Omega \subset X$. If $f_{1}\left(\alpha_{*}, \beta ; \mathfrak{h}\right), f_{2}\left(\alpha_{*}, \beta ; \mathfrak{h}\right), V\left(\alpha_{*}, \beta ; \mathfrak{h}\right)$ are history complete functions that are supported in $\Omega$ (i.e. $\left.f_{i}\right|_{\Omega}=f_{i}$ ) we define

$$
\begin{aligned}
\overline{\mathfrak{R}}_{\Omega ; t}(V ; & \left.f_{1}, f_{2}\right)\left(\alpha_{*}, \beta ; \mathfrak{h}\right) \\
=\{ & f_{1}\left(\alpha_{*}, j_{\mathfrak{c}}(t) \beta\right)+f_{2}\left(j_{\mathfrak{c}}(t) \alpha_{*}, \beta\right)+\log \frac{\int d \mu_{\Omega, \mathrm{r}(t)}\left(z^{*}, z\right) e^{\mathcal{A}\left(\alpha_{*}, \beta ; z^{*}, z\right)}}{\int d \mu_{\Omega, \mathrm{r}(t)}\left(z^{*}, z\right)} \\
\quad-\left\langle\left[j(t)-j_{\mathfrak{c}}(t)\right] \alpha_{*},\left[j(t)-j_{\mathfrak{c}}(t)\right] \beta\right\rangle & \left.\quad+V\left(\alpha_{*}, j_{\mathfrak{c}}(t) \beta\right)-V\left(\alpha_{*}, j(t) \beta\right)+V\left(j_{\mathfrak{c}}(t) \alpha_{*}, \beta\right)-V\left(j(t) \alpha_{*}, \beta\right)\right\}\left.\right|_{\Omega}
\end{aligned}
$$

where $\mathcal{A}\left(\alpha_{*}, \beta ; z_{*}, z\right)$ is

$$
\begin{aligned}
{\left[f _ { 1 } \left(\alpha_{*}, z\right.\right.} & \left.\left.+j_{\mathfrak{c}}(t) \beta\right)-f_{1}\left(\alpha_{*}, j_{\mathfrak{c}}(t) \beta\right)\right]+\left[f_{2}\left(z_{*}+j_{\mathfrak{c}}(t) \alpha_{*}, \beta\right)-f_{2}\left(j_{\mathfrak{c}}(t) \alpha_{*}, \beta\right)\right] \\
& +\left\langle\left[j(t)-j_{\mathfrak{c}}(t)\right] \alpha_{*}, z\right\rangle+\left\langle z_{*},\left[j(t)-j_{\mathfrak{c}}(t)\right] \beta\right\rangle \\
& +\left[V\left(\alpha_{*}, z+j_{\mathfrak{c}}(t) \beta\right)-V\left(\alpha_{*}, j_{\mathfrak{c}}(t) \beta\right)\right]+\left[V\left(z_{*}+j_{\mathfrak{c}}(t) \alpha_{*}, \beta\right)-V\left(j_{\mathfrak{c}}(t) \alpha_{*}, \beta\right)\right]
\end{aligned}
$$


Since $\left.\left.j(t)\right|_{\Omega}\right|_{\mathfrak{h}=1}=j_{(\Omega)}(t)$ and $\left.\left.j_{\mathfrak{c}}(t)\right|_{\Omega}\right|_{\mathfrak{h}=1}=j_{(\Omega), \mathfrak{c}}(t)$,

$$
\left.\overline{\mathfrak{R}}_{\Omega ; t}\left(V ; f_{1}, f_{2}\right)\right|_{\mathfrak{h}=1}=\mathfrak{R}_{\Omega ; t}\left(\left.V\right|_{\mathfrak{h}=1} ;\left.f_{1}\right|_{\mathfrak{h}=1},\left.f_{2}\right|_{\mathfrak{h}=1}\right)
$$

\section{Remark III.23 If $\Omega^{\prime} \subset \Omega$}

$$
\bar{\Re}_{\Omega^{\prime} ; t}\left(\left.V\right|_{\Omega^{\prime}} ;\left.f_{1}\right|_{\Omega^{\prime}},\left.f_{2}\right|_{\Omega^{\prime}}\right)=\left.\bar{\Re}_{\Omega ; t}\left(V ; f_{1}, f_{2}\right)\right|_{\Omega^{\prime}}
$$

We control the small field part in one decimation step by the following

Theorem III.24 Set $K_{R}=2^{12} K_{j}^{2}$ and $K_{E}=2^{23}$. There are constants $\Theta, \mathfrak{v}_{0}>0$ such that, for all $\delta \leq \frac{1}{2} \Theta$ and $\mathfrak{v} \leq \mathfrak{v}_{0}$, the following holds for all $\Omega \subset X$ :

Let $\mathcal{R}_{1}\left(\alpha_{*}, \beta ; \mathfrak{h}\right), \mathcal{R}_{2}\left(\alpha_{*}, \beta ; \mathfrak{h}\right)$ and $\mathcal{E}_{1}\left(\alpha_{*}, \beta ; \mathfrak{h}\right), \mathcal{E}_{2}\left(\alpha_{*}, \beta ; \mathfrak{h}\right)$ be history complete functions that are supported on $\Omega$ (that is $\left.\mathcal{R}_{i}\right|_{\Omega}=\mathcal{R}_{i},\left.\mathcal{E}_{i}\right|_{\Omega}=\mathcal{E}_{i}$ for $i=1,2$ ) with the following properties

$\circ \mathcal{R}_{1}$ and $\mathcal{R}_{2}$ are both bilinear in $\alpha_{*}$ and $\beta$ and fulfill the estimates

$$
\left\|\mathcal{R}_{i}\right\|_{2 \mathrm{R}(\delta), 2 \mathrm{~m}} \leq K_{R} \delta^{2} \mathrm{r}(\delta)^{2} \mathrm{R}(\delta)^{2} e^{-2 \mathrm{~m} \mathfrak{c}}
$$

$\circ \mathcal{E}_{1}$ and $\mathcal{E}_{2}$ both have degree at least two both in $\alpha_{*}$ and in $\beta$ and fulfill the estimates

$$
\left\|\mathcal{E}_{i}\right\|_{2 \mathrm{R}(\delta), 2 \mathrm{~m}} \leq K_{E}(\delta \mathfrak{v})^{2} \mathrm{r}(\delta)^{2} \mathrm{R}(\delta)^{6}
$$

- All four of these functions are invariant under $\alpha_{*} \rightarrow e^{-i \theta} \alpha_{*}, \beta \rightarrow e^{i \theta} \beta$.

Let $\varepsilon$ be a divisor of $\delta$. Then there are history complete functions $\mathcal{R}\left(\alpha_{*}, \beta ; \mathfrak{h}\right)$ and $\mathcal{E}\left(\alpha_{*}, \beta ; \mathfrak{h}\right)$ that are supported on $\Omega$ such that

$$
\overline{\mathfrak{R}}_{\Omega ; \delta}\left(V_{\Omega, \delta}(\varepsilon ; \cdot) ; \mathcal{R}_{1}+\mathcal{E}_{1}, \mathcal{R}_{2}+\mathcal{E}_{2}\right)=\mathcal{R}+\mathcal{E}
$$

and which have the following properties:

$\circ \mathcal{R}$ is bilinear in $\alpha_{*}$ and $\beta$ and fulfills the estimates

$$
\|\mathcal{R}\|_{2 \mathrm{R}(2 \delta), 2 \mathrm{~m}} \leq K_{R}(2 \delta)^{2} \mathrm{r}(2 \delta)^{2} \mathrm{R}(2 \delta)^{2} e^{-2 \mathrm{~m} \mathrm{c}}
$$

Furthermore $\mathcal{R}$ is the quadratic part of $\overline{\mathfrak{R}}_{\Omega ; \delta}\left(0 ; \mathcal{R}_{1}, \mathcal{R}_{2}\right)$ and, in particular, is independent of $V_{\Omega, \delta}(\varepsilon ; \cdot), \mathcal{E}_{1}$ and $\mathcal{E}_{2}$. 
$\circ \mathcal{E}$ has degree at least two both in $\alpha_{*}$ and in $\beta$ and fulfills the estimates

$$
\|\mathcal{E}\|_{2 \mathrm{R}(2 \delta), 2 \mathrm{~m}} \leq K_{E}(2 \delta \mathfrak{v})^{2} \mathrm{r}(2 \delta)^{2} \mathrm{R}(2 \delta)^{6}
$$

○ Both functions are invariant under $\alpha_{*} \rightarrow e^{-i \theta} \alpha_{*}, \beta \rightarrow e^{i \theta} \beta$.

The proof of Theorem III.24 is similar to that of [BFKT5, Proposition III.3] and is given in Chapter IV.

Remark III.25 By the definitions (II.18)

$$
\begin{aligned}
\delta^{2} \mathrm{r}(\delta)^{2} \mathrm{R}(\delta)^{2} e^{-2 \mathrm{~m} \mathfrak{c}} & =(\delta \mathfrak{v})^{2\left(1-e_{\mathrm{R}}-2 e_{\mathrm{r}}\right)} \frac{e^{-2 \mathrm{~m} \mathfrak{c}}}{\mathfrak{v}^{2}} \\
(\delta \mathfrak{v})^{2} \mathrm{r}(\delta)^{2} \mathrm{R}(\delta)^{6} & =(\delta \mathfrak{v})^{2\left(1-3 e_{\mathrm{R}}-4 e_{\mathrm{r}}\right)}
\end{aligned}
$$

Both quantities go to zero with $\delta$ by (II.17).

The last Theorem allows us to recursively control the "small field parts". We now consider the full model.

Theorem III.26 Set $K_{D}=2^{35} e^{6 K_{j}}$ and $K_{L}=2^{48} e^{6 K_{j}}$. There are constants $\Theta, \mathfrak{v}_{0}>0$ such that, for all $\delta \leq \frac{1}{2} \Theta$ and $\mathfrak{v} \leq \mathfrak{v}_{0}$, the following holds:

Let $\mathfrak{S}_{1}$ and $\mathfrak{S}_{2}$ be hierarchies for scale $\delta$ with summits $\Omega_{1}=\Omega_{\mathfrak{S}_{1}}$ and $\Omega_{2}=\Omega_{\mathfrak{S}_{2}}$. Let $\varepsilon=2^{-n} \delta$ with $n \geq \max \left\{\operatorname{depth}\left(\mathfrak{S}_{1}\right), \operatorname{depth}\left(\mathfrak{S}_{2}\right)\right\}$.

Furthermore let $\mathcal{D}_{1}\left(\alpha_{*}, \beta ; \vec{\rho} ; \mathfrak{h}\right), \mathcal{D}_{2}\left(\alpha_{*}, \beta ; \vec{\rho} ; \mathfrak{h}\right), b_{1}\left(\alpha_{*}, \beta ; \vec{\rho} ; \mathfrak{h}\right)$ and $b_{2}\left(\alpha_{*}, \beta ; \vec{\rho} ; \mathfrak{h}\right)$ be history complete functions with the following properties

○ For $i=1,2, \mathcal{D}_{i}(0,0 ; \overrightarrow{0} ; 0)=0$ and

$$
\left\|\mathcal{D}_{i}\right\|_{\mathfrak{S}_{i}} \leq 1 \quad\left\|\left.\mathcal{D}_{i}\right|_{\Omega_{i}}\right\|_{\mathfrak{S}_{i}} \leq 2^{-20}
$$

- The "pure large field parts" $\left.\mathcal{D}_{1}\right|_{\Omega_{1}^{c}}$ and $\left.\mathcal{D}_{2}\right|_{\Omega_{2}^{c}}$ vanish. On the other hand, $b_{1}$ and $b_{2}$ are purely large field. That $i s, b_{1}=\left.b_{1}\right|_{\Omega_{1}^{c}}$ and $b_{2}=\left.b_{2}\right|_{\Omega_{2}^{c}}$.

- $\mathcal{D}_{1}, \mathcal{D}_{2}$ are invariant under $\alpha_{*} \rightarrow e^{-i \theta} \alpha_{*}, \beta \rightarrow e^{i \theta} \beta, \vec{\rho}=\left(\vec{\alpha}_{*}, \vec{\alpha}, \vec{z}_{*}, \vec{z}\right) \rightarrow$ $\left(e^{-i \theta} \vec{\alpha}_{*}, e^{i \theta} \vec{\alpha}, e^{-i \theta} \vec{z}_{*}, e^{i \theta} \vec{z}\right)$

Set, for $i=1,2$,

$$
\begin{aligned}
& I_{i}\left(\alpha^{*}, \beta\right)= \\
& \quad \mathcal{Z}_{\delta}^{\left|\Omega_{i}\right|} \chi_{\delta}\left(\Omega_{i} ; \alpha, \beta\right) \mathcal{I}_{\left(\mathfrak{S}_{i} ; \alpha^{*}, \beta\right)}\left(e^{-Q_{\mathfrak{S}_{i}}\left(\alpha^{*}, \beta ; \vec{\alpha}^{*}, \vec{\alpha}\right)+\mathcal{V}_{\mathfrak{S}_{i}}\left(\varepsilon ; \alpha^{*}, \beta ; \vec{\alpha}^{*}, \vec{\alpha}\right)+\mathcal{D}_{i}\left(\alpha^{*}, \beta ; \overrightarrow{\rho_{\uparrow}}\right)} b_{i}\left(\alpha^{*}, \beta ; \overrightarrow{\rho_{\uparrow}}\right)\right)
\end{aligned}
$$


where $\chi_{\delta}\left(\Omega_{i} ; \alpha, \beta\right)$ is the characteristic function introduced in Theorem II.16. Also set

$$
I\left(\alpha^{*}, \beta\right)=\int d \mu_{\mathrm{R}(\varepsilon)}\left(\phi^{*}, \phi\right) I_{1}\left(\alpha^{*}, \phi\right) I_{2}\left(\phi^{*}, \beta\right)
$$

Then

$$
\begin{array}{r}
I\left(\alpha^{*}, \beta\right)=\sum_{\substack{\text { hierarchies } \\
\text { for scale } \\
\left(\mathfrak{S}_{1}, \mathfrak{S}_{2}\right) \prec \mathfrak{S}}} \mathcal{Z}_{2 \delta}^{\left|\Omega_{\mathfrak{S}}\right|} \chi_{2 \delta}\left(\Omega_{\mathfrak{S}} ; \alpha, \beta\right) \mathcal{I}_{\left(\mathfrak{S} ; \alpha^{*}, \beta\right)}\left(e^{-Q_{\mathfrak{S}}\left(\alpha^{*}, \beta ; \vec{\alpha}^{*}, \vec{\alpha}\right)+\mathcal{V}_{\mathfrak{S}}\left(\varepsilon ; \alpha^{*}, \beta ; \vec{\alpha}^{*}, \vec{\alpha}\right)}\right. \\
\left.e^{\mathcal{D}_{\mathfrak{S}}\left(\alpha^{*}, \beta ; \overrightarrow{\rho_{\uparrow}}\right)} b_{1}\left(\alpha^{*}, \alpha_{\delta} ; \overrightarrow{\rho_{\uparrow l} l}\right) b_{2}\left(\alpha_{\delta}^{*}, \beta ; \overrightarrow{\rho_{\uparrow r}}\right) e^{\mathcal{L}_{\mathfrak{S}}^{\prime}\left(\alpha^{*}, \beta ; \overrightarrow{\rho_{\uparrow}}\right)}\right)
\end{array}
$$

where, for each hierarchy $\mathfrak{S}$ that is preceded by $\left(\mathfrak{S}_{1}, \mathfrak{S}_{2}\right)$ as in Definition III.1, $\mathcal{D}_{\mathfrak{S}}$ and $\mathcal{L}_{\mathfrak{S}}^{\prime}$ are analytic history complete functions that fulfill

- The "pure large field part" $\left.\mathcal{D}_{\mathfrak{S}}\right|_{\Omega_{\mathfrak{S}}^{c}}$ of $\mathcal{D}_{\mathfrak{S}}$ vanishes. Also,

$$
\begin{aligned}
\left\|\mathcal{D}_{\mathfrak{S}}\right\|_{\mathfrak{S}} \leq \frac{1}{2} K_{D}(2 \delta \mathfrak{v}) \mathrm{r}(2 \delta) \mathrm{R}(2 \delta)^{3} & +2^{14} e^{-\operatorname{mc}(\delta)}\left(\left\|\mathcal{D}_{1}\right\|_{\mathfrak{S}_{1}}+\left\|\mathcal{D}_{2}\right\|_{\mathfrak{S}_{2}}\right) \\
& +2^{14}\left(\left\|\left.\mathcal{D}_{1}\right|_{\Omega_{1}}\right\|_{\mathfrak{S}_{1}}+\left\|\left.\mathcal{D}_{2}\right|_{\Omega_{2}}\right\|_{\mathfrak{S}_{2}}\right)
\end{aligned}
$$

The "pure small field part" $\left.\mathcal{D}_{\mathfrak{S}}\right|_{\Omega_{\mathfrak{S}}}$ is determined by $\left.\mathcal{D}_{1}\right|_{\Omega_{\mathfrak{S}}}$ and $\left.\mathcal{D}_{2}\right|_{\Omega_{\mathfrak{S}}}$ through

$$
\left.\mathcal{D}_{\mathfrak{S}}\right|_{\Omega_{\mathfrak{S}}}=\overline{\mathfrak{R}}_{\Omega_{\mathfrak{S}} ; \delta}\left(\mathcal{V}_{\Omega_{\mathfrak{S}} ; \delta}(\varepsilon ; \cdot) ;\left.\mathcal{D}_{1}\right|_{\Omega_{\mathfrak{S}}},\left.\mathcal{D}_{2}\right|_{\Omega_{\mathfrak{S}}}\right)
$$

$\circ \mathcal{L}_{\mathfrak{S}}^{\prime}$ is "pure large field" (that is $\mathcal{L}_{\mathfrak{S}}^{\prime}=\left.\mathcal{L}_{\mathfrak{S}}^{\prime}\right|_{\Omega_{\mathfrak{S}}^{c}}$ ) and fulfills the estimate

$$
\left\|\mathcal{L}_{\mathfrak{S}}^{\prime}\right\|_{\mathfrak{S}} \leq \frac{1}{2} K_{L}(2 \delta \mathfrak{v}) r(2 \delta) \mathrm{R}(2 \delta)^{3}+2^{8}\left(\left\|\mathcal{D}_{1}\right\|_{\mathfrak{S}_{1}}+\left\|\mathcal{D}_{2}\right\|_{\mathfrak{S}_{2}}\right)
$$

$\circ \mathcal{D}_{\mathfrak{S}}$ and $\mathcal{L}_{\mathfrak{S}}^{\prime}$ are invariant under $\alpha_{*} \rightarrow e^{-i \theta} \alpha_{*}, \beta \rightarrow e^{i \theta} \beta, \vec{\rho}=\left(\vec{\alpha}_{*}, \vec{\alpha}, \vec{z}_{*}, \vec{z}\right) \rightarrow$ $\left(e^{-i \theta} \vec{\alpha}_{*}, e^{i \theta} \vec{\alpha}, e^{-i \theta} \vec{z}_{*}, e^{i \theta} \vec{z}\right)$

This Theorem allows the construction of $I_{m}\left(2^{-m} \theta ; \cdot, \cdot\right)$ for each fixed $m$, and Theorem III.24 provides control of its small field parts. See Propositions III.32 and III.29, with $n=m$, below. The second step in the proof of Theorem II.16 is the comparison of $I_{m}\left(2^{-m} \theta ; \cdot, \cdot\right)$ and $I_{m+1}\left(2^{-(m+1)} \theta ; \cdot, \cdot\right)$, leading to the limit $m \rightarrow \infty$. To do this, we compare $I_{n}\left(2^{-m} \theta ; \cdot, \cdot\right)$ and $I_{n+1}\left(2^{-(m+1)} \theta ; \cdot, \cdot\right)$ for $1 \leq n \leq m$.

For the pure small field part the essential comparison step is

Theorem III.27 Under the hypotheses of Theorem III.24, assume that there is a second set $\tilde{\mathcal{R}}_{1}\left(\alpha_{*}, \beta ; \mathfrak{h}\right), \tilde{\mathcal{R}}_{2}\left(\alpha_{*}, \beta ; \mathfrak{h}\right)$ and $\tilde{\mathcal{E}}_{1}\left(\alpha_{*}, \beta ; \mathfrak{h}\right), \tilde{\mathcal{E}}_{2}\left(\alpha_{*}, \beta ; \mathfrak{h}\right)$ of history complete functions 
that have similar properties to $\mathcal{R}_{1}, \mathcal{R}_{2}, \mathcal{E}_{1}, \mathcal{E}_{2}$ and are close to these functions. Precisely, we assume that

$\circ \tilde{\mathcal{R}}_{1}$ and $\tilde{\mathcal{R}}_{2}$ are both bilinear in $\alpha_{*}$ and $\beta$ and fulfill the estimates

$$
\left\|\tilde{\mathcal{R}}_{i}\right\|_{2 \mathrm{R}(\delta), 2 \mathrm{~m}} \leq K_{R} \delta^{2} \mathrm{r}(\delta)^{2} \mathrm{R}(\delta)^{2} e^{-2 \mathrm{~m} \mathfrak{c}}
$$

$\circ \mathcal{E}_{1}$ and $\mathcal{E}_{2}$ both have degree at least two both in $\alpha_{*}$ and in $\beta$ and fulfill the estimates

$$
\left\|\tilde{\mathcal{E}}_{i}\right\|_{2 \mathrm{R}(\delta), 2 \mathrm{~m}} \leq K_{E}(\delta \mathfrak{v})^{2} \mathrm{r}(\delta)^{2} \mathrm{R}(\delta)^{6}
$$

Let

$$
\overline{\mathfrak{R}}_{\Omega ; \delta}\left(V_{\Omega, \delta}\left(\frac{\varepsilon}{2} ; \cdot\right) ; \tilde{\mathcal{R}}_{1}+\tilde{\mathcal{E}}_{1}, \tilde{\mathcal{R}}_{2}+\tilde{\mathcal{E}}_{2}\right)=\tilde{\mathcal{R}}+\tilde{\mathcal{E}}
$$

be the decomposition of Theorem III.24. Then

$$
\begin{aligned}
\|\tilde{\mathcal{R}}-\mathcal{R}\|_{2 \mathrm{R}(2 \delta), 2 \mathrm{~m}} & \leq 2 \Delta_{\mathcal{R}} \\
\|\tilde{\mathcal{E}}-\mathcal{E}\|_{2 \mathrm{R}(2 \delta), 2 \mathrm{~m}} & \leq \Delta_{\mathcal{E}}+K_{\Delta}(2 \delta \mathfrak{v}) \mathrm{r}(2 \delta)^{2} \mathrm{R}(2 \delta)^{2}\left(\Delta_{\mathcal{R}}+\varepsilon(2 \delta \mathfrak{v}) \mathrm{R}(2 \delta)^{4}\right)
\end{aligned}
$$

where $K_{\Delta}=2^{40} e^{10 K_{j}}$ and

$$
\begin{aligned}
\Delta_{\mathcal{R}} & =\frac{1}{2}\left(\left\|\tilde{\mathcal{R}}_{1}-\mathcal{R}_{1}\right\|_{2 \mathrm{R}(\delta), 2 \mathrm{~m}}+\left\|\tilde{\mathcal{R}}_{2}-\mathcal{R}_{2}\right\|_{2 \mathrm{R}(\delta), 2 \mathrm{~m}}\right) \\
\Delta_{\mathcal{E}} & =\frac{1}{2}\left(\left\|\tilde{\mathcal{E}}_{1}-\mathcal{E}_{1}\right\|_{2 \mathrm{R}(\delta), 2 \mathrm{~m}}+\left\|\tilde{\mathcal{E}}_{2}-\mathcal{E}_{2}\right\|_{2 \mathrm{R}(\delta), 2 \mathrm{~m}}\right)
\end{aligned}
$$

For the full model the essential comparison step is

Theorem III.28 Under the hypotheses of Theorem III.26, assume that there is a second set $\tilde{\mathcal{D}}_{1}\left(\alpha_{*}, \beta ; \vec{\rho} ; \mathfrak{h}\right), \tilde{\mathcal{D}}_{2}\left(\alpha_{*}, \beta ; \vec{\rho} ; \mathfrak{h}\right), \tilde{b}_{1}\left(\alpha_{*}, \beta ; \vec{\rho} ; \mathfrak{h}\right)$ and $\tilde{b}_{2}\left(\alpha_{*}, \beta ; \vec{\rho} ; \mathfrak{h}\right)$ of history complete functions such that $\left\|\tilde{\mathcal{D}}_{i}\right\|_{\mathfrak{S}_{i}} \leq 1$ and $\left\|\left.\tilde{\mathcal{D}}_{i}\right|_{\Omega_{i}}\right\|_{\mathfrak{S}_{i}} \leq 2^{-20}$. Set

$$
\begin{aligned}
\tilde{I}_{i}\left(\alpha^{*}, \beta\right) & =\mathcal{Z}_{\delta}^{\left|\Omega_{i}\right|} \chi_{\delta}\left(\Omega_{i} ; \alpha, \beta\right) \mathcal{I}_{\left(\mathfrak{S}_{i} ; \alpha^{*}, \beta\right)}\left(e^{-Q_{\mathfrak{S}_{i}}+\mathcal{V}_{\mathfrak{S}_{i}}(\varepsilon / 2 ; \cdot)+\tilde{\mathcal{D}}_{i}} \tilde{b}_{i}\right) \\
\tilde{I}\left(\alpha^{*}, \beta\right) & =\int d \mu_{\mathrm{R}\left(\frac{\varepsilon}{2}\right)}\left(\phi^{*}, \phi\right) \tilde{I}_{1}\left(\alpha^{*}, \phi\right) \tilde{I}_{2}\left(\phi^{*}, \beta\right)
\end{aligned}
$$

and let

$$
\tilde{I}\left(\alpha^{*}, \beta\right)=\sum_{\substack{\text { hierarchies } \\ \text { for scale } 2 \delta \\\left(\mathfrak{S}_{1}, \mathfrak{S}_{2}\right) \prec \mathfrak{S}}} \mathcal{Z}_{2 \delta}^{\left|\Omega_{\mathfrak{S}}\right|} \chi_{2 \delta}\left(\Omega_{\mathfrak{S}} ; \alpha, \beta\right) \mathcal{I}_{\left(\mathfrak{S} ; \alpha^{*}, \beta\right)}\left(e^{-Q_{\mathfrak{S}}+\mathcal{V}_{\mathfrak{S}}(\varepsilon / 2 ; \cdot)} e^{\tilde{\mathcal{D}}_{\mathfrak{S}}} \tilde{b}_{1} \tilde{b}_{2} e^{\tilde{\mathcal{L}}_{\mathfrak{S}}^{\prime}}\right)
$$


be its representation as in Theorem III.26. Then

$$
\begin{gathered}
\left\|\mathcal{D}_{\mathfrak{S}}-\tilde{\mathcal{D}}_{\mathfrak{S}}\right\|_{\mathfrak{S}} \leq \frac{1}{2} K_{D} \varepsilon(2 \delta \mathfrak{v}) \mathrm{r}(2 \delta) \mathrm{R}(2 \delta)^{3}+2^{16} e^{-\mathrm{mc}(\delta)}\left(\left\|\mathcal{D}_{1}-\tilde{\mathcal{D}}_{1}\right\|_{\mathfrak{S}_{1}}+\left\|\mathcal{D}_{2}-\tilde{\mathcal{D}}_{2}\right\|_{\mathfrak{S}_{2}}\right) \\
+2^{16}\left(\left\|\left.\left(\mathcal{D}_{1}-\tilde{\mathcal{D}}_{1}\right)\right|_{\Omega_{1}}\right\|_{\mathfrak{S}_{1}}+\left\|\left.\left(\mathcal{D}_{2}-\tilde{\mathcal{D}}_{2}\right)\right|_{\Omega_{2}}\right\|_{\mathfrak{S}_{2}}\right) \\
\left\|\mathcal{L}_{\mathfrak{S}}^{\prime}-\tilde{\mathcal{L}}_{\mathfrak{S}}^{\prime}\right\|_{\mathfrak{S}} \leq \frac{1}{2} K_{L} \varepsilon(2 \delta \mathfrak{v}) \mathrm{r}(2 \delta) \mathrm{R}(2 \delta)^{3}+2^{8}\left(\left\|\mathcal{D}_{1}-\tilde{\mathcal{D}}_{1}\right\|_{\mathfrak{S}_{1}}+\left\|\mathcal{D}_{2}-\tilde{\mathcal{D}}_{2}\right\|_{\mathfrak{S}_{2}}\right)
\end{gathered}
$$

Theorems III.24 and III.27 will be proven in Chapter IV. Theorems III.26 and III.28 will be proven in Chapter V.

In the proof of Theorem II.16, the analysis of the "pure small field part" can be treated almost independently from the rest. To do this, we define, as in $§ I I .1$ (but now with history complete functions)

$$
\begin{aligned}
\mathcal{D}_{\Omega ; 0}\left(\varepsilon ; \alpha_{*}, \beta\right) & =0 \\
\mathcal{D}_{\Omega ; n+1}\left(\varepsilon ; \alpha_{*}, \beta\right) & =\overline{\mathfrak{R}}_{\Omega ; 2^{n} \varepsilon}\left(V_{\Omega ; 2^{n} \varepsilon}(\varepsilon ; \cdot, \cdot) ; \mathcal{D}_{\Omega ; n}(\varepsilon ; \cdot, \cdot), \mathcal{D}_{\Omega ; n}(\varepsilon ; \cdot, \cdot)\right)
\end{aligned}
$$

By Remark III.23

$$
\mathcal{D}_{\Omega^{\prime} ; n}(\varepsilon ; \cdot)=\left.\mathcal{D}_{\Omega ; n}(\varepsilon ; \cdot)\right|_{\Omega^{\prime}} \quad \text { when } \Omega^{\prime} \subset \Omega
$$

Proposition III.29 There are constants $\Theta, \mathfrak{v}_{0}>0$ such that, for all $\theta \leq \Theta$ and $\mathfrak{v} \leq \mathfrak{v}_{0}$, the following holds for all $\Omega \subset X$ :

For each $\varepsilon>0$ and each integer $1 \leq n \leq \log _{2} \frac{\theta}{\varepsilon}$ the function $\mathcal{D}_{\Omega ; n}(\varepsilon ; \cdot)$ is the sum of a function $\mathcal{R}_{\Omega ; n}(\varepsilon ; \cdot)$ that is bilinear in $\alpha_{*}$ and $\beta$ and an analytic function $\mathcal{E}_{\Omega ; n}(\varepsilon ; \cdot)$ that has degree at least two both in $\alpha_{*}$ and in $\beta$. They fulfill the estimates

$$
\begin{aligned}
\left\|\mathcal{R}_{\Omega ; n}(\varepsilon ; \cdot)\right\|_{2 \mathrm{R}\left(2^{n} \varepsilon\right), 2 \mathrm{~m}} & \leq K_{R}\left(2^{n} \varepsilon\right)^{2} \mathrm{r}\left(2^{n} \varepsilon\right)^{2} \mathrm{R}\left(2^{n} \varepsilon\right)^{2} e^{-2 \mathrm{~m} \mathfrak{c}} \\
\left\|\mathcal{E}_{\Omega ; n}(\varepsilon ; \cdot)\right\|_{2 \mathrm{R}\left(2^{n} \varepsilon\right), 2 \mathrm{~m}} & \leq K_{E}\left(2^{n} \varepsilon \mathfrak{v}\right)^{2} \mathrm{r}\left(2^{n} \varepsilon\right)^{2} \mathrm{R}\left(2^{n} \varepsilon\right)^{6}
\end{aligned}
$$

Furthermore

$$
\begin{aligned}
\left\|\mathcal{R}_{\Omega ; n}(\varepsilon ; \cdot)-\mathcal{R}_{\Omega ; n+1}\left(\frac{\varepsilon}{2} ; \cdot\right)\right\|_{2 \mathrm{R}\left(2^{n} \varepsilon\right), 2 \mathrm{~m}} & \leq K_{R} 2^{n} \varepsilon^{2} \mathrm{r}(\varepsilon)^{2} \mathrm{R}(\varepsilon)^{2} e^{-2 \mathrm{~m} \mathfrak{c}} \\
\left\|\mathcal{E}_{\Omega ; n}(\varepsilon ; \cdot)-\mathcal{E}_{\Omega ; n+1}\left(\frac{\varepsilon}{2} ; \cdot\right)\right\|_{2 \mathrm{R}\left(2^{n} \varepsilon\right), 2 \mathrm{~m}} & \leq 2 K_{E}(\varepsilon \mathfrak{v})^{2} \mathrm{r}(\varepsilon)^{2} \mathrm{R}(\varepsilon)^{6}
\end{aligned}
$$

Proof: We first prove, by induction on $n$, the statement of the Proposition but with the second line of (III.10) replaced by

$$
\begin{aligned}
\left\|\mathcal{E}_{\Omega ; n}(\varepsilon ; \cdot)-\mathcal{E}_{\Omega ; n+1}\left(\frac{\varepsilon}{2} ; \cdot\right)\right\|_{2 \mathrm{R}\left(2^{n} \varepsilon\right), 2 \mathrm{~m}} \leq & K_{E}(\varepsilon \mathfrak{v})^{2} \mathrm{r}(\varepsilon)^{2} \mathrm{R}(\varepsilon)^{6} \\
& +K_{\Delta} \varepsilon^{1-2 e_{\mathrm{R}}-4 e_{\mathrm{r}}}\left(\sum_{k=1}^{n}\left(2^{k} \varepsilon \mathfrak{v}\right)^{2} \mathrm{r}\left(2^{k} \varepsilon\right)^{2} \mathrm{R}\left(2^{k} \varepsilon\right)^{6}\right)
\end{aligned}
$$


This induction argument is similar to that of Theorems I.3 and I.4 in [BFKT5].

The induction starts with $n=0$. Observe that

$$
\mathcal{R}_{\Omega ; 0}(\varepsilon ; \cdot)=\mathcal{E}_{\Omega ; 0}(\varepsilon ; \cdot)=0
$$

while

$$
\mathcal{R}_{\Omega ; 1}\left(\frac{\varepsilon}{2} ; \cdot\right)+\mathcal{E}_{\Omega ; 1}\left(\frac{\varepsilon}{2} ; \cdot\right)=\bar{\Re}_{\Omega ; \frac{\varepsilon}{2}}\left(V_{\Omega, \frac{\varepsilon}{2}}\left(\frac{\varepsilon}{2} ; \cdot\right) ; 0,0\right)
$$

By Theorem III.24, with $\delta=\frac{\varepsilon}{2}$,

$$
\begin{aligned}
\left\|\mathcal{R}_{\Omega ; 1}\left(\frac{\varepsilon}{2} ; \cdot\right)\right\|_{2 \mathrm{R}(\varepsilon), 2 \mathrm{~m}} & \leq K_{R} \varepsilon^{2} \mathrm{r}(\varepsilon)^{2} \mathrm{R}(\varepsilon)^{2} e^{-2 \mathrm{~m} \mathfrak{c}} \\
\left\|\mathcal{E}_{\Omega ; 1}\left(\frac{\varepsilon}{2} ; \cdot\right)\right\|_{2 \mathrm{R}(\varepsilon), 2 \mathrm{~m}} & \leq K_{E}(\varepsilon \mathfrak{v})^{2} \mathrm{r}(\varepsilon)^{2} \mathrm{R}(\varepsilon)^{6}
\end{aligned}
$$

For the induction step from $n$ to $n+1$ we apply Theorems III.24 and III.27 with $\delta=2^{n} \varepsilon$

$$
\begin{aligned}
\mathcal{R}_{1}=\mathcal{R}_{2}=\mathcal{R}_{\Omega ; n}(\varepsilon ; \cdot, \cdot) & \tilde{\mathcal{R}}_{1}=\tilde{\mathcal{R}}_{2}=\mathcal{R}_{\Omega ; n+1}\left(\frac{\varepsilon}{2} ; \cdot, \cdot\right) \\
\mathcal{E}_{1}=\mathcal{E}_{2}=\mathcal{E}_{\Omega ; n}(\varepsilon ; \cdot, \cdot) & \tilde{\mathcal{E}}_{1}=\tilde{\mathcal{E}}_{2}=\mathcal{E}_{\Omega ; n+1}\left(\frac{\varepsilon}{2} ; \cdot, \cdot\right)
\end{aligned}
$$

By the inductive hypothesis and Theorem III.24,

$$
\begin{aligned}
& \mathcal{D}_{\Omega ; n+1}\left(\varepsilon ; \alpha_{*}, \beta\right)=\overline{\mathfrak{R}}_{\Omega ; 2^{n} \varepsilon}\left(V_{\Omega ; 2^{n} \varepsilon}(\varepsilon ; \cdot, \cdot) ;\right. \mathcal{R}_{\Omega ; n}(\varepsilon ; \cdot, \cdot)+\mathcal{E}_{\Omega ; n}(\varepsilon ; \cdot, \cdot), \\
&\left.\mathcal{R}_{\Omega ; n}(\varepsilon ; \cdot, \cdot)+\mathcal{E}_{\Omega ; n}(\varepsilon ; \cdot, \cdot)\right)
\end{aligned}
$$

has the decomposition

$$
\mathcal{D}_{\Omega ; n+1}\left(\varepsilon ; \alpha_{*}, \beta\right)=\mathcal{R}_{\Omega ; n+1}\left(\varepsilon ; \alpha_{*}, \beta\right)+\mathcal{E}_{\Omega ; n+1}\left(\varepsilon ; \alpha_{*}, \beta\right)
$$

where $\mathcal{R}_{\Omega ; n+1}(\varepsilon ; \cdot)$ and $\mathcal{E}_{\Omega ; n+1}(\varepsilon ; \cdot)$ have all of the required properties. Furthermore, by Theorem III.27,

$$
\begin{aligned}
\left\|\mathcal{R}_{\Omega ; n+1}(\varepsilon ; \cdot)-\mathcal{R}_{\Omega ; n+2}\left(\frac{\varepsilon}{2} ; \cdot\right)\right\|_{2 \mathrm{R}\left(2^{n+1} \varepsilon\right), 2 \mathrm{~m}} & \leq 2\left\|\mathcal{R}_{\Omega ; n}(\varepsilon ; \cdot)-\mathcal{R}_{\Omega ; n+1}\left(\frac{\varepsilon}{2} ; \cdot\right)\right\|_{2 \mathrm{R}\left(2^{n} \varepsilon\right), 2 \mathrm{~m}} \\
& \leq K_{R} 2^{n+1} \varepsilon^{2} \mathrm{r}(\varepsilon)^{2} \mathrm{R}(\varepsilon)^{2} e^{-2 \mathrm{~m} \mathfrak{c}}
\end{aligned}
$$

and

$$
\begin{aligned}
\| \mathcal{E}_{\Omega ; n+1}(\varepsilon ; \cdot)-\mathcal{E}_{\Omega ; n+2}\left(\frac{\varepsilon}{2} ; \cdot\right) & \|_{2 \mathrm{R}\left(2^{n+1} \varepsilon\right), 2 \mathrm{~m}} \\
\leq & \left\|\mathcal{E}_{\Omega ; n}(\varepsilon ; \cdot)-\mathcal{E}_{\Omega ; n+1}\left(\frac{\varepsilon}{2} ; \cdot\right)\right\|_{2 \mathrm{R}\left(2^{n} \varepsilon\right), 2 \mathrm{~m}} \\
\quad & \quad+K_{\Delta}(2 \delta \mathfrak{v}) \mathrm{r}(2 \delta)^{2} \mathrm{R}(2 \delta)^{2}\left(\left\|\mathcal{R}_{\Omega ; n}(\varepsilon ; \cdot)-\mathcal{R}_{\Omega ; n+1}\left(\frac{\varepsilon}{2} ; \cdot\right)\right\|_{2 \mathrm{R}\left(2^{n} \varepsilon\right), 2 \mathrm{~m}}+\varepsilon(2 \delta \mathfrak{v}) \mathrm{R}(2 \delta)^{4}\right) \\
\leq & \left\|\mathcal{E}_{\Omega ; n}(\varepsilon ; \cdot)-\mathcal{E}_{\Omega ; n+1}\left(\frac{\varepsilon}{2} ; \cdot\right)\right\|_{2 \mathrm{R}\left(2^{n} \varepsilon\right), 2 \mathrm{~m}} \\
\quad & +K_{\Delta}(2 \delta \mathfrak{v}) \mathrm{r}(2 \delta)^{2} \mathrm{R}(2 \delta)^{2}\left(K_{R} \delta \varepsilon \mathrm{r}(\varepsilon)^{2} \mathrm{R}(\varepsilon)^{2} e^{-2 \mathrm{~m} \mathfrak{c}}+\varepsilon(2 \delta \mathfrak{v}) \mathrm{R}(2 \delta)^{4}\right)
\end{aligned}
$$




$$
\begin{aligned}
\leq & \left\|\mathcal{E}_{\Omega ; n}(\varepsilon ; \cdot)-\mathcal{E}_{\Omega ; n+1}\left(\frac{\varepsilon}{2} ; \cdot\right)\right\|_{2 \mathrm{R}\left(2^{n} \varepsilon\right), 2 \mathrm{~m}} \\
& +K_{\Delta} \varepsilon(2 \delta \mathfrak{v})^{2} \mathrm{r}(2 \delta)^{2} \mathrm{R}(2 \delta)^{2}\left(\frac{1}{2} K_{R} \mathrm{r}(\varepsilon)^{2} \mathrm{R}(\varepsilon)^{2} \frac{e^{-2 \mathrm{~m} \mathfrak{c}}}{\mathfrak{v}}+\mathrm{R}(2 \delta)^{4}\right) \\
\leq & \left\|\mathcal{E}_{\Omega ; n}(\varepsilon ; \cdot)-\mathcal{E}_{\Omega ; n+1}\left(\frac{\varepsilon}{2} ; \cdot\right)\right\|_{2 \mathrm{R}\left(2^{n} \varepsilon\right), 2 \mathrm{~m}} \\
& +K_{\Delta} \varepsilon(2 \delta \mathfrak{v})^{2} \mathrm{r}(2 \delta)^{2} \mathrm{R}(2 \delta)^{2}\left(\frac{1}{\varepsilon^{2 e_{\mathrm{R}}+4 e_{\mathrm{r}}}}+\mathrm{R}(2 \delta)^{4}\right) \\
\leq & K_{E}(\varepsilon \mathfrak{v})^{2} \mathrm{r}(\varepsilon)^{2} \mathrm{R}(\varepsilon)^{6}+K_{\Delta} \varepsilon^{1-2 e_{\mathrm{R}}-4 e_{\mathrm{r}}}\left(\sum_{k=1}^{n}\left(2^{k} \varepsilon \mathfrak{v}\right)^{2} \mathrm{r}\left(2^{k} \varepsilon\right)^{2} \mathrm{R}\left(2^{k} \varepsilon\right)^{6}\right) \\
& +K_{\Delta} \varepsilon^{1-2 e_{\mathrm{R}}-4 e_{\mathrm{r}}}(2 \delta \mathfrak{v})^{2} \mathrm{r}(2 \delta)^{2} \mathrm{R}(2 \delta)^{6} \\
= & K_{E}(\varepsilon \mathfrak{v})^{2} \mathrm{r}(\varepsilon)^{2} \mathrm{R}(\varepsilon)^{6}+K_{\Delta} \varepsilon^{1-2 e_{\mathrm{R}}-4 e_{\mathrm{r}}}\left(\sum_{k=1}^{n+1}\left(2^{k} \varepsilon \mathfrak{v}\right)^{2} \mathrm{r}\left(2^{k} \varepsilon\right)^{2} \mathrm{R}\left(2^{k} \varepsilon\right)^{6}\right)
\end{aligned}
$$

For the fourth inequality, we used that, by (F.4.b) and Hypothesis F.7.ii,

$$
\frac{1}{2} K_{R} \mathrm{r}(\varepsilon)^{2} \mathrm{R}(\varepsilon)^{2} \frac{e^{-2 \mathrm{~m} \mathfrak{c}}}{\mathfrak{v}}=\frac{1}{\varepsilon^{2 e_{\mathrm{R}}+4 e_{\mathrm{r}}}} \frac{1}{2} K_{R} \mathfrak{v}^{1-2 e_{\mathrm{R}}-4 e_{\mathrm{r}}} \frac{e^{-2 \mathrm{~m} \mathfrak{c}}}{\mathfrak{v}^{2}} \leq \frac{1}{\varepsilon^{2 e_{\mathrm{R}}+4 e_{\mathrm{r}}}}
$$

This completes the induction argument.

It remains only to prove that (III.11) implies the second line of (III.10). Summing the geometric series in (III.11) gives

$$
\begin{aligned}
\left\|\mathcal{E}_{\Omega ; n}(\varepsilon ; \cdot)-\mathcal{E}_{\Omega ; n+1}\left(\frac{\varepsilon}{2} ; \cdot\right)\right\|_{2 \mathrm{R}\left(2^{n} \varepsilon\right), 2 \mathrm{~m}} \\
\quad \leq K_{E}(\varepsilon \mathfrak{v})^{2-6 e_{\mathrm{R}}-8 e_{\mathrm{r}}}+\frac{K{ }^{-}}{1-2^{-\left(2-6 e_{\mathrm{R}}-8 e_{\mathrm{r}}\right)}} \varepsilon^{1-2 e_{\mathrm{R}}-4 e_{\mathrm{r}}}\left(2^{n} \varepsilon \mathfrak{v}\right)^{2-6 e_{\mathrm{R}}-8 e_{\mathrm{r}}} \\
\quad=(\varepsilon \mathfrak{v})^{2-6 e_{\mathrm{R}}-8 e_{\mathrm{r}}}\left(K_{E}+\frac{2^{17} e^{10 K_{j}} K_{E}}{1-2^{-\left(2-6 e_{\mathrm{R}}-8 e_{\mathrm{r}}\right)}} \varepsilon^{1-2 e_{\mathrm{R}}-4 e_{\mathrm{r}}}\left(2^{n}\right)^{2-6 e_{\mathrm{R}}-8 e_{\mathrm{r}}}\right) \\
\quad \leq(\varepsilon \mathfrak{v})^{2-6 e_{\mathrm{R}}-8 e_{\mathrm{r}}} K_{E}\left(1+\frac{2^{17} e^{10 K_{j}}}{1-2^{-\left(2-6 e_{\mathrm{R}}-8 e_{\mathrm{r}}\right)}}\left(2^{n} \varepsilon\right)^{1-2 e_{\mathrm{R}}-4 e_{\mathrm{r}}}\right) \\
\quad \leq 2 K_{E}(\varepsilon \mathfrak{v})^{2-6 e_{\mathrm{R}}-8 e_{\mathrm{r}}}
\end{aligned}
$$

by (II.17) and Hypothesis F.7.i.

Corollary III.30 For each subset $\Omega$ of $X$

$$
\mathcal{D}_{\Omega, \theta}\left(\alpha_{*}, \beta\right)=\lim _{m \rightarrow \infty} \mathcal{D}_{\Omega, m}\left(2^{-m} \theta ; \alpha_{*}, \beta\right)
$$

exists $^{(1)}$. It has a decomposition

$$
\mathcal{D}_{\Omega, \theta}=\mathcal{R}_{\Omega, \theta}+\mathcal{E}_{\Omega, \theta}
$$

where

(1) The convergence is with respect to the norm $\|\cdot\|_{2 \mathrm{R}(\theta), 2 \mathrm{~m}}$. 
- $\mathcal{R}_{\Omega ; \theta}\left(\alpha_{*}, \beta\right)$ is bilinear in $\alpha_{*}$ and $\beta$ and fulfills the estimate

$$
\left\|\mathcal{R}_{\Omega ; \theta}\right\|_{2 \mathrm{R}(\theta), 2 \mathrm{~m}} \leq K_{R} \theta^{2} \mathrm{r}(\theta)^{2} \mathrm{R}(\theta)^{2} e^{-2 \mathrm{~m} \mathfrak{c}}
$$

- $\mathcal{E}_{\Omega ; \theta}\left(\alpha_{*}, \beta\right)$ has degree at least two both in $\alpha_{*}$ and in $\beta$ and fulfills the estimate

$$
\left\|\mathcal{E}_{\Omega ; \theta}\right\|_{2 \mathrm{R}(\theta), 2 \mathrm{~m}} \leq K_{E}(\theta \mathfrak{v})^{2} \mathrm{r}(\theta)^{2} \mathrm{R}(\theta)^{6}
$$

Proof: As

$$
2^{n} \varepsilon^{2} \mathrm{r}(\varepsilon)^{2} \mathrm{R}(\varepsilon)^{2} e^{-2 \mathrm{~m} \mathfrak{c}}=2^{n}(\varepsilon \mathfrak{v})^{2-2 e_{\mathrm{R}}-4 e_{\mathrm{r}}} \frac{e^{-2 \mathrm{~m} \mathfrak{c}}}{\mathfrak{v}^{2}} \quad(\varepsilon \mathfrak{v})^{2} \mathrm{r}(\varepsilon)^{2} \mathrm{R}(\varepsilon)^{6}=(\varepsilon \mathfrak{v})^{2-6 e_{\mathrm{R}}-8 e_{\mathrm{r}}}
$$

and the exponents $2-2 e_{\mathrm{R}}-4 e_{\mathrm{r}}$ and $2-6 e_{\mathrm{R}}-8 e_{\mathrm{r}}$ are strictly positive, Proposition III.29, with $n=m$, implies that the limits

$$
\lim _{m \rightarrow \infty} \mathcal{R}_{\Omega ; m}\left(2^{-m} \theta ; \cdot\right) \quad \lim _{m \rightarrow \infty} \mathcal{E}_{\Omega ; m}\left(2^{-m} \theta ; \cdot\right)
$$

exist.

We now describe the construction of the functions $\mathcal{D}_{\Omega ; \theta}, \mathcal{B}_{\mathfrak{S}}$ and $\mathcal{L}_{\mathfrak{S}}$ of Theorem II.16 from Theorems III.24, III.26, III.27 and III.28.

In each of the four theorems mentioned above we assert the existence of constants $\mathfrak{v}_{0}$ and $\Theta$ such that for all interactions and "times" bounded by $\mathfrak{v}_{0}$ resp. $\Theta$, the conclusions are true. Now choose $\mathfrak{v}_{0}$ and $\Theta$ as the smallest of the constants from the three theorems.

Fix an interaction $v$ obeying Hypothesis II.14. For each $0<\varepsilon<\Theta / 2$ and each natural number $1 \leq n \leq \log _{2} \frac{\Theta}{\varepsilon}$ define, for each $\alpha$ and $\beta$ obeying $|\alpha(\mathbf{x})|,|\beta(\mathbf{x})| \leq \mathrm{R}(\varepsilon)$ for all $\mathrm{x} \in X$, the effective density $I_{n}^{\bullet}\left(\varepsilon ; \alpha^{*}, \beta\right)$ recursively by

$$
\begin{aligned}
I_{1}^{\bullet}\left(\varepsilon ; \alpha^{*}, \beta\right)=\mathcal{Z}_{\varepsilon}^{2|X|} \int d \mu_{\mathrm{R}(\varepsilon)}\left(\phi^{*}, \phi\right) \zeta_{\varepsilon}\left(\alpha^{*}, \phi\right) e^{\left\langle\alpha^{*}, j(\varepsilon) \phi\right\rangle+\left\langle\phi^{*}, j(\varepsilon) \beta\right\rangle} \\
e^{-\varepsilon\left(\left\langle\alpha^{*} \phi, v \alpha^{*} \phi\right\rangle+\left\langle\phi^{*} \beta, v \phi^{*} \beta\right\rangle\right)} \zeta_{\varepsilon}\left(\phi^{*}, \beta\right) \\
I_{n+1}^{\bullet}\left(\varepsilon ; \alpha^{*}, \beta\right)=\int d \mu_{\mathrm{R}(\varepsilon)}\left(\phi^{*}, \phi\right) I_{n}^{\bullet}\left(\varepsilon ; \alpha^{*}, \phi\right) I_{n}^{\bullet}\left(\varepsilon ; \phi^{*}, \beta\right)
\end{aligned}
$$

with the $\mathcal{Z}_{\varepsilon}$ of Lemma II.7. 
Remark III.31 For each $n \geq 1$ we have

$$
\begin{aligned}
& I_{n}^{\bullet}\left(\varepsilon ; \alpha^{*}, \beta\right) \\
& \left.\quad=\mathcal{Z}_{\varepsilon}^{2^{n}|X|} \int_{\tau \in \varepsilon \mathbb{Z} \cap\left(0,2^{n} \varepsilon\right)} \prod_{\mathrm{R}(\varepsilon)} d \alpha_{\tau}^{*}, \alpha_{\tau}\right) \prod_{\tau \in \varepsilon \mathbb{Z} \cap\left(0,2^{n} \varepsilon\right]}\left[\zeta_{\varepsilon}\left(\alpha_{\tau-\varepsilon}, \alpha_{\tau}\right) e^{\left\langle\alpha_{\tau-\varepsilon}^{*}, j(\varepsilon) \alpha_{\tau}\right\rangle-\varepsilon\left\langle\alpha_{\tau-\varepsilon}^{*} \alpha_{\tau} v \alpha_{\tau-\varepsilon}^{*} \alpha_{\tau}\right\rangle}\right]
\end{aligned}
$$

with $\alpha_{0}=\alpha$ and $\alpha_{2^{n} \varepsilon}=\beta$. Comparing this with (I.3), we see that

$$
\left.I_{n}^{\bullet}\left(\varepsilon ; \alpha^{*}, \beta\right)\right|_{\mathfrak{h}=1}=\mathcal{Z}_{\varepsilon}^{2^{n}|X|} I_{n}\left(\varepsilon ; \alpha^{*}, \beta\right)
$$

In $§ I I I .7$, we shall prove that

$$
I_{\theta}^{\bullet}\left(\alpha^{*}, \beta\right)=\left.\lim _{m \rightarrow \infty} I_{m}^{\bullet}\left(2^{-m} \theta ; \alpha^{*}, \beta\right)\right|_{\mathfrak{h}=1}
$$

exists. Using the initial condition in Lemma II.7, it will then follow that $I_{\theta}\left(\alpha^{*}, \beta\right)=$ $\lim _{m \rightarrow \infty} I_{m}\left(2^{-m} \theta ; \alpha^{*}, \beta\right)$ also exists and

$$
I_{\theta}\left(\alpha^{*}, \beta\right)=I_{\theta}^{\bullet}\left(\alpha^{*}, \beta\right)
$$

Theorem III.26 allows us to recursively construct a representation of $I_{n}^{\bullet}(\varepsilon ; \cdot)$ similar to that of Theorem II.16. To do so, fix $\mathfrak{v} \leq \mathfrak{v}_{0}$ and an interaction $v$ obeying Hypothesis II.14. In the following, we use the constants $K_{D}$ and $K_{L}$ of Theorem III.26.

Proposition III.32 For each $\varepsilon$, sufficiently small, and integer $n \geq 0$ with $2^{n} \varepsilon \leq \Theta$, the effective density $I_{n}^{\bullet}\left(\varepsilon ; \alpha^{*}, \beta\right)$ has, for all $\alpha, \beta$ obeying $\sup _{\mathbf{x} \in X}|\alpha(\mathbf{x})|, \sup _{\mathbf{x} \in X}|\beta(\mathbf{x})| \leq \mathrm{R}(\varepsilon)$, a representation

$$
\begin{aligned}
I_{n}^{\bullet}\left(\varepsilon ; \alpha_{*}, \beta\right)= & \sum_{\begin{array}{c}
\mathfrak{S} \text { hierarchy } \\
\text { for scale } 2^{2} \varepsilon \\
\text { of depth at most } n
\end{array}} \mathcal{Z}_{2^{n} \varepsilon}^{\left|\Omega_{\mathfrak{S}}\right|} \chi_{2^{n} \varepsilon}\left(\Omega_{\mathfrak{S}} ; \alpha, \beta\right) \\
& \mathcal{I}_{\left(\mathfrak{S} ; \alpha^{*}, \beta\right)}\left(e^{-Q_{\mathfrak{S}}\left(\alpha^{*}, \beta ; \vec{\alpha}^{*}, \vec{\alpha}\right)+\mathcal{V}_{\mathfrak{S}}\left(\varepsilon ; \alpha^{*}, \beta ; \vec{\alpha}^{*}, \vec{\alpha}\right)} e^{\mathcal{D}_{\mathfrak{S}}\left(\varepsilon ; \alpha^{*}, \beta ; \overrightarrow{\rho_{\uparrow}}\right)+\mathcal{L}_{\mathfrak{S}}\left(\varepsilon ; \alpha^{*}, \beta ; \overrightarrow{\rho^{\prime}}\right)}\right)
\end{aligned}
$$

Here, for each $\varepsilon>0$, sufficiently small, and hierarchy $\mathfrak{S}$ for scale $2^{n} \varepsilon \leq \Theta$, with depth at most $n, \mathcal{D}_{\mathfrak{S}}\left(\varepsilon ; \alpha^{*}, \beta ; \vec{\rho}\right)$ and $\mathcal{L}_{\mathfrak{S}}\left(\varepsilon ; \alpha^{*}, \beta ; \vec{\rho}\right)$ are analytic functions that have the following properties

- The "pure large field part of $\mathcal{D}_{\mathfrak{S}}(\varepsilon ; \cdot)$ vanishes, that is $\left.\mathcal{D}_{\mathfrak{S}}\right|_{\Omega_{\mathfrak{S}}^{c}}=0$. The "pure small field part"

$$
\left.\mathcal{D}_{\mathfrak{S}}(\varepsilon ; \cdot)\right|_{\Omega_{\mathfrak{S}}}=\mathcal{D}_{\Omega_{\mathfrak{S}}, n}(\varepsilon ; \cdot)
$$


as in (III.8) and Proposition III.29. Also

$$
\left\|\mathcal{D}_{\mathfrak{S}}(\varepsilon ; \cdot)\right\|_{\mathfrak{S}} \leq K_{D}\left(2^{n} \varepsilon \mathfrak{v}\right) \mathrm{r}\left(2^{n} \varepsilon\right) \mathrm{R}\left(2^{n} \varepsilon\right)^{3}
$$

- The "pure large field part" $\mathcal{L}_{\mathfrak{S}}$ has the decomposition

$$
\mathcal{L}_{\mathfrak{S}}\left(\varepsilon ; \alpha_{*}, \beta ; \vec{\rho}\right)=\sum_{\substack{\text { decimation intervals } \mathcal{J} \\ \text { for }\left[0,2^{n} \varepsilon \text { of length } \\ \text { at least } 2^{n-\operatorname{depth}(\mathcal{S})+1}\right.}} \mathcal{L}_{\mathfrak{S}}\left(\mathcal{J}, \varepsilon ; \alpha_{*}, \beta ; \vec{\rho}\right)
$$

where, for each decimation interval $\mathcal{J}$ in the sum, the function $\mathcal{L}_{\mathfrak{S}}\left(\mathcal{J}, \varepsilon ; \alpha_{*}, \beta ; \vec{\rho}\right)$ is an analytic function of its arguments that

- is "large field with respect to the interval $\mathcal{J}$ " (that is, it depends only on values of the fields at points $\mathbf{x} \in X \backslash \Omega_{\mathfrak{S}}(\mathcal{J})$ and depends only on the variables $\alpha_{* \tau}, \alpha_{\tau}, z_{* \tau}, z_{\tau}$ with $\tau \in \mathcal{J} \cap\left(2^{-\operatorname{depth}(\mathfrak{S})} \theta\right) \mathbb{Z}$.

- and fulfills the estimate

$$
\left\|\mathcal{L}_{\mathfrak{S}}(\mathcal{J}, \varepsilon ; \cdot)\right\|_{\mathfrak{S}} \leq K_{L} \delta \mathfrak{v} \mathrm{r}(\delta) \mathrm{R}(\delta)^{3}
$$

where $\delta$ is the length of the time interval $\mathcal{J}$.

- The functions $\mathcal{D}_{\mathfrak{S}}$ and $\mathcal{L}_{\mathfrak{S}}(\mathcal{J})$ are all history complete and invariant under $\alpha_{*} \rightarrow$ $e^{-i \theta} \alpha_{*}, \beta \rightarrow e^{i \theta} \beta, \vec{\rho}=\left(\vec{\alpha}_{*}, \vec{\alpha}, \vec{z}_{*}, \vec{z}\right) \rightarrow\left(e^{-i \theta} \vec{\alpha}_{*}, e^{i \theta} \vec{\alpha}, e^{-i \theta} \vec{z}_{*}, e^{i \theta} \vec{z}\right)$

Furthermore, for each hierarchy $\mathfrak{S}$ for scale $2^{n} \varepsilon$, of depth at most $n$, and each decimation interval $\mathcal{J} \subset\left[0,2^{n} \varepsilon\right]$, of length at least $2^{n-\operatorname{depth}(\mathfrak{S})+1} \varepsilon$,

$$
\begin{aligned}
\left\|\mathcal{D}_{\mathfrak{S}}(\varepsilon ; \cdot)-\mathcal{D}_{\mathfrak{S}}\left(\frac{\varepsilon}{2} ; \cdot\right)\right\|_{\mathfrak{S}} & \leq\left(\varepsilon^{2} \mathfrak{v}\right)^{1-3 e_{\mathrm{R}}-4 e_{\mathrm{r}}} \\
\left\|\mathcal{L}_{\mathfrak{S}}(\mathcal{J}, \varepsilon ; \cdot)-\mathcal{L}_{\mathfrak{S}}\left(\mathcal{J}, \frac{\varepsilon}{2} ; \cdot\right)\right\|_{\mathfrak{S}} & \leq 2^{10}\left(\varepsilon^{2} \mathfrak{v}\right)^{1-3 e_{\mathrm{R}}-4 e_{\mathrm{r}}}
\end{aligned}
$$

Proof of Proposition III.32 from Theorems III.24, III.26, III.27 and III.28: We introduce, in addition to the effective densities $I_{1}, I_{2}, \cdots$ of (III.12), the initial effective density

$$
I_{0}^{\bullet}\left(\varepsilon ; \alpha^{*}, \beta\right)=\mathcal{Z}_{\varepsilon}^{|X|} \chi_{\mathrm{R}(\varepsilon)}(X, \alpha) \chi_{\mathrm{R}(\varepsilon)}(X, \beta) \zeta_{\varepsilon}\left(\alpha^{*}, \beta\right) e^{\left\langle\alpha^{*}, j(\varepsilon) \beta\right\rangle} e^{-\varepsilon\left\langle\alpha^{*} \beta, v \alpha^{*} \beta\right\rangle}
$$

where $\chi_{\mathrm{R}}(X, \alpha)$ is the characteristic function which restricts $|\alpha(\mathbf{x})| \leq \mathrm{R}$ for each $\mathbf{x} \in X$. Then the recursion relation of (III.12) still holds for the step from $n=0$ to $n=1$.

We prove the Proposition by induction on $n$, starting with $n=0$. There is only a single hierarchy $\mathfrak{S}$ of scale $\varepsilon$ and depth 0 , namely that with $\Omega_{\mathfrak{S}}=X$. For $\varepsilon$ sufficiently small, the initial effective density $I_{0}$ may also be written

$$
I_{0}^{\bullet}\left(\varepsilon ; \alpha^{*}, \beta\right)=\mathcal{Z}_{\varepsilon}^{|X|} \chi_{\varepsilon}(X ; \alpha, \beta) e^{\left\langle\alpha^{*}, j(\varepsilon) \beta\right\rangle} e^{-\varepsilon\left\langle\alpha^{*} \beta, v \alpha^{*} \beta\right\rangle}
$$


because $|\nabla \alpha(b)|,|\nabla \beta(b)| \leq 2 \mathrm{R}(\varepsilon) \leq \mathrm{R}^{\prime}(\varepsilon)$ for all $b \in X^{*}$. It satisfies the conclusions of the Proposition, with $\mathcal{D}_{\mathfrak{S}}(\varepsilon)=\mathcal{L}_{\mathfrak{S}}(\varepsilon)=0$ and $\mathcal{I}_{\left(\mathfrak{S} ; \alpha^{*}, \beta\right)}$ the identity operator. Since $\Omega_{\mathfrak{S}}=X$, we have $\mathcal{L}_{\mathfrak{S}}\left(\frac{\varepsilon}{2}\right)=0$ and, in the notation of Proposition III.29,

$$
\mathcal{D}_{\mathfrak{S}}\left(\frac{\varepsilon}{2}\right)=\mathcal{D}_{X, 1}\left(\frac{\varepsilon}{2}\right)=\mathcal{R}_{X, 1}\left(\frac{\varepsilon}{2}\right)+\mathcal{E}_{X, 1}\left(\frac{\varepsilon}{2}\right)
$$

so that

$$
\begin{aligned}
\left\|\mathcal{D}_{\mathfrak{S}}(\varepsilon)-\mathcal{D}_{\mathfrak{S}}\left(\frac{\varepsilon}{2}\right)\right\|_{\mathfrak{S}} & =\left\|\mathcal{D}_{\mathfrak{S}}\left(\frac{\varepsilon}{2}\right)\right\|_{2 \mathrm{R}(\varepsilon), 2 \mathrm{~m}} \leq K_{R} \varepsilon^{2} \mathrm{r}(\varepsilon)^{2} \mathrm{R}(\varepsilon)^{2} e^{-2 \mathrm{~m} \mathfrak{c}}+K_{E}(\varepsilon \mathfrak{v})^{2} \mathrm{r}(\varepsilon)^{2} \mathrm{R}(\varepsilon)^{6} \\
& =K_{R}(\varepsilon \mathfrak{v})^{2-2 e_{\mathrm{R}}-4 e_{\mathrm{r}}} \frac{e^{-2 \mathrm{~m} \mathfrak{c}}}{\mathfrak{v}^{2}}+K_{E}(\varepsilon \mathfrak{v})^{2-6 e_{\mathrm{R}}-8 e_{\mathrm{r}}} \\
& \leq\left(\varepsilon^{2} \mathfrak{v}\right)^{1-3 e_{\mathrm{R}}-4 e_{\mathrm{r}}}
\end{aligned}
$$

by (F.6.a) and (F.4.b), if $\varepsilon$ is small enough.

For the induction step from $n$ to $n+1$, set $\delta=2^{n} \varepsilon$. By definition and the induction hypothesis

$$
I_{n+1}^{\bullet}\left(\varepsilon ; \alpha^{*}, \beta\right)=\sum_{\substack{\mathfrak{S}_{1}, \mathfrak{S}_{2} \text { hierarchies } \\ \text { for scale } \text { ond } \\ \text { depth at most } n}} \int d \mu_{\mathrm{R}(\varepsilon)}\left(\phi^{*}, \phi\right) I_{\mathfrak{S}_{1}}\left(\varepsilon ; \alpha^{*}, \phi\right) I_{\mathfrak{S}_{2}}\left(\varepsilon ; \phi^{*}, \beta\right)
$$

where

$$
\begin{aligned}
I_{\mathfrak{S}_{i}}\left(\varepsilon ; \alpha^{*}, \beta\right)= & \mathcal{Z}_{\delta}^{\left|\Omega_{\mathfrak{S}_{i}}\right|} \chi_{\delta}\left(\Omega_{\mathfrak{S}_{i}} ; \alpha, \beta\right) \\
& \mathcal{I}_{\left(\mathfrak{S}_{i} ; \alpha^{*}, \beta\right)}\left(e^{-Q_{\mathfrak{S}_{i}}\left(\alpha^{*}, \beta ; \vec{\alpha}^{*}, \vec{\alpha}\right)+\mathcal{V}_{\mathfrak{S}_{i}}\left(\varepsilon ; \alpha^{*}, \beta ; \vec{\alpha}^{*}, \vec{\alpha}\right)+\mathcal{D}_{\mathfrak{S}_{i}}\left(\varepsilon ; \alpha^{*}, \beta ; \overrightarrow{\rho_{\uparrow}}\right)} e^{\mathcal{L}_{\mathfrak{S}_{i}}\left(\varepsilon ; \alpha^{*}, \beta ; \overrightarrow{\rho_{\uparrow}}\right)}\right)
\end{aligned}
$$

By the induction hypothesis, Remark III.16, Proposition III.29, (F.4.b) and (F.6.a),

$$
\begin{aligned}
& \left\|\mathcal{D}_{\mathfrak{S}_{i}}(\varepsilon ; \cdot)\right\|_{\mathfrak{S}_{i}} \leq K_{D}(\delta \mathfrak{v}) \mathrm{r}(\delta) \mathrm{R}(\delta)^{3} \leq 1 \\
& \left\|\left.\mathcal{D}_{\mathfrak{S}_{i}}(\varepsilon ; \cdot)\right|_{\Omega_{\mathfrak{S}_{i}}}\right\|_{\mathfrak{S}_{i}} \leq\left\|\mathcal{D}_{\Omega_{\mathfrak{S}_{i}}, n}(\varepsilon ; \cdot)\right\|_{2 \mathrm{R}(\delta), 2 \mathrm{~m}}
\end{aligned}
$$

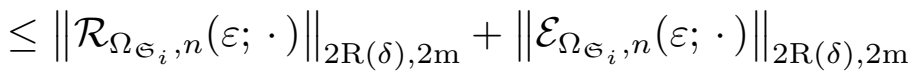

$$
\begin{aligned}
& \leq K_{R} \delta^{2} \mathrm{r}(\delta)^{2} \mathrm{R}(\delta)^{2} e^{-2 \mathrm{~m} \mathfrak{c}}+K_{E}(\delta \mathfrak{v})^{2} \mathrm{r}(\delta)^{2} \mathrm{R}(\delta)^{6} \\
& \leq 2^{-17} K_{D}(2 \delta \mathfrak{v}) \mathrm{r}(2 \delta) \mathrm{R}(2 \delta)^{3} \leq 2^{-20}
\end{aligned}
$$

Hence, for each pair of hierarchies $\mathfrak{S}_{1}$ and $\mathfrak{S}_{2}$, we may apply Theorem III.26 to the integral $\int d \mu_{\mathrm{R}(\varepsilon)}\left(\phi^{*}, \phi\right) I_{\mathfrak{S}_{1}}\left(\varepsilon ; \alpha^{*}, \phi\right) I_{\mathfrak{S}_{2}}\left(\varepsilon ; \phi^{*}, \beta\right)$. We apply it with $\mathcal{D}_{i}=\mathcal{D}_{\mathfrak{S}_{i}}$ and $b_{i}=e^{\mathcal{L}_{i}}$. It gives the representation

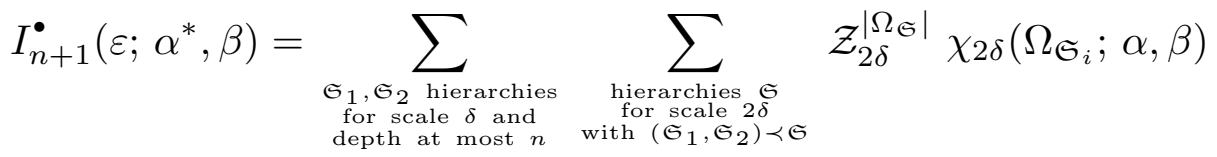

$$
\begin{aligned}
& \mathcal{I}_{\left(\mathfrak{S} ; \alpha^{*}, \beta\right)}\left(e^{-Q_{\mathfrak{S}}\left(\alpha^{*}, \beta ; \vec{\alpha}^{*}, \vec{\alpha}\right)+\mathcal{V}_{\mathfrak{S}}\left(\varepsilon ; \alpha^{*}, \beta ; \vec{\alpha}^{*}, \vec{\alpha}\right)+\mathcal{D}_{\mathfrak{S}}\left(\varepsilon ; \alpha^{*}, \beta ; \overrightarrow{p_{\mathfrak{f}}}\right)}\right. \\
& e^{\mathcal{L}_{\mathfrak{S}}^{\prime}\left(\varepsilon ; \alpha^{*}, \beta ; \overrightarrow{\rho_{\uparrow}}\right)+\mathcal{L}_{\mathfrak{S}_{1}}\left(\varepsilon ; \alpha^{*}, \alpha_{\delta} ; \overrightarrow{\rho_{\uparrow} l}\right)+\mathcal{L}_{\mathfrak{S}_{2}}\left(\varepsilon ; \alpha_{\delta}^{*}, \beta ; \overrightarrow{\rho_{\uparrow} r}\right)}
\end{aligned}
$$


The resulting functions $\mathcal{D}_{\mathfrak{S}}$ fulfill $\left.\mathcal{D}_{\mathfrak{S}}(\varepsilon ; \cdot)\right|_{\Omega_{\mathfrak{G}}^{c}}=0$,

$$
\begin{aligned}
\left.\mathcal{D}_{\mathfrak{S}}(\varepsilon ; \cdot)\right|_{\Omega_{\mathfrak{S}}} & =\bar{\Re}_{\Omega_{\mathfrak{S}} ; \delta}\left(V_{\Omega_{\mathfrak{S}} ; \delta}(\varepsilon ; \cdot) ;\left.\mathcal{D}_{\Omega_{\mathfrak{S}_{1}, n}}(\varepsilon ; \cdot)\right|_{\Omega_{\mathfrak{S}}},\left.\mathcal{D}_{\Omega_{\mathfrak{S}_{2}}, n}(\varepsilon ; \cdot)\right|_{\Omega_{\mathfrak{S}}}\right) \\
& =\bar{\Re}_{\Omega_{\mathfrak{S}} ; \delta}\left(V_{\Omega_{\mathfrak{F}} ; \delta}(\varepsilon ; \cdot) ; \mathcal{D}_{\Omega_{\mathfrak{S}}, n}(\varepsilon ; \cdot), \mathcal{D}_{\Omega_{\mathfrak{S}}, n}(\varepsilon ; \cdot)\right) \\
& =\mathcal{D}_{\Omega_{\mathfrak{S}}, n+1}(\varepsilon ; \cdot)
\end{aligned}
$$

by the inductive hypothesis, (III.9) and (III.8), and

$$
\begin{aligned}
& \left\|\mathcal{D}_{\mathfrak{S}}(\varepsilon ; \cdot)\right\|_{\mathfrak{S}} \leq \frac{1}{2} K_{D}(2 \delta \mathfrak{v}) \mathrm{r}(2 \delta) \mathrm{R}(2 \delta)^{3}+2^{14} e^{-\mathrm{mc}(\delta)}\left(\left\|\mathcal{D}_{\mathfrak{S}_{1}}(\varepsilon ; \cdot)\right\|_{\mathfrak{S}_{1}}+\left\|\mathcal{D}_{\mathfrak{S}_{2}}(\varepsilon ; \cdot)\right\|_{\mathfrak{S}_{2}}\right) \\
& \quad+2^{14}\left(\left\|\left.\mathcal{D}_{\mathfrak{S}_{1}}(\varepsilon ; \cdot)\right|_{\Omega_{\mathfrak{S}_{1}}}\right\|_{\mathfrak{S}_{1}}+\left\|\left.\mathcal{D}_{\mathfrak{S}_{2}}(\varepsilon ; \cdot)\right|_{\Omega_{\mathfrak{S}_{2}}}\right\|_{\mathfrak{S}_{2}}\right) \\
& \leq \frac{1}{2} K_{D}(2 \delta \mathfrak{v}) \mathrm{r}(2 \delta) \mathrm{R}(2 \delta)^{3}+2^{15} e^{-\mathrm{m} \mathfrak{c}(\delta)} K_{D}(\delta \mathfrak{v}) \mathrm{r}(\delta) \mathrm{R}(\delta)^{3}+\frac{1}{4} K_{D}(2 \delta \mathfrak{v}) \mathrm{r}(2 \delta) \mathrm{R}(2 \delta)^{3} \\
& \leq K_{D}(2 \delta \mathfrak{v}) \mathrm{r}(2 \delta) \mathrm{R}(2 \delta)^{3}
\end{aligned}
$$

by (F.4.c). The resulting functions $\mathcal{L}_{\mathfrak{S}}^{\prime}$ fulfill $\mathcal{L}_{\mathfrak{S}}^{\prime}=\left.\mathcal{L}_{\mathfrak{S}}^{\prime}\right|_{\Omega_{\mathfrak{S}}^{c}}$ and

$$
\begin{aligned}
\left\|\mathcal{L}_{\mathfrak{S}}^{\prime}\right\|_{\mathfrak{S}} & \leq \frac{1}{2} K_{L}(2 \delta \mathfrak{v}) r(2 \delta) \mathrm{R}(2 \delta)^{3}+2^{8}\left(\left\|\mathcal{D}_{1}\right\|_{\mathfrak{S}_{1}}+\left\|\mathcal{D}_{2}\right\|_{\mathfrak{S}_{2}}\right) \\
& \leq \frac{1}{2} K_{L}(2 \delta \mathfrak{v}) r(2 \delta) \mathrm{R}(2 \delta)^{3}+2^{9} K_{D}(\delta \mathfrak{v}) \mathrm{r}(\delta) \mathrm{R}(\delta)^{3} \\
& \leq K_{L}(2 \delta \mathfrak{v}) r(2 \delta) \mathrm{R}(2 \delta)^{3}
\end{aligned}
$$

since $K_{L} \geq 2{ }^{13} K_{D}$. If $\mathcal{J}$ is a decimation interval for $\mathfrak{S}$, we set

$$
\mathcal{L}_{\mathfrak{S}}\left(\mathcal{J}, \varepsilon ; \alpha_{*}, \beta ; \vec{\rho}\right)= \begin{cases}\mathcal{L}_{\mathfrak{S}_{1}}\left(\mathcal{J}, \varepsilon ; \alpha_{*}, \alpha_{\delta} ; \vec{\rho}_{l}\right) & \text { if } \mathcal{J} \subset[0, \delta] \\ \mathcal{L}_{\mathfrak{S}_{2}}\left(\mathcal{J}-\delta, \varepsilon ; \alpha_{* \delta}, \beta ; \vec{\rho}_{r}\right) & \text { if } \mathcal{J} \subset[\delta, 2 \delta] \\ \mathcal{L}_{\mathfrak{S}}^{\prime}(\varepsilon ; \alpha, \beta ; \vec{\rho}) & \text { if } \mathcal{J}=[0,2 \delta]\end{cases}
$$

Now let $\mathfrak{S}, \mathfrak{S}_{1}$ and $\mathfrak{S}_{2}$ be hierarchies with $\left(\mathfrak{S}_{1}, \mathfrak{S}_{2}\right) \prec \mathfrak{S}$. By the induction hypothesis and Proposition III.29,

$$
\begin{aligned}
& \left\|\mathcal{D}_{\mathfrak{S}_{i}}(\varepsilon ; \cdot)-\mathcal{D}_{\mathfrak{S}_{i}}\left(\frac{\varepsilon}{2} ; \cdot\right)\right\|_{\mathfrak{S}_{i}} \leq\left(\varepsilon^{2} \mathfrak{v}\right)^{1-3 e_{\mathrm{R}}-4 e_{\mathrm{r}}} \\
& \left\|\left.\mathcal{D}_{\mathfrak{S}_{i}}(\varepsilon ; \cdot)\right|_{\Omega_{\mathfrak{S}_{i}}}-\left.\mathcal{D}_{\mathfrak{S}_{i}}\left(\frac{\varepsilon}{2} ; \cdot\right)\right|_{\Omega_{\mathfrak{S}_{i}}}\right\|_{\mathfrak{S}_{i}} \leq\left\|\mathcal{R}_{\Omega_{\mathfrak{S}_{i}}, n}(\varepsilon ; \cdot)-\mathcal{R}_{\Omega_{\mathfrak{S}_{i}}, n}\left(\frac{\varepsilon}{2} ; \cdot\right)\right\|_{2 \mathrm{R}(\delta), 2 \mathrm{~m}}
\end{aligned}
$$

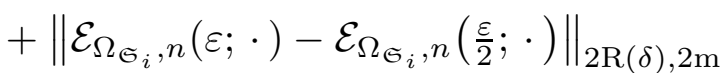

$$
\begin{aligned}
& \leq K_{R} \varepsilon \delta \mathrm{r}(\varepsilon)^{2} \mathrm{R}(\varepsilon)^{2} e^{-2 \mathrm{~m} \mathfrak{c}}+2 K_{E}(\varepsilon \mathfrak{v})^{2} \mathrm{r}(\varepsilon)^{2} \mathrm{R}(\varepsilon)^{6} \\
& =K_{R} \delta(\varepsilon \mathfrak{v})^{1-2 e_{\mathrm{R}}-4 e_{\mathrm{r}}} \frac{e^{-2 \mathrm{~m} \mathfrak{c}}}{\mathfrak{v}}+2 K_{E}(\varepsilon \mathfrak{v})^{2-6 e_{\mathrm{R}}-8 e_{\mathrm{r}}} \\
& \leq 3 K_{E}(\varepsilon \mathfrak{v})^{2-6 e_{\mathrm{R}}-8 e_{\mathrm{r}}}
\end{aligned}
$$

if $\varepsilon$ is small enough. We apply Theorem III.28 with $\mathcal{D}_{i}=\mathcal{D}_{\mathfrak{S}_{i}}(\varepsilon ; \cdot), \tilde{\mathcal{D}}_{i}=\mathcal{D}_{\mathfrak{S}_{i}}\left(\frac{\varepsilon}{2} ; \cdot\right)$, $b_{i}=e^{\mathcal{L}_{\mathfrak{S}_{i}}(\varepsilon ; \cdot)}$ and $\tilde{b}_{i}=e^{\mathcal{L}_{\mathfrak{S}_{i}}\left(\frac{\varepsilon}{2} ; \cdot\right)}$. It gives

$$
\begin{aligned}
\left\|\mathcal{D}_{\mathfrak{S}}(\varepsilon ; \cdot)-\mathcal{D}_{\mathfrak{S}}\left(\frac{\varepsilon}{2} ; \cdot\right)\right\|_{\mathfrak{S}} \leq & \frac{1}{2} K_{D} \varepsilon(2 \delta \mathfrak{v}) \mathrm{r}(2 \delta) \mathrm{R}(2 \delta)^{3}+2^{17} e^{-\mathrm{m} \mathfrak{c}(\delta)}\left(\varepsilon^{2} \mathfrak{v}\right)^{1-3 e_{\mathrm{R}}-4 e_{\mathrm{r}}} \\
& \quad+2^{17}\left(3 K_{E}(\varepsilon \mathfrak{v})^{2-6 e_{\mathrm{R}}-8 e_{\mathrm{r}}}\right) \\
= & \frac{1}{2} K_{D}(2 \delta)^{1-3 e_{\mathrm{R}}-4 e_{\mathrm{r}}} \varepsilon \mathfrak{v}^{1-3 e_{\mathrm{R}}-4 e_{\mathrm{r}}}+2^{17} e^{-\mathrm{m} \mathfrak{c}(\delta)}\left(\varepsilon^{2} \mathfrak{v}\right)^{1-3 e_{\mathrm{R}}-4 e_{\mathrm{r}}} \\
& \quad+3 \cdot 2^{17} K_{E} \mathfrak{v}^{1-3 e_{\mathrm{R}}-4 e_{\mathrm{r}}}\left(\varepsilon^{2} \mathfrak{v}\right)^{1-3 e_{\mathrm{R}}-4 e_{\mathrm{r}}} \\
\leq & \left(\varepsilon^{2} \mathfrak{v}\right)^{1-3 e_{\mathrm{R}}-4 e_{\mathrm{r}}}
\end{aligned}
$$


by (F.4.c) and (F.6.a), if $\varepsilon$ is small enough, and

$$
\begin{aligned}
\left\|\mathcal{L}_{\mathfrak{S}}([0,2 \delta], \varepsilon ; \cdot)-\mathcal{L}_{\mathfrak{S}}\left([0,2 \delta], \frac{\varepsilon}{2} ; \cdot\right)\right\|_{\mathfrak{S}} & \leq \frac{1}{2} K_{L} \varepsilon(2 \delta \mathfrak{v}) \mathrm{r}(2 \delta) \mathrm{R}(2 \delta)^{3}+2^{9}\left(\varepsilon^{2} \mathfrak{v}\right)^{1-3 e_{\mathrm{R}}-4 e_{\mathrm{r}}} \\
& \leq 2^{10}\left(\varepsilon^{2} \mathfrak{v}\right)^{1-3 e_{\mathrm{R}}-4 e_{\mathrm{r}}}
\end{aligned}
$$

if $\varepsilon$ is small enough.

Corollary III.33 Let $\mathfrak{S}$ be a hierarchy of scale $\theta$. Then the limit

$$
\mathcal{D}_{\mathfrak{S}}\left(\alpha_{*}, \beta ; \vec{\rho}\right)=\lim _{m \rightarrow \infty} \mathcal{D}_{\mathfrak{S} ; m}\left(2^{-m} \theta ; \alpha_{*}, \beta ; \vec{\rho}\right)
$$

exists $^{(2)}$. Its "pure large field part" vanishes, that is $\left.\mathcal{D}_{\mathfrak{S}}\right|_{\Omega_{\mathfrak{S}}^{c}}=0$. The "pure small field part"

$$
\left.\mathcal{D}_{\mathfrak{S}}\right|_{\Omega_{\mathfrak{S}}}=\mathcal{D}_{\Omega_{\mathfrak{S}}, \theta}
$$

as in Corollary III.30. Also

$$
\left\|\mathcal{D}_{\mathfrak{S}}\right\|_{\mathfrak{S}} \leq K_{D}(\theta \mathfrak{v}) \mathrm{r}(\theta) \mathrm{R}(\theta)^{3}
$$

For each decimation interval $\mathcal{J}$ in $[0, \theta]$, the limit

$$
\mathcal{L}_{\mathfrak{S}}\left(\mathcal{J} ; \alpha_{*}, \beta ; \vec{\rho}\right)=\lim _{m \rightarrow \infty} \mathcal{L}_{\mathfrak{S} ; m}\left(\mathcal{J}, 2^{-m} \theta ; \alpha_{*}, \beta ; \vec{\rho}\right)
$$

exists $^{(2)}$. The function $\mathcal{L}_{\mathfrak{S}}\left(\mathcal{J} ; \alpha_{*}, \beta ; \vec{\rho}\right)$ is an analytic function of its arguments that

- is "large field with respect to the interval $\mathcal{J}$ " (that is, it depends only on values of the fields at points $\mathbf{x} \in X \backslash \Omega_{\mathfrak{S}}(\mathcal{J})$ and depends only on the variables $\alpha_{* \tau}, \alpha_{\tau}, z_{* \tau}, z_{\tau}$ with $\tau \in \mathcal{J} \cap\left(2^{-\operatorname{depth}(\mathfrak{S})} \theta\right) \mathbb{Z}$.

- and fulfills the estimate

$$
\left\|\mathcal{L}_{\mathfrak{S}}(\mathcal{J} ; \cdot)\right\|_{\mathfrak{S}} \leq K_{L} \delta\|v\| \mathrm{r}(\delta) \mathrm{R}(\delta)^{3}
$$

where $\delta$ is the length of the time interval $\mathcal{J}$.

The functions $\mathcal{D}_{\mathfrak{S}}$ and $\mathcal{L}_{\mathfrak{S}}(\mathcal{J})$ are all history complete and invariant under $\alpha_{*} \rightarrow e^{-i \theta} \alpha_{*}$, $\beta \rightarrow e^{i \theta} \beta, \vec{\rho}=\left(\vec{\alpha}_{*}, \vec{\alpha}, \vec{z}_{*}, \vec{z}\right) \rightarrow\left(e^{-i \theta} \vec{\alpha}_{*}, e^{i \theta} \vec{\alpha}, e^{-i \theta} \vec{z}_{*}, e^{i \theta} \vec{z}\right)$.

The remaining $\varepsilon$ dependent term in the representation of Proposition III.32 is $\mathcal{V}_{\mathfrak{S}}\left(\varepsilon ; \alpha^{*}, \beta ; \vec{\alpha}^{*}, \vec{\alpha}\right)$. We now show that its limit as $\varepsilon$ tends to zero is the interaction

$$
\mathcal{V}_{\mathfrak{S}}\left(\alpha_{*}, \beta ; \vec{\alpha}_{*}, \vec{\alpha}\right)=-\int_{0}^{\delta} d \tau\left\langle\Gamma_{* \mathfrak{S}}\left(\tau ; \alpha_{*}, \vec{\alpha}_{*}\right) \Gamma_{\mathfrak{S}}(\tau ; \vec{\alpha}, \beta), v \Gamma_{* \mathfrak{S}}\left(\tau ; \alpha_{*}, \vec{\alpha}_{*}\right) \Gamma_{\mathfrak{S}}(\tau ; \vec{\alpha}, \beta)\right\rangle
$$

This agrees with (II.11), though of course $v$ now implicitly depends on $\mathfrak{h}$.

(2) The convergence is with respect to the norm $\|\cdot\| \mathfrak{S}$. 
Lemma III.34 Let $\mathfrak{S}$ be a hierarchy for scale $\theta$. Then, for each $\alpha^{*}, \beta, \vec{\alpha}^{*}, \vec{\alpha}$,

$$
\lim _{m \rightarrow \infty} \mathcal{V}_{\mathfrak{S}}\left(2^{-m} \theta ; \alpha_{*}, \beta ; \vec{\alpha}_{*}, \vec{\alpha}\right)=\mathcal{V}_{\mathfrak{S}}\left(\alpha_{*}, \beta ; \vec{\alpha}_{*}, \vec{\alpha}\right)
$$

The convergence is uniform on compact sets. Furthermore

$$
\left.\mathcal{V}_{\mathfrak{S}}\left(\alpha_{*}, \beta ; \vec{\alpha}_{*}, \vec{\alpha}\right)\right|_{\Omega_{\mathfrak{S}}}=V_{\Omega_{\mathfrak{S}} ; \theta}\left(\alpha_{*}, \beta\right)
$$

where $V_{\Omega ; \theta}\left(\alpha_{*}, \beta\right)$ is defined as in (II.4), but with history fields included.

Proof: We use the shorthand notations

$$
\gamma_{* \tau}=\left\{\begin{array}{ll}
\Gamma_{* \mathfrak{S}}\left(\tau ; \alpha_{*}, \vec{\alpha}_{*}\right) & \text { if } \tau \in(0, \theta) \\
\alpha_{* 0}=\alpha_{*} & \text { if } \tau=0
\end{array}\right\} \quad \gamma_{\tau}=\left\{\begin{array}{ll}
\Gamma_{\mathfrak{S}}(\tau ; \vec{\alpha}, \beta) & \text { if } \tau \in(0, \theta) \\
\alpha_{\theta}=\beta & \text { if } \tau=\theta
\end{array}\right\}
$$

Set $\varepsilon=2^{-m} \theta$ and write

$$
\begin{aligned}
& \mathcal{V}_{\mathfrak{S}}\left(\alpha^{*}, \beta ; \vec{\alpha}^{*}, \vec{\alpha}\right)-\mathcal{V}_{\mathfrak{S}}\left(\varepsilon ; \alpha^{*}, \beta ; \vec{\alpha}^{*}, \vec{\alpha}\right) \\
& =-\int_{0}^{\theta} d \tau\left\langle\gamma_{* \tau} \gamma_{\tau}, v \gamma_{* \tau} \gamma_{\tau}\right\rangle+\varepsilon \sum_{\tau \in \varepsilon \mathbb{Z} \cap[0, \theta)}\left\langle\gamma_{* \tau} \gamma_{\tau+\varepsilon}, v \gamma_{* \tau} \gamma_{\tau+\varepsilon}\right\rangle \\
& \quad=\sum_{\tau \in \varepsilon \mathbb{Z} \cap[0, \theta)} \int_{0}^{\varepsilon} d t\left[\left\langle\gamma_{* \tau} \gamma_{\tau+\varepsilon}, v \gamma_{* \tau} \gamma_{\tau+\varepsilon}\right\rangle-\left\langle\gamma_{* \tau+t} \gamma_{\tau+t}, v \gamma_{* \tau+t} \gamma_{\tau+t}\right\rangle\right]
\end{aligned}
$$

Consequently

$$
\begin{aligned}
& \left|\mathcal{V}_{\mathfrak{S}}\left(\alpha^{*}, \beta ; \vec{\alpha}^{*}, \vec{\alpha}\right)-\mathcal{V}_{\mathfrak{S}}\left(\varepsilon ; \alpha^{*}, \beta ; \vec{\alpha}^{*}, \vec{\alpha}\right)\right| \\
& \quad \leq \theta \sup _{\substack{\tau \in \varepsilon \mathbb{Z} \cap[0, \theta) \\
0 \leq t \leq \varepsilon}}\left|\left\langle\gamma_{* \tau} \gamma_{\tau+\varepsilon}, v \gamma_{* \tau} \gamma_{\tau+\varepsilon}\right\rangle-\left\langle\gamma_{* \tau+t} \gamma_{\tau+t}, v \gamma_{* \tau+t} \gamma_{\tau+t}\right\rangle\right| \\
& \quad \leq \theta \sup _{\substack{\tau \in \mathbb{Z} \cap[0, \theta) \\
0 \leq t \leq \varepsilon}}\left\{\left|\left\langle\left(\gamma_{* \tau}-\gamma_{* \tau+t}\right) \gamma_{\tau+\varepsilon}, v \gamma_{* \tau} \gamma_{\tau+\varepsilon}\right\rangle\right|+\left|\left\langle\gamma_{* \tau+t}\left(\gamma_{\tau+\varepsilon}-\gamma_{\tau+t}\right), v \gamma_{* \tau} \gamma_{\tau+\varepsilon}\right\rangle\right|\right. \\
& \left.\quad+\left|\left\langle\gamma_{* \tau+t} \gamma_{\tau+t}, v\left(\gamma_{* \tau}-\gamma_{* \tau+t}\right) \gamma_{\tau+\varepsilon}\right\rangle\right|+\left|\left\langle\gamma_{* \tau+t} \gamma_{\tau+t}, v \gamma_{* \tau+t}\left(\gamma_{\tau+\varepsilon}-\gamma_{\tau+t}\right)\right\rangle\right|\right\}
\end{aligned}
$$

We bound the first term. The bounds on the remaining three terms are virtually identical. For all $\tau \in \varepsilon \cap[0, \theta)$,

$$
\begin{aligned}
\left|\left\langle\left(\gamma_{* \tau}-\gamma_{* \tau+t}\right) \gamma_{\tau+\varepsilon}, v \gamma_{* \tau} \gamma_{\tau+\varepsilon}\right\rangle\right| & =\left|\left\langle\left([j(t)-\mathfrak{h}] \gamma_{* \tau}\right) \gamma_{\tau+\varepsilon}, v \gamma_{* \tau} \gamma_{\tau+\varepsilon}\right\rangle\right| \\
& \leq K_{j} t e^{K_{j} t}|| v|\|||X| \max _{\mathbf{x} \in X}\left|\gamma_{* \tau}(\mathbf{x})\right|^{2} \max _{\mathbf{x} \in X}\left|\gamma_{\tau+\varepsilon}(\mathbf{x})\right|^{2}
\end{aligned}
$$

by Lemmas E.14 and III.21.ii, and

$$
\begin{gathered}
\max _{\mathbf{x} \in X}\left|\gamma_{\tau+\varepsilon}(\mathbf{x})\right| \leq K_{\alpha} \equiv 2+5 e^{K_{j}} \max _{\tau^{\prime} \in \cap(0, \theta]} \max _{\mathbf{x} \in \Lambda_{\tau^{\prime}}^{c}}\left|\alpha_{\tau^{\prime}}(\mathbf{x})\right| \\
\max _{\mathbf{x} \in X}\left|\gamma_{* \tau}(\mathbf{x})\right| \leq K_{\alpha *} \equiv 2+5 e^{K_{j}} \max _{\tau^{\prime} \in \cap[0, \theta)} \max _{\mathbf{x} \in \Lambda_{\tau^{\prime}}^{c}}\left|\alpha_{* \tau^{\prime}}(\mathbf{x})\right|
\end{gathered}
$$

by Lemma E.8. Hence

$$
\left|\mathcal{V}_{\mathfrak{S}}\left(\alpha^{*}, \beta ; \vec{\alpha}^{*}, \vec{\alpha}\right)-\mathcal{V}_{\mathfrak{S}}\left(\varepsilon ; \alpha^{*}, \beta ; \vec{\alpha}^{*}, \vec{\alpha}\right)\right| \leq\left(4 \theta e^{2 K_{j}}\left\||\|\|| X \mid K_{\alpha}^{2} K_{\alpha *}^{2}\right) \varepsilon\right.
$$

clearly converges to zero, uniformly on compacta, as $\varepsilon \rightarrow 0$. 


\section{III.7 Bounds on the Large Field Integral Operator}

In this section we bound the large field integral operators $\mathcal{I}_{\left(\mathfrak{S} ; \alpha^{*}, \beta\right)}$ when the history field is identically one. So we set $\mathfrak{h} \equiv 1$ throughout this section. Recall that $\mathcal{I}_{\left(\mathfrak{S} ; \alpha^{*}, \beta\right)}$ and its absolute value $\left|\mathcal{I}_{\left(\mathfrak{S} ; \alpha^{*}, \beta\right)}\right|$ were defined in Definition II.8.

Proposition III.32 gives a representation for $I_{m}^{\bullet}\left(2^{-m} \theta ; \alpha^{*}, \beta\right)$ that is analogous to the representation for $I_{\theta}\left(\alpha^{*}, \beta\right)=\left.\lim _{m \rightarrow \infty} I_{m}^{\bullet}\left(2^{-m} \theta ; \alpha^{*}, \beta\right)\right|_{\mathfrak{h}=1}$ given in our main Theorem II.16. To take the limit and prove Theorem II.16, we shall apply the dominated convergence theorem to the sum over hierarchies in Proposition III.32. To do so, we apply a result, Theorem III.35, below, that is slightly more general than Theorem II.18.

Fix $0<\theta \leq \Theta$. Let $m \in \mathbb{N}$ and set $\varepsilon=2^{-m} \theta$. Assume that $\alpha$ and $\beta$ obey the small field conditions $\chi_{\theta}(\Omega ; \alpha, \beta)=1$.

Theorem III.35 For any bounded measurable function $f(\alpha, \beta ; \vec{\rho})$ and subset $\Omega \subset X$,

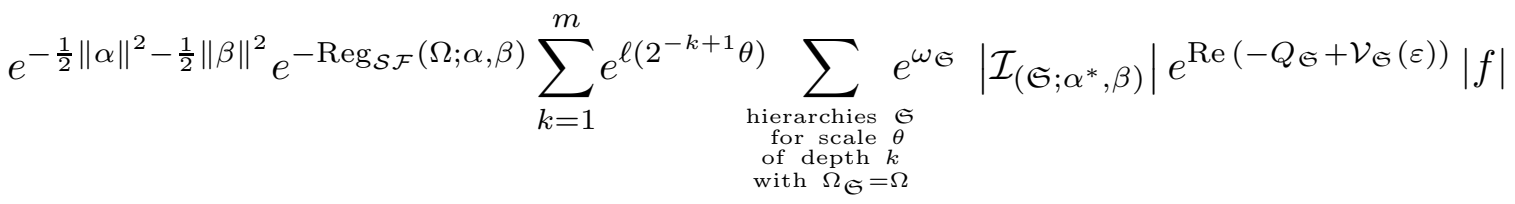

$$
\begin{aligned}
& \leq e^{-\frac{1}{4} \ell(\theta)\left|\Omega^{c}\right|}\left[\prod_{\mathbf{x} \in \Omega^{c}} \frac{1}{1+|\alpha(\mathbf{x})|^{3}} \frac{1}{1+|\beta(\mathbf{x})|^{3}}\right] \sup |f|
\end{aligned}
$$

with the $\operatorname{Reg}_{\mathcal{S F}}(\Omega ; \alpha, \beta)$ of Definition II.17 and

$$
\omega_{\mathfrak{S}}=\sum_{\substack{\text { decimation } \\ \text { intervals } \\ \mathcal{J} \subset[0, \theta]}} \frac{1}{2}\left|\Omega(\mathcal{J})^{c}\right|
$$

The small factors that lead to Theorem III.35 arise from three sources.

- For each decimation interval $\mathcal{J}$, there is a large positive contribution to the main quadratic part of the action, $Q_{\mathfrak{S}}\left(\alpha^{*}, \beta ; \vec{\alpha}^{*}, \vec{\alpha}\right)$, for each point of the "large field sets of the first kind" $P_{\alpha}^{\prime}(\mathcal{J}), P_{\beta}^{\prime}(\mathcal{J})$ and $Q(\mathcal{J})$ that were introduced in the Definition II.4 of a hierarchy. This is made precise in Proposition III.36, below.

- For each decimation interval $\mathcal{J}$, there is a large negative contribution to the main quartic part of the action, $\mathcal{V}_{\mathfrak{S}}\left(\varepsilon ; \alpha^{*}, \beta ; \vec{\alpha}^{*}, \vec{\alpha}\right)$, for each point of the "large field sets of the first kind" $P_{\alpha}(\mathcal{J})$ and $P_{\beta}(\mathcal{J})$, that were introduced in the Definition II.4 of a hierarchy. This is made precise in Proposition III.37, below.

- For each decimation interval $\mathcal{J}$ of length $2 s$, the integral operator $\mathcal{I}_{\left(\mathcal{J}, \mathfrak{S} ; \alpha^{*}, \beta\right)}$, of Definition II.8, includes an integral

$$
\int_{C_{s}\left(\mathbf{x} ; \alpha^{*}, \beta\right)} \frac{d z_{* \tau}(\mathbf{x}) \wedge d z_{\tau}(\mathbf{x})}{2 \pi i} e^{-z_{* \tau}(\mathbf{x}) z_{\tau}(\mathbf{x})}
$$


for each point $\mathrm{x}$ in the "large field set of the second kind" $R(\mathcal{J})$. Proposition III.38, below, shows that we may choose the domain of integration $C_{s}\left(\mathbf{x} ; \alpha^{*}, \beta\right)$ in such a way that $\operatorname{Re} z_{* \tau}(\mathbf{x}) z_{\tau}(\mathbf{x})$ is large on the entire surface $C_{s}\left(\mathbf{x} ; \alpha^{*}, \beta\right)$.

At the end of this section, we show how Theorem III.35 is deduced from these three propositions, which, in turn, will be proven in $\S \mathrm{VI}$. The three propositions involve a constant $C_{L}>0$, that is defined in Lemma F.5 and depends only on $\mathrm{h}$ and the constants of Hypothesis II.14, (II.18), (II.19) and (II.20). For these three propositions, we fix an integer $0 \leq n \leq m$ and a hierarchy $\mathfrak{S}$ for scale $\delta=2^{n} \varepsilon$ of depth at most $n$ and write

$$
\gamma_{* \tau}=\left\{\begin{array}{ll}
\alpha^{*} & \text { if } \tau=0 \\
\Gamma_{* \mathfrak{S}}\left(\tau ; \alpha^{*}, \vec{\alpha}^{*}\right) & \text { if } \tau \in(0, \delta) \\
\beta^{*} & \text { if } \tau=\delta
\end{array}\right\} \quad \gamma_{\tau}=\left\{\begin{array}{ll}
\alpha & \text { if } \tau=0 \\
\Gamma_{\mathfrak{S}}(\tau ; \vec{\alpha}, \beta) & \text { if } \tau \in(0, \delta) \\
\beta & \text { if } \tau=\delta
\end{array}\right\}
$$

\section{Proposition III.36}

$$
\begin{aligned}
& -\operatorname{Re}\left[\frac{1}{2}\|\alpha\|^{2}+Q_{\mathfrak{S}}\left(\alpha^{*}, \beta ; \vec{\alpha}^{*}, \vec{\alpha}\right)+\frac{1}{2}\|\beta\|^{2}\right] \\
& \leq-C_{L} \sum_{\substack{\text { decimation } \\
\text { intervals } \\
\mathcal{J} \subset[0, \theta]}} \mathrm{r}(|\mathcal{J}|)^{2}\left\{|\tilde{Q}(\mathcal{J})|+\left|\tilde{P}_{\alpha}^{\prime}(\mathcal{J})\right|+\left|\tilde{P}_{\beta}^{\prime}(\mathcal{J})\right|\right\}-\frac{1}{16} \sum_{\tau \in[0, \delta)}\left\|\gamma_{* \tau}^{*}-\gamma_{\tau+\varepsilon}\right\|_{\Lambda_{\tau}^{c} \cup \Lambda_{\tau+\varepsilon}^{c}}^{2} \\
& \quad+\sum_{\substack{\text { decimation } \\
\text { intervals } \\
\mathcal{J} \subset[0, \delta]}} \frac{1}{8}\left|\Omega(\mathcal{J})^{c}\right|+\left|e^{\varepsilon \mu}-1\right| \sum_{\tau \in[0, \delta]}\left[\left\|\gamma_{\tau}\right\|_{\Omega^{c}}^{2}+\left\|\gamma_{* \tau}\right\|_{\Omega^{c}}^{2}\right]+\operatorname{Reg}_{\mathcal{S} \mathcal{F}}^{(2)}(\Omega ; \alpha, \beta)
\end{aligned}
$$

where $\operatorname{Reg}_{\mathcal{S} \mathcal{F}}^{(2)}(\Omega ; \alpha, \beta)$ is given in Definition II.17 and, for each decimation interval $\mathcal{J}$,

$$
\begin{aligned}
\tilde{P}_{\alpha}^{\prime}(\mathcal{J}) & =\left\{b \in P_{\alpha}^{\prime}(\mathcal{J}) \mid d\left(b, \Lambda\left(\mathcal{J}^{-}\right)^{c} \cup \Lambda\left(\mathcal{J}^{+}\right)^{c}\right)>2 \mathfrak{c}\left(\left|\mathcal{J}^{ \pm}\right|\right)\right\} \\
\tilde{P}_{\beta}^{\prime}(\mathcal{J}) & =\left\{b \in P_{\beta}^{\prime}(\mathcal{J}) \mid d\left(b, \Lambda\left(\mathcal{J}^{-}\right)^{c} \cup \Lambda\left(\mathcal{J}^{+}\right)^{c}\right)>2 \mathfrak{c}\left(\left|\mathcal{J}^{ \pm}\right|\right)\right\} \\
\tilde{Q}(\mathcal{J}) & =\left\{\mathbf{x} \in Q(\mathcal{J}) \mid d\left(\mathbf{x}, \Lambda\left(\mathcal{J}^{-}\right)^{c} \cup \Lambda\left(\mathcal{J}^{+}\right)^{c}\right)>2 \mathfrak{c}\left(\left|\mathcal{J}^{ \pm}\right|\right)\right\}
\end{aligned}
$$

\section{Proposition III.37}

$$
\begin{aligned}
\operatorname{Re}\left[\mathcal{V}_{\mathfrak{S}}\left(\varepsilon ; \alpha^{*}, \beta ; \vec{\alpha}^{*}, \vec{\alpha}\right)-\right. & \left.\operatorname{Reg}_{\mathcal{S} \mathcal{F}}^{(4)}(\alpha, \beta)\right] \\
\leq-C_{L} & \sum_{\substack{\text { decimation } \\
\text { intervals } \\
\mathcal{J} \subset[0, \delta]}} \mathrm{r}(|\mathcal{J}|)^{2}\left\{\left|\tilde{P}_{\alpha}(\mathcal{J})\right|+\left|\tilde{P}_{\beta}(\mathcal{J})\right|\right\} \\
& -\frac{1}{4} \sum_{\tau \in[0, \delta)} \varepsilon\left\langle\gamma_{* \tau}^{*} \gamma_{* \tau}, v \gamma_{* \tau}^{*} \gamma_{* \tau}\right\rangle-\frac{1}{4} \sum_{\tau \in(0, \delta]} \varepsilon\left\langle\gamma_{\tau}^{*} \gamma_{\tau}, v \gamma_{\tau}^{*} \gamma_{\tau}\right\rangle \\
& +\sum_{\substack{\text { decimation } \\
\text { intervals } \\
\mathcal{J} \subset[0, \delta]}} \frac{1}{8}\left|\Omega(\mathcal{J})^{c}\right|+\sum_{\tau \in[0, \delta)} \frac{1}{16}\left\|\gamma_{* \tau}^{*}-\gamma_{\tau+\varepsilon}\right\|_{\Lambda_{\tau}^{c} \cup \Lambda_{\tau+\varepsilon}^{c}}^{2}
\end{aligned}
$$


where $\operatorname{Reg}_{\mathcal{S} \mathcal{F}}^{(4)}(\alpha, \beta)$ is given in Definition II.17 and, for each decimation interval $\mathcal{J}$,

$$
\begin{aligned}
& \tilde{P}_{\alpha}(\mathcal{J})=\left\{\mathbf{x} \in P_{\alpha}(\mathcal{J}) \mid d\left(\mathbf{x}, \Lambda\left(\mathcal{J}^{-}\right)^{c} \cup \Lambda\left(\mathcal{J}^{+}\right)^{c}\right)>2 \mathfrak{c}\left(\frac{1}{2}|\mathcal{J}|\right)\right\} \\
& \tilde{P}_{\beta}(\mathcal{J})=\left\{\mathbf{x} \in P_{\beta}(\mathcal{J}) \mid d\left(\mathbf{x}, \Lambda\left(\mathcal{J}^{-}\right)^{c} \cup \Lambda\left(\mathcal{J}^{+}\right)^{c}\right)>2 \mathfrak{c}\left(\frac{1}{2}|\mathcal{J}|\right)\right\}
\end{aligned}
$$

Proposition III.38 Let $\mathcal{J}$ be a decimation interval for $\mathfrak{S}$ with length $2 s$ and centre $\tau$, and $\mathbf{x} \in \Lambda(\mathcal{J})$. We may choose the surface $C_{s}\left(\mathbf{x} ; \alpha^{*}, \beta\right)$ of Definition II.8 so that the following holds. Assume that $\alpha$ and $\beta$ are such that the characteristic functions $\chi_{2 s}(\Lambda(\mathcal{J}) ; \alpha, \beta)$ and $\chi_{\mathcal{J}}\left(\alpha, \alpha_{\tau}, \beta\right)$ are nonzero. Then

(i) $C_{s}\left(\mathbf{x} ; \alpha^{*}, \beta\right) \subset\left\{\left(z_{*}, z\right) \in \mathbb{C}^{2}|| z_{*}|| z \mid, \leq 2 \mathrm{r}(s)\right\}$.

(ii) For all $\left(z_{*}, z\right) \in C_{s}\left(\mathbf{x} ; \alpha^{*}, \beta\right)$,

$$
\operatorname{Re}\left(z_{*} z\right) \geq C_{L} \mathrm{r}(s)^{2}
$$

(iii) The area of $C_{s}\left(\mathbf{x} ; \alpha^{*}, \beta\right)$ is bounded by $40 \pi \mathrm{r}(s)^{2}$.

Proof of Theorem III.35: We still fix a hierarchy $\mathfrak{S}$ for scale $\delta=2^{n} \varepsilon$. For bounding the "absolute value" $\left|\mathcal{I}_{\mathfrak{S}}\right|=\left|\mathcal{I}_{\left(\mathfrak{S} ; \alpha^{*}, \beta\right)}\right|$, we introduce the auxiliary integral operator

$$
\overline{\mathcal{I}}_{\mathfrak{S}}=\prod_{k=0, \cdots, \operatorname{depth}(\mathfrak{S})} \prod_{\substack{\text { decimation intervals } \\ \mathcal{J}\left[\tau_{1}, r_{r}\right][0, \delta] \\ \text { of length } 2-k_{\delta}}} \overline{\mathcal{I}}_{\left(\mathcal{J} ; \alpha_{\tau_{\ell}}^{*}, \alpha_{\tau_{r}}\right)}
$$

where $\overline{\mathcal{I}}_{\left(\mathcal{J} ; \alpha^{*}, \beta\right)}$ is constructed by replacing

$$
\int_{C_{s}\left(\mathbf{x} ; \alpha^{*}, \beta\right)} \frac{d z_{* \tau}(\mathbf{x}) \wedge d z_{\tau}(\mathbf{x})}{2 \pi i} e^{-z_{* \tau}(\mathbf{x}) z_{\tau}(\mathbf{x})} \quad \text { by } \quad \int_{C_{s}\left(\mathbf{x} ; \alpha^{*}, \beta\right)}\left|\frac{d z_{* \tau}(\mathbf{x}) \wedge d z_{\tau}(\mathbf{x})}{2 \pi i}\right|
$$

in the formula for $\mathcal{I}_{\left(\mathcal{J} ; \alpha^{*}, \beta\right)}$ in Definition II.8. Then,

$$
\left|\mathcal{I}_{\mathfrak{S}}\right|=\overline{\mathcal{I}}_{\mathfrak{S}} \prod_{\substack{\text { decimation intervals } \\ \mathcal{J} \subset[0, \delta] \text { with centre } \tau}} \prod_{\mathbf{x} \in R(\mathcal{J})} e^{-\operatorname{Re} z_{* \tau}(\mathbf{x}) z_{\tau}(\mathbf{x})}
$$

By Proposition III.38,

$$
\prod_{\substack{\text { decimation intervals } \\ \mathcal{J} \subset[0, \delta] \text { with centre } \tau}} \prod_{\mathbf{x} \in R(\mathcal{J})} e^{-\operatorname{Re} z_{* \tau}(\mathbf{x}) z_{\mathcal{\tau}}(\mathbf{x})} \leq \prod_{\substack{\text { decimation } \\ \text { intervals } \\ \mathcal{J} \subset[0, \delta]}} e^{-C_{L}|R(\mathcal{J})| \mathrm{r}\left(\frac{1}{2}|\mathcal{J}|\right)^{2}}
$$

on the domain of integration. 


\section{Lemma III.39}

$$
\begin{aligned}
\operatorname{Re}\left[-\frac{1}{2}\|\alpha\|^{2}-Q_{\mathfrak{S}}\left(\alpha^{*}, \beta ; \vec{\alpha}^{*}, \vec{\alpha}\right)-\frac{1}{2}\|\beta\|^{2}+\mathcal{V}_{\mathfrak{S}}\left(\varepsilon ; \alpha^{*}, \beta ; \vec{\alpha}^{*}, \vec{\alpha}\right)\right]-\operatorname{Reg}_{\mathcal{S F}}(\Omega ; \alpha, \beta) \\
\leq-C_{L} \sum_{\substack{\text { decimation } \\
\text { intervals } \\
\mathcal{J} \subset[0, \delta]}} \mathrm{r}(|\mathcal{J}|)^{2}\left\{|\tilde{Q}(\mathcal{J})|+\left|\tilde{P}_{\alpha}^{\prime}(\mathcal{J})\right|+\left|\tilde{P}_{\beta}^{\prime}(\mathcal{J})\right|+\left|\tilde{P}_{\alpha}(\mathcal{J})\right|+\left|\tilde{P}_{\beta}(\mathcal{J})\right|\right\} \\
+\sum_{\substack{\text { decimation } \\
\text { intervals } \\
\mathcal{J} \subset[0, \delta]}} \frac{1}{2}\left|\Omega(\mathcal{J})^{c}\right|
\end{aligned}
$$

Proof: We assume that $\varepsilon$ is sufficiently small that $\left|e^{\varepsilon \mu}-1\right| \leq \frac{1}{8}$, which implies that $\left|e^{\varepsilon \mu}-1\right| \leq 2 \varepsilon|\mu|$. It suffices to apply Propositions III.36 and III.37 together with

$$
\begin{aligned}
&-\frac{1}{4} \varepsilon v_{1} \sum_{\tau \in(0, \delta]}\left\|\gamma_{\tau}^{*} \gamma_{\tau}\right\|_{\Omega^{c}}^{2}+2\left|e^{\varepsilon \mu}-1\right| \sum_{\tau \in(0, \delta]}\left\|\gamma_{\tau}\right\|_{\Omega^{c}}^{2} \\
& \quad=-\frac{1}{4} \varepsilon v_{1} \sum_{\tau \in(0, \delta]} \sum_{\mathbf{x} \in \Omega^{c}}\left\{\left|\gamma_{\tau}(\mathbf{x})\right|^{4}-8 \frac{\left|e^{\varepsilon \mu}-1\right|}{\varepsilon v_{1}}\left|\gamma_{\tau}(\mathbf{x})\right|^{2}\right\} \\
&=-\frac{1}{4} \varepsilon v_{1} \sum_{\tau \in(0, \delta]} \sum_{\mathbf{x} \in \Omega^{c}}\left\{\left[\left|\gamma_{\tau}(\mathbf{x})\right|^{2}-4 \frac{\left|e^{\varepsilon \mu}-1\right|}{\varepsilon v_{1}}\right]^{2}-16 \frac{\left|e^{\varepsilon \mu}-1\right|^{2}}{\varepsilon^{2} v_{1}^{2}}\right\} \\
& \leq 4 \varepsilon v_{1} \sum_{\tau \in(0, \delta]} \sum_{\mathbf{x} \in \Omega^{c}} \frac{\left|e^{\varepsilon \mu}-1\right|^{2}}{\varepsilon^{2} v_{1}^{2}}=4 \delta \frac{\left|e^{\varepsilon \mu}-1\right|^{2}}{\varepsilon^{2} v_{1}}\left|\Omega^{c}\right| \leq 16 \delta \frac{\mu^{2}}{v_{1}}\left|\Omega^{c}\right| \\
& \leq 64 \frac{K_{\mu}^{2}}{c_{v}} \delta \mathfrak{v}^{2 e_{\mu}-1}\left|\Omega^{c}\right| \quad \text { by Hypothesis II.14 } \\
& \leq \frac{1}{8}\left|\Omega^{c}\right| \quad \text { by Hypothesis F.7.i and the fact that } e_{\mu}>\frac{1}{2}
\end{aligned}
$$

and

$$
-\frac{1}{4} \varepsilon v_{1} \sum_{\tau \in[0, \delta)}\left\|\gamma_{* \tau}^{*} \gamma_{* \tau}\right\|_{\Omega^{c}}^{2}+2\left|e^{\varepsilon \mu}-1\right| \sum_{\tau \in[0, \delta)}\left\|\gamma_{* \tau}\right\|_{\Omega^{c}}^{2} \leq 4 \delta \frac{\left|e^{\varepsilon \mu}-1\right|^{2}}{\varepsilon^{2} v_{1}}\left|\Omega^{c}\right| \leq \frac{1}{8}\left|\Omega^{c}\right|
$$

Consequently, by (III.13) and Lemma III.39,

$$
e^{-\frac{1}{2}\|\alpha\|^{2}-\frac{1}{2}\|\beta\|^{2}} e^{-\operatorname{Reg}_{\mathcal{S F}}(\Omega ; \alpha, \beta)} e^{\omega_{\mathfrak{S}}}\left|\mathcal{I}_{\left(\mathfrak{S} ; \alpha^{*}, \beta\right)}\right| e^{\operatorname{Re}\left(-Q_{\mathfrak{S}}+\mathcal{V}_{\mathfrak{S}}(\varepsilon)\right)}|f| \leq e^{-L(\mathfrak{S})} \overline{\mathcal{I}}_{\mathfrak{S}}|f|
$$

where

$$
\begin{aligned}
L(\mathfrak{S})=C_{L} & \sum_{\substack{\text { decimation } \\
\text { intervals } \\
\mathcal{J} \subset[0, \delta]}} \mathrm{r}(|\mathcal{J}|)^{2}\left\{|\tilde{Q}(\mathcal{J})|+\left|\tilde{P}_{\alpha}^{\prime}(\mathcal{J})\right|+\left|\tilde{P}_{\beta}^{\prime}(\mathcal{J})\right|+\left|\tilde{P}_{\alpha}(\mathcal{J})\right|+\left|\tilde{P}_{\beta}(\mathcal{J})\right|+|R(\mathcal{J})|\right\} \\
& -\sum_{\substack{\text { decimation } \\
\text { intervals } \\
\mathcal{J} \subset[0, \delta]}}\left|\Omega(\mathcal{J})^{c}\right|
\end{aligned}
$$


The quantity $L(\mathfrak{S})$ is defined in terms of the number of points at which there are violations of the various small field conditions (the sets $Q(\mathcal{J})$, etc.). Lemma III.40, which is proven in $\S \mathrm{VI}$, provides a lower bound for $L(\mathfrak{S})$ purely in terms of the large field sets $\Omega(\mathcal{J})^{c}$.

Lemma III.40 We have

$$
\begin{aligned}
& C_{L} \sum_{\substack{\text { decimation } \\
\text { intervals } \\
\mathcal{J} \subset[0, \delta]}} \mathrm{r}(|\mathcal{J}|)^{2}\left\{|\tilde{Q}(\mathcal{J})|+\left|\tilde{P}_{\alpha}^{\prime}(\mathcal{J})\right|+\left|\tilde{P}_{\beta}^{\prime}(\mathcal{J})\right|+\left|\tilde{P}_{\alpha}(\mathcal{J})\right|+\left|\tilde{P}_{\beta}(\mathcal{J})\right|+|R(\mathcal{J})|\right\} \\
& \geq \ell(\mathfrak{S})+\sum_{\substack{\text { decimation } \\
\text { intervals } \\
\mathcal{J} \subset[0, \delta]}}\left|\Omega(\mathcal{J})^{c}\right|
\end{aligned}
$$

where

$$
\ell(\mathfrak{S})=\sum_{\substack{\text { decimation } \\ \text { intervals } \\ \mathcal{J} \subset[0, \delta]}} \ell(|\mathcal{J}|)\left|\Omega(\mathcal{J})^{c}\right|
$$

Applying (III.14) and Lemma III.40, we have

$$
e^{-\frac{1}{2}\|\alpha\|^{2}-\frac{1}{2}\|\beta\|^{2}} e^{-\operatorname{Reg}_{\mathcal{S} \mathcal{F}}(\Omega ; \alpha, \beta)} e^{\omega_{\mathfrak{S}}}\left|\mathcal{I}_{\left(\mathfrak{S} ; \alpha^{*}, \beta\right)}\right| e^{\operatorname{Re}\left(-Q_{\mathfrak{S}}+\mathcal{V}_{\mathfrak{S}}(\varepsilon)\right)}|f| \leq e^{-\ell(\mathfrak{S})} \overline{\mathcal{I}}_{\mathfrak{S}}|f|
$$

Theorem III.35 is an immediate consequence of (III.15) and

Proposition III.41 For any (small field) subset $\Omega \subset X$, any $0 \leq n \leq m$, and any bounded function $f(\alpha, \beta ; \vec{\rho})$,

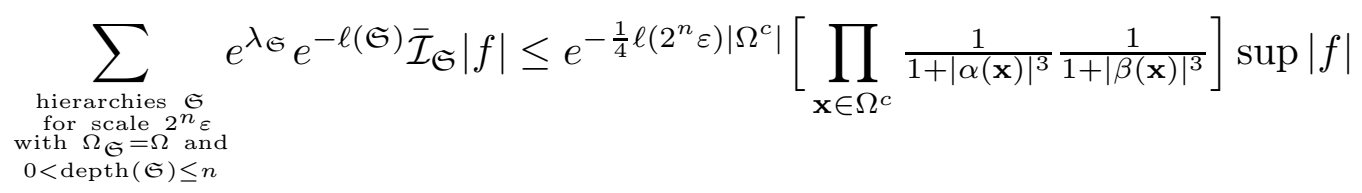

where

$$
\lambda_{\mathfrak{S}}= \begin{cases}\ell\left(2^{n-\operatorname{depth}(\mathfrak{S})+1} \varepsilon\right) & \text { if } \operatorname{depth}(\mathfrak{S})>0 \\ 0 & \text { if } \operatorname{depth}(\mathfrak{S})=0\end{cases}
$$

Here $2^{n-\operatorname{depth}(\mathfrak{S})+1} \varepsilon$ is the length of the shortest decimation interval $\mathcal{J}$ with $\Omega_{\mathfrak{S}}(\mathcal{J}) \neq X$. 
Proof: The proof is by induction on $n$. The case $n=0$ is trivial as is the case that $\Omega^{c}=\emptyset$.

Assume that the statement holds for some $0 \leq n<m$ and that $\Omega^{c} \neq \emptyset$. Set $\delta=2^{n} \varepsilon$. Given subsets $\Omega_{1}, \Omega_{2}, \Lambda$ of $X$ containing $\Omega$ and hierarchies $\mathfrak{S}_{1}, \mathfrak{S}_{2}$ for scale $\delta$ with $\Omega_{\mathfrak{S}_{1}}=\Omega_{1}$ and $\Omega_{\mathfrak{S}_{2}}=\Omega_{2}$, a hierarchy $\mathfrak{S}$ with $\Lambda_{\mathfrak{S}}=\Lambda, \Omega_{\mathfrak{S}}=\Omega$ and $\left(\mathfrak{S}_{1}, \mathfrak{S}_{2}\right) \prec \mathfrak{S}$ is specified by the sets

$$
P_{\alpha}, P_{\beta} \subset \Omega_{1} \cap \Omega_{2} \cap \Lambda^{c} \quad P_{\alpha}^{\prime}, P_{\beta}^{\prime} \subset\left(\Omega_{1} \cap \Omega_{2} \cap \Lambda^{c}\right)^{*} \quad Q \subset\left(\Omega_{1} \cap \Omega_{2}\right)^{\star} \cap \Lambda^{c}
$$

and $R \subset \Lambda \cap \Omega^{c}$

See Definition II.4. There are at most

$$
2^{2\left|\Omega_{1} \cap \Omega_{2} \cap \Lambda^{c}\right|} 2^{4 \mathrm{D}\left|\Omega_{1} \cap \Omega_{2} \cap \Lambda^{c}\right|} 2^{2\left|\Omega_{1}^{\star} \cap \Omega_{2}^{\star} \cap \Lambda^{c}\right|} 2^{\left|\Lambda \cap \Omega^{c}\right|} \leq e^{(5+4 \mathrm{D})\left|\Omega^{c}\right|}
$$

(where D is the dimension of space) such choices. For each hierarchy $\mathfrak{S}$ as above (see Definition II.8 and Appendix A)

$$
\begin{aligned}
& \overline{\mathcal{I}}_{\mathfrak{S}}|f|=(\left.\prod_{\mathbf{x} \in \Lambda \backslash(R \cup \Omega)} \int_{\left|z_{\delta}(\mathbf{x})\right| \leq \mathrm{r}(\delta)} \frac{d z_{\delta}(\mathbf{x})^{*} \wedge d z_{\delta}(\mathbf{x})}{2 \pi i} e^{-z_{\delta}(\mathbf{x})^{*} z_{\delta}(\mathbf{x})}\right) \\
&\left(\prod_{\mathbf{x} \in R} \int_{C_{\delta}\left(\mathbf{x} ; \alpha^{*}, \beta\right)}\left|\frac{d z_{* \delta}(\mathbf{x}) \wedge d z_{\delta}(\mathbf{x})}{2 \pi i}\right|\right) \\
&\left(\prod_{\mathbf{x} \in X \backslash \Lambda} \int_{\left|\alpha_{\delta}(\mathbf{x})\right| \leq \mathrm{R}(\varepsilon)} \frac{d \alpha_{\delta}(\mathbf{x})^{*} \wedge d \alpha_{\delta}(\mathbf{x})}{2 \pi i}\right) \chi_{[0,2 \delta]}\left(\alpha, \alpha_{\delta}, \beta\right) \\
& \mathcal{Z}_{\delta}^{\left|\Omega_{1} \backslash \Omega\right|} \mathcal{Z}_{\delta}^{\left|\Omega_{2} \backslash \Omega\right|} \overline{\mathcal{I}}_{\mathfrak{S}_{1}} \overline{\mathcal{I}}_{\mathfrak{S}_{2}} \sup |f| \\
& \leq\left(40 \pi \mathrm{r}(\delta)^{2}\right)^{|R|} \chi_{\mathrm{R}(\delta)}\left(\Omega_{1} \cap \Lambda^{c}, \alpha\right) \chi_{\mathrm{R}(2 \delta)}\left(\Lambda \cap \Omega^{c}, \alpha\right) \chi_{\mathrm{R}(\delta)}\left(\Omega_{2} \cap \Lambda^{c}, \beta\right) \chi_{\mathrm{R}(2 \delta)}\left(\Lambda \cap \Omega^{c}, \beta\right) \\
&\left(\prod_{\mathbf{x} \in X \backslash \Lambda} \int_{\left|\alpha_{\delta}(\mathbf{x})\right| \leq \mathrm{R}(\varepsilon)} \frac{d \alpha_{\delta}(\mathbf{x})^{*} \wedge d \alpha_{\delta}(\mathbf{x})}{2 \pi i}\right) \chi_{\mathrm{R}(\delta)}\left(\left(\Omega_{1} \cup \Omega_{2}\right) \cap \Lambda^{c}, \alpha_{\delta}\right) \overline{\mathcal{I}}_{\mathfrak{S}_{1}} \overline{\mathcal{I}}_{\mathfrak{S}_{2}} \sup |f|
\end{aligned}
$$

Here we used Lemma A.4.a, which ensures that the integral over $\alpha_{\delta}(\mathbf{x})$ is restricted to $\left|\alpha_{\delta}(\mathbf{x})\right| \leq \mathrm{R}(\varepsilon)$. For the inequality, we used that $\int_{\mathbb{C}} \frac{d z_{\delta}(\mathbf{x})^{*} \wedge d z_{\delta}(\mathbf{x})}{2 \pi i} e^{-z_{\delta}(\mathbf{x})^{*} z_{\delta}(\mathbf{x})}=1$, that the area of $C_{\delta}\left(\mathbf{x} ; \alpha^{*}, \beta\right)$ is at most $40 \pi \mathrm{r}(\delta)^{2}$ (by Lemma III.38.iii), that $\mathcal{Z}_{\delta} \leq 1$, and the definition of $\chi_{[0,2 \delta]}\left(\alpha, \alpha_{\delta}, \beta\right)$ (given in Appendix A).

Since

$$
\begin{aligned}
\lambda_{\mathfrak{S}}-\ell(\mathfrak{S}) & =-\ell(2 \delta)\left|\Omega^{c}\right|-\ell\left(\mathfrak{S}_{1}\right)-\ell\left(\mathfrak{S}_{2}\right)+ \begin{cases}\max \left\{\lambda_{\mathfrak{S}_{1}}, \lambda_{\mathfrak{S}_{2}}\right\} & \text { if } \operatorname{depth}(\mathfrak{S})>1 \\
\ell(2 \delta) & \text { if } \operatorname{depth}(\mathfrak{S})=1\end{cases} \\
& \leq-\ell(2 \delta)\left[\left|\Omega^{c}\right|-1\right]+\lambda_{\mathfrak{S}_{1}}-\ell\left(\mathfrak{S}_{1}\right)+\lambda_{\mathfrak{S}_{2}}-\ell\left(\mathfrak{S}_{2}\right)
\end{aligned}
$$


(III.16), (III.17) and the induction hypothesis give that

$$
\begin{aligned}
& \sum_{\substack{\mathfrak{S}_{1}, \mathfrak{S}_{2} \\
\Omega_{\mathfrak{S}_{1}}=\Omega_{1} \\
\Omega_{\mathfrak{S}_{2}=\Omega_{2}}}} \sum_{\substack{\left(\mathfrak{S}_{1}, \mathfrak{S}_{2}\right) \prec \mathfrak{S} \\
\Omega_{\mathfrak{S}}=\Omega, \Lambda_{\mathfrak{S}}=\Lambda}} e^{\lambda \mathfrak{S}-\ell(\mathfrak{S})} \overline{\mathcal{I}}_{\mathfrak{S}}|f| \\
& \leq e^{-\ell(2 \delta)\left[\left|\Omega^{c}\right|-1\right]} e^{(5+4 \mathrm{D})\left|\Omega^{c}\right|}\left(40 \pi \mathrm{r}(\delta)^{2}\right)^{|R|} \\
& \chi_{\mathrm{R}(\delta)}\left(\Omega_{1} \cap \Lambda^{c}, \alpha\right) \chi_{\mathrm{R}(2 \delta)}\left(\Lambda \cap \Omega^{c}, \alpha\right) \chi_{\mathrm{R}(\delta)}\left(\Omega_{2} \cap \Lambda^{c}, \beta\right) \chi_{\mathrm{R}(2 \delta)}\left(\Lambda \cap \Omega^{c}, \beta\right) \\
& \left(\prod_{\mathbf{x} \in X \backslash \Lambda} \int_{\left|\alpha_{\delta}(\mathbf{x})\right| \leq \mathrm{R}(\varepsilon)} \frac{d \alpha_{\delta}(\mathbf{x})^{*} \wedge d \alpha_{\delta}(\mathbf{x})}{2 \pi i}\right) \quad \chi_{\mathrm{R}(\delta)}\left(\left(\Omega_{1} \cup \Omega_{2}\right) \cap \Lambda^{c}, \alpha_{\delta}\right) \\
& \left\{\sum_{\substack{\mathfrak{S}_{1} \\
\Omega_{\mathfrak{S}_{1}}=\Omega_{1}}} e^{\lambda_{\mathfrak{S}_{1}}-\ell\left(\mathfrak{S}_{1}\right)} \overline{\mathcal{I}}_{\mathfrak{S}_{1}}\right\}\left\{\sum_{\substack{\mathfrak{S}_{2} \\
\Omega_{\mathfrak{S}_{2}}=\Omega_{2}}} e^{\lambda_{\mathfrak{S}_{2}}-\ell\left(\mathfrak{S}_{2}\right)} \overline{\mathcal{I}}_{\mathfrak{S}_{2}}\right\} \sup |f| \\
& \leq e^{-\ell(2 \delta)\left[\left|\Omega^{c}\right|-1\right]} e^{(5+4 \mathrm{D})\left|\Omega^{c}\right|} e^{[5+2 \ln \mathrm{r}(\delta)]\left|\Omega^{c}\right|} \\
& \chi_{\mathrm{R}(\delta)}\left(\Omega_{1} \cap \Lambda^{c}, \alpha\right) \chi_{\mathrm{R}(2 \delta)}\left(\Lambda \cap \Omega^{c}, \alpha\right) \chi_{\mathrm{R}(\delta)}\left(\Omega_{2} \cap \Lambda^{c}, \beta\right) \chi_{\mathrm{R}(2 \delta)}\left(\Lambda \cap \Omega^{c}, \beta\right) \\
& \left(\prod_{\mathbf{x} \in X \backslash \Lambda} \int_{\left|\alpha_{\delta}(\mathbf{x})\right| \leq \mathrm{R}(\varepsilon)} \frac{d \alpha_{\delta}(\mathbf{x})^{*} \wedge d \alpha_{\delta}(\mathbf{x})}{2 \pi i}\right) \quad \chi_{\mathrm{R}(\delta)}\left(\left(\Omega_{1} \cup \Omega_{2}\right) \cap \Lambda^{c}, \alpha_{\delta}\right) \\
& e^{-\frac{1}{4} \ell(\delta)\left|\Omega_{1}^{c}\right|}\left[\prod_{\mathbf{x} \in \Omega_{1}^{c}} \frac{1}{1+|\alpha(\mathbf{x})|^{3}} \frac{1}{1+\left|\alpha_{\delta}(\mathbf{x})\right|^{3}}\right] e^{-\frac{1}{4} \ell(\delta)\left|\Omega_{2}^{c}\right|}\left[\prod_{\mathbf{x} \in \Omega_{2}^{c}} \frac{1}{1+\left|\alpha_{\delta}(\mathbf{x})\right|^{3}} \frac{1}{1+|\beta(\mathbf{x})|^{3}}\right] \sup |f|
\end{aligned}
$$

For any $t>1$,

$$
\chi_{t}(\alpha) \leq \frac{(1+t)^{3}}{1+|\alpha|^{3}} \leq \frac{8 t^{3}}{1+|\alpha|^{3}} \leq e^{\ln 8+3 \ln t} \frac{1}{1+|\alpha|^{3}}
$$

which yields the bound

$$
\begin{aligned}
\chi_{\mathrm{R}(\delta)}\left(\Omega_{1} \cap \Lambda^{c}, \alpha\right) \chi_{\mathrm{R}(2 \delta)}\left(\Lambda \cap \Omega^{c}, \alpha\right) \prod_{\mathbf{x} \in \Omega_{1}^{c}} \frac{1}{1+|\alpha(\mathbf{x})|^{3}} & \leq \chi_{\mathrm{R}(\delta)}\left(\Omega_{1} \cap \Omega^{c}, \alpha\right) \prod_{\mathbf{x} \in \Omega_{1}^{c}} \frac{1}{1+|\alpha(\mathbf{x})|^{3}} \\
& \leq e^{[\ln 8+3 \ln \mathrm{R}(\delta)]\left|\Omega^{c}\right|} \prod_{\mathbf{x} \in \Omega^{c}} \frac{1}{1+|\alpha(\mathbf{x})|^{3}}
\end{aligned}
$$

Similarly

$$
\chi_{\mathrm{R}(\delta)}\left(\Omega_{2} \cap \Lambda^{c}, \beta\right) \chi_{\mathrm{R}(2 \delta)}\left(\Lambda \cap \Omega^{c}, \beta\right) \prod_{\mathbf{x} \in \Omega_{2}^{c}} \frac{1}{1+|\beta(\mathbf{x})|^{3}} \leq e^{[\ln 8+3 \ln \mathrm{R}(\delta)]\left|\Omega^{c}\right|} \prod_{\mathbf{x} \in \Omega^{c}} \frac{1}{1+|\beta(\mathbf{x})|^{3}}
$$

To bound the $\alpha_{\delta}$ integrals, we use

$$
\begin{aligned}
& \left(\prod_{\mathbf{x} \in \Lambda^{c}} \int_{\left|\alpha_{\delta}(\mathbf{x})\right| \leq \mathrm{R}(\varepsilon)^{2 \pi i}} \frac{d \alpha_{\delta}(\mathbf{x})^{*} \wedge d \alpha_{\delta}(\mathbf{x})}{2 \pi}\right) \chi_{\mathrm{R}(\delta)}\left(\left(\Omega_{1} \cup \Omega_{2}\right) \cap \Lambda^{c}, \alpha_{\delta}\right) \prod_{\mathbf{x} \in \Omega_{1}^{c}} \frac{1}{1+\left|\alpha_{\delta}(\mathbf{x})\right|^{3}} \prod_{\mathbf{x} \in \Omega_{2}^{c}} \frac{1}{1+\left|\alpha_{\delta}(\mathbf{x})\right|^{3}} \\
& \leq \prod_{\mathbf{x} \in\left(\Omega_{1} \cap \Omega_{2}\right)^{c}} \frac{1}{\pi} \int \frac{d^{2} \alpha_{\delta}(\mathbf{x})}{1+\left|\alpha_{\delta}(\mathbf{x})\right|^{3}} \prod_{\mathbf{x} \in \Omega_{1} \cap \Omega_{2} \cap \Lambda^{c}} \frac{1}{\pi} \int_{\left|\alpha_{\delta}(\mathbf{x})\right| \leq \mathrm{R}(\delta)} d^{2} \alpha_{\delta}(\mathbf{x}) \\
& \leq \quad 3^{\left|\left(\Omega_{1} \cap \Omega_{2}\right)^{c}\right|} \quad \mathrm{R}(\delta)^{2\left|\Omega_{1} \cap \Omega_{2} \cap \Lambda^{c}\right|} \\
& \leq e^{[\ln 3+2 \ln \mathrm{R}(\delta)]\left|\Lambda^{c}\right|}
\end{aligned}
$$


Inserting the last estimates into (III.18) gives

$$
\begin{aligned}
& \sum_{\substack{\Omega_{\mathfrak{S}}=\Omega_{\Lambda}, \Lambda_{\mathfrak{S}}=\Lambda \\
\Omega_{\mathfrak{S}}([0, \delta])=\Omega_{1} \\
\Omega_{\mathfrak{S}([\delta, 2 \delta])=\Omega_{2}}}} e^{\lambda_{\mathfrak{S}}-\ell(\mathfrak{S})} \overline{\mathcal{I}}_{\mathfrak{S}}|f| \\
& \leq e^{-\ell(2 \delta)\left[\left|\Omega^{c}\right|-1\right]} e^{(5+4 \mathrm{D})\left|\Omega^{c}\right|} e^{[5+2 \ln \mathrm{r}(\delta)]\left|\Omega^{c}\right|} \\
& \quad e^{2[\ln 8+3 \ln \mathrm{R}(\delta)]\left|\Omega^{c}\right|} \prod_{\mathbf{x} \in \Omega^{c}} \frac{1}{1+|\alpha(\mathbf{x})|^{3}} \frac{1}{1+|\beta(\mathbf{x})|^{3}} e^{[\ln 3+2 \ln \mathrm{R}(\delta)]\left|\Lambda^{c}\right|} \sup |f| \\
& \leq e^{-\frac{1}{2} \ell(2 \delta)\left|\Omega^{c}\right|} e^{[16+4 \mathrm{D}+10 \ln \mathrm{R}(\delta)]\left|\Omega^{c}\right|} \prod_{\mathbf{x} \in \Omega^{c}} \frac{1}{1+|\alpha(\mathbf{x})|^{3}} \frac{1}{1+|\beta(\mathbf{x})|^{3}} \sup |f|
\end{aligned}
$$

Summing over the subsets $\Omega_{1}^{c}, \Omega_{2}^{c}, \Lambda^{c} \subset \Omega^{c}$,

$$
\begin{aligned}
\sum_{\substack{\mathfrak{S} \\
\Omega_{\mathfrak{S}}=\Omega}} e^{\lambda_{\mathfrak{S}}-\ell(\mathfrak{S})} \overline{\mathcal{I}}_{\mathfrak{S}}|f| & \leq e^{-\frac{1}{2} \ell(2 \delta)\left|\Omega^{c}\right|} e^{[19+4 \mathrm{D}+10 \ln \mathrm{R}(\delta)]\left|\Omega^{c}\right|} \prod_{\mathbf{x} \in \Omega^{c}} \frac{1}{1+|\alpha(\mathbf{x})|^{3}} \frac{1}{1+|\beta(\mathbf{x})|^{3}} \sup |f| \\
& \leq e^{-\frac{1}{4} \ell(2 \delta)\left|\Omega^{c}\right|} \prod_{\mathbf{x} \in \Omega^{c}} \frac{1}{1+|\alpha(\mathbf{x})|^{3}} \frac{1}{1+|\beta(\mathbf{x})|^{3}} \sup |f|
\end{aligned}
$$

by (F.6.d).

\section{III.8 Proof of Theorems II.16 and II.18}

As we saw in Remark III.31, it suffices, for the proof of Theorems II.16, to prove the convergence of $\left.I_{m}^{\bullet}\left(2^{-m} \theta ; \cdot\right)\right|_{\mathfrak{h}=1}$ to the desired limit. By Proposition III.32, with $\varepsilon=2^{-m} \theta$ and $n=m$,

$$
\begin{aligned}
& I_{m}^{\bullet}\left(2^{-m} \theta ; \alpha_{*}, \beta\right)= \sum_{\Omega \subset X} \mathcal{Z}_{\theta}^{|\Omega|} e^{\left.\left\langle\alpha_{*}, j(\theta) \beta\right\rangle\right|_{\Omega}+V_{\Omega, \theta}\left(\varepsilon ; \alpha^{*}, \beta\right)+\mathcal{D}_{\Omega, m}\left(\varepsilon ; \alpha^{*}, \beta\right)} \chi_{\theta}(\Omega ; \alpha, \beta) \\
& \sum_{\begin{array}{c}
\mathfrak{S} \text { hierarchy } \\
\text { for scale } \theta \\
\text { depth }(\mathfrak{S}) \leq m \\
\text { with } \Omega \mathfrak{S}=\Omega
\end{array}} \mathcal{I}_{\left(\mathfrak{S} ; \alpha^{*}, \beta\right)}\left(e^{-Q_{\mathfrak{S}}^{r e s}\left(\alpha^{*}, \beta ; \vec{\alpha}^{*}, \vec{\alpha}\right)+\mathcal{V}_{\mathfrak{S}}^{r e s}\left(\varepsilon ; \alpha^{*}, \beta ; \vec{\alpha}^{*}, \vec{\alpha}\right)}\right. \\
&\left.e^{\mathcal{B}_{\mathfrak{S}}\left(\varepsilon ; \alpha^{*}, \beta ; \overrightarrow{\rho_{\uparrow}}\right)+\mathcal{L}_{\mathfrak{S}}\left(\varepsilon ; \alpha^{*}, \beta ; \overrightarrow{\rho_{\uparrow}}\right)}\right)\left.\right|_{\varepsilon=2^{-m} \theta}
\end{aligned}
$$

where

$$
\begin{aligned}
Q_{\mathfrak{S}}^{r e s}\left(\alpha^{*}, \beta ; \vec{\alpha}^{*}, \vec{\alpha}\right) & =Q_{\mathfrak{S}}\left(\alpha^{*}, \beta ; \vec{\alpha}^{*}, \vec{\alpha}\right)+\left.\left\langle\alpha_{*}, j(\theta) \beta\right\rangle\right|_{\Omega_{\mathfrak{S}}} \\
\mathcal{V}_{\mathfrak{S}}^{r e s}\left(\varepsilon ; \alpha^{*}, \beta ; \vec{\alpha}^{*}, \vec{\alpha}\right) & =\mathcal{V}_{\mathfrak{S}}\left(\varepsilon ; \alpha^{*}, \beta ; \vec{\alpha}^{*}, \vec{\alpha}\right)-V_{\Omega_{\mathfrak{S}}, \theta}\left(\varepsilon ; \alpha^{*}, \beta\right) \\
\mathcal{B}_{\mathfrak{S}}\left(\varepsilon ; \alpha^{*}, \beta ; \vec{\rho}\right) & =\mathcal{D}_{\mathfrak{S}}\left(\varepsilon ; \alpha^{*}, \beta ; \vec{\rho}\right)-\mathcal{D}_{\Omega_{\mathfrak{S}}, m}\left(\varepsilon ; \alpha^{*}, \beta\right)
\end{aligned}
$$

with the $V_{\Omega, \theta}(\varepsilon ; \cdot)$ of Definition III.8.ii and the $\mathcal{D}_{\Omega, m}(\varepsilon ; \cdot)$ of (III.8). 
By (II.4) and Corollary III.30, for any fixed $\Omega \subset X$,

$$
\lim _{m \rightarrow \infty} \chi_{\theta}(\Omega ; \alpha, \beta) e^{V_{\Omega, \theta}\left(2^{-m} \theta ; \alpha^{*}, \beta\right)+\mathcal{D}_{\Omega, m}\left(2^{-m} \theta ; \alpha^{*}, \beta\right)}=\chi_{\theta}(\Omega ; \alpha, \beta) e^{V_{\Omega ; \theta}\left(\alpha^{*}, \beta\right)+\mathcal{D}_{\Omega, \theta}\left(\alpha^{*}, \beta\right)}
$$

uniformly in $\alpha$ and $\beta$. Fix any hierarchy $\mathfrak{S}$ for scale $\theta$ with $\Omega_{\mathfrak{S}}=\Omega$ and set

$$
\begin{aligned}
\mathcal{V}_{\mathfrak{S}}^{r e s}\left(\alpha^{*}, \beta ; \vec{\alpha}^{*}, \vec{\alpha}\right) & =\mathcal{V}_{\mathfrak{S}}\left(\alpha^{*}, \beta ; \vec{\alpha}^{*}, \vec{\alpha}\right)-V_{\Omega_{\mathfrak{S}}, \theta}\left(\alpha^{*}, \beta\right) \\
\mathcal{B}_{\mathfrak{S}}\left(\alpha^{*}, \beta ; \vec{\rho}\right) & =\mathcal{D}_{\mathfrak{S}}\left(\alpha^{*}, \beta ; \vec{\rho}\right)-\mathcal{D}_{\Omega_{\mathfrak{S}}, \theta}\left(\alpha^{*}, \beta\right)
\end{aligned}
$$

By Lemma III.34 and Corollary III.33,

$$
\chi_{\theta}(\Omega ; \alpha, \beta) e^{\mathcal{V}_{\mathfrak{S}}^{r e s}\left(2^{-m} \theta ; \alpha^{*}, \beta ; \vec{\alpha}^{*}, \vec{\alpha}\right)} e^{\mathcal{B}_{\mathfrak{S}}\left(2^{-m} \theta ; \alpha^{*}, \beta ; \vec{\rho}\right)+\mathcal{L}_{\mathfrak{S}}\left(2^{-m} \theta ; \alpha^{*}, \beta ; \vec{\rho}\right)}
$$

converges as $m \rightarrow \infty$ to

$$
\chi_{\theta}(\Omega ; \alpha, \beta) e^{\mathcal{V}_{\mathfrak{S}}^{r e s}\left(\alpha^{*}, \beta ; \vec{\alpha}^{*}, \vec{\alpha}\right)} e^{\mathcal{B}_{\mathfrak{S}}\left(\alpha^{*}, \beta ; \vec{\rho}\right)+\mathcal{L}_{\mathfrak{S}}\left(\alpha^{*}, \beta ; \vec{\rho}\right)}
$$

uniformly for $\vec{\rho}$ in the domain of integration of $\mathcal{I}_{\left(\mathfrak{S} ; \alpha^{*}, \beta\right)}$ and $(\alpha, \beta)$ in the support of $\chi_{\theta}(\Omega ; \alpha, \beta)$. By Remark III.11, the domain of integration for $\mathcal{I}_{\left(\mathfrak{S} ; \alpha^{*}, \beta\right)}$ is compact. Consequently, as $Q_{\mathfrak{S}}^{r e s}$ is a polynomial,

$$
\begin{gathered}
\lim _{m \rightarrow \infty} \chi_{\theta}(\Omega ; \alpha, \beta) \mathcal{I}_{\left(\mathfrak{S} ; \alpha^{*}, \beta\right)}\left(e^{-Q_{\mathfrak{S}}^{r e s}\left(\alpha^{*}, \beta ; \vec{\alpha}^{*}, \vec{\alpha}\right)+\mathcal{V}_{\mathfrak{S}}^{r e s}\left(2^{-m} \theta ; \alpha^{*}, \beta ; \vec{\alpha}^{*}, \vec{\alpha}\right)}\right. \\
\left.e^{\mathcal{B}_{\mathfrak{S}}\left(2^{-m} \theta ; \alpha^{*}, \beta ; \overrightarrow{\rho_{\uparrow}}\right)+\mathcal{L}_{\mathfrak{S}}\left(2^{-m} \theta ; \alpha^{*}, \beta ; \overrightarrow{\rho_{\uparrow}}\right)}\right) \\
=\chi_{\theta}(\Omega ; \alpha, \beta) \mathcal{I}_{\left(\mathfrak{S} ; \alpha^{*}, \beta\right)}\left(e^{-Q_{\mathfrak{S}}^{r e s}\left(\alpha^{*}, \beta ; \vec{\alpha}^{*}, \vec{\alpha}\right)+\mathcal{V}_{\mathfrak{S}}^{r e s}\left(\alpha^{*}, \beta ; \vec{\alpha}^{*}, \vec{\alpha}\right)}\right. \\
\left.e^{\mathcal{B}_{\mathfrak{S}}\left(\alpha^{*}, \beta ; \overrightarrow{\rho_{\uparrow}}\right)+\mathcal{L}_{\mathfrak{S}}\left(\alpha^{*}, \beta ; \overrightarrow{\rho_{\uparrow}}\right)}\right)
\end{gathered}
$$

uniformly for $(\alpha, \beta)$ in the support of $\chi_{\theta}(\Omega ; \alpha, \beta)$.

By (III.20) and (III.21), the $(\Omega, \mathfrak{S})$ term on the right hand side of (III.19) converges to

$$
\begin{aligned}
\mathcal{Z}_{\theta}^{|\Omega|} e^{\left.\left\langle\alpha_{*}, j(\theta) \beta\right\rangle\right|_{\Omega}+V_{\Omega, \theta}\left(\alpha^{*}, \beta\right)+\mathcal{D}_{\Omega, \theta}\left(\alpha^{*}, \beta\right)} \chi_{\theta}(\Omega ; \alpha, \beta) \\
\mathcal{I}_{\left(\mathfrak{S} ; \alpha^{*}, \beta\right)}\left(e^{-Q_{\mathfrak{S}}^{r e s}\left(\alpha^{*}, \beta ; \vec{\alpha}^{*}, \vec{\alpha}\right)+\mathcal{V}_{\mathfrak{S}}^{r e s}\left(\alpha^{*}, \beta ; \vec{\alpha}^{*}, \vec{\alpha}\right)} e^{\mathcal{B}_{\mathfrak{S}}\left(\alpha^{*}, \beta ; \overrightarrow{\rho_{\uparrow}}\right)+\mathcal{L}_{\mathfrak{S}}\left(\alpha^{*}, \beta ; \overrightarrow{\rho_{\uparrow}}\right)}\right)
\end{aligned}
$$

as $m \rightarrow \infty$. Setting $\mathfrak{h}=1$ gives the $(\Omega, \mathfrak{S})$ term on the right hand side of the representation of $I_{\theta}\left(\alpha^{*}, \beta\right)$ in Theorem II.16. The properties and estimates of the various functions in Theorem II.16 follow from Corollaries III.30 and III.33. It remains to prove the convergence of the sum over $\Omega$ and $\mathfrak{S}$.

Recall that $X$ is a finite set. We saw in Remark II.6 that, for any $\emptyset \neq \Omega \subset X$, the corridor condition in the definition of a hierarchy ensures that there are only finitely many 
hierarchies $\mathfrak{S}$ with $\Omega_{\mathfrak{S}}=\Omega$. If $\Omega=\emptyset$, then $Q_{\mathfrak{S}}^{r e s}=Q_{\mathfrak{S}}, \mathcal{V}_{\mathfrak{S}}^{r e s}=\mathcal{V}_{\mathfrak{S}}$ and $\mathcal{B}_{\mathfrak{S}}=\mathcal{D}_{\mathfrak{S}}=0$. Therefore it suffices to prove the convergence, as $m \rightarrow \infty$, of

$$
\sum_{k=1}^{m} \mathcal{F}_{k, m}\left(\alpha^{*}, \beta\right)
$$

where

$$
\mathcal{F}_{k, m}\left(\alpha^{*}, \beta\right)=\left.\sum_{\substack{\mathfrak{S} \text { hierarchy } \\ \text { for scale } \theta \\ \text { depth( })=k \\ \text { with } \Omega_{\mathfrak{S}}=\emptyset}} \mathcal{I}_{\left(\mathfrak{S} ; \alpha^{*}, \beta\right)}\left(e^{-Q_{\mathfrak{S}}\left(\alpha^{*}, \beta ; \vec{\alpha}^{*}, \vec{\alpha}\right)+\mathcal{V}_{\mathfrak{S}}\left(2^{-m} \theta ; \alpha^{*}, \beta ; \vec{\alpha}^{*}, \vec{\alpha}\right)} e^{\mathcal{L}_{\mathfrak{S}}\left(2^{-m} \theta ; \alpha^{*}, \beta ; \overrightarrow{\rho_{\uparrow}}\right)}\right)\right|_{\mathfrak{h}=1}
$$

We already know the convergence, for each fixed $k$, of $\mathcal{F}_{k, m}\left(\alpha^{*}, \beta\right)$ as $m \rightarrow \infty$. By Theorem III.35 and Lemma III.42, below,

$$
\left|\mathcal{F}_{k, m}\left(\alpha^{*}, \beta\right)\right| \leq e^{\frac{1}{2}\|\alpha\|^{2}+\frac{1}{2}\|\beta\|^{2}} e^{-\ell\left(2^{-k+1} \theta\right)}
$$

As this bound is summable in $k$, the dominated convergence theorem gives the desired limit. This completes the proof of Theorems II.16.

Lemma III.42 Let $\mathfrak{S}$ be a hierarchy for scale $\theta$. Then, on the domain of integration for $\mathcal{I}_{\mathfrak{S}}$,

$$
\left|\mathcal{B}_{\mathfrak{S}}\right|+\left|\mathcal{L}_{\mathfrak{S}}\right| \leq \omega_{\mathfrak{S}}
$$

where $\omega_{\mathfrak{S}}$ was defined in Theorem III.35.

Proof: Let $\varepsilon=2^{-\operatorname{depth}(\mathfrak{S})} \theta$. On the domain of integration,

$$
\left|\alpha_{\tau}(\mathbf{x})\right| \leq \min \left\{\kappa_{\mathfrak{S}, \tau}(\mathbf{x}), \kappa_{* \mathfrak{S}, \tau}(\mathbf{x})\right\} \quad|\alpha(\mathbf{x})| \leq \kappa_{* \mathfrak{S}, 0}(\mathbf{x}) \quad|\beta(\mathbf{x})| \leq \kappa_{\mathfrak{S}, \theta}(\mathbf{x})
$$

for all $\tau \in \varepsilon \mathbb{Z} \cap(0, \theta)$ and $\mathbf{x} \in X$. By Proposition III.32 and [BFKT4, Lemma B.1],

$$
\begin{aligned}
& \left|\mathcal{B}_{\mathfrak{S}}\right| \leq K_{d} K_{D}(\theta \mathfrak{v}) \mathrm{r}(\theta) \mathrm{R}(\theta)^{3}\left|\Omega_{\mathfrak{S}}^{c}\right| \\
& \left|\mathcal{L}_{\mathfrak{S}}\right| \leq \sum_{\substack{\text { decimation } \\
\text { intervals } \\
\mathcal{J} \subset[0, \theta]}} K_{d} K_{L}(|\mathcal{J}| \mathfrak{v}) \mathrm{r}(|\mathcal{J}|) \mathrm{R}(|\mathcal{J}|)^{3}\left|\Omega_{\mathfrak{S}}(\mathcal{J})^{c}\right|
\end{aligned}
$$

where $K_{d}=\sup _{\mathbf{y} \in X} \sum_{\mathbf{x} \in X} e^{-d(\mathbf{x}, \mathbf{y})}$. The result now follows by (F.6.a). 
Proof of Theorem II.18: Set $\mathfrak{h}=1$ and let $f$ be any bounded measurable function. First consider any fixed hierarchy $\mathfrak{S}$ for scale $\theta$ with $\Omega_{\mathfrak{S}}=\Omega$. By Remark III.11, the domain of integration for the large field integral operator $\left|\mathcal{I}_{\left(\mathfrak{S} ; \alpha^{*}, \beta\right)}\right|$ is compact. By Lemma III.34, $\mathcal{V}_{\mathfrak{S}}\left(2^{-m} \theta ; \alpha_{*}, \beta ; \vec{\alpha}_{*}, \vec{\alpha}\right)$ converges uniformly on this domain to

$$
\left.\mathcal{V}_{\mathfrak{S}}\left(\alpha_{*}, \beta ; \vec{\alpha}_{*}, \vec{\alpha}\right)=V_{\Omega ; \theta}\left(\alpha_{*}, \beta\right)\right)+\mathcal{V}_{\mathfrak{S}}^{r e s}\left(\alpha_{*}, \beta ; \vec{\alpha}_{*}, \vec{\alpha}\right)
$$

as $m \rightarrow \infty$. Consequently,

$$
\begin{aligned}
& \lim _{m \rightarrow \infty}\left|\mathcal{I}_{\left(\mathfrak{S} ; \alpha^{*}, \beta\right)}\right| e^{\operatorname{Re}\left(-Q_{\mathfrak{S}}+\mathcal{V}_{\mathfrak{S}}\left(2^{-m} \theta\right)\right)}|f| \\
& =e^{\operatorname{Re}\left(\left\langle\alpha^{*}, j_{(\Omega)}(\theta) \beta\right\rangle+V_{\Omega ; \theta}\left(\alpha_{*}, \beta\right)\right)}\left|\mathcal{I}_{\left(\mathfrak{S} ; \alpha^{*}, \beta\right)}\right| e^{\operatorname{Re}\left(-Q_{\mathfrak{S}}^{r e s}+\mathcal{V}_{\mathfrak{S}}^{r e s}\right)}|f|
\end{aligned}
$$

For any natural number $k$, there are only finitely many hierarchies for scale $\theta$ of depth $k$. So, by Theorem III.35,

$$
\begin{aligned}
& e^{-\frac{1}{2}\|\alpha\|^{2}-\frac{1}{2}\|\beta\|^{2}} e^{-\operatorname{Reg}_{\mathcal{S F}}(\Omega ; \alpha, \beta)} \sum_{k=1}^{k_{0}} e^{\ell\left(2^{-k+1} \theta\right)} \\
& \sum_{\begin{array}{c}
\text { hirchies } \\
\text { for scale } \\
\text { of depth } k \\
\text { with } \Omega_{\mathfrak{S}=\Omega}=\Omega
\end{array}} e^{\omega_{\mathfrak{S}}} e^{\operatorname{Re}\left(\left\langle\alpha^{*}, j_{(\Omega)}(\theta) \beta\right\rangle+V_{\Omega ; \theta}\left(\alpha_{*}, \beta\right)\right)}\left|\mathcal{I}_{\left(\mathfrak{S} ; \alpha^{*}, \beta\right)}\right| e^{\operatorname{Re}\left(-Q_{\mathfrak{S}}^{r e s}+\mathcal{V}_{\mathfrak{S}}^{\text {res }}\right)}|f| \\
& \qquad e^{-\frac{1}{4} \ell(\theta)\left|\Omega^{c}\right|}\left[\prod_{\mathbf{x} \in \Omega^{c}} \frac{1}{1+|\alpha(\mathbf{x})|^{3}} \frac{1}{1+|\beta(\mathbf{x})|^{3}}\right] \sup |f|
\end{aligned}
$$

for all $k_{0} \in \mathbb{N}$. As all terms on the left hand side are nonnegative,

$$
\begin{aligned}
& e^{-\frac{1}{2}\|\alpha\|^{2}-\frac{1}{2}\|\beta\|^{2}} e^{\operatorname{Re}\left(\left\langle\alpha^{*}, j_{(\Omega)}(\theta) \beta\right\rangle+V_{\Omega ; \theta}\left(\alpha_{*}, \beta\right)\right)} e^{-\operatorname{Reg}_{\mathcal{S F}}(\Omega ; \alpha, \beta)} \\
& \sum_{k=1}^{\infty} e^{\ell\left(2^{-k+1} \theta\right)} \sum_{\substack{\text { hierarchies } \\
\text { for scale } \theta \\
\text { of depth } k \\
\text { with } \Omega \mathfrak{S}=\Omega}}\left|\mathcal{I}_{\left(\mathfrak{S} ; \alpha^{*}, \beta\right)}\right| e^{\operatorname{Re}\left(-Q_{\mathfrak{S}}^{\text {res }}+\mathcal{V}_{\mathfrak{S}}^{\text {res }}\right)+\omega_{\mathfrak{S}}}|f| \\
& \leq e^{-\frac{1}{4} \ell(\theta)\left|\Omega^{c}\right|}\left[\prod_{\mathbf{x} \in \Omega^{c}} \frac{1}{1+|\alpha(\mathbf{x})|^{3}} \frac{1}{1+|\beta(\mathbf{x})|^{3}}\right] \sup |f|
\end{aligned}
$$

The theorem now follows by setting $f \equiv 1$ and applying Lemma III.42. 


\section{The "Small Field" Part of the Decimation Step}

The "small field decimation step" is formulated in Theorems III.24 and III.27, which we are going to prove in this chapter. It deals with the "stationary phase approximation" to the construction on a fixed subset $\Omega$ of $X$. As the estimates do not depend on $\Omega$, we may, for simplicity of notation, assume that $\Omega=X$. We shall write $\overline{\mathfrak{R}}_{\delta}$ in place of $\overline{\mathfrak{R}}_{\Omega ; \delta}$ and $V_{\delta}$ in place of $V_{\Omega, \delta}$. Also write

$$
\begin{array}{cc}
\mathrm{r}=\mathrm{r}(\delta) & \mathrm{R}=\mathrm{R}(\delta) \\
\mathrm{r}_{+}=\mathrm{r}(2 \delta) & \mathrm{R}_{+}=\mathrm{R}(2 \delta)
\end{array}
$$

Observe that, by (II.18)

$$
\frac{\mathrm{r}_{+}}{\mathrm{r}}=\frac{1}{2^{e_{\mathrm{r}}}} \quad \frac{\mathrm{R}_{+}}{\mathrm{R}}=\frac{1}{2^{e_{\mathrm{R}}+e_{\mathrm{r}}}}
$$

We also write $\|\cdot\|_{\delta}$ for $\|\cdot\|_{2 R(\delta), 2 \mathrm{~m}}$.

The proofs of Theorem III.24 and III.27 are similar to [BFKT5]. The main technical differences are the presence of the history field and the additional terms created by the fact that the cut off propagator $j_{\mathfrak{c}}(t)$ of (III.2) is not a semigroup - in contrast to the original propagator $j(t)=\operatorname{exph}(-t(\mathrm{~h}-\mu))$.

It turns out that the dominant contribution to $\overline{\mathfrak{R}}_{\delta}\left(V_{\delta}(\varepsilon ; \cdot) ; f_{1}, f_{2}\right)$ in Definition III.22 is $f_{1}\left(\alpha_{*}, j_{\mathfrak{c}}(t) \beta\right)+f_{2}\left(j_{\mathfrak{c}}(t) \alpha_{*}, \beta\right)$ To estimate it, we use

Lemma IV.1 Let $f\left(\alpha_{*}, \beta ; \mathfrak{h}\right)$ be an analytic function. Let $\delta \leq \Theta$.

(i) If $f$ is bilinear in $\alpha_{*}, \beta$ then

$$
\left\|f\left(\alpha_{*}, j_{\mathfrak{c}}(\delta) \beta\right)\right\|_{2 \delta},\left\|f\left(j_{\mathfrak{c}}(\delta) \alpha_{*}, \beta\right)\right\|_{2 \delta} \leq e^{\delta K_{j}}\left(\frac{R_{+}}{\mathrm{R}}\right)^{2}\|f\|_{\delta}
$$

(ii) If $f$ has degree at least two both in $\alpha_{*}$ and $\beta$

$$
\left\|f\left(\alpha_{*}, j_{\mathfrak{c}}(\delta) \beta\right)\right\|_{2 \delta},\left\|f\left(j_{\mathfrak{c}}(\delta) \alpha_{*}, \beta\right)\right\|_{2 \delta} \leq e^{2 \delta K_{j}}\left(\frac{R_{+}}{\mathrm{R}}\right)^{4}\|f\|_{\delta}
$$

Proof: Both in cases (i) and (ii), we prove the first inequality. To do so, we introduce the auxiliary weight system $w_{\text {aux }}$ with metric $2 \mathrm{~m} d$ that associates the constant weight factor $2 \mathrm{R}$ to the field $\alpha_{*}$ and the constant weight factor $2 e^{-\delta K_{j}} \mathrm{R}$ to the field $\beta$. To control the change of variables from $f\left(\alpha_{*}, \beta\right)$ to $f\left(\alpha_{*}, j_{\mathfrak{c}}(\delta) \beta\right)$, we use the weighted $L^{1}-L^{\infty}$ operator norm $N_{2 m d}\left(j_{\mathfrak{c}}(\delta) ; 2 \mathrm{R}, 2 e^{-\delta K_{j}} \mathrm{R}\right)$ of [BFKT4, Definition IV.2]. In the notation of this paper,

$$
N_{2 \mathrm{~m} d}\left(j(\delta) ; 2 \mathrm{R}, 2 e^{-\delta K_{j}} \mathrm{R}\right) \leq e^{-\delta K_{j}}\|\| j(\delta) \| \mid \leq 1
$$


by Lemma III.21.i. Hence, by [BFKT4, Proposition IV.4]

$$
\left\|f\left(\alpha_{*}, j_{\mathfrak{c}}(\delta) \beta\right)\right\|_{w_{\mathrm{aux}}} \leq\|f\|_{\delta}
$$

If $f$ is bilinear in $\alpha_{*}, \beta$ then

$$
\left\|f\left(\alpha_{*}, j_{\mathfrak{c}}(\delta) \beta\right)\right\|_{2 \delta}=\left(\frac{2 \mathrm{R}_{+}}{2 \mathrm{R}}\right)\left(\frac{2 \mathrm{R}_{+}}{2 e^{-\delta K_{j} \mathrm{R}}}\right)\left\|f\left(\alpha_{*}, j_{\mathfrak{c}}(\delta) \beta\right)\right\|_{w_{\mathrm{aux}}} \leq e^{\delta K_{j}}\left(\frac{\mathrm{R}_{+}}{\mathrm{R}}\right)^{2}\|f\|_{\delta}
$$

If $f$ has degree at least two both in $\alpha_{*}$ and $\beta$

$$
\left\|f\left(\alpha_{*}, j_{\mathfrak{c}}(\delta) \beta\right)\right\|_{2 \delta} \leq\left(\frac{2 \mathrm{R}_{+}}{2 \mathrm{R}}\right)^{2}\left(\frac{2 \mathrm{R}_{+}}{2 e^{-\delta K_{j} \mathrm{R}}}\right)^{2}\left\|f\left(\alpha_{*}, j_{\mathfrak{c}}(\delta) \beta\right)\right\|_{w_{\text {aux }}} \leq e^{2 \delta K_{j}}\left(\frac{\mathrm{R}_{+}}{\mathrm{R}}\right)^{4}\|f\|_{\delta}
$$

since $e^{-\delta K_{j}} \geq \frac{\mathrm{R}_{+}}{\mathrm{R}}=\frac{1}{2^{e_{\mathrm{R}}+e_{\mathrm{r}}}}$ by Hypothesis F.7.i.

To treat the fluctuation integral in Definition III.22, we introduce a second auxiliary weight system $w_{\text {fluct }}$ with metric $2 \mathrm{~m} d$ that gives weight $2 \mathrm{R}_{+}$both to $\alpha_{*}$ and $\beta$, and weight $32 \mathrm{r}$ to the fields $z_{*}$ and $z$. We write $\|\cdot\|_{\text {fluct }}$ for $\|\cdot\|_{w_{\text {fluct }}}$. If $f\left(\alpha_{*}, \beta, z_{*}, z\right)$ happens to be independent of $z_{*}$ and $z$, then $\|f\|_{\text {fluct }}=\|f\|_{2 \delta}$. For the first two terms in the effective action $\mathcal{A}$ of Definition III.22, we have

Lemma IV.2 For any history complete analytic function $f\left(\alpha_{*}, \beta\right)$

$$
\begin{aligned}
\left\|f\left(\alpha_{*}, z+j_{\mathfrak{c}}(\delta) \beta\right)-f\left(\alpha_{*}, j_{\mathfrak{c}}(\delta) \beta\right)\right\|_{\text {fluct }} & \leq\|f\|_{\delta} \\
\left.\| f\left(z_{*}+j_{\mathfrak{c}}(\delta) \alpha_{*}, \beta\right)-f\left(j_{\mathfrak{c}}(\delta) \alpha_{*}, \beta\right)\right) \|_{\text {fluct }} & \leq\|f\|_{\delta}
\end{aligned}
$$

for all $\delta \leq \Theta$.

Proof: We prove the first inequality. By [BFKT4, Corollary IV.6],

$$
\left\|f\left(\alpha_{*}, z+j_{\mathfrak{c}}(\delta) \beta\right)-f\left(\alpha_{*}, j_{\mathfrak{c}}(\delta) \beta\right)\right\|_{\text {fluct }} \leq\left\|f\left(\alpha_{*}, z+j_{\mathfrak{c}}(\delta) \beta\right)\right\|_{\text {fluct }} \leq\left\|f\left(\alpha_{*}, \beta\right)\right\|_{\delta}
$$

since, by Lemma III.21.i

$$
\begin{aligned}
N_{2 \mathrm{~m} d}(1 \mathbb{1} ; 2 \mathrm{R}, 32 \mathrm{r})+N_{2 \mathrm{~m} d}\left(j_{\mathfrak{c}}(\delta) ; 2 \mathrm{R}, 2 \mathrm{R}+\right) & \leq\|1\|\left\|\frac{32 \mathrm{r}}{2 \mathrm{R}}+\right\| j_{\mathfrak{c}}(\delta) \| \frac{\mathrm{R}_{+}}{\mathrm{R}} \\
& \leq \frac{16 \mathrm{r}}{\mathrm{R}}+e^{\delta K_{j}} \frac{\mathrm{R}_{+}}{\mathrm{R}}=16(\delta \mathfrak{v})^{e_{\mathrm{R}}}+\frac{e^{\delta K_{j}}}{2^{e_{\mathrm{R}}+e_{\mathrm{r}}}} \leq 1
\end{aligned}
$$

by Hypothesis F.7.i 
The remaining summands in Definition III.22 are explicit quadratic and quartic terms. The quadratic terms all involve the difference $j_{\mathfrak{c}}(\delta)-j(\delta)$ and are consequently exponentially small with $\mathfrak{c}$.

Lemma IV.3

$$
\begin{array}{r}
\left\|\left\langle\left[j(\delta)-j_{\mathfrak{c}}(\delta)\right] \alpha_{*},\left[j(\delta)-j_{\mathfrak{c}}(\delta)\right] \beta\right\rangle\right\|_{2 \delta} \leq 4 \delta^{2} K_{j}^{2} e^{2 K_{j} \delta} \mathrm{R}_{+}^{2} e^{-2 \mathrm{~m} \mathfrak{c}} \\
\left\|\left\langle\left[j(\delta)-j_{\mathfrak{c}}(\delta)\right] \alpha_{*}, z\right\rangle\right\|_{\text {fluct }}, \quad\left\|\left\langle z_{*},\left[j(\delta)-j_{\mathfrak{c}}(\delta)\right] \beta\right\rangle\right\|_{\text {fluct }} \leq 64 \delta K_{j} e^{K_{j} \delta} \mathrm{r} \mathrm{R}_{+} e^{-\mathrm{m} \mathfrak{c}}
\end{array}
$$

Proof: By definition

$$
\left\|\left\langle\alpha_{*}, \beta\right\rangle\right\|_{2 \delta}=4 \mathrm{R}_{+}^{2}
$$

Therefore, by Lemma G.2.a,

$$
\begin{aligned}
\left\|\left\langle\left[j(\delta)-j_{\mathfrak{c}}(\delta)\right] \alpha_{*},\left[j(\delta)-j_{\mathfrak{c}}(\delta)\right] \beta\right\rangle\right\|_{2 \delta} & \leq 4 \mathrm{R}_{+}^{2} N_{2 \mathrm{~m} d}\left(j(\delta)-j_{\mathfrak{c}}(\delta) ; 2 \mathrm{R}_{+}, 2 \mathrm{R}_{+}\right)^{2} \\
& =4 \mathrm{R}_{+}^{2} N_{2 \mathrm{~m} d}\left(j(\delta)-j_{\mathfrak{c}}(\delta) ; 1,1\right)^{2} \\
& \leq 4 \mathrm{R}_{+}^{2}\left\|j(\delta)-j_{\mathfrak{c}}(\delta)\right\|^{2} \\
& \leq 4 \delta^{2} K_{j}^{2} e^{2 K_{j} \delta} \mathrm{R}_{+}^{2} e^{-2 \mathrm{~m} \mathfrak{c}}
\end{aligned}
$$

In the last line, we used Lemma III.21.iii. Similarly

$$
\begin{aligned}
\left\|\left\langle\left[j(\delta)-j_{\mathfrak{c}}(\delta)\right] \alpha_{*}, z\right\rangle\right\|_{\text {fluct }} & \leq 64 \mathrm{R}_{+} \mathrm{r} N_{2 \mathrm{~m} d}\left(j(\delta)-j_{\mathfrak{c}}(\delta) ; 2 \mathrm{R}_{+}, 2 \mathrm{R}_{+}\right) \\
& \leq 64 \delta K_{j} e^{K_{j} \delta} \mathrm{r} \mathrm{R} e^{-\mathrm{m} \mathfrak{c}}
\end{aligned}
$$

The explicit quartic terms in $\overline{\mathfrak{R}}_{\delta}\left(V_{\delta}(\varepsilon ; \cdot) ; \mathcal{R}_{1}+\mathcal{E}_{1}, \mathcal{R}_{2}+\mathcal{E}_{2}\right)$ are

$$
\left[V_{\delta}\left(\varepsilon ; \alpha_{*}, j_{\mathfrak{c}}(\delta) \beta\right)-V_{\delta}\left(\varepsilon ; \alpha_{*}, j(\delta) \beta\right)\right]+\left[V_{\delta}\left(\varepsilon ; j_{\mathfrak{c}}(\delta) \alpha_{*}, \beta\right)-V_{\delta}\left(\varepsilon ; j(\delta) \alpha_{*}, \beta\right)\right]
$$

"downstairs", and

$$
\left[V_{\delta}\left(\varepsilon ; \alpha_{*}, z+j_{\mathfrak{c}}(\delta) \beta\right)-V_{\delta}\left(\varepsilon ; \alpha_{*}, j_{\mathfrak{c}}(\delta) \beta\right)\right]+\left[V_{\delta}\left(\varepsilon ; z_{*}+j_{\mathfrak{c}}(\delta) \alpha_{*}, \beta\right)-V_{\delta}\left(\varepsilon ; j_{\mathfrak{c}}(\delta) \alpha_{*}, \beta\right)\right]
$$

as a contribution to the effective action. The term "downstairs" again involves the difference $j_{\mathfrak{c}}(\delta)-j(\delta)$ and is exponentially small with $\mathfrak{c}$.

Lemma IV.4 If $\delta \leq \Theta$, then

$$
\begin{aligned}
& \left\|V_{\delta}\left(\varepsilon ; \alpha_{*}, j_{\mathfrak{c}}(\delta) \beta\right)-V_{\delta}\left(\varepsilon ; \alpha_{*}, j(\delta) \beta\right)\right\|_{2 \delta} \leq 64 K_{j} e^{8 K_{j} \delta} \delta^{2}\|v\| \mathrm{R}_{+}^{4} e^{-\mathrm{m} \mathfrak{c}} \\
& \left\|V_{\delta}\left(\varepsilon ; j_{\mathfrak{c}}(\delta) \alpha_{*}, \beta\right)-V_{\delta}\left(\varepsilon ; j(\delta) \alpha_{*}, \beta\right)\right\|_{2 \delta} \leq 64 K_{j} e^{8 K_{j} \delta} \delta^{2}\|v\| \mathrm{R}_{+}^{4} e^{-\mathrm{m} \mathfrak{c}}
\end{aligned}
$$

and

$$
\begin{gathered}
\left\|V_{\delta}\left(\varepsilon ; \alpha_{*}, z+j_{\mathfrak{c}}(\delta) \beta\right)-V_{\delta}\left(\varepsilon ; \alpha_{*}, j_{\mathfrak{c}}(\delta) \beta\right)\right\|_{\text {fluct }} \leq 2^{11} \delta\|v\| \mathrm{r} \mathrm{R}_{+}^{3} \\
\left\|V_{\delta}\left(\varepsilon ; z_{*}+j_{\mathfrak{c}}(\delta) \alpha_{*}, \beta\right)-V_{\delta}\left(\varepsilon ; j_{\mathfrak{c}}(\delta) \alpha_{*}, \beta\right)\right\|_{\text {fluct }} \leq 2^{11} \delta\|v\| \mathrm{r} \mathrm{R}_{+}^{3}
\end{gathered}
$$


Proof: We prove the first in each pair of inequalities. By Definition III.8

$$
V_{\delta}\left(\varepsilon ; \alpha_{*}, \beta\right)=-\varepsilon \sum_{\tau \in \varepsilon \mathbb{Z} \cap[0, \delta)}\left\langle\gamma_{* \tau} \gamma_{\tau+\varepsilon}, v \gamma_{* \tau} \gamma_{\tau+\varepsilon}\right\rangle
$$

with

$$
\gamma_{* \tau}=j(\tau) \alpha_{*} \quad \gamma_{\tau}=j(\delta-\tau) \beta
$$

For the first inequality, we write

$V_{\delta}\left(\varepsilon ; \alpha_{*}, j_{\mathfrak{c}}(\delta) \beta\right)-V_{\delta}\left(\varepsilon ; \alpha_{*}, j(\delta) \beta\right)=\varepsilon \sum_{\tau \in \varepsilon \mathbb{Z} \cap[0, \delta)}\left[\left\langle\gamma_{* \tau} g_{\tau+\varepsilon}, v \gamma_{* \tau} g_{\tau+\varepsilon}\right\rangle-\left\langle\gamma_{* \tau} \tilde{g}_{\tau+\varepsilon}, v \gamma_{* \tau} \tilde{g}_{\tau+\varepsilon}\right\rangle\right]$

with

$$
g_{\tau}=j(\delta-\tau) j(\delta) \beta \quad \quad \tilde{g}_{\tau}=j(\delta-\tau) j_{\mathfrak{c}}(\delta) \beta
$$

By definition

$$
\left\|\left\langle\alpha_{*} \beta, v \alpha_{*} \beta\right\rangle\right\|_{2 \delta} \leq 16\|\| v \| \mathrm{R}_{+}^{4}
$$

We apply Corollary G.3.ii, with $d$ and $d^{\prime}$ both replaced by $2 \mathrm{~m} d, \delta=0, r=4, s=2$, $h\left(\gamma_{1}, \cdots, \gamma_{4}\right)=\left\langle\gamma_{1} \gamma_{2}, v \gamma_{3} \gamma_{4}\right\rangle, \alpha_{1}=\alpha_{*}, \alpha_{2}=\beta$ and

$$
\begin{array}{llll}
\Gamma_{1}^{1}=\Gamma_{3}^{1}=j(\tau) & \Gamma_{1}^{2}=\Gamma_{3}^{2}=0 & \Gamma_{2}^{1}=\Gamma_{4}^{1}=0 & \Gamma_{2}^{2}=\Gamma_{4}^{2}=j(\delta-\tau-\varepsilon) j(\delta) \\
\tilde{\Gamma}_{1}^{1}=\tilde{\Gamma}_{3}^{1}=j(\tau) & \tilde{\Gamma}_{1}^{2}=\tilde{\Gamma}_{3}^{2}=0 & \tilde{\Gamma}_{2}^{1}=\tilde{\Gamma}_{4}^{1}=0 & \tilde{\Gamma}_{2}^{2}=\tilde{\Gamma}_{4}^{2}=j(\delta-\tau-\varepsilon) j_{\mathfrak{c}}(\delta)
\end{array}
$$

As

$$
\begin{aligned}
\sigma & =\max \left\{N_{2 \mathrm{~m} d}\left(j(\tau) ; 2 R_{+}, 2 \mathrm{R}_{+}\right), \quad N_{2 \mathrm{~m} d}\left(j(\delta-\tau-\varepsilon) j(\delta) ; 2 R_{+}, 2 \mathrm{R}_{+}\right),\right. \\
& \leq \max \left\{\|j(\tau)\|, \quad\|j(\delta-\tau-\varepsilon) j(\delta)\|, \quad\left\|j(\delta-\tau-\varepsilon) j_{\mathfrak{c}}(\delta)\right\|\right\} \\
& \leq e^{2 K_{j} \delta} \\
\sigma_{\delta} & =N_{2 \mathrm{~m} d}\left(j(\delta-\tau-\varepsilon)\left[j(\delta)-j_{\mathfrak{c}}(\delta)\right] ; 2 R_{+}, 2 \mathrm{R}_{+}\right) \\
& \leq\|j(\delta-\tau-\varepsilon)\|\left\|j(\delta)-j_{\mathfrak{c}}(\delta)\right\| \\
& \leq \delta K_{j} e^{2 K_{j} \delta} e^{-\mathrm{m} \mathfrak{c}}
\end{aligned}
$$

by Lemma III.21, it gives, for each $\tau \in \varepsilon \mathbb{Z} \cap[0, \delta)$

$$
\begin{aligned}
\left\|\left\langle\gamma_{* \tau} g_{\tau+\varepsilon}, v \gamma_{* \tau} g_{\tau+\varepsilon}\right\rangle-\left\langle\gamma_{* \tau} \tilde{g}_{\tau+\varepsilon}, v \gamma_{* \tau} \tilde{g}_{\tau+\varepsilon}\right\rangle\right\|_{2 \delta} & \leq 64\|v\| \mathrm{R}_{+}^{4} \sigma_{\delta} \sigma^{3} \\
& \leq 64\|v\| \mathrm{R}_{+}^{4} \delta K_{j} e^{8 K_{j} \delta} e^{-\mathrm{m} \mathfrak{c}}
\end{aligned}
$$

Summing over $\tau$ and multiplying with $\varepsilon$ gives the desired estimate. 
For the third inequality, we write

$$
\begin{aligned}
V_{\delta}\left(\varepsilon ; \alpha_{*}, z+j_{\mathfrak{c}}(\delta) \beta\right)-V_{\delta} & \left(\varepsilon ; \alpha_{*}, j_{\mathfrak{c}}(\delta) \beta\right) \\
& =-\varepsilon \sum_{\tau \in \varepsilon \mathbb{Z} \cap[0, \delta)}\left[\left\langle\gamma_{* \tau} \hat{g}_{\tau+\varepsilon}, v \gamma_{* \tau} \hat{g}_{\tau+\varepsilon}\right\rangle-\left\langle\gamma_{* \tau} \tilde{g}_{\tau+\varepsilon}, v \gamma_{* \tau} \tilde{g}_{\tau+\varepsilon}\right\rangle\right]
\end{aligned}
$$

with

$$
\hat{g}_{\tau}=j(\delta-\tau)\left(z+j_{\mathfrak{c}}(\delta) \beta\right)=j(\delta-\tau) z+j(\delta-\tau) j_{\mathfrak{c}}(\delta) \beta
$$

This time, we apply Corollary G.3.ii, with $d$ and $d^{\prime}$ both replaced by $2 \mathrm{~m} d, \delta=0, r=4$, $s=3, h\left(\gamma_{1}, \cdots, \gamma_{4}\right)=\left\langle\gamma_{1} \gamma_{2}, v \gamma_{3} \gamma_{4}\right\rangle, \alpha_{1}=\alpha_{*}, \alpha_{2}=\beta, \alpha_{3}=z$ and

$$
\begin{array}{lll}
\hat{\Gamma}_{1}^{1}=\hat{\Gamma}_{3}^{1}=j(\tau) & \hat{\Gamma}_{2}^{2}=\hat{\Gamma}_{4}^{2}=j(\delta-\tau-\varepsilon) j_{\mathfrak{c}}(\delta) & \hat{\Gamma}_{2}^{3}=\hat{\Gamma}_{4}^{3}=j(\delta-\tau-\varepsilon) \\
\hat{\tilde{\Gamma}}_{1}^{1}=\hat{\tilde{\Gamma}}_{3}^{1}=j(\tau) & \hat{\tilde{\Gamma}}_{2}^{2}=\hat{\tilde{\Gamma}}_{4}^{2}=j(\delta-\tau-\varepsilon) j_{\mathfrak{c}}(\delta) & \hat{\tilde{\Gamma}}_{2}^{3}=\hat{\tilde{\Gamma}}_{4}^{3}=0
\end{array}
$$

with all other $\hat{\Gamma}_{i}^{j}$ s and $\hat{\tilde{\Gamma}}_{i}^{j}$ 's being zero. Then

$$
\begin{aligned}
& \hat{\sigma}=\max \left\{N_{2 \mathrm{~m} d}\left(j(\tau) ; 2 R_{+}, 2 \mathrm{R}_{+}\right), \quad N_{2 \mathrm{~m} d}\left(j(\delta-\tau-\varepsilon) j_{\mathfrak{c}}(\delta) ; 2 R_{+}, 2 \mathrm{R}_{+}\right),\right. \\
& \left.N_{2 \mathrm{~m} d}\left(j(\delta-\tau-\varepsilon) ; 2 \mathrm{R}_{+}, 32 \mathrm{r}\right)+N_{2 \mathrm{~m} d}\left(j(\delta-\tau-\varepsilon) j_{\mathfrak{c}}(\delta) ; 2 R_{+}, 2 \mathrm{R}_{+}\right)\right\} \\
& \leq \max \left\{\|j(\tau)\|\|, \quad\| j(\delta-\tau-\varepsilon) j_{\mathfrak{c}}(\delta)\left\|, \quad 16 \frac{\mathrm{r}}{\mathrm{R}_{+}}\right\| j(\delta-\tau-\varepsilon)\|\|+\left\|j(\delta-\tau-\varepsilon) j_{\mathfrak{c}}(\delta)\right\|\right\} \\
& \leq\left(1+16(\delta \mathfrak{v})^{e_{\mathrm{R}}}\right) e^{2 K_{j} \delta} \\
& \hat{\sigma}_{\delta}=N_{2 \mathrm{~m} d}\left(j(\delta-\tau-\varepsilon) ; 2 R_{+}, 32 \mathrm{r}\right) \\
& \leq 16 \frac{\mathrm{r}}{\mathrm{R}_{+}}\|j(\delta-\tau-\varepsilon)\| \| \\
& \leq 16 \frac{\mathrm{r}}{\mathrm{R}_{+}} e^{K_{j} \delta}
\end{aligned}
$$

So, as before, for each $\tau \in \varepsilon \mathbb{Z} \cap[0, \delta)$

$$
\begin{aligned}
\left\|\left\langle\gamma_{* \tau} \hat{g}_{\tau+\varepsilon}, v \gamma_{* \tau} \hat{g}_{\tau+\varepsilon}\right\rangle-\left\langle\gamma_{* \tau} \tilde{g}_{\tau+\varepsilon}, v \gamma_{* \tau} \tilde{g}_{\tau+\varepsilon}\right\rangle\right\|_{\text {fluct }} & \leq 64\|v\| \mathrm{R}_{+}^{4} \hat{\sigma}_{\delta} \hat{\sigma}^{3} \\
& \leq 2^{11}\|v\| \| \mathrm{rR}_{+}^{3}
\end{aligned}
$$

by Hypothesis F.7.i. Summing over $\tau$ gives the desired result.

Proof of Theorem III.24: Set

$$
\begin{aligned}
\mathcal{R}^{(1)}\left(\alpha_{*}, \beta\right) & =\mathcal{R}_{1}\left(\alpha_{*}, j_{\mathfrak{c}}(\delta) \beta\right)+\mathcal{R}_{2}\left(j_{\mathfrak{c}}(\delta) \alpha_{*}, \beta\right)-\left\langle\left[j(\delta)-j_{\mathfrak{c}}(\delta)\right] \alpha_{*},\left[j(\delta)-j_{\mathfrak{c}}(\delta)\right] \beta\right\rangle \\
\mathcal{E}^{(1)}\left(\alpha_{*}, \beta\right) & =\mathcal{E}_{1}\left(\alpha_{*}, j_{\mathfrak{c}}(\delta) \beta\right)+\mathcal{E}_{2}\left(j_{\mathfrak{c}}(\delta) \alpha_{*}, \beta\right) \\
& +V_{\delta}\left(\varepsilon ; \alpha_{*}, j_{\mathfrak{c}}(\delta) \beta\right)-V_{\delta}\left(\varepsilon ; \alpha_{*}, j(\delta) \beta\right)+V_{\delta}\left(\varepsilon ; j_{\mathfrak{c}}(\delta) \alpha_{*}, \beta\right)-V_{\delta}\left(\varepsilon ; j(\delta) \alpha_{*}, \beta\right)
\end{aligned}
$$


and

$$
\begin{aligned}
\mathcal{R}_{l}\left(\alpha_{*}, z\right) & =\mathcal{R}_{1}\left(\alpha_{*}, z\right)+\left\langle\left[j(\delta)-j_{\mathfrak{c}}(\delta] \alpha_{*}, z\right\rangle\right. \\
\mathcal{R}_{r}\left(\alpha_{*}, z\right) & =\mathcal{R}_{2}\left(z_{*}, \beta\right)+\left\langle z_{*},\left[j(\delta)-j_{\mathfrak{c}}(\delta] \beta\right\rangle\right. \\
\mathcal{E}_{l}\left(\alpha_{*}, \beta, z\right)=\left[\mathcal{E}_{1}\left(\alpha_{*}, z+j_{\mathfrak{c}}(\delta) \beta\right)-\mathcal{E}_{1}\left(\alpha_{*}, j_{\mathfrak{c}}(\delta) \beta\right)\right] & +\left[V_{\delta}\left(\varepsilon ; \alpha_{*}, z+j_{\mathfrak{c}}(\delta) \beta\right)-V_{\delta}\left(\varepsilon ; \alpha_{*}, j_{\mathfrak{c}}(\delta) \beta\right)\right] \\
\mathcal{E}_{r}\left(z_{*}, \alpha_{*}, \beta\right)=\left[\mathcal{E}_{2}\left(z_{*}+j_{\mathfrak{c}}(\delta) \alpha_{*}, \beta\right)-\mathcal{E}_{2}\left(z_{*}+j_{\mathfrak{c}}(\delta) \alpha_{*}, \beta\right)\right] & +\left[V_{\delta}\left(\varepsilon ; z_{*}+j_{\mathfrak{c}}(\delta) \alpha_{*}, \beta\right)-V_{\delta}\left(\varepsilon ; j_{\mathfrak{c}}(\delta) \alpha_{*}, \beta\right]\right.
\end{aligned}
$$

Clearly, $\mathcal{R}^{(1)}\left(\alpha_{*}, \beta\right)$ is bilinear in $\alpha_{*}, \beta$ and $\mathcal{E}^{(1)}\left(\alpha_{*}, \beta\right)$ has degree at least two both in $\alpha_{*}$ and $\beta$. Furthermore, by Lemmas IV.1, IV.3 and IV.4

$$
\begin{aligned}
\left\|\mathcal{R}^{(1)}\right\|_{2 \delta} & \leq e^{\delta K_{j}}\left(\frac{R_{+}}{\mathrm{R}}\right)^{2}\left(\left\|\mathcal{R}_{1}\right\|_{\delta}+\left\|\mathcal{R}_{2}\right\|_{\delta}\right)+4 \delta^{2} K_{j}^{2} e^{2 K_{j} \delta} \mathrm{R}_{+}^{2} e^{-2 \mathrm{~m} \mathfrak{c}} \\
\left\|\mathcal{E}^{(1)}\right\|_{2 \delta} & \leq e^{2 \delta K_{j}}\left(\frac{R_{+}}{\mathrm{R}}\right)^{4}\left(\left\|\mathcal{E}_{1}\right\|_{\delta}+\left\|\mathcal{E}_{2}\right\|_{\delta}\right)+128 K_{j} e^{8 K_{j} \delta} \delta^{2}\|v\| \mathrm{R}_{+}^{4} e^{-\mathrm{m} \mathfrak{c}}
\end{aligned}
$$

Also, $\mathcal{R}_{l}$ is bilinear in $\alpha_{*}, z$, and $\mathcal{R}_{r}$ is bilinear in $z_{*}, \beta$. By Lemma IV.2 and Lemma IV.3

$$
\begin{aligned}
& \left\|\mathcal{R}_{l}\right\|_{\text {fluct }} \leq\left\|\mathcal{R}_{1}\right\|_{\delta}+64 \delta K_{j} e^{K_{j} \delta} \mathrm{rR}_{+} e^{-\mathrm{m} \mathfrak{c}} \\
& \left\|\mathcal{R}_{r}\right\|_{\text {fluct }} \leq\left\|\mathcal{R}_{2}\right\|_{\delta}+64 \delta K_{j} e^{K_{j} \delta} \mathrm{rR}_{+} e^{-\mathrm{m} \mathfrak{c}}
\end{aligned}
$$

Furthermore, $\mathcal{E}_{l}\left(\alpha_{*}, \beta, z\right)$ has degree at least two in the variable $\alpha_{*}$; and the sums of the degrees of the variables $\beta$ and $z$ in each monomial of its power series expansion is also at least two. Also, by Lemma IV.2 and Lemma IV.4

$$
\left\|\mathcal{E}_{l}\right\|_{\text {fluct }} \leq\left\|\mathcal{E}_{1}\right\|_{\delta}+2^{11} \delta\|v\| \| \mathrm{r} \mathrm{R}_{+}^{3}
$$

There is the analogous statement for $\mathcal{E}_{r}$.

By construction, the effective density in Definition III.22 in the situation of Theorem III.24 is

$$
\mathcal{A}\left(\alpha_{*}, \beta ; z_{*}, z\right)=\mathcal{R}_{l}\left(\alpha_{*}, z\right)+\mathcal{E}_{l}\left(\alpha_{*}, \beta, z\right)+\mathcal{R}_{r}\left(z_{*}, \beta\right)+\mathcal{E}_{r}\left(z_{*}, \alpha_{*}, \beta\right)
$$

so that

$$
\begin{aligned}
\overline{\mathfrak{R}}_{\delta}\left(V_{\delta}(\varepsilon ; \cdot) ; \mathcal{R}_{1}+\mathcal{E}_{1}, \mathcal{R}_{2}+\right. & \left.\mathcal{E}_{2}\right)=\mathcal{R}^{(1)}+\mathcal{E}^{(1)} \\
& +\log \frac{1}{Z} \int d \mu_{\mathrm{r}}\left(z^{*}, z\right) e^{\mathcal{R}_{l}\left(\alpha_{*}, \beta, z\right)+\mathcal{E}_{l}\left(\alpha_{*}, \beta, z\right)+\mathcal{R}_{r}\left(z^{*}, \beta\right)+\mathcal{E}_{r}\left(z^{*}, \alpha_{*}, \beta\right)}
\end{aligned}
$$

where $Z=\int d \mu_{\mathrm{r}}\left(z^{*}, z\right)$. Set

$$
\mathcal{R}^{(2)}\left(\alpha_{*}, \beta\right)=\frac{1}{Z} \int d \mu_{\mathrm{r}}\left(z^{*}, z\right) \mathcal{R}_{l}\left(\alpha_{*}, z\right) \mathcal{R}_{r}\left(z^{*}, \beta\right)
$$


By [BFKT4, Remark III.3.ii]

$$
\left\|\mathcal{R}^{(2)}\right\|_{2 \delta}=\left\|\mathcal{R}^{(2)}\right\|_{\text {fluct }} \leq\left\|\mathcal{R}_{l}\right\|_{\text {fluct }}\left\|\mathcal{R}_{r}\right\|_{\text {fluct }}
$$

Expanding the exponential in Definition III.22 and using the fact that the $d \mu_{\mathrm{r}}\left(z^{*}, z\right)$ integral is zero unless there are the same number of $z$ 's and $z^{*}$ 's, one sees that

$$
\mathcal{E}^{(2)}\left(\alpha_{*}, \beta\right)=\log \frac{1}{Z} \int d \mu_{\mathrm{r}}\left(z^{*}, z\right) e^{\mathcal{A}\left(\alpha_{*}, \beta ; z^{*}, z\right)}-\mathcal{R}^{(2)}\left(\alpha_{*}, \beta\right)
$$

has degree at least two both in $\alpha_{*}$ and in $\beta$. By [BFKT4, Corollary III.5], with $n=2$, there is a function $\mathcal{E}^{\prime}\left(\alpha_{*}, \beta\right)$ such that

$$
\begin{aligned}
\log \frac{1}{Z} \int d \mu_{\mathrm{r}}\left(z^{*}, z\right) e^{\mathcal{A}\left(\alpha_{*}, \beta ; z^{*}, z\right)}=\mathcal{E}^{\prime}\left(\alpha_{*}, \beta\right)+\frac{1}{2 Z} \int d \mu_{\mathrm{r}}\left(z^{*}, z\right)\left[\mathcal{R}_{l}+\mathcal{E}_{l}+\mathcal{R}_{r}+\mathcal{E}_{r}\right]^{2} \\
=\mathcal{E}^{\prime}\left(\alpha_{*}, \beta\right)+\mathcal{R}^{(2)}\left(\alpha_{*}, \beta\right)+\frac{1}{Z} \int d \mu_{\mathrm{r}}\left(z^{*}, z\right)\left[\mathcal{R}_{l} \mathcal{E}_{r}+\mathcal{E}_{l} \mathcal{R}_{r}+\mathcal{E}_{l} \mathcal{E}_{r}\right]
\end{aligned}
$$

and

$$
\left\|\mathcal{E}^{\prime}\right\|_{2 \delta} \leq\left(\frac{\|\mathcal{A}\|_{\text {fluct }}}{\frac{1}{20}-\|\mathcal{A}\|_{\text {fluct }}}\right)^{3}
$$

(The hypothesis that $\|\mathcal{A}\|_{\text {fluct }}<\frac{1}{32}$ is verified below.) Clearly

$$
\mathcal{E}^{(2)}=\mathcal{E}^{\prime}+\frac{1}{Z} \int d \mu_{\mathrm{r}}\left(z^{*}, z\right)\left[\mathcal{R}_{l} \mathcal{E}_{r}+\mathcal{E}_{l} \mathcal{R}_{r}+\mathcal{E}_{l} \mathcal{E}_{r}\right]
$$

and, by [BFKT4, Remark III.3.ii]

$$
\left\|\mathcal{E}^{(2)}\right\|_{2 \delta} \leq\left\|\mathcal{E}^{\prime}\right\|_{2 \delta}+\left\|\mathcal{R}_{l}\right\|_{\text {fluct }}\left\|\mathcal{E}_{r}\right\|_{\text {fluct }}+\left\|\mathcal{E}_{l}\right\|_{\text {fluct }}\left\|\mathcal{R}_{r}\right\|_{\text {fluct }}+\left\|\mathcal{E}_{l}\right\|_{\text {fluct }}\left\|\mathcal{E}_{r}\right\|_{\text {fluct }}
$$

We set

$$
\mathcal{R}=\mathcal{R}^{(1)}+\mathcal{R}^{(2)} \quad, \quad \mathcal{E}=\mathcal{E}^{(1)}+\mathcal{E}^{(2)}
$$

By construction

$$
\overline{\mathfrak{R}}_{\delta}\left(V_{\delta}(\varepsilon ; \cdot) ; \mathcal{R}_{1}+\mathcal{E}_{1}, \mathcal{R}_{2}+\mathcal{E}_{2}\right)=\mathcal{R}+\mathcal{E}
$$

Furthermore, $\mathcal{R}\left(\alpha_{*}, \beta\right)$ is bilinear in $\alpha_{*}, \beta$, and $\mathcal{E}\left(\alpha_{*}, \beta\right)$ has degree at least two both in $\alpha_{*}$ and $\beta$. Hence $\mathcal{R}$ is the quadratic part of $\overline{\mathfrak{R}}_{\delta}\left(V_{\delta}(\varepsilon ; \cdot) ; \mathcal{R}_{1}+\mathcal{E}_{1}, \mathcal{R}_{2}+\mathcal{E}_{2}\right)$, which coincides with the quadratic part of $\overline{\mathfrak{R}}_{\delta}\left(0 ; \mathcal{R}_{1}, \mathcal{R}_{2}\right)$. Also

$$
\begin{aligned}
\|\mathcal{R}\|_{2 \delta} \leq & \left\|\mathcal{R}^{(1)}\right\|_{2 \delta}+\left\|\mathcal{R}^{(2)}\right\|_{2 \delta} \\
\leq & \left\|\mathcal{R}^{(1)}\right\|_{2 \delta}+\left\|\mathcal{R}_{l}\right\|_{\text {fluct }}\left\|\mathcal{R}_{r}\right\|_{\text {fluct }} \\
\leq & e^{\delta K_{j}}\left(\frac{R_{+}}{\mathrm{R}}\right)^{2}\left(\left\|\mathcal{R}_{1}\right\|_{\delta}+\left\|\mathcal{R}_{2}\right\|_{\delta}\right)+(2 \delta)^{2} K_{j}^{2} e^{2 K_{j} \delta} \mathrm{R}_{+}^{2} e^{-2 \mathrm{~m} \mathfrak{c}} \\
& \quad+\left(\left\|\mathcal{R}_{1}\right\|_{\delta}+64 \delta K_{j} e^{K_{j} \delta} \mathrm{r} \mathrm{R}_{+} e^{-\mathrm{m} \mathfrak{c}}\right)\left(\left\|\mathcal{R}_{2}\right\|_{\delta}+64 \delta K_{j} e^{K_{j} \delta} \mathrm{r} \mathrm{R}_{+} e^{-\mathrm{m} \mathfrak{c}}\right)
\end{aligned}
$$


The hypotheses on $\left\|\mathcal{R}_{1}\right\|_{\delta}$ and $\left\|\mathcal{R}_{2}\right\|_{\delta}$ imply that

$$
\begin{aligned}
& \|\mathcal{R}\|_{2 \delta} \leq 2 e^{\delta K_{j}}\left(\frac{R_{+}}{\mathrm{R}}\right)^{2} K_{R} \delta^{2} \mathrm{r}^{2} \mathrm{R}^{2} e^{-2 \mathrm{~m} \mathfrak{c}}+(2 \delta)^{2} K_{j}^{2} e^{2 K_{j} \delta} \mathrm{R}_{+}^{2} e^{-2 \mathrm{~m} \mathfrak{c}} \\
& +\left(K_{R} \delta^{2} \mathrm{r}^{2} \mathrm{R}^{2} e^{-2 \mathrm{~m} \mathfrak{c}}+64 \delta K_{j} e^{K_{j} \delta} \mathrm{r} \mathrm{R}_{+} e^{-\mathrm{m} \mathfrak{c}}\right)^{2} \\
& \leq K_{R}(2 \delta)^{2} \mathrm{r}_{+}^{2} \mathrm{R}_{+}^{2} e^{-2 \mathrm{~m} \mathfrak{c}}\left[\frac{e^{\delta K_{j}}}{2^{1-2 e_{\mathrm{r}}}}+\frac{K_{j}^{2} e^{2 K_{j} \delta}}{K_{R}}(2 \delta \mathfrak{v})^{2 e_{\mathrm{r}}}+\left(\frac{\sqrt{K_{R}}}{2^{1-e_{\mathrm{R}}-2 e_{\mathrm{r}}}} \frac{\delta e^{-\mathrm{m} \mathfrak{c}}}{(\delta \mathfrak{v})^{e^{2}+2 e_{\mathrm{r}}}}+\frac{2^{5+e_{\mathrm{r}}} K_{j} e^{K_{j} \delta}}{\sqrt{K_{R}}}\right)^{2}\right] \\
& \leq K_{R}(2 \delta)^{2} \mathrm{r}_{+}^{2} \mathrm{R}_{+}^{2} e^{-2 \mathrm{~m} \mathfrak{c}}
\end{aligned}
$$

by (F.8.a). Similarly

$$
\begin{aligned}
\|\mathcal{E}\|_{2 \delta} & \leq\left\|\mathcal{E}^{(1)}\right\|_{2 \delta}+\left\|\mathcal{E}^{(2)}\right\|_{2 \delta} \\
& \leq\left\|\mathcal{E}^{(1)}\right\|_{2 \delta}+\left\|\mathcal{E}^{\prime}\right\|_{2 \delta}+\left\|\mathcal{R}_{l}\right\|_{\text {fluct }}\left\|\mathcal{E}_{r}\right\|_{\text {fluct }}+\left\|\mathcal{E}_{l}\right\|_{\text {fluct }}\left\|\mathcal{R}_{r}\right\|_{\text {fluct }}+\left\|\mathcal{E}_{l}\right\|_{\text {fluct }}\left\|\mathcal{E}_{r}\right\|_{\text {fluct }}
\end{aligned}
$$

By (IV.5) and the hypotheses in Theorem III.24

$$
\begin{aligned}
\left\|\mathcal{E}^{(1)}\right\|_{2 \delta} & \leq 2 e^{2 \delta K_{j}}\left(\frac{R_{+}}{\mathrm{R}}\right)^{4} K_{E}(\delta \mathfrak{v})^{2} \mathrm{r}^{2} \mathrm{R}^{6}+64 K_{j} e^{8 K_{j} \delta} \delta^{2} \mathfrak{v} \mathrm{R}_{+}^{4} e^{-\mathrm{m} \mathfrak{c}} \\
& \leq(2 \delta \mathfrak{v})^{2} \mathrm{r}_{+}^{2} \mathrm{R}_{+}^{6}\left(K_{E} \frac{e^{2 \delta K_{j}}}{2^{1-\left(2 e_{\mathrm{R}}+4 e_{\mathrm{r}}\right)}}+\frac{16 K_{j} e^{8 K_{j} \delta} e^{-\mathrm{mc}}}{\mathrm{r}_{+}^{2} \mathrm{R}_{+}^{2} \mathfrak{v}}\right)
\end{aligned}
$$

By (IV.6), (IV.7) and the hypotheses of the Theorem

$$
\begin{aligned}
\left\|\mathcal{R}_{l}\right\|_{\text {fluct }},\left\|\mathcal{R}_{r}\right\|_{\text {fluct }} & \leq K_{R} \delta^{2} \mathrm{r}^{2} \mathrm{R}^{2} e^{-2 \mathrm{~m} \mathfrak{c}}+64 \delta K_{j} e^{K_{j} \delta} \mathrm{rR} \mathrm{R}_{+} e^{-\mathrm{m} \mathfrak{c}} \\
& \leq \delta \mathrm{r}_{+} \mathrm{R}_{+} e^{-\mathrm{m} \mathfrak{c}}\left(2^{e_{\mathrm{R}}+2 e_{\mathrm{r}}} K_{\mathrm{R}} \frac{\delta e^{-\mathrm{m} \mathfrak{c}}}{(\delta \mathfrak{v})^{e_{\mathrm{R}}+2 e_{\mathrm{r}}}}+64 K_{j} 2^{e_{\mathrm{r}}} e^{\delta K_{j}}\right) \\
& \leq \delta \mathrm{r}_{+} \mathrm{R}_{+} e^{-\frac{1}{2} \mathrm{~m} \mathfrak{c}} \\
\left\|\mathcal{E}_{l}\right\|_{\text {fluct }},\left\|\mathcal{E}_{r}\right\|_{\text {fluct }} & \leq K_{E}(\delta \mathfrak{v})^{2} \mathrm{r}^{2} \mathrm{R}^{6}+2^{11} \delta\|v\| \| \mathrm{r} \mathrm{R}_{+}^{3} \\
& \leq(2 \delta \mathfrak{v}) \mathrm{r}_{+} \mathrm{R}_{+}^{3}\left(K_{E} 2^{3 e_{\mathrm{R}}+4 e_{\mathrm{r}}-1}(\delta \mathfrak{v}) \mathrm{r} \mathrm{R}^{3}+2^{9} e^{e_{\mathrm{r}}}\right) \\
& \leq 2^{10}(2 \delta \mathfrak{v}) \mathrm{r}_{+} \mathrm{R}_{+}^{3}
\end{aligned}
$$

by (F.4.b), Hypothesis F.7.i,ii, (F.6.a) and the fact that $e_{\mathrm{r}} \leq$ 0.1. Consequently, by (F.4.b),

$$
\begin{aligned}
\|\mathcal{A}\|_{\text {fluct }} & =\left\|\mathcal{R}_{l}+\mathcal{R}_{r}+\mathcal{E}_{l}+\mathcal{E}_{r}\right\|_{\text {fluct }} \leq(2 \delta \mathfrak{v}) \mathrm{r}_{+} \mathrm{R}_{+}^{3}\left(\frac{e^{-\frac{1}{2} \mathrm{~m} \mathfrak{c}}}{\mathfrak{v} \mathrm{R}_{+}^{2}}+2^{11}\right) \\
& \leq 2^{12}(2 \delta \mathfrak{v}) \mathrm{r}_{+} \mathrm{R}_{+}^{3}
\end{aligned}
$$

By (F.6.a), this number is smaller than $\frac{1}{60}$. Therefore, by (IV.8),

$$
\left\|\mathcal{E}^{\prime}\right\|_{2 \delta} \leq(30)^{3}\|\mathcal{A}\|_{\text {fluct }}^{3} \leq 2^{51}(2 \delta \mathfrak{v})^{3} \mathrm{r}_{+}^{3} \mathrm{R}_{+}^{9}
$$

Inserting all these estimates into (IV.9) gives

$$
\begin{aligned}
\|\mathcal{E}\|_{2 \delta} & \leq(2 \delta \mathfrak{v})^{2} \mathrm{r}_{+}^{2} \mathrm{R}_{+}^{6}\left[K_{E} \frac{e^{2 \delta K_{j}}}{2^{1-\left(2 e_{\mathrm{R}}+4 e_{\mathrm{r}}\right)}}+\frac{16 K_{j} e^{8 K_{j} \delta} e^{-\mathrm{mc}}}{\mathrm{r}_{+}^{2} \mathrm{R}_{+}^{2} \mathfrak{v}}+2^{51}(2 \delta \mathfrak{v}) \mathrm{r}_{+} \mathrm{R}_{+}^{3}+\frac{2^{10} e^{-\frac{1}{2} \mathrm{~m} \mathfrak{c}}}{\mathfrak{v R}_{+}^{2}}+2^{20}\right] \\
& \leq K_{E}(2 \delta \mathfrak{v})^{2} \mathrm{r}_{+}^{2} \mathrm{R}_{+}^{6}
\end{aligned}
$$

by (F.8.b). 
The additional ingredient that we need for the proof of Theorem III.27 is

Lemma IV.5 Set

$$
W\left(\alpha_{*}, \beta\right)=V_{\delta}\left(\varepsilon ; \alpha_{*}, \beta\right)-V_{\delta}\left(\frac{\varepsilon}{2} ; \alpha_{*}, \beta\right)
$$

Then

$$
\begin{aligned}
\left\|W\left(\alpha_{*}, j(\delta) \beta\right)-W\left(\alpha_{*}, j_{\mathfrak{c}}(\delta) \beta\right)\right\|_{2 \delta} & \leq 2^{8} e^{10 K_{j}} \varepsilon \delta^{2}\|v\| \mathrm{R}_{+}^{4} e^{-\mathrm{m} \mathfrak{c}} \\
\left\|W\left(j(\delta) \alpha_{*}, \beta\right)-W\left(j_{\mathfrak{c}}(\delta) \alpha_{*}, \beta\right)\right\|_{2 \delta} & \leq 2^{8} e^{10 K_{j}} \varepsilon \delta^{2}\|v\| \mathrm{R}_{+}^{4} e^{-\mathrm{m} \mathfrak{c}}
\end{aligned}
$$

and

$$
\begin{aligned}
\left\|W\left(\alpha_{*}, z+j_{\mathfrak{c}}(\delta) \beta\right)-W\left(\alpha_{*}, j_{\mathfrak{c}}(\delta) \beta\right)\right\|_{\text {fluct }} & \leq 2^{12} e^{9 K_{j}} \varepsilon \delta\|v\| \mathrm{rR}_{+}^{3} \\
\left\|W\left(z_{*}+j_{\mathfrak{c}}(\delta) \alpha_{*}, \beta\right)-W\left(j_{\mathfrak{c}}(\delta) \alpha_{*}, \beta\right)\right\|_{\text {fluct }} & \leq 2^{12} e^{9 K_{j}} \varepsilon \delta\|v\| \mathrm{rR}_{+}^{3}
\end{aligned}
$$

Proof: We prove the first of each of the two pairs of inequalities and use the same notation as in Lemma IV.4. By definition

$$
W\left(\alpha_{*}, \beta\right)=W_{1}\left(\alpha_{*}, \beta\right)+W_{2}\left(\alpha_{*}, \beta\right)
$$

with

$$
\begin{aligned}
& W_{1}\left(\alpha_{*}, \beta\right)=\frac{\varepsilon}{2} \sum_{\tau \in \varepsilon \mathbb{Z} \cap[0, \delta)}\left[\left\langle\gamma_{* \tau} \gamma_{\tau+\frac{\varepsilon}{2}}, v \gamma_{* \tau} \gamma_{\tau+\frac{\varepsilon}{2}}\right\rangle-\left\langle\gamma_{* \tau} \gamma_{\tau+\varepsilon}, v \gamma_{* \tau} \gamma_{\tau+\varepsilon}\right\rangle\right] \\
& W_{2}\left(\alpha_{*}, \beta\right)=\frac{\varepsilon}{2} \sum_{\tau \in \varepsilon \mathbb{Z} \cap[0, \delta)}\left[\left\langle\gamma_{* \tau+\frac{\varepsilon}{2}} \gamma_{\tau+\varepsilon}, v \gamma_{* \tau+\frac{\varepsilon}{2}} \gamma_{\tau+\varepsilon}\right\rangle-\left\langle\gamma_{* \tau} \gamma_{\tau+\varepsilon}, v \gamma_{* \tau} \gamma_{\tau+\varepsilon}\right\rangle\right]
\end{aligned}
$$

Now, using the $g_{\tau}$ and $\tilde{g}_{\tau}$ of Lemma IV.4,

$$
\begin{aligned}
W_{1}\left(\alpha_{*}, j(\delta) \beta\right)- & W_{1}\left(\alpha_{*}, j_{\mathfrak{c}}(\delta) \beta\right) \\
=\frac{\varepsilon}{2} \sum_{\tau \in \varepsilon \mathbb{Z} \cap[0, \delta)} & {\left[\left\langle\gamma_{* \tau} g_{\tau+\frac{\varepsilon}{2}}, v \gamma_{* \tau} g_{\tau+\frac{\varepsilon}{2}}\right\rangle-\left\langle\gamma_{* \tau} g_{\tau+\varepsilon}, v \gamma_{* \tau} g_{\tau+\varepsilon}\right\rangle\right.} \\
& \left.\quad-\left\langle\gamma_{* \tau} \tilde{g}_{\tau+\frac{\varepsilon}{2}}, v \gamma_{* \tau} \tilde{g}_{\tau+\frac{\varepsilon}{2}}\right\rangle+\left\langle\gamma_{* \tau} \tilde{g}_{\tau+\varepsilon}, v \gamma_{* \tau} \tilde{g}_{\tau+\varepsilon}\right\rangle\right] \\
=\frac{\varepsilon}{2} \sum_{\tau \in \varepsilon \mathbb{Z} \cap[0, \delta)} & {\left[\left\langle\gamma_{* \tau} j\left(\frac{\varepsilon}{2}\right) g_{\tau+\varepsilon}, v \gamma_{* \tau} j\left(\frac{\varepsilon}{2}\right) g_{\tau+\varepsilon}\right\rangle-\left\langle\gamma_{* \tau} g_{\tau+\varepsilon}, v \gamma_{* \tau} g_{\tau+\varepsilon}\right\rangle\right.} \\
& \left.\quad-\left\langle\gamma_{* \tau} j\left(\frac{\varepsilon}{2}\right) \tilde{g}_{\tau+\varepsilon}, v \gamma_{* \tau} j\left(\frac{\varepsilon}{2}\right) \tilde{g}_{\tau+\varepsilon}\right\rangle+\left\langle\gamma_{* \tau} \tilde{g}_{\tau+\varepsilon}, v \gamma_{* \tau} \tilde{g}_{\tau+\varepsilon}\right\rangle\right]
\end{aligned}
$$

We apply Corollary G.3.iii, with the metrics $d$ and $d^{\prime}$ both replaced by $2 \mathrm{~m} d$, the metric $\delta=0$, and with the same substitutions as in the first bound on Lemma IV.4, namely,

$$
\begin{array}{llll}
\Gamma_{1}^{1}=\Gamma_{3}^{1}=j(\tau) & \Gamma_{1}^{2}=\Gamma_{3}^{2}=0 & \Gamma_{2}^{1}=\Gamma_{4}^{1}=0 & \Gamma_{2}^{2}=\Gamma_{4}^{2}=j(\delta-\tau-\varepsilon) j(\delta) \\
\tilde{\Gamma}_{1}^{1}=\tilde{\Gamma}_{3}^{1}=j(\tau) & \tilde{\Gamma}_{1}^{2}=\tilde{\Gamma}_{3}^{2}=0 & \tilde{\Gamma}_{2}^{1}=\tilde{\Gamma}_{4}^{1}=0 & \tilde{\Gamma}_{2}^{2}=\tilde{\Gamma}_{4}^{2}=j(\delta-\tau-\varepsilon) j_{\mathfrak{c}}(\delta)
\end{array}
$$


and, in addition,

$$
A_{1}=\tilde{A}_{1}=A_{3}=\tilde{A}_{3}=11 \quad A_{2}=A_{4}=j\left(\frac{\varepsilon}{2}\right) \quad \tilde{A}_{2}=\tilde{A}_{4}=\mathfrak{h}
$$

The Corollary bounds the $\|\cdot\|_{2 \delta}$ norm of the $\tau$ term in terms of the $\sigma$ and $\sigma_{\delta}$ of (IV.2) and

$$
\begin{aligned}
a & \leq \max \left\{\|11\|, \quad\left\|j\left(\frac{\varepsilon}{2}\right)\right\|\right\} \leq e^{K_{j} \frac{\varepsilon}{2}} \\
a_{\delta} & =N_{2 \mathrm{~m} d}\left(j\left(\frac{\varepsilon}{2}\right)-\mathfrak{h} ; 2 R_{+}, 2 \mathrm{R}_{+}\right) \leq\left\|j\left(\frac{\varepsilon}{2}\right)-\mathfrak{h}\right\| \| \leq \frac{\varepsilon}{2} K_{j} e^{K_{j} \frac{\varepsilon}{2}}
\end{aligned}
$$

by Lemma III.21. Summing over $\tau$ and multiplying by $\frac{\varepsilon}{2}$, we get

$$
\begin{aligned}
\left\|W_{1}\left(\alpha_{*}, j_{\mathfrak{c}}(\delta) \beta\right)-W_{1}\left(\alpha_{*}, j(\delta) \beta\right)\right\|_{2 \delta} & \leq \frac{\varepsilon}{2} \frac{\delta}{\varepsilon} 4^{2} 16\|v\| \mathrm{R}_{+}^{4} \sigma_{\delta} a_{\delta}(\sigma a)^{3} \\
& \leq 2^{7} e^{10 K_{j}} \varepsilon \delta^{2}\|v\| \mathrm{R}_{+}^{4} e^{-\mathrm{m} \mathfrak{c}}
\end{aligned}
$$

The same estimate holds for $\left\|W_{2}\left(\alpha_{*}, j_{\mathfrak{c}}(\delta) \beta\right)-W_{2}\left(\alpha_{*}, j(\delta) \beta\right)\right\|_{2 \delta}$.

For the first of the second pair of inequalities, write, using the $\hat{g}_{\tau}$ and $\tilde{g}_{\tau}$ of Lemma IV.4,

$$
\begin{aligned}
W_{1}\left(\alpha_{*}, z+j_{\mathfrak{c}}(\delta) \beta\right)- & W_{1}\left(\alpha_{*}, j_{\mathfrak{c}}(\delta) \beta\right) \\
=\frac{\varepsilon}{2} \sum_{\tau \in \varepsilon \mathbb{Z} \cap[0, \delta)} & {\left[\left\langle\gamma_{* \tau} \hat{g}_{\tau+\frac{\varepsilon}{2}}, v \gamma_{* \tau} \hat{g}_{\tau+\frac{\varepsilon}{2}}\right\rangle-\left\langle\gamma_{* \tau} \hat{g}_{\tau+\varepsilon}, v \gamma_{* \tau} \hat{g}_{\tau+\varepsilon}\right\rangle\right.} \\
& \left.\quad-\left\langle\gamma_{* \tau} \tilde{g}_{\tau+\frac{\varepsilon}{2}}, v \gamma_{* \tau} \tilde{g}_{\tau+\frac{\varepsilon}{2}}\right\rangle+\left\langle\gamma_{* \tau} \tilde{g}_{\tau+\varepsilon}, v \gamma_{* \tau} \tilde{g}_{\tau+\varepsilon}\right\rangle\right] \\
=\frac{\varepsilon}{2} \sum_{\tau \in \varepsilon \mathbb{Z} \cap[0, \delta)} & {\left[\left\langle\gamma_{* \tau} j\left(\frac{\varepsilon}{2}\right) \hat{g}_{\tau+\varepsilon}, v \gamma_{* \tau} j\left(\frac{\varepsilon}{2}\right) \hat{g}_{\tau+\varepsilon}\right\rangle-\left\langle\gamma_{* \tau} \hat{g}_{\tau+\varepsilon}, v \gamma_{* \tau} \hat{g}_{\tau+\varepsilon}\right\rangle\right.} \\
& \left.\quad\left\langle\gamma_{* \tau} j\left(\frac{\varepsilon}{2}\right) \tilde{g}_{\tau+\varepsilon}, v \gamma_{* \tau} j\left(\frac{\varepsilon}{2}\right) \tilde{g}_{\tau+\varepsilon}\right\rangle+\left\langle\gamma_{* \tau} \tilde{g}_{\tau+\varepsilon}, v \gamma_{* \tau} \tilde{g}_{\tau+\varepsilon}\right\rangle\right]
\end{aligned}
$$

This time, we apply Corollary G.3.iii using the $\hat{\Gamma}_{i}^{j}$ 's and $\hat{\tilde{\Gamma}}_{i}^{j}$ 's of (IV.3) together with the $A_{i}$ 's and $\tilde{A}_{i}$ 's of (IV.11). By (IV.4) and (IV.12)

$$
\begin{aligned}
\left\|W_{1}\left(\alpha_{*}, z+j_{\mathfrak{c}}(\delta) \beta\right)-W_{1}\left(\alpha_{*}, j_{\mathfrak{c}}(\delta) \beta\right)\right\|_{\text {fluct }} & \leq \frac{\varepsilon}{2} \frac{\delta}{\varepsilon} 4^{2} 16\|v\| \mathrm{R}_{+}^{4} \hat{\sigma}_{\delta} a_{\delta}(\hat{\sigma} a)^{3} \\
& \leq 2^{11} e^{9 K_{j}} \varepsilon \delta\|v\| \mathrm{rR}_{+}^{3}
\end{aligned}
$$

The same estimate holds for $\left\|W_{2}\left(\alpha_{*}, z+j_{\mathfrak{c}}(\delta) \beta\right)-W_{2}\left(\alpha_{*}, j_{\mathfrak{c}}(\delta) \beta\right)\right\|_{\text {fluct }}$.

Proof of Theorem III.27: We use the same notation as in the proof of Theorem III.24. Define $\tilde{\mathcal{R}}^{(1)}, \tilde{\mathcal{E}}^{(1)}, \tilde{\mathcal{R}}_{l}, \tilde{\mathcal{R}}_{r}, \tilde{\mathcal{E}}_{l}, \tilde{\mathcal{E}}_{r}$ and $\tilde{\mathcal{A}}$ in the same way as the corresponding "untilded" 
quantities were defined at the beginning of the proof of Theorem III.24. They fulfill the same bounds as their untilded siblings. Furthermore

$$
\begin{aligned}
& \left\|\tilde{\mathcal{R}}^{(1)}-\mathcal{R}^{(1)}\right\|_{2 \delta} \\
& \quad \leq\left\|\left(\tilde{\mathcal{R}}_{1}-\mathcal{R}_{1}\right)\left(\alpha_{*}, j_{\mathfrak{c}}(\delta) \beta\right)\right\|_{2 \delta}+\left\|\left(\tilde{\mathcal{R}}_{2}-\mathcal{R}_{2}\right)\left(j_{\mathfrak{c}}(\delta) \alpha_{*}, \beta\right)\right\|_{2 \delta} \\
& \quad \leq 2 e^{\delta K_{j}}\left(\frac{R_{+}}{\mathrm{R}}\right)^{2} \Delta_{\mathcal{R}} \\
& \left\|\tilde{\mathcal{E}}^{(1)}-\mathcal{E}^{(1)}\right\|_{2 \delta} \\
& \leq\left\|\left(\tilde{\mathcal{E}}_{1}-\mathcal{E}_{1}\right)\left(\alpha_{*}, j_{\mathfrak{c}}(\delta) \beta\right)\right\|_{2 \delta}+\left\|\left(\tilde{\mathcal{E}}_{2}-\mathcal{E}_{2}\right)\left(j_{\mathfrak{c}}(\delta) \alpha_{*}, \beta\right)\right\|_{2 \delta} \\
& \quad+\left\|W\left(\alpha_{*}, j_{\mathfrak{c}}(\delta) \beta\right)-W\left(\alpha_{*}, j(\delta) \beta\right)\right\|_{2 \delta}+\left\|W\left(j_{\mathfrak{c}}(\delta) \alpha_{*}, \beta\right)-W\left(j(\delta) \alpha_{*}, \beta\right)\right\|_{2 \delta} \\
& \leq 2 e^{2 \delta K_{j}}\left(\frac{R_{+}}{\mathrm{R}}\right)^{4} \Delta_{\mathcal{E}}+2^{9} e^{10 K_{j}} \varepsilon \delta^{2}\|v\| \mathrm{R}_{+}^{4} e^{-\mathrm{m} \mathfrak{c}}
\end{aligned}
$$

by Lemmas IV.1 and IV.5. Also

$$
\begin{aligned}
\left\|\tilde{\mathcal{R}}_{l}-\mathcal{R}_{l}\right\|_{\text {fluct }}= & \left\|\tilde{\mathcal{R}}_{1}\left(\alpha_{*}, z\right)-\mathcal{R}_{1}\left(\alpha_{*}, z\right)\right\|_{\text {fluct }} \\
= & \frac{\mathrm{R}_{+}}{\mathrm{R}} \frac{16 \mathrm{r}}{\mathrm{R}}\left\|\tilde{\mathcal{R}}_{1}\left(\alpha_{*}, \beta\right)-\mathcal{R}_{1}\left(\alpha_{*}, \beta\right)\right\|_{\delta} \\
\left\|\tilde{\mathcal{E}}_{l}-\mathcal{E}_{l}\right\|_{\text {fluct }} \leq & \left\|\left(\tilde{\mathcal{E}}_{1}-\mathcal{E}_{1}\right)\left(\alpha_{*}, z+j_{\mathfrak{c}}(\delta) \beta\right)-\left(\tilde{\mathcal{E}}_{1}-\mathcal{E}_{1}\right)\left(\alpha_{*}, j_{\mathfrak{c}}(\delta) \beta\right)\right\|_{\text {fluct }} \\
& \quad+\left\|W\left(\alpha_{*}, z+j_{\mathfrak{c}}(\delta) \beta\right)-W\left(\alpha_{*}, j(\delta) \beta\right)\right\|_{\text {fluct }} \\
\leq & \left\|\tilde{\mathcal{E}}_{1}-\mathcal{E}_{1}\right\|_{\delta}+2^{12} e^{9 K_{j}} \varepsilon \delta\|v\| \mathrm{rR}_{+}^{3}
\end{aligned}
$$

by Lemmas IV.2 and IV.5. Similarly

$$
\begin{aligned}
\left\|\tilde{\mathcal{R}}_{r}-\mathcal{R}_{r}\right\|_{\text {fluct }} & =\frac{\mathrm{R}_{+}}{\mathrm{R}} \frac{16 \mathrm{r}}{\mathrm{R}}\left\|\tilde{\mathcal{R}}_{2}-\mathcal{R}_{2}\right\|_{\delta} \\
\left\|\tilde{\mathcal{E}}_{r}-\mathcal{E}_{r}\right\|_{\text {fluct }} & \leq\left\|\tilde{\mathcal{E}}_{2}-\mathcal{E}_{2}\right\|_{\delta}+2^{12} e^{9 K_{j}} \varepsilon \delta\|v\| \mathrm{rR}_{+}^{3}
\end{aligned}
$$

Consequently

$$
\|\tilde{\mathcal{A}}-\mathcal{A}\|_{\text {fluct }} \leq \frac{32 \mathrm{r}}{\mathrm{R}} \Delta_{\mathcal{R}}+2 \Delta_{\mathcal{E}}+2^{13} e^{9 K_{j}} \varepsilon \delta\|v\| \| \mathrm{rR}_{+}^{3}
$$

For the fluctuation integral, we have

$$
\tilde{\mathcal{R}}^{(2)}-\mathcal{R}^{(2)}=\frac{1}{Z} \int d \mu_{\mathrm{r}}\left(z^{*}, z\right)\left[\left(\tilde{\mathcal{R}}_{l}-\mathcal{R}_{l}\right)\left(\alpha_{*}, z\right) \tilde{\mathcal{R}}_{r}\left(z^{*}, \beta\right)+\mathcal{R}_{l}\left(\alpha_{*}, z\right)\left(\tilde{\mathcal{R}}_{r}-\mathcal{R}_{r}\right)\left(z^{*}, \beta\right)\right]
$$

By [BFKT4, Remark III.3.ii] and (IV.6),

$$
\begin{aligned}
\left\|\tilde{\mathcal{R}}^{(2)}-\mathcal{R}^{(2)}\right\|_{2 \delta} & =\left\|\tilde{\mathcal{R}}^{(2)}-\mathcal{R}^{(2)}\right\|_{\text {fluct }} \\
& \leq\left\|\tilde{\mathcal{R}}_{l}-\mathcal{R}_{l}\right\|_{\text {fluct }}\left\|\tilde{\mathcal{R}}_{r}\right\|_{\text {fluct }}+\left\|\mathcal{R}_{l}\right\|_{\text {fluct }}\left\|\tilde{\mathcal{R}}_{r}-\mathcal{R}_{r}\right\|_{\text {fluct }} \\
& \leq\left(K_{R} \delta^{2} \mathrm{r}^{2} \mathrm{R}^{2} e^{-2 \mathrm{mc}}+64 \delta K_{j} e^{K_{j} \delta} \mathrm{r} \mathrm{R}_{+} e^{-\mathrm{m} \mathfrak{c}}\right) \frac{32 \mathrm{r}}{\mathrm{R}} \Delta_{\mathcal{R}} \\
& \leq 2^{12} e^{2 K_{j}} \delta \mathrm{r}_{+}^{2} e^{-\mathrm{m} \mathfrak{c}} \Delta_{\mathcal{R}}
\end{aligned}
$$


by (F.4.b) and (F.6.a). Furthermore

$$
\begin{gathered}
\left(\tilde{\mathcal{E}}^{(2)}-\mathcal{E}^{(2)}\right)\left(\alpha_{*}, \beta\right)=\log \frac{1}{Z} \int d \mu_{\mathrm{r}}\left(z^{*}, z\right) e^{\tilde{\mathcal{A}}\left(\alpha_{*}, \beta ; z^{*}, z\right)}-\log \frac{1}{Z} \int d \mu_{\mathrm{r}}\left(z^{*}, z\right) e^{\mathcal{A}\left(\alpha_{*}, \beta ; z^{*}, z\right)} \\
-\left(\tilde{\mathcal{R}}^{(2)}-\mathcal{R}^{(2)}\right)\left(\alpha_{*}, \beta\right)
\end{gathered}
$$

We apply [BFKT4, Corollary III.6] to the difference of the two logarithms of integrals. Since each monomial of $\mathcal{A}$ and $\tilde{A}$ contains either only $z$ 's or only $z^{*}$ 's, and hence integrates to zero, the first hypothesis of this corollary is satisfied. For the second hypothesis, we have, by (IV.10),

$$
\begin{aligned}
\|\mathcal{A}\|_{\text {fluct }}+\|\tilde{\mathcal{A}}-\mathcal{A}\|_{\text {fluct }} \leq & 2^{12}(2 \delta \mathfrak{v}) \mathrm{r}_{+} \mathrm{R}_{+}^{3}+\frac{16 \mathrm{r}}{\mathrm{R}}\left(\left\|\tilde{\mathcal{R}}_{1}-\mathcal{R}_{1}\right\|_{\delta}+\left\|\tilde{\mathcal{R}}_{2}-\mathcal{R}_{2}\right\|_{\delta}\right) \\
& +\left(\left\|\tilde{\mathcal{E}}_{1}-\mathcal{E}_{1}\right\|_{\delta}+\left\|\tilde{\mathcal{E}}_{2}-\mathcal{E}_{2}\right\|_{\delta}\right)+2^{13} e^{9 K_{j}} \varepsilon \delta\|v\| \mathrm{rR}_{+}^{3} \\
\leq & 2^{13}(2 \delta \mathfrak{v}) \mathrm{r}_{+} \mathrm{R}_{+}^{3}+\frac{16 \mathrm{r}}{\mathrm{R}}\left(\left\|\tilde{\mathcal{R}}_{1}\right\|_{\delta}+\left\|\mathcal{R}_{1}\right\|_{\delta}+\left\|\tilde{\mathcal{R}}_{2}\right\|_{\delta}+\left\|\mathcal{R}_{2}\right\|_{\delta}\right) \\
& +\left(\left\|\tilde{\mathcal{E}}_{1}\right\|_{\delta}+\left\|\mathcal{E}_{1}\right\|_{\delta}+\left\|\tilde{\mathcal{E}}_{2}\right\|_{\delta}+\left\|\mathcal{E}_{2}\right\|_{\delta}\right) \\
\leq & 2^{14}(2 \delta \mathfrak{v}) \mathrm{r}_{+} \mathrm{R}_{+}^{3} \leq \frac{1}{34}
\end{aligned}
$$

by Hypothesis F.7.i and (F.6.a). The corollary gives

$$
\begin{aligned}
&\left\|\tilde{\mathcal{E}}^{(2)}-\mathcal{E}^{(2)}\right\|_{2 \delta} \leq 4(34)^{2}\left(2^{14}(2 \delta \mathfrak{v}) \mathrm{r}_{+} \mathrm{R}_{+}^{3}\right)\left(\frac{32 \mathrm{r}}{\mathrm{R}} \Delta_{\mathcal{R}}+2 \Delta_{\mathcal{E}}+2^{13} e^{9 K_{j}} \varepsilon \delta\|v\| \mathrm{rR}_{+}^{3}\right)+\left\|\tilde{\mathcal{R}}^{(2)}-\mathcal{R}^{(2)}\right\|_{2 \delta} \\
& \leq 2^{33} e^{2 K_{j}}(2 \delta \mathfrak{v}) \mathrm{r}_{+}^{2} \mathrm{R}_{+}^{2} \Delta_{\mathcal{R}}+2^{28}(2 \delta \mathfrak{v}) \mathrm{r}_{+} \mathrm{R}_{+}^{3} \Delta_{\mathcal{E}}+2^{39} e^{9 K_{j}} \varepsilon(2 \delta \mathfrak{v})^{2} \mathrm{r}_{+}^{2} \mathrm{R}_{+}^{6}
\end{aligned}
$$

Combining the above bounds, we have

$$
\begin{aligned}
\|\tilde{\mathcal{R}}-\mathcal{R}\|_{2 \delta} & \leq\left\|\tilde{\mathcal{R}}^{(1)}-\mathcal{R}^{(1)}\right\|_{2 \delta}+\left\|\tilde{\mathcal{R}}^{(2)}-\mathcal{R}^{(2)}\right\|_{2 \delta} \\
& \leq\left(2 e^{\delta K_{j}}\left(\frac{R_{+}}{\mathrm{R}}\right)^{2}+2^{12} e^{2 K_{j}} \delta \mathrm{r}_{+}^{2} e^{-\mathrm{m} \mathfrak{c}}\right) \Delta_{\mathcal{R}} \\
& \leq 2 \Delta_{\mathcal{R}} \\
\|\tilde{\mathcal{E}}-\mathcal{E}\|_{2 \delta} & \leq\left\|\tilde{\mathcal{E}}^{(1)}-\mathcal{E}^{(1)}\right\|_{2 \delta}+\left\|\tilde{\mathcal{E}}^{(2)}-\mathcal{E}^{(2)}\right\|_{2 \delta} \\
& \leq\left(2 e^{2 \delta K_{j}}\left(\frac{R_{+}}{\mathrm{R}}\right)^{4}+2^{28}(2 \delta \mathfrak{v}) \mathrm{r}_{+} \mathrm{R}_{+}^{3}\right) \Delta_{\mathcal{E}}+2^{33} e^{2 K_{j}}(2 \delta \mathfrak{v}) \mathrm{r}_{+}^{2} \mathrm{R}_{+}^{2} \Delta_{\mathcal{R}} \\
& \quad+2^{9} e^{10 K_{j}} \varepsilon \delta^{2}\|v\| \mathrm{R}_{+}^{4} e^{-\mathrm{m} \mathfrak{c}}+2^{39} e^{9 K_{j}} \varepsilon(2 \delta \mathfrak{v})^{2} \mathrm{r}_{+}^{2} \mathrm{R}_{+}^{6} \\
& \leq \Delta_{\mathcal{E}}+2^{33} e^{2 K_{j}}(2 \delta \mathfrak{v}) \mathrm{r}_{+}^{2} \mathrm{R}_{+}^{2} \Delta_{\mathcal{R}}+2^{40} e^{10 K_{j}} \varepsilon(2 \delta \mathfrak{v})^{2} \mathrm{r}_{+}^{2} \mathrm{R}_{+}^{6}
\end{aligned}
$$

by (F.4.b), (F.6.a), Hypothesis F.7.i and (F.8.c). 


\section{The Decimation Step in all of Space}

The "decimation step" for all large and small field regions is formulated in Theorems III.26 and III.28. We shall prove them in this chapter. As in Chapter IV, write

$$
\begin{array}{ccc}
\mathrm{r}=\mathrm{r}(\delta) & \mathrm{R}=\mathrm{R}(\delta) & \mathrm{R}^{\prime}=\mathrm{R}^{\prime}(\delta) \\
\mathrm{r}_{+}=\mathrm{r}(2 \delta) & \mathrm{R}_{+}=\mathrm{R}(2 \delta) & \mathrm{R}_{+}^{\prime}=\mathrm{R}^{\prime}(2 \delta)
\end{array}
$$

Recall that, by (II.18),

$$
\frac{\mathrm{r}_{+}}{\mathrm{r}}=\frac{1}{2^{e_{\mathrm{r}}}} \quad \frac{\mathrm{R}_{+}}{\mathrm{R}}=\frac{1}{2^{e} \mathrm{R}^{+e_{\mathrm{r}}}} \quad \frac{\mathrm{R}_{+}^{\prime}}{\mathrm{R}^{\prime}}=\frac{1}{2^{e^{\prime}}+e_{\mathrm{r}}}
$$

The hierarchies $\mathfrak{S}_{1}$ and $\mathfrak{S}_{1}$ of Theorem III.26 each specify the large and small field sets for decimation intervals of length at most $\delta$. For a decimation interval $\mathcal{J}$ of length at most $\delta$ contained in $[0,2 \delta]$, we set, for $\mathcal{X}=\Lambda, \Omega, P_{\alpha}, P_{\beta}, P_{\alpha}^{\prime}, P_{\beta}^{\prime}, Q, R$

$$
\mathcal{X}(\mathcal{J})= \begin{cases}\mathcal{X}_{\mathfrak{S}_{1}}(\mathcal{J}) & \text { if } \mathcal{J} \subset[0, \delta] \\ \mathcal{X}_{\mathfrak{S}_{2}}(\mathcal{J}-\delta) & \text { if } \mathcal{J} \subset[\delta, 2 \delta]\end{cases}
$$

Then $\mathcal{X}(\mathcal{J})=\mathcal{X}_{\mathfrak{S}}(\mathcal{J})$ for all hierarchies $\mathfrak{S}$ of scale $2 \delta$ with $\left(\mathfrak{S}_{1}, \mathfrak{S}_{2}\right) \prec \mathfrak{S}$ and all $\mathcal{J}$ contained in either $[0, \delta]$ or $[\delta, 2 \delta]$.

We also set $\Omega_{0}=\Omega_{1} \cap \Omega_{2}$.

\section{V.1 The new small field/large field decomposition of the first kind}

By hypothesis, the integrand of the integral defining $I\left(\alpha^{*}, \beta\right)$ in Theorem III.26 contains the product $\chi_{\delta}\left(\Omega_{1} ; \alpha^{*}, \phi\right) \chi_{\delta}\left(\Omega_{2} ; \phi^{*}, \beta\right)$ of characteristic functions. These characteristic functions impose small field conditions for the decimation intervals $[0, \delta]$ and $[\delta, 2 \delta]$. For example, the first characteristic function contains a factor which vanishes unless $|\alpha(\mathbf{x})| \leq \mathrm{R}(\delta)$ for each $\mathbf{x} \in \Omega_{1}$. The representation for $I\left(\alpha^{*}, \beta\right)$ in the conclusion of Theorem III.26 contains the characteristic function $\chi_{2 \delta}\left(\Omega_{\mathfrak{S}} ; \alpha, \beta\right)$ which imposes small field conditions for the decimation interval $[0,2 \delta]$. For example, it vanishes unless $|\alpha(\mathbf{x})| \leq \mathrm{R}(2 \delta)$ for each $\mathbf{x} \in \Omega_{\mathfrak{S}}$.

The first step in the proof of Theorem III.26 builds $\chi_{2 \delta}\left(\Omega_{\mathfrak{S}} ; \alpha, \beta\right)$ from the product $\chi_{\delta}\left(\Omega_{1} ; \alpha^{*}, \phi\right) \chi_{\delta}\left(\Omega_{2} ; \phi^{*}, \beta\right)$. To illustrate the construction procedure, we consider the conditions on $|\alpha(\mathbf{x})|$. We expand the existing conditions on $|\alpha(\mathbf{x})|$, for $\mathbf{x} \in \Omega_{0}=\Omega_{1} \cap \Omega_{2}$, 
to

$$
\begin{aligned}
\chi_{\mathrm{R}(\delta)}\left(\Omega_{0}, \alpha\right) & =\prod_{\mathbf{x} \in \Omega_{0}} \chi_{\mathrm{R}(\delta)}(\alpha(\mathbf{x})) \\
& =\prod_{\mathbf{x} \in \Omega_{0}}\left[\chi_{\mathrm{R}(2 \delta)}(\alpha(\mathbf{x}))+\chi_{\mathrm{R}(2 \delta), \mathrm{R}(\delta)}(\alpha(\mathbf{x}))\right] \\
& =\sum_{P_{\alpha} \subset \Omega_{0}} \chi_{\mathrm{R}(2 \delta)}\left(\Omega_{0} \backslash P_{\alpha}, \alpha\right) \chi_{\mathrm{R}(2 \delta), \mathrm{R}(\delta)}\left(P_{\alpha}, \alpha\right)
\end{aligned}
$$

where we are using

Notation V.1 Set, for $0<r<R, t \in \mathbb{C}$, any set $Y$ and any complex valued function $f$ on $Y$

$$
\chi_{r}(t)=\left\{\begin{array}{ll}
1 & \text { if }|t| \leq r \\
0 & \text { otherwise }
\end{array}\right\} \quad \chi_{r, R}(t)=\left\{\begin{array}{ll}
1 & \text { if } r<|t| \leq R \\
0 & \text { otherwise }
\end{array}\right\}
$$

and

$$
\chi_{r}(Y, f)=\prod_{x \in Y} \chi_{r}(f(x)) \quad \chi_{r, R}(Y, f)=\prod_{x \in Y} \chi_{r, R}(f(x))
$$

The characteristic function $\chi_{\mathrm{R}(2 \delta)}\left(\Omega_{0} \backslash P_{\alpha}, \alpha\right)$ successfully imposes the new small field condition of scale $2 \delta$ on $|\alpha(\mathbf{x})|$ at each point of $\Omega_{0} \backslash P_{\alpha}$. The characteristic function $\chi_{\mathrm{R}(2 \delta), \mathrm{R}(\delta)}\left(P_{\alpha}, \alpha\right)$ says that $|\alpha(\mathbf{x})|$ violates the new small field condition at each $\mathbf{x} \in P_{\alpha}$. In Lemma A.3, we perform a similar expansion for the other small field conditions as well. The conclusion of that Lemma is

$$
\begin{aligned}
& \chi_{\delta}\left(\Omega_{1} ; \alpha, \phi\right) \chi_{\delta}\left(\Omega_{2} ; \phi, \beta\right) \\
& \quad=\sum_{\mathfrak{A} \in F_{\delta}\left(\Omega_{0}\right)} \chi_{2 \delta}\left(\Lambda_{\mathfrak{A}} ; \alpha, \beta\right) \chi_{\mathrm{r}}\left(\Lambda_{\mathfrak{A}}, \alpha-\phi\right) \chi_{\mathrm{r}}\left(\Lambda_{\mathfrak{A}}, \phi-\beta\right) \chi_{\mathfrak{A}, \delta}\left(\Omega_{1}, \Omega_{2} ; \alpha, \phi, \beta\right)
\end{aligned}
$$

where $F_{\delta}\left(\Omega_{0}\right)$ is the set of possible configurations of large field/small field sets for the decimation interval $[0,2 \delta]$ that are compatible with $\left(\mathfrak{S}_{1}, \mathfrak{S}_{2}\right)$ (see Definition III.2) and the associated characteristic function $\chi_{\mathfrak{A}, \delta}\left(\Omega_{1}, \Omega_{2} ; \alpha, \phi, \beta\right)$ is given in Definition A.1. Note, for future reference, that the factor $\chi_{\mathfrak{A}, \delta}\left(\Omega_{1}, \Omega_{2} ; \alpha, \phi, \beta\right)$ does not depend on the values of the fields $\alpha, \phi$ and $\beta$ at points of $\Lambda$.

Recall, from Definition III.1, the notation

$$
\vec{\alpha}_{l}=\left(\alpha_{\tau}(\mathbf{x})\right) \underset{\substack{\tau \in \varepsilon \mathbb{Z} \cap(0, \delta) \\ \mathbf{x} \in X}}{ } \quad \vec{\alpha}_{r}=\left(\alpha_{\tau+\delta}(\mathbf{x})\right)_{\substack{\tau \in \varepsilon \mathbb{Z} \cap(0, \delta) \\ \mathbf{x} \in X}}
$$

for a system $\vec{\alpha}=\left(\alpha_{\tau}(\mathbf{x})\right)_{\substack{\tau \in \varepsilon \mathbb{Z} \cap(0,2 \delta) \\ \mathbf{x} \in X}}$ of fields. 
As an immediate consequence of $(\mathrm{V} .2)$ we have that the $I\left(\alpha^{*}, \beta\right)$ of Theorem III.26 is

$$
\begin{gathered}
I\left(\alpha^{*}, \beta\right)=\sum_{\mathfrak{A} \in F_{\delta}\left(\Omega_{0}\right)} \mathcal{Z}_{\delta}^{2\left|\Lambda_{\mathfrak{A}}\right|} \int \prod_{\mathbf{x} \in \Lambda_{\mathfrak{A}}} \frac{d \phi(\mathbf{x})^{*} \wedge d \phi(\mathbf{x})}{2 \pi i} \operatorname{Int}_{\left(\mathfrak{A} ; \alpha^{*}, \beta\right)} e^{-\left\langle\phi^{*}, \phi\right\rangle} \\
\chi_{2 \delta}\left(\Lambda_{\mathfrak{A}} ; \alpha, \beta\right) \chi_{\mathrm{r}}\left(\Lambda_{\mathfrak{A}}, \alpha-\phi\right) \chi_{\mathrm{r}}\left(\Lambda_{\mathfrak{A}}, \phi-\beta\right) \\
e^{-Q_{\mathfrak{S}_{1}}\left(\alpha^{*}, \phi ; \vec{\alpha}_{l}^{*}, \vec{\alpha}_{l}\right)-Q_{\mathfrak{S}_{2}}\left(\phi^{*}, \beta ; \vec{\alpha}_{r}^{*}, \vec{\alpha}_{r}\right)} e^{\mathcal{V}_{\mathfrak{S}_{1}}\left(\varepsilon ; \alpha^{*}, \phi ; \vec{\alpha}_{l}^{*}, \vec{\alpha}_{l}\right)+\mathcal{V}_{\mathfrak{S}_{2}}\left(\varepsilon ; \phi^{*}, \beta ; \vec{\alpha}_{r}^{*}, \vec{\alpha}_{r}\right)} \\
e^{\mathcal{D}_{1}\left(\alpha^{*}, \phi ; \overrightarrow{\rho_{\uparrow l}}\right)+\mathcal{D}_{2}\left(\phi^{*}, \beta ; \overrightarrow{\rho_{\uparrow}}\right)} b_{1}\left(\alpha^{*}, \phi ; \overrightarrow{\rho_{\uparrow l}}\right) b_{2}\left(\phi^{*}, \beta ; \overrightarrow{\rho_{\uparrow r}}\right)
\end{gathered}
$$

where $\operatorname{Int}_{\left(\mathfrak{A} ; \alpha^{*}, \beta\right)}$ is the integral operator

$$
\begin{aligned}
& \operatorname{Int}_{\left(\mathfrak{A} ; \alpha^{*}, \beta\right)}\left[d \mu\left(\phi^{*}, \phi ; \vec{\alpha}, \vec{z}_{*}, \vec{z}\right)\right] \\
& =\mathcal{Z}_{\delta}^{\left|\Omega_{1} \backslash \Lambda_{\mathfrak{A}}\right|} \mathcal{Z}_{\delta}^{\left|\Omega_{2} \backslash \Lambda_{\mathfrak{A}}\right|} \int \prod_{\mathbf{x} \in X \backslash \Lambda_{\mathfrak{A}}} \frac{d \phi(\mathbf{x})^{*} \wedge d \phi(\mathbf{x})}{2 \pi i} \chi_{\mathrm{R}(\varepsilon)}(\phi(\mathbf{x})) \chi_{\mathfrak{A}, \delta}\left(\Omega_{1}, \Omega_{2} ; \alpha, \phi, \beta\right) \\
& \mathcal{I}_{\left(\mathfrak{S}_{1} ; \alpha^{*}, \phi\right)}\left[d \mu\left(\vec{\alpha}_{l}, \vec{z}_{* l}, \vec{z}_{l}\right)\right] \mathcal{I}_{\left(\mathfrak{S}_{2} ; \phi^{*}, \beta\right)}\left[d \mu\left(\vec{\alpha}_{r}, \vec{z}_{* r}, \vec{z}_{r}\right)\right] \\
& =\mathcal{Z}_{\delta}^{\left|\Omega_{1} \backslash \Lambda_{\mathfrak{A}}\right|} \mathcal{Z}_{\delta}^{\left|\Omega_{2} \backslash \Lambda_{\mathfrak{A}}\right|} \int \prod_{\mathbf{x} \in X \backslash \Lambda_{\mathfrak{A}}} \frac{d \phi(\mathbf{x})^{*} \wedge d \phi(\mathbf{x})}{2 \pi i} \chi_{\mathfrak{A}, \delta}\left(\Omega_{1}, \Omega_{2} ; \alpha, \phi, \beta\right) \\
& \mathcal{I}_{\left(\mathfrak{S}_{1} ; \alpha^{*}, \phi\right)}\left[d \mu\left(\vec{\alpha}_{l}, \vec{z}_{* l}, \vec{z}_{l}\right)\right] \mathcal{I}_{\left(\mathfrak{S}_{2} ; \phi^{*}, \beta\right)}\left[d \mu\left(\vec{\alpha}_{r}, \vec{z}_{* r}, \vec{z}_{r}\right)\right]
\end{aligned}
$$

by Lemma A.4.b. By Lemma III.10.ii, $\mathcal{I}_{\left(\mathfrak{S}_{1} ; \alpha^{*}, \phi\right)}$ and $\mathcal{I}_{\left(\mathfrak{S}_{2} ; \phi^{*}, \beta\right)}$ are independent of $\phi(\mathbf{x})$ for all $\mathbf{x} \in \Lambda_{\mathfrak{A}}$. Inspection of the definition of $\chi_{\mathfrak{A}, \delta}\left(\Omega_{1}, \Omega_{2} ; \alpha, \phi, \beta\right)$ in Definition A.1 shows that it is also independent of $\phi(\mathbf{x})$ for all $\mathbf{x} \in \Lambda_{\mathfrak{A}}$. By hypothesis, $b_{1}\left(\alpha_{*}, \phi ; \vec{\rho}_{l}\right)$ and $b_{2}\left(\phi_{*}, \beta ; \vec{\rho}_{r}\right)$ are pure large field and thus independent of $\phi_{*}(\mathbf{x}), \phi(\mathbf{x})$ for all $\mathbf{x} \in \Lambda_{\mathfrak{A}}$. Therefore we may move the integral $\int \prod_{\mathbf{x} \in \Lambda_{\mathfrak{A}}} \frac{d \phi(\mathbf{x})^{*} \wedge d \phi(\mathbf{x})}{2 \pi i}$ to the right to give

\section{Corollary V.2}

$$
\begin{gathered}
I\left(\alpha^{*}, \beta\right)=\sum_{\mathfrak{A} \in F_{\delta}\left(\Omega_{0}\right)} \operatorname{Int}_{\left(\mathfrak{A} ; \alpha^{*}, \beta\right)} b_{1}\left(\alpha^{*}, \phi ; \overrightarrow{\rho_{\imath l}}\right) b_{2}\left(\phi^{*}, \beta ; \overrightarrow{\rho_{\uparrow r}}\right) \chi_{2 \delta}\left(\Lambda_{\mathfrak{A}} ; \alpha, \beta\right) \\
\mathcal{Z}_{\delta}^{2\left|\Lambda_{\mathfrak{A}}\right|} \int \prod_{\mathbf{x} \in \Lambda_{\mathfrak{A}}} \frac{d \phi(\mathbf{x})^{*} \wedge d \phi(\mathbf{x})}{2 \pi i} e^{-\left\langle\phi^{*}, \phi\right\rangle} \chi_{\mathrm{r}}\left(\Lambda_{\mathfrak{A}}, \alpha-\phi\right) \chi_{\mathrm{r}}\left(\Lambda_{\mathfrak{A}}, \phi-\beta\right) \\
e^{-Q_{\mathfrak{S}_{1}}\left(\alpha^{*}, \phi ; \vec{\alpha}_{l}^{*}, \vec{\alpha}_{l}\right)-Q_{\mathfrak{S}_{2}}\left(\phi^{*}, \beta ; \vec{\alpha}_{r}^{*}, \vec{\alpha}_{r}\right)} e^{\mathcal{V}_{\mathfrak{S}_{1}}\left(\varepsilon ; \alpha^{*}, \phi ; \vec{\alpha}_{l}^{*}, \vec{\alpha}_{l}\right)+\mathcal{V}_{\mathfrak{S}_{2}}\left(\varepsilon ; \phi^{*}, \beta ; \vec{\alpha}_{r}^{*}, \vec{\alpha}_{r}\right)} \\
e^{\mathcal{D}_{1}\left(\alpha^{*}, \phi ; \overrightarrow{\rho_{l} l}\right)+\mathcal{D}_{2}\left(\phi^{*}, \beta ; \overrightarrow{\rho_{\uparrow}}\right)}
\end{gathered}
$$

Observe that the integrand above is independent of the variables $z_{* \delta}, z_{\delta}$ and $\alpha_{* \delta}, \alpha_{\delta}$. We will shortly introduce $z_{*}=z_{* \delta}$ and $z=z_{\delta}$ as the fluctuation fields associated to $\phi^{*}, \phi$, and $\alpha_{* \delta}, \alpha_{\delta}$ as the values of $\phi^{*}, \phi$ in the new large field region of the second kind where no fluctuation fields are introduced. 


\section{V.2 Approximate diagonalization of the quadratic form}

In this subsection, we fix $\mathfrak{A} \in F_{\delta}\left(\Omega_{0}\right)$ and study the small field integral, that is the integral over the variables $\phi(\mathbf{x}), \mathbf{x} \in \Lambda_{\mathfrak{A}}$, in the conclusion of Corollary V.2. To simplify notation, write $\Lambda=\Lambda_{\mathfrak{A}}$. So, we study the integral

$$
\begin{aligned}
& J_{\mathfrak{A}}\left(\alpha_{*}, \beta ; \vec{\rho}_{l}, \vec{\rho}_{r} ; \Lambda^{c} \phi^{*}, \Lambda^{c} \phi\right) \\
& =\int \prod_{\mathbf{x} \in \Lambda} \frac{d \phi(\mathbf{x})^{*} \wedge d \phi(\mathbf{x})}{2 \pi i} \chi_{\mathrm{r}}(\Lambda, \alpha-\phi) \chi_{\mathrm{r}}(\Lambda, \phi-\beta) e^{-Q_{\mathfrak{S}_{1}}\left(\alpha_{*}, \phi ; \vec{\alpha}_{* l}, \vec{\alpha}_{l}\right)-\left\langle\phi^{*}, \phi\right\rangle-Q_{\mathfrak{S}_{2}}\left(\phi^{*}, \beta ; \vec{\alpha}_{* r}, \vec{\alpha}_{r}\right)} \\
& e^{\mathcal{V}_{\mathfrak{S}_{1}}\left(\varepsilon ; \alpha_{*}, \phi ; \vec{\alpha}_{* l}, \vec{\alpha}_{l}\right)+\mathcal{V}_{\mathfrak{S}_{2}}\left(\varepsilon ; \phi^{*}, \beta ; \vec{\alpha}_{* r}, \vec{\alpha}_{r}\right)} e^{\mathcal{D}_{1}\left(\alpha_{*}, \phi ; \vec{\rho}_{l}\right)+\mathcal{D}_{2}\left(\phi^{*}, \beta ; \vec{\rho}_{r}\right)}
\end{aligned}
$$

To "compute" this integral, we use "stationary phase" in many complex variables as discussed in $\S \mathrm{I}$. As we saw there, if we treat $\phi^{*}$ and $\phi$ as independent variables, the critical value of $\phi^{*}$ for the quadratic part of the effective action is not the complex conjugate of the critical value of $\phi$. To produce a mathematically rigorous argument we introduce independent complex variables $\phi_{*}(\mathbf{x}), \phi(\mathbf{x})$ in $\mathbb{C}^{\Lambda}$ and write (V.3) as

$$
\begin{aligned}
J_{\mathfrak{A}}=\int_{D\left(\alpha_{*}, \beta\right)} \prod_{\mathbf{x} \in \Lambda} \frac{d \phi_{*}(\mathbf{x}) \wedge d \phi(\mathbf{x})}{2 \pi i} e^{-Q_{\mathfrak{S}_{1}}\left(\alpha_{*}, \phi ; \vec{\alpha}_{* l}, \vec{\alpha}_{l}\right)-\left\langle\phi_{*}, \phi\right\rangle-Q_{\mathfrak{S}_{2}}\left(\phi_{*}, \beta ; \vec{\alpha}_{* r}, \vec{\alpha}_{r}\right)} \\
e^{\mathcal{V}_{\mathfrak{S}_{1}}\left(\varepsilon ; \alpha_{*}, \phi ; \vec{\alpha}_{* l}, \vec{\alpha}_{l}\right)+\mathcal{V}_{\mathfrak{S}_{2}}\left(\varepsilon ; \phi_{*}, \beta ; \vec{\alpha}_{* r}, \vec{\alpha}_{r}\right)} e^{\mathcal{D}_{1}\left(\alpha_{*}, \phi ; \vec{\rho}_{l}\right)+\mathcal{D}_{2}\left(\phi_{*}, \beta ; \vec{\rho}_{r}\right)}
\end{aligned}
$$

where the domain of integration is $D\left(\alpha_{*}, \beta\right)=\mathbf{X}_{\mathbf{x} \in \Lambda} D\left(\mathbf{x} ; \alpha_{*}, \beta\right)$ with

$$
\begin{aligned}
& D\left(\mathbf{x} ; \alpha_{*}, \beta\right)=\left\{\left(\phi_{*}(\mathbf{x}), \phi(\mathbf{x})\right) \in \mathbb{C}^{2} \mid \phi_{*}^{*}(\mathbf{x})=\phi(\mathbf{x})\right. \\
& \left.\left|\phi_{*}(\mathbf{x})-\alpha_{*}(\mathbf{x})\right| \leq \mathrm{r},|\phi(\mathbf{x})-\beta(\mathbf{x})| \leq \mathrm{r}\right\}
\end{aligned}
$$

A good approximation to the critical point of the bilinear form

$$
Q_{\mathfrak{S}_{1}}\left(\alpha_{*}, \phi ; \vec{\alpha}_{* l}, \vec{\alpha}_{l}\right)+\left\langle\phi_{*}, \phi\right\rangle+Q_{\mathfrak{S}_{2}}\left(\phi_{*}, \beta ; \vec{\alpha}_{* r}, \vec{\alpha}_{r}\right)
$$

(in the variables $\phi_{*}, \phi$, with $\alpha_{*}, \vec{\alpha}_{*}, \vec{\alpha}, \beta$ considered as parameters) is ${ }^{(1)}$

$$
\phi_{*}^{\mathrm{cr}}=\Lambda j_{\mathfrak{c}}(\delta) \alpha_{*} \quad \phi^{\mathrm{cr}}=\Lambda j_{\mathfrak{c}}(\delta) \beta
$$

(1) If one approximated $Q_{\mathfrak{S}_{1}}\left(\alpha_{*}, \phi ; \cdot\right)$ and $Q_{\mathfrak{S}_{2}}\left(\phi_{*}, \beta ; \cdot\right)$ by their pure small field parts $-\left\langle\alpha_{*}, j(\delta) \phi\right\rangle$ and $-\left\langle\phi_{*}, j(\delta) \beta\right\rangle$, respectively (see Lemma III.7.ii), the critical point would be exactly $\phi_{*}^{\text {crit }}=$ $j(\delta) \alpha_{*}, \phi^{\text {crit }}=j(\delta) \beta$ as in (I.13). The cutoff $j_{\mathfrak{c}}(\delta)$ was motivated near (I.24). See also Lemma III.10.ii, which was used to give Corollary V.2. 
where the cut off propagator $j_{\mathfrak{c}}$ was defined in (I.24). We shall expand around the approximate critical point. Therefore we make a change of variables from $\phi_{*}(\mathbf{x}), \phi(\mathbf{x}), \mathbf{x} \in \Lambda$ to variables $z_{*}(\mathbf{x}), z(\mathbf{x})$ with $\mathbf{x} \in \Lambda$ by

$$
\phi_{*}(\mathbf{x})=z_{*}(x)+\left[j_{\mathfrak{c}}(\delta) \alpha_{*}\right](\mathbf{x}), \quad \phi(\mathbf{x})=z(x)+\left[j_{\mathfrak{c}}(\delta) \beta\right](\mathbf{x}) \quad \text { when } \mathbf{x} \in \Lambda
$$

Under this change of variables, we have, on $\Lambda$,

$$
\begin{aligned}
\phi_{*}^{*}-\phi & =z_{*}^{*}-z+j_{\mathfrak{c}}(\delta)\left[\alpha_{*}^{*}-\beta\right] \\
\phi_{*}-\alpha_{*} & =z_{*}-\left[1-j_{\mathfrak{c}}(\delta)\right] \alpha_{*} \\
\phi-\beta & =z-\left[1-j_{\mathfrak{c}}(\delta)\right] \beta
\end{aligned}
$$

Thus the change of variables transforms the domain $D\left(\mathbf{x} ; \alpha_{*}, \beta\right)$ of (V.5) into

$$
\begin{array}{r}
D^{\prime}\left(\mathbf{x} ; \alpha_{*}, \beta\right)=\left\{\left(z_{*}(\mathbf{x}), z(\mathbf{x})\right) \in \mathbb{C}^{2}|| z_{*}(\mathbf{x})-\left(\left[1-j_{\mathfrak{c}}(\delta)\right] \alpha_{*}\right)(\mathbf{x}) \mid \leq \mathrm{r},\right. \\
\left|z(\mathbf{x})-\left(\left[1-j_{\mathfrak{c}}(\delta)\right] \beta\right)(\mathbf{x})\right| \leq \mathrm{r}, \\
\left.z(\mathbf{x})-z_{*}(\mathbf{x})^{*}=\left(j_{\mathfrak{c}}(\delta)\left[\alpha_{*}^{*}-\beta\right]\right)(\mathbf{x})\right\}
\end{array}
$$

Observe that $D^{\prime}\left(\mathbf{x} ; \alpha_{*}, \beta\right)$ depends only on the values of the fields $\alpha_{*}$ and $\beta$ at points $\mathbf{y} \in X$ with $d(\mathbf{x}, \mathbf{y}) \leq \mathfrak{c}$. Also $D^{\prime}\left(\mathbf{x} ; \alpha_{*}, \beta\right)$ depends on $\mathfrak{h}$ through $j_{\mathfrak{c}}(\delta)$.

For convenience, we rename $\phi(\mathbf{x})=\alpha_{\delta}(\mathbf{x})$ and $\phi_{*}(\mathbf{x})=\alpha_{* \delta}(\mathbf{x})$ when $\mathbf{x} \in \Lambda^{c}$, and also define $z(\mathbf{x})=z_{*}(\mathbf{x})=0$ for all $\mathbf{x} \in \Lambda^{c}$ and $\alpha_{* \delta}(\mathbf{x})=\alpha_{\delta}(\mathbf{x})=0$ for $\mathbf{x} \in \Lambda$.

To this point, we have obtained the following expression for the function $J_{\mathfrak{A}}$ of (V.3)

Lemma V.3 Set $D^{\prime}\left(\alpha_{*}, \beta\right)=\mathbf{X}_{\mathbf{x} \in \Lambda} D^{\prime}\left(\mathbf{x} ; \alpha_{*}, \beta\right)$. Then

$$
J_{\mathfrak{A}}\left(\alpha_{*}, \beta ; \vec{\rho}_{l}, \vec{\rho}_{r}, \alpha_{* \delta}, \alpha_{\delta}\right)=\int_{D^{\prime}\left(\alpha_{*}, \beta\right)} \prod_{\mathbf{x} \in \Lambda}\left[\frac{d z_{*}(\mathbf{x}) \wedge d z(\mathbf{x})}{2 \pi i} e^{-z_{*}(\mathbf{x}) z(\mathbf{x})}\right] e^{f_{\mathfrak{A}}\left(\alpha_{*}, \beta ; \vec{\rho} ; z_{*}, z\right)}
$$

where

$$
\begin{aligned}
& f_{\mathfrak{A}}\left(\alpha_{*}, \beta ; \vec{\rho} ; z_{*}, z\right)=f_{\mathfrak{A}}\left(\alpha_{*}, \beta ; \vec{\alpha}_{*}, \vec{\alpha}, \vec{z}_{*}, \vec{z} ; z_{*}, z\right) \\
&=-\left\langle z_{*}, \Lambda j_{\mathfrak{c}}(\delta) \beta\right\rangle-\left\langle\Lambda j_{\mathfrak{c}}(\delta) \alpha_{*}, z\right\rangle-\left\langle\alpha_{* \delta}^{\mathrm{cr}}, \alpha_{\delta}^{\mathrm{cr}}\right\rangle \\
&-Q_{\mathfrak{S}_{1}}\left(\alpha_{*}, z+\alpha_{\delta}^{\mathrm{cr}} ; \vec{\alpha}_{l}^{*}, \vec{\alpha}_{l}\right)-Q_{\mathfrak{S}_{2}}\left(z_{*}+\alpha_{* \delta}^{\mathrm{cr}}, \beta ; \vec{\alpha}_{* r}, \vec{\alpha}_{r}\right) \\
&+\mathcal{V}_{\mathfrak{S}_{1}}\left(\varepsilon ; \alpha_{*}, z+\alpha_{\delta}^{\mathrm{cr}} ; \vec{\alpha}_{l}^{*}, \vec{\alpha}_{l}\right)+\mathcal{V}_{\mathfrak{S}_{2}}\left(\varepsilon ; z_{*}+\alpha_{* \delta}^{\mathrm{cr}}, \beta ; \vec{\alpha}_{* r}, \vec{\alpha}_{r}\right) \\
&+\mathcal{D}_{1}\left(\alpha_{*}, z+\alpha_{\delta}^{\mathrm{cr}} ; \vec{\rho}_{l}\right)+\mathcal{D}_{2}\left(z_{*}+\alpha_{* \delta}^{\mathrm{cr}}, \beta ; \vec{\rho}_{r}\right)
\end{aligned}
$$

and

$$
\alpha_{* \delta}^{\mathrm{cr}}=\alpha_{* \delta}^{\mathrm{cr}}\left(\alpha_{*}, \alpha_{* \delta}\right)=\Lambda j_{\mathfrak{c}}(\delta) \alpha_{*}+\Lambda^{c} \alpha_{* \delta} \quad \alpha_{\delta}^{\mathrm{cr}}=\alpha_{\delta}^{\mathrm{cr}}\left(\alpha_{\delta}, \beta\right)=\Lambda j_{\mathfrak{c}}(\delta) \beta+\Lambda^{c} \alpha_{\delta}
$$

By Lemma III.7.ii,

$$
\left.f_{\mathfrak{A}}\right|_{\mathfrak{h}=0}=-\sum_{\tau \in \varepsilon \mathbb{Z} \cap(0,2 \delta)}\left\langle\Lambda_{\tau}^{c} \alpha_{* \tau}, \Lambda_{\tau}^{c} \alpha_{\tau}\right\rangle
$$

Furthermore, $f_{\mathfrak{A}}+\sum_{\tau}\left\langle\Lambda_{\tau}^{c} \alpha_{* \tau}, \Lambda_{\tau}^{c} \alpha_{\tau}\right\rangle$ is history complete. 


\section{V.3 Stokes' theorem and the small field/large field decomposition of the second kind}

We apply [BFKT5, Lemma A.1] to the integral in Lemma V.3 with $X$ replaced by $\Lambda$, with $\rho(\mathbf{x})=\left(j_{\mathfrak{c}}(\delta)\left[\alpha_{*}^{*}-\beta\right]\right)(\mathbf{x}), \sigma_{*}(\mathbf{x})=\left(\left[1-j_{\mathfrak{c}}(\delta)\right] \alpha_{*}\right)(\mathbf{x}), \sigma(\mathbf{x})=\left(\left[1-j_{\mathfrak{c}}(\delta)\right] \beta\right)(\mathbf{x})$ and with $r=\mathrm{r}$. We block the resulting sum over $R$ into

$$
\sum_{R \subset \Lambda}=\sum_{\Omega \subset \Lambda} \sum_{\substack{R \subset \Lambda \\ \Omega=\Lambda \backslash L(\mathfrak{c}(\delta), R)}}
$$

where $L(\mathfrak{c}(\delta), R)$ is the set of points of $X$ that are within a distance $\mathfrak{c}(\delta)$ of $R$. This gives

$$
\begin{aligned}
J_{\mathfrak{A}}= & \sum_{\Omega \subset \Lambda} \sum_{\substack{R \subset \Lambda \\
\Omega=\Lambda \backslash L(\mathbf{c}(\delta), R)}} \prod_{\mathbf{x} \in R}\left(\int_{C\left(\mathbf{x} ; \alpha_{*}, \beta\right)} \frac{d z_{*}(\mathbf{x}) \wedge d z(\mathbf{x})}{2 \pi i} e^{-z_{*}(\mathbf{x}) z(\mathbf{x})}\right) \\
& \left.\prod_{\mathbf{x} \in \Lambda \backslash R}\left(\int_{|z(\mathbf{x})| \leq \mathrm{r}} \frac{d z(\mathbf{x})^{*} \wedge d z(\mathbf{x})}{2 \pi i} e^{-z(\mathbf{x})^{*} z(\mathbf{x})}\right) e^{f_{\mathfrak{A}}\left(\alpha_{*}, \beta ; \vec{\rho} ; z_{*}, z\right)}\right|_{\substack{z_{*}(\mathbf{x})=z(\mathbf{x})^{*} \\
\text { for } \mathbf{x} \in \Lambda \backslash \mathrm{R}}}
\end{aligned}
$$

with, for each $\mathbf{x} \in \Lambda, C\left(\mathbf{x} ; \alpha_{*}, \beta\right)$ a two real dimensional surface in

$$
\mathcal{P}(\mathbf{x})=\left\{\left(z_{*}(\mathbf{x}), z(\mathbf{x})\right) \in \mathbb{C}^{2}|| z_{*}(\mathbf{x})|,| z(\mathbf{x}) \mid<\mathrm{R}\right\}
$$

whose boundary is the union of the circle $\left\{\left(z_{*}(\mathbf{x}), z(\mathbf{x})\right) \in \mathbb{C}^{2}\left|z_{*}^{*}(\mathbf{x})=z(\mathbf{x}),\right| z(\mathbf{x}) \mid=\mathrm{r}\right\}$ and the curve bounding $D^{\prime}\left(\mathbf{x} ; \alpha_{*}, \beta\right)$.

We now verify the hypotheses of [BFKT5, Lemma A.1], for arguments that appear in the integral operator. For this, we must show that the function $f_{\mathfrak{A}}\left(\alpha^{*}, \beta ; \overrightarrow{\rho_{\uparrow}} ; z_{*}, z\right)$ is analytic in $\mathbf{X}_{\mathbf{x} \in \Lambda} \mathcal{P}(\mathbf{x})$, for all allowed $\left(\alpha^{*}, \beta ; \overrightarrow{\rho_{\uparrow}}\right)$, and that, for each $\mathbf{x} \in \Lambda$, the two boundary curves are contained in $\mathcal{P}(\mathbf{x})$.

○ As $\left\|\mathcal{D}_{i}\right\|_{\mathfrak{S}_{i}}, i=1,2$ are finite, $\mathbf{X}_{\mathbf{x} \in \Lambda} \mathcal{P}(\mathbf{x})$ will be contained in the domain of analyticity of $f_{\mathfrak{A}}\left(\alpha_{*}, \beta ; \vec{\rho} ; z_{*}, z\right)$ provided

$$
\left|z_{*}(\mathbf{x})+\alpha_{* \delta}^{\mathrm{cr}}(\mathbf{x})\right|<\kappa_{* \mathfrak{S}_{2}, 0}(\mathbf{x})=2 \mathrm{R} \quad\left|z(\mathbf{x})+\alpha_{\delta}^{\mathrm{cr}}(\mathbf{x})\right|<\kappa_{\mathfrak{S}_{1}, \delta}(\mathbf{x})=2 \mathrm{R}
$$

for all $\mathbf{x} \in \Lambda$ and $\left(z_{*}(\mathbf{x}), z(\mathbf{x})\right) \in \mathcal{P}(\mathbf{x})$. This is the case because

$$
\left|\alpha_{* \delta}^{\mathrm{cr}}(\mathbf{x})\right|=\left|\left(j_{\mathfrak{c}}(\delta) \alpha^{*}\right)(\mathbf{x})\right| \leq\left|\left\|j_{\mathfrak{c}}(\delta)\right\|\right| \sup _{\substack{\mathbf{y} \in X \\(\mathbf{x}, \mathbf{y}) \leq \mathfrak{c}}}|\alpha(\mathbf{y})| \leq\left\|j_{\mathfrak{c}}(\delta)\right\| \mathrm{R}_{+} \leq e^{K_{j} \delta} \mathrm{R}_{+}<\mathrm{R}
$$

The second inequality follows from the observation that $|\alpha(\mathbf{y})| \leq \mathrm{R}_{+}$for all $\mathbf{y}$ within a distance $\mathfrak{c}$ of $\Lambda$. When $\mathbf{y} \in \Lambda$, this is enforced by the characteristic function $\chi_{2 \delta}\left(\Lambda_{\mathfrak{A}} ; \alpha, \beta\right)$ in Corollary V.2. When $\mathbf{y}$ is not in $\Lambda$, but is within a distance $\mathfrak{c}$ of $\Lambda$, 
this is enforced by the factor of $\chi_{\mathrm{R}_{2 \delta}}\left(\Omega_{0} \backslash\left(P_{\alpha} \cup \Lambda\right), \alpha\right)$ in the characteristic function ${ }^{(2)}$ $\chi_{\mathfrak{A}, \delta}\left(\Omega_{1}, \Omega_{2} ; \alpha, \alpha_{\delta}, \beta\right)$ appearing in the definition of $\operatorname{Int}_{\mathfrak{A}, \varepsilon}$ in Corollary V.2. (Recall that $d\left(\Lambda, P_{\alpha} \cup \Omega_{0}^{c}\right)>\mathfrak{c}(\delta) \geq \mathfrak{c}$. The third inequality follows from Lemma III.21.i. The fourth is implied by Hypothesis F.7.i.

○ Since $\mathrm{r}<\mathrm{R}$, the boundary circle $\left\{z_{*}^{*}(\mathbf{x})=z(\mathbf{x}),|z(\mathbf{x})|=\mathrm{r}\right\} \subset \mathcal{P}(\mathbf{x})$.

○ The boundary of $D^{\prime}\left(\mathbf{x} ; \alpha^{*}, \beta\right)$ is contained in $\mathcal{P}(\mathbf{x})$ provided

$$
\left|\left(\left[1-j_{\mathfrak{c}}(\delta)\right] \alpha^{*}\right)(\mathbf{x})\right|+\mathrm{r},\left|\left(\left[11-j_{\mathfrak{c}}(\delta)\right] \beta\right)(\mathbf{x})\right|+\mathrm{r}<\mathrm{R}
$$

If $\mathfrak{h}(\mathbf{x})=0$, this condition reduces to $|\alpha(\mathbf{x})|+\mathrm{r}<\mathrm{R}$ and $|\beta(\mathbf{x})|+\mathrm{r}<\mathrm{R}$. Since $\mathbf{x} \in \Lambda$, both follow from $R_{+}+r<$ R. See (F.3.d) in Appendix F. If $\mathfrak{h}(\mathbf{x})=1$,

$$
\left|\left(\left[\mathbb{1}-j_{\mathfrak{c}}(\delta)\right] \alpha^{*}\right)(\mathbf{x})\right| \leq\left\|\left|\mathfrak{h}-j_{\mathfrak{c}}(\delta) \|\right| \sup _{\substack{\mathbf{y} \in X \\ d(\mathbf{x}, \mathbf{y}) \leq \mathfrak{c}}}\left|\alpha^{*}(\mathbf{y})\right| \leq \delta K_{j} e^{K_{j} \delta} \mathrm{R}_{+}<\mathrm{R}-\mathrm{r}\right.
$$

by Lemma III.21.ii, (F.3.d) and Hypothesis F.7.i.

Under Stokes' theorem we may choose any surface $C\left(\mathbf{x} ; \alpha^{*}, \beta\right)$ that has the specified boundary and lies in the domain of analyticity of the integrand. We choose $C\left(\mathbf{x} ; \alpha^{*}, \beta\right)$ to depend only on the values of the fields $\alpha$ and $\beta$ at points $\mathbf{y} \in X$ with $d(\mathbf{x}, \mathbf{y}) \leq \mathfrak{c}$. This is possible because $D^{\prime}\left(\mathbf{x} ; \alpha^{*}, \beta\right)$ has the same property.

Combining Corollary V.2, (V.3), and (V.7) gives

$$
\begin{aligned}
& I\left(\alpha^{*}, \beta\right)=\sum_{\mathfrak{A} \in F_{\delta}\left(\Omega_{0}\right)} \operatorname{Int}_{\left(\mathfrak{A} ; \alpha^{*}, \beta\right)} b_{1}\left(\alpha^{*}, \alpha_{\delta}, \overrightarrow{\rho_{l} l}\right) b_{2}\left(\alpha_{\delta}^{*}, \beta, \overrightarrow{\rho_{\uparrow r}}\right) \chi_{2 \delta}\left(\Lambda_{\mathfrak{A}} ; \alpha, \beta\right) \\
& \mathcal{Z}_{\delta}^{2\left|\Lambda_{\mathfrak{A}}\right|} \sum_{\Omega \subset \Lambda_{\mathfrak{A}}} \sum_{\substack{R \subset \Lambda_{\mathfrak{A}} \\
\Omega=\Lambda_{\mathfrak{A} \backslash} \backslash L(\mathbf{c}(\delta), R)}} \prod_{\mathbf{x} \in R}\left(\int_{C\left(\mathbf{x} ; \alpha^{*}, \beta\right)} \frac{d z_{*}(\mathbf{x}) \wedge d z(\mathbf{x})}{2 \pi i} e^{-z_{*}(\mathbf{x}) z(\mathbf{x})}\right) \\
& \left.\int d \mu_{\left(\Lambda_{\mathfrak{A l}} \backslash R\right), \mathrm{r}}\left(z^{*}, z\right) e^{f_{\mathfrak{P l}}\left(\alpha^{*}, \beta ; \overrightarrow{\rho_{\mathfrak{l}}} ; z_{*}, z\right)}\right|_{\substack{z_{*}(\mathbf{x})=z(\mathbf{x})^{*} \\
\text { for } \mathbf{x} \in \Lambda_{\mathfrak{P}} \backslash \mathbb{R}}}
\end{aligned}
$$

with, for each $\mathbf{x} \in X \backslash \Lambda_{\mathfrak{A}}$, the integration variable $\phi(\mathbf{x})$ of $\operatorname{Int}_{\left(\mathfrak{A} ; \alpha^{*}, \beta\right)}$ renamed to $\alpha_{\delta}(\mathbf{x})$. Renaming the fields $z_{*}(\mathbf{x}), z(\mathbf{x})$ with $\mathbf{x} \in \Lambda_{\mathfrak{A}} \backslash \Omega$ to $z_{* \delta}(\mathbf{x}), z_{\delta}(\mathbf{x})$,

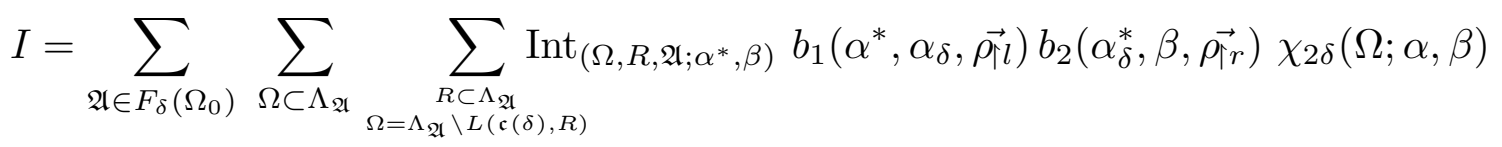

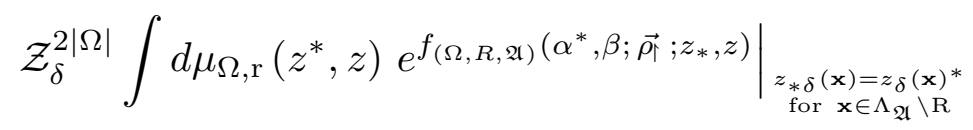

(2) The characteristic function $\chi_{\mathfrak{A}, \delta}\left(\Omega_{1}, \Omega_{2} ; \alpha, \alpha_{\delta}, \beta\right)$ is defined in Definition A.1. 
where

$$
\begin{aligned}
\operatorname{Int}_{\left(\Omega, R, \mathfrak{A} ; \alpha^{*}, \beta\right)}=\operatorname{Int}_{\left(\mathfrak{A} ; \alpha^{*}, \beta\right)} & \mathcal{Z}_{\delta}^{2\left|\Lambda_{\mathfrak{A}} \backslash \Omega\right|} \chi_{2 \delta}\left(\Lambda_{\mathfrak{A}} \backslash \Omega ; \alpha, \beta\right) \\
& \prod_{\mathbf{x} \in R}\left(\int_{C\left(\mathbf{x} ; \alpha^{*}, \beta\right)} \frac{d z_{* \delta}(\mathbf{x}) \wedge d z_{\delta}(\mathbf{x})}{2 \pi i} e^{-z_{* \delta}(\mathbf{x}) z_{\delta}(\mathbf{x})}\right) \int d \mu_{\Lambda_{\mathfrak{A}} \backslash(R \cup \Omega), \mathrm{r}}\left(z_{\delta}^{*}, z_{\delta}\right)
\end{aligned}
$$

and $f_{(\Omega, R, \mathfrak{A})}$ is obtained from the function $f_{\mathfrak{A}}$ of Lemma V.3 by

$$
f_{(\Omega, R, \mathfrak{A})}\left(\alpha_{*}, \beta ; \vec{\rho} ; z_{*}, z\right)=f_{\mathfrak{A}}\left(\alpha_{*}, \beta ; \vec{\rho} ;(\Lambda \backslash \Omega) z_{* \delta}+\Omega z_{*},(\Lambda \backslash \Omega) z_{\delta}+\Omega z\right)
$$

$z_{* \delta}(\mathbf{x}), z_{\delta}(\mathbf{x})$ with $\mathbf{x} \in \Lambda \backslash \Omega$ are also "residual variables" and subsumed in $\vec{\rho}$. The fields $z_{*}(\mathbf{x}), z(\mathbf{x})$ with $\mathbf{x} \in \Omega$ are the "fluctuation fields" to be integrated out.

For each $\Omega, R, \mathfrak{A}=\left(\Lambda, P_{\alpha}, \cdots, Q\right)$ in the above sum, we define the hierarchy $\mathfrak{S}$ with $\left(\mathfrak{S}_{1}, \mathfrak{S}_{2}\right)<\mathfrak{S}$ by setting $\mathcal{X}([0,2 \delta])=\mathcal{X}$ for $\mathcal{X}=\Omega, R, \Lambda, \cdots, Q$. Using the recursion relation of Lemma III.10.i, we have $\operatorname{Int}_{\left(\Omega, R, \mathfrak{A} ; \alpha^{*}, \beta\right)}=I_{\left(\mathfrak{S} ; \alpha^{*}, \beta\right)}$. We set $f_{\mathfrak{S}}=f_{(\Omega, R, \mathfrak{A})}$. Then

\section{Lemma V.4}

$$
\begin{aligned}
& I\left(\alpha^{*}, \beta\right)=\sum_{\substack{\text { hierarchies } \\
\text { for scale } \\
\left(\mathfrak{S}_{1}, \mathfrak{S}_{2}\right) \prec \mathfrak{S}}} I_{\left(\mathfrak{S} ; \alpha^{*}, \beta\right)} b_{1}\left(\alpha^{*}, \alpha_{\delta}, \overrightarrow{\rho_{\uparrow l}}\right) b_{2}\left(\alpha_{\delta}^{*}, \beta, \overrightarrow{\rho_{\uparrow r}}\right) \chi_{2 \delta}\left(\Omega_{\mathfrak{S}} ; \alpha, \beta\right) \\
& \left.\mathcal{Z}_{\delta}^{2\left|\Omega_{\mathfrak{S}}\right|} \int d \mu_{\Omega_{\mathfrak{S}}, \mathrm{r}}\left(z^{*}, z\right) e^{f_{\mathfrak{S}}\left(\alpha^{*}, \beta ; \overrightarrow{\rho_{\uparrow}} ; z^{*}, z\right)}\right|_{\substack{z_{* \delta}(\mathbf{x})=z_{\delta}(\mathbf{x}) * \\
\text { for } \mathbf{x} \in \Lambda_{\mathfrak{S}} \backslash \mathrm{R}}}
\end{aligned}
$$

where $f_{\mathfrak{S}}$ is given by (V.8).

Under the hypotheses of Theorem III.28, we have the analogous representation

$$
\begin{aligned}
& \tilde{I}\left(\alpha^{*}, \beta\right)=\sum_{\substack{\text { hierarchies } \\
\text { for scale } \\
\left(\mathfrak{S}_{1}, \mathfrak{S}_{2}\right) \prec \mathfrak{S}^{\delta}}} I_{\left(\mathfrak{S} ; \alpha^{*}, \beta\right)} \tilde{b}_{1}\left(\alpha^{*}, \alpha_{\delta}, \overrightarrow{\rho_{\uparrow l}}\right) \tilde{b}_{2}\left(\alpha_{\delta}^{*}, \beta, \overrightarrow{\rho_{\uparrow}}\right) \chi_{2 \delta}\left(\Omega_{\mathfrak{S}} ; \alpha, \beta\right) \\
& \left.\mathcal{Z}_{\delta}^{2\left|\Omega_{\mathfrak{S}}\right|} \int d \mu_{\Omega_{\mathfrak{S}}, \mathrm{r}}\left(z^{*}, z\right) e^{\tilde{f}_{\mathfrak{S}}\left(\alpha^{*}, \beta ; \overrightarrow{\rho_{\uparrow}} ; z^{*}, z\right)}\right|_{\substack{\begin{subarray}{c}{* \delta \\
\text { for } \mathbf{x} \in \Lambda_{\mathfrak{S}} \backslash \mathrm{R}} }} \\
{\mid}\end{subarray}}
\end{aligned}
$$

In Remark V.5, below, we give explicit descriptions of $f_{\mathfrak{S}}$ and $\tilde{f}_{\mathfrak{S}}$.

\section{V.4 Preparing for the analysis of the fluctuation integral}

Fix a hierarchy $\mathfrak{S}$ of scale $2 \delta$. If $\mathcal{J}$ is a decimation interval for $\mathfrak{S}$, write $\Omega(\mathcal{J})=\Omega_{\mathfrak{S}}(\mathcal{J})$ and $\Lambda(\mathcal{J})=\Lambda_{\mathfrak{S}}(\mathcal{J})$. Again, we use the notation $\Omega=\Omega_{\mathfrak{S}}([0,2 \delta]), \Lambda=\Lambda_{\mathfrak{S}}([0,2 \delta])$. We will sometimes also shorten $\Omega z_{*}+(\Lambda \backslash \Omega) z_{* \delta}$ to $z_{*}+z_{* \delta}$ and $\Omega z+(\Lambda \backslash \Omega) z_{\delta}$ to $z+z_{\delta}$. 
Remark V.5 The function $f_{\mathfrak{S}}\left(\alpha_{*}, \beta ; \vec{\rho} ; z_{*}, z\right)$ of (V.8) that appears in Lemma V.4 is the sum of

(i) the quadratic part

$$
\begin{aligned}
-\left\langle z_{*}+z_{* \delta}, \Lambda j_{\mathfrak{c}}(\delta) \beta\right\rangle-\left\langle\Lambda j_{\mathfrak{c}}(\delta) \alpha_{*}, z+z_{\delta}\right\rangle-\left\langle\alpha_{* \delta}^{\mathrm{cr}}, \alpha_{\delta}^{\mathrm{cr}}\right\rangle \\
-Q_{\mathfrak{S}_{1}}\left(\alpha_{*}, z+z_{\delta}+\alpha_{\delta}^{\mathrm{cr}} ; \vec{\alpha}_{*_{l}}, \vec{\alpha}_{l}\right)-Q_{\mathfrak{S}_{2}}\left(z_{*}+z_{* \delta}+\alpha_{* \delta}^{\mathrm{cr}}, \beta ; \vec{\alpha}_{*_{r}}, \vec{\alpha}_{r}\right)
\end{aligned}
$$

which, by the bilinearity of $Q$ and the observation that

$$
\begin{aligned}
\Gamma_{* \mathfrak{S}_{2}}\left(\cdot ; z_{*}+z_{* \delta}+\alpha_{* \delta}^{\mathrm{cr}}, \vec{\alpha}_{* r}\right) & =\Gamma_{* \mathfrak{S}_{2}}\left(\cdot ; z_{*}, 0\right)+\Gamma_{* \mathfrak{S}_{2}}\left(\cdot ; z_{* \delta}+\alpha_{* \delta}^{\mathrm{cr}}, \vec{\alpha}_{* r}\right) \\
\Gamma_{\mathfrak{S}_{1}}\left(\cdot ; \vec{\alpha}_{l}, z+z_{\delta}+\alpha_{\delta}^{\mathrm{cr}}\right) & =\Gamma_{\mathfrak{S}_{1}}(\cdot ; 0, z)+\Gamma_{\mathfrak{S}_{1}}\left(\cdot ; \vec{\alpha}_{l}, z_{\delta}+\alpha_{\delta}^{\mathrm{cr}}\right)
\end{aligned}
$$

can be written in the form

$$
\begin{aligned}
& -Q_{\mathfrak{S}}\left(\alpha_{*}, \beta ; \vec{\alpha}_{*}, \vec{\alpha}\right)+\delta Q\left(\alpha_{*}, \beta ; \vec{\rho}\right) \\
& \quad-\left[\left\langle z_{*}, \Omega j_{\mathfrak{c}}(\delta) \beta\right\rangle+Q_{\mathfrak{S}_{2}}\left(z_{*}, \beta ; 0, \vec{\alpha}_{r}\right)\right]-\left[\left\langle\Omega j_{\mathfrak{c}}(\delta) \alpha_{*}, z\right\rangle+Q_{\mathfrak{S}_{1}}\left(\alpha_{*}, z ; \vec{\alpha}_{*_{l}}, 0\right)\right]
\end{aligned}
$$

where

$$
\begin{aligned}
\delta Q\left(\alpha_{*}, \beta ; \vec{\rho}\right) & \\
= & Q_{\mathfrak{S}}\left(\alpha_{*}, \beta ; \vec{\alpha}_{*}, \vec{\alpha}\right)-Q_{\mathfrak{S}_{1}}\left(\alpha_{*}, z_{\delta}+\alpha_{\delta}^{\mathrm{cr}} ; \vec{\alpha}_{*_{l}}, \vec{\alpha}_{l}\right)-Q_{\mathfrak{S}_{2}}\left(z_{* \delta}+\alpha_{* \delta}^{\mathrm{cr}}, \beta ; \vec{\alpha}_{*_{r}}, \vec{\alpha}_{r}\right) \\
-\left\langle\alpha_{* \delta}^{\mathrm{cr}}, \alpha_{\delta}^{\mathrm{cr}}\right\rangle-\left\langle z_{* \delta},(\Lambda \backslash \Omega) j_{\mathfrak{c}}(\delta) \beta\right\rangle-\left\langle(\Lambda \backslash \Omega) j_{\mathfrak{c}}(\delta) \alpha_{*}, z_{\delta}\right\rangle & \\
= & {\left[Q_{\mathfrak{S}}\left(\alpha_{*}, \beta ; \vec{\alpha}_{*}, \vec{\alpha}\right)-Q_{\mathfrak{S}_{1}}\left(\alpha_{*}, \alpha_{\delta}^{\mathrm{cr}} ; \vec{\alpha}_{*_{l}}, \vec{\alpha}_{l}\right)-Q_{\mathfrak{S}_{2}}\left(\alpha_{* \delta}^{\mathrm{cr}}, \beta ; \vec{\alpha}_{*_{r}}, \vec{\alpha}_{r}\right)-\left\langle\alpha_{* \delta}^{\mathrm{cr}}, \alpha_{\delta}^{\mathrm{cr}}\right\rangle\right] } \\
& \quad-\left[\left\langle z_{* \delta},(\Lambda \backslash \Omega) j_{\mathfrak{c}}(\delta) \beta\right\rangle+Q_{\mathfrak{S}_{2}}\left(z_{* \delta}, \beta ; 0, \vec{\alpha}_{r}\right)\right] \\
& -\left[\left\langle(\Lambda \backslash \Omega) j_{\mathfrak{c}}(\delta) \alpha_{*}, z_{\delta}\right\rangle+Q_{\mathfrak{S}_{1}}\left(\alpha_{*}, z_{\delta} ; \vec{\alpha}_{*_{l}}, 0\right)\right]
\end{aligned}
$$

(ii) the quartic part $\mathcal{V}_{\mathfrak{S}_{1}}\left(\varepsilon ; \alpha_{*}, z+z_{\delta}+\alpha_{\delta}^{\mathrm{cr}} ; \vec{\alpha}_{*_{l}}, \vec{\alpha}_{l}\right)+\mathcal{V}_{\mathfrak{S}_{2}}\left(\varepsilon ; z_{*}+z_{* \delta}+\alpha_{* \delta}^{\mathrm{cr}}, \beta ; \vec{\alpha}_{*_{r}}, \vec{\alpha}_{r}\right)$ and

(iii) $\mathcal{D}_{1}\left(\alpha_{*}, z+z_{\delta}+\alpha_{\delta}^{\mathrm{cr}} ; \vec{\rho}_{l}\right)+\mathcal{D}_{2}\left(z_{*}+z_{* \delta}+\alpha_{* \delta}^{\mathrm{cr}}, \beta ; \vec{\rho}_{r}\right)$ from the non large field terms.

\section{Remark V.6}

$$
\begin{aligned}
\left.f_{\mathfrak{S}}\right|_{\Omega}=\langle & \left.\alpha_{*}, j(2 \delta) \beta\right\rangle\left.\right|_{\Omega}-\left.\left\langle\left[j(\delta)-j_{\mathfrak{c}}(\delta)\right] \alpha_{*},\left[j(\delta)-j_{\mathfrak{c}}(\delta)\right] \beta\right\rangle\right|_{\Omega} \\
& +\left.\left\langle z_{*},\left[j(\delta)-j_{\mathfrak{c}}(\delta)\right] \beta\right\rangle\right|_{\Omega}+\left.\left\langle\left[j(\delta)-j_{\mathfrak{c}}(\delta)\right] \alpha_{*}, z\right\rangle\right|_{\Omega}-\sum_{\tau \in \varepsilon \mathbb{Z} \cap(0,2 \delta)}\left\langle\Lambda_{\tau}^{c} \alpha_{* \tau}, \Lambda_{\tau}^{c} \alpha_{\tau}\right\rangle \\
& +\left.\left[V_{\Omega, \delta}\left(\varepsilon ; \alpha_{*}, z+j_{\mathfrak{c}}(\delta) \beta\right)+V_{\Omega, \delta}\left(\varepsilon ; z_{*}+j_{\mathfrak{c}}(\delta) \alpha_{*}, \beta\right)\right]\right|_{\Omega} \\
& +\left.\left[\mathcal{D}_{1}\left(\alpha_{*}, z+j_{\mathfrak{c}}(\delta) \beta ; 0\right)+\mathcal{D}_{2}\left(z_{*}+j_{\mathfrak{c}}(\delta) \alpha_{*}, \beta ; 0\right)\right]\right|_{\Omega}
\end{aligned}
$$


Proof: By its definition in Lemma V.3

$$
\left.\alpha_{* \delta}^{\mathrm{cr}}\right|_{\Omega}=\left.j_{\mathfrak{c}}(\delta) \alpha_{*}\right|_{\Omega}+\left.\Lambda^{c} \alpha_{* \delta} \quad \alpha_{\delta}^{\mathrm{cr}}\right|_{\Omega}=\left.j_{\mathfrak{c}}(\delta) \beta\right|_{\Omega}+\Lambda^{c} \alpha_{\delta}
$$

By Lemma III.7.ii, the quadratic part of $\left.f_{\mathfrak{S}}\right|_{\Omega}$ is equal to

$$
\begin{aligned}
& \quad-\left.\left\langle z_{*}, j_{\mathfrak{c}}(\delta) \beta\right\rangle\right|_{\Omega}-\left.\left\langle j_{\mathfrak{c}}(\delta) \alpha_{*}, z\right\rangle\right|_{\Omega}-\left.\left\langle j_{\mathfrak{c}}(\delta) \alpha_{*}, j_{\mathfrak{c}}(\delta) \beta\right\rangle\right|_{\Omega}-\left\langle\Lambda^{c} \alpha_{* \delta}, \Lambda^{c} \alpha_{\delta}\right\rangle \\
& +\left.\left\langle\alpha_{*}, j(\delta)\left(z+j_{\mathfrak{c}}(\delta) \beta\right)\right\rangle\right|_{\Omega}-\sum_{\tau \in(0, \delta)}\left\langle\Lambda_{\tau}^{c} \alpha_{* \tau}, \Lambda_{\tau}^{c} \alpha_{\tau}\right\rangle \\
& \left.+\left\langle z_{*}+j_{\mathfrak{c}}(\delta) \alpha_{*}, j(\delta) \beta\right)\right\rangle\left.\right|_{\Omega}-\sum_{\tau \in(\delta, 2 \delta)}\left\langle\Lambda_{\tau}^{c} \alpha_{* \tau}, \Lambda_{\tau}^{c} \alpha_{\tau}\right\rangle \\
& =\left.\left\langle\alpha_{*}, j(2 \delta) \beta\right\rangle\right|_{\Omega}-\left.\left\langle\left[j(\delta)-j_{\mathfrak{c}}(\delta)\right] \alpha_{*},\left[j(\delta)-j_{\mathfrak{c}}(\delta)\right] \beta\right\rangle\right|_{\Omega} \\
& \quad+\left.\left\langle z_{*},\left[j(\delta)-j_{\mathfrak{c}}(\delta)\right] \beta\right\rangle\right|_{\Omega}+\left.\left\langle\left[j(\delta)-j_{\mathfrak{c}}(\delta)\right] \alpha_{*}, z\right\rangle\right|_{\Omega}-\sum_{\tau \in(0,2 \delta)}\left\langle\Lambda_{\tau}^{c} \alpha_{* \tau}, \Lambda_{\tau}^{c} \alpha_{\tau}\right\rangle
\end{aligned}
$$

By Remark III.9.i and (V.11), the restriction of the explicit quartic part in Remark V.5.ii is $\left.\left[V_{\Omega, \delta}\left(\varepsilon ; \alpha_{*}, z+j_{\mathfrak{c}}(\delta) \beta\right)+\mathcal{V}_{\Omega, \delta}\left(\varepsilon ; z_{*}+j_{\mathfrak{c}}(\delta) \alpha_{*}, \beta\right)\right]\right|_{\Omega}$, and the restriction of the term in Remark V.5.iii is obvious.

This enables us to control the small field part in Lemma V.4.

\section{Corollary V.7}

$$
\left.\int d \mu_{\Omega, \mathrm{r}}\left(z^{*}, z\right) e^{f_{\mathfrak{S}}\left(\alpha_{*}, \beta ; \vec{\rho} ; z^{*}, z\right)}\right|_{\Omega}=e^{\left.\left\langle\alpha_{*}, j(2 \delta) \beta\right\rangle\right|_{\Omega}+V_{\Omega, 2 \delta}\left(\varepsilon ; \alpha_{*}, \beta\right)} e^{D\left(\alpha_{*}, \beta\right)} \prod_{\tau \in(0,2 \delta)} e^{-\left\langle\Lambda_{\tau}^{c} \alpha_{* \tau}, \Lambda_{\tau}^{c} \alpha_{\tau}\right\rangle}
$$

where

$$
D=\overline{\mathfrak{R}}_{\Omega ; \delta}\left(V_{\Omega, \delta}(\varepsilon ; \cdot) ;\left.\mathcal{D}_{1}(\cdot ; 0)\right|_{\Omega},\left.\mathcal{D}_{2}(\cdot ; 0)\right|_{\Omega}\right)
$$

Proof: By Remark V.6 and Definition III.22,

$$
\begin{aligned}
& \left.\int d \mu_{\Omega, \mathrm{r}}\left(z^{*}, z\right) e^{f_{\mathfrak{S}}\left(\alpha_{*}, \beta ; \vec{\rho} ; z^{*}, z\right)}\right|_{\Omega} \\
& \quad=e^{\left.\left\langle\alpha_{*}, j(2 \delta) \beta\right\rangle\right|_{\Omega}+V_{\Omega, \delta}\left(\varepsilon ; \alpha_{*}, j(\delta) \beta\right)+V_{\Omega, \delta}\left(\varepsilon ; j(\delta) \alpha_{*}, \beta\right)} e^{D\left(\alpha_{*}, \beta\right)} \prod_{\tau \in(0,2 \delta)} e^{-\left\langle\Lambda_{\tau}^{c} \alpha_{* \tau}, \Lambda_{\tau}^{c} \alpha_{\tau}\right\rangle}
\end{aligned}
$$

Now apply Remark III.9.ii. 
From the contributions in Remark V.5, we shall split off the part that is independent of the fluctuation fields $z_{*}, z$. The remaining parts, that truly contain fluctuation fields, will be integrated out. Proposition V.14 below gives the decomposition just mentioned and thus prepares for the fluctuation integral. Lemmas V.10 and V.11 and Propositions V.12 and V.13 below are used in the proof of Proposition V.14.

To estimate the fluctuation integral we shall apply Theorem III.14 with the weight system of the following definition.

Definition V.8 (Fluctuation integral weight systems) Let $w_{\text {fluct }}$ be the weight system with core $\Omega$ that associates

$\circ$ the weight factors $\left(\kappa_{* \tau}\right)_{\tau \in[0,2 \delta)}$ to the fields $\alpha_{*}, \vec{\alpha}_{*}$, the weight factors $\left(\kappa_{\tau}\right)_{\tau \in(0,2 \delta]}$ to the fields $\vec{\alpha}, \beta$,

$\circ$ the weight factors $\lambda_{\tau}$ to the fields $z_{* \tau}$ and $z_{\tau}$ with $\tau \in(0,2 \delta)$, and

$\circ$ to the fluctuation fields $z_{*}, z$ the weight factor

$$
\tilde{\lambda}(\mathbf{x})= \begin{cases}32 \mathrm{r} & \text { if } \mathbf{x} \in \Omega \\ \infty & \text { otherwise }\end{cases}
$$

$\circ$ and the constant weight factor 1 to the history field $\mathfrak{h}$.

We write $\|f\|_{\text {fluct }}$ instead of $\|f\|_{w_{\text {fluct }}}$.

Remark V.9 $w_{\text {fluct }}$ extends the weight system $w_{\mathfrak{S}}$ of Definition III.15.i by weight factors for the fluctuation fields. Thus, for a function $h$ that is independent of the fluctuation fields, $\|h\|_{\text {fluct }}=\|h\|_{\mathfrak{S}}$. Also, it is an extension of the weight system $w_{\text {fluct }}$ introduced just before Lemma IV.2.

The following Lemma shall be applied with $g_{i}=\mathcal{D}_{i}$ or $g_{i}=\mathcal{D}_{i}-\tilde{\mathcal{D}}_{i}$.

Lemma V.10 Let $g_{1}\left(\alpha_{*}, \beta ; \vec{\rho}_{l}\right)$ and $g_{2}\left(\alpha_{*}, \beta ; \vec{\rho}_{r}\right)$ be history complete analytic functions. Then

$$
\begin{gathered}
\left\|\left.g_{1}\left(\alpha_{*}, z_{\delta}+\alpha_{\delta}^{\mathrm{cr}} ; \vec{\rho}_{l}\right)\right|_{\Omega^{c}}\right\|_{\mathfrak{S}} \leq 2^{8}\left\|g_{1}\right\|_{\mathfrak{S}_{1}} \\
\left\|\left.g_{2}\left(z_{* \delta}+\alpha_{* \delta}^{\mathrm{cr}}, \beta ; \vec{\rho}_{r}\right)\right|_{\Omega^{c}}\right\|_{\mathfrak{S}} \leq 2^{8}\left\|g_{2}\right\|_{\mathfrak{S}_{2}}
\end{gathered}
$$

and

$$
\begin{array}{r}
\left\|g_{1}\left(\alpha_{*}, z_{\delta}+\alpha_{\delta}^{\mathrm{cr}} ; \vec{\rho}_{l}\right)-\left.g_{1}\left(\alpha_{*}, z_{\delta}+\alpha_{\delta}^{\mathrm{cr}} ; \vec{\rho}_{l}\right)\right|_{\Omega^{c}}\right\|_{\mathfrak{S}} \leq 2^{8}\left(e^{-\mathrm{mc}(\delta)}\left\|g_{1}\right\| \mathfrak{S}_{1}+\left\|\left.g_{1}\right|_{\Omega_{1}}\right\|_{\mathfrak{S}_{1}}\right) \\
\left\|g_{2}\left(z_{* \delta}+\alpha_{* \delta}^{\mathrm{cr}}, \beta ; \vec{\rho}_{r}\right)-\left.g_{2}\left(z_{* \delta}+\alpha_{* \delta}^{\mathrm{cr}}, \beta ; \vec{\rho}_{r}\right)\right|_{\Omega^{c}}\right\|_{\mathfrak{S}} \leq 2^{8}\left(e^{-\mathrm{mc}(\delta)}\left\|g_{2}\right\| \mathfrak{S}_{2}+\left\|\left.g_{2}\right|_{\Omega_{2}}\right\|_{\mathfrak{S}_{2}}\right)
\end{array}
$$


Also

$$
\begin{aligned}
& \left\|g_{1}\left(\alpha_{*}, z+z_{\delta}+\alpha_{\delta}^{\mathrm{cr}} ; \vec{\rho}_{l}\right)-g_{1}\left(\alpha_{*}, z_{\delta}+\alpha_{\delta}^{\mathrm{cr}} ; \vec{\rho}_{l}\right)\right\|_{\text {fluct }} \leq 2^{8}\left(e^{-\mathrm{mc}(\delta)}\left\|g_{1}\right\| \mathfrak{S}_{1}+\left\|\left.g_{1}\right|_{\Omega_{1}}\right\|_{\mathfrak{S}_{1}}\right) \\
& \left\|g_{2}\left(z_{*}+z_{* \delta}+\alpha_{* \delta}^{\mathrm{cr}}, \beta ; \vec{\rho}_{r}\right)-g_{2}\left(z_{* \delta}+\alpha_{* \delta}^{\mathrm{cr}}, \beta ; \vec{\rho}_{r}\right)\right\|_{\text {fluct }} \leq 2^{8}\left(e^{-\mathrm{mc}(\delta)}\left\|g_{2}\right\|_{\mathfrak{S}_{2}}+\left\|\left.g_{2}\right|_{\Omega_{2}}\right\|_{\mathfrak{S}_{2}}\right)
\end{aligned}
$$

Proof: We estimate the $g_{2}$-terms. Observe that $g_{2}\left(z_{*}+z_{* \delta}+\alpha_{* \delta}^{\mathrm{cr}}, \beta ; \vec{\rho}_{r}\right)$ is obtained from $g_{2}\left(\alpha_{*}, \beta ; \vec{\rho}_{r}\right)$ by substituting

$$
\mathfrak{h}\left[\Omega z_{*}+(\Lambda \backslash \Omega) z_{* \delta}+\Lambda j_{\mathfrak{c}}(\delta) \alpha_{*}+\Lambda^{c} \alpha_{* \delta}\right]
$$

for $\alpha_{*}$. Introduce the auxiliary weight system $\tilde{w}_{\text {aux }}$ that has the same weight factors as $w_{\text {fluct }}$, but core $\Omega_{2}$ instead of $\Omega=\Omega_{\mathfrak{S}}$. We shall apply Proposition G.1, with $\nu=\frac{20}{19}$ and $C_{\nu}=\frac{4 \nu}{(e \ln \nu)^{2}}<2^{8}$, to prove

$$
\left\|g_{2}\left(z_{*}+z_{* \delta}+\alpha_{*}^{\mathrm{cr}}, \beta ; \vec{\rho}_{r}\right)\right\|_{\tilde{w}_{\mathrm{aux}}} \leq 2^{8}\left\|g_{2}\right\|_{\mathfrak{S}_{2}}
$$

The weight factors for the fields $\beta, \vec{\rho}_{r}$ in $\tilde{w}_{\text {aux }}$ (namely $\kappa_{\mathfrak{S}, 2 \delta}$ and $\kappa_{* \mathfrak{S}, \tau}, \kappa_{\mathfrak{S}, \tau}, \lambda_{\mathfrak{S}, \tau}$ with $\delta<\tau<2 \delta)$ are smaller than the weight factors for the corresponding fields in $w_{\mathfrak{S}_{2}}$ (namely $\kappa_{\mathfrak{S}_{2}, \delta}$ and $\kappa_{* \mathfrak{S}_{2}, \tau}, \kappa_{\mathfrak{S}_{2}, \tau}, \lambda_{\mathfrak{S}_{2}, \tau}$ with $0<\tau<\delta$ ). Consequently, the hypothesis of Proposition G.1 is satisfied if

$$
\begin{aligned}
N_{d_{\Omega_{2}}}\left(\Omega ; \kappa_{* \mathfrak{S}_{2}, 0}, \tilde{\lambda}\right)+N_{d_{\Omega_{2}}}\left(\Lambda \backslash \Omega ; \kappa_{* \mathfrak{S}_{2}, 0}, \lambda_{\mathfrak{S}, \delta}\right)+N_{d_{\Omega_{2}}}\left(\Lambda j_{\mathfrak{c}}(\delta) ; \kappa_{* \mathfrak{S}_{2}, 0}, \kappa_{* \mathfrak{S}, 0}\right) & \leq \frac{19}{20} \\
N_{d_{\Omega_{2}}}\left(\Lambda^{c} ; \kappa_{* \mathfrak{S}_{2}, 0}, \kappa_{* \mathfrak{S}, \delta}\right) & \leq \frac{19}{20}
\end{aligned}
$$

Since $\tilde{\lambda}(\mathbf{x})=32 \mathrm{r}$ for all $\mathbf{x} \in \Omega, \kappa_{* \mathfrak{S}_{2}, 0}(\mathbf{x})=2 \mathrm{R}$ for all $\mathbf{x} \in \Omega_{\mathfrak{S}_{2}}, \lambda_{\mathfrak{S}, \delta}=32 \mathrm{r}$ for all $\mathbf{x} \in \Lambda \backslash \Omega, \kappa_{* \mathfrak{S}, 0}(\mathbf{x})=2 \mathrm{R}_{+}$for all $\mathbf{x} \in \Lambda$ and, by Remark III.17, $\kappa_{* \mathfrak{S}, \delta}(\mathbf{x})=\frac{1}{2} \kappa_{* \mathfrak{S}_{2}, 0}(\mathbf{x})$ for all $\mathrm{x} \in \Lambda^{c}$, (III.6) yields

$$
\begin{aligned}
& \mathcal{N}_{2 \mathrm{~m}}\left(\Omega ; \kappa_{* \mathfrak{S}_{2}, 0}, \tilde{\lambda}\right) \leq \frac{32 \mathrm{r}}{2 \mathrm{R}} \quad \mathcal{N}_{2 \mathrm{~m}}\left(\Lambda \backslash \Omega ; \kappa_{* \mathfrak{S}_{2}, 0}, \lambda_{\mathfrak{S}, \delta}\right) \leq \frac{32 \mathrm{r}}{2 \mathrm{R}} \\
& \mathcal{N}_{2 \mathrm{~m}}\left(\Lambda \mathfrak{h} ; \kappa_{* \mathfrak{S}_{2}, 0}, \kappa_{* \mathfrak{S}, 0}\right) \leq \frac{\mathrm{R}_{+}}{\mathrm{R}} \quad \mathcal{N}_{2 \mathrm{~m}}\left(\Lambda^{c} ; \kappa_{* \mathfrak{S}_{2}, 0}, \kappa_{* \mathfrak{S}, \delta}\right) \leq \frac{1}{2}
\end{aligned}
$$

By Remark G.4.iii, Lemma B.1.i and Lemma III.21.ii,

$$
\begin{aligned}
\mathcal{N}_{2 \mathrm{~m}}\left(\Lambda\left(j_{\mathfrak{c}}(\delta)-\mathfrak{h}\right) ; \kappa_{* \mathfrak{S}_{2}, 0}, \kappa_{* \mathfrak{S}, 0}\right) & =\frac{\mathrm{R}_{+}}{\mathrm{R}} \mathcal{N}_{2 \mathrm{~m}}\left(\Lambda\left(j_{\mathfrak{c}}(\delta)-\mathfrak{h}\right) ; \kappa_{* \mathfrak{S}, 0}, \kappa_{* \mathfrak{S}, 0}\right) \\
& \leq 2 \frac{\mathrm{R}_{+}}{\mathrm{R}} \mathcal{N}_{\frac{7}{3} \mathrm{~m}}\left(\Lambda\left(j_{\mathfrak{c}}(\delta)-\mathfrak{h}\right) ; 1,1\right) \\
& \leq 2 \frac{\mathrm{R}_{+}}{\mathrm{R}} K_{j} \delta e^{K_{j} \delta}
\end{aligned}
$$

Since $d_{\Omega_{2}} \leq 2 \mathrm{~m} d$, the left hand side of the top inequality in (V.14) is bounded by

$$
\begin{aligned}
\frac{16 \mathrm{r}}{\mathrm{R}}+\frac{16 \mathrm{r}}{\mathrm{R}}+\mathcal{N}_{2 \mathrm{~m}}\left(\Lambda \mathfrak{h} ; \kappa_{* \mathfrak{S}_{2}, 0}\right. & \left., \kappa_{* \mathfrak{S}, 0}\right)+\mathcal{N}_{2 \mathrm{~m}}\left(\Lambda\left(j_{\mathfrak{c}}(\delta)-\mathfrak{h}\right) ; \kappa_{* \mathfrak{S}_{2}, 0}, \kappa_{* \mathfrak{S}, 0}\right) \\
& \leq \frac{32 \mathrm{r}}{\mathrm{R}}+\frac{\mathrm{R}_{+}}{\mathrm{R}}+2 \frac{\mathrm{R}_{+}}{\mathrm{R}} K_{j} \delta e^{K_{j} \delta} \\
& \leq \frac{19}{20}
\end{aligned}
$$


by (F.3.f) and Hypothesis F.7.i, since $\frac{\mathrm{R}_{+}}{\mathrm{R}}=\frac{1}{2^{e \mathrm{R}_{\mathrm{r}}}} \leq \frac{1}{\sqrt[4]{2}}<\frac{19}{20}-\frac{1}{16}$, by (II.17). The left hand side of the bottom inequality in (V.14) is bounded by $\frac{1}{2}<\frac{19}{20}$. This proves (V.13).

Since $d_{\Omega} \leq d_{\Omega_{2}},(\mathrm{~V} .13)$ gives that

$$
\begin{aligned}
\left\|\left.g_{2}\left(z_{* \delta}+\alpha_{* \delta}^{\mathrm{cr}}, \beta ; \vec{\rho}_{r}\right)\right|_{\Omega^{c}}\right\|_{\mathfrak{S}} & =\left\|\left.g_{2}\left(z_{*}+z_{* \delta}+\alpha_{* \delta}^{\mathrm{cr}}, \beta ; \vec{\rho}_{r}\right)\right|_{\Omega^{c}}\right\|_{\text {fluct }} \\
& \leq\left\|g_{2}\left(z_{*}+z_{* \delta}+\alpha_{* \delta}^{\mathrm{cr}}, \beta ; \vec{\rho}_{r}\right)\right\|_{\tilde{w}_{\mathrm{aux}}} \leq 2^{8}\left\|g_{2}\right\| \mathfrak{S}_{2}
\end{aligned}
$$

As

$$
\begin{aligned}
& \left\|g_{2}\left(z_{*}+z_{* \delta}+\alpha_{* \delta}^{\mathrm{cr}}, \beta ; \vec{\rho}_{r}\right)-g_{2}\left(z_{* \delta}+\alpha_{* \delta}^{\mathrm{cr}}, \beta ; \vec{\rho}_{r}\right)\right\|_{\text {fluct }} \\
& \quad+\left\|g_{2}\left(z_{* \delta}+\alpha_{* \delta}^{\mathrm{cr}}, \beta ; \vec{\rho}_{r}\right)-\left.g_{2}\left(z_{* \delta}+\alpha_{* \delta}^{\mathrm{cr}}, \beta ; \vec{\rho}_{r}\right)\right|_{\Omega^{c}}\right\|_{\text {fluct }} \\
& \quad=\left\|g_{2}\left(z+z_{* \delta}+\alpha_{* \delta}^{\mathrm{cr}}, \beta ; \vec{\rho}_{r}\right)-\left.g_{2}\left(z_{* \delta}+\alpha_{* \delta}^{\mathrm{cr}}, \beta ; \vec{\rho}_{r}\right)\right|_{\Omega^{c}}\right\|_{\text {fluct }}
\end{aligned}
$$

it now suffices to prove that

$$
\begin{aligned}
\| g_{2}\left(z_{*}+z_{* \delta}+\alpha_{* \delta}^{\mathrm{cr}}, \beta ; \vec{\rho}_{r}\right)-\left.g_{2}\left(z_{* \delta}+\alpha_{* \delta}^{\mathrm{cr}}, \beta ; \vec{\rho}_{r}\right)\right|_{\Omega^{c}} & \|_{\text {fluct }} \\
& \leq 2^{8}\left(e^{-\mathrm{mc}(\delta)}\left\|g_{2}\right\| \mathfrak{S}_{2}+\left\|\left.g_{2}\right|_{\Omega_{2}}\right\|_{\mathfrak{S}_{2}}\right)
\end{aligned}
$$

Write

$$
g_{2}=\mathcal{B}+\mathcal{S}
$$

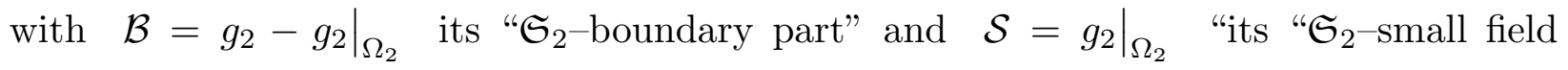
part". Each nonzero monomial in the power series expansion of $\mathcal{B}$ contains at least one factor $\mathfrak{h}(\mathbf{x})$ with $\mathbf{x} \in \Omega_{2}^{c}$. Also, each nonzero monomial in the power series expansion of $\mathcal{B}\left(z_{*}+z_{* \delta}+\alpha_{* \delta}^{\mathrm{cr}}, \beta ; \vec{\rho}_{r}\right)-\left.\mathcal{B}\left(z_{* \delta}+\alpha_{* \delta}^{\mathrm{cr}}, \beta ; \vec{\rho}_{r}\right)\right|_{\Omega^{c}}$ contains at least one factor $\mathfrak{h}(\mathbf{y})$ with $\mathbf{y} \in \Omega$. Therefore, by (II.15) and (V.13)

$$
\begin{aligned}
\| \mathcal{B}\left(z_{*}\right. & \left.+z_{* \delta}+\alpha_{* \delta}^{\mathrm{cr}}, \beta ; \vec{\rho}_{r}\right)-\left.\mathcal{B}\left(z_{* \delta}+\alpha_{* \delta}^{\mathrm{cr}}, \beta ; \vec{\rho}_{r}\right)\right|_{\Omega^{c}} \|_{\text {fluct }} \\
& \leq e^{-\mathrm{m} d\left(\bar{\Omega}, \bar{\Omega}_{2}^{c}\right)}\left\|\mathcal{B}\left(z_{*}+z_{* \delta}+\alpha_{* \delta}^{\mathrm{cr}}, \beta ; \vec{\rho}_{r}\right)-\left.\mathcal{B}\left(z_{* \delta}+\alpha_{* \delta}^{\mathrm{cr}}, \beta ; \vec{\rho}_{r}\right)\right|_{\Omega^{c}}\right\|_{\tilde{w}_{\mathrm{aux}}} \\
& \leq e^{-\mathrm{m} d\left(\bar{\Omega}, \bar{\Omega}_{2}^{c}\right)}\left\|\mathcal{B}\left(z_{*}+z_{* \delta}+\alpha_{* \delta}^{\mathrm{cr}}, \beta ; \vec{\rho}_{r}\right)\right\|_{\tilde{w}_{\mathrm{aux}}} \\
& \leq e^{-\mathrm{m} d\left(\bar{\Omega}, \bar{\Omega}_{2}^{c}\right)}\left\|g_{2}\left(z_{*}+z_{* \delta}+\alpha_{* \delta}^{\mathrm{cr}}, \beta ; \vec{\rho}_{r}\right)\right\|_{\tilde{w}_{\mathrm{aux}}} \\
& \leq 2^{8} e^{-\mathrm{mc}(\delta)}\left\|g_{2}\right\|_{\mathfrak{S}_{2}}
\end{aligned}
$$

Finally

$$
\begin{aligned}
\left\|\mathcal{S}\left(z_{*}+z_{* \delta}+\alpha_{* \delta}^{\mathrm{cr}}, \beta ; \vec{\rho}_{r}\right)-\left.\mathcal{S}\left(z_{* \delta}+\alpha_{* \delta}^{\mathrm{cr}}, \beta ; \vec{\rho}_{r}\right)\right|_{\Omega^{c}}\right\|_{\text {fluct }} & \leq\left\|\mathcal{S}\left(z_{*}+z_{* \delta}+\alpha_{* \delta}^{\mathrm{cr}}, \beta ; \vec{\rho}_{r}\right)\right\|_{w_{\text {aux }}} \\
& \leq 2^{8}\|\mathcal{S}\|_{\mathfrak{S}_{2}}
\end{aligned}
$$

by (V.13), with $g_{2}$ replaced by $\mathcal{S}$. 
Parts (i) and (ii) of Remark V.5 (quadratic and quartic terms) implicitly involve the substitution of the critical fields $\alpha_{\delta}^{\mathrm{cr}}, \alpha_{* \delta}^{\mathrm{cr}}$ (defined in Lemma V.3) in the concrete background fields $\Gamma_{* \mathfrak{S}_{1}}, \Gamma_{\mathfrak{S}_{1}}$ and $\Gamma_{* \mathfrak{S}_{2}}, \Gamma_{\mathfrak{S}_{2}}$. To control it, set

$$
\begin{array}{r}
\tilde{\Gamma}_{*}\left(\tau ; \alpha_{*}, \vec{\alpha}_{*}\right)= \begin{cases}\Gamma_{* \mathfrak{S}_{1}}\left(\tau ; \alpha_{*}, \vec{\alpha}_{* l}\right) & \text { if } \tau \in \varepsilon \mathbb{Z} \cap(0, \delta) \\
\alpha_{* \delta}^{\mathrm{cr}} & \text { if } \tau=\delta \\
\Gamma_{* \mathfrak{S}_{2}}\left(\tau-\delta ; \alpha_{* \delta}^{\mathrm{cr}}, \vec{\alpha}_{* r}\right) & \text { if } \tau \in \varepsilon \mathbb{Z} \cap(\delta, 2 \delta)\end{cases} \\
\tilde{\Gamma}(\tau ; \vec{\alpha}, \beta)= \begin{cases}\Gamma_{\mathfrak{S}_{1}}\left(\tau ;, \vec{\alpha}_{l}, \alpha_{\delta}^{\mathrm{cr}}\right) & \text { if } \tau \in \varepsilon \mathbb{Z} \cap(0, \delta) \\
\alpha_{\delta}^{\mathrm{cr}} & \text { if } \tau=\delta \\
\Gamma_{\mathfrak{S}_{2}}\left(\tau-\delta ; \vec{\alpha}_{r}, \beta\right) & \text { if } \tau \in \varepsilon \mathbb{Z} \cap(\delta, 2 \delta)\end{cases}
\end{array}
$$

Observe that (V.16) is very similar to the recursion relation of Proposition III.6. Indeed, replacing $\alpha_{* \delta}^{\mathrm{cr}}$ and $\alpha_{\delta}^{\mathrm{cr}}$ by

$\Lambda j(\delta) \alpha_{*}+\Lambda^{c} \alpha_{* \delta}=\alpha_{* \delta}^{\mathrm{cr}}+\Lambda\left[j(\delta)-j_{\mathfrak{c}}(\delta)\right] \alpha_{*} \quad$ and $\quad \Lambda j(\delta) \beta+\Lambda^{c} \alpha_{\delta}=\alpha_{\delta}^{\mathrm{cr}}+\Lambda\left[j(\delta)-j_{\mathfrak{c}}(\delta)\right] \beta$

respectively, in (V.16) gives the recursion relation of Proposition III.6, except for the $\partial \Gamma_{* \tau} \alpha_{*}$ and $\partial \Gamma_{\tau} \beta$ terms. For convenience, we set

$$
j_{\delta}=j(\delta)-j_{\mathfrak{c}}(\delta)
$$

Lemma III.21.iii gives the estimate $\left\|j_{\delta}\right\| \leq \leq K_{j} e^{K_{j} \delta} e^{-\mathrm{mc}}$. This discussion shows that

$$
\begin{aligned}
\Gamma_{* \mathfrak{S}}\left(\tau ; \alpha_{*}, \vec{\alpha}_{*}\right)-\tilde{\Gamma}_{*}\left(\tau ; \alpha_{*}, \vec{\alpha}_{*}\right) & =\partial_{\mathfrak{c}} \Gamma_{* \tau} \alpha_{*} \\
\Gamma_{\mathfrak{S}}(\tau ; \vec{\alpha}, \beta)-\tilde{\Gamma}(\tau ; \vec{\alpha}, \beta) & =\partial_{\mathfrak{c}} \Gamma_{\tau} \beta
\end{aligned}
$$

where we set

$$
\begin{gathered}
\partial_{\mathfrak{c}} \Gamma_{* \tau}= \begin{cases}0 & \text { if } \tau \in[0, \delta) \text { or } \tau=2 \delta \\
\Lambda j_{\delta} & \text { if } \tau=\delta \\
\partial \Gamma_{* \tau}+\Gamma_{* \tau-\delta}^{0}\left(\mathfrak{S}_{2}\right) \Lambda j_{\delta} & \text { if } \tau \in(\delta, 2 \delta)\end{cases} \\
\partial_{\mathfrak{c}} \Gamma_{\tau}= \begin{cases}\partial \Gamma_{\tau}+\Gamma_{\tau}^{\delta}\left(\mathfrak{S}_{1}\right) \Lambda j_{\delta} & \text { if } \tau \in(0, \delta) \\
\Lambda j_{\delta} & \text { if } \tau=\delta \\
0 & \text { if } \tau \in(\delta, 2 \delta] \text { or } \tau=0\end{cases}
\end{gathered}
$$

Under the conventions of parts (iii) and (iv) of Lemma III.7, we may also write $\partial_{\mathfrak{c}} \Gamma_{* \tau}=$ $\partial \Gamma_{* \tau}+\Gamma_{* \tau-\delta}^{0}\left(\mathfrak{S}_{2}\right) \Lambda j_{\delta}$ for $\tau=\delta, 2 \delta$ and $\partial_{\mathfrak{c}} \Gamma_{\tau}=\partial \Gamma_{\tau}+\Gamma_{\tau}^{\delta}\left(\mathfrak{S}_{1}\right) \Lambda j_{\delta}$ for $\tau=0, \delta$. The operators $\partial_{\mathfrak{c}} \Gamma_{* \tau}, \partial_{\mathfrak{c}} \Gamma_{\tau}$ are estimated in Lemma E.18. 
We use the shorthand notations

$$
\gamma_{* \tau}=\left\{\begin{array}{ll}
\Gamma_{* \mathfrak{S}}\left(\tau ; \alpha_{*}, \vec{\alpha}_{*}\right) & \text { if } \tau \in(0,2 \delta) \\
\alpha_{*} & \text { if } \tau=0
\end{array}\right\} \quad \gamma_{\tau}=\left\{\begin{array}{ll}
\Gamma_{\mathfrak{S}}(\tau ; \vec{\alpha}, \beta) & \text { if } \tau \in(0,2 \delta) \\
\beta & \text { if } \tau=2 \delta
\end{array}\right\}
$$

By the recursion relation Proposition III.6,

$$
\Gamma_{* \mathfrak{S}_{1}}\left(\tau ; \alpha_{*}, \vec{\alpha}_{*}\right)=\gamma_{* \tau} \quad \Gamma_{\mathfrak{S}_{2}}(\tau ; \vec{\alpha}, \beta)=\gamma_{\tau+\delta} \quad \text { for all } 0<\tau<\delta
$$

\section{Bound on the Quadratic Part of the Fluctuation Action}

In part (i) of Remark V.5 we wrote the "quadratic part" as the sum of $-Q_{\mathfrak{S}}, \delta Q$,

$$
-\left\langle z_{*}, \Omega j_{\mathfrak{c}}(\delta) \beta\right\rangle-Q_{\mathfrak{S}_{2}}\left(z_{*}, \beta ; 0, \vec{\alpha}_{r}\right)=\left\langle z_{*}, \Omega j_{\delta} \beta\right\rangle-\left\langle z_{*}, \Omega j(\delta) \beta\right\rangle-Q_{\mathfrak{S}_{2}}\left(z_{*}, \beta ; 0, \vec{\alpha}_{r}\right)
$$

and

$$
-\left\langle\Omega j_{\mathfrak{c}}(\delta) \alpha_{*}, z\right\rangle-Q_{\mathfrak{S}_{1}}\left(\alpha_{*}, z ; \vec{\alpha}_{*_{l}}, 0\right)=\left\langle\Omega j_{\delta} \alpha_{*}, z\right\rangle-\left\langle\Omega j(\delta) \alpha_{*}, z\right\rangle-Q_{\mathfrak{S}_{1}}\left(\alpha_{*}, z ; \vec{\alpha}_{*_{l}}, 0\right)
$$

We bound these quantities in Proposition V.12, below. By way of preparation for the bound on $\delta Q$, recall from (V.10) that

$$
\begin{aligned}
\delta Q=Q_{\mathfrak{S}} & \left(\alpha_{*}, \beta ; \vec{\alpha}_{*}, \vec{\alpha}\right)-Q_{\mathfrak{S}_{1}}\left(\alpha_{*}, \alpha_{\delta}^{\mathrm{cr}} ; \vec{\alpha}_{*_{l}}, \vec{\alpha}_{l}\right)-Q_{\mathfrak{S}_{2}}\left(\alpha_{* \delta}^{\mathrm{cr}}, \beta ; \vec{\alpha}_{*_{r}}, \vec{\alpha}_{r}\right)-\left\langle\alpha_{* \delta}^{\mathrm{cr}}, \alpha_{\delta}^{\mathrm{cr}}\right\rangle \\
- & {\left[\left\langle z_{* \delta},(\Lambda \backslash \Omega) j(\delta) \beta\right\rangle+Q_{\mathfrak{S}_{2}}\left(z_{* \delta}, \beta ; 0, \vec{\alpha}_{r}\right)\right]+\left\langle z_{* \delta},(\Lambda \backslash \Omega) j_{\delta}(\delta) \beta\right\rangle } \\
- & {\left[\left\langle(\Lambda \backslash \Omega) j(\delta) \alpha_{*}, z_{\delta}\right\rangle+Q_{\mathfrak{S}_{1}}\left(\alpha_{*}, z_{\delta} ; \vec{\alpha}_{*_{l}}, 0\right)\right]+\left\langle(\Lambda \backslash \Omega) j_{\delta}(\delta) \alpha_{*}, z_{\delta}\right\rangle }
\end{aligned}
$$

We start by rewriting the first line of the right hand side.

\section{Lemma V.11}

$$
\begin{aligned}
& Q_{\mathfrak{S}}\left(\alpha_{*}, \beta ; \vec{\alpha}_{*}, \vec{\alpha}\right)-Q_{\mathfrak{S}_{1}}\left(\alpha_{*}, \alpha_{\delta}^{\mathrm{cr}} ; \vec{\alpha}_{*_{l}}, \vec{\alpha}_{l}\right)-Q_{\mathfrak{S}_{2}}\left(\alpha_{* \delta}^{\mathrm{cr}}, \beta ; \vec{\alpha}_{*_{r}}, \vec{\alpha}_{r}\right)-\left\langle\alpha_{* \delta}^{\mathrm{cr}}, \alpha_{\delta}^{\mathrm{cr}}\right\rangle \\
& \quad=\sum_{\tau \in(0,2 \delta]}\left\langle\partial_{j} \Gamma_{* \tau} \alpha_{*}, \gamma_{\tau}\right\rangle+\sum_{\tau \in[0,2 \delta)}\left\langle\gamma_{* \tau}, \partial_{j} \Gamma_{\tau} \beta\right\rangle-\left\langle\Lambda j_{\delta} \alpha_{*}, \Lambda j_{\delta} \beta\right\rangle
\end{aligned}
$$

where

$$
\partial_{j} \Gamma_{* \tau}=\partial_{\mathfrak{c}} \Gamma_{* \tau}-j(\varepsilon) \partial_{\mathfrak{c}} \Gamma_{* \tau-\varepsilon} \quad \partial_{j} \Gamma_{\tau}=\partial_{\mathfrak{c}} \Gamma_{\tau}-j(\varepsilon) \partial_{\mathfrak{c}} \Gamma_{\tau+\varepsilon}
$$

Proof: By parts (iii) and (iv) of Lemma III.7 and (V.21), 


$$
\begin{aligned}
& Q_{\mathfrak{S}}\left(\alpha_{*}, \beta ; \vec{\alpha}_{*}, \vec{\alpha}\right)-Q_{\mathfrak{S}_{1}}\left(\alpha_{*}, \alpha_{\delta}^{\mathrm{cr}} ; \vec{\alpha}_{*_{l}}, \vec{\alpha}_{l}\right)-Q_{\mathfrak{S}_{2}}\left(\alpha_{* \delta}^{\mathrm{cr}}, \beta ; \vec{\alpha}_{*_{r}}, \vec{\alpha}_{r}\right)-\left\langle\alpha_{* \delta}^{\mathrm{cr}}, \alpha_{\delta}^{\mathrm{cr}}\right\rangle \\
& =Q_{\mathfrak{S}_{1}}\left(\alpha_{*}, \Lambda j(\delta) \beta+\Lambda^{c} \alpha_{\delta} ; \vec{\alpha}_{* l}, \vec{\alpha}_{l}\right)-Q_{\mathfrak{S}_{1}}\left(\alpha_{*}, \Lambda j_{\mathfrak{c}}(\delta) \beta+\Lambda^{c} \alpha_{\delta} ; \vec{\alpha}_{*_{l}}, \vec{\alpha}_{l}\right) \\
& +Q_{\mathfrak{S}_{2}}\left(\Lambda j(\delta) \alpha_{*}+\Lambda^{c} \alpha_{* \delta}, \beta ; \vec{\alpha}_{* r}, \vec{\alpha}_{r}\right)-Q_{\mathfrak{S}_{2}}\left(\Lambda j_{\mathfrak{c}}(\delta) \alpha_{*}+\Lambda^{c} \alpha_{* \delta}, \beta ; \vec{\alpha}_{*_{r}}, \vec{\alpha}_{r}\right) \\
& +\left\langle\Lambda j(\delta) \alpha_{*}, \Lambda j(\delta) \beta\right\rangle+\left\langle\Lambda^{c} \alpha_{* \delta}, \Lambda^{c} \alpha_{\delta}\right\rangle-\left\langle\Lambda j_{\mathfrak{c}}(\delta) \alpha_{*}+\Lambda^{c} \alpha_{* \delta}, \Lambda j_{\mathfrak{c}}(\delta) \beta+\Lambda^{c} \alpha_{\delta}\right\rangle \\
& +\sum_{\tau \in \varepsilon \mathbb{Z} \cap[0, \delta)}\left\langle\gamma_{* \tau},\left(\partial \Gamma_{\tau}-j(\varepsilon) \partial \Gamma_{\tau+\varepsilon}\right) \beta\right\rangle+\sum_{\tau \in \varepsilon \mathbb{Z} \cap(0, \delta]}\left\langle\left(\partial \Gamma_{* \delta+\tau}-j(\varepsilon) \partial \Gamma_{* \delta+\tau-\varepsilon}\right) \alpha_{*}, \gamma_{\delta+\tau}\right\rangle \\
& =\sum_{\tau \in \varepsilon \mathbb{Z} \cap[0, \delta)}\left\langle\gamma_{* \tau},\left(\Gamma_{\tau}^{\delta}\left(\mathfrak{S}_{1}\right)-j(\varepsilon) \Gamma_{\tau+\varepsilon}^{\delta}\left(\mathfrak{S}_{1}\right)\right) \Lambda j_{\delta} \beta\right\rangle \\
& +\sum_{\tau \in \varepsilon \mathbb{Z} \cap(0, \delta]}\left\langle\left(\Gamma_{* \tau}^{0}\left(\mathfrak{S}_{2}\right)-j(\varepsilon) \Gamma_{* \tau-\varepsilon}^{0}\left(\mathfrak{S}_{2}\right)\right) \Lambda j_{\delta} \alpha_{*}, \gamma_{\delta+\tau}\right\rangle \\
& +\left\langle\Lambda j(\delta) \alpha_{*}, \Lambda j(\delta) \beta\right\rangle-\left\langle\Lambda j_{\mathfrak{c}}(\delta) \alpha_{*}, \Lambda j_{\mathfrak{c}}(\delta) \beta\right\rangle \\
& +\sum_{\tau \in \varepsilon \mathbb{Z} \cap[0, \delta)}\left\langle\gamma_{* \tau},\left(\partial \Gamma_{\tau}-j(\varepsilon) \partial \Gamma_{\tau+\varepsilon}\right) \beta\right\rangle+\sum_{\tau \in \varepsilon \mathbb{Z} \cap(0, \delta]}\left\langle\left(\partial \Gamma_{* \delta+\tau}-j(\varepsilon) \partial \Gamma_{* \delta+\tau-\varepsilon}\right) \alpha_{*}, \gamma_{\delta+\tau}\right\rangle \\
& =\sum_{\tau \in \varepsilon \mathbb{Z} \cap[0, \delta)}\left\langle\gamma_{* \tau},\left(\partial_{\mathfrak{c}} \Gamma_{\tau}-j(\varepsilon) \partial_{\mathfrak{c}} \Gamma_{\tau+\varepsilon}\right) \beta\right\rangle+\sum_{\tau \in \varepsilon \mathbb{Z} \cap(\delta, 2 \delta]}\left\langle\left(\partial_{\mathfrak{c}} \Gamma_{* \tau}-j(\varepsilon) \partial_{\mathfrak{c}} \Gamma_{* \tau-\varepsilon}\right) \alpha_{*}, \gamma_{\tau}\right\rangle \\
& +\left\langle\Lambda j(\delta) \alpha_{*}, \Lambda j(\delta) \beta\right\rangle-\left\langle\Lambda j_{\mathfrak{c}}(\delta) \alpha_{*}, \Lambda j_{\mathfrak{c}}(\delta) \beta\right\rangle
\end{aligned}
$$

This is the desired equation, since

$$
\begin{aligned}
\partial_{j} \Gamma_{* \tau} & =\left\{\begin{array}{ll}
0 & \text { if } 0<\tau<\delta \\
\Lambda j_{\delta} & \text { if } \tau=\delta
\end{array}\right\} & \partial_{j} \Gamma_{\tau} & =\left\{\begin{array}{ll}
0 & \text { if } \delta<\tau<2 \delta \\
\Lambda j_{\delta} & \text { if } \tau=\delta
\end{array}\right\} \\
\gamma_{\delta} & =\Lambda^{c} \alpha_{\delta}+\Lambda j(\delta) \beta & \gamma_{* \delta} & =\Lambda^{c} \alpha_{* \delta}+\Lambda j(\delta) \alpha_{*}
\end{aligned}
$$

so that

$$
\begin{aligned}
\left\langle\partial_{j} \Gamma_{* \tau} \alpha_{*}, \gamma_{\tau}\right\rangle & =\left\{\begin{array}{ll}
0 & \text { if } 0<\tau<\delta \\
\left\langle\Lambda j_{\delta} \alpha_{*}, \Lambda j(\delta) \beta\right\rangle & \text { if } \tau=\delta
\end{array}\right\} \\
\left\langle\gamma_{* \tau}, \partial_{j} \Gamma_{\tau} \beta\right\rangle & =\left\{\begin{array}{ll}
0 & \text { if } \delta<\tau<2 \delta \\
\left\langle\Lambda j(\delta) \alpha_{*}, \Lambda j_{\delta} \beta\right\rangle & \text { if } \tau=\delta
\end{array}\right\}
\end{aligned}
$$

Proposition V.12 Set $K_{Q}=2^{9} e^{2 K_{j}}$.

(i)

$$
\begin{aligned}
& \left\|\left\langle z_{*}, \Omega j_{\mathfrak{c}}(\delta) \beta\right\rangle+Q_{\mathfrak{S}_{2}}\left(z_{*}, \beta ; 0, \vec{\alpha}_{r}\right)\right\|_{\text {fluct }} \leq K_{Q}\left[e^{-\mathrm{mc}(\delta)}+\delta \mathrm{rR}_{+} e^{-\mathrm{m} \mathfrak{c}}\right] \\
& \left\|\left\langle\Omega j_{\mathfrak{c}}(\delta) \alpha_{*}, z\right\rangle+Q_{\mathfrak{S}_{1}}\left(\alpha_{*}, z ; \vec{\alpha}_{* l}, 0\right)\right\|_{\text {fluct }} \leq K_{Q}\left[e^{-\mathrm{m} \mathfrak{c}(\delta)}+\delta \mathrm{rR}+e^{-\mathrm{m} \mathfrak{c}}\right]
\end{aligned}
$$

(ii)

$$
\left\|\delta Q\left(\alpha_{*}, \beta ; \vec{\rho}\right)\right\|_{\mathfrak{S}} \leq K_{Q}\left[e^{-\frac{1}{4} \mathrm{~m} \mathfrak{c}(\delta)}+\delta \mathrm{rR}_{+} e^{-\mathrm{m} \mathfrak{c}}\right]
$$


Proof: (i) We prove the second inequality. The proof of the first is analogous. Observe that, by the definition (III.3),

$$
Q_{\mathfrak{S}_{1}}\left(\alpha_{*}, z ; \vec{\alpha}_{* l}, 0\right)=Q_{\varepsilon, \delta}\left(\alpha_{*}, z ; \Gamma_{* \mathfrak{S}_{1}}\left(\cdot ; \alpha_{*}, \vec{\alpha}_{* l}\right), \Gamma_{\mathfrak{S}_{1}}(\cdot ; 0, z)\right)
$$

with the $Q_{\varepsilon, \delta}$ of (II.10). Since $z$ is supported in $\Omega$,

$$
\begin{aligned}
\left\langle j_{\delta} \alpha_{*}, z\right\rangle+[ & \left.\left\langle\Omega j_{\mathfrak{c}}(\delta) \alpha_{*}, z\right\rangle+Q_{\varepsilon, \delta}\left(\alpha_{*}, z ; \Gamma_{* \mathfrak{S}_{1}}\left(\cdot ; \alpha_{*}, \vec{\alpha}_{* l}\right), \Gamma_{\mathfrak{S}_{1}}(\cdot ; 0, z)\right)\right] \\
& =\left\langle\Omega j(\delta) \alpha_{*}, z\right\rangle+Q_{\varepsilon, \delta}\left(\alpha_{*}, z ; \Gamma_{* \mathfrak{S}_{1}}\left(\cdot ; \alpha_{*}, \vec{\alpha}_{* l}\right), \Gamma_{\mathfrak{S}_{1}}(\cdot ; 0, z)\right) \\
& =\sum_{\tau \in[0, \delta)}\left\langle\gamma_{* \tau},\left(\Gamma_{\tau}^{\delta}\left(\mathfrak{S}_{1}\right)-j(\varepsilon) \Gamma_{\tau+\varepsilon}^{\delta}\left(\mathfrak{S}_{1}\right)\right) \Omega z\right\rangle
\end{aligned}
$$

evaluated at

$$
\gamma_{* \tau}= \begin{cases}\alpha_{*} & \text { if } \tau=0 \\ \Gamma_{* \mathfrak{S}_{1}}\left(\tau ; \alpha_{*}, \vec{\alpha}_{* l}\right)=\Gamma_{* \tau}^{0}\left(\mathfrak{S}_{1}\right) \alpha_{*}+\sum_{\tau^{\prime} \in(0, \tau] \cap \mathbb{Z}} \Gamma_{* \tau}^{\tau^{\prime}}\left(\mathfrak{S}_{1}\right) \alpha_{* \tau^{\prime}} & \text { if } \tau \in(0, \delta)\end{cases}
$$

and with the conventions that $\Gamma_{0}^{\delta}\left(\mathfrak{S}_{1}\right)=j(\delta)$ and $\Gamma_{\delta}^{\delta}\left(\mathfrak{S}_{1}\right)=\mathfrak{h}$.

To bound this, we first claim that for any two $\mathfrak{h}$-operators $A$ and $A^{\prime}$

$$
\left\|\left\langle A^{\prime} \alpha_{* \tau^{\prime}}, A z\right\rangle\right\|_{\text {fluct }} \leq N_{d_{\Omega}}\left(A^{\prime} ; e^{\mathrm{m} d(\mathbf{x}, \Omega)}, \kappa_{* \tau^{\prime}}\right) N_{d_{\Omega}}\left(A ; e^{-\mathrm{m} d(\mathbf{x}, \Omega)}, \tilde{\lambda}\right)
$$

As $\left\langle A^{\prime} \alpha_{* \tau^{\prime}}, A z\right\rangle$ is obtained from $\langle\varphi, \psi\rangle$ by the substitution $\varphi=A^{\prime} \alpha_{* \tau^{\prime}}, \psi=A z$, this inequality follows Lemma G.2.a with $s=2, d=d_{\Omega}, \kappa_{1}^{\prime}=\kappa_{* \tau}, \kappa_{2}^{\prime}=\tilde{\lambda}, \kappa_{1}(\mathbf{x})=e^{\mathrm{m} d(\mathbf{x}, \Omega)}$ and $\kappa_{2}(\mathbf{x})=e^{-\operatorname{md}(\mathbf{x}, \Omega)}$.

Applying the estimates (V.24) and (III.7) we get

$$
\begin{aligned}
\|\left\langle\Omega j(\delta) \alpha_{*}, z\right\rangle+ & Q_{\varepsilon, \delta}\left(\alpha_{*}, z ; \Gamma_{* \mathfrak{S}_{1}}\left(\cdot ; \alpha_{*}, \vec{\alpha}_{* l}\right), \Gamma_{\mathfrak{S}_{1}}(\cdot ; 0, z)\right) \|_{\text {fluct }} \\
& \leq \sum_{\tau \in[0, \delta)} a_{\tau} \mathcal{N}_{2 \mathrm{~m}}\left(\left(\Gamma_{\tau}^{\delta}\left(\mathfrak{S}_{1}\right)-j(\varepsilon) \Gamma_{\tau+\varepsilon}^{\delta}\left(\mathfrak{S}_{1}\right)\right) \Omega ; e^{-\mathrm{m} d(\mathbf{x}, \Omega)}, \tilde{\lambda}\right)
\end{aligned}
$$

where

$$
a_{\tau}= \begin{cases}\mathcal{N}_{2 \mathrm{~m}}\left(\mathbb{1} ; e^{\mathrm{m} d(\mathbf{x}, \Omega)}, \kappa_{* 0}\right) & \text { if } \tau=0 \\ \sum_{\tau^{\prime} \in[0, \delta)} \mathcal{N}_{2 \mathrm{~m}}\left(\Gamma_{* \tau}^{\tau^{\prime}}\left(\mathfrak{S}_{1}\right) ; e^{\mathrm{m} d(\mathbf{x}, \Omega)}, \kappa_{* \tau^{\prime}}\right) & \text { if } \tau \in(0, \delta)\end{cases}
$$

By Lemma B.1.ii, Lemma E.13 and the observation that $e^{-\operatorname{md}(\mathbf{x}, \Omega)} \leq e^{-\frac{\mathrm{m}}{2} d(\mathbf{x}, \Omega)} \leq$ $e^{-\frac{\mathrm{m}}{2} d\left(\mathbf{x}, \Lambda_{\mathfrak{S}_{1}}\right)}$, we have $a_{\tau} \leq 16 e^{K_{j}} \mathrm{R}$, for all $\tau \in[0, \delta)$. Therefore, by Lemma E.15.ii with $\lambda=\tilde{\lambda}, O=\Omega$ and $\Omega_{\mathfrak{S}}$ replaced by $\Omega_{1}$

$$
\begin{aligned}
\|\left\langle j_{\delta} \alpha_{*},\right. & z\rangle+\left[\left\langle\Omega j_{\mathfrak{c}}(\delta) \alpha_{*}, z\right\rangle+Q_{\varepsilon, \delta}\left(\alpha_{*}, z ; \Gamma_{* \mathfrak{S}_{1}}\left(\cdot ; \alpha_{*}, \vec{\alpha}_{* l}\right), \Gamma_{\mathfrak{S}_{1}}(\cdot ; 0, z)\right)\right] \|_{\text {fluct }} \\
& \leq 16 e^{K_{j}} \mathrm{R} \sum_{\tau \in[0, \delta)} \mathcal{N}_{2 \mathrm{~m}}\left(\left(\Gamma_{\tau}^{\delta}\left(\mathfrak{S}_{1}\right)-j(\varepsilon) \Gamma_{\tau+\varepsilon}^{\delta}\left(\mathfrak{S}_{1}\right)\right) \Omega ; e^{-\mathrm{m} d(\mathbf{x}, \Omega)}, \tilde{\lambda}\right) \\
& \leq 2^{11} e^{2 K_{j}} \operatorname{rRe} e^{-2 \mathrm{~m} d\left(\Omega, \Omega_{1}^{c}\right)} \\
& \leq e^{-\mathrm{mc}(\delta)}
\end{aligned}
$$


since $8 \leq 16 e^{K_{j}} \mathrm{r} \leq 16 e^{K_{j}} \mathrm{R} \leq e^{\frac{1}{4} \mathrm{~m} \mathfrak{c}(\delta)}$, by (F.6.b), and $d\left(\Omega, \Omega_{1}^{c}\right) \geq \mathfrak{c}(\delta)$.

The function $\left\langle j_{\delta} \alpha_{*}, z\right\rangle$ is obtained from $\langle\varphi, z\rangle$ by the linear substitution $\varphi=$ $\Omega j_{\delta} \alpha_{*}$. Let $\omega$ be the weight system with core $\Omega$ that associates the constant weight factor 1 to the fields $\varphi$ and $z$. Clearly, $\|\langle\varphi, z\rangle\|_{\omega}=1$. It follows from Lemma G.2.a (with $\left.s=2, \tilde{w}=w_{\text {fluct }}, w=\omega\right)$ that

$$
\begin{aligned}
\left\|\left\langle j_{\delta} \alpha_{*}, z\right\rangle\right\|_{\text {fluct }}=\left\|\left\langle\Lambda j_{\delta} \alpha_{*}, \Omega z\right\rangle\right\|_{\text {fluct }} & \leq N_{d_{\Omega}}\left(\Lambda j_{\delta} ; 1, \kappa_{* \mathfrak{S}, 0}\right) N_{d_{\Omega}}(\Omega ; 1, \tilde{\lambda}) \\
& \leq 4 K_{j} e^{K_{j} \delta} \delta \mathrm{R}_{+} e^{-\mathrm{m} \mathfrak{c}} 32 \mathrm{r}
\end{aligned}
$$

since, by (III.7), Remark G.4.i, Lemma B.1.i and Lemma III.21.iii,

$$
N_{d_{\Omega}}\left(\Lambda j_{\delta} ; 1, \kappa_{* \mathfrak{S}, 0}\right) \leq \mathcal{N}_{2 \mathrm{~m}}\left(\Lambda j_{\delta} ; 1, \kappa_{* \mathfrak{S}, 0}\right) \leq 4\left\|j_{\delta}\right\| \mathrm{R}_{+} \leq 4 K_{j} e^{K_{j} \delta} \delta \mathrm{R}_{+} e^{-\mathrm{m} \mathfrak{c}}
$$

This completes the proof of the second inequality.

(ii) As in part (i) one shows that

$$
\begin{aligned}
\left\|\left\langle z_{* \delta},(\Lambda \backslash \Omega) j_{\delta} \beta\right\rangle\right\|_{\mathfrak{S}} & \leq 2^{7} K_{j} e^{K_{j}} \delta \mathrm{rR}_{+} e^{-\mathrm{m} \mathfrak{c}} \\
\left\|\left\langle z_{* \delta},(\Lambda \backslash \Omega) j(\delta) \beta\right\rangle+Q_{\mathfrak{S}_{2}}\left(z_{* \delta}, \beta ; 0, \vec{\alpha}_{r}\right)\right\|_{\mathfrak{S}} & \leq e^{-\mathrm{m} \mathfrak{c}(\delta)} \\
\left\|\left\langle(\Lambda \backslash \Omega) j_{\delta} \alpha_{*}, z_{\delta}\right\rangle\right\|_{\mathfrak{S}} & \leq 2^{7} K_{j} e^{K_{j}} \delta \mathrm{rR}_{+} e^{-\mathrm{m} \mathfrak{c}} \\
\left\|\left\langle(\Lambda \backslash \Omega) j(\delta) \alpha_{*}, z_{\delta}\right\rangle+Q_{\mathfrak{S}_{1}}\left(\alpha_{*}, z_{\delta} ; \vec{\alpha}_{*_{l}}, 0\right)\right\|_{\mathfrak{S}} & \leq e^{-\mathrm{m} \mathfrak{c}(\delta)}
\end{aligned}
$$

This bounds the last four terms of (V.22).

It remains to bound the first four terms of (V.22), which form the left hand side of Lemma V.11. By (V.23), the first sum on the right hand side of Lemma V.11 is

$$
\begin{aligned}
\sum_{\tau \in(0,2 \delta]}\left\langle\partial_{j} \Gamma_{* \tau} \alpha_{*}, \gamma_{\tau}\right\rangle & =\left\langle j(\delta) \Lambda j_{\delta} \alpha_{*}, \beta\right\rangle+\sum_{\tau \in(\delta, 2 \delta)}\left\langle\partial_{j} \Gamma_{* \tau} \alpha_{*}, \gamma_{\tau}\right\rangle+\left\langle\partial_{j} \Gamma_{* 2 \delta} \alpha_{*}, \gamma_{2 \delta}\right\rangle \\
& =\sum_{\tau \in(\delta, 2 \delta)}\left\langle\partial_{j} \Gamma_{* \tau} \alpha_{*}, \gamma_{\tau}\right\rangle+\left\langle\left(\partial_{j} \Gamma_{* 2 \delta}+j(\delta) \Lambda j_{\delta}\right) \alpha_{*}, \gamma_{2 \delta}\right\rangle
\end{aligned}
$$

As in (V.24) one sees for all $\mathfrak{h}$-operators $A$ and $B$

$$
\left\|\left\langle A \alpha_{*}, B \alpha_{\tau^{\prime}}\right\rangle\right\|_{\mathfrak{S}} \leq \mathcal{N}_{d_{\Omega}}\left(A ; e^{-\frac{\mathrm{m}}{2} d(\mathbf{x}, \Lambda)}, \kappa_{* 0}\right) \mathcal{N}_{d_{\Omega}}\left(B ; e^{\frac{\mathrm{m}}{2} d(\mathbf{x}, \Lambda)}, \kappa_{\tau^{\prime}}\right)
$$

Hence,

$$
\begin{aligned}
\| \sum_{\tau \in(\delta, 2 \delta)}\left\langle\partial_{j} \Gamma_{* \tau} \alpha_{*}, \gamma_{\tau}\right\rangle & +\left\langle\left(\partial_{j} \Gamma_{* 2 \delta}+j(\delta) \Lambda j_{\delta}\right) \alpha_{*}, \gamma_{2 \delta}\right\rangle \|_{\mathfrak{S}} \\
\leq & \sum_{\tau \in(\delta, 2 \delta)} a_{\tau} \mathcal{N}_{2 \mathrm{~m}}\left(\partial_{j} \Gamma_{* \tau} ; e^{-\frac{\mathrm{m}}{2} d(\mathbf{x}, \Lambda)}, \kappa_{* 0}\right) \\
& \quad a_{2 \delta} \mathcal{N}_{2 \mathrm{~m}}\left(\partial_{j} \Gamma_{* 2 \delta}+j(\delta) \Lambda j_{\delta} ; e^{-\frac{\mathrm{m}}{2} d(\mathbf{x}, \Lambda)}, \kappa_{* 0}\right)
\end{aligned}
$$


where

$$
a_{\tau}= \begin{cases}\mathcal{N}_{2 \mathrm{~m}}\left(\mathbb{1} ; e^{\frac{\mathrm{m}}{2} d(\mathbf{x}, \Lambda)}, \kappa_{2 \delta}\right) & \text { if } \tau=2 \delta \\ \sum_{\tau^{\prime} \in(0,2 \delta]} \mathcal{N}_{2 \mathrm{~m}}\left(\Gamma_{\tau}^{\tau^{\prime}} ; e^{\frac{\mathrm{m}}{2} d(\mathbf{x}, \Lambda)}, \kappa_{\tau^{\prime}}\right) & \text { if } \tau \in(\delta, 2 \delta)\end{cases}
$$

By Lemma B.1.ii and Lemma E.13, we have $a_{\tau} \leq 16 e^{K_{j}} \mathrm{R}_{+}$for all $\tau \in(0,2 \delta]$. Therefore, by (V.26), and Lemma E.20.ii, the first sum on the right hand side of Lemma V.11 is bounded by

$$
\left\|\sum_{\tau \in(0,2 \delta]}\left\langle\partial_{j} \Gamma_{* \tau} \alpha_{*}, \gamma_{\tau}\right\rangle\right\|_{\mathfrak{S}} \leq 16 e^{K_{j}} \mathrm{R}_{+} e^{-\frac{1}{2} \mathrm{mc}(\delta)} \leq e^{-\frac{1}{4} \mathrm{mc}(\delta)}
$$

by (F.6.b). The second sum is bounded in the same way. Combining this with (V.22) and (V.25) gives

$$
\left\|\delta Q\left(\alpha_{*}, \beta ; \vec{\rho}\right)+\left\langle\Lambda j_{\delta} \alpha_{*}, \Lambda j_{\delta} \beta\right\rangle\right\|_{\mathfrak{S}} \leq 2\left\{e^{-\frac{1}{4} \mathrm{~m} \mathfrak{c}(\delta)}+e^{-\mathrm{m} \mathfrak{c}(\delta)}+2^{7} K_{j} e^{K_{j}} \delta \mathrm{rR}+e^{-\mathrm{mc}}\right\}
$$

As $\left\langle\Lambda j_{\delta} \alpha_{*}, \Lambda j_{\delta} \beta\right\rangle$ is obtained from $\langle\varphi, \psi\rangle$ by the substitution $\varphi=\Lambda j_{\delta} \alpha_{*}, \psi=\Lambda j_{\delta} \beta$, an application of Lemma G.2.a with $s=2, d=d_{\Omega}, \kappa_{1}^{\prime}=\kappa_{* 0}, \kappa_{2}^{\prime}=\kappa_{2 \delta}$, and $\kappa_{1}(\mathbf{x})=$ $\kappa_{2}(\mathbf{x})=1$ yields

$$
\begin{aligned}
\left\|\left\langle\Lambda j_{\delta} \alpha_{*}, \Lambda j_{\delta} \beta\right\rangle\right\|_{\mathfrak{S}} & \leq N_{d_{\Omega}}\left(\Lambda j_{\delta} ; 1, \kappa_{* 0}\right) N_{d_{\Omega}}\left(\Lambda j_{\delta} ; 1, \kappa_{2 \delta}\right) \\
& \leq\left(4 \mathrm{R}_{+}\left\|j_{\delta}\right\|\right)^{2} \leq 16 K_{j}^{2} e^{2 K_{j}} \delta^{2} e^{-2 \mathrm{mc}} \mathrm{R}_{+}^{2}
\end{aligned}
$$

by (III.7), Remark G.4.i, Lemma B.1.i and Lemma III.21.iii.

Combining (V.27) and (V.28), we have

$$
\begin{aligned}
\left\|\delta Q\left(\alpha_{*}, \beta ; \vec{\rho}\right)\right\|_{\mathfrak{S}} & \leq 4 e^{-\frac{1}{4} \mathrm{~m} \mathfrak{c}(\delta)}+\left\{2^{8} K_{j} e^{K_{j}}+16 K_{j}^{2} e^{2 K_{j}} \delta \mathrm{R}_{+} e^{-\mathrm{m} \mathfrak{c}}\right\} \delta \mathrm{rR}+e^{-\mathrm{m} \mathfrak{c}} \\
& \leq 4 e^{-\frac{1}{4} \mathrm{~m} \mathfrak{c}(\delta)}+\left\{2^{8} K_{j} e^{K_{j}}+\frac{1}{40}\right\} \delta \mathrm{rR}_{+} e^{-\mathrm{m} \mathfrak{c}}
\end{aligned}
$$

by (F.4.b) and (F.6.a).

\section{Bound on the Quartic Part of the Fluctuation Action}

Finally, we treat the quartic contribution to $f_{\mathfrak{S}}\left(\alpha_{*}, \beta ; \vec{\rho} ; z_{*}, z\right)$ identified in Remark V.5.ii. We write it as

$$
\begin{aligned}
\mathcal{V}_{\mathfrak{S}_{1}}\left(\varepsilon ; \alpha_{*}, z+z_{\delta}+\alpha_{\delta}^{\mathrm{cr}} ; \vec{\alpha}_{*_{l}}, \vec{\alpha}_{l}\right)+\mathcal{V}_{\mathfrak{S}_{2}}\left(\varepsilon ; z_{*}+z_{* \delta}+\alpha_{* \delta}^{\mathrm{cr}}, \beta ; \vec{\alpha}_{*_{r}}, \vec{\alpha}_{r}\right) \\
\quad=\mathcal{V}_{\mathfrak{S}}\left(\varepsilon ; \alpha_{*}, \beta ; \vec{\alpha}_{*}, \vec{\alpha}\right)+\delta \mathcal{V}\left(\varepsilon ; \alpha_{*}, \beta ; \vec{\rho}\right)+\mathcal{V}_{l}\left(\varepsilon ; \alpha_{*}, \beta ; \vec{\rho} ; z\right)+\mathcal{V}_{r}\left(\varepsilon ; \alpha_{*}, \beta ; \vec{\rho} ; z_{*}\right)
\end{aligned}
$$

where

$$
\begin{aligned}
\delta \mathcal{V} & =-\mathcal{V}_{\mathfrak{S}}\left(\varepsilon ; \alpha_{*}, \beta ; \vec{\alpha}_{*}, \vec{\alpha}\right)+\mathcal{V}_{\mathfrak{S}_{1}}\left(\varepsilon ; \alpha_{*}, z_{\delta}+\alpha_{\delta}^{\mathrm{cr}} ; \vec{\alpha}_{*_{l}}, \vec{\alpha}_{l}\right)+\mathcal{V}_{\mathfrak{S}_{2}}\left(\varepsilon ; z_{* \delta}+\alpha_{* \delta}^{\mathrm{cr}}, \beta ; \vec{\alpha}_{*_{r}}, \vec{\alpha}_{r}\right) \\
\mathcal{V}_{l} & =\mathcal{V}_{\mathfrak{S}_{1}}\left(\varepsilon ; \alpha_{*}, z+z_{\delta}+\alpha_{\delta}^{\mathrm{cr}} ; \vec{\alpha}_{*_{l}}, \vec{\alpha}_{l}\right)-\mathcal{V}_{\mathfrak{S}_{1}}\left(\varepsilon ; \alpha_{*}, z_{\delta}+\alpha_{\delta}^{\mathrm{cr}} ; \vec{\alpha}_{*_{l}}, \vec{\alpha}_{l}\right) \\
\mathcal{V}_{r} & =\mathcal{V}_{\mathfrak{S}_{2}}\left(\varepsilon ; z_{*}+z_{* \delta}+\alpha_{* \delta}^{\mathrm{cr}}, \beta ; \vec{\alpha}_{*_{r}}, \vec{\alpha}_{r}\right)-\mathcal{V}_{\mathfrak{S}_{2}}\left(\varepsilon ; z_{* \delta}+\alpha_{* \delta}^{\mathrm{cr}}, \beta ; \vec{\alpha}_{*_{r}}, \vec{\alpha}_{r}\right)
\end{aligned}
$$


Similarly,

$$
\mathcal{V}_{\mathfrak{S}_{1}}\left(\frac{\varepsilon}{2} ; \alpha_{*}, z+z_{\delta}+\alpha_{\delta}^{\mathrm{cr}} ; \cdots\right)+\mathcal{V}_{\mathfrak{S}_{2}}\left(\frac{\varepsilon}{2} ; z_{*}+z_{* \delta}+\alpha_{* \delta}^{\mathrm{cr}}, \beta ; \cdots\right)=\mathcal{V}_{\mathfrak{S}}\left(\frac{\varepsilon}{2}\right)+\widetilde{\delta \mathcal{V}}+\tilde{\mathcal{V}}_{l}+\tilde{\mathcal{V}}_{r}
$$

where

$$
\begin{aligned}
\widetilde{\delta \mathcal{V}} & =-\mathcal{V}_{\mathfrak{S}}\left(\frac{\varepsilon}{2} ; \alpha_{*}, \beta ; \vec{\alpha}_{*}, \vec{\alpha}\right)+\mathcal{V}_{\mathfrak{S}_{1}}\left(\frac{\varepsilon}{2} ; \alpha_{*}, z_{\delta}+\alpha_{\delta}^{\mathrm{cr}} ; \vec{\alpha}_{*_{l}}, \vec{\alpha}_{l}\right)+\mathcal{V}_{\mathfrak{S}_{2}}\left(\frac{\varepsilon}{2} ; z_{* \delta}+\alpha_{* \delta}^{\mathrm{cr}}, \beta ; \vec{\alpha}_{*_{r}}, \vec{\alpha}_{r}\right) \\
\tilde{\mathcal{V}}_{l} & =\mathcal{V}_{\mathfrak{S}_{1}}\left(\frac{\varepsilon}{2} ; \alpha_{*}, z+z_{\delta}+\alpha_{\delta}^{\mathrm{cr}} ; \vec{\alpha}_{*_{l}}, \vec{\alpha}_{l}\right)-\mathcal{V}_{\mathfrak{S}_{1}}\left(\frac{\varepsilon}{2} ; \alpha_{*}, z_{\delta}+\alpha_{\delta}^{\mathrm{cr}} ; \vec{\alpha}_{*_{l}}, \vec{\alpha}_{l}\right) \\
\tilde{\mathcal{V}}_{r} & =\mathcal{V}_{\mathfrak{S}_{2}}\left(\frac{\varepsilon}{2} ; z_{*}+z_{* \delta}+\alpha_{* \delta}^{\mathrm{cr}}, \beta ; \vec{\alpha}_{*_{r}}, \vec{\alpha}_{r}\right)-\mathcal{V}_{\mathfrak{S}_{2}}\left(\frac{\varepsilon}{2} ; z_{* \delta}+\alpha_{* \delta}^{\mathrm{cr}}, \beta ; \vec{\alpha}_{*_{r}}, \vec{\alpha}_{r}\right)
\end{aligned}
$$

Proposition V.13 Set $K_{\mathcal{V}}=2^{25} e^{6 K_{j}}$

(i)

$$
\begin{array}{r}
\left\|\mathcal{V}_{l}\right\|_{\text {fluct }},\left\|\mathcal{V}_{r}\right\|_{\text {fluct }} \leq K_{\mathcal{V}} \delta\|v\| \mathrm{r} \mathrm{R}_{+}^{3} \\
\left\|\mathcal{V}_{l}-\tilde{\mathcal{V}}_{l}\right\|_{\text {fluct }},\left\|\mathcal{V}_{r}-\tilde{\mathcal{V}}_{r}\right\|_{\text {fluct }} \leq K_{\mathcal{V}} \varepsilon \delta\|v\| \mathrm{r} \mathrm{R}_{+}^{3}
\end{array}
$$

(ii)

$$
\begin{aligned}
\|\delta \mathcal{V}\|_{\mathfrak{S}} & \leq K_{\mathcal{V}} \delta\|v\| \mathrm{rR}_{+}^{3} \\
\|\delta \mathcal{V}-\widetilde{\delta \mathcal{V}}\|_{\mathfrak{S}} & \leq K_{\mathcal{V}} \varepsilon \delta\|v\| \mathrm{rR}_{+}^{3}
\end{aligned}
$$

\section{Proof:}

(i) We treat $\mathcal{V}_{l}$. By (V.16), (V.18) and (V.20)

$$
\begin{aligned}
\Gamma_{* \mathfrak{S}_{1}}\left(\tau ; \alpha_{*}, \vec{\alpha}_{* l}\right) & =\tilde{\Gamma}_{*}\left(\tau ; \alpha_{*}, \vec{\alpha}_{*}\right)=\Gamma_{* \mathfrak{S}}\left(\tau ; \alpha_{*}, \vec{\alpha}_{*}\right)=\gamma_{* \tau} \\
\Gamma_{\mathfrak{S}_{1}}\left(\tau ; \vec{\alpha}_{l}, z+z_{\delta}+\alpha_{\delta}^{\mathrm{cr}}\right) & =\Gamma_{\tau}^{\delta}\left(\mathfrak{S}_{1}\right) z+\Gamma_{\tau}^{\delta}\left(\mathfrak{S}_{1}\right) z_{\delta}+\Gamma_{\mathfrak{S}_{1}}\left(\tau ; \vec{\alpha}_{l}, \alpha_{\delta}^{\mathrm{cr}}\right) \\
& =\Gamma_{\tau}^{\delta}\left(\mathfrak{S}_{1}\right) z+\Gamma_{\tau}^{\delta}\left(\mathfrak{S}_{1}\right) z_{\delta}+\tilde{\Gamma}(\tau ; \vec{\alpha}, \beta) \\
& =\Gamma_{\tau}^{\delta}\left(\mathfrak{S}_{1}\right) z+\Gamma_{\tau}^{\delta}\left(\mathfrak{S}_{1}\right) z_{\delta}+\Gamma_{\mathfrak{S}}(\tau ; \vec{\alpha}, \beta)-\partial_{\mathfrak{c}} \Gamma_{\tau} \beta \\
& =\Gamma_{\tau}^{\delta}\left(\mathfrak{S}_{1}\right) z+\Gamma_{\tau}^{\delta}\left(\mathfrak{S}_{1}\right) z_{\delta}+\gamma_{\tau}-\partial_{\mathfrak{c}} \Gamma_{\tau} \beta
\end{aligned}
$$

for all $\tau \in(0, \delta)$. When $\tau=0$, the right hand side $\gamma_{* \tau}=\alpha_{*}$ and, with the convention $\Gamma_{\delta}^{\delta}\left(\mathfrak{S}_{1}\right)=\mathbb{1}$, when $\tau=\delta$ the right hand side $\Gamma_{\tau}^{\delta}\left(\mathfrak{S}_{1}\right) z+\Gamma_{\tau}^{\delta}\left(\mathfrak{S}_{1}\right) z_{\delta}+\tilde{\Gamma}(\delta ; \vec{\alpha}, \beta)=z+z_{\delta}+\alpha_{\delta}^{\mathrm{cr}}$. Similarly,

$$
\begin{aligned}
\Gamma_{* \mathfrak{S}_{2}}\left(\tau-\delta ; z_{*}+z_{* \delta}+\alpha_{* \delta}^{\mathrm{cr}}, \vec{\alpha}_{* r}\right) & =\Gamma_{* \tau-\delta}^{0}\left(\mathfrak{S}_{2}\right) z_{*}+\Gamma_{* \tau-\delta}^{0}\left(\mathfrak{S}_{2}\right) z_{* \delta}+\gamma_{* \tau}-\partial_{\mathfrak{c}} \Gamma_{* \tau} \alpha_{*} \\
\Gamma_{\mathfrak{S}_{2}}\left(\tau-\delta ; \vec{\alpha}_{r}, \beta\right) & =\gamma_{\tau}
\end{aligned}
$$

for all $\tau \in(\delta, 2 \delta)$. So, by the Definition III.8.i of $\mathcal{V}_{\mathfrak{S}_{1}}$,

$$
\begin{aligned}
\mathcal{V}_{\mathfrak{S}_{1}}\left(\varepsilon ; \alpha_{*}, z+z_{\delta}+\alpha_{\delta}^{\mathrm{cr}} ; \vec{\alpha}_{* l}, \vec{\alpha}_{l}\right) & \\
\quad=-\varepsilon & \sum_{\tau \in \varepsilon \mathbb{Z} \cap[0, \delta)}\left\langle\gamma_{* \tau}\left(\Gamma_{\tau+\varepsilon}^{\delta}\left(\mathfrak{S}_{1}\right) z+g_{\tau+\varepsilon}\right), v \gamma_{* \tau}\left(\Gamma_{\tau+\varepsilon}^{\delta}\left(\mathfrak{S}_{1}\right) z+g_{\tau+\varepsilon}\right)\right\rangle
\end{aligned}
$$


where

$$
\begin{aligned}
g_{\tau} & =\Gamma_{\tau}^{\delta}\left(\mathfrak{S}_{1}\right) z_{\delta}+\gamma_{\tau}-\partial_{\mathfrak{c}} \Gamma_{\tau} \beta \\
g_{* \tau} & =\Gamma_{* \tau-\delta}^{0}\left(\mathfrak{S}_{2}\right) z_{* \delta}+\gamma_{* \tau}-\partial_{\mathfrak{c}} \Gamma_{* \tau} \alpha_{*}
\end{aligned}
$$

Consequently,

$$
\begin{gathered}
\mathcal{V}_{l}=-\varepsilon \sum_{\tau \in \varepsilon \mathbb{Z} \cap[0, \delta)}\left[\left\langle\gamma_{* \tau}\left(\Gamma_{\tau+\varepsilon}^{\delta}\left(\mathfrak{S}_{1}\right) z+g_{\tau+\varepsilon}\right), v \gamma_{* \tau}\left(\Gamma_{\tau+\varepsilon}^{\delta}\left(\mathfrak{S}_{1}\right) z+g_{\tau+\varepsilon}\right)\right\rangle\right. \\
\left.-\left\langle\gamma_{* \tau} g_{\tau+\varepsilon}, v \gamma_{* \tau} g_{\tau+\varepsilon}\right\rangle\right]
\end{gathered}
$$

To each term, we apply Corollary G.3.ii, $r=4$,

$$
h\left(\gamma_{1}, \cdots, \gamma_{4}\right)=\left\langle\gamma_{1} \gamma_{2}, v \gamma_{3} \gamma_{4}\right\rangle \quad \lambda_{1}=\lambda_{2}=\lambda_{3}=\lambda_{4}=1
$$

and the $s$ fields $\alpha_{1}, \cdots, \alpha_{s}$ being $z, z_{\delta}, \beta, \alpha_{*}, \alpha_{* \varepsilon}, \cdots, \alpha_{* \delta-\varepsilon}, \alpha_{\varepsilon}, \cdots, \alpha_{2 \delta-\varepsilon}$. Recalling that

$$
\begin{aligned}
\gamma_{* \tau} & =\Gamma_{* \tau}^{0}(\mathfrak{S}) \alpha_{*}+\sum_{\tau^{\prime} \in \varepsilon \mathbb{Z} \cap(0, \delta)} \Gamma_{* \tau}^{\tau^{\prime}}(\mathfrak{S}) \alpha_{* \tau^{\prime}} \\
g_{\tau+\varepsilon} & =\Gamma_{\tau+\varepsilon}^{\delta}\left(\mathfrak{S}_{1}\right) z_{\delta}+\sum_{\tau^{\prime \prime} \in \varepsilon \mathbb{Z} \cap(0,2 \delta)} \Gamma_{\tau+\varepsilon}^{\tau^{\prime \prime}}(\mathfrak{S}) \alpha_{\tau^{\prime \prime}}+\Gamma_{\tau+\varepsilon}^{2 \delta}(\mathfrak{S}) \beta-\partial_{\mathfrak{c}} \Gamma_{\tau+\varepsilon} \beta
\end{aligned}
$$

we have, as coefficients for the substitution,

$$
\begin{array}{llrl}
\Gamma_{1}^{\alpha_{*}}=\Gamma_{3}^{\alpha_{*}}=\Gamma_{* \tau}^{0}(\mathfrak{S}) & =\tilde{\Gamma}_{1}^{\alpha_{*}}=\tilde{\Gamma}_{3}^{\alpha_{*}} \\
\Gamma_{1}^{\alpha_{* \tau^{\prime}}}=\Gamma_{3}^{\alpha_{* \tau^{\prime}}}=\Gamma_{* \tau}^{\tau^{\prime}}(\mathfrak{S}) & =\tilde{\Gamma}_{1}^{\alpha_{* \tau^{\prime}}}=\tilde{\Gamma}_{3}^{\alpha_{* \tau^{\prime}}} \\
\Gamma_{2}^{z}=\Gamma_{4}^{z}=\Gamma_{\tau+\varepsilon}^{\delta}\left(\mathfrak{S}_{1}\right) \Omega & \tilde{\Gamma}_{2}^{z}=\tilde{\Gamma}_{4}^{z}=0 \\
\Gamma_{2}^{z}=\Gamma_{4}^{z}=\Gamma_{\tau+\varepsilon}^{\delta}\left(\mathfrak{S}_{1}\right)(\Lambda \backslash \Omega) & =\tilde{\Gamma}_{2}^{z}=\tilde{\Gamma}_{4}^{z_{\delta}} \\
\Gamma_{2}^{\beta}=\Gamma_{4}^{\beta}=\Gamma_{\tau+\varepsilon}^{2 \delta}(\mathfrak{S})-\partial_{\mathfrak{c}} \Gamma_{\tau+\varepsilon} & =\tilde{\Gamma}_{2}^{\beta}=\tilde{\Gamma}_{4}^{\beta} \\
\Gamma_{2}^{\alpha_{\tau^{\prime \prime}}}=\Gamma_{4}^{\alpha_{\tau^{\prime \prime}}}=\Gamma_{\tau+\varepsilon}^{\tau^{\prime \prime}}(\mathfrak{S}) & & =\tilde{\Gamma}_{2}^{\alpha_{\tau^{\prime \prime}}}=\tilde{\Gamma}_{4}^{\alpha_{\tau^{\prime \prime}}}
\end{array}
$$

with $\tau^{\prime} \in \varepsilon \mathbb{Z} \cap(0, \delta), \tau^{\prime \prime} \in \varepsilon \mathbb{Z} \cap(0,2 \delta)$ and with all other $\Gamma_{i}^{j}$ 's and $\tilde{\Gamma}_{i}^{j}$ 's being zero. We apply Corollary G.3.ii with $L=\Lambda, d$ replaced by $4 \mathrm{~m} d, d^{\prime}=d_{\Omega}$ and $\delta=\frac{\mathrm{m}}{2} d$. The Corollary gives

$$
\begin{aligned}
& \|\left\langle\gamma_{* \tau}\left(\Gamma_{\tau+\varepsilon}^{\delta}\left(\mathfrak{S}_{1}\right) z+g_{\tau+\varepsilon}\right), v \gamma_{* \tau}\right.\left.\left(\Gamma_{\tau+\varepsilon}^{\delta}\left(\mathfrak{S}_{1}\right) z+g_{\tau+\varepsilon}\right)\right\rangle-\left\langle\gamma_{* \tau} g_{\tau+\varepsilon}, v \gamma_{* \tau} g_{\tau+\varepsilon}\right\rangle \|_{\text {fluct }} \\
& \leq 4\|v\| \sigma_{\delta} \sigma^{3}
\end{aligned}
$$

where

$$
\begin{aligned}
\sigma & \leq \max \left\{\sigma_{* \tau}, \sigma_{\tau+\varepsilon}\right\} \\
\sigma_{\delta} & \leq N_{d_{\Omega}}\left(\Gamma_{\tau+\varepsilon}^{\delta}\left(\mathfrak{S}_{1}\right) \Omega ; e^{-\frac{3}{2} \mathrm{~m} d(\mathbf{x}, \Lambda)}, \tilde{\lambda}\right)
\end{aligned}
$$


with

$$
\begin{aligned}
\sigma_{* \tau}= & \sum_{\tau^{\prime} \in[0, \delta)} N_{d_{\Omega}}\left(\Gamma_{* \tau}^{\tau^{\prime}}(\mathfrak{S}) ; e^{\frac{\mathrm{m}}{2} d(\mathbf{x}, \Lambda)}, \kappa_{* \tau^{\prime}}\right) \\
\sigma_{\tau}= & N_{d_{\Omega}}\left(\Gamma_{\tau}^{\delta}\left(\mathfrak{S}_{1}\right) \Omega ; e^{\frac{\mathrm{m}}{2} d(\mathbf{x}, \Lambda)}, \tilde{\lambda}\right)+N_{d_{\Omega}}\left(\Gamma_{\tau}^{\delta}\left(\mathfrak{S}_{1}\right)(\Lambda \backslash \Omega) ; e^{\frac{\mathrm{m}}{2} d(\mathbf{x}, \Lambda)}, \lambda_{\delta}\right) \\
& +N_{d_{\Omega}}\left(\partial_{\mathfrak{c}} \Gamma_{\tau} ; e^{\frac{\mathrm{m}}{2} d(\mathbf{x}, \Lambda)}, \kappa_{2 \delta}\right)+\sum_{\tau^{\prime \prime} \in(0,2 \delta]} N_{d_{\Omega}}\left(\Gamma_{\tau}^{\tau^{\prime \prime}}(\mathfrak{S}) ; e^{\frac{\mathrm{m}}{2} d(\mathbf{x}, \Lambda)}, \kappa_{\tau^{\prime \prime}}\right)
\end{aligned}
$$

Below we prove that

$$
\begin{aligned}
\sigma_{\delta} & \leq 32 e^{K_{j}} \mathrm{r} \\
\sigma_{* \tau} & \leq 16 e^{K_{j} \tau} \mathrm{R}_{+} \\
\sigma_{\tau} & \leq 32 e^{K_{j}} \mathrm{R}_{+}
\end{aligned}
$$

Consequently, the fluctuation norm of each term in (V.31) is bounded by

$$
4\|v\|\left(32 e^{K_{j}} \mathrm{r}\right)\left(32 e^{K_{j}} \mathrm{R}_{+}\right)^{3} \leq 2^{22} e^{4 K_{j}}\|v\| \| \mathrm{r} \mathrm{R}_{+}^{3}
$$

Summing over $\tau$ and multiplying by $\varepsilon$ gives the desired bound on $\mathcal{V}_{l}$.

By (V.31),

$$
\mathcal{V}_{l}-\tilde{\mathcal{V}}_{l}=\mathcal{W}_{1}+\mathcal{W}_{2}
$$

where

$$
\begin{array}{r}
\mathcal{W}_{1}=-\frac{\varepsilon}{2} \sum_{\tau \in \varepsilon \mathbb{Z} \cap[0, \delta)}\left[\left\langle\gamma_{* \tau}\left(\Gamma_{\tau+\varepsilon}^{\delta}\left(\mathfrak{S}_{1}\right) z+g_{\tau+\varepsilon}\right), v \gamma_{* \tau}\left(\Gamma_{\tau+\varepsilon}^{\delta}\left(\mathfrak{S}_{1}\right) z+g_{\tau+\varepsilon}\right)\right\rangle\right. \\
-\left\langle\gamma_{* \tau}\left(\Gamma_{\tau+\frac{\varepsilon}{2}}^{\delta}\left(\mathfrak{S}_{1}\right) z+g_{\tau+\frac{\varepsilon}{2}}\right), v \gamma_{* \tau}\left(\Gamma_{\tau+\frac{\varepsilon}{2}}^{\delta}\left(\mathfrak{S}_{1}\right) z+g_{\tau+\frac{\varepsilon}{2}}\right)\right\rangle \\
\left.\quad-\left\langle\gamma_{* \tau} g_{\tau+\varepsilon}, v \gamma_{* \tau} g_{\tau+\varepsilon}\right\rangle+\left\langle\gamma_{* \tau} g_{\tau+\frac{\varepsilon}{2}}, v \gamma_{* \tau} g_{\tau+\frac{\varepsilon}{2}}\right\rangle\right]
\end{array}
$$

and

$$
\begin{aligned}
\mathcal{W}_{2}=-\frac{\varepsilon}{2} \sum_{\tau \in \varepsilon \mathbb{Z} \cap[0, \delta)}\left[\left\langle\gamma_{* \tau}\left(\Gamma_{\tau+\varepsilon}^{\delta}\left(\mathfrak{S}_{1}\right) z+g_{\tau+\varepsilon}\right), v \gamma_{* \tau}\left(\Gamma_{\tau+\varepsilon}^{\delta}\left(\mathfrak{S}_{1}\right) z+g_{\tau+\varepsilon}\right)\right\rangle\right. \\
-\left\langle\gamma_{* \tau+\frac{\varepsilon}{2}}\left(\Gamma_{\tau+\varepsilon}^{\delta}\left(\mathfrak{S}_{1}\right) z+g_{\tau+\varepsilon}\right), v \gamma_{* \tau+\frac{\varepsilon}{2}}\left(\Gamma_{\tau+\varepsilon}^{\delta}\left(\mathfrak{S}_{1}\right) z+g_{\tau+\varepsilon}\right)\right\rangle \\
\left.-\left\langle\gamma_{* \tau} g_{\tau+\varepsilon}, v \gamma_{* \tau} g_{\tau+\varepsilon}\right\rangle+\left\langle\gamma_{* \tau+\frac{\varepsilon}{2}} g_{\tau+\varepsilon}, v \gamma_{* \tau+\frac{\varepsilon}{2}} g_{\tau+\varepsilon}\right\rangle\right]
\end{aligned}
$$

By Lemma E.14, (V.30), (V.19) and Remark E.19,

$$
\Gamma_{\tau+\frac{\varepsilon}{2}}^{\delta}\left(\mathfrak{S}_{1}\right)=j\left(\frac{\varepsilon}{2}\right) \Gamma_{\tau+\varepsilon}^{\delta}\left(\mathfrak{S}_{1}\right) \quad g_{\tau+\frac{\varepsilon}{2}}=j\left(\frac{\varepsilon}{2}\right) g_{\tau+\varepsilon} \quad \gamma_{* \tau+\frac{\varepsilon}{2}}=j\left(\frac{\varepsilon}{2}\right) \gamma_{* \tau}
$$


for all $\tau \in \varepsilon \mathbb{Z} \cap[0, \delta)$. Therefore the $\tau$ term of $\mathcal{W}_{1}$ is

$$
\begin{aligned}
& \left\langle\gamma_{* \tau}\left(\Gamma_{\tau+\varepsilon}^{\delta}\left(\mathfrak{S}_{1}\right) z+g_{\tau+\varepsilon}\right), v \gamma_{* \tau}\left(\Gamma_{\tau+\varepsilon}^{\delta}\left(\mathfrak{S}_{1}\right) z+g_{\tau+\varepsilon}\right)\right\rangle \\
& -\left\langle\gamma_{* \tau}\left(j\left(\frac{\varepsilon}{2}\right) \Gamma_{\tau+\varepsilon}^{\delta}\left(\mathfrak{S}_{1}\right) z+j\left(\frac{\varepsilon}{2}\right) g_{\tau+\varepsilon}\right), v \gamma_{* \tau}\left(j\left(\frac{\varepsilon}{2}\right) \Gamma_{\tau+\varepsilon}^{\delta}\left(\mathfrak{S}_{1}\right) z+j\left(\frac{\varepsilon}{2}\right) g_{\tau+\varepsilon}\right)\right\rangle \\
& \quad-\left\langle\gamma_{* \tau} g_{\tau+\varepsilon}, v \gamma_{* \tau} g_{\tau+\varepsilon}\right\rangle+\left\langle\gamma_{* \tau} j\left(\frac{\varepsilon}{2}\right) g_{\tau+\varepsilon}, v \gamma_{* \tau} j\left(\frac{\varepsilon}{2}\right) g_{\tau+\varepsilon}\right\rangle
\end{aligned}
$$

We again apply Corollary G.3, this time part (iii), but with the same metrics, $h, \Gamma_{i}^{j}$,s and $\tilde{\Gamma}_{i}^{j}$ 's as before and with, in addition,

$$
A_{1}=\tilde{A}_{1}=A_{3}=\tilde{A}_{3}=\mathbb{1} \quad A_{2}=A_{4}=\mathfrak{h} \quad \tilde{A}_{2}=\tilde{A}_{4}=j\left(\frac{\varepsilon}{2}\right)
$$

The corollary bounds the fluctuation norm of the $\tau$ term by $16\|v\| \sigma_{\delta} a_{\delta}(\sigma a)^{3}$ where

$$
\begin{aligned}
a & \leq \max \left\{\|1\|\|,\| j\left(\frac{\varepsilon}{2}\right)\|\|\right\} \leq e^{K_{j} \frac{\varepsilon}{2}} \\
a_{\delta} & \leq\left\|j\left(\frac{\varepsilon}{2}\right)-\mathfrak{h}\right\| \|=K_{j} \frac{\varepsilon}{2} e^{K_{j} \frac{\varepsilon}{2}}
\end{aligned}
$$

by Lemma III.21. By this and (V.32)

$$
\begin{aligned}
16\|v\| \sigma_{\delta} a_{\delta}(\sigma a)^{3} & \leq 16\|v\|\left(32 e^{K_{j}} \mathrm{r}\right)\left(K_{j} \frac{\varepsilon}{2} e^{K_{j} \frac{\varepsilon}{2}}\right)\left(32 e^{K_{j}} \mathrm{R}_{+} e^{K_{j} \frac{\varepsilon}{2}}\right)^{3} \\
& \leq 2^{23} e^{6 K_{j}} \varepsilon\|v\| \mathrm{r} \mathrm{R}_{+}^{3}
\end{aligned}
$$

Summing over $\tau$ and multiplying by $\frac{\varepsilon}{2}$ shows that

$$
\left\|\mathcal{W}_{1}\right\|_{\text {fluct }} \leq 2^{22} e^{6 K_{j}} \varepsilon \delta\|v\| \| \mathrm{r}_{+}^{3}
$$

The same bound applies to $\left\|\mathcal{W}_{2}\right\|_{\text {fluct }}$. This gives the desired bound on $\mathcal{V}_{l}-\tilde{\mathcal{V}}_{l}$.

Finally, we prove (V.32). By (III.7) and Lemma E.13,

$$
\begin{gathered}
\sum_{\tau^{\prime} \in[0,2 \delta)} N_{d_{\Omega}}\left(\Gamma_{* \tau}^{\tau^{\prime}}(\mathfrak{S}) ; e^{\frac{\mathrm{m}}{2} d(\mathbf{x}, \Lambda)}, \kappa_{* \tau^{\prime}}\right) \leq 16 e^{K_{j} \tau} \mathrm{R}_{+} \\
\sum_{\tau^{\prime} \in(0,2 \delta]} N_{d_{\Omega}}\left(\Gamma_{\tau}^{\tau^{\prime}}(\mathfrak{S}) ; e^{\frac{\mathrm{m}}{2} d(\mathbf{x}, \Lambda)}, \kappa_{\tau^{\prime}}\right) \leq 16 e^{K_{j} \tau} \mathrm{R}_{+}
\end{gathered}
$$

This gives the second line of (V.32).

If $\mathcal{J}$ is the smallest decimation interval that strictly contains $[\tau, \delta]$ and has $\delta$ as its right endpoint and if $\tau_{\mathcal{J}}$ denotes the midpoint of $\mathcal{J}$, then

$$
\begin{aligned}
N_{d_{\Omega}}\left(\Gamma_{\tau}^{\delta}\left(\mathfrak{S}_{1}\right) \Omega ; e^{-\frac{3}{2} \mathrm{~m} d(\mathbf{x}, \Lambda)}, \tilde{\lambda}\right) & =N_{d_{\Omega}}\left(j\left(\tau_{\mathcal{J}}-\tau\right) \Lambda(\mathcal{J}) j\left(\delta-\tau_{\mathcal{J}}\right) \Omega ; e^{-\frac{3}{2} \mathrm{~m} d(\mathbf{x}, \Lambda)}, \tilde{\lambda}\right) \\
& \leq 32 \mathrm{r}\left\|j\left(\tau_{\mathcal{J}}-\tau\right)\right\|\|\| j\left(\delta-\tau_{\mathcal{J}}\right)\|\| \\
& \leq 32 e^{K_{j}} \mathrm{r}
\end{aligned}
$$


by (III.7) and Lemma G.5.ii with $d$ replaced by $d_{\Omega}$, R replaced by $32 \mathrm{r}$ and

$$
\begin{gathered}
L_{1}=X, L_{2}=\Lambda(\mathcal{J}), L_{3}=\Omega, \quad O_{1}=\Lambda, O_{2}=X \\
\delta_{1}=\frac{3}{2} \mathrm{~m} d, \quad \delta_{2}=0, \delta=0, \tilde{d}=4 \mathrm{~m} d, \quad \kappa=\tilde{\lambda}
\end{gathered}
$$

(The hypothesis that $\kappa(\mathbf{x})=\tilde{\lambda}(\mathbf{x}) \leq \mathrm{R}=32 \mathrm{r}$ for all $\mathbf{x} \in L_{3}=\Omega$ is fulfilled by the definition (V.12) of $\tilde{\lambda}$ ). This gives the first line of (V.32).

Similarly

$$
N_{d_{\Omega}}\left(\Gamma_{\tau}^{\delta}\left(\mathfrak{S}_{1}\right)(\Lambda \backslash \Omega) ; e^{\frac{\mathrm{m}}{2} d(\mathbf{x}, \Lambda)}, \lambda_{\delta}\right) \leq N_{d_{\Omega}}\left(\Gamma_{\tau}^{\delta}\left(\mathfrak{S}_{1}\right)(\Lambda \backslash \Omega) ; e^{-\frac{3}{2} \mathrm{~m} d(\mathbf{x}, \Lambda)}, \lambda_{\delta}\right) \leq 32 e^{K_{j}} \mathrm{r}
$$

By Lemma E.18

$$
N_{d_{\Omega}}\left(\partial_{\mathfrak{c}} \Gamma_{\tau} ; e^{\frac{\mathrm{m}}{2} d(\mathbf{x}, \Lambda)}, \kappa_{2 \delta}\right) \leq N_{d_{\Omega}}\left(\partial_{\mathfrak{c}} \Gamma_{\tau} ; e^{-\frac{3}{2} \mathrm{~m} d(\mathbf{x}, \Lambda)}, \kappa_{2 \delta}\right) \leq 4 e^{2 K_{j}} \mathrm{R}_{+}\left(\delta e^{-\mathrm{m} \mathfrak{c}}+e^{-\operatorname{mc}(\delta)}\right)
$$

Combining these two bounds with (V.34) and (V.35) gives

$$
\begin{aligned}
\sigma_{\tau} & \leq 32 e^{K_{j}} \mathrm{r}+32 e^{K_{j}} \mathrm{r}+4 e^{2 K_{j}} \mathrm{R}_{+}\left(\delta e^{-\mathrm{m} \mathfrak{c}}+e^{-\mathrm{mc}(\delta)}\right)+16 e^{K_{j} \tau} \mathrm{R}_{+} \\
& \leq 32 e^{K_{j}} \mathrm{R}_{+}
\end{aligned}
$$

This completes the proof of part (i) of the proposition.

(ii) With the notation (V.30) we have

$$
\delta \mathcal{V}=\delta \mathcal{V}_{1}(\varepsilon)+\delta \mathcal{V}_{2}(\varepsilon)
$$

where

$$
\begin{aligned}
& \delta \mathcal{V}_{1}(\varepsilon)=\varepsilon \sum_{\tau \in[0, \delta)}\left\{\left\langle\gamma_{* \tau} \gamma_{\tau+\varepsilon}, v \gamma_{* \tau} \gamma_{\tau+\varepsilon}\right\rangle-\left\langle\gamma_{* \tau} g_{\tau+\varepsilon}, v \gamma_{* \tau} g_{\tau+\varepsilon}\right\rangle\right\} \\
& \delta \mathcal{V}_{2}(\varepsilon)=\varepsilon \sum_{\tau \in[\delta, 2 \delta)}\left\{\left\langle\gamma_{* \tau} \gamma_{\tau+\varepsilon}, v \gamma_{* \tau} \gamma_{\tau+\varepsilon}\right\rangle-\left\langle g_{* \tau} \gamma_{\tau+\varepsilon}, v g_{* \tau} \gamma_{\tau+\varepsilon}\right\rangle\right\}
\end{aligned}
$$

We bound $\delta \mathcal{V}_{1}$. To treat the $\tau^{\text {th }}$ term, we again apply Corollary G.3.ii with $r=4$,

$$
h\left(\gamma_{1}, \cdots, \gamma_{4}\right)=\left\langle\gamma_{1} \gamma_{2}, v \gamma_{3} \gamma_{4}\right\rangle \quad \lambda_{1}=\lambda_{2}=\lambda_{3}=\lambda_{4}=1
$$

This time the $s$ fields $\alpha_{1}, \cdots, \alpha_{s}$ are $z_{\delta}, \beta, \alpha_{*}, \alpha_{* \varepsilon}, \cdots, \alpha_{* \delta-\varepsilon}, \alpha_{\varepsilon}, \cdots, \alpha_{2 \delta-\varepsilon}$. Recalling that

$$
\begin{aligned}
\gamma_{* \tau} & =\Gamma_{* \tau}^{0}(\mathfrak{S}) \alpha_{*}+\sum_{\tau^{\prime} \in \varepsilon \mathbb{Z} \cap(0, \delta)} \Gamma_{* \tau}^{\tau^{\prime}}(\mathfrak{S}) \alpha_{* \tau^{\prime}} \\
\gamma_{\tau+\varepsilon} & =\sum_{\tau^{\prime} \in \varepsilon \mathbb{Z} \cap(0,2 \delta)} \Gamma_{\tau+\varepsilon}^{\tau^{\prime}}(\mathfrak{S}) \alpha_{\tau^{\prime}}+\Gamma_{\tau+\varepsilon}^{2 \delta}(\mathfrak{S}) \beta \\
g_{\tau+\varepsilon} & =\Gamma_{\tau+\varepsilon}^{\delta}\left(\mathfrak{S}_{1}\right) z_{\delta}+\sum_{\tau^{\prime} \in \varepsilon \mathbb{Z} \cap(0,2 \delta)} \Gamma_{\tau+\varepsilon}^{\tau^{\prime}}(\mathfrak{S}) \alpha_{\tau^{\prime}}+\Gamma_{\tau+\varepsilon}^{2 \delta}(\mathfrak{S}) \beta-\partial_{\mathfrak{c}} \Gamma_{\tau+\varepsilon} \beta
\end{aligned}
$$


we have, as coefficients for the substitution,

$$
\begin{array}{ll}
\Gamma_{1}^{\alpha_{*}}=\Gamma_{3}^{\alpha_{*}}=\Gamma_{* \tau}^{0}(\mathfrak{S}) & =\tilde{\Gamma}_{1}^{\alpha_{*}}=\tilde{\Gamma}_{3}^{\alpha_{*}} \\
\Gamma_{1}^{\alpha_{* \tau^{\prime}}}=\Gamma_{3}^{\alpha_{* \tau^{\prime}}}=\Gamma_{* \tau}^{\tau^{\prime}}(\mathfrak{S}) & =\tilde{\Gamma}_{1}^{\alpha_{* \tau^{\prime}}}=\tilde{\Gamma}_{3}^{\alpha_{* \tau^{\prime}}} \\
\Gamma_{2}^{z_{\delta}}=\Gamma_{4}^{z_{\delta}}=0 & \tilde{\Gamma}_{2}^{z_{\delta}}=\tilde{\Gamma}_{4}^{z_{\delta}}=\Gamma_{\tau+\varepsilon}^{\delta}\left(\mathfrak{S}_{1}\right)(\Lambda \backslash \Omega) \\
\Gamma_{2}^{\beta}=\Gamma_{4}^{\beta}=\Gamma_{\tau+\varepsilon}^{2 \delta}(\mathfrak{S}) & \tilde{\Gamma}_{2}^{\beta}=\tilde{\Gamma}_{4}^{\beta}=\Gamma_{\tau+\varepsilon}^{2 \delta}(\mathfrak{S})-\partial_{\mathfrak{c}} \Gamma_{\tau+\varepsilon} \\
\Gamma_{2}^{\alpha_{\tau^{\prime \prime}}}=\Gamma_{4}^{\alpha_{\tau^{\prime \prime}}}=\Gamma_{\tau+\varepsilon}^{\tau^{\prime \prime}}(\mathfrak{S}) & =\tilde{\Gamma}_{2}^{\alpha_{\tau^{\prime \prime}}}=\tilde{\Gamma}_{4}^{\alpha_{\tau^{\prime \prime}}}
\end{array}
$$

with $\tau^{\prime} \in \varepsilon \mathbb{Z} \cap(0, \delta), \tau^{\prime \prime} \in \varepsilon \mathbb{Z} \cap(0,2 \delta)$ and with all other $\Gamma_{i}^{j}$, s and $\tilde{\Gamma}_{i}^{j}$, s being zero. We apply Corollary G.3.ii with $L=\Lambda, d$ replaced by $4 \mathrm{~m} d, d^{\prime}=d_{\Omega}$ and $\delta=\frac{\mathrm{m}}{2} d$. The Corollary gives

$$
\left\|\left\langle\gamma_{* \tau} \gamma_{\tau+\varepsilon}, v \gamma_{* \tau} \gamma_{\tau+\varepsilon}\right\rangle-\left\langle\gamma_{* \tau} g_{\tau+\varepsilon}, v \gamma_{* \tau} g_{\tau+\varepsilon}\right\rangle\right\|_{\text {fluct }} \leq 4\|v\| \mid \sigma_{\delta}^{\prime} \sigma^{\prime 3}
$$

where

$$
\begin{aligned}
\sigma^{\prime} & \leq \max \left\{\sigma_{* \tau}^{\prime}, \sigma_{\tau+\varepsilon}^{\prime}\right\} \\
\sigma_{\delta}^{\prime} & =N_{d_{\Omega}}\left(\Gamma_{\tau+\varepsilon}^{\delta}\left(\mathfrak{S}_{1}\right)(\Lambda \backslash \Omega) ; e^{-\frac{3}{2} \mathrm{~m} d(\mathbf{x}, \Lambda)}, \lambda_{\delta}\right)+N_{d_{\Omega}}\left(\partial_{\mathfrak{c}} \Gamma_{\tau} ; e^{-\frac{3}{2} \mathrm{~m} d(\mathbf{x}, \Lambda)}, \kappa_{2 \delta}\right) \\
& \leq 32 e^{K_{j}} \mathrm{r}+4 e^{2 K_{j}} \mathrm{R}_{+}\left(\delta e^{-\mathrm{mc}}+e^{-\mathrm{mc}(\delta)}\right) \quad \text { by (V.36) and (V.37) }
\end{aligned}
$$

with

$$
\begin{aligned}
\sigma_{* \tau}^{\prime}= & \sum_{\tau^{\prime} \in[0, \delta)} N_{d_{\Omega}}\left(\Gamma_{* \tau}^{\tau^{\prime}}(\mathfrak{S}) ; e^{\frac{\mathrm{m}}{2} d(\mathbf{x}, \Lambda)}, \kappa_{* \tau^{\prime}}\right)=\sigma_{* \tau} \leq 16 e^{K_{j} \tau} \mathrm{R}_{+} \\
\sigma_{\tau}^{\prime}= & N_{d_{\Omega}}\left(\Gamma_{\tau}^{\delta}\left(\mathfrak{S}_{1}\right)(\Lambda \backslash \Omega) ; e^{\frac{\mathrm{m}}{2} d(\mathbf{x}, \Lambda)}, \lambda_{\delta}\right)+N_{d_{\Omega}}\left(\partial_{\mathfrak{c}} \Gamma_{\tau} ; e^{\frac{\mathrm{m}}{2} d(\mathbf{x}, \Lambda)}, \kappa_{2 \delta}\right) \\
& +\sum N_{d_{\Omega}^{\prime \prime} \in(0,2 \delta]}\left(\Gamma_{\tau}^{\tau^{\prime}}(\mathfrak{S}) ; e^{\frac{\mathrm{m}}{2} d(\mathbf{x}, \Lambda)}, \kappa_{\tau^{\prime \prime}}\right) \\
\leq & \sigma_{\tau} \leq 32 e^{K_{j}} \mathrm{R}_{+}
\end{aligned}
$$

by (V.32), twice. Hence

$$
\begin{aligned}
\|\left\langle\gamma_{* \tau} \gamma_{\tau+\varepsilon}, v \gamma_{* \tau}\right. & \left.\gamma_{\tau+\varepsilon}\right\rangle-\left\langle\gamma_{* \tau} g_{\tau+\varepsilon}, v \gamma_{* \tau} g_{\tau+\varepsilon}\right\rangle \|_{\text {fluct }} \\
& \leq 4\|v\|\left[32 e^{K_{j}} \mathrm{r}+4 e^{2 K_{j}} \mathrm{R}_{+}\left(\delta e^{-\mathrm{mc}}+e^{-\mathrm{mc}(\delta)}\right)\right]\left[32 e^{K_{j}} \mathrm{R}_{+}\right]^{3} \\
& \leq 2^{22} e^{4 K_{j}}\|v\| \|\left[\mathrm{r}+e^{K_{j}} \mathrm{R}_{+}\left(\delta e^{-\mathrm{mc}}+e^{-\mathrm{mc}(\delta)}\right)\right] \mathrm{R}_{+}^{3} \\
& \leq 2^{23} e^{4 K_{j}}\|v\| \mathrm{rR}_{+}^{3}
\end{aligned}
$$

by (F.4.b), (F.6.a) and (F.6.b). Summing over $\tau$ and multiplying by $\varepsilon$ bounds $\left\|\delta \mathcal{V}_{1}\right\|_{\text {fluct }}$ by $2^{23} e^{4 K_{j}} \delta\|v\| \| \mathrm{rR}_{+}^{3}$. The $\delta V_{2}$ contribution obeys the same bound.

We now move onto the bound of $\|\delta \mathcal{V}-\widetilde{\delta \mathcal{V}}\|_{\mathfrak{S}}$. Then

$$
\delta \mathcal{V}-\widetilde{\delta \mathcal{V}}=\delta \mathcal{V}_{1}(\varepsilon)-\delta \mathcal{V}_{1}\left(\frac{\varepsilon}{2}\right)+\delta \mathcal{V}_{2}(\varepsilon)-\delta \mathcal{V}_{2}\left(\frac{\varepsilon}{2}\right)
$$


Write

$$
\delta \mathcal{V}_{1}(\varepsilon)-\delta \mathcal{V}_{1}\left(\frac{\varepsilon}{2}\right)=\delta \mathcal{W}_{1}+\delta \mathcal{W}_{2}
$$

where

$$
\begin{aligned}
& \delta \mathcal{W}_{1}\left(\alpha_{*}, \beta ; \vec{\alpha}_{*}, \vec{\alpha}\right)=\frac{\varepsilon}{2} \sum_{\tau \in \varepsilon \mathbb{Z} \cap[0, \delta)}[\left\langle\gamma_{* \tau} \gamma_{\tau+\varepsilon}, v \gamma_{* \tau} \gamma_{\tau+\varepsilon}\right\rangle-\left\langle\gamma_{* \tau} \gamma_{\tau+\frac{\varepsilon}{2}}, v \gamma_{* \tau} \gamma_{\tau+\frac{\varepsilon}{2}}\right\rangle \\
&\left.-\left\langle\gamma_{* \tau} g_{\tau+\varepsilon}, v \gamma_{* \tau} g_{\tau+\varepsilon}\right\rangle+\left\langle\gamma_{* \tau} g_{\tau+\frac{\varepsilon}{2}}, v \gamma_{* \tau} g_{\tau+\frac{\varepsilon}{2}}\right\rangle\right] \\
& \delta \mathcal{W}_{2}\left(\alpha_{*}, \beta ; \vec{\alpha}_{*}, \vec{\alpha}\right)=\frac{\varepsilon}{2} \sum_{\tau \in \varepsilon \mathbb{Z} \cap[0, \delta)}\left[\left\langle\gamma_{* \tau} \gamma_{\tau+\varepsilon}, v \gamma_{* \tau} \gamma_{\tau+\varepsilon}\right\rangle-\left\langle\gamma_{* \tau+\frac{\varepsilon}{2}} \gamma_{\tau+\varepsilon}, v \gamma_{* \tau+\frac{\varepsilon}{2}} \gamma_{\tau+\varepsilon}\right\rangle\right. \\
&\left.-\left\langle g_{* \tau} \gamma_{\tau+\varepsilon}, v g_{* \tau} \gamma_{\tau+\varepsilon}\right\rangle+\left\langle g_{* \tau+\frac{\varepsilon}{2}} \gamma_{\tau+\varepsilon}, v g_{* \tau+\frac{\varepsilon}{2}} \gamma_{\tau+\varepsilon}\right\rangle\right]
\end{aligned}
$$

We estimate $\delta \mathcal{W}_{1}$. The term of $\delta \mathcal{W}_{1}$ with index $\tau$ is

$$
\begin{aligned}
& {\left[\left\langle\gamma_{* \tau} \gamma_{\tau+\varepsilon}, v \gamma_{* \tau} \gamma_{\tau+\varepsilon}\right\rangle-\left\langle\gamma_{* \tau} j\left(\frac{\varepsilon}{2}\right) \gamma_{\tau+\varepsilon}, v \gamma_{* \tau} j\left(\frac{\varepsilon}{2}\right) \gamma_{\tau+\varepsilon}\right\rangle\right.} \\
& \left.\quad-\left\langle\gamma_{* \tau} g_{\tau+\varepsilon}, v \gamma_{* \tau} g_{\tau+\varepsilon}\right\rangle+\left\langle\gamma_{* \tau} j\left(\frac{\varepsilon}{2}\right) g_{\tau+\varepsilon}, v \gamma_{* \tau} j\left(\frac{\varepsilon}{2}\right) g_{\tau+\varepsilon}\right\rangle\right]
\end{aligned}
$$

since, by Lemma E.14, (V.30), (V.19) and Remark E.19,

$$
\gamma_{\tau+\frac{\varepsilon}{2}}=j\left(\frac{\varepsilon}{2}\right) \gamma_{\tau+\varepsilon} \quad g_{\tau+\frac{\varepsilon}{2}}=j\left(\frac{\varepsilon}{2}\right) g_{\tau+\varepsilon}
$$

We again apply Corollary G.3with the $\Gamma_{i}^{j}$ 's and $\tilde{\Gamma}_{i}^{j}$ 's of (V.38). This time we use part (iii) with

$$
A_{1}=\tilde{A}_{1}=A_{3}=\tilde{A}_{3}=\mathbb{1} \quad A_{2}=A_{4}=\mathfrak{h} \quad \tilde{A}_{2}=\tilde{A}_{4}=j\left(\frac{\varepsilon}{2}\right)
$$

as coefficients for the substitution. The Corollary, with $L=\Lambda, d$ replaced by $4 \mathrm{~m} d, d^{\prime}=d_{\Omega}$ and $\delta=\frac{\mathrm{m}}{2} d$, gives

$$
\begin{aligned}
& \|\left\langle\gamma_{* \tau} \gamma_{\tau+\varepsilon}, v \gamma_{* \tau} \gamma_{\tau+\varepsilon}\right\rangle-\left\langle\gamma_{* \tau} j\left(\frac{\varepsilon}{2}\right) \gamma_{\tau+\varepsilon}, v \gamma_{* \tau} j\left(\frac{\varepsilon}{2}\right) \gamma_{\tau+\varepsilon}\right\rangle \\
& \quad-\left\langle\gamma_{* \tau} g_{\tau+\varepsilon}, v \gamma_{* \tau} g_{\tau+\varepsilon}\right\rangle+\left\langle\gamma_{* \tau} j\left(\frac{\varepsilon}{2}\right) g_{\tau+\varepsilon}, v \gamma_{* \tau} j\left(\frac{\varepsilon}{2}\right) g_{\tau+\varepsilon}\right\rangle\left\|_{\text {fluct }} \leq 16\right\| v \| \sigma_{\delta}^{\prime} a_{\delta}\left(\sigma^{\prime} a\right)^{3}
\end{aligned}
$$

where, by Lemma III.21,

$$
\begin{aligned}
a & \leq \max \left\{\|1\|\|,\| j\left(\frac{\varepsilon}{2}\right)\|\|\right\} \leq e^{K_{j} \frac{\varepsilon}{2}} \\
a_{\delta} & \leq\left\|j\left(\frac{\varepsilon}{2}\right)-\mathfrak{h}\right\| \|=K_{j} \frac{\varepsilon}{2} e^{K_{j} \frac{\varepsilon}{2}}
\end{aligned}
$$

Thus

$$
\left\|\delta \mathcal{W}_{1}\right\|_{\text {fluct }} \leq \frac{\varepsilon}{2} \frac{\delta}{\varepsilon} 16\|\| v\left\|\sigma_{\delta}^{\prime} a_{\delta}\left(\sigma^{\prime} a\right)^{3} \leq \varepsilon \delta K_{j} e^{2 K_{j} \varepsilon} 4\right\| v\|\| \sigma_{\delta}^{\prime} \sigma^{\prime 3} \leq 2^{23} e^{6 K_{j}} \varepsilon \delta\|v\| \| \mathrm{rR}_{+}^{3}
$$

since the right hand side of (V.39) is a bound on $4\|v\| \sigma_{\delta}^{\prime} \sigma^{\prime 3}$. The bounds on $\delta \mathcal{W}_{1}$ and the two corresponding terms of $\delta \mathcal{V}_{2}(\varepsilon)-\delta \mathcal{V}_{2}\left(\frac{\varepsilon}{2}\right)$ are the same. 


\section{The Structure of the Fluctuation Integrand}

Proposition V.14 The function $f_{\mathfrak{S}}\left(\alpha_{*}, \beta ; \vec{\rho} ; z_{*}, z\right)$ of (V.8) that appears in Lemma V.4 and the function $\tilde{f}_{\mathfrak{S}}$ of (V.9) can be written in the form

$$
\begin{aligned}
f_{\mathfrak{S}}\left(\alpha_{*}, \beta ; \vec{\rho} ; z_{*}, z\right)=- & Q_{\mathfrak{S}}\left(\alpha_{*}, \beta ; \vec{\alpha}_{*}, \vec{\alpha}\right)+\mathcal{V}_{\mathfrak{S}}\left(\varepsilon ; \alpha_{*}, \beta ; \vec{\alpha}_{*}, \vec{\alpha}\right) \\
& +\mathcal{F}_{l}\left(\alpha_{*}, \beta ; \vec{\rho} ; z\right)+\mathcal{F}_{r}\left(\alpha_{*}, \beta ; \vec{\rho} ; z_{*}\right)+\mathcal{D}^{(1)}\left(\alpha_{*}, \beta ; \vec{\rho}\right)+\mathcal{L}_{\mathfrak{S}}^{\prime}\left(\alpha_{*}, \beta ; \vec{\rho}\right)
\end{aligned}
$$

and

$$
\tilde{f}_{\mathfrak{S}}=-Q_{\mathfrak{S}}+\mathcal{V}_{\mathfrak{S}}\left(\frac{\varepsilon}{2} ; \cdots\right)+\tilde{\mathcal{F}}_{l}+\tilde{\mathcal{F}}_{r}+\tilde{\mathcal{D}}^{(1)}+\tilde{\mathcal{L}}_{\mathfrak{S}}^{\prime}
$$

respectively, with history complete analytic functions $\mathcal{F}_{l}, \mathcal{F}_{r}, \mathcal{D}^{(1)}, \mathcal{L}_{\mathfrak{S}}^{\prime}, \tilde{\mathcal{F}}_{l}, \tilde{\mathcal{F}}_{r}, \tilde{\mathcal{D}}^{(1)}, \tilde{\mathcal{L}}_{\mathfrak{S}}^{\prime}$ that have the following properties.

(i) $\mathcal{F}_{l}\left(\alpha_{*}, \beta ; \vec{\rho} ; 0\right)=\mathcal{F}_{r}\left(\alpha_{*}, \beta ; \vec{\rho} ; 0\right)=0$ and

$$
\begin{aligned}
& \left\|\mathcal{F}_{l}\right\|_{\text {fluct }} \leq K_{\mathcal{V}} \delta \mathfrak{v} \mathrm{rR}_{+}^{3}+2^{8}\left(e^{-\mathrm{mc}(\delta)}\left\|\mathcal{D}_{1}\right\|_{\mathfrak{S}_{1}}+\left\|\left.\mathcal{D}_{1}\right|_{\Omega_{1}}\right\|_{\mathfrak{S}_{1}}\right) \\
& \left\|\mathcal{F}_{r}\right\|_{\text {fluct }} \leq K_{\mathcal{V}} \delta \mathfrak{v} \mathrm{rR}_{+}^{3}+2^{8}\left(e^{-\operatorname{mc}(\delta)}\left\|\mathcal{D}_{2}\right\|_{\mathfrak{S}_{2}}+\left\|\left.\mathcal{D}_{2}\right|_{\Omega_{2}}\right\|_{\mathfrak{S}_{2}}\right)
\end{aligned}
$$

Similarly, $\tilde{\mathcal{F}}_{l}\left(\alpha_{*}, \beta ; \vec{\rho} ; 0\right)=\tilde{\mathcal{F}}_{r}\left(\alpha_{*}, \beta ; \vec{\rho} ; 0\right)=0$ and

$$
\begin{aligned}
& \left\|\tilde{\mathcal{F}}_{\|}\right\|_{\text {fluct }} \leq K_{\mathcal{V}} \delta \mathfrak{v} \mathrm{rR}_{+}^{3}+2^{8}\left(e^{-\mathrm{mc}(\delta)}\left\|\tilde{\mathcal{D}}_{1}\right\|_{\mathfrak{S}_{1}}+\left\|\left.\tilde{\mathcal{D}}_{1}\right|_{\Omega_{1}}\right\|_{\mathfrak{S}_{1}}\right) \\
& \left\|\tilde{\mathcal{F}}_{r}\right\|_{\text {fluct }} \leq K_{\mathcal{V}} \delta \mathfrak{v} \mathrm{rR}_{+}^{3}+2^{8}\left(e^{-\mathrm{m} \mathfrak{c}(\delta)}\left\|\tilde{\mathcal{D}}_{2}\right\|_{\mathfrak{S}_{2}}+\left\|\left.\tilde{\mathcal{D}}_{2}\right|_{\Omega_{2}}\right\|_{\mathfrak{S}_{2}}\right)
\end{aligned}
$$

Furthermore

$$
\begin{aligned}
& \left\|\mathcal{F}_{l}-\tilde{\mathcal{F}}_{l}\right\|_{\text {fluct }} \leq K_{\mathcal{V}} \varepsilon \delta \mathfrak{v} \mathrm{rR}_{+}^{3}+2^{8}\left(e^{-\mathrm{mc}(\delta)}\left\|\mathcal{D}_{1}-\tilde{\mathcal{D}}_{1}\right\|_{\mathfrak{S}_{1}}+\left\|\left.\left(\mathcal{D}_{1}-\tilde{\mathcal{D}}_{1}\right)\right|_{\Omega_{1}}\right\|_{\mathfrak{S}_{1}}\right) \\
& \left\|\mathcal{F}_{r}-\tilde{\mathcal{F}}_{r}\right\|_{\text {fluct }} \leq K_{\mathcal{V}} \varepsilon \delta \mathfrak{v} \mathrm{rR}_{+}^{3}+2^{8}\left(e^{-\mathrm{mc}(\delta)}\left\|\mathcal{D}_{2}-\tilde{\mathcal{D}}_{2}\right\|_{\mathfrak{S}_{2}}+\left\|\left.\left(\mathcal{D}_{2}-\tilde{\mathcal{D}}_{2}\right)\right|_{\Omega_{2}}\right\|_{\mathfrak{S}_{2}}\right)
\end{aligned}
$$

(ii) $\left.\mathcal{D}^{(1)}\right|_{\Omega^{c}}=\left.\tilde{\mathcal{D}}^{(1)}\right|_{\Omega^{c}}=0$ and

$$
\begin{aligned}
& \left\|\mathcal{D}^{(1)}\right\|_{\mathfrak{S}} \leq K_{\mathcal{V}}(2 \delta \mathfrak{v}) \mathrm{r}_{+} \mathrm{R}_{+}^{3}+2^{8} e^{-\mathrm{mc}(\delta)} \Sigma_{\mathcal{D}}+2^{8} \Sigma_{\mathcal{D}}^{(S F)} \\
& \left\|\tilde{\mathcal{D}}^{(1)}\right\|_{\mathfrak{S}} \leq K_{\mathcal{V}}(2 \delta \mathfrak{v}) \mathrm{r}_{+} \mathrm{R}_{+}^{3}+2^{8} e^{-\mathrm{mc}(\delta)} \tilde{\Sigma}_{\mathcal{D}}+2^{8} \tilde{\Sigma}_{\mathcal{D}}^{(S F)}
\end{aligned}
$$

where

$$
\begin{array}{ll}
\Sigma_{\mathcal{D}}=\left\|\mathcal{D}_{1}\right\|_{\mathfrak{S}_{1}}+\left\|\mathcal{D}_{2}\right\|_{\mathfrak{S}_{2}} & \Sigma_{\mathcal{D}}^{(S F)}=\left\|\left.\mathcal{D}_{1}\right|_{\Omega_{1}}\right\|_{\mathfrak{S}_{1}}+\left\|\left.\mathcal{D}_{2}\right|_{\Omega_{2}}\right\|_{\mathfrak{S}_{2}} \\
\tilde{\Sigma}_{\mathcal{D}}=\left\|\tilde{\mathcal{D}}_{1}\right\|_{\mathfrak{S}_{1}}+\left\|\tilde{\mathcal{D}}_{2}\right\|_{\mathfrak{S}_{2}} & \tilde{\Sigma}_{\mathcal{D}}^{(S F)}=\left\|\left.\tilde{\mathcal{D}}_{1}\right|_{\Omega_{1}}\right\|_{\mathfrak{S}_{1}}+\left\|\left.\tilde{\mathcal{D}}_{2}\right|_{\Omega_{2}}\right\|_{\mathfrak{S}_{2}}
\end{array}
$$

Furthermore

$$
\left\|\mathcal{D}^{(1)}-\tilde{\mathcal{D}}^{(1)}\right\|_{\mathfrak{S}} \leq K_{\mathcal{V}} \varepsilon \delta \mathfrak{v} \mathrm{r}_{+}^{3}+2^{8} e^{-\mathrm{mc}(\delta)} \Delta_{\mathcal{D}}+2^{8} \Delta_{\mathcal{D}}^{(S F)}
$$


where

$$
\begin{aligned}
\Delta_{\mathcal{D}} & =\left\|\mathcal{D}_{1}-\tilde{\mathcal{D}}_{1}\right\|_{\mathfrak{S}_{1}}+\left\|\mathcal{D}_{2}-\tilde{\mathcal{D}}_{2}\right\|_{\mathfrak{S}_{2}} \\
\Delta_{\mathcal{D}}^{(S F)} & =\left\|\left.\left(\mathcal{D}_{1}-\tilde{\mathcal{D}}_{1}\right)\right|_{\Omega_{1}}\right\|_{\mathfrak{S}_{1}}+\left\|\left.\left(\mathcal{D}_{2}-\tilde{\mathcal{D}}_{2}\right)\right|_{\Omega_{2}}\right\|_{\mathfrak{S}_{2}}
\end{aligned}
$$

(iii) $\left.\mathcal{L}_{\mathfrak{S}}^{\prime}\right|_{\Omega^{c}}=\mathcal{L}_{\mathfrak{S}}^{\prime}$ and $\left.\tilde{\mathcal{L}}_{\mathfrak{S}}^{\prime}\right|_{\Omega^{c}}=\tilde{\mathcal{L}}_{\mathfrak{S}}^{\prime}$ and

$$
\begin{aligned}
\left\|\mathcal{L}_{\mathfrak{S}}^{\prime}\right\|_{\mathfrak{S}} & \leq K_{\mathcal{V}}(2 \delta \mathfrak{v}) \mathrm{r}_{+} \mathrm{R}_{+}^{3}+2^{8} \Sigma_{\mathcal{D}} \\
\left\|\tilde{\mathcal{L}}_{\mathfrak{S}}^{\prime}\right\|_{\mathfrak{S}} & \leq K_{\mathcal{V}}(2 \delta \mathfrak{v}) \mathrm{r}_{+} \mathrm{R}_{+}^{3}+2^{8} \tilde{\Sigma}_{\mathcal{D}}
\end{aligned}
$$

and

$$
\left\|\mathcal{L}_{\mathfrak{S}}^{\prime}-\tilde{\mathcal{L}}_{\mathfrak{S}}^{\prime}\right\|_{\mathfrak{S}} \leq K_{\mathcal{V}} \varepsilon \delta \mathfrak{v} r \mathrm{R}_{+}^{3}+2^{8} \Delta_{\mathcal{D}}
$$

Proof: We set

$$
\begin{aligned}
\mathcal{F}_{l}\left(\alpha_{*}, \beta ; \vec{\rho} ; z\right)=- & {\left[\left\langle\Omega j_{\mathfrak{c}}(\delta) \alpha_{*}, z\right\rangle+Q_{\mathfrak{S}_{1}}\left(\alpha_{*}, z ; \vec{\alpha}_{* l}, 0\right)\right]+\mathcal{V}_{l}\left(\varepsilon ; \alpha_{*}, \beta ; \vec{\rho} ; z\right) } \\
& +\mathcal{D}_{1}\left(\alpha_{*}, z+z_{\delta}+\alpha_{\delta}^{\mathrm{cr}} ; \vec{\rho}_{l}\right)-\mathcal{D}_{1}\left(\alpha_{*}, z_{\delta}+\alpha_{\delta}^{\mathrm{cr}} ; \vec{\rho}_{l}\right) \\
\mathcal{F}_{r}\left(\alpha_{*}, \beta ; \vec{\rho} ; z_{*}\right)=- & {\left[\left\langle z_{*}, \Omega j_{\mathfrak{c}}(\delta) \beta\right\rangle+Q_{\mathfrak{S}_{2}}\left(z_{*}, \beta ; 0, \vec{\alpha}_{r}\right)\right]+\mathcal{V}_{r}\left(\varepsilon ; \alpha_{*}, \beta ; \vec{\rho} ; z_{*}\right) } \\
& +\mathcal{D}_{2}\left(z_{*}+z_{* \delta}+\alpha_{* \delta}^{\mathrm{cr}}, \beta ; \vec{\rho}_{r}\right)-\mathcal{D}_{2}\left(z_{* \delta}+\alpha_{* \delta}^{\mathrm{cr}}, \beta ; \vec{\rho}_{r}\right) \\
\mathcal{D}^{\prime}\left(\alpha_{*}, \beta ; \vec{\rho}\right)= & \delta Q\left(\alpha_{*}, \beta ; \vec{\rho}\right)+\delta \mathcal{V}\left(\varepsilon ; \alpha_{*}, \beta ; \vec{\rho}\right) \\
& +\mathcal{D}_{1}\left(\alpha_{*}, z_{\delta}+\alpha_{\delta}^{\mathrm{cr}} ; \vec{\alpha}_{*_{l}}, \vec{\alpha}_{l}\right)+\mathcal{D}_{2}\left(z_{* \delta}+\alpha_{* \delta}^{\mathrm{cr}}, \beta ; \vec{\alpha}_{*_{r}}, \vec{\alpha}_{r}\right)
\end{aligned}
$$

and

$$
\mathcal{L}_{\mathfrak{S}}^{\prime}=\left.\mathcal{D}^{\prime}\right|_{\Omega^{c}} \quad, \quad \mathcal{D}^{(1)}=\mathcal{D}^{\prime}-\mathcal{L}_{\mathfrak{S}}^{\prime}
$$

The fact that $f_{\mathfrak{S}}=-Q_{\mathfrak{S}}+\mathcal{V}_{\mathfrak{S}}+\mathcal{F}_{l}+\mathcal{F}_{r}+\mathcal{D}^{(1)}+\mathcal{L}_{\mathfrak{S}}^{\prime}$ is immediate from Remark V.5 and (V.29). Similarly, we set

$$
\begin{aligned}
\tilde{\mathcal{F}}_{l}\left(\alpha_{*}, \beta ; \vec{\rho} ; z\right)=- & {\left[\left\langle\Omega j_{\mathfrak{c}}(\delta) \alpha_{*}, z\right\rangle+Q_{\mathfrak{S}_{1}}\left(\alpha_{*}, z ; \vec{\alpha}_{*_{l}}, 0\right)\right]+\mathcal{V}_{l}\left(\frac{\varepsilon}{2} ; \alpha_{*}, \beta ; \vec{\rho} ; z\right) } \\
& +\tilde{\mathcal{D}}_{1}\left(\alpha_{*}, z+z_{\delta}+\alpha_{\delta}^{\mathrm{cr}} ; \vec{\rho}_{l}\right)-\tilde{\mathcal{D}}_{1}\left(\alpha_{*}, z_{\delta}+\alpha_{\delta}^{\mathrm{cr}} ; \vec{\rho}_{l}\right) \\
\tilde{\mathcal{F}}_{r}\left(\alpha_{*}, \beta ; \vec{\rho} ; z_{*}\right)=- & {\left[\left\langle z_{*}, \Omega j_{\mathfrak{c}}(\delta) \beta\right\rangle+Q_{\mathfrak{S}_{2}}\left(z_{*}, \beta ; 0, \vec{\alpha}_{r}\right)\right]+\mathcal{V}_{r}\left(\frac{\varepsilon}{2} ; \alpha_{*}, \beta ; \vec{\rho} ; z_{*}\right) } \\
& +\tilde{\mathcal{D}}_{2}\left(z_{*}+z_{* \delta}+\alpha_{* \delta}^{\mathrm{cr}}, \beta ; \vec{\rho}_{r}\right)-\tilde{\mathcal{D}}_{2}\left(z_{* \delta}+\alpha_{* \delta}^{\mathrm{cr}}, \beta ; \vec{\rho}_{r}\right) \\
\tilde{\mathcal{D}}^{\prime}\left(\alpha_{*}, \beta ; \vec{\rho}\right)= & \delta Q\left(\alpha_{*}, \beta ; \vec{\rho}\right)+\widetilde{\delta \mathcal{V}}\left(\frac{\varepsilon}{2} ; \alpha_{*}, \beta ; \vec{\rho}\right) \\
& +\tilde{\mathcal{D}}_{1}\left(\alpha_{*}, z_{\delta}+\alpha_{\delta}^{\mathrm{cr}} ; \vec{\alpha}_{*_{l}}, \vec{\alpha}_{l}\right)+\tilde{\mathcal{D}}_{2}\left(z_{* \delta}+\alpha_{* \delta}^{\mathrm{cr}}, \beta ; \vec{\alpha}_{*_{r}}, \vec{\alpha}_{r}\right)
\end{aligned}
$$

and $\tilde{\mathcal{L}}_{\mathfrak{S}}^{\prime}=\left.\tilde{\mathcal{D}}^{\prime}\right|_{\Omega^{c}}, \tilde{\mathcal{D}}^{(1)}=\tilde{\mathcal{D}}^{\prime}-\tilde{\mathcal{L}}_{\mathfrak{S}}^{\prime}$.

(i) By Propositions V.12.i, V.13.i and Lemma V.10, with $g_{1}=\mathcal{D}_{1}$,

$$
\begin{aligned}
\left\|\mathcal{F}_{l}\right\|_{\text {fluct }} \leq K_{Q}\left[e^{-\mathrm{mc}(\delta)}+\delta \mathrm{rR}+e^{-\mathrm{m} \mathfrak{c}}\right]+K_{\mathcal{V}} \delta\|v\| \mathrm{r} \mathrm{R}_{+}^{3} & \\
& +2^{8}\left(e^{-\mathrm{mc}(\delta)}\left\|\mathcal{D}_{1}\right\|_{\mathfrak{S}_{1}}+\left\|\left.\mathcal{D}_{1}\right|_{\Omega_{1}}\right\|_{\mathfrak{S}_{1}}\right) \\
\leq & K_{\mathcal{V}} \delta \mathrm{rR}_{+}^{3} \mathfrak{v}+2^{8}\left(e^{-\mathrm{mc}(\delta)}\left\|\mathcal{D}_{1}\right\|_{\mathfrak{S}_{1}}+\left\|\left.\mathcal{D}_{1}\right|_{\Omega_{1}}\right\|_{\mathfrak{S}_{1}}\right)
\end{aligned}
$$


by (F.4.b,c) and the hypothesis that $\|v\| \leq \frac{\mathfrak{v}}{2}$. Also, by construction,

$$
\begin{aligned}
\mathcal{F}_{l}-\tilde{\mathcal{F}}_{l}= & \mathcal{V}_{l}\left(\varepsilon ; \alpha_{*}, \beta ; \vec{\rho} ; z\right)-\mathcal{V}_{l}\left(\frac{\varepsilon}{2} ; \alpha_{*}, \beta ; \vec{\rho} ; z\right) \\
& +\left(\mathcal{D}_{1}-\tilde{\mathcal{D}}_{1}\right)\left(\alpha_{*}, z+z_{\delta}+\alpha_{\delta}^{\mathrm{cr}} ; \vec{\rho}_{l}\right)-\left(\mathcal{D}_{1}-\tilde{\mathcal{D}}_{1}\right)\left(\alpha_{*}, z_{\delta}+\alpha_{\delta}^{\mathrm{cr}} ; \vec{\rho}_{l}\right)
\end{aligned}
$$

By Proposition V.13.i and Lemma V.10, with $g_{1}=\mathcal{D}_{1}-\tilde{\mathcal{D}}_{1}$,

$$
\left\|\mathcal{F}_{l}-\tilde{\mathcal{F}}_{l}\right\|_{\text {fluct }} \leq K_{\mathcal{V}} \varepsilon \delta \mathfrak{v} \mathrm{rR}_{+}^{3}+2^{8}\left(e^{-\mathrm{mc}(\delta)}\left\|\mathcal{D}_{1}-\tilde{\mathcal{D}}_{1}\right\|_{\mathfrak{S}_{1}}+\left\|\left.\left(\mathcal{D}_{1}-\tilde{\mathcal{D}}_{1}\right)\right|_{\Omega_{1}}\right\|_{\mathfrak{S}_{1}}\right)
$$

(ii) $\mathcal{D}^{(1)}$ and $\mathcal{L}_{\mathfrak{S}}^{\prime}$ are constructed so that $\left.\mathcal{D}^{(1)}\right|_{\Omega^{c}}=0$ and $\mathcal{L}_{\mathfrak{S}}^{\prime}=\left.\mathcal{L}_{\mathfrak{S}}^{\prime}\right|_{\Omega^{c}}$. Also

$$
\begin{aligned}
\mathcal{D}^{(1)}=\delta Q-\left.\delta Q\right|_{\Omega^{c}}+\delta \mathcal{V}-\left.\delta \mathcal{V}\right|_{\Omega^{c}} & +\mathcal{D}_{1}\left(\alpha_{*}, z_{\delta}+\alpha_{\delta}^{\mathrm{cr}} ; \cdot\right)-\left.\mathcal{D}_{1}\left(\alpha_{*}, z_{\delta}+\alpha_{\delta}^{\mathrm{cr}} ; \cdot\right)\right|_{\Omega^{c}} \\
& +\mathcal{D}_{2}\left(z_{* \delta}+\alpha_{* \delta}^{\mathrm{cr}}, \beta ; \cdot\right)-\left.\mathcal{D}_{2}\left(z_{* \delta}+\alpha_{* \delta}^{\mathrm{cr}}, \beta ; \cdot\right)\right|_{\Omega^{c}}
\end{aligned}
$$

Therefore, by Propositions V.12.ii, V.13.ii and Lemma V.10

$$
\begin{gathered}
\left\|\mathcal{D}^{(1)}\right\|_{\mathfrak{S}} \leq K_{Q}\left[e^{-\frac{1}{4} \mathrm{mc}(\delta)}+\delta \mathrm{r} \mathrm{R}_{+} e^{-\mathrm{m} \mathfrak{c}}\right]+K_{\mathcal{V}} \delta\|v\| \| \mathrm{R}_{+}^{3} \\
\quad+2^{8}\left(e^{-\mathrm{m} \mathfrak{c}(\delta)}\left(\left\|\mathcal{D}_{1}\right\|_{\mathfrak{S}_{1}}+\left\|\mathcal{D}_{2}\right\|_{\mathfrak{S}_{2}}\right)+\left(\left\|\left.\mathcal{D}_{1}\right|_{\Omega_{1}}\right\|_{\mathfrak{S}_{1}}+\left\|\left.\mathcal{D}_{2}\right|_{\Omega_{2}}\right\|_{\mathfrak{S}_{2}}\right)\right) \\
\leq K_{\mathcal{V}} 2 \delta \mathrm{r}_{+} \mathrm{R}_{+}^{3} \mathfrak{v}+2^{8}\left(e^{-\mathrm{mc}(\delta)} \Sigma_{\mathcal{D}}+\Sigma_{\mathcal{D}}^{(S F)}\right)
\end{gathered}
$$

by (F.3.b), (F.4.b,c). Also

$$
\begin{aligned}
\mathcal{D}^{(1)}-\tilde{\mathcal{D}}^{(1)}=(\delta \mathcal{V} & -\widetilde{\delta \mathcal{V}})-\left.(\delta \mathcal{V}-\widetilde{\delta \mathcal{V}})\right|_{\Omega^{c}} \\
& +\left(\mathcal{D}_{1}-\tilde{\mathcal{D}}_{1}\right)\left(\alpha_{*}, z_{\delta}+\alpha_{\delta}^{\mathrm{cr}} ; \cdot\right)-\left.\left(\mathcal{D}_{1}-\tilde{\mathcal{D}}_{1}\right)\left(\alpha_{*}, z_{\delta}+\alpha_{\delta}^{\mathrm{cr}} ; \cdot\right)\right|_{\Omega^{c}} \\
& +\left(\mathcal{D}_{2}-\tilde{\mathcal{D}}_{2}\right)\left(z_{* \delta}+\alpha_{* \delta}^{\mathrm{cr}}, \beta ; \cdot\right)-\left.\left(\mathcal{D}_{2}-\tilde{\mathcal{D}}_{2}\right)\left(z_{* \delta}+\alpha_{* \delta}^{\mathrm{cr}}, \beta ; \cdot\right)\right|_{\Omega^{c}}
\end{aligned}
$$

Therefore, the desired estimate on $\left\|\mathcal{D}^{(1)}-\tilde{\mathcal{D}}^{(1)}\right\|_{\mathfrak{S}}$ follows from Proposition V.13.ii and Lemma V.10.

(iii) By Propositions V.12.ii, V.13.ii and Lemma V.10

$$
\begin{aligned}
& \left\|\mathcal{L}_{\mathfrak{S}}^{\prime}\right\|_{\mathfrak{S}}=\left\|\left.\mathcal{D}^{\prime}\right|_{\Omega^{c}}\right\|_{\mathfrak{S}} \\
& \quad \leq K_{Q}\left[e^{-\frac{1}{4} \mathrm{mc}(\delta)}+\delta \mathrm{rR}_{+} e^{-\mathrm{m} \mathfrak{c}}\right]+K_{\mathcal{V}} \delta\|v\| \mathrm{r} \mathrm{R}_{+}^{3}+2^{8}\left\|\mathcal{D}_{1}\right\|_{\mathfrak{S}_{1}}+2^{8}\left\|\mathcal{D}_{2}\right\|_{\mathfrak{S}_{2}} \\
& \quad \leq K_{\mathcal{V}} 2 \delta \mathrm{r}_{+} \mathrm{R}_{+}^{3} \mathfrak{v}+2^{8} \Sigma_{\mathcal{D}}
\end{aligned}
$$

by (F.3.b), (F.4.b,c). Furthermore,

$$
\mathcal{D}^{\prime}-\tilde{\mathcal{D}}^{\prime}=\delta \mathcal{V}-\widetilde{\delta \mathcal{V}}+\left(\mathcal{D}_{1}-\tilde{\mathcal{D}}_{1}\right)\left(\alpha_{*}, z_{\delta}+\alpha_{\delta}^{\mathrm{cr}} ; \vec{\alpha}_{*_{l}}, \vec{\alpha}_{l}\right)+\left(\mathcal{D}_{2}-\tilde{\mathcal{D}}_{2}\right)\left(z_{* \delta}+\alpha_{* \delta}^{\mathrm{cr}}, \beta ; \vec{\alpha}_{*_{r}}, \vec{\alpha}_{r}\right)
$$

The desired bound on $\left\|\mathcal{L}_{\mathfrak{S}}^{\prime}-\tilde{\mathcal{L}}_{\mathfrak{S}}^{\prime}\right\|_{\mathfrak{S}}$ now follows from Proposition V.13.ii and Lemma V.10. 


\section{V.5 The fluctuation integral - proofs of Theorems III.26 and III.28}

Recall from Lemma V.4 that

$$
\begin{aligned}
I\left(\alpha^{*}, \beta\right)=\sum_{\substack{\text { hierarchies } \\
\text { forr scale } \\
\left(\mathfrak{S}_{1}, \mathfrak{S}_{2}\right) \prec \mathfrak{S}}} I_{\left(\mathfrak{S} ; \alpha^{*}, \beta\right)} & b_{1}\left(\alpha^{*}, \alpha_{\delta}, \overrightarrow{\rho_{\mid l}}\right) b_{2}\left(\alpha_{\delta}^{*}, \beta, \overrightarrow{\rho_{\mid r}}\right) \chi_{2 \delta}\left(\Omega_{\mathfrak{S}} ; \alpha, \beta\right) \\
& \left.\mathcal{Z}_{\delta}^{2\left|\Omega_{\mathfrak{S}}\right|} \int d \mu_{\Omega_{\mathfrak{S}}, \mathrm{r}}\left(z^{*}, z\right) e^{f_{\mathfrak{S}}\left(\alpha^{*}, \beta ; \overrightarrow{\rho^{\prime}} ; z^{*}, z\right)}\right|_{\substack{z_{* \delta}(\mathbf{x})=z_{\delta}(\mathbf{x}) * \\
\text { for } \mathbf{x} \in \Lambda_{\mathfrak{S}} \backslash \mathrm{R}}}
\end{aligned}
$$

In this section we perform the fluctuation integral to give the proofs of Theorems III.26 and III.28.

For each hierarchy $\mathfrak{S}$ preceded by $\mathfrak{S}_{1}$ and $\mathfrak{S}_{2}$, by Proposition V.14 and Lemma II.7,

$$
\begin{aligned}
& \mathcal{Z}_{\delta}^{2\left|\Omega_{\mathfrak{S}}\right|} \int d \mu_{\Omega_{\mathfrak{S}, \mathrm{r}}}\left(z^{*}, z\right) e^{f_{\mathfrak{S}}\left(\alpha^{*}, \beta ; \vec{\rho} ; z^{*}, z\right)} \\
& =e^{-Q_{\mathfrak{S}}+\mathcal{V}_{\mathfrak{S}}+\mathcal{D}^{(1)}+\mathcal{L}_{\mathfrak{S}}^{\prime}} \mathcal{Z}_{\delta}^{2\left|\Omega_{\mathfrak{S}}\right|} \int d \mu_{\Omega_{\mathfrak{S}, \mathrm{r}}}\left(z^{*}, z\right) e^{\mathcal{F}_{l}\left(\alpha_{*}, \beta ; \vec{\rho} ; z\right)+\mathcal{F}_{r}\left(\alpha_{*}, \beta ; \vec{\rho} ; z^{*}\right)} \\
& =e^{-Q_{\mathfrak{S}}+\mathcal{V}_{\mathfrak{S}}+\mathcal{D}^{(1)}+\mathcal{L}_{\mathfrak{S}}^{\prime}} \mathcal{Z}_{2 \delta}^{\left|\Omega_{\mathfrak{S}}\right|} \frac{\int d \mu_{\Omega_{\mathfrak{S}, \mathrm{r}}}\left(z^{*}, z\right) e^{\mathcal{F}_{l}\left(\alpha_{*}, \beta ; \vec{\rho} ; z\right)+\mathcal{F}_{r}\left(\alpha_{*}, \beta ; \vec{\rho} ; z^{*}\right)}}{\int d \mu_{\Omega_{\mathfrak{S}}, \mathrm{r}}\left(z^{*}, z\right)}
\end{aligned}
$$

By Proposition V.14.i and the hypotheses of Theorem III.26,

$$
\begin{aligned}
\left\|\mathcal{F}_{l}+\mathcal{F}_{r}\right\|_{\text {fluct }} & \leq 2 K_{\mathcal{V}} \delta \mathfrak{v} \mathrm{rR}_{+}^{3}+2^{8} e^{-\mathrm{mc}(\delta)} \Sigma_{\mathcal{D}}+2^{8} \Sigma_{\mathcal{D}}^{(S F)} \\
& \leq \frac{1}{40}
\end{aligned}
$$

by (F.4.c) and (F.6.a). So we can apply Theorem III.14 with $w=w_{\text {fluct }}$ and $f=\mathcal{F}_{l}+\mathcal{F}_{r}$. It gives the existence of a function $\mathcal{D}^{(2)}\left(\alpha_{*}, \beta ; \vec{\rho}\right)$ such that

$$
\frac{\int d \mu_{\Omega_{\mathfrak{S}, \mathrm{r}}}\left(z^{*}, z\right) e^{\mathcal{F}_{l}\left(\alpha_{*}, \beta ; \vec{\rho} ; z\right)+\mathcal{F}_{r}\left(\alpha_{*}, \beta ; \vec{\rho} ; z^{*}\right)}}{\int d \mu_{\Omega_{\mathfrak{S}, \mathrm{r}}}\left(z^{*}, z\right)}=e^{\mathcal{D}^{(2)}\left(\alpha_{*}, \beta ; \vec{\rho}\right)}
$$

since $\mathcal{F}_{l}(0,0 ; \overrightarrow{0} ; z)=\mathcal{F}_{r}\left(0,0 ; \overrightarrow{0} ; z^{*}\right)=0$. The estimate (III.5) in Theorem III.14 applies and

$$
\begin{aligned}
\left\|\mathcal{D}^{(2)}\right\|_{\mathfrak{S}}=\left\|\mathcal{D}^{(2)}\right\|_{\text {fluct }} & \leq 1600\left(\left\|\mathcal{F}_{l}+\mathcal{F}_{r}\right\|_{\text {fluct }}\right)^{2} \\
& \leq 40\left(2 K_{\mathcal{V}} \delta \mathfrak{v} \mathrm{rR}_{+}^{3}+2^{8} e^{-\operatorname{mc}(\delta)} \Sigma_{\mathcal{D}}+2^{8} \Sigma_{\mathcal{D}}^{(S F)}\right)
\end{aligned}
$$

by $(\mathrm{V} .40)$. As $\left.\left[\mathcal{F}_{l}+\mathcal{F}_{r}\right]\right|_{\Omega^{c}}=0$, also $\left.\mathcal{D}^{(2)}\right|_{\Omega^{c}}=0$. We set

$$
\mathcal{D}_{\mathfrak{S}}=\mathcal{D}^{(1)}+\mathcal{D}^{(2)}
$$


By Proposition V.14.ii and (V.42)

$$
\left\|\mathcal{D}_{\mathfrak{S}}\right\|_{\mathfrak{S}} \leq 2^{7} K_{\mathcal{V}}(2 \delta) \mathrm{r}_{+} \mathrm{R}_{+}^{3} \mathfrak{v}+2^{14} e^{-\mathrm{m} \mathfrak{c}(\delta)} \Sigma_{\mathcal{D}}+2^{14} \Sigma_{\mathcal{D}}^{(S F)}
$$

By Corollary V.7,

$$
\left.\mathcal{D}_{\mathfrak{S}}\right|_{\Omega}=\overline{\mathfrak{R}}_{\Omega ; \delta}\left(V_{\Omega, \delta}(\varepsilon ; \cdot) ;\left.\mathcal{D}_{1}(\cdot ; 0)\right|_{\Omega},\left.\mathcal{D}_{2}(\cdot ; 0)\right|_{\Omega}\right)
$$

The desired bound on $\mathcal{L}_{\mathfrak{S}}^{\prime}$ was proven in Proposition V.14. Since $K_{D} \geq 2^{8} K_{\mathcal{V}}$ and $K_{L} \geq$ $2 K_{\mathcal{V}}$ the proof of Theorem III.26 is now complete.

We now move on to the proof of Theorem III.28. The functions $\tilde{\mathcal{D}}^{(2)}$ and $\tilde{\mathcal{D}}_{\mathfrak{S}}$ are constructed as above and obey the corresponding estimates. By Proposition V.14.i and the hypotheses of Theorem III.28,

$$
\left\|\left(\mathcal{F}_{l}+\mathcal{F}_{r}\right)-\left(\tilde{\mathcal{F}}_{l}+\tilde{\mathcal{F}}_{r}\right)\right\|_{\text {fluct }} \leq 2 K_{\mathcal{V}} \varepsilon \delta \mathrm{rv} \mathrm{R}_{+}^{3}+2^{8} e^{-\mathrm{mc}(\delta)} \Delta_{\mathcal{D}}+2^{8} \Delta_{\mathcal{D}}^{(S F)} \leq \frac{1}{2^{8}}
$$

by (F.4.c) and (F.6.a). We apply [BFKT4, Corollary III.6] with $f=\mathcal{F}_{l}+\mathcal{F}_{r}$ and $f^{\prime}=$ $\tilde{\mathcal{F}}_{r}+\tilde{\mathcal{F}}_{r}$. Since

$$
\left\|\mathcal{F}_{l}+\mathcal{F}_{r}\right\|_{\text {fluct }}+\left\|\left(\mathcal{F}_{l}+\mathcal{F}_{r}\right)-\left(\tilde{\mathcal{F}}_{l}+\tilde{\mathcal{F}}_{r}\right)\right\|_{\text {fluct }} \leq \frac{1}{40}+\frac{1}{2^{8}} \leq \frac{1}{34}
$$

it shows that

$$
\left\|\mathcal{D}^{(2)}-\tilde{\mathcal{D}}^{(2)}\right\|_{\mathfrak{S}} \leq 4(34)\left(2 K_{\mathcal{V}} \varepsilon \delta \mathfrak{v} \mathrm{rR}_{+}^{3}+2^{8} e^{-\mathrm{m} \mathfrak{c}(\delta)} \Delta_{\mathcal{D}}+2^{8} \Delta_{\mathcal{D}}^{(S F)}\right)
$$

We combine this with the estimate of Proposition V.14.ii to give

$$
\begin{aligned}
\left\|\mathcal{D}_{\mathfrak{S}}-\tilde{\mathcal{D}}_{\mathfrak{S}}\right\|_{\mathfrak{S}} & \leq[(4)(34)(2)+1] K_{\mathcal{V}} \varepsilon \delta \mathfrak{v} \mathrm{rR}_{+}^{3}+[(4)(34)+1] 2^{8}\left(e^{-\mathrm{mc}(\delta)} \Delta_{\mathcal{D}}+\Delta_{\mathcal{D}}^{(S F)}\right) \\
& \leq 2^{9} K_{\mathcal{V}} \varepsilon(2 \delta \mathfrak{v}) \mathrm{r}_{+} \mathrm{R}_{+}^{3}+2^{16} e^{-\mathrm{mc}(\delta)} \Delta_{\mathcal{D}}+2^{16} \Delta_{\mathcal{D}}^{(S F)}
\end{aligned}
$$

The desired bound on $\left\|\mathcal{L}_{\mathfrak{S}}^{\prime}-\tilde{\mathcal{L}}_{\mathfrak{S}}^{\prime}\right\|_{\mathfrak{S}}$ was proven in Proposition V.14.iii. Since $K_{D} \geq 2{ }^{10} K_{\mathcal{V}}$ and $K_{L} \geq 2 K_{\mathcal{V}}$ the proof of Theorem III.28 is now complete too. 


\section{Large Field Bounds}

In this chapter we prove the large field bounds stated in Propositions III.36, III.37 and III.38 and Lemma III.40. Fix a $0<\delta \leq \Theta$ and an integer $m \geq 0$ and set $\varepsilon=2^{-m} \delta$, as in Theorem III.35. We shall assume that $\varepsilon$ is small enough that $\left|e^{\varepsilon \mu}-1\right| \leq \frac{1}{8}$. For notational compactness, we set

$$
\mathrm{R}_{n}=\mathrm{R}\left(2^{n} \varepsilon\right) \quad \mathrm{R}_{n}^{\prime}=\mathrm{R}^{\prime}\left(2^{n} \varepsilon\right) \quad \mathrm{r}_{n}=\mathrm{r}\left(2^{n} \varepsilon\right) \quad \mathfrak{c}_{n}=\mathfrak{c}\left(2^{n} \varepsilon\right)
$$

as well as $\chi_{n}(\Omega ; \alpha, \beta)=\chi_{2^{n} \varepsilon}(\Omega ; \alpha, \beta)$ and $F_{n}\left(\Omega_{0}\right)=F_{2^{n} \varepsilon}\left(\Omega_{0}\right)$.

Fix a hierarchy $\mathfrak{S}$ for scale $\delta=2^{m} \varepsilon$ with depth at most $m$. For a decimation interval $\mathcal{J} \subset[0, \delta]$, write $\Omega(\mathcal{J})=\Omega_{\mathfrak{S}}(\mathcal{J})$ and $\Lambda(\mathcal{J})=\Lambda_{\mathfrak{S}}(\mathcal{J})$. Similarly, $\Gamma_{* \mathfrak{S}}\left(\tau ; \alpha^{*}, \vec{\alpha}^{*}\right)=$ $\Gamma_{*}\left(\tau ; \alpha^{*}, \vec{\alpha}^{*}\right), \Gamma_{\mathfrak{S}}(\tau ; \vec{\alpha}, \beta)=\Gamma(\tau ; \vec{\alpha}, \beta)$ and $\Omega=\Omega_{\mathfrak{S}}([0, \delta]), \Lambda=\Lambda_{\mathfrak{S}}([0, \delta])$. Recall that, for each decimation interval $\mathcal{J}=\left[t_{-}, t_{+}\right] \subset[0, \delta]$, with midpoint $t, \mathcal{J}^{-}=\left[t_{-}, t\right]$ and $\mathcal{J}^{+}=\left[t, t_{+}\right]$. Also recall, from Notation II.5, that $\Lambda_{0}=\Lambda_{\delta}=\emptyset$ and, for each $\tau \in \varepsilon \mathbb{Z} \cap(0, \delta)$, $\Lambda_{\tau}=\Lambda\left(\mathcal{J}_{\tau}\right)=\Lambda\left(\left[\tau-2^{-\mathfrak{d}(\tau)} \delta, \tau+2^{-\mathfrak{d}(\tau)} \delta\right]\right)$, where $\mathcal{J}_{\tau}$ is the unique decimation interval centred on $\tau$ and the decimation index $\mathfrak{d}(\tau)$ is the smallest integer $k \geq 0$ such that $\tau \in \frac{\delta}{2^{k}} \mathbb{Z}$.

We shall consistently use the notation

$$
\gamma_{* \tau}=\left\{\begin{array}{ll}
\alpha^{*} & \text { if } \tau=0 \\
\Gamma_{*}\left(\tau ; \alpha^{*}, \vec{\alpha}^{*}\right) & \text { if } \tau \in(0, \delta) \\
\beta^{*} & \text { if } \tau=\delta
\end{array}\right\} \quad \gamma_{\tau}=\left\{\begin{array}{ll}
\alpha & \text { if } \tau=0 \\
\Gamma(\tau ; \vec{\alpha}, \beta) & \text { if } \tau \in(0, \delta) \\
\beta & \text { if } \tau=\delta
\end{array}\right\}
$$

Using this notation

$$
\begin{aligned}
-\left[\frac{1}{2}\|\alpha\|^{2}+Q_{\mathfrak{S}}\right. & \left.\left(\alpha^{*}, \beta ; \vec{\alpha}^{*}, \vec{\alpha}\right)+\frac{1}{2}\|\beta\|^{2}\right] \\
& =\sum_{\tau \in \varepsilon \mathbb{Z} \cap[0, \delta)}\left[-\frac{1}{2}\left\langle\gamma_{* \tau}, \gamma_{\tau}\right\rangle+\left\langle\gamma_{* \tau}, j(\varepsilon) \gamma_{\tau+\varepsilon}\right\rangle-\frac{1}{2}\left\langle\gamma_{* \tau+\varepsilon}, \gamma_{\tau+\varepsilon}\right\rangle\right]
\end{aligned}
$$

and

$$
\mathcal{V}_{\mathfrak{S}}\left(\varepsilon ; \alpha^{*}, \beta ; \vec{\alpha}^{*}, \vec{\alpha}\right)=-\varepsilon \sum_{\tau \in \varepsilon \mathbb{Z} \cap[0, \delta)}\left\langle\gamma_{* \tau} \gamma_{\tau+\varepsilon}, v \gamma_{* \tau} \gamma_{\tau+\varepsilon}\right\rangle
$$

Throughout this chapter we assume that the field $\alpha_{\tau}$ is compatible with $\mathfrak{S}$ in the sense of Definition E.1, as is the case in the domain of the integral operator $\mathcal{I}_{\left(\mathfrak{S}, \varepsilon ; \alpha^{*}, \beta\right)}$. In particular,

$$
\left|\alpha_{\tau}(\mathbf{x})\right| \leq \min \left\{\kappa_{\tau}(\mathbf{x}), \kappa_{* \tau}(\mathbf{x})\right\} \quad|\alpha(\mathbf{x})| \leq \kappa_{* 0}(\mathbf{x}) \quad|\beta(\mathbf{x})| \leq \kappa_{\delta}(\mathbf{x})
$$

for all $\tau \in \varepsilon \mathbb{Z} \cap(0, \delta)$ and $\mathbf{x} \in X$. We also assume that $\mathfrak{h} \equiv 1$, as we did in $\S I I I .7$ and, in particular, in Theorem III.35. 
In Propositions III.36 and III.37, we introduced restricted large fields regions $\tilde{P}_{\alpha}(\mathcal{J})$, $\cdots, \tilde{Q}(\mathcal{J})$. The reason for introducing these smaller large field sets is the following. When we are decimating at time $t$, the centre of $\mathcal{J}$, we need to extract a small factor for certain points in $\Lambda(\mathcal{J})^{c}$ that are not in $\Lambda\left(\mathcal{J}^{-}\right)^{c} \cup \Lambda\left(\mathcal{J}^{+}\right)^{c}$. Small factors were already extracted from the latter regions in previous decimation steps. Each time we extract a small factor associated with a point $\mathbf{x}$ we will distribute it amongst all nearby points $\mathbf{y}$. As a result, when we are decimating at time $t$, it is not necessary to extract small factors from points that are within a distance $2 \mathfrak{c}\left(\left|\mathcal{J}^{ \pm}\right|\right)$of $\Lambda\left(\mathcal{J}^{-}\right)^{c} \cup \Lambda\left(\mathcal{J}^{+}\right)^{c}$. So, for example, $\tilde{P}_{\alpha}(\mathcal{J})$ consists of those points of $P_{\alpha}(\mathcal{J})$ whose distance from $\Lambda\left(\mathcal{J}^{-}\right)^{c} \cup \Lambda\left(\mathcal{J}^{+}\right)^{c}$ is greater than $2 \mathfrak{c}\left(\left|\mathcal{J}^{ \pm}\right|\right)$.

Remark VI.1 For each decimation interval $\mathcal{J}=\left[t_{-}, t_{+}\right]$,

$$
\begin{aligned}
& \tilde{P}_{\alpha}(\mathcal{J})=\left\{\mathbf{x} \in \Omega_{0}(\mathcal{J})|| \alpha_{t_{-}}(\mathbf{x}) \mid>\mathrm{R}(|\mathcal{J}|), \begin{array}{c}
d(\mathbf{x}, \Lambda(\mathcal{J}))>\mathfrak{c}\left(\frac{1}{2}|\mathcal{J}|\right) \\
d\left(\mathbf{x}, \Lambda\left(\mathcal{J}^{-}\right)^{c} \cup \Lambda\left(\mathcal{J}^{+}\right)^{c}>2 \mathfrak{c}\left(\frac{1}{2}|\mathcal{J}|\right)\right.
\end{array}\right\} \\
& \tilde{P}_{\beta}(\mathcal{J})=\left\{\mathbf{x} \in \Omega_{0}(\mathcal{J})|| \alpha_{t_{+}}(\mathbf{x}) \mid>\mathrm{R}(|\mathcal{J}|), \begin{array}{c}
d(b, \Lambda(\mathcal{J}))>\mathfrak{c}\left(\frac{1}{2}|\mathcal{J}|\right) \\
d\left(b, \Lambda\left(\mathcal{J}^{-}\right)^{c} \cup \Lambda\left(\mathcal{J}^{+}\right)^{c}>2 \mathfrak{c}\left(\frac{1}{2}|\mathcal{J}|\right)\right.
\end{array}\right\} \\
& \tilde{P}_{\alpha}^{\prime}(\mathcal{J})=\left\{b \in \Omega_{0}(\mathcal{J})^{*}||\left(\nabla \alpha_{t_{-}}\right)(b) \mid>\mathrm{R}^{\prime}(|\mathcal{J}|), \begin{array}{c}
d(b, \Lambda(\mathcal{J}))>\mathfrak{c}\left(\frac{1}{2}|\mathcal{J}|\right) \\
d\left(b, \Lambda\left(\mathcal{J}^{-}\right)^{c} \cup \Lambda\left(\mathcal{J}^{+}\right)^{c}\right)>2 \mathfrak{c}\left(\frac{1}{2}|\mathcal{J}|\right)
\end{array}\right\} \\
& \tilde{P}_{\beta}^{\prime}(\mathcal{J})=\left\{b \in \Omega_{0}(\mathcal{J})^{*}||\left(\nabla \alpha_{t_{+}}\right)(b) \mid>\mathrm{R}^{\prime}(|\mathcal{J}|), \begin{array}{c}
d\left(b, \Lambda(\mathcal{J})>>\mathfrak{c}\left(\frac{1}{2}|\mathcal{J}|\right)\right. \\
d\left(b, \Lambda\left(\mathcal{J}^{-}\right)^{c} \cup \Lambda\left(\mathcal{J}^{+}\right)^{c}\right)>2 \mathfrak{c}\left(\frac{1}{2}|\mathcal{J}|\right)
\end{array}\right\} \\
& \tilde{Q}(\mathcal{J})=\left\{\mathbf{x} \in \Omega_{0}(\mathcal{J})^{\star}|| \alpha_{t_{+}}(\mathbf{x})-\alpha_{t_{-}}(\mathbf{x}) \mid>\mathrm{r}(|\mathcal{J}|), \begin{array}{c}
d(\mathbf{x}, \Lambda(\mathcal{J}))>\mathfrak{c}\left(\frac{1}{2}|\mathcal{J}|\right) \\
d\left(\mathbf{x}, \Lambda\left(\mathcal{J}^{-}\right)^{c} \cup \Lambda\left(\mathcal{J}^{+}\right)^{c}\right)>2 \mathfrak{c}\left(\frac{1}{2}|\mathcal{J}|\right)
\end{array}\right\}
\end{aligned}
$$

where $\Omega_{0}(\mathcal{J})=\Omega\left(\mathcal{J}^{-}\right) \cap \Omega\left(\mathcal{J}^{+}\right)$.

\section{VI.1 Extracting Small Factors from the Quadratic Form}

In this subsection, we prove Proposition III.36. The main ingredient is

Lemma VI.2 Set $\mathfrak{j}(\varepsilon)=e^{-\varepsilon \mu} j(\varepsilon)=e^{-\varepsilon h}$ and $c_{\mathfrak{j}, \varepsilon}=\|1-\mathfrak{j}(\varepsilon)\|$. Assume that $c_{\mathfrak{j}, \varepsilon} \leq \frac{1}{9}$.

Then

$$
\begin{aligned}
& \operatorname{Re} \sum_{\tau \in \varepsilon \mathbb{Z} \cap[0, \delta)}\left[-\frac{1}{2}\left\langle\gamma_{* \tau}, \gamma_{\tau}\right\rangle+\left\langle\gamma_{* \tau}, j(\varepsilon) \gamma_{\tau+\varepsilon}\right\rangle-\frac{1}{2}\left\langle\gamma_{* \tau+\varepsilon}, \gamma_{\tau+\varepsilon}\right\rangle\right] \\
& \leq-\frac{1}{4}\left\{\sum_{\tau \in[0, \delta)} \frac{1}{2}\left\|\gamma_{* \tau}^{*}-\gamma_{\tau+\varepsilon}\right\|_{\Lambda_{\tau}^{c} \cup \Lambda_{\tau+\varepsilon}^{c}}^{2}+\sum_{\tau \in(0, \delta]}\left\langle\gamma_{\tau}^{*},[1-j(\varepsilon)] \gamma_{\tau}\right\rangle+\sum_{\tau \in[0, \delta)}\left\langle\gamma_{* \tau}^{*},[1-j(\varepsilon)] \gamma_{* \tau}\right\rangle\right\} \\
& \quad+\frac{1}{2} \sum_{\tau \in(0, \delta)}\left|\left\langle\gamma_{* \tau}-\gamma_{\tau}^{*}, \Lambda_{\tau}\left(\gamma_{\tau}-j(\varepsilon) \gamma_{\tau+\varepsilon}\right)\right\rangle\right|+\frac{1}{2} \sum_{\tau \in(0, \delta)}\left|\left\langle\gamma_{\tau}-\gamma_{* \tau}^{*}, \Lambda_{\tau}\left(\gamma_{* \tau}-j(\varepsilon) \gamma_{* \tau-\varepsilon}\right)\right\rangle\right| \\
& \quad+\frac{1}{2} \sum_{\tau \in(0, \delta)}\left\|\Lambda_{\tau}^{c}\left(\gamma_{* \tau}^{*}-\gamma_{\tau}\right)\right\|^{2}+\left|e^{\varepsilon \mu}-1\right| \sum_{\tau \in[0, \delta]}\left[\left\|\gamma_{\tau}\right\|^{2}+\left\|\gamma_{* \tau}\right\|^{2}\right]
\end{aligned}
$$


where, for each subset $S \subset X,\|u\|_{S}^{2}=\sum_{\mathbf{x} \in S}|u(\mathbf{x})|^{2}=\left\langle S u^{*}, S u\right\rangle$.

Proof: $\quad$ Recall that $\|\alpha\|^{*}=\left\langle\alpha^{*}, \alpha\right\rangle$ and $\gamma_{* 0}=\gamma_{0}^{*}, \gamma_{* \delta}=\gamma_{\delta}^{*}$ so that $\left\langle\gamma_{* 0}, \gamma_{0}\right\rangle=\left\|\gamma_{0}\right\|^{2}=$ $\left\|\gamma_{* 0}^{*}\right\|^{2}$ and $\left\langle\gamma_{* \delta}, \gamma_{\delta}\right\rangle=\left\|\gamma_{\delta}\right\|^{2}$. The real part of

$$
\begin{aligned}
& \sum_{\tau \in[0, \delta)}\left[-\frac{1}{2}\left\langle\gamma_{* \tau}, \gamma_{\tau}\right\rangle+\left\langle\gamma_{* \tau}, j(\varepsilon) \gamma_{\tau+\varepsilon}\right\rangle-\frac{1}{2}\left\langle\gamma_{* \tau+\varepsilon}, \gamma_{\tau+\varepsilon}\right\rangle\right] \\
& =-\frac{1}{2}\left\|\gamma_{0}\right\|^{2}-\sum_{\tau \in(0, \delta)}\left\langle\gamma_{* \tau}, \gamma_{\tau}\right\rangle-\frac{1}{2}\left\|\gamma_{\delta}\right\|^{2}+\sum_{\tau \in[0, \delta)}\left\langle\gamma_{* \tau}, j(\varepsilon) \gamma_{\tau+\varepsilon}\right\rangle \\
& =-\frac{1}{2}\left\|\gamma_{0}\right\|^{2}-\sum_{\tau \in(0, \delta)}\left\langle\gamma_{* \tau}, \gamma_{\tau}\right\rangle-\frac{1}{2}\left\|\gamma_{\delta}\right\|^{2}+\sum_{\tau \in[0, \delta)}\left\langle\gamma_{* \tau}, \gamma_{\tau+\varepsilon}\right\rangle-\sum_{\tau \in[0, \delta)}\left\langle\gamma_{* \tau},[1-j(\varepsilon)] \gamma_{\tau+\varepsilon}\right\rangle \\
& =\sum_{\tau \in(0, \delta)}\left[\frac{1}{2}\left\|\gamma_{* \tau}^{*}\right\|^{2}-\left\langle\gamma_{* \tau}, \gamma_{\tau}\right\rangle+\frac{1}{2}\left\|\gamma_{\tau}\right\|^{2}\right]+\sum_{\tau \in[0, \delta)}\left[-\frac{1}{2}\left\|\gamma_{* \tau}^{*}\right\|^{2}+\left\langle\gamma_{* \tau}, \gamma_{\tau+\varepsilon}\right\rangle-\frac{1}{2}\left\|\gamma_{\tau+\varepsilon}\right\|^{2}\right] \\
& -\sum_{\tau \in[0, \delta)}\left\langle\gamma_{* \tau},[1-j(\varepsilon)] \gamma_{\tau+\varepsilon}\right\rangle
\end{aligned}
$$

is

$$
\begin{aligned}
& \operatorname{Re} \sum_{\tau \in[0, \delta)}\left[-\frac{1}{2}\left\langle\gamma_{* \tau}, \gamma_{\tau}\right\rangle+\left\langle\gamma_{* \tau}, j(\varepsilon) \gamma_{\tau+\varepsilon}\right\rangle-\frac{1}{2}\left\langle\gamma_{* \tau+\varepsilon}, \gamma_{\tau+\varepsilon}\right\rangle\right] \\
& \quad=\frac{1}{2} \sum_{\tau \in(0, \delta)}\left\|\gamma_{* \tau}^{*}-\gamma_{\tau}\right\|^{2}-\frac{1}{2} \sum_{\tau \in[0, \delta)}\left\|\gamma_{* \tau}^{*}-\gamma_{\tau+\varepsilon}\right\|^{2}-\sum_{\tau \in[0, \delta)} \operatorname{Re}\left\langle\gamma_{* \tau},[\mathbb{1}-j(\varepsilon)] \gamma_{\tau+\varepsilon}\right\rangle \\
& \quad=\sum_{\tau \in[0, \delta)}\left\{\frac{1}{2}\left\|\gamma_{* \tau}^{*}-\gamma_{\tau}\right\|^{2}-\frac{1}{2}\left\|\gamma_{* \tau}^{*}-\gamma_{\tau+\varepsilon}\right\|^{2}-\operatorname{Re}\left\langle\gamma_{* \tau},[\mathbb{1}-j(\varepsilon)] \gamma_{\tau+\varepsilon}\right\rangle\right\}
\end{aligned}
$$

because $\|\alpha-\beta\|^{2}=\|\alpha\|^{2}+\|\beta\|^{2}-2 \operatorname{Re}\left\langle\alpha^{*}, \beta\right\rangle$. Now

$$
\begin{aligned}
& \left\|\gamma_{* \tau}^{*}-\gamma_{\tau}\right\|_{\Lambda_{\tau}}^{2}-\left\|\gamma_{* \tau}^{*}-\gamma_{\tau+\varepsilon}\right\|_{\Lambda_{\tau}}^{2} \\
& =\left\|\Lambda_{\tau} \gamma_{* \tau}^{*}-\Lambda_{\tau} j(\varepsilon) \gamma_{\tau+\varepsilon}-\Lambda_{\tau}\left(\gamma_{\tau}-j(\varepsilon) \gamma_{\tau+\varepsilon}\right)\right\|^{2}-\left\|\gamma_{* \tau}^{*}-\gamma_{\tau+\varepsilon}\right\|_{\Lambda_{\tau}}^{2} \\
& =\left\|\Lambda_{\tau} \gamma_{* \tau}^{*}-\Lambda_{\tau} j(\varepsilon) \gamma_{\tau+\varepsilon}\right\|^{2}-\left\|\gamma_{* \tau}^{*}-\gamma_{\tau+\varepsilon}\right\|_{\Lambda_{\tau}}^{2} \\
& \quad+\left\|\Lambda_{\tau}\left(\gamma_{\tau}-j(\varepsilon) \gamma_{\tau+\varepsilon}\right)\right\|^{2}-2 \operatorname{Re}\left\langle\gamma_{* \tau}-j(\varepsilon) \gamma_{\tau+\varepsilon}^{*}, \Lambda_{\tau}\left(\gamma_{\tau}-j(\varepsilon) \gamma_{\tau+\varepsilon}\right)\right\rangle \\
& =\left\|\Lambda_{\tau}\left(\gamma_{* \tau}^{*}-\gamma_{\tau+\varepsilon}\right)+\Lambda_{\tau}[\mathbb{1}-j(\varepsilon)] \gamma_{\tau+\varepsilon}\right\|^{2}-\left\|\gamma_{* \tau}^{*}-\gamma_{\tau+\varepsilon}\right\|_{\Lambda_{\tau}}^{2} \\
& \quad+\left\|\Lambda_{\tau}\left(\gamma_{\tau}-j(\varepsilon) \gamma_{\tau+\varepsilon}\right)\right\|^{2}-2 \operatorname{Re}\left\langle\gamma_{* \tau}-j(\varepsilon) \gamma_{\tau+\varepsilon}^{*}, \Lambda_{\tau}\left(\gamma_{\tau}-j(\varepsilon) \gamma_{\tau+\varepsilon}\right)\right\rangle \\
& =2 \operatorname{Re}\left\langle\Lambda_{\tau}\left(\gamma_{* \tau}-\gamma_{\tau+\varepsilon}^{*}\right),[\mathbb{1}-j(\varepsilon)] \gamma_{\tau+\varepsilon}\right\rangle+\left\|\Lambda_{\tau}[\mathbb{1}-j(\varepsilon)] \gamma_{\tau+\varepsilon}\right\|^{2} \\
& \quad+\left\|\Lambda_{\tau}\left(\gamma_{\tau}-j(\varepsilon) \gamma_{\tau+\varepsilon}\right)\right\|^{2}-2 \operatorname{Re}\left\langle\gamma_{* \tau}-\gamma_{\tau}^{*}+\gamma_{\tau}^{*}-j(\varepsilon) \gamma_{\tau+\varepsilon}^{*}, \Lambda_{\tau}\left(\gamma_{\tau}-j(\varepsilon) \gamma_{\tau+\varepsilon}\right)\right\rangle \\
& =2 \operatorname{Re}\left\langle\Lambda_{\tau}\left(\gamma_{* \tau}-\gamma_{\tau+\varepsilon}^{*}\right),[\mathbb{1}-j(\varepsilon)] \gamma_{\tau+\varepsilon}\right\rangle+\left\|\Lambda_{\tau}[\mathbb{1}-j(\varepsilon)] \gamma_{\tau+\varepsilon}\right\|^{2} \\
& \quad-\left\|\Lambda_{\tau}\left(\gamma_{\tau}-j(\varepsilon) \gamma_{\tau+\varepsilon}\right)\right\|^{2}-2 \operatorname{Re}\left\langle\gamma_{* \tau}-\gamma_{\tau}^{*}, \Lambda_{\tau}\left(\gamma_{\tau}-j(\varepsilon) \gamma_{\tau+\varepsilon}\right)\right\rangle
\end{aligned}
$$


so that

$$
\begin{aligned}
& \frac{1}{2}\left\|\gamma_{* \tau}^{*}-\gamma_{\tau}\right\|^{2}-\frac{1}{2}\left\|\gamma_{* \tau}^{*}-\gamma_{\tau+\varepsilon}\right\|^{2}-\operatorname{Re}\left\langle\gamma_{* \tau},[1-j(\varepsilon)] \gamma_{\tau+\varepsilon}\right\rangle \\
& =-\frac{1}{2}\left\|\gamma_{* \tau}^{*}-\gamma_{\tau+\varepsilon}\right\|_{\Lambda_{\tau}^{c}}^{2}-\operatorname{Re}\left\langle\Lambda_{\tau} \gamma_{\tau+\varepsilon}^{*}+\Lambda_{\tau}^{c} \gamma_{* \tau},[1-j(\varepsilon)] \gamma_{\tau+\varepsilon}\right\rangle+\frac{1}{2}\left\|\Lambda_{\tau}[1-j(\varepsilon)] \gamma_{\tau+\varepsilon}\right\|^{2} \\
& \quad-\frac{1}{2}\left\|\Lambda_{\tau}\left(\gamma_{\tau}-j(\varepsilon) \gamma_{\tau+\varepsilon}\right)\right\|^{2}-\operatorname{Re}\left\langle\gamma_{* \tau}-\gamma_{\tau}^{*}, \Lambda_{\tau}\left(\gamma_{\tau}-j(\varepsilon) \gamma_{\tau+\varepsilon}\right)\right\rangle+\frac{1}{2}\left\|\gamma_{* \tau}^{*}-\gamma_{\tau}\right\|_{\Lambda_{\tau}^{c}}^{2} \\
& =-\frac{1}{2}\left\|\gamma_{* \tau}^{*}-\gamma_{\tau+\varepsilon}\right\|_{\Lambda_{\tau}^{c}}^{2}-\left\langle\gamma_{\tau+\varepsilon}^{*},[11-j(\varepsilon)] \gamma_{\tau+\varepsilon}\right\rangle-\frac{1}{2}\left\|\gamma_{\tau}-j(\varepsilon) \gamma_{\tau+\varepsilon}\right\|_{\Lambda_{\tau}}^{2} \\
& \quad-\operatorname{Re}\left\langle\Lambda_{\tau}^{c}\left(\gamma_{* \tau}-\gamma_{\tau+\varepsilon}^{*}\right),[11-j(\varepsilon)] \gamma_{\tau+\varepsilon}\right\rangle+\frac{1}{2}\left\|\Lambda_{\tau}[1-j(\varepsilon)] \gamma_{\tau+\varepsilon}\right\|^{2} \\
& \quad-\operatorname{Re}\left\langle\gamma_{* \tau}-\gamma_{\tau}^{*}, \Lambda_{\tau}\left(\gamma_{\tau}-j(\varepsilon) \gamma_{\tau+\varepsilon}\right)\right\rangle+\frac{1}{2}\left\|\gamma_{* \tau}^{*}-\gamma_{\tau}\right\|_{\Lambda_{\tau}^{c}}^{2} \\
& =-\frac{1}{2}\left\|\gamma_{* \tau}^{*}-\gamma_{\tau+\varepsilon}\right\|_{\Lambda_{\tau}^{c}}^{2}-e^{\varepsilon \mu}\left\langle\gamma_{\tau+\varepsilon}^{*},[1-j(\varepsilon)] \gamma_{\tau+\varepsilon}\right\rangle \\
& \quad-e^{\varepsilon \mu} \operatorname{Re}\left\langle\Lambda_{\tau}^{c}\left(\gamma_{* \tau}-\gamma_{\tau+\varepsilon}^{*}\right),[1-j(\varepsilon)] \gamma_{\tau+\varepsilon}\right\rangle+\frac{1}{2}\left\|\Lambda_{\tau}[1-j(\varepsilon)] \gamma_{\tau+\varepsilon}\right\|^{2} \\
& \quad+\left(e^{\varepsilon \mu}-1\right)\left\|\gamma_{\tau+\varepsilon}\right\|^{2}+\left(e^{\varepsilon \mu}-1\right) \operatorname{Re}\left\langle\Lambda_{\tau}^{c}\left(\gamma_{* \tau}-\gamma_{\tau+\varepsilon}^{*}\right), \gamma_{\tau+\varepsilon}\right\rangle \\
& \quad-\frac{1}{2}\left\|\gamma_{\tau}-j(\varepsilon) \gamma_{\tau+\varepsilon}\right\|_{\Lambda_{\tau}}^{2}-\operatorname{Re}\left\langle\gamma_{* \tau}-\gamma_{\tau}^{*}, \Lambda_{\tau}\left(\gamma_{\tau}-j(\varepsilon) \gamma_{\tau+\varepsilon}\right)\right\rangle+\frac{1}{2}\left\|\gamma_{* \tau}^{*}-\gamma_{\tau}\right\|_{\Lambda_{\tau}^{c}}^{2}
\end{aligned}
$$

Using Cauchy-Schwarz and $|A B| \leq \frac{1}{2}\left(A^{2}+B^{2}\right)$,

$$
\begin{aligned}
& \left|\left\langle\Lambda_{\tau}^{c}\left(\gamma_{* \tau}-\gamma_{\tau+\varepsilon}^{*}\right),[1-\mathfrak{j}(\varepsilon)] \gamma_{\tau+\varepsilon}\right\rangle\right| \leq\left\|\gamma_{* \tau}^{*}-\gamma_{\tau+\varepsilon}\right\|_{\Lambda_{\tau}^{c}}\|1-\mathfrak{j}(\varepsilon)\|^{1 / 2}\left\|[1-\mathfrak{j}(\varepsilon)]^{1 / 2} \gamma_{\tau+\varepsilon}\right\| \\
& \leq \sqrt{c_{\mathfrak{j}, \varepsilon}} \frac{1}{2}\left(\left\|\gamma_{* \tau}^{*}-\gamma_{\tau+\varepsilon}\right\|_{\Lambda_{\tau}^{c}}^{2}+\left\langle\gamma_{\tau+\varepsilon}^{*},[11-\mathfrak{j}(\varepsilon)] \gamma_{\tau+\varepsilon}\right\rangle\right) \\
& \left|\left(e^{\varepsilon \mu}-1\right) \operatorname{Re}\left\langle\Lambda_{\tau}^{c}\left(\gamma_{* \tau}-\gamma_{\tau+\varepsilon}^{*}\right), \gamma_{\tau+\varepsilon}\right\rangle\right| \leq \frac{1}{2}\left|e^{\varepsilon \mu}-1\right|\left(\left\|\gamma_{* \tau}^{*}-\gamma_{\tau+\varepsilon}\right\|_{\Lambda_{\tau}^{c}}^{2}+\left\|\gamma_{\tau+\varepsilon}\right\|^{2}\right) \\
& \frac{1}{2}\left\|\Lambda_{\tau}[\mathbb{1}-j(\varepsilon)] \gamma_{\tau+\varepsilon}\right\|^{2} \leq\left\|[\mathbb{1}-\mathfrak{j}(\varepsilon)] \gamma_{\tau+\varepsilon}\right\|^{2}+\left\|[j(\varepsilon)-\mathfrak{j}(\varepsilon)] \gamma_{\tau+\varepsilon}\right\|^{2} \\
& \leq\|\mathbb{1}-\mathfrak{j}(\varepsilon)\|\left\|[1 \mathbb{1}-\mathfrak{j}(\varepsilon)]^{\frac{1}{2}} \gamma_{\tau+\varepsilon}\right\|^{2}+\left[e^{\varepsilon \mu}-1\right]^{2}\left\|\mathfrak{j}(\varepsilon) \gamma_{\tau+\varepsilon}\right\|^{2} \\
& \leq c_{\mathfrak{j}, \varepsilon}\left\langle\gamma_{\tau+\varepsilon}^{*},[1-\mathfrak{j}(\varepsilon)] \gamma_{\tau+\varepsilon}\right\rangle+\left[e^{\varepsilon \mu}-1\right]^{2}\left\|\gamma_{\tau+\varepsilon}\right\|^{2}
\end{aligned}
$$

Thus we have

$$
\begin{aligned}
\operatorname{Re} & \sum_{\tau \in[0, \delta)}\left[-\frac{1}{2}\left\langle\gamma_{* \tau}, \gamma_{\tau}\right\rangle+\left\langle\gamma_{* \tau}, j(\varepsilon) \gamma_{\tau+\varepsilon}\right\rangle-\frac{1}{2}\left\langle\gamma_{* \tau+\varepsilon}, \gamma_{\tau+\varepsilon}\right\rangle\right] \\
\leq- & \sum_{\tau \in[0, \delta)}\left\{\frac{1}{2}\left(1-e^{\varepsilon \mu} \sqrt{c_{\mathfrak{j}, \varepsilon}}-\left|e^{\varepsilon \mu}-1\right|\right)\left\|\gamma_{* \tau}^{*}-\gamma_{\tau+\varepsilon}\right\|_{\Lambda_{\tau}^{c}}^{2}\right. \\
& \left.-\sum_{\tau \in[0, \delta)}\left(e^{\varepsilon \mu}-\frac{1}{2} e^{\varepsilon \mu} \sqrt{c_{\mathfrak{j}, \varepsilon}}-c_{\mathfrak{j}, \varepsilon}\right)\left\langle\gamma_{\tau+\varepsilon}^{*},[1-j(\varepsilon)] \gamma_{\tau+\varepsilon}\right\rangle\right\} \\
& +\sum_{\tau \in[0, \delta)}\left(\frac{3}{2}\left|e^{\varepsilon \mu}-1\right|+\left[e^{\varepsilon \mu}-1\right]^{2}\right)\left\|\gamma_{\tau+\varepsilon}\right\|^{2} \\
& -\sum_{\tau \in[0, \delta)} \frac{1}{2}\left\|\gamma_{\tau}-j(\varepsilon) \gamma_{\tau+\varepsilon}\right\|_{\Lambda_{\tau}}^{2}-\sum_{\tau \in[0, \delta)} \operatorname{Re}\left\langle\gamma_{* \tau}-\gamma_{\tau}^{*}, \Lambda_{\tau}\left(\gamma_{\tau}-j(\varepsilon) \gamma_{\tau+\varepsilon}\right)\right\rangle \\
& +\frac{1}{2} \sum_{\tau \in[0, \delta)}\left\|\gamma_{* \tau}^{*}-\gamma_{\tau}\right\|_{\Lambda_{\tau}^{c}}^{2}
\end{aligned}
$$


and hence, since $1-e^{\varepsilon \mu} \sqrt{c_{\mathfrak{j}, \varepsilon}}-\left|e^{\varepsilon \mu}-1\right| \geq \frac{1}{2}$ and $e^{\varepsilon \mu}-\frac{1}{2} e^{\varepsilon \mu} \sqrt{c_{\mathfrak{j}, \varepsilon}}-c_{\mathfrak{j}, \varepsilon} \geq \frac{1}{2}$,

$$
\begin{aligned}
\operatorname{Re} & \sum_{\tau \in[0, \delta)}\left[-\frac{1}{2}\left\langle\gamma_{* \tau}, \gamma_{\tau}\right\rangle+\left\langle\gamma_{* \tau}, j(\varepsilon) \gamma_{\tau+\varepsilon}\right\rangle-\frac{1}{2}\left\langle\gamma_{* \tau+\varepsilon}, \gamma_{\tau+\varepsilon}\right\rangle\right] \\
\leq- & \sum_{\tau \in[0, \delta)}\left\{\frac{1}{4}\left\|\gamma_{* \tau}^{*}-\gamma_{\tau+\varepsilon}\right\|_{\Lambda_{\tau}^{c}}^{2}+\frac{1}{2}\left\langle\gamma_{\tau+\varepsilon}^{*},[1-j(\varepsilon)] \gamma_{\tau+\varepsilon}\right\rangle\right\} \\
& -\sum_{\tau \in(0, \delta)} \operatorname{Re}\left\langle\gamma_{* \tau}-\gamma_{\tau}^{*}, \Lambda_{\tau}\left(\gamma_{\tau}-j(\varepsilon) \gamma_{\tau+\varepsilon}\right)\right\rangle+2\left|e^{\varepsilon \mu}-1\right| \sum_{\tau \in[0, \delta]}\left\|\gamma_{\tau}\right\|^{2} \\
& +\frac{1}{2} \sum_{\tau \in(0, \delta)}\left\|\gamma_{* \tau}^{*}-\gamma_{\tau}\right\|_{\Lambda_{\tau}^{c}}^{2}
\end{aligned}
$$

The bound

$$
\begin{aligned}
& \operatorname{Re} \sum_{\tau \in[0, \delta)}\left[-\frac{1}{2}\left\langle\gamma_{* \tau}, \gamma_{\tau}\right\rangle+\left\langle\gamma_{* \tau}, j(\varepsilon) \gamma_{\tau+\varepsilon}\right\rangle-\frac{1}{2}\left\langle\gamma_{* \tau+\varepsilon}, \gamma_{\tau+\varepsilon}\right\rangle\right] \\
& \leq-\sum_{\tau \in(0, \delta]}\left\{\frac{1}{4}\left\|\gamma_{* \tau-\varepsilon}^{*}-\gamma_{\tau}\right\|_{\Lambda_{\tau}^{c}}^{2}+\frac{1}{2}\left\langle\gamma_{* \tau-\varepsilon}^{*},[1-j(\varepsilon)] \gamma_{* \tau-\varepsilon}\right\rangle\right\} \\
& \quad-\sum_{\tau \in(0, \delta)} \operatorname{Re}\left\langle\Lambda_{\tau}\left(\gamma_{* \tau}-j(\varepsilon) \gamma_{* \tau-\varepsilon}\right), \gamma_{\tau}-\gamma_{* \tau}^{*}\right\rangle+2\left|e^{\varepsilon \mu}-1\right| \sum_{\tau \in[0, \delta]}\left\|\gamma_{* \tau}\right\|^{2} \\
& \quad+\frac{1}{2} \sum_{\tau \in(0, \delta)}\left\|\gamma_{* \tau}^{*}-\gamma_{\tau}\right\|_{\Lambda_{\tau}^{c}}^{2}
\end{aligned}
$$

is proven similarly. Taking the average of these two bounds and using

$$
\frac{1}{8}\left\|\gamma_{* \tau}^{*}-\gamma_{\tau+\varepsilon}\right\|_{\Lambda_{\tau}^{c}}^{2}+\frac{1}{8}\left\|\gamma_{* \tau}^{*}-\gamma_{\tau+\varepsilon}\right\|_{\Lambda_{\tau+\varepsilon}^{c}}^{2} \geq \frac{1}{8}\left\|\gamma_{* \tau}^{*}-\gamma_{\tau+\varepsilon}\right\|_{\Lambda_{\tau}^{c} \cup \Lambda_{\tau+\varepsilon}^{c}}^{2}
$$

gives the bound of the Lemma.

The first line of the right hand side of the conclusion of VI.2, consists of terms that are invariably negative. The first can be thought of as a time derivative term and the other two as space derivative terms. These three terms are responsible for the contributions $\mathrm{r}(|\mathcal{J}|)^{2}\left\{|\tilde{Q}(\mathcal{J})|+\left|\tilde{P}_{\alpha}^{\prime}(\mathcal{J})\right|+\left|\tilde{P}_{\beta}^{\prime}(\mathcal{J})\right|\right\}$ to Proposition III.36. See (VI.8,a,b,c).

The terms on the other two lines are all positive. The terms on the second line will be controlled using the "smallness" of $\gamma_{\tau}-j(\varepsilon) \gamma_{\tau+\varepsilon}$ and $\gamma_{* \tau}-j(\varepsilon) \gamma_{* \tau-\varepsilon}$. See Lemma E.17. The first term on the third line is controlled in Proposition E.11.ii, using the smallness of $\gamma_{* \tau}^{*}-\gamma_{\tau}$. The small field part of the second term in the third line is bounded in the following lemma and gives the small field regulator $\operatorname{Reg}_{\mathcal{S F}}^{(2)}(\Omega ; \alpha, \beta)$ in Proposition III.36. The large field part is left explicitly in Proposition III.36. In the proof of Theorem III.35, it is canceled by quartic contributions. 


\section{Lemma VI.3}

$$
\left|e^{\varepsilon \mu}-1\right| \sum_{\tau \in[0, \delta]}\left[\left\|\gamma_{\tau}\right\|_{\Omega}^{2}+\left\|\gamma_{* \tau}\right\|_{\Omega}^{2}\right] \leq K_{\mathrm{reg}} \delta|\mu|\left[\|\alpha\|_{\Omega}^{2}+\|\beta\|_{\Omega}^{2}\right]+\frac{1}{16}\left|\Omega^{c}\right|
$$

with the $K_{\text {reg }}$ of Definition II.1\%.

Proof: Write, using the notation of Definition E.3,

$$
\sum_{\tau \in(0, \delta)}\left\|\gamma_{\tau}\right\|_{\Omega}^{2}=\sum_{\substack{\tau \in(0, \delta) \\ \tau_{1}, \tau_{2} \in \mathcal{T}_{r}(\tau, \delta)}} \sum_{\mathbf{x} \in \Omega} \overline{\left(\Gamma_{\tau}^{\tau_{1}} \alpha_{\tau_{1}}\right)(\mathbf{x})}\left(\Gamma_{\tau}^{\tau_{2}} \alpha_{\tau_{2}}\right)(\mathbf{x})
$$

We bound the terms with $\left(\tau_{1}, \tau_{2}\right) \neq(\delta, \delta)$ using

$$
\begin{aligned}
& \sum_{\substack{\tau \in(0, \delta) \\
\tau_{1}, \tau_{2} \in \mathcal{T}_{r}(\tau, \delta) \\
\left(\tau_{1}, \tau_{2}\right) \neq(\delta, \delta)}} \sum_{\mathbf{x} \in \Omega} \overline{\left(\Gamma_{\tau}^{\tau_{1}} \alpha_{\tau_{1}}\right)(\mathbf{x})}\left(\Gamma_{\tau}^{\tau_{2}} \alpha_{\tau_{2}}\right)(\mathbf{x}) \mid \\
& \leq \sum_{\substack{\tau \in(0, \delta) \\
\tau_{1}, \tau_{2} \in \mathcal{T}_{r}(\tau, \delta) \\
\left(\tau_{1}, \tau_{2}\right) \neq(\delta, \delta)}} \sum_{\substack{\mathbf{y} \in \Lambda_{\tau_{1}, \mathbf{z} \in \Lambda_{\tau_{2}}}^{c} \\
\leq}}\left|\Gamma_{\tau}^{\tau_{1}}(\mathbf{x}, \mathbf{y})\right| \kappa_{\tau_{1}}(\mathbf{y})\left|\Gamma_{\tau}^{\tau_{2}}(\mathbf{x}, \mathbf{z})\right| \kappa_{\tau_{2}}(\mathbf{z}) \\
& \leq \sum_{\substack{\tau \in(0, \delta) \\
\tau_{1}, \tau_{2} \in \mathcal{T}_{r}(\tau, \delta) \\
\left(\tau_{1}, \tau_{2}\right) \neq(\delta, \delta)}} N_{0}\left(\Gamma_{\tau}^{\tau_{1}} ; e^{\frac{\mathrm{m}}{2} d(\mathbf{x}, \Lambda)}, \kappa_{\tau_{1}}\right) N_{0}\left(\Gamma_{\tau}^{\tau_{2}} ; e^{\frac{\mathrm{m}}{2} d(\mathbf{x}, \Lambda)}, \kappa_{\tau_{2}}\right) \min \left\{\left|\Lambda_{\tau_{1}}^{c}\right|,\left|\Lambda_{\tau_{2}}^{c}\right|\right\} \\
& \leq\left|\Omega^{c}\right| \sum_{\substack{\tau \in(0, \delta) \\
\tau, \tau^{\prime} \in(0, \delta]}}\left[N_{0}\left[\Gamma_{\tau}^{\tau^{\prime}} ; e^{\frac{\mathrm{m}}{2} d(\mathbf{x}, \Lambda)}, \kappa_{\tau^{\prime}}\right)\right]^{2} \\
& \leq \frac{\delta}{\varepsilon}\left|\Omega^{c}\right|\left(16 e^{K_{j}} \mathrm{R}(\delta)\right)^{2} \text { by Lemma E.13 }
\end{aligned}
$$

and we bound the term with $\tau_{1}=\tau_{2}=\delta$ using

$$
\begin{aligned}
& \sum_{\tau \in(0, \delta)} \sum_{\mathbf{x} \in \Omega} \overline{\left(\Gamma_{\tau}^{\delta} \alpha_{\delta}\right)(\mathbf{x})}\left(\Gamma_{\tau}^{\delta} \alpha_{\delta}\right)(\mathbf{x})=\sum_{\tau \in(0, \delta)} \sum_{\substack{\mathbf{x} \in \Omega \\
\mathbf{y}, \mathbf{z} \in X}} \overline{\Gamma_{\tau}^{\delta}(\mathbf{x}, \mathbf{y}) \beta(\mathbf{y})} \Gamma_{\tau}^{\delta}(\mathbf{x}, \mathbf{z}) \beta(\mathbf{z}) \\
& \leq \sum_{\tau \in(0, \delta)} \sum_{\mathbf{x}, \mathbf{y}, \mathbf{z} \in \Omega} \overline{\Gamma_{\tau}^{\delta}(\mathbf{x}, \mathbf{y}) \beta(\mathbf{y})} \Gamma_{\tau}^{\delta}(\mathbf{x}, \mathbf{z}) \beta(\mathbf{z})+2\left|\Omega^{c}\right| \sum_{\tau \in(0, \delta)} N_{0}\left(\Gamma_{\tau}^{\delta} ; e^{\frac{\mathrm{m}}{2} d(\mathbf{x}, \Lambda)}, \kappa_{\delta}\right)^{2} \\
& \leq \sum_{\tau \in(0, \delta)} N_{0}\left(\Gamma_{\tau}^{\delta} ; 1,1\right)^{2}\|\beta\|_{\Omega}^{2}+2\left|\Omega^{c}\right| \sum_{\tau \in(0, \delta)} N_{0}\left(\Gamma_{\tau}^{\delta} ; e^{\frac{\mathrm{m}}{2} d(\mathbf{x}, \Lambda)}, \kappa_{\delta}\right)^{2} \\
& \leq \frac{\delta}{\varepsilon} e^{2 K_{j}}\|\beta\|_{\Omega}^{2}+2 \frac{\delta}{\varepsilon}\left|\Omega^{c}\right|\left(16 e^{K_{j}} \mathrm{R}(\delta)\right)^{2}
\end{aligned}
$$

We used that the operator on $L^{2}(\Omega)$ with kernel $\Gamma_{\tau}^{\delta}(\mathbf{x}, \mathbf{y})$ has norm at most $N_{0}\left(\Gamma_{\tau}^{\delta} ; 1,1\right)$. 
We have also used that $\left|e^{\varepsilon \mu}-1\right| \leq \frac{1}{8}$. Consequently $\left|e^{\varepsilon \mu}-1\right| \leq 2 \varepsilon|\mu|$ and, all together,

$$
\begin{aligned}
& \left|e^{\varepsilon \mu}-1\right| \sum_{\tau \in[0, \delta]}\left[\left\|\gamma_{\tau}\right\|_{\Omega}^{2}+\left\|\gamma_{* \tau}\right\|_{\Omega}^{2}\right] \\
& \quad \leq\left|e^{\varepsilon \mu}-1\right|\left\{2\|\alpha\|_{\Omega}^{2}+2\|\beta\|_{\Omega}^{2}+\frac{\delta}{\varepsilon} e^{2 K_{j}}\|\alpha\|_{\Omega}^{2}+\frac{\delta}{\varepsilon} e^{2 K_{j}}\|\beta\|_{\Omega}^{2}+6 \frac{\delta}{\varepsilon}\left|\Omega^{c}\right|\left(16 e^{K_{j}} \mathrm{R}(\delta)\right)^{2}\right\} \\
& \quad \leq\left(4+2 e^{2 K_{j}}\right) \delta|\mu|\left[\|\alpha\|_{\Omega}^{2}+\|\beta\|_{\Omega}^{2}\right]+2^{12} e^{2 K_{j}} \delta|\mu| \mathrm{R}(\delta)^{2}\left|\Omega^{c}\right|
\end{aligned}
$$

By Hypothesis F.7.i, (II.19) and (II.17),

$$
2^{12} e^{2 K_{j}} \delta|\mu| \mathrm{R}(\delta)^{2}=2^{12} e^{2 K_{j}} K_{\mu} \delta^{1-2 e_{\mathrm{R}}-2 e_{\mathrm{r}}} \mathfrak{v}^{e_{\mu}-2 e_{\mathrm{R}}-2 e_{\mathrm{r}}} \leq \frac{1}{16}
$$

The claim now follows from Remark D.3.

Proof of Proposition III.36: Define, for each $t \in(0, \delta) \cap \varepsilon \mathbb{Z}$,

$$
B_{t}=\Lambda\left(\mathcal{J}_{t}\right)^{c} \cap \Lambda\left(\mathcal{J}_{t}^{-}\right) \cap \Lambda\left(\mathcal{J}_{t}^{+}\right)
$$

Observe that, for all $t \in(0, \delta) \cap \varepsilon \mathbb{Z}$,

$$
\begin{aligned}
\operatorname{supp} \tilde{P}_{\alpha}^{\prime}\left(\mathcal{J}_{t}\right) & \subset B_{t} \\
\operatorname{supp} \tilde{P}_{\beta}^{\prime}\left(\mathcal{J}_{t}\right) & \subset B_{t} \\
\tilde{Q}\left(\mathcal{J}_{t}\right) & \subset B_{t} \\
B_{t} \cap B_{t^{\prime}} & =\emptyset \quad \text { for all } t \neq t^{\prime} \in \mathcal{J}_{t}^{\circ}
\end{aligned}
$$

where $\mathcal{J}_{t}^{\circ}=\mathcal{J}_{t} \backslash\left\{t \pm \frac{1}{2}\left|\mathcal{J}_{t}\right|\right\}$ is the interior of the interval $\mathcal{J}_{t}$. To see the last line, observe that if $t^{\prime}$ is strictly between $t$ and $t \pm \frac{1}{2}\left|\mathcal{J}_{t}\right|$, then $\Lambda\left(\mathcal{J}_{t}^{ \pm}\right) \subset \Lambda_{t^{\prime}}$ so that $B_{t} \subset \Lambda\left(\mathcal{J}_{t}^{ \pm}\right)$cannot intersect $B_{t^{\prime}} \subset \Lambda_{t^{\prime}}^{c}$.

Now fix any $0 \leq p, p^{\prime}<m$. Suppose that $t, t^{\prime} \in \varepsilon \mathbb{Z} \cap(0, \delta]$ have $\mathfrak{d}(t)=p, \mathfrak{o}\left(t^{\prime}\right)=p^{\prime}$ and $t \neq t^{\prime}$ and suppose that $B_{t} \cap B_{t^{\prime}} \neq \emptyset$. We claim that

$$
\begin{aligned}
& \left\{\tau \in \varepsilon \mathbb{Z} \mid \tau \in \mathcal{J}_{t}^{-}, \tau \neq t\right\} \cap\left\{\tau \in \varepsilon \mathbb{Z} \mid \tau \in \mathcal{J}_{t^{\prime}}^{-}, \tau \neq t^{\prime}\right\}=\emptyset \\
& \left\{\tau \in \varepsilon \mathbb{Z} \mid \tau \in \mathcal{J}_{t}^{+}, \tau \neq t\right\} \cap\left\{\tau \in \varepsilon \mathbb{Z} \mid \tau \in \mathcal{J}_{t^{\prime}}^{+}, \tau \neq t^{\prime}\right\}=\emptyset
\end{aligned}
$$

To see the upper claim, first consider $p=p^{\prime}$, so that $\left|\mathcal{J}_{t}^{ \pm}\right|=\left|\mathcal{J}_{t^{\prime}}^{ \pm}\right|=\frac{1}{2^{p}} \delta$. Then either $t=t^{\prime}$ or (VI.7) is satisfied. So, without loss of generality, we may assume that $p<p^{\prime}$ so that $\left|\mathcal{J}_{t^{\prime}}^{-}\right|<\left|\mathcal{J}_{t}^{-}\right|$. If the upper claim of (VI.7) is to be violated, then it is necessary that $t^{\prime}$ be in the interior of $\mathcal{J}_{t}^{-}$. But then (VI.6) provides the contradiction that $B_{t} \cap B_{t^{\prime}}=\emptyset$.

By Lemma D.4,

$$
\begin{gathered}
\sum_{\tau \in(0, \delta]}\left\langle\gamma_{\tau}^{*},[11-j(\varepsilon)] \gamma_{\tau}\right\rangle=\sum_{\tau \in(0, \delta]}\left\langle\gamma_{\tau}^{*},\left[11-e^{-\varepsilon \nabla^{*} \mathcal{H} \nabla}\right] \gamma_{\tau}\right\rangle \geq c_{\mathcal{H}} e^{-4 \mathrm{D} C_{\mathcal{H}}} \sum_{\tau \in(0, \delta]} \varepsilon\left\|\nabla \gamma_{\tau}\right\|^{2} \\
=c_{\mathcal{H}} e^{-4 \mathrm{D} C_{\mathcal{H}}} \sum_{b \in X^{*}} \sum_{\tau \in(0, \delta]} \varepsilon\left|\nabla \gamma_{\tau}(b)\right|^{2}
\end{gathered}
$$


Applying (VI.7),

$$
\begin{aligned}
\sum_{\tau \in(0, \delta]}\left\langle\gamma_{\tau}^{*},[\mathbb{1}-\mathfrak{j}(\varepsilon)] \gamma_{\tau}\right\rangle & \geq c_{\mathcal{H}} e^{-4 \mathrm{D} C_{\mathcal{H}}} \sum_{b \in X^{*}} \sum_{\substack{t \in(0, \delta) \\
\operatorname{supp} b B_{t} \\
t+\left|\mathcal{J}_{t}^{+}\right|}} \sum_{\tau=t+\varepsilon}^{t+\left|\mathcal{J}_{t}^{+}\right|} \varepsilon\left|\nabla \gamma_{\tau}(b)\right|^{2} \\
& =c_{\mathcal{H}} e^{-4 \mathrm{D} C_{\mathcal{H}}} \sum_{t \in(0, \delta)} \sum_{\tau=t+\varepsilon} \sum_{\substack{b \in X^{*} \\
\text { supp } b B_{t}}} \varepsilon\left|\nabla \gamma_{\tau}(b)\right|^{2} \\
& \geq c_{\mathcal{H}} e^{-4 \mathrm{D} C_{\mathcal{H}}} \sum_{t \in(0, \delta)} \frac{1}{8}\left|\mathcal{J}_{t}\right| \mathrm{R}^{\prime}\left(\left|\mathcal{J}_{t}\right|\right)^{2}\left|\tilde{P}_{\beta}^{\prime}\left(\mathcal{J}_{t}\right)\right|
\end{aligned}
$$

by Lemma E.12.ii. Similarly

$$
\begin{aligned}
\sum_{\tau \in[0, \delta)}\left\langle\gamma_{* \tau}^{*},[11-\mathfrak{j}(\varepsilon)] \gamma_{* \tau}\right\rangle & \geq c_{\mathcal{H}} e^{-4 \mathrm{D} C_{\mathcal{H}}} \sum_{t \in(0, \delta)} \sum_{\tau=t-\left|\mathcal{J}_{t}^{-}\right|} \sum_{\substack{b \in X^{*} \\
\operatorname{supp} b \subset B_{t}}} \varepsilon\left|\nabla \gamma_{* \tau}(b)\right|^{2} \\
& \geq c_{\mathcal{H}} e^{-4 \mathrm{D} C_{\mathcal{H}}} \sum_{t \in(0, \delta)} \frac{1}{8}\left|\mathcal{J}_{t}\right| \mathrm{R}^{\prime}\left(\left|\mathcal{J}_{t}\right|\right)^{2}\left|\tilde{P}_{\alpha}^{\prime}\left(\mathcal{J}_{t}\right)\right|
\end{aligned}
$$

and

$$
\begin{aligned}
\sum_{\tau \in[0, \delta)} 2\left\|\gamma_{* \tau}^{*}-\gamma_{\tau+\varepsilon}\right\|_{\Lambda_{\tau}^{c} \cup \Lambda_{\tau+\varepsilon}^{c}}^{2} \geq \sum_{\tau \in(0, \delta)}\left\{\left\|\gamma_{* \tau}^{*}-\gamma_{\tau+\varepsilon}\right\|_{\Lambda_{\tau}^{c} \cup \Lambda_{\tau+\varepsilon}^{c}}^{2}+\left\|\gamma_{* \tau-\varepsilon}^{*}-\gamma_{\tau}\right\|_{\Lambda_{\tau-\varepsilon}^{c} \cup \Lambda_{\tau}^{c}}^{2}\right\} \\
\quad=\sum_{t \in(0, \delta)}\left\{\left\|\gamma_{* t}^{*}-\gamma_{t+\varepsilon}\right\|_{\Lambda_{t}^{c} \cup \Lambda_{t+\varepsilon}^{c}}^{2}+\left\|\gamma_{* t-\varepsilon}^{*}-\gamma_{t}\right\|_{\Lambda_{t-\varepsilon}^{c} \cup \Lambda_{t}^{c}}^{2}\right\} \\
\geq \sum_{t \in(0, \delta)}\left\{\left\|\gamma_{* t}^{*}-\gamma_{t+\varepsilon}\right\|_{\tilde{Q}\left(\mathcal{J}_{t}\right)}^{2}+\left\|\gamma_{* t-\varepsilon}^{*}-\gamma_{t}\right\|_{\tilde{Q}\left(\mathcal{J}_{t}\right)}^{2}\right\} \\
\geq \sum_{t \in(0, \delta)} \frac{1}{32} \mathrm{r}\left(\left|\mathcal{J}_{t}\right|\right)^{2}\left|\tilde{Q}\left(\mathcal{J}_{t}\right)\right|
\end{aligned}
$$

Hence, by Lemmas VI.2 and E.17and Proposition E.11.ii,

$$
\begin{aligned}
& \operatorname{Re} \sum_{\tau \in[0, \delta)}\left[-\frac{1}{2}\left\langle\gamma_{* \tau}, \gamma_{\tau}\right\rangle+\left\langle\gamma_{* \tau}, j(\varepsilon) \gamma_{\tau+\varepsilon}\right\rangle-\frac{1}{2}\left\langle\gamma_{* \tau+\varepsilon}, \gamma_{\tau+\varepsilon}\right\rangle\right] \\
& \leq-\frac{1}{4}\left\{\sum_{\tau \in[0, \delta)} \frac{1}{2}\left\|\gamma_{* \tau}^{*}-\gamma_{\tau+\varepsilon}\right\|_{\Lambda_{\tau}^{c} \cup \Lambda_{\tau+\varepsilon}^{c}}^{2}+\sum_{\tau \in(0, \delta]}\left\langle\gamma_{\tau}^{*},[1-j(\varepsilon)] \gamma_{\tau}\right\rangle+\sum_{\tau \in[0, \delta)}\left\langle\gamma_{* \tau}^{*},[1-j(\varepsilon)] \gamma_{* \tau}\right\rangle\right\} \\
& \quad+\sum_{\tau \in(0, \delta)} e^{-2 \operatorname{mc}\left(\frac{1}{2}\left|\mathcal{J}_{\tau}\right|\right)}\left|\Lambda_{\tau}^{c}\right|+\left|e^{\varepsilon \mu}-1\right| \sum_{\tau \in[0, \delta]}\left[\left\|\gamma_{\tau}\right\|^{2}+\left\|\gamma_{* \tau}\right\|^{2}\right]
\end{aligned}
$$




$$
\begin{aligned}
\leq-\frac{1}{16} & \sum_{\tau \in[0, \delta)}\left\|\gamma_{* \tau}^{*}-\gamma_{\tau+\varepsilon}\right\|_{\Lambda_{\tau}^{c} \cup \Lambda_{\tau+\varepsilon}^{c}}^{2} \\
& -K_{L 2} \sum_{t \in(0, \delta)}\left\{\mathrm{r}\left(\left|\mathcal{J}_{t}\right|\right)^{2}\left|\tilde{Q}\left(\mathcal{J}_{t}\right)\right|+\left|\mathcal{J}_{t}\right| \mathrm{R}^{\prime}\left(\mathcal{J}_{t}\right)^{2}\left|\tilde{P}_{\alpha}^{\prime}\left(\mathcal{J}_{t}\right)\right|+\left|\mathcal{J}_{t}\right| \mathrm{R}^{\prime}\left(\mathcal{J}_{t}\right)^{2}\left|\tilde{P}_{\beta}^{\prime}\left(\mathcal{J}_{t}\right)\right|\right\} \\
& +\sum_{\tau \in(0, \delta)} e^{-2 \operatorname{mc}\left(\frac{1}{2}\left|\mathcal{J}_{\tau}\right|\right)}\left|\Lambda_{\tau}^{c}\right|+\left|e^{\varepsilon \mu}-1\right| \sum_{\tau \in[0, \delta]}\left[\left\|\gamma_{\tau}\right\|^{2}+\left\|\gamma_{* \tau}\right\|^{2}\right]
\end{aligned}
$$

where $K_{L 2}=\frac{1}{32} \min \left\{c_{\mathcal{H}} e^{-4 \mathrm{D} C_{\mathcal{H}}}, \frac{1}{32}\right\}$ with $c_{\mathcal{H}}$ and $C_{\mathcal{H}}$ being the smallest and largest eigenvalues of $\mathcal{H}$, respectively. The claim follows from (F.4.c), (F.7.a,b) and Lemma VI.3.

\section{VI.2 Extracting Small Factors from the Quartic Form}

In this section, we prove Proposition III.37. Recall that

$$
\mathcal{V}_{\mathfrak{S}}=-\varepsilon \sum_{\tau \in \varepsilon \mathbb{Z} \cap[0, \delta)}\left\langle\gamma_{* \tau} \gamma_{\tau+\varepsilon}, v \gamma_{* \tau} \gamma_{\tau+\varepsilon}\right\rangle
$$

If we could replace $\gamma_{* \tau}$ by $\gamma_{\tau}^{*}$ and $\gamma_{\tau+\varepsilon}$ by $\gamma_{\tau}$, we would have

$$
-\varepsilon \sum_{\tau \in \varepsilon \mathbb{Z} \cap[0, \delta)}\left\langle\gamma_{\tau}^{*} \gamma_{\tau}, v \gamma_{\tau}^{*} \gamma_{\tau}\right\rangle \leq-\varepsilon \sum_{\tau \in \varepsilon \mathbb{Z} \cap[0, \delta)} v_{1}\left\|\gamma_{\tau}\right\|_{L^{4}(X)}^{2}=-\varepsilon v_{1} \sum_{\substack{\tau \in \varepsilon \mathbb{Z} \cap[0, \delta) \\ \mathbf{x} \in X}}\left|\gamma_{\tau}(\mathbf{x})\right|^{4}
$$

which is very negative when some x's are in large field regions. The following lemma expresses the error introduced by such a replacement as a sum of two terms, $\widetilde{\mathcal{E}}_{4, \mathfrak{S}}$ and $\widehat{\mathcal{V}}_{\mathfrak{S}}$. The first, $\widetilde{\mathcal{E}}_{4, \mathfrak{S}}$ is a pure small field contribution, which will be bounded by the "small field regulator" $\operatorname{Reg}_{\mathcal{S} \mathcal{F}}^{(4)}$. See Lemma VI.7. The second, $\widehat{\mathcal{V}}_{\mathfrak{S}}$, is a large field contribution and is bounded by the two terms in the third line of the right hand side in Proposition III.37.

\section{Lemma VI.4}

$$
\begin{array}{r}
\mathcal{V}_{\mathfrak{S}}\left(\alpha_{*}, \beta ; \vec{\alpha}_{*}, \vec{\alpha}\right)=-\frac{1}{2} \sum_{\tau \in[0, \delta)} \varepsilon\left\langle\gamma_{* \tau}^{*} \gamma_{* \tau}, v \gamma_{* \tau}^{*} \gamma_{* \tau}\right\rangle-\frac{1}{2} \sum_{\tau \in(0, \delta]} \varepsilon\left\langle\gamma_{\tau}^{*} \gamma_{\tau}, v \gamma_{\tau}^{*} \gamma_{\tau}\right\rangle \\
+\widetilde{\mathcal{E}}_{4, \mathfrak{S}}(\alpha, \beta)+\widehat{\mathcal{V}}_{\mathfrak{S}}\left(\alpha_{*}, \beta ; \vec{\alpha}_{*}, \vec{\alpha}\right)
\end{array}
$$

where $\widehat{\mathcal{V}}_{\mathfrak{S}}\left(\alpha_{*}, \beta ; \vec{\alpha}_{*}, \vec{\alpha}\right)$ is defined in (VI.14) and bounded in Lemma VI.6 and $\widetilde{\mathcal{E}}_{4, \mathfrak{S}}(\alpha, \beta)$ is defined in (VI.12) and bounded in Lemma VI.7. The first two terms on the right hand side are bounded in Lemma VI.5. 
Proof: We start with the difference

$$
\mathcal{V}_{\mathfrak{S}}-\left[-\frac{1}{2} \sum_{\tau \in[0, \delta)} \varepsilon\left\langle\gamma_{* \tau}^{*} \gamma_{* \tau}, v \gamma_{* \tau}^{*} \gamma_{* \tau}\right\rangle-\frac{1}{2} \sum_{\tau \in(0, \delta]} \varepsilon\left\langle\gamma_{\tau}^{*} \gamma_{\tau}, v \gamma_{\tau}^{*} \gamma_{\tau}\right\rangle\right]
$$

between $\mathcal{V}_{\mathfrak{S}}$ and the expressions which are manifestly large and negative in the large field region. (See Lemma VI.5). From this, we successively pull off four controllable pieces, $\widehat{\mathcal{V}}_{1, \mathfrak{S}}, \cdots, \widehat{\mathcal{V}}_{4, \mathfrak{S}}$, leaving the small field contribution $\widetilde{\mathcal{E}}_{4, \mathfrak{S}}(\alpha, \beta)$. The first step is

$$
\begin{aligned}
\mathcal{V}_{\mathfrak{S}}\left(\alpha_{*}, \beta ;\right. & \left.\vec{\alpha}_{*}, \vec{\alpha}\right)+\frac{1}{2} \sum_{\tau \in[0, \delta)} \varepsilon\left\langle\gamma_{* \tau}^{*} \gamma_{* \tau}, v \gamma_{* \tau}^{*} \gamma_{* \tau}\right\rangle+\frac{1}{2} \sum_{\tau \in(0, \delta]} \varepsilon\left\langle\gamma_{\tau}^{*} \gamma_{\tau}, v \gamma_{\tau}^{*} \gamma_{\tau}\right\rangle \\
= & -\frac{\varepsilon}{2} \sum_{\tau \in[0, \delta)}\left\{\left\langle\gamma_{* \tau}\left(\gamma_{\tau+\varepsilon}-\gamma_{* \tau}^{*}\right), v \gamma_{* \tau} \gamma_{\tau+\varepsilon}\right\rangle+\left\langle\gamma_{* \tau} \gamma_{* \tau}^{*}, v \gamma_{* \tau}\left(\gamma_{\tau+\varepsilon}-\gamma_{* \tau}^{*}\right)\right\rangle\right\} \\
& -\frac{\varepsilon}{2} \sum_{\tau \in[0, \delta)}\left\{\left\langle\left(\gamma_{* \tau}-\gamma_{\tau+\varepsilon}^{*}\right) \gamma_{\tau+\varepsilon}, v \gamma_{* \tau} \gamma_{\tau+\varepsilon}\right\rangle+\left\langle\gamma_{\tau+\varepsilon}^{*} \gamma_{\tau+\varepsilon}, v\left(\gamma_{* \tau}-\gamma_{\tau+\varepsilon}^{*}\right) \gamma_{\tau+\varepsilon}\right\rangle\right\} \\
= & \mathcal{V}_{\mathfrak{S}}^{\prime}\left(\alpha_{*}, \beta ; \vec{\alpha}_{*}, \vec{\alpha}\right)+\widehat{\mathcal{V}}_{1, \mathfrak{S}}\left(\alpha_{*}, \beta ; \vec{\alpha}_{*}, \vec{\alpha}\right)
\end{aligned}
$$

where

$$
\begin{aligned}
& \mathcal{V}_{\mathfrak{S}}^{\prime}\left(\alpha_{*}, \beta ; \vec{\alpha}_{*}, \vec{\alpha}\right) \\
& =-\frac{\varepsilon}{2} \sum_{\tau \in[0, \delta)}\left\{\left\langle\Lambda \gamma_{* \tau}\left(\gamma_{\tau+\varepsilon}-\gamma_{* \tau}^{*}\right), v \Lambda \gamma_{* \tau} \gamma_{\tau+\varepsilon}\right\rangle+\left\langle\Lambda \gamma_{* \tau} \gamma_{* \tau}^{*}, v \Lambda \gamma_{* \tau}\left(\gamma_{\tau+\varepsilon}-\gamma_{* \tau}^{*}\right)\right\rangle\right\} \\
& \quad-\frac{\varepsilon}{2} \sum_{\tau \in[0, \delta)}\left\{\left\langle\Lambda\left(\gamma_{* \tau}-\gamma_{\tau+\varepsilon}^{*}\right) \gamma_{\tau+\varepsilon}, v \Lambda \gamma_{* \tau} \gamma_{\tau+\varepsilon}\right\rangle+\left\langle\Lambda \gamma_{\tau+\varepsilon}^{*} \gamma_{\tau+\varepsilon}, v \Lambda\left(\gamma_{* \tau}-\gamma_{\tau+\varepsilon}^{*}\right) \gamma_{\tau+\varepsilon}\right\rangle\right\}
\end{aligned}
$$

and

$$
\begin{aligned}
& \widehat{\mathcal{V}}_{1, \mathfrak{S}}\left(\alpha_{*}, \beta ; \vec{\alpha}_{*}, \vec{\alpha}\right) \\
& =-\frac{\varepsilon}{2} \sum_{\tau \in[0, \delta)}\left\{\left\langle\Lambda^{c} \gamma_{* \tau}\left(\gamma_{\tau+\varepsilon}-\gamma_{* \tau}^{*}\right), v \gamma_{* \tau} \gamma_{\tau+\varepsilon}\right\rangle+\left\langle\gamma_{* \tau} \gamma_{* \tau}^{*}, v \Lambda^{c} \gamma_{* \tau}\left(\gamma_{\tau+\varepsilon}-\gamma_{* \tau}^{*}\right)\right\rangle\right\} \\
& \quad-\frac{\varepsilon}{2} \sum_{\tau \in[0, \delta)}\left\{\left\langle\Lambda^{c}\left(\gamma_{* \tau}-\gamma_{\tau+\varepsilon}^{*}\right) \gamma_{\tau+\varepsilon}, v \gamma_{* \tau} \gamma_{\tau+\varepsilon}\right\rangle+\left\langle\gamma_{\tau+\varepsilon}^{*} \gamma_{\tau+\varepsilon}, v \Lambda^{c}\left(\gamma_{* \tau}-\gamma_{\tau+\varepsilon}^{*}\right) \gamma_{\tau+\varepsilon}\right\rangle\right\} \\
& \quad-\frac{\varepsilon}{2} \sum_{\tau \in[0, \delta)}\left\{\left\langle\Lambda \gamma_{* \tau}\left(\gamma_{\tau+\varepsilon}-\gamma_{* \tau}^{*}\right), v \Lambda^{c} \gamma_{* \tau} \gamma_{\tau+\varepsilon}\right\rangle+\left\langle\Lambda^{c} \gamma_{* \tau} \gamma_{* \tau}^{*}, v \Lambda \gamma_{* \tau}\left(\gamma_{\tau+\varepsilon}-\gamma_{* \tau}^{*}\right)\right\rangle\right\} \\
& \quad-\frac{\varepsilon}{2} \sum_{\tau \in[0, \delta)}\left\{\left\langle\Lambda\left(\gamma_{* \tau}-\gamma_{\tau+\varepsilon}^{*}\right) \gamma_{\tau+\varepsilon}, v \Lambda^{c} \gamma_{* \tau} \gamma_{\tau+\varepsilon}\right\rangle+\left\langle\Lambda^{c} \gamma_{\tau+\varepsilon}^{*} \gamma_{\tau+\varepsilon}, v \Lambda\left(\gamma_{* \tau}-\gamma_{\tau+\varepsilon}^{*}\right) \gamma_{\tau+\varepsilon}\right\rangle\right\}
\end{aligned}
$$

Next write

$$
\mathcal{V}_{\mathfrak{S}}^{\prime}\left(\alpha_{*}, \beta ; \vec{\alpha}_{*}, \vec{\alpha}\right)=\mathcal{V}_{\mathfrak{S}}^{\prime \prime}\left(\alpha_{*}, \beta ; \vec{\alpha}_{*}, \vec{\alpha}\right)+\widehat{\mathcal{V}}_{2, \mathfrak{S}}\left(\alpha_{*}, \beta ; \vec{\alpha}_{*}, \vec{\alpha}\right)
$$


where, in the notation of Definition II.9 and Lemma E.4.i,

$$
\begin{aligned}
\mathcal{V}_{\mathfrak{S}}^{\prime \prime}\left(\alpha_{*}, \beta ; \vec{\alpha}_{*}, \vec{\alpha}\right)= & -\frac{\varepsilon}{2} \sum_{\tau \in[0, \delta)}\left\langle\Lambda\left(\Gamma_{* \tau}^{0} \alpha^{*}\right)\left(\gamma_{\tau+\varepsilon}-\gamma_{* \tau}^{*}\right), v \Lambda\left(\Gamma_{* \tau}^{0} \alpha^{*}\right)\left(\Gamma_{\tau+\varepsilon}^{\delta} \beta\right)\right\rangle \\
& -\frac{\varepsilon}{2} \sum_{\tau \in[0, \delta)}\left\langle\Lambda\left(\Gamma_{* \tau}^{0} \alpha^{*}\right)\left(\Gamma_{* \tau}^{0} \alpha\right), v \Lambda\left(\Gamma_{* \tau}^{0} \alpha^{*}\right)\left(\gamma_{\tau+\varepsilon}-\gamma_{* \tau}^{*}\right)\right\rangle \\
& -\frac{\varepsilon}{2} \sum_{\tau \in[0, \delta)}\left\langle\Lambda\left(\gamma_{* \tau}-\gamma_{\tau+\varepsilon}^{*}\right)\left(\Gamma_{\tau+\varepsilon}^{\delta} \beta\right), v \Lambda\left(\Gamma_{* \tau}^{0} \alpha^{*}\right)\left(\Gamma_{\tau+\varepsilon}^{\delta} \beta\right)\right\rangle \\
& -\frac{\varepsilon}{2} \sum_{\tau \in[0, \delta)}\left\langle\Lambda\left(\Gamma_{\tau+\varepsilon}^{\delta} \beta^{*}\right)\left(\Gamma_{\tau+\varepsilon}^{\delta} \beta\right), v \Lambda\left(\gamma_{* \tau}-\gamma_{\tau+\varepsilon}^{*}\right)\left(\Gamma_{\tau+\varepsilon}^{\delta} \beta\right)\right\rangle
\end{aligned}
$$

and, using the notation of Definition E.3,

$$
\begin{aligned}
& \widehat{\mathcal{V}}_{2, \mathfrak{S}}\left(\alpha_{*}, \beta ; \vec{\alpha}_{*}, \vec{\alpha}\right) \\
& =-\frac{\varepsilon}{2} \sum_{\tau \in[0, \delta)} \sum_{\substack{\tau_{1}, \tau_{2} \in \mathcal{T}_{l}(\tau, \delta) \\
\tau_{3} \in \mathcal{T}_{r}(\tau+\varepsilon, \delta) \\
\left(\tau_{1}, \tau_{2}, \tau_{3}\right) \neq(0,0, \delta)}}\left\langle\Lambda\left(\Gamma_{* \tau}^{\tau_{1}} \alpha_{\tau_{1}}^{*}\right)\left(\gamma_{\tau+\varepsilon}-\gamma_{* \tau}^{*}\right), v \Lambda\left(\Gamma_{* \tau}^{\tau_{2}} \alpha_{\tau_{2}}^{*}\right)\left(\Gamma_{\tau+\varepsilon}^{\tau_{3}} \alpha_{\tau_{3}}\right)\right\rangle \\
& -\frac{\varepsilon}{2} \sum_{\tau \in[0, \delta)} \sum_{\substack{\tau_{1}, \tau_{2}, \tau_{3} \in \mathcal{T}_{l}(\tau, \delta) \\
\left(\tau_{1}, \tau_{2}, \tau_{3}\right) \neq(0,0,0)}}\left\langle\Lambda\left(\Gamma_{* \tau}^{\tau_{1}} \alpha_{\tau_{1}}^{*}\right)\left(\Gamma_{* \tau}^{\tau_{2}} \alpha_{\tau_{2}}\right), v \Lambda\left(\Gamma_{* \tau}^{\tau_{3}} \alpha_{\tau_{3}}^{*}\right)\left(\gamma_{\tau+\varepsilon}-\gamma_{* \tau}^{*}\right)\right\rangle \\
& -\frac{\varepsilon}{2} \sum_{\tau \in[0, \delta)} \sum_{\substack{\tau_{1}, \tau_{2} \in \mathcal{T}_{r}(\tau+\varepsilon, \delta) \\
\tau_{3} \in \mathcal{T}_{l}(\tau, \delta) \\
\left(\tau_{1}, \tau_{2}, \tau_{3}\right) \neq(\delta, \delta, 0)}}\left\langle\Lambda\left(\gamma_{* \tau}-\gamma_{\tau+\varepsilon}^{*}\right)\left(\Gamma_{\tau+\varepsilon}^{\tau_{1}} \alpha_{\tau_{1}}\right), v \Lambda\left(\Gamma_{* \tau}^{\tau_{3}} \alpha_{\tau_{3}}^{*}\right)\left(\Gamma_{\tau+\varepsilon}^{\tau_{2}} \alpha_{\tau_{2}}\right)\right\rangle \\
& -\frac{\varepsilon}{2} \sum_{\tau \in[0, \delta)} \sum_{\substack{\tau_{1}, \tau_{2}, \tau_{3} \in \mathcal{T}_{r}(\tau+\varepsilon, \delta) \\
\left(\tau_{1}, \tau_{2}, \tau_{3}\right) \neq(\delta, \delta, \delta)}}\left\langle\Lambda\left(\Gamma_{\tau+\varepsilon}^{\tau_{1}} \alpha_{\tau_{1}}^{*}\right)\left(\Gamma_{\tau+\varepsilon}^{\tau_{2}} \alpha_{\tau_{2}}\right), v \Lambda\left(\gamma_{* \tau}-\gamma_{\tau+\varepsilon}^{*}\right)\left(\Gamma_{\tau+\varepsilon}^{\tau_{3}} \alpha_{\tau_{3}}\right)\right\rangle
\end{aligned}
$$

Next write

$$
\mathcal{V}_{\mathfrak{S}}^{\prime \prime}\left(\alpha_{*}, \beta ; \vec{\alpha}_{*}, \vec{\alpha}\right)=\mathcal{V}_{\mathfrak{S}}^{\prime \prime \prime}\left(\alpha_{*}, \beta ; \vec{\alpha}_{*}, \vec{\alpha}\right)+\widehat{\mathcal{V}}_{3, \mathfrak{S}}\left(\alpha_{*}, \beta ; \vec{\alpha}_{*}, \vec{\alpha}\right)
$$

where

$$
\begin{aligned}
\mathcal{V}_{\mathfrak{S}}^{\prime \prime \prime}\left(\alpha_{*}, \beta ; \vec{\alpha}_{*}, \vec{\alpha}\right)= & -\frac{\varepsilon}{2} \sum_{\tau \in[0, \delta)}\left\langle\Lambda\left(\Gamma_{* \tau}^{0} \Omega \alpha^{*}\right)\left(\gamma_{\tau+\varepsilon}-\gamma_{* \tau}^{*}\right), v \Lambda\left(\Gamma_{* \tau}^{0} \Omega \alpha^{*}\right)\left(\Gamma_{\tau+\varepsilon}^{\delta} \Omega \beta\right)\right\rangle \\
& -\frac{\varepsilon}{2} \sum_{\tau \in[0, \delta)}\left\langle\Lambda\left(\Gamma_{* \tau}^{0} \Omega \alpha^{*}\right)\left(\Gamma_{* \tau}^{0} \Omega \alpha\right), v \Lambda\left(\Gamma_{* \tau}^{0} \Omega \alpha^{*}\right)\left(\gamma_{\tau+\varepsilon}-\gamma_{* \tau}^{*}\right)\right\rangle \\
& -\frac{\varepsilon}{2} \sum_{\tau \in[0, \delta)}\left\langle\Lambda\left(\gamma_{* \tau}-\gamma_{\tau+\varepsilon}^{*}\right)\left(\Gamma_{\tau+\varepsilon}^{\delta} \Omega \beta\right), v \Lambda\left(\Gamma_{* \tau}^{0} \Omega \alpha^{*}\right)\left(\Gamma_{\tau+\varepsilon}^{\delta} \Omega \beta\right)\right\rangle \\
& -\frac{\varepsilon}{2} \sum_{\tau \in[0, \delta)}\left\langle\Lambda\left(\Gamma_{\tau+\varepsilon}^{\delta} \Omega \beta^{*}\right)\left(\Gamma_{\tau+\varepsilon}^{\delta} \Omega \beta\right), v \Lambda\left(\gamma_{* \tau}-\gamma_{\tau+\varepsilon}^{*}\right)\left(\Gamma_{\tau+\varepsilon}^{\delta} \Omega \beta\right)\right\rangle
\end{aligned}
$$


and, using $\Omega^{(0)}=\Omega$ and $\Omega^{(1)}=\Omega^{c}$,

$$
\begin{aligned}
\widehat{\mathcal{V}}_{3, \mathfrak{S}}\left(\alpha_{*}, \beta ; \vec{\alpha}_{*}, \vec{\alpha}\right) & -\frac{\varepsilon}{2} \sum_{\tau \in[0, \delta)} \sum_{\substack{i, j, k \in\{0,1\} \\
(i, j, k) \neq(0,0,0)}}\left\langle\Lambda\left(\Gamma_{* \tau}^{0} \Omega^{(i)} \alpha^{*}\right)\left(\gamma_{\tau+\varepsilon}-\gamma_{* \tau}^{*}\right), v \Lambda\left(\Gamma_{* \tau}^{0} \Omega^{(j)} \alpha^{*}\right)\left(\Gamma_{\tau+\varepsilon}^{\delta} \Omega^{(k)} \beta\right)\right\rangle \\
& -\frac{\varepsilon}{2} \sum_{\tau \in[0, \delta)} \sum_{\substack{i, j, k \in\{0,1\} \\
(i, j, k) \neq(0,0,0)}}\left\langle\Lambda\left(\Gamma_{* \tau}^{0} \Omega^{(i)} \alpha^{*}\right)\left(\Gamma_{* \tau}^{0} \Omega^{(j)} \alpha\right), v \Lambda\left(\Gamma_{* \tau}^{0} \Omega^{(k)} \alpha^{*}\right)\left(\gamma_{\tau+\varepsilon}-\gamma_{* \tau}^{*}\right)\right\rangle \\
& -\frac{\varepsilon}{2} \sum_{\tau \in[0, \delta)} \sum_{\substack{i, j, k \in\{0,1\} \\
(i, j, k) \neq(0,0,0)}}\left\langle\Lambda\left(\gamma_{* \tau}-\gamma_{\tau+\varepsilon}^{*}\right)\left(\Gamma_{\tau+\varepsilon}^{\delta} \Omega^{(i)} \beta\right), v \Lambda\left(\Gamma_{* \tau}^{0} \Omega^{(j)} \alpha^{*}\right)\left(\Gamma_{\tau+\varepsilon}^{\delta} \Omega^{(k)} \beta\right)\right\rangle \\
& -\frac{\varepsilon}{2} \sum_{\tau \in[0, \delta)} \sum_{\substack{i, j, k \in\{0,1\} \\
(i, j, k) \neq(0,0,0)}}\left\langle\Lambda\left(\Gamma_{\tau+\varepsilon}^{\delta} \Omega^{(i)} \beta^{*}\right)\left(\Gamma_{\tau+\varepsilon}^{\delta} \Omega^{(j)} \beta\right), v \Lambda\left(\gamma_{* \tau}-\gamma_{\tau+\varepsilon}^{*}\right)\left(\Gamma_{\tau+\varepsilon}^{\delta} \Omega^{(k)} \beta\right)\right\rangle
\end{aligned}
$$

Finally substitute, in $\mathcal{V}_{\mathfrak{S}}^{\prime \prime \prime}\left(\alpha_{*}, \beta ; \vec{\alpha}_{*}, \vec{\alpha}\right)$,

$$
\gamma_{* \tau}^{*}-\gamma_{\tau+\varepsilon}=\tilde{\tilde{\gamma}}_{\tau, \varepsilon}+\tilde{\gamma}_{\tau, \varepsilon}^{(1)}+\tilde{\gamma}_{\tau, \varepsilon}^{(2)}
$$

where

$$
\tilde{\tilde{\gamma}}_{\tau, \varepsilon}=j(\tau) \tilde{\Omega} \alpha-j(\delta-\tau-\varepsilon) \tilde{\Omega} \beta \quad \tilde{\gamma}_{\tau, \varepsilon}^{(1)}=\gamma_{* \tau}^{*}-\gamma_{\tau+\varepsilon}-\tilde{\gamma}_{\tau, \varepsilon} \quad \tilde{\gamma}_{\tau, \varepsilon}^{(2)}=\tilde{\gamma}_{\tau, \varepsilon}-\tilde{\tilde{\gamma}}_{\tau, \varepsilon}
$$

where $\tilde{\Omega}$ the set of all point in $X$ that are within a distance $\mathfrak{c}(\delta)$ of $\Omega$ and $\tilde{\gamma}_{\tau, \varepsilon}$ was defined in Corollary E.9, and write

$$
\mathcal{V}_{\mathfrak{S}}^{\prime \prime \prime}\left(\alpha_{*}, \beta ; \vec{\alpha}_{*}, \vec{\alpha}\right)=\widetilde{\mathcal{E}}_{4, \mathfrak{S}}(\alpha, \beta)+\widehat{\mathcal{V}}_{4, \mathfrak{S}}\left(\alpha_{*}, \beta ; \vec{\alpha}_{*}, \vec{\alpha}\right)
$$

where

$$
\begin{aligned}
\widetilde{\mathcal{E}}_{4, \mathfrak{S}}(\alpha, \beta)= & \frac{\varepsilon}{2} \sum_{\tau \in[0, \delta)}\left\langle\Lambda\left(\Gamma_{* \tau}^{0} \Omega \alpha^{*}\right) \tilde{\tilde{\gamma}}_{\tau, \varepsilon}, v \Lambda\left(\Gamma_{* \tau}^{0} \Omega \alpha^{*}\right)\left(\Gamma_{\tau+\varepsilon}^{\delta} \Omega \beta\right)\right\rangle \\
& +\frac{\varepsilon}{2} \sum_{\tau \in[0, \delta)}\left\langle\Lambda\left(\Gamma_{* \tau}^{0} \Omega \alpha^{*}\right)\left(\Gamma_{* \tau}^{0} \Omega \alpha\right), v \Lambda\left(\Gamma_{* \tau}^{0} \Omega \alpha^{*}\right) \tilde{\tilde{\gamma}}_{\tau, \varepsilon}\right\rangle \\
& -\frac{\varepsilon}{2} \sum_{\tau \in[0, \delta)}\left\langle\Lambda \tilde{\tilde{\gamma}}_{\tau, \varepsilon}^{*}\left(\Gamma_{\tau+\varepsilon}^{\delta} \Omega \beta\right), v \Lambda\left(\Gamma_{* \tau}^{0} \Omega \alpha^{*}\right)\left(\Gamma_{\tau+\varepsilon}^{\delta} \Omega \beta\right)\right\rangle \\
& -\frac{\varepsilon}{2} \sum_{\tau \in[0, \delta)}\left\langle\Lambda\left(\Gamma_{\tau+\varepsilon}^{\delta} \Omega \beta^{*}\right)\left(\Gamma_{\tau+\varepsilon}^{\delta} \Omega \beta\right), v \Lambda \tilde{\tilde{\gamma}}_{\tau, \varepsilon}^{*}\left(\Gamma_{\tau+\varepsilon}^{\delta} \Omega \beta\right)\right\rangle
\end{aligned}
$$


and

$$
\begin{aligned}
\widehat{\mathcal{V}}_{4, \mathfrak{S}}\left(\alpha_{*}, \beta ; \vec{\alpha}_{*}, \vec{\alpha}\right)= & \frac{\varepsilon}{2} \sum_{\tau \in[0, \delta)}\left\langle\Lambda\left(\Gamma_{* \tau}^{0} \Omega \alpha^{*}\right)\left(\tilde{\gamma}_{\tau, \varepsilon}^{(1)}+\tilde{\gamma}_{\tau, \varepsilon}^{(2)}\right), v \Lambda\left(\Gamma_{* \tau}^{0} \Omega \alpha^{*}\right)\left(\Gamma_{\tau+\varepsilon}^{\delta} \Omega \beta\right)\right\rangle \\
& +\frac{\varepsilon}{2} \sum_{\tau \in[0, \delta)}\left\langle\Lambda\left(\Gamma_{* \tau}^{0} \Omega \alpha^{*}\right)\left(\Gamma_{* \tau}^{0} \Omega \alpha\right), v \Lambda\left(\Gamma_{* \tau}^{0} \Omega \alpha^{*}\right)\left(\tilde{\gamma}_{\tau, \varepsilon}^{(1)}+\tilde{\gamma}_{\tau, \varepsilon}^{(2)}\right)\right\rangle \\
& -\frac{\varepsilon}{2} \sum_{\tau \in[0, \delta)}\left\langle\Lambda\left(\tilde{\gamma}_{\tau, \varepsilon}^{(1)}+\tilde{\gamma}_{\tau, \varepsilon}^{(2)}\right)^{*}\left(\Gamma_{\tau+\varepsilon}^{\delta} \beta\right), v \Lambda\left(\Gamma_{* \tau}^{0} \Omega \alpha^{*}\right)\left(\Gamma_{\tau+\varepsilon}^{\delta} \Omega \beta\right)\right\rangle \\
& -\frac{\varepsilon}{2} \sum_{\tau \in[0, \delta)}\left\langle\Lambda\left(\Gamma_{\tau+\varepsilon}^{\delta} \Omega \beta^{*}\right)\left(\Gamma_{\tau+\varepsilon}^{\delta} \Omega \beta\right), v \Lambda\left(\tilde{\gamma}_{\tau, \varepsilon}^{(1)}+\tilde{\gamma}_{\tau, \varepsilon}^{(2)}\right)^{*}\left(\Gamma_{\tau+\varepsilon}^{\delta} \Omega \beta\right)\right\rangle
\end{aligned}
$$

Of course

$$
\widehat{\mathcal{V}}_{\mathfrak{S}}\left(\alpha_{*}, \beta ; \vec{\alpha}_{*}, \vec{\alpha}\right)=\sum_{i=1}^{4} \widehat{\mathcal{V}}_{i, \mathfrak{S}}\left(\alpha_{*}, \beta ; \vec{\alpha}_{*}, \vec{\alpha}\right)
$$

Lemma VI.5 We have

$$
\begin{gathered}
\sum_{\tau \in(0, \delta]} \varepsilon\left\langle\gamma_{\tau}^{*} \gamma_{\tau}, v \gamma_{\tau}^{*} \gamma_{\tau}\right\rangle \geq 4 C_{L} \sum_{\substack{\text { decimation } \\
\text { intervals } \\
\mathcal{J} \subset[0, \delta]}} \mathrm{r}(|\mathcal{J}|)^{2} \# \tilde{P}_{\beta}(\mathcal{J}) \\
\sum_{\tau \in[0, \delta)} \varepsilon\left\langle\gamma_{* \tau}^{*} \gamma_{* \tau}, v \gamma_{* \tau}^{*} \gamma_{* \tau}\right\rangle \geq 4 C_{L} \sum_{\substack{\text { decimation } \\
\text { intervals } \\
\mathcal{J} \subset[0, \delta]}} \mathrm{r}(|\mathcal{J}|)^{2} \# \tilde{P}_{\alpha}(\mathcal{J})
\end{gathered}
$$

with the $C_{L}$ of Lemma F.5.

Proof: We again use, for each $t \in(0, \delta) \cap \varepsilon \mathbb{Z}$, the notation

$$
B_{t}=\Lambda\left(\mathcal{J}_{t}\right)^{c} \cap \Lambda\left(\mathcal{J}_{t}^{-}\right) \cap \Lambda\left(\mathcal{J}_{t}^{+}\right)
$$

of (VI.5). Since $v$ is repulsive, its smallest eigenvalue $v_{1}>0$, so that

$$
\begin{aligned}
& \sum_{\tau \in(0, \delta]} \varepsilon\left\langle\gamma_{\tau}^{*} \gamma_{\tau}, v \gamma_{\tau}^{*} \gamma_{\tau}\right\rangle \geq \varepsilon v_{1} \sum_{\tau \in(0, \delta]}\left\|\gamma_{\tau}^{*} \gamma_{\tau}\right\|^{2}=\sum_{\mathbf{x} \in X} \sum_{\tau \in(0, \delta]} \varepsilon v_{1}\left|\gamma_{\tau}(\mathbf{x})\right|^{4} \\
& \geq \sum_{\mathbf{x} \in X} \sum_{\substack{t \in(0, \delta) \\
\mathbf{x} \in B_{t}}} \sum_{\tau=t+\varepsilon}^{t+\left|\mathcal{J}_{t}^{+}\right|} \varepsilon v_{1}\left|\gamma_{\tau}(\mathbf{x})\right|^{4} \quad \text { by (VI.7) } \\
&=\sum_{t \in(0, \delta)} \sum_{\tau=t+\varepsilon}^{t+\left|\mathcal{J}_{t}^{+}\right|} \sum_{\mathbf{x} \in B_{t}} \varepsilon v_{1}\left|\gamma_{\tau}(\mathbf{x})\right|^{4} \\
& \geq \frac{1}{16} \sum_{t \in(0, \delta)}\left|\mathcal{J}_{t}^{+}\right| v_{1} \mathrm{R}\left(\left|\mathcal{J}_{t}\right|\right)^{4} \# \tilde{P}_{\beta}\left(\mathcal{J}_{t}\right) \quad \text { by Lemma E.12.v }
\end{aligned}
$$


Similarly

$$
\sum_{\tau \in[0, \delta)} \varepsilon\left\langle\gamma_{* \tau}^{*} \gamma_{* \tau}, v \gamma_{* \tau}^{*} \gamma_{* \tau}\right\rangle \geq \frac{1}{16} \sum_{t \in(0, \delta)}\left|\mathcal{J}_{t}^{-}\right| v_{1} \mathrm{R}\left(\left|\mathcal{J}_{t}\right|\right)^{4} \# \tilde{P}_{\alpha}\left(\left[\mathcal{J}_{t}\right)\right.
$$

By Hypothesis II.14, $\frac{1}{16}\left|\mathcal{J}_{t}^{ \pm}\right| v_{1} \geq \frac{c_{v}}{16}\left|\mathcal{J}_{t}^{ \pm}\right||| v\left|\| \geq \frac{c_{v}}{64}\right| \mathcal{J}_{t}^{ \pm} \mid \mathfrak{v}$, and the claim now follows from (F.7.c).

Lemma VI.6 Recall that $\widehat{\mathcal{V}}_{1, \mathfrak{S}}, \widehat{\mathcal{V}}_{2, \mathfrak{S}}, \widehat{\mathcal{V}}_{3, \mathfrak{S}}$ and $\widehat{\mathcal{V}}_{4, \mathfrak{S}}$ were defined in (VI.9), (VI.10), (VI.11) and (VI.13) respectively.

$$
\begin{aligned}
\left|\widehat{\mathcal{V}}_{1, \mathfrak{S}}\left(\alpha_{*}, \beta ; \vec{\alpha}_{*}, \vec{\alpha}\right)\right| \leq & \sum_{\tau \in[0, \delta)} 2^{19} e^{3 K_{j}} \varepsilon \mathfrak{v R}(\varepsilon)^{3}\left\|\gamma_{* \tau}^{*}-\gamma_{\tau+\varepsilon}\right\|_{\Lambda_{\tau}^{c} \cup \Lambda_{\tau+\varepsilon}^{c}}^{2} \\
& \quad+\sum_{\tau \in(0, \delta)}\left(2^{21} e^{3 K_{j}} \varepsilon \mathfrak{v} \mathrm{R}(\varepsilon)^{3}+2^{28} e^{4 K_{j}}\left|\mathcal{J}_{\tau}\right| \mathfrak{v} \mathrm{r}\left(\left|\mathcal{J}_{\tau}\right|\right) \mathrm{R}\left(\left|\mathcal{J}_{\tau}\right|\right)^{3}\right)\left|\Lambda_{\tau}^{c}\right| \\
\left|\widehat{\mathcal{V}}_{2, \mathfrak{S}}\left(\alpha_{*}, \beta ; \vec{\alpha}_{*}, \vec{\alpha}\right)\right| \leq & 2^{17} e^{4 K_{j}} \delta \mathfrak{v} \mathrm{r}(\delta) \mathrm{R}(\delta)^{3}\left|\Lambda^{c}\right| \\
\left|\widehat{\mathcal{V}}_{3, \mathfrak{S}}\left(\alpha_{*}, \beta ; \vec{\alpha}_{*}, \vec{\alpha}\right)\right| \leq & 2^{17} e^{4 K_{j}} \delta \mathfrak{v} \mathrm{r}(\delta) \mathrm{R}(\delta)^{3}\left|\Omega^{c}\right| \\
\left|\widehat{\mathcal{V}}_{4, \mathfrak{S}}\left(\alpha_{*}, \beta ; \vec{\alpha}_{*}, \vec{\alpha}\right)\right| \leq & 2^{16} e^{4 K_{j}} \delta \mathfrak{v} \mathrm{r}(\delta) \mathrm{R}(\delta)^{3}\left|\Omega^{c}\right|
\end{aligned}
$$

Proof: We prove that

$$
\begin{aligned}
\varepsilon \sum_{\tau \in[0, \delta)}\left|\left\langle\Lambda^{c}\left(\Lambda_{\tau}^{c} \cup \Lambda_{\tau+\varepsilon}^{c}\right)\left(\gamma_{* \tau}-\gamma_{\tau+\varepsilon}^{*}\right) \gamma_{\tau+\varepsilon}, v \gamma_{* \tau} \gamma_{\tau+\varepsilon}\right\rangle\right| \\
\leq \sum_{\tau \in[0, \delta)} 2^{18} e^{3 K_{j}} \varepsilon \mathfrak{v R}(\varepsilon)^{3}\left\|\gamma_{* \tau}^{*}-\gamma_{\tau+\varepsilon}\right\|_{\Lambda_{\tau}^{c} \cup \Lambda_{\tau+\varepsilon}^{c}}^{2}+\sum_{\tau \in(0, \delta)} 2^{20} e^{3 K_{j}} \varepsilon \mathfrak{v R}(\varepsilon)^{3}\left|\Lambda_{\tau}^{c}\right|
\end{aligned}
$$

and

$$
\begin{aligned}
\varepsilon \sum_{\tau \in \varepsilon \mathbb{Z} \cap[0, \delta)} \mid\left\langle\Lambda ^ { c } \Lambda _ { \tau } \Lambda _ { \tau + \varepsilon } \left(\gamma_{* \tau}-\right.\right. & \left.\left.\gamma_{\tau+\varepsilon}^{*}\right) \gamma_{\tau+\varepsilon}, v \gamma_{* \tau} \gamma_{\tau+\varepsilon}\right\rangle \mid \\
& \leq \sum_{\tau^{\prime} \in(0, \delta)} 2^{26} e^{4 K_{j}}\left|\mathcal{J}_{\tau^{\prime}}\right| \mathfrak{v} \mathrm{r}\left(\left|\mathcal{J}_{\tau^{\prime}}\right|\right) \mathrm{R}\left(\left|\mathcal{J}_{\tau^{\prime}}\right|\right)^{3}\left|\Lambda_{\tau^{\prime}}^{c}\right|
\end{aligned}
$$

and

$$
\varepsilon \sum_{\tau \in[0, \delta)}\left|\left\langle\Lambda\left(\gamma_{* \tau}-\gamma_{\tau+\varepsilon}^{*}\right) \gamma_{\tau+\varepsilon}, v \Lambda^{c} \gamma_{* \tau} \gamma_{\tau+\varepsilon}\right\rangle\right| \leq 2^{14} e^{4 K_{j}} \delta \mathfrak{v} \mathrm{r}(\delta) \mathrm{R}(\delta)^{3}\left|\Lambda_{\frac{\delta}{2}}^{c}\right|
$$

and

$$
\begin{aligned}
\varepsilon \sum_{\tau \in[0, \delta)} \sum_{\substack{\tau_{1}, \tau_{2} \in \mathcal{T}_{r}(\tau+\varepsilon, \delta) \\
\tau_{3} \mathcal{T}_{\mathcal{T}}(\tau, \delta) \\
\left(\tau_{1}, \tau_{2}, \tau_{3}\right) \neq(\delta, \delta, 0)}}\left|\left\langle\Lambda\left(\gamma_{* \tau}-\gamma_{\tau+\varepsilon}^{*}\right)\left(\Gamma_{\tau+\varepsilon}^{\tau_{1}} \alpha_{\tau_{1}}\right), v \Lambda\left(\Gamma_{* \tau}^{\tau_{3}} \alpha_{\tau_{3}}^{*}\right)\left(\Gamma_{\tau+\varepsilon}^{\tau_{2}} \alpha_{\tau_{2}}\right)\right\rangle\right| \\
\leq 2^{16} e^{4 K_{j}} \delta \mathfrak{v} \mathrm{r}(\delta) \mathrm{R}(\delta)^{3}\left|\Lambda^{c}\right|
\end{aligned}
$$


and

$$
\begin{aligned}
\varepsilon \sum_{\tau \in[0, \delta)}\left|\left\langle\Lambda\left|\gamma_{* \tau}-\gamma_{\tau+\varepsilon}^{*}\right|\left(\Gamma_{\tau+\varepsilon}^{\delta} \Omega^{c}|\beta|\right), v \Lambda\left(\Gamma_{* \tau}^{0}|\alpha|\right)\left(\Gamma_{\tau+\varepsilon}^{\delta}|\beta|\right)\right\rangle\right| & \leq 2^{14} e^{4 K_{j}} \delta \mathfrak{v} \mathrm{r}(\delta) \mathrm{R}(\delta)^{3}\left|\Omega^{c}\right|
\end{aligned}
$$

and

$$
\varepsilon \sum_{\tau \in[0, \delta)}\left|\left\langle\Lambda\left(\Gamma_{* \tau}^{0} \Omega \alpha^{*}\right) \tilde{\gamma}_{\tau, \varepsilon}^{(1)}, v \Lambda\left(\Gamma_{* \tau}^{0} \Omega \alpha^{*}\right)\left(\Gamma_{\tau+\varepsilon}^{\delta} \Omega \beta\right)\right\rangle\right| \leq 2^{14} e^{4 K_{j}} \delta \mathfrak{v} \mathrm{r}(\delta) \mathrm{R}(\delta)^{3}\left|\Lambda^{c}\right|
$$

and

$$
\varepsilon \sum_{\tau \in[0, \delta)}\left|\left\langle\Lambda\left(\Gamma_{* \tau}^{0} \Omega \alpha^{*}\right) \tilde{\gamma}_{\tau, \varepsilon}^{(2)}, v \Lambda\left(\Gamma_{* \tau}^{0} \Omega \alpha^{*}\right)\left(\Gamma_{\tau+\varepsilon}^{\delta} \Omega \beta\right)\right\rangle\right| \leq \frac{1}{8} e^{-2 \mathrm{~m} \mathfrak{c}(\delta)} \delta \mathfrak{v}\left|\tilde{\Omega}^{c}\right|
$$

Four copies of (minor variants of) $\frac{1}{2}[(\mathrm{VI} .15)+($ VI.16) $]$ bounds the first two lines of the definition of $\widehat{\mathcal{V}}_{1, \mathfrak{S}}\left(\alpha_{*}, \beta ; \vec{\alpha}_{*}, \vec{\alpha}\right)$ in (VI.9). Four copies of (minor variants of) $\frac{1}{2}$ (VI.17) bounds the last two lines of the definition of $\widehat{\mathcal{V}}_{1, \mathfrak{S}}\left(\alpha_{*}, \beta ; \vec{\alpha}_{*}, \vec{\alpha}\right)$ in (VI.9). Four copies of (minor variants of $\frac{1}{2}$ (VI.18) bounds $\widehat{\mathcal{V}}_{2, \mathfrak{S}}\left(\alpha_{*}, \beta ; \vec{\alpha}_{*}, \vec{\alpha}\right)$, which was defined in (VI.10). Twelve copies of (minor variants of) $\frac{1}{2}$ (VI.19) bounds $\widehat{\mathcal{V}}_{3, \mathfrak{S}}\left(\alpha_{*}, \beta ; \vec{\alpha}_{*}, \vec{\alpha}\right)$, which was defined in (VI.11). Four copies of (minor variants of) $\frac{1}{2}[(\mathrm{VI} .20)+(\mathrm{VI} .21)]$ bounds $\widehat{\mathcal{V}}_{4, \mathfrak{S}}\left(\alpha_{*}, \beta ; \vec{\alpha}_{*}, \vec{\alpha}\right)$, which was defined in (VI.13).

Proof of (VI.15): By Proposition E.11.i,ii with $\mathcal{J}=\mathcal{J}_{\tau}$, and (VI.4),

$$
\left|\gamma_{\tau}(\mathbf{y})\right|,\left|\gamma_{* \tau}(\mathbf{y})\right| \leq 2^{6} e^{K_{j}} \mathrm{R}(\varepsilon) \text { for all } \mathbf{y} \in X
$$

for all $\tau \in \varepsilon \mathbb{Z} \cap[0, \delta]$. Hence, since $\sum_{\mathbf{y} \in X}|v(\mathbf{x}, \mathbf{y})| \leq \mathfrak{v}$,

$$
\begin{aligned}
\varepsilon \sum_{\tau \in[0, \delta)}\left|\left\langle\Lambda^{c}\left(\Lambda_{\tau}^{c} \cup \Lambda_{\tau+\varepsilon}^{c}\right)\left(\gamma_{* \tau}-\gamma_{\tau+\varepsilon}^{*}\right) \gamma_{\tau+\varepsilon}, v \gamma_{* \tau} \gamma_{\tau+\varepsilon}\right\rangle\right| \\
\leq \sum_{\substack{\tau \in[0, \delta) \\
\mathbf{x} \in\left(\Lambda_{\tau}^{c} \cup \Lambda_{\tau+\varepsilon}^{c}\right) \cap \Lambda^{c}}} \varepsilon \mathfrak{v} 2^{18} e^{3 K_{j}} \mathrm{R}(\varepsilon)^{3}\left|\gamma_{* \tau}^{*}(\mathbf{x})-\gamma_{\tau+\varepsilon}(\mathbf{x})\right| \\
\leq \sum_{\substack{\tau \in[0, \delta) \\
\mathbf{x} \in\left(\Lambda_{\tau}^{c} \cup \Lambda_{\tau+\varepsilon}^{c}\right) \cap \Lambda^{c}}} 2^{18} e^{3 K_{j}} \varepsilon \mathfrak{v} \mathrm{R}(\varepsilon)^{3}\left\{\left|\gamma_{* \tau}^{*}(\mathbf{x})-\gamma_{\tau+\varepsilon}(\mathbf{x})\right|^{2}+1\right\} \\
\leq \sum_{\tau \in[0, \delta)} 2^{18} e^{3 K_{j}} \varepsilon \mathfrak{v R}(\varepsilon)^{3}\left\{\left\|\gamma_{* \tau}^{*}-\gamma_{\tau+\varepsilon}\right\|_{\Lambda_{\tau}^{c} \cup \Lambda_{\tau+\varepsilon}^{c}}^{2}+\left|\Lambda_{\tau}^{c} \cap \Lambda_{\frac{\delta}{2}}^{c}\right|+\left|\Lambda_{\tau+\varepsilon}^{c} \cap \Lambda_{\frac{\delta}{2}}^{c}\right|\right\} \\
\leq \sum_{\tau \in[0, \delta)} 2^{18} e^{3 K_{j}} \varepsilon \mathfrak{v R}(\varepsilon)^{3}\left\|\gamma_{* \tau}^{*}-\gamma_{\tau+\varepsilon}\right\|_{\Lambda_{\tau}^{c} \cup \Lambda_{\tau+\varepsilon}^{c}}^{2}+\sum_{\tau \in(0, \delta)} 2^{20} e^{3 K_{j}} \varepsilon \mathfrak{v R}(\varepsilon)^{3}\left|\Lambda_{\tau}^{c}\right|
\end{aligned}
$$


Proof of (VI.16): We actually prove that

$$
\begin{aligned}
\varepsilon \sum_{\substack{\tau \in[0, \delta) \\
\tau \in 2 \varepsilon \mathbb{Z}}}\left|\left\langle\Lambda^{c} \Lambda_{\tau} \Lambda_{\tau+\varepsilon}\left(\gamma_{* \tau}-\gamma_{\tau+\varepsilon}^{*}\right) \gamma_{\tau+\varepsilon}, v \gamma_{* \tau} \gamma_{\tau+\varepsilon}\right\rangle\right| \\
\leq \sum_{\tau^{\prime} \in(0, \delta)} 2^{25} e^{4 K_{j}}\left|\mathcal{J}_{\tau^{\prime}}\right| \mathfrak{v} \mathrm{r}\left(\left|\mathcal{J}_{\tau^{\prime}}\right|\right) \mathrm{R}\left(\left|\mathcal{J}_{\tau^{\prime}}\right|\right)^{3}\left|\Lambda_{\tau^{\prime}}^{c}\right|
\end{aligned}
$$

The proof that

$$
\begin{aligned}
& \varepsilon \sum_{\substack{\tau \in[0, \delta) \\
\tau \in \varepsilon \mathbb{Z} \backslash 2 \varepsilon \mathbb{Z}}}\left|\left\langle\Lambda^{c} \Lambda_{\tau} \Lambda_{\tau+\varepsilon}\left(\gamma_{* \tau}-\gamma_{\tau+\varepsilon}^{*}\right) \gamma_{\tau+\varepsilon}, v \gamma_{* \tau} \gamma_{\tau+\varepsilon}\right\rangle\right| \\
& =\underset{\substack{\tau \in(0, \delta] \\
\tau \in 2 \varepsilon \mathbb{Z}}}{\varepsilon}\left|\left\langle\Lambda^{c} \Lambda_{\tau} \Lambda_{\tau-\varepsilon}\left(\gamma_{* \tau-\varepsilon}-\gamma_{\tau}^{*}\right) \gamma_{\tau}, v \gamma_{* \tau-\varepsilon} \gamma_{\tau}\right\rangle\right| \\
& \leq \sum_{\tau^{\prime} \in(0, \delta)} 2^{25} e^{4 K_{j}}\left|\mathcal{J}_{\tau^{\prime}}\right| \mathfrak{v} \mathrm{r}\left(\left|\mathcal{J}_{\tau^{\prime}}\right|\right) \operatorname{R}\left(\left|\mathcal{J}_{\tau^{\prime}}\right|\right)^{3}\left|\Lambda_{\tau^{\prime}}^{c}\right|
\end{aligned}
$$

is similar.

Now for (VI.16.a). For any $\tau \in 2 \varepsilon \mathbb{Z} \cap(0, \delta)$, we necessarily have $\mathcal{J}_{\tau+\varepsilon}=[\tau, \tau+2 \varepsilon] \subset \mathcal{J}_{\tau}$, so that $\Lambda_{\tau+\varepsilon} \supset \Lambda_{\tau}$. Hence, by Lemma E.4.ii, and recalling that $\Lambda_{0}=\emptyset$,

$$
\begin{aligned}
& \varepsilon \sum_{\tau \in 2 \varepsilon \mathbb{Z} \cap[0, \delta)}\left|\left\langle\Lambda^{c} \Lambda_{\tau} \Lambda_{\tau+\varepsilon}\left(\gamma_{* \tau}-\gamma_{\tau+\varepsilon}^{*}\right) \gamma_{\tau+\varepsilon}, v \gamma_{* \tau} \gamma_{\tau+\varepsilon}\right\rangle\right| \\
& \quad=\varepsilon \sum_{\tau \in 2 \varepsilon \mathbb{Z} \cap[0, \delta)}\left|\left\langle\Lambda^{c} \Lambda_{\tau}\left(\gamma_{* \tau}-\gamma_{\tau+\varepsilon}^{*}\right) \gamma_{\tau+\varepsilon}, v \gamma_{* \tau} \gamma_{\tau+\varepsilon}\right\rangle\right| \\
& \quad \leq \varepsilon \sum_{\tau \in 2 \varepsilon \mathbb{Z} \cap(0, \delta)} \sum_{\substack{\tau_{r} \in \mathcal{T}_{r}(\tau, \delta) \\
\tau_{l} \in \mathcal{T}_{l}(\tau, \delta)}}\left|\left\langle\Lambda^{c} \Lambda_{\pi\left(\tau_{r}\right)} \Lambda_{\tau_{r}}^{c} \Lambda_{\sigma\left(\tau_{l}\right)} \Lambda_{\tau_{l}}^{c}\left(\gamma_{* \tau}-\gamma_{\tau+\varepsilon}^{*}\right) \gamma_{\tau+\varepsilon}, v \gamma_{* \tau} \gamma_{\tau+\varepsilon}\right\rangle\right| \\
& \leq \varepsilon \sum_{\tau \in 2 \varepsilon \mathbb{Z} \cap(0, \delta)} \sum_{\left[\tau_{r}, \tau_{l}\right] \in \mathcal{T}_{l r}(\tau, \delta)}\left|\left\langle\Lambda^{c} \Lambda\left(\left[\tau_{l}, \tau_{r}\right]\right) \Lambda_{\tau_{r}}^{c} \Lambda_{\tau_{l}}^{c}\left(\gamma_{* \tau}-\gamma_{\tau+\varepsilon}^{*}\right) \gamma_{\tau+\varepsilon}, v \gamma_{* \tau} \gamma_{\tau+\varepsilon}\right\rangle\right|
\end{aligned}
$$

where $\mathcal{T}_{l r}(\tau, \delta)$ is the set of all decimation intervals (in the sense of Notation II.2) $\left[\tau_{l}, \tau_{r}\right]$ with $\tau_{l} \in \mathcal{T}_{l}(\tau, \delta), \tau_{r} \in \mathcal{T}_{r}(\tau, \delta)$ and $\Lambda_{\pi\left(\tau_{r}\right)} \cap \Lambda_{\tau_{r}}^{c} \cap \Lambda_{\sigma\left(\tau_{l}\right)} \cap \Lambda_{\tau_{l}}^{c} \neq \emptyset$. If $\left[\tau_{l}, \tau_{r}\right] \in \mathcal{T}_{l r}(\tau, \delta)$, then $\tau \in\left(\tau_{l}, \tau_{r}\right)$ so, given any decimation interval $\left[\tau_{l}, \tau_{r}\right]$, the number of $\tau \in 2 \varepsilon \mathbb{Z} \cap(0, \delta)$ with $\left[\tau_{l}, \tau_{r}\right] \in \mathcal{T}_{l r}(\tau)$ is less than $\frac{\tau_{r}-\tau_{l}}{2 \varepsilon}$ and

$$
\begin{aligned}
& \varepsilon \sum_{\substack{\tau \in 2 \varepsilon \mathbb{Z} \cap(0, \delta) \\
\left[\tau_{l}, \tau_{r}\right] \in \mathcal{T}_{l r}(\tau, \delta)}}\left|\left\langle\Lambda^{c} \Lambda\left(\left[\tau_{l}, \tau_{r}\right]\right) \Lambda_{\tau_{l}}^{c} \Lambda_{\tau_{r}}^{c}\left(\gamma_{* \tau}-\gamma_{\tau+\varepsilon}^{*}\right) \gamma_{\tau+\varepsilon}, v \gamma_{* \tau} \gamma_{\tau+\varepsilon}\right\rangle\right| \\
& \leq \frac{\tau_{r}-\tau_{l}}{2} \sup _{\substack{\tau \in 2 \varepsilon \mathbb{Z n}\left(\pi_{0}, \delta\right) \\
\left[\tau_{l}, \tau_{r}\right] \in \mathcal{T}_{l r}(\tau, \delta)}}\left|\left\langle\Lambda^{c} \Lambda\left(\left[\tau_{l}, \tau_{r}\right]\right) \Lambda_{\tau_{l}}^{c} \Lambda_{\tau_{r}}^{c}\left(\gamma_{* \tau}-\gamma_{\tau+\varepsilon}^{*}\right) \gamma_{\tau+\varepsilon}, v \gamma_{* \tau} \gamma_{\tau+\varepsilon}\right\rangle\right|
\end{aligned}
$$


We now fix any decimation interval $\left[\tau_{l}, \tau_{r}\right]$ and any $\tau \in 2 \varepsilon \mathbb{Z} \cap(0, \delta)$ for which $\left[\tau_{l}, \tau_{r}\right] \in$ $\mathcal{T}_{l r}(\tau, \delta)$ and bound

$$
\begin{aligned}
& \frac{\tau_{r}-\tau_{l}}{2}\left|\left\langle\Lambda^{c} \Lambda\left(\left[\tau_{l}, \tau_{r}\right]\right) \Lambda_{\tau_{l}}^{c} \Lambda_{\tau_{r}}^{c}\left(\gamma_{* \tau}-\gamma_{\tau+\varepsilon}^{*}\right) \gamma_{\tau+\varepsilon}, v \gamma_{* \tau} \gamma_{\tau+\varepsilon}\right\rangle\right| \\
& \leq \frac{\tau_{r}-\tau_{l}}{2}\left|\Lambda^{c} \cap \Lambda_{\tau_{l}}^{c} \cap \Lambda_{\tau_{r}}^{c}\right|\left(4 e^{K_{j}}+3\right) \mathrm{r}\left(\tau_{r}-\tau_{l}\right) \sup _{\mathbf{x} \in \Lambda\left(\left[\tau_{l}, \tau_{r}\right]\right)} \sum_{\mathbf{y} \in X}\left|\gamma_{\tau+\varepsilon}(\mathbf{x}) v(\mathbf{x}, \mathbf{y}) \gamma_{* \tau}(\mathbf{y}) \gamma_{\tau+\varepsilon}(\mathbf{y})\right| \\
& \leq \frac{\tau_{r}-\tau_{l}}{2}\left|\Lambda^{c} \cap \Lambda_{\tau_{l}}^{c} \cap \Lambda_{\tau_{r}}^{c}\right|\left(4 e^{K_{j}}+3\right) \mathrm{r}\left(\tau_{r}-\tau_{l}\right)\|v \mid\|\left[\sum_{\tau^{\prime} \in(0, \delta]} N_{0}\left(\Gamma_{\tau+\varepsilon}^{\tau^{\prime}}(\mathfrak{S}) ; e^{\frac{\mathrm{m}}{2} d\left(\mathbf{x}, \Lambda\left(\left[\tau_{l}, \tau_{r}\right]\right)\right)}, \kappa_{\tau^{\prime}}\right)\right]^{2} \\
& \\
& {\left[\sum_{\tau^{\prime} \in[0, \delta)} N_{0}\left(\Gamma_{* \tau}^{\tau^{\prime}}(\mathfrak{S}) ; e^{\frac{\mathrm{m}}{2} d\left(\mathbf{x}, \Lambda\left(\left[\tau_{l}, \tau_{r}\right]\right)\right)}, \kappa_{* \tau^{\prime}}\right)\right] }
\end{aligned}
$$

by Corollary E.9.b. Since $\tau$ is in the interior of $\left[\tau_{l}, \tau_{r}\right]$ and $\tau+\varepsilon$ is its neighbour and has $\mathfrak{d}(\tau+\varepsilon)=m>\mathfrak{d}\left(\tau_{r}\right), \tau+\varepsilon$ is also in the interior of $\left[\tau_{l}, \tau_{r}\right]$. Hence by Lemma E.13,

$$
\begin{aligned}
\frac{\tau_{r}-\tau_{l}}{2}\left|\left\langle\Lambda^{c} \Lambda\left(\left[\tau_{l}, \tau_{r}\right]\right) \Lambda_{\tau_{l}}^{c} \Lambda_{\tau_{r}}^{c}\left(\gamma_{* \tau}-\gamma_{\tau+\varepsilon}^{*}\right) \gamma_{\tau+\varepsilon}, v \gamma_{* \tau} \gamma_{\tau+\varepsilon}\right\rangle\right| \\
\quad \leq \frac{\tau_{r}-\tau_{l}}{2}\left|\Lambda^{c} \cap \Lambda_{\tau_{l}}^{c} \cap \Lambda_{\tau_{r}}^{c}\right|\left(4 e^{K_{j}}+3\right) \mathrm{r}\left(\tau_{r}-\tau_{l}\right)\|v \mid\| 40^{3} e^{3 K_{j}} \mathrm{R}\left(\tau_{r}-\tau_{l}\right)^{3} \\
\quad \leq 2^{20} e^{4 K_{j}}\left(\tau_{r}-\tau_{l}\right) \mathfrak{v} \mathrm{r}\left(\tau_{r}-\tau_{l}\right) \mathrm{R}\left(\tau_{r}-\tau_{l}\right)^{3}\left|\Lambda^{c} \cap \Lambda_{\tau_{l}}^{c} \cap \Lambda_{\tau_{r}}^{c}\right|
\end{aligned}
$$

and

$$
\begin{aligned}
& \varepsilon \sum_{\tau \in \varepsilon 2 \mathbb{Z} \cap[0, \delta)}\left|\left\langle\Lambda^{c} \Lambda_{\tau} \Lambda_{\tau+\varepsilon}\left(\gamma_{* \tau}-\gamma_{\tau+\varepsilon}^{*}\right) \gamma_{\tau+\varepsilon}, v \gamma_{* \tau} \gamma_{\tau+\varepsilon}\right\rangle\right| \\
& \quad \leq \sum_{\substack{\text { decimation } \\
\text { intervals } \\
\left[\tau_{l}, \tau_{r}\right]}} 2^{20} e^{4 K_{j}}\left(\tau_{r}-\tau_{l}\right) \mathfrak{v} \mathrm{r}\left(\tau_{r}-\tau_{l}\right) \mathrm{R}\left(\tau_{r}-\tau_{l}\right)^{3}\left|\Lambda^{c} \cap \Lambda_{\tau_{l}}^{c} \cap \Lambda_{\tau_{r}}^{c}\right| \\
& \leq \sum_{\tau^{\prime} \in \varepsilon \mathbb{Z} \cap(0, \delta)} 2^{25} e^{4 K_{j}}\left|\mathcal{J}_{\tau^{\prime}}\right| \mathfrak{v} \mathrm{r}\left(\left|\mathcal{J}_{\tau^{\prime}}\right|\right) \mathrm{R}\left(\left|\mathcal{J}_{\tau^{\prime}}\right|\right)^{3}\left|\Lambda_{\tau^{\prime}}^{c}\right|
\end{aligned}
$$

For the last inequality, each decimation interval $\left[\tau_{l}, \tau_{r}\right]$ was assigned to a $\tau^{\prime} \in \varepsilon \mathbb{Z} \cap(0, \delta)$ by

$\circ$ if $\left[\tau_{l}, \tau_{r}\right]=[0, \delta]$, the assigned $\tau^{\prime}$ is $\frac{\delta}{2}$.

$\circ$ if $\left[\tau_{l}, \tau_{r}\right] \neq[0, \delta]$, then $\left[\tau_{l}, \tau_{r}\right]$ is an interval of length $\frac{1}{2^{p}} \delta$ for some scale $1 \leq p<m$. In this case $\tau^{\prime}$ is assigned to the unique end point, $\tau_{l}$ or $\tau_{r}$, whose decimation index is $p$. At most $3 \leq 2^{2}$ intervals are assigned to each $\tau^{\prime}$ and, for each decimation interval,

$$
\left(\tau_{r}-\tau_{l}\right) \mathfrak{v} \mathrm{r}\left(\tau_{r}-\tau_{l}\right) \mathrm{R}\left(\tau_{r}-\tau_{l}\right)^{3}\left|\Lambda^{c} \cap \Lambda_{\tau_{l}}^{c} \cap \Lambda_{\tau_{r}}^{c}\right| \leq 2^{3}\left|\mathcal{J}_{\tau^{\prime}}\right| \mathfrak{v} \mathrm{r}\left(\left|\mathcal{J}_{\tau^{\prime}}\right|\right) \mathrm{R}\left(\left|\mathcal{J}_{\tau^{\prime}}\right|\right)^{3}\left|\Lambda_{\tau^{\prime}}^{c}\right|
$$


Proof of (VI.17):

$$
\begin{aligned}
\varepsilon \sum_{\tau \in[0, \delta)} \mid & \left\langle\Lambda\left(\gamma_{* \tau}-\gamma_{\tau+\varepsilon}^{*}\right) \gamma_{\tau+\varepsilon}, v \Lambda^{c} \gamma_{* \tau} \gamma_{\tau+\varepsilon}\right\rangle \mid \\
& \leq \max _{\tau \in[0, \delta)} \delta\left|\left\langle\Lambda\left(\gamma_{* \tau}-\gamma_{\tau+\varepsilon}^{*}\right) \gamma_{\tau+\varepsilon}, v \Lambda^{c} \gamma_{* \tau} \gamma_{\tau+\varepsilon}\right\rangle\right| \\
& \leq \max _{\tau \in[0, \delta)} \delta\left|\Lambda^{c}\right| \sup _{\mathbf{y} \in \Lambda^{c}} \sum_{\mathbf{x} \in \Lambda}\left|\left(\gamma_{* \tau}(\mathbf{x})-\gamma_{\tau+\varepsilon}^{*}(\mathbf{x})\right) \gamma_{\tau+\varepsilon}(\mathbf{x}) v(\mathbf{x}, \mathbf{y}) \gamma_{* \tau}(\mathbf{y}) \gamma_{\tau+\varepsilon}(\mathbf{y})\right| \\
& \leq \max _{\tau \in[0, \delta)} \delta\left|\Lambda^{c}\right|\left(4 e^{K_{j}}+3\right) \mathrm{r}(\delta)|\|v\||\left[\sum_{\tau^{\prime} \in(0, \delta]} N_{0}\left(\Gamma_{\tau+\varepsilon}^{\tau^{\prime}}(\mathfrak{S}) ; e^{\frac{m}{2} d(\mathbf{x}, \Lambda)}, \kappa_{\tau^{\prime}}\right)\right]^{2} \\
& \quad\left[\sum_{\tau^{\prime} \in[0, \delta)} N_{0}\left(\Gamma_{* \tau}^{\tau^{\prime}}(\mathfrak{S}) ; e^{\frac{\mathbf{m}}{2} d(\mathbf{x}, \Lambda)}, \kappa_{* \tau^{\prime}}\right)\right] \\
& \leq \delta\left(4 e^{K_{j}}+3\right) \mathrm{r}(\delta) \frac{\mathfrak{v}}{2}\left(2^{4} e^{K_{j}} \mathrm{R}(\delta)\right)^{3}\left|\Lambda^{c}\right| \\
&
\end{aligned}
$$

by Corollary E.9.b and Lemma E.13 (When $\tau+\varepsilon=\delta$, use $\Gamma_{\delta}^{\delta}(\mathfrak{S})=\mathbb{1}$ and $\Gamma_{\delta}^{\tau^{\prime}}(\mathfrak{S})=0$ for $\tau^{\prime} \in(0, \delta)$, and when $\tau=0$, use $\Gamma_{* 0}^{0}(\mathfrak{S})=\mathbb{1}$ and $\Gamma_{* 0}^{\tau^{\prime}}(\mathfrak{S})=0$ for $\tau^{\prime} \in(0, \delta)$. In both cases, apply Lemma B.1.)

Proof of (VI.18): We are to sum over $\left(\tau_{1}, \tau_{2}, \tau_{3}\right)$ excluding $(\delta, \delta, 0)$. We treat the case $\tau_{3} \neq 0$. The other cases are similar.

$$
\begin{aligned}
& \varepsilon \sum_{\tau \in[0, \delta)} \sum_{\substack{\tau_{1}, \tau_{2} \in \mathcal{T}_{r}(\tau+\varepsilon, \delta) \\
\tau_{3} \in \mathcal{T}_{l}(\tau, \delta) \backslash\{0\}}}\left|\left\langle\Lambda\left(\gamma_{* \tau}-\gamma_{\tau+\varepsilon}^{*}\right)\left(\Gamma_{\tau+\varepsilon}^{\tau_{1}} \alpha_{\tau_{1}}\right), v \Lambda\left(\Gamma_{* \tau}^{\tau_{3}} \alpha_{\tau_{3}}^{*}\right)\left(\Gamma_{\tau+\varepsilon}^{\tau_{2}} \alpha_{\tau_{2}}\right)\right\rangle\right| \\
& \leq \max _{\tau \in[0, \delta)} \delta \sum_{\substack{\tau_{1}, \tau_{2} \in \mathcal{T}_{r}(\tau+\varepsilon, \delta) \\
\tau_{3} \in \mathcal{T}_{l}(\tau, \delta) \backslash\{0\}}}\left|\left\langle\Lambda\left(\gamma_{* \tau}-\gamma_{\tau+\varepsilon}^{*}\right)\left(\Gamma_{\tau+\varepsilon}^{\tau_{1}} \alpha_{\tau_{1}}\right), v \Lambda\left(\Gamma_{* \tau}^{\tau_{3}} \alpha_{\tau_{3}}^{*}\right)\left(\Gamma_{\tau+\varepsilon}^{\tau_{2}} \alpha_{\tau_{2}}\right)\right\rangle\right|
\end{aligned}
$$

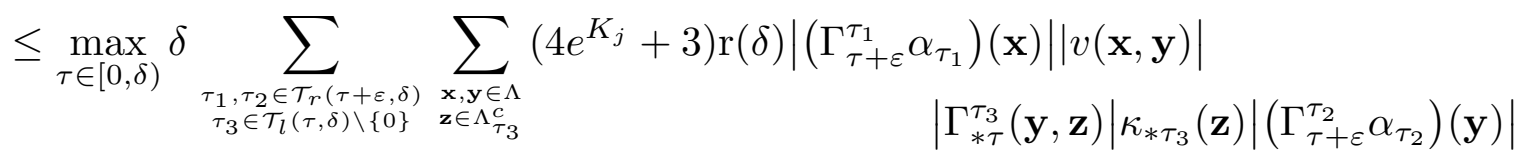

$$
\begin{aligned}
& \leq\left(4 e^{K_{j}}+3\right) \delta \mathrm{r}(\delta) \max _{\tau \in[0, \delta)}\|v\| \|\left[\sum_{\tau^{\prime} \in(0, \delta]} N_{0}\left(\Gamma_{\tau+\varepsilon}^{\tau^{\prime}}(\mathfrak{S}) ; e^{\frac{\mathrm{m}}{2} d(\mathbf{x}, \Lambda)}, \kappa_{\tau^{\prime}}\right)\right]^{2} \\
& {\left[\sum_{\tau_{3} \in(0, \delta)}\left|\Lambda_{\tau_{3}}^{c}\right| N_{0}\left(\Gamma_{* \tau}^{\tau_{3}}(\mathfrak{S}) ; e^{\left.\frac{\mathbf{m}}{2} d(\mathbf{x}, \Lambda)\right)}, \kappa_{* \tau_{3}}\right)\right]} \\
& \leq\left(4 e^{K_{j}}+3\right) \delta \mathrm{r}(\delta) \frac{\mathfrak{v}}{2}\left(2^{4} e^{K_{j}} \mathrm{R}(\delta)\right)^{3}\left|\Lambda^{c}\right| \\
& \leq 2^{14} e^{4 K_{j}} \delta \operatorname{vr}(\delta) \mathrm{R}(\delta)^{3}\left|\Lambda^{c}\right|
\end{aligned}
$$

by Lemma E.13 and Corollary E.9.b. 
Proof of (VI.19): By Corollary E.9.b and Lemma E.13,

$$
\begin{aligned}
& \varepsilon \sum_{\tau \in[0, \delta)}\left|\left\langle\Lambda\left|\gamma_{* \tau}-\gamma_{\tau+\varepsilon}^{*}\right|\left(\Gamma_{\tau+\varepsilon}^{\delta} \Omega^{c}|\beta|\right), v \Lambda\left(\Gamma_{* \tau}^{0}|\alpha|\right)\left(\Gamma_{\tau+\varepsilon}^{\delta}|\beta|\right)\right\rangle\right| \\
& \leq \max _{\tau \in[0, \delta)} \delta\left|\left\langle\Lambda\left|\gamma_{* \tau}-\gamma_{\tau+\varepsilon}^{*}\right|\left(\Gamma_{\tau+\varepsilon}^{\delta} \Omega^{c}|\beta|\right), v \Lambda\left(\Gamma_{* \tau}^{0}|\alpha|\right)\left(\Gamma_{\tau+\varepsilon}^{\delta}|\beta|\right)\right\rangle\right|
\end{aligned}
$$

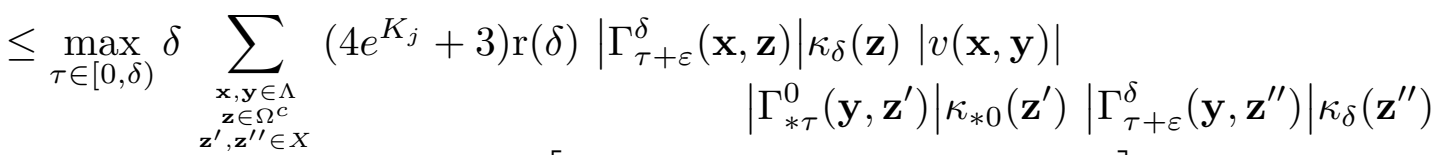

$$
\begin{aligned}
& \leq\left(4 e^{K_{j}}+3\right) \delta \mathrm{r}(\delta) \max _{\tau \in[0, \delta)}\|v \mid\|\left[\left|\Omega^{c}\right| N_{0}\left(\Gamma_{\tau+\varepsilon}^{\delta}(\mathfrak{S}) ; e^{\frac{\mathrm{m}}{2} d(\mathbf{x}, \Lambda)}, \kappa_{\delta}\right)\right] \\
& {\left[N_{0}\left(\Gamma_{* \tau}^{0}(\mathfrak{S}) ; e^{\left.\frac{\mathrm{m}}{2} d(\mathbf{x}, \Lambda)\right)}, \kappa_{* 0}\right)\right]\left[N_{0}\left(\Gamma_{\tau+\varepsilon}^{\delta}(\mathfrak{S}) ; e^{\left.\frac{\mathrm{m}}{2} d(\mathbf{x}, \Lambda)\right)}, \kappa_{\delta}\right)\right]} \\
& \leq\left(4 e^{K_{j}}+3\right) \delta \mathrm{r}(\delta) \frac{\mathfrak{v}}{2}\left(2^{4} e^{K_{j}} \mathrm{R}(\delta)\right)^{3}\left|\Omega^{c}\right| \\
& \leq 2^{14} e^{4 K_{j}} \delta \operatorname{vr}(\delta) \mathrm{R}(\delta)^{3}\left|\Omega^{c}\right|
\end{aligned}
$$

Proof of (VI.20): $\quad$ By Corollary E.9.c

$$
\begin{aligned}
& \varepsilon \sum_{\tau \in[0, \delta)}\left|\left\langle\Lambda\left(\Gamma_{* \tau}^{0} \Omega \alpha^{*}\right) \tilde{\gamma}_{\tau, \varepsilon}^{(1)}, v \Lambda\left(\Gamma_{* \tau}^{0} \Omega \alpha^{*}\right)\left(\Gamma_{\tau+\varepsilon}^{\delta} \Omega \beta\right)\right\rangle\right| \\
& \quad \leq \max _{\tau \in[0, \delta)} \delta \sum_{\mathbf{x}, \mathbf{y} \in \Lambda}\left|\left(\gamma_{\tau+\varepsilon}-\gamma_{* \tau}^{*}+\tilde{\gamma}_{\tau, \varepsilon}\right)(\mathbf{x})\right|\left|\left(\Gamma_{* \tau}^{0} \Omega \alpha^{*}\right)(\mathbf{x})\right||v(\mathbf{x}, \mathbf{y})| \\
& \quad \leq \delta 3 e^{K_{j}} \mathrm{r}(\delta)\left|\left(\Gamma_{* \tau}^{0} \Omega \alpha^{c} \mid \max _{\tau \in[0, \delta)} \| \mathbf{y}\right)\right|\left|\left(\Gamma_{\tau+\varepsilon}^{\delta} \Omega \beta\right)(\mathbf{y})\right| \\
& \quad \leq 3 e^{K_{j}} \delta \mathrm{r}(\delta) \mathfrak{v}\left(N_{0}\left(\Gamma_{* \tau}^{0} e^{K_{j}} \mathrm{R}(\delta)\right)^{3}\left|\Lambda^{c}\right|\right. \\
& \quad \leq 2^{14} e^{4 K_{j}} \delta \mathfrak{v} \mathrm{r}(\delta) \operatorname{R}(\delta)^{3}\left|\Lambda^{c}\right|
\end{aligned}
$$

by Lemma E.13.

Proof of (VI.21): By Corollary E.9.c

$$
\begin{aligned}
& \varepsilon \sum_{\tau \in[0, \delta)}\left|\left\langle\Lambda\left(\Gamma_{* \tau}^{0} \Omega \alpha^{*}\right) \tilde{\gamma}_{\tau, \varepsilon}^{(2)}, v \Lambda\left(\Gamma_{* \tau}^{0} \Omega \alpha^{*}\right)\left(\Gamma_{\tau+\varepsilon}^{\delta} \Omega \beta\right)\right\rangle\right| \\
& \begin{array}{r}
\leq \max _{\tau \in[0, \delta)} \delta \sum_{\mathbf{x}, \mathbf{y} \in \Lambda} e^{-4 \mathrm{~m} d(\mathbf{x}, \Omega)}\left|\left(\tilde{\gamma}_{\tau, \varepsilon}-\tilde{\tilde{\gamma}}_{\tau, \varepsilon}\right)(\mathbf{x})\right| e^{4 \mathrm{~m} d(\mathbf{x}, \Omega)}\left|\left(\Gamma_{* \tau}^{0} \Omega \alpha^{*}\right)(\mathbf{x})\right||v(\mathbf{x}, \mathbf{y})| \\
\left|\left(\Gamma_{* \tau}^{0} \Omega \alpha^{*}\right)(\mathbf{y})\right|\left|\left(\Gamma_{\tau+\varepsilon}^{\delta} \Omega \beta\right)(\mathbf{y})\right|
\end{array} \\
& \leq \delta \frac{1}{8} e^{-3 \mathrm{mc}(\delta)}\left|\tilde{\Omega}^{c}\right| \max _{\tau \in[0, \delta)}\||v|\| N_{4 \mathrm{~m}}\left(\Gamma_{* \tau}^{0}(\mathfrak{S}) ; e^{\frac{\mathrm{m}}{2} d(\mathbf{x}, \Lambda)}, \kappa_{* 0}\right) \\
& N_{0}\left(\Gamma_{* \tau}^{0}(\mathfrak{S}) ; e^{\frac{\mathrm{m}}{2} d(\mathbf{x}, \Lambda)}, \kappa_{* 0}\right) N_{0}\left(\Gamma_{\tau+\varepsilon}^{\delta}(\mathfrak{S}) ; e^{\left.\frac{\mathrm{m}}{2} d(\mathbf{x}, \Lambda)\right)}, \kappa_{\delta}\right) \\
& \leq \frac{1}{8} e^{-3 \mathrm{mc}(\delta)} \delta \mathfrak{v}\left(2^{4} e^{K_{j}} \mathrm{R}(\delta)\right)^{3}\left|\tilde{\Omega}^{c}\right| \\
& \leq \frac{1}{8} e^{-2 \mathrm{~m} \mathfrak{c}(\delta)} \delta \mathfrak{v}\left|\tilde{\Omega}^{c}\right|
\end{aligned}
$$

by Lemma E.13, followed by (F.6.b). 


\section{Lemma VI.7}

$$
\left|\widetilde{\mathcal{E}}_{4, \mathfrak{S}}(\alpha, \beta)\right| \leq \operatorname{Reg}_{\mathcal{S F}}^{(4)}(\alpha, \beta)
$$

with the $\widetilde{\mathcal{E}}_{4, \mathfrak{S}}(\alpha, \beta)$ defined in (VI.12) and the $\operatorname{Reg}_{\mathcal{S} \mathcal{F}}^{(4)}(\Omega ; \alpha, \beta)$ and $K_{\text {reg }}$ of Definition II.17.

Proof: Applying that

$$
\langle f, v g\rangle \leq\|v\|\|\| f\left\|_{L^{2}(X)}\right\| g \|_{L^{2}(X)}
$$

and that

$$
\begin{aligned}
\|f g\|_{L^{2}(X)} & \leq\left\|e^{-5 \mathrm{~m} d(\mathbf{x}, \Omega)} f\right\|_{L^{4}(X)}\left\|e^{5 \mathrm{~m} d(\mathbf{x}, \Omega)} g\right\|_{L^{4}(X)} \\
& \leq\left\|e^{5 \mathrm{~m} d(\mathbf{x}, \Omega)} f\right\|_{L^{4}(X)}\left\|e^{5 \mathrm{~m} d(\mathbf{x}, \Omega)} g\right\|_{L^{4}(X)}
\end{aligned}
$$

we have

$$
\begin{aligned}
&\left|\widetilde{\mathcal{E}}_{4, \mathfrak{S}}(\alpha, \beta)\right| \leq 2 \delta \max _{\tau \in[0, \delta)}\|v \mid\|\left\|e^{-5 \mathrm{~m} d(\mathbf{x}, \Omega)} \tilde{\tilde{\gamma}}_{\tau, \varepsilon}\right\|_{L^{4}(X)} \\
& \max \left\{\left\|e^{5 \mathrm{~m} d(\mathbf{x}, \Omega)} \Gamma_{* \tau}^{0} \Omega \alpha^{*}\right\|_{L^{4}(X)}^{3},\left\|e^{5 \mathrm{~m} d(\mathbf{x}, \Omega)} \Gamma_{\tau+\varepsilon}^{\delta} \Omega \beta\right\|_{L^{4}(X)}^{3}\right\}
\end{aligned}
$$

Next use the fact [E, Theorem 9.5.1] that, for any function $A: X \times X \rightarrow \mathbb{C}$, the norm of the operator $f \in L^{4}(X) \mapsto(A f)(\mathbf{x})=\sum_{\mathbf{y} \in X} A(\mathbf{x}, \mathbf{y}) f(\mathbf{y}) \in L^{4}(X)$ is bounded by

$$
\max \left\{\max _{\mathbf{x} \in X} \sum_{\mathbf{y} \in X}|A(\mathbf{x}, \mathbf{y})|, \max _{\mathbf{y} \in X} \sum_{\mathbf{x} \in X}|A(\mathbf{x}, \mathbf{y})|\right\}
$$

and the trivial observation that, for $\mathbf{y} \in \Omega,\left|e^{5 \mathrm{~m} d(\mathbf{x}, \Omega)} A(\mathbf{x}, \mathbf{y})\right| \leq\left|e^{5 \mathrm{~m} d(\mathbf{x}, \mathbf{y})} A(\mathbf{x}, \mathbf{y})\right|$. Consequently

$$
\begin{aligned}
& \left\|e^{5 \mathrm{~m} d(\mathbf{x}, \Omega)} \Gamma_{* \tau}^{0} \Omega \alpha^{*}\right\|_{L^{4}(X)} \leq\left\|\Gamma_{* \tau}^{0}\right\|\|\alpha\|_{L^{4}(\Omega)} \leq e^{K_{j} \delta}\|\alpha\|_{L^{4}(\Omega)} \\
& \left\|e^{5 \mathrm{~m} d(\mathbf{x}, \Omega)} \Gamma_{\tau+\varepsilon}^{\delta} \Omega \beta\right\|_{L^{4}(X)} \leq\left\|\Gamma_{\tau+\varepsilon}^{\delta}\right\|\|\beta\|_{L^{4}(\Omega)} \leq e^{K_{j} \delta}\|\beta\|_{L^{4}(\Omega)}
\end{aligned}
$$

To bound $\left\|e^{-5 \mathrm{~m} d(\mathbf{x}, \Omega)} \tilde{\tilde{\gamma}}_{\tau, \varepsilon}\right\|_{L^{4}(X)}$, write

$$
\tilde{\tilde{\gamma}}_{\tau, \varepsilon}=\tilde{\Omega}(\alpha-\beta)+(j(\tau)-\mathbb{1}) \tilde{\Omega} \alpha-(j(\delta-\tau-\varepsilon)-\mathbb{1}) \tilde{\Omega} \beta
$$

Set

$$
\begin{aligned}
& \mathcal{B}^{(0)}=\left\{b=(\mathbf{u}, \mathbf{v}) \in \tilde{\Omega}^{*} \mid \mathbf{u}, \mathbf{v} \in \tilde{\Omega}\right\} \\
& \mathcal{B}_{b 1}=\left\{b=(\mathbf{u}, \mathbf{v}) \in \tilde{\Omega}^{*} \mid \mathbf{u} \in \tilde{\Omega}, \mathbf{v} \in \tilde{\Omega}^{c}\right\} \\
& \mathcal{B}_{b 2}=\left\{b=(\mathbf{u}, \mathbf{v}) \in \tilde{\Omega}^{*} \mid \mathbf{u} \in \tilde{\Omega}^{c}, \mathbf{v} \in \tilde{\Omega}\right\}
\end{aligned}
$$

and write

$$
e^{\mu \tau} \int_{0}^{\tau} d \tau^{\prime}\left(\left[e^{-\tau^{\prime} \nabla^{*} \mathcal{H} \nabla} \nabla^{*} \mathcal{H} \nabla\right] \tilde{\Omega} \alpha\right)(\mathbf{x})=a_{1}(\mathbf{x})+a_{2}(\mathbf{x})+a_{3}(\mathbf{x})
$$


with

$$
\begin{aligned}
& a_{1}(\mathbf{x})=\sum_{\substack{\mathbf{z} \in X \\
b \in \mathcal{B}^{(0)}}} e^{\mu \tau} \int_{0}^{\tau} d \tau^{\prime} e^{-\tau^{\prime} \nabla^{*} \mathcal{H} \nabla}(\mathbf{x}, \mathbf{z})\left(\nabla^{*} \mathcal{H}\right)(\mathbf{z}, b)(\nabla \alpha)(b) \\
& a_{2}(\mathbf{x})=\sum_{\substack{\mathbf{z} \in X \\
b=(\mathbf{u}, \mathbf{v}) \in \mathcal{B}_{b 1}}} e^{\mu \tau} \int_{0}^{\tau} d \tau^{\prime} e^{-\tau^{\prime} \nabla^{*} \mathcal{H} \nabla}(\mathbf{x}, \mathbf{z})\left(\nabla^{*} \mathcal{H}\right)(\mathbf{z}, b)\{-\alpha(\mathbf{u})\} \\
& a_{3}(\mathbf{x})=\sum_{\substack{\mathbf{z} \in X \\
b=(\mathbf{u}, \mathbf{v}) \in \mathcal{B}_{b 2}}} e^{\mu \tau} \int_{0}^{\tau} d \tau^{\prime} e^{-\tau^{\prime} \nabla^{*} \mathcal{H} \nabla}(\mathbf{x}, \mathbf{z})\left(\nabla^{*} \mathcal{H}\right)(\mathbf{z}, b)\{\alpha(\mathbf{v})\}
\end{aligned}
$$

By (D.4)

$$
\left\|\left(1-e^{\mu \tau}\right) \tilde{\Omega} \alpha\right\|_{L^{4}(X)} \leq|\mu| \delta e^{K_{j}^{\prime}}\|\alpha\|_{L^{4}(\tilde{\Omega})}
$$

By (D.3), and the corresponding bounds with the sup over the right argument and the sum over the left argument,

$$
\begin{aligned}
\left\|a_{1}\right\|_{L^{4}(X)} & \leq \delta K_{j}^{\prime} e^{K_{j}^{\prime}}\|\nabla \alpha\|_{L^{4}\left(\tilde{\Omega}^{*}\right)} \\
\left\|e^{-5 \operatorname{md}(\mathbf{x}, \Omega)} a_{2}\right\|_{L^{4}(X)} & \leq e^{-5 \operatorname{m} d\left(\Omega, \tilde{\Omega}^{c}\right)} \delta K_{j}^{\prime} e^{K_{j}^{\prime}}\|\alpha\|_{L^{4}(\tilde{\Omega})} \\
\left\|e^{-5 \operatorname{md} d(\mathbf{x}, \Omega)} a_{3}\right\|_{L^{4}(X)} & \leq e^{-5 \operatorname{md} d\left(\Omega, \tilde{\Omega}^{c}\right)} \delta K_{j}^{\prime} e^{K_{j}^{\prime}}\|\alpha\|_{L^{4}(\tilde{\Omega})}
\end{aligned}
$$

All together, using (D.2),

$$
\begin{aligned}
& \left\|e^{-5 \mathrm{~m} d(\mathbf{x}, \Omega)} \tilde{\tilde{\gamma}}\right\|_{L^{4}(X)} \leq\|\alpha-\beta\|_{L^{4}(X)}+2 K_{j}^{\prime} e^{K_{j}^{\prime}}\left\{\delta\left[\mu+e^{-5 \mathrm{~m} d\left(\Omega, \tilde{\Omega}^{c}\right)}\right]\left[\|\alpha\|_{L^{4}(\tilde{\Omega})}+\|\beta\|_{L^{4}(\tilde{\Omega})}\right]\right. \\
& \left.+\delta\left[\|\nabla \alpha\|_{L^{4}\left(\tilde{\Omega}^{*}\right)}+\|\nabla \beta\|_{L^{4}\left(\tilde{\Omega}^{*}\right)}\right]\right\}
\end{aligned}
$$

and

$$
\begin{aligned}
\left|\widetilde{\mathcal{E}}_{4, \mathfrak{S}}(\alpha, \beta)\right| \leq \delta \mathfrak{v} 2 K_{j}^{\prime} e^{3 K_{j}+K_{j}^{\prime} \max \left\{\|\alpha\|_{L^{4}(\Omega)}^{3},\|\beta\|_{L^{4}(\Omega)}^{3}\right\}} \\
\begin{aligned}
\left\{\|\alpha-\beta\|_{L^{4}(X)}+\delta\left[\mu+e^{-5 \operatorname{mc}(\delta)}\right]\left[\|\alpha\|_{L^{4}(\tilde{\Omega})}+\|\beta\|_{L^{4}(\tilde{\Omega})}\right]\right. \\
\left.\quad+\delta\left[\|\nabla \alpha\|_{L^{4}\left(\tilde{\Omega}^{*}\right)}+\|\nabla \beta\|_{L^{4}\left(\tilde{\Omega}^{*}\right)}\right]\right\}
\end{aligned}
\end{aligned}
$$

The lemma now follows by Remark D.3.

Proof of Proposition III.37: It suffices to apply Lemmas VI.4, VI.5, VI.6 and VI.7 and the observations, from (F.6.a), that

$$
\begin{aligned}
2^{19} e^{3 K_{j}} \varepsilon \mathfrak{v R}(\varepsilon)^{3} & \leq \frac{1}{16} \\
2^{21} e^{3 K_{j}} \varepsilon \mathfrak{v R}(\varepsilon)^{3}+\left(2^{28} e^{4 K_{j}}+2^{19} e^{4 K_{j}}\right)|\mathcal{J}| \mathfrak{v} \mathrm{r}(|\mathcal{J}|) \mathrm{R}(|\mathcal{J}|)^{3} & \leq \frac{1}{8}
\end{aligned}
$$


Remark VI.8 Since Proposition III.37 is an upper bound, rather than an equality, the specific choice of $\operatorname{Reg}_{\mathcal{S} \mathcal{F}}^{(4)}(\alpha, \beta)$ that we have made is far from the only possibility. We have chosen it to be relatively simple.

\section{VI.3 Extracting Small Factors from the Stokes' Cylinder}

Proof of Proposition III.38: In this section we prove the required bounds involving the Stokes' cylinder $C_{s}\left(\mathbf{x} ; \alpha^{*}, \beta\right)$ introduced in Definition II.8. For simplicity of notation, write

$$
\mathrm{r}=\mathrm{r}(s) \quad \mathrm{R}=\mathrm{R}(s) \quad \mathrm{R}^{\prime}=\mathrm{R}^{\prime}(s) \quad \mathrm{r}_{+}=\mathrm{r}(2 s) \quad \mathrm{R}_{+}=\mathrm{R}(2 s) \quad \mathrm{R}_{+}^{\prime}=\mathrm{R}^{\prime}(2 s)
$$

and

$$
\Omega_{1}=\Omega\left(\mathcal{J}^{-}\right) \quad \Omega_{2}=\Omega\left(\mathcal{J}^{+}\right) \quad \Omega_{0}=\Omega_{1} \cap \Omega_{2} \quad \Lambda=\Lambda(\mathcal{J}) \quad P_{\alpha}=P_{\alpha}(\mathcal{J}) \quad \ldots
$$

As pointed out in Definition II.8, we may choose for this cylinder any two real dimensional surface in

$$
\left\{\left(z_{*}, z\right) \in \mathbb{C}^{2}|| z_{*}|,| z \mid<\mathrm{R}\right\}
$$

whose boundary is the union of the circle $\left\{\left(z_{*}, z\right) \in \mathbb{C}^{2}\left|z_{*}^{*}=z,\right| z \mid=\mathrm{r}\right\}$ and the curve bounding

$$
D=\left\{\left(z_{*}, z\right) \in \mathbb{C}^{2}|| z_{*}-\sigma_{*}|\leq \mathrm{r},| z-\sigma \mid \leq \mathrm{r}, z-z_{*}^{*}=\rho\right\}
$$

where

$$
\begin{aligned}
\sigma & =\left(\left[1-j_{\mathfrak{c}}(s)\right] \beta\right)(\mathbf{x}) \\
\sigma_{*} & =\left(\left[1-j_{\mathfrak{c}}(s)\right] \alpha^{*}\right)(\mathbf{x}) \\
\rho & =\left(j_{\mathfrak{c}}(s)[\alpha-\beta]\right)(\mathbf{x})
\end{aligned}
$$

For the estimates, we make the special choice suggested by [BFKT5, Example A.2]. We introduce the interpolating set $\mathcal{B}=\bigcup_{0 \leq t \leq 1} D_{t}$ where

$$
D_{t}=\left\{\left(z_{*}, z\right) \in \mathbb{C}^{2}|| z_{*}-t \sigma_{*}|\leq \mathrm{r},| z-t \sigma \mid \leq \mathrm{r}, z-z_{*}^{*}=t \rho\right\}
$$

Set $C_{s}\left(\mathbf{x} ; \alpha^{*}, \beta\right)=\bigcup_{0<t<1} \partial D_{t}$. Obviously, $C_{s}\left(\mathbf{x} ; \alpha^{*}, \beta\right)$ has the required boundary.

We start by proving

$$
\begin{aligned}
\left|\sigma_{*}\right|,|\sigma| & \leq s e^{2 K_{j}^{\prime} s}\left(\left[|\mu|+e^{-5 \mathrm{mc}}\right] \mathrm{R}_{+}+\mathrm{R}_{+}^{\prime}\right) \leq \mathrm{r} \\
|\rho| & \leq \mathrm{r}_{+}+|\sigma|+\left|\sigma_{*}\right|
\end{aligned}
$$


For the first bound, by Corollary D.2,

$$
\begin{aligned}
\left|\sigma_{*}\right| & =\left|\left(\left[\mathbb{1}-j_{\mathfrak{c}}(s)\right] \alpha^{*}\right)(\mathbf{x})\right| \\
& \leq s K_{j}^{\prime} e^{K_{j}^{\prime} s}\left(\left[|\mu|+e^{-5 \mathrm{mc}}\right] \max _{\substack{\mathbf{y} \in \mathbf{X} \\
d(\mathbf{x}, \mathbf{y}) \leq \mathfrak{c}}}|\alpha(\mathbf{y})|+\max _{\substack{b \in X^{*} \\
d(\mathbf{x}, b) \leq \mathfrak{c}}}|\nabla \alpha(b)|\right) \\
& \leq s e^{2 K_{j}^{\prime} s}\left(\left[|\mu|+e^{-5 \mathrm{mc}}\right] \mathrm{R}_{+}+\mathrm{R}_{+}^{\prime}\right) \leq \mathrm{r}
\end{aligned}
$$

The second inequality follows from the observations that $|\alpha(\mathbf{y})| \leq \mathbf{R}_{+}$for all $\mathbf{y}$ within a distance $\mathfrak{c}$ of $\Lambda$ and $|\nabla \alpha(b)| \leq \mathrm{R}_{+}^{\prime}$ for all bonds $b$ within a distance $\mathfrak{c}$ of $\Lambda$. When $\mathbf{y} \in \Lambda$, this is enforced by the characteristic function $\chi_{2 s}(\Lambda ; \alpha, \beta)$. When $\mathbf{y}$ is not in $\Lambda$, but is within a distance $\mathfrak{c}$ of $\Lambda$, this is enforced by the factor of $\chi_{\mathrm{R}_{+}}\left(\Omega_{0} \backslash\left(P_{\alpha} \cup \Lambda\right), \alpha\right)$ in the characteristic function $\chi_{\mathfrak{A}, s}\left(\Omega_{1}, \Omega_{2} ; \alpha, \phi, \beta\right)$. (Recall that $d\left(\Lambda, P_{\alpha} \cup \Omega_{0}^{c}\right)>\mathfrak{c}(s)>\mathfrak{c}$, by (F.4.a).) When $b \in \Lambda^{*}$, this is enforced by the characteristic function $\chi_{2 s}(\Lambda ; \alpha, \beta)$. When $b$ is not in $\Lambda^{*}$, but is within a distance $\mathfrak{c}$ of $\Lambda$, this is enforced by the factor of $\chi_{\mathrm{R}^{\prime}+}\left(\Omega_{0}^{*} \backslash\left(P_{\alpha}^{\prime} \cup \Lambda^{*}\right), \nabla \alpha\right)$ in the characteristic function $\chi_{\mathfrak{A}, s}\left(\Omega_{1}, \Omega_{2} ; \alpha, \phi, \beta\right)$. The third inequality follows immediately from (F.7.d).

For the bound on $\rho$ observe

$$
|\rho| \leq|\beta(\mathbf{x})-\alpha(\mathbf{x})|+\left|\left(\left[\mathbb{1}-j_{\mathfrak{c}}(s)\right][\beta-\alpha]\right)(\mathbf{x})\right| \leq \mathrm{r}_{+}+\left|\sigma_{*}\right|+|\sigma|
$$

Since $C_{s}\left(\mathbf{x} ; \alpha^{*}, \beta\right) \subset \bigcup_{0 \leq t \leq 1} D_{t}$, part (i) follows from (VI.22) and (F.3.f). By [BFKT5, Remark A.3], on $C_{s}\left(\mathbf{x} ; \alpha^{*}, \beta\right)$,

$$
\begin{aligned}
\operatorname{Re}\left(z_{*} z\right) & \geq \frac{1}{2}\left(\mathrm{r}^{2}-|\rho|^{2}\right)-\mathrm{r}\left(|\sigma|+\left|\sigma_{*}\right|\right) \\
& \geq \frac{1}{2}\left(\mathrm{r}^{2}-\mathrm{r}_{+}^{2}\right)-\left(\mathrm{r}_{+}+\frac{|\sigma|+\left|\sigma_{*}\right|}{2}+\mathrm{r}\right)\left(|\sigma|+\left|\sigma_{*}\right|\right) \\
& \geq \frac{1}{2}\left(\mathrm{r}-\mathrm{r}_{+}\right)\left(\mathrm{r}+\mathrm{r}_{+}\right)-3 \mathrm{r}\left(|\sigma|+\left|\sigma_{*}\right|\right) \\
& \geq \frac{3}{4} \mathrm{r}\left(\mathrm{r}-\mathrm{r}_{+}\right)-6 \mathrm{r} s e^{2 K_{j}^{\prime}}\left(\left[|\mu|+e^{-5 \mathrm{mc}}\right] \mathrm{R}_{+}+\mathrm{R}_{+}^{\prime}\right) \\
& \geq C_{L} \mathrm{r}^{2}
\end{aligned}
$$

The second and third inequality follow from (VI.22), the fourth inequality from (F.3.b) and (VI.22) and the last from (F.7.d).

By [BFKT5, Remark A.3], the area of $C_{s}\left(\mathbf{x} ; \alpha^{*}, \beta\right)$ is bounded by

$$
8 \pi \mathrm{r}\left[|\sigma|+\left|\sigma_{*}\right|+|\rho|\right] \leq 40 \pi \mathrm{r}^{2}
$$




\section{VI.4 Relative Sizes of Large Field Sets}

In this subsection, we prove Lemma III.40, which is used in the proof of Theorem III.35. As in the part of the proof of Theorem III.35 where Lemma III.40 is used, we fix a hierarchy for scale $\delta=2^{n} \varepsilon$. By way of preparation, we have

Lemma VI.9 Let $\mathcal{J}$ be a decimation interval of length $2^{p} \varepsilon$, for some $0 \leq p \leq n$, and $\mathbf{x} \in \Omega(\mathcal{J})^{c}$. Then there is a decimation interval $\mathcal{J}^{\prime} \subset \mathcal{J}$, of length $2^{q} \varepsilon$, with $1 \leq q \leq p$, such that

$$
d\left(\mathbf{x}, \tilde{Q}\left(\mathcal{J}^{\prime}\right) \cup \operatorname{supp} \tilde{P}_{\alpha}^{\prime}\left(\mathcal{J}^{\prime}\right) \cup \operatorname{supp} \tilde{P}_{\beta}^{\prime}\left(\mathcal{J}^{\prime}\right) \cup \tilde{P}_{\alpha}\left(\mathcal{J}^{\prime}\right) \cup \tilde{P}_{\beta}\left(\mathcal{J}^{\prime}\right) \cup R\left(\mathcal{J}^{\prime}\right)\right) \leq 4 \sum_{k=q-1}^{p-1} \mathfrak{c}\left(2^{k} \varepsilon\right)
$$

Proof: The proof is by induction on $p$. If $p=0$, then $\Omega(\mathcal{J})^{c}=\emptyset$ and the statement is vacuous. Assume that the statement is satisfied for some $p \geq 0$. Let $\mathcal{J}$ be a decimation interval of length $2^{p+1} \varepsilon$ and $\mathbf{x} \in \Omega(\mathcal{J})^{c}$. By Definition II.4, there is a point $\mathbf{y}_{1} \in \Omega\left(\mathcal{J}^{-}\right)^{c} \cup$ $\Omega\left(\mathcal{J}^{+}\right)^{c} \cup P_{\alpha}(\mathcal{J}) \cup P_{\beta}(\mathcal{J}) \cup \operatorname{supp} P_{\alpha}^{\prime}(\mathcal{J}) \cup \operatorname{supp} P_{\beta}^{\prime}(\mathcal{J}) \cup Q(\mathcal{J}) \cup R(\mathcal{J})$ such that $d\left(\mathbf{x}, \mathbf{y}_{1}\right) \leq$ $\mathfrak{c}\left(2^{p} \varepsilon\right)$.

- If $\mathbf{y}_{1} \in \Omega\left(\mathcal{J}^{-}\right)^{c} \cup \Omega\left(\mathcal{J}^{+}\right)^{c}$, then by the induction hypothesis, there is a decimation interval $\mathcal{J}^{\prime}$ of length $2^{q} \varepsilon$, with $1 \leq q \leq p$, that is contained either in $\mathcal{J}^{-}$or $\mathcal{J}^{+}$, such that

$$
d\left(\mathbf{y}_{1}, \tilde{Q}\left(\mathcal{J}^{\prime}\right) \cup \cdots \cup \tilde{P}_{\beta}\left(\mathcal{J}^{\prime}\right) \cup R\left(\mathcal{J}^{\prime}\right)\right) \leq 4 \sum_{k=q-1}^{p-1} \mathfrak{c}\left(2^{k} \varepsilon\right)
$$

As $d\left(\mathbf{x}, \mathbf{y}_{1}\right) \leq \mathfrak{c}\left(2^{p} \varepsilon\right)$, we are finished.

- If $\mathbf{y}_{1} \in \tilde{P}_{\alpha}(\mathcal{J}) \cup \tilde{P}_{\beta}(\mathcal{J}) \cup \operatorname{supp} \tilde{P}_{\alpha}^{\prime}(\mathcal{J}) \cup \operatorname{supp} \tilde{P}_{\beta}^{\prime}(\mathcal{J}) \cup \tilde{Q}(\mathcal{J}) \cup R(\mathcal{J})$, we set $\mathcal{J}^{\prime}=\mathcal{J}$ and are finished.

- If $\mathbf{y}_{1} \in \mathcal{X}(\mathcal{J}) \backslash \tilde{\mathcal{X}}(\mathcal{J})$, for some $\mathcal{X} \in\left\{P_{\alpha}, P_{\beta}\right.$, supp $\left.P_{\alpha}^{\prime}, \operatorname{supp} P_{\beta}^{\prime}, Q\right\}$, then there is a $\mathbf{y}_{2} \in \Lambda\left(\mathcal{J}^{-}\right)^{c} \cup \Lambda\left(\mathcal{J}^{+}\right)^{c} \subset \Omega\left(\mathcal{J}^{-}\right)^{c} \cup \Omega\left(\mathcal{J}^{+}\right)^{c}$ with $d\left(\mathbf{y}_{1}, \mathbf{y}_{2}\right) \leq 2 \mathfrak{c}\left(2^{p} \varepsilon\right)+1 \leq 3 \mathfrak{c}\left(2^{p} \varepsilon\right)$.

By the induction hypothesis, there is a decimation interval $\mathcal{J}^{\prime}$ of length $2^{q} \varepsilon$, with $1 \leq q \leq p$, that is contained either in $\mathcal{J}^{-}$or $\mathcal{J}^{+}$, such that

$$
d\left(\mathbf{y}_{2}, \tilde{Q}\left(\mathcal{J}^{\prime}\right) \cup \cdots \cup \tilde{P}_{\beta}\left(\mathcal{J}^{\prime}\right) \cup R\left(\mathcal{J}^{\prime}\right)\right) \leq 4 \sum_{k=q-1}^{p-1} \mathfrak{c}\left(2^{k} \varepsilon\right)
$$

As $d\left(\mathbf{x}, \mathbf{y}_{2}\right) \leq 4 \mathfrak{c}\left(2^{p} \varepsilon\right)$, we are finished. 
Proof of Lemma III.40: By Lemma VI.9, for each decimation interval $\mathcal{J}$ of length $2^{p} \varepsilon$, with $1 \leq p \leq n$,

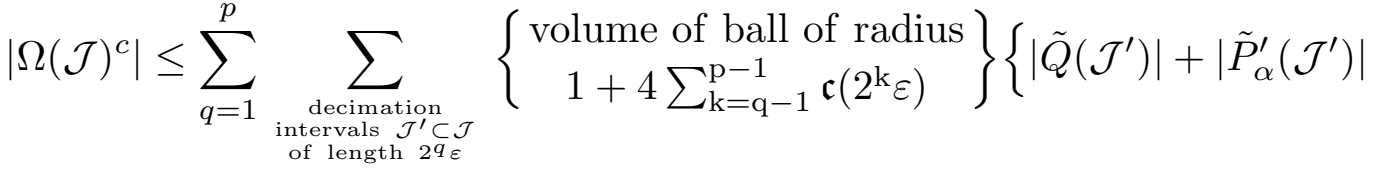

$$
\begin{aligned}
& \left.+\left|\tilde{P}_{\beta}^{\prime}\left(\mathcal{J}^{\prime}\right)\right|+\left|\tilde{P}_{\alpha}\left(\mathcal{J}^{\prime}\right)\right|+\left|\tilde{P}_{\beta}\left(\mathcal{J}^{\prime}\right)\right|+\left|\mathcal{R}\left(\mathcal{J}^{\prime}\right)\right|\right\}
\end{aligned}
$$

Since, for each $q \leq p$, any decimation interval $\mathcal{J}^{\prime}$ of length $2^{q} \varepsilon$ is contained in a unique decimation interval of length $2^{p} \varepsilon$,

$$
\begin{aligned}
& \sum_{p=1}^{n} \sum_{\substack{\text { decimation } \\
\text { intervals } \\
\text { of length } 2_{\varepsilon}}}\left(\ell\left(2^{p} \varepsilon\right)+1\right)\left|\Omega(\mathcal{J})^{c}\right| \\
& \leq \sum_{q=1}^{n} \sum_{\substack{\text { decimation } \\
\text { intervals } \mathcal{J}^{\prime} \\
\text { of length } 2 q_{\varepsilon}}}\left\{\left|\tilde{Q}\left(\mathcal{J}^{\prime}\right)\right|+\cdots+\left|\mathcal{R}\left(\mathcal{J}^{\prime}\right)\right|\right\} \sum_{p=q}^{n}\left(\ell\left(2^{p} \varepsilon\right)+1\right)\left\{\begin{array}{c}
\text { volume of ball of radius } \\
1+4 \sum_{\mathrm{k}=\mathrm{q}-1}^{\mathrm{p}-1} \mathfrak{c}\left(2^{\mathrm{k}} \varepsilon\right)
\end{array}\right\} \\
& \leq \sum_{q=1}^{n} \sum_{\substack{\text { decimation } \\
\text { intervals } \\
\text { of length } 2^{\prime} q_{\varepsilon}}} C_{L} \mathrm{r}\left(2^{q} \varepsilon\right)^{2}\left\{\left|\tilde{Q}\left(\mathcal{J}^{\prime}\right)\right|+\cdots+\left|\mathcal{R}\left(\mathcal{J}^{\prime}\right)\right|\right\}
\end{aligned}
$$

by (F.7.e). 


\section{Appendix A: Large/Small Field Characteristic Functions}

The representation of the effective density given in Theorem II.16 involves the "large field integral operator" introduced in Definition II.8. In part (i) of this definition we associate to a hierarchy $\mathfrak{S}$ and a decimation interval $\mathcal{J}$ an integral operator $\mathcal{I}_{\left(\mathcal{J} ; \alpha^{*}, \beta\right)}$. Its definition involves a characteristic function $\chi_{\mathcal{J}}\left(\alpha, \alpha_{\tau}, \beta\right)=\chi_{\mathcal{J}, \mathfrak{S}}\left(\alpha, \alpha_{\tau}, \beta\right)$ implementing large and small field conditions. Here, we are going to define this characteristic function.

For this definition, we use the notation that, for $0<r<R, z \in \mathbb{C}$, any set $Y$ and any complex valued function $f$ on $Y$

and

$$
\chi_{r}(z)=\left\{\begin{array}{ll}
1 & \text { if }|z| \leq r \\
0 & \text { otherwise }
\end{array}\right\} \quad \chi_{r, R}(t)=\left\{\begin{array}{ll}
1 & \text { if } r \leq|z| \leq R \\
0 & \text { otherwise }
\end{array}\right\}
$$

$$
\chi_{r}(Y, f)=\prod_{x \in Y} \chi_{r}(f(x)) \quad \chi_{r, R}(Y, f)=\prod_{x \in Y} \chi_{r, R}(f(x))
$$

As pointed out in the leadup to Definition III.2, the data associated to an interval in a hierarchy naturally split into two parts, the "first kind" of natural large/small field conditions and the "second kind" associated to the stationary phase construction. The following definition collects the conditions of the "first kind" that arise in Lemma A.3, below.

Definition A.1 Let $\Omega_{1}, \Omega_{2} \subset X, \delta>0$ and let $\mathfrak{A}=\left(\Lambda, P_{\alpha}, P_{\beta}, P_{\alpha}^{\prime}, P_{\beta}^{\prime}, Q\right) \in F_{\delta}\left(\Omega_{1} \cap \Omega_{2}\right)$ be a choice of "small/large field sets of the first kind" as in Definition III.2. The characteristic function for the small/large field sets of the first kind is

$$
\chi_{\mathfrak{A}, \delta}\left(\Omega_{1}, \Omega_{2} ; \alpha, \phi, \beta\right)=\chi^{(1)}\left(\Omega_{1}, \Omega_{2} ; \alpha, \phi, \beta\right) \chi_{\mathfrak{A}}^{(2)}(\alpha, \beta) \chi_{\mathfrak{A}}^{(3)}(\alpha, \phi, \beta)
$$

where

$$
\begin{aligned}
\chi^{(1)}\left(\Omega_{1}, \Omega_{2} ; \alpha, \phi, \beta\right)= & \chi_{\mathrm{R}(\delta)}\left(\Omega_{1} \backslash \Omega_{0}, \alpha\right) \chi_{\mathrm{R}(\delta)}\left(\Omega_{2} \backslash \Omega_{0}, \beta\right) \chi_{\mathrm{R}(\delta)}\left(\left(\Omega_{1} \cup \Omega_{2}\right) \backslash \Omega_{0}, \phi\right) \\
& \chi_{\mathrm{R}^{\prime}(\delta)}\left(\Omega_{1}^{*} \backslash \Omega_{0}^{*}, \nabla \alpha\right) \chi_{\mathrm{R}^{\prime}(\delta)}\left(\Omega_{2}^{*} \backslash \Omega_{0}^{*}, \nabla \beta\right) \chi_{\mathrm{R}^{\prime}(\delta)}\left(\left(\Omega_{1}^{*} \cup \Omega_{2}^{*}\right) \backslash \Omega_{0}^{*}, \nabla \phi\right) \\
& \chi_{\mathrm{r}(\delta)}\left(\Omega_{1}^{\star} \backslash \Omega_{0}^{\star}, \alpha-\phi\right) \chi_{\mathrm{r}(\delta)}\left(\Omega_{2}^{\star} \backslash \Omega_{0}^{\star}, \phi-\beta\right)
\end{aligned}
$$

and

$$
\begin{array}{rlr}
\chi_{\mathfrak{A}}^{(2)}(\alpha, \beta)= & \chi_{\mathrm{R}(2 \delta)}\left(\Omega_{0} \backslash\left(P_{\alpha} \cup \Lambda\right), \alpha\right) & \\
& \chi_{\mathrm{R}(2 \delta)}\left(\Omega_{0} \backslash\left(P_{\beta} \cup \Lambda\right), \beta\right) & \chi_{\mathrm{R}(2 \delta), \mathrm{R}(\delta)}\left(P_{\beta}, \beta\right) \\
& \chi_{\mathrm{R}^{\prime}(2 \delta)}\left(\Omega_{0}^{*} \backslash\left(P_{\alpha}^{\prime} \cup \Lambda^{*}\right), \nabla \alpha\right) & \chi_{\mathrm{R}^{\prime}(2 \delta), \mathrm{R}^{\prime}(\delta)}\left(P_{\alpha}^{\prime}, \nabla \alpha\right) \\
& \chi_{\mathrm{R}^{\prime}(2 \delta)}\left(\Omega_{0}^{*} \backslash\left(P_{\beta}^{\prime} \cup \Lambda^{*}\right), \nabla \beta\right) & \chi_{\mathrm{R}^{\prime}(2 \delta), \mathrm{R}^{\prime}(\delta)}\left(P_{\beta}^{\prime}, \nabla \beta\right) \\
& \chi_{\mathrm{r}(2 \delta)}\left(\Omega_{0}^{\star} \backslash\left(Q \cup \Lambda^{\star}\right), \alpha-\beta\right) & \chi_{\mathrm{r}(2 \delta), \infty}(Q, \alpha-\beta)
\end{array}
$$


and

$$
\begin{aligned}
\chi_{\mathfrak{A}}^{(3)}(\alpha, \phi, \beta)= & \chi_{\mathrm{R}(\delta)}\left(\Omega_{0} \backslash \Lambda, \phi\right) \chi_{\mathrm{R}^{\prime}(\delta)}\left(\Omega_{0}^{*} \backslash \Lambda^{*}, \nabla \phi\right) \\
& \chi_{\mathrm{r}(\delta)}\left(\Omega_{0}^{\star} \backslash \Lambda, \alpha-\phi\right) \chi_{\mathrm{r}(\delta)}\left(\Omega_{0}^{\star} \backslash \Lambda, \phi-\beta\right)
\end{aligned}
$$

with $\Omega_{0}=\Omega_{1} \cap \Omega_{2}$.

If $\mathfrak{A}$ is part of the data associated to a decimation interval of length $2 \delta$ in a hierarchy $\mathfrak{S}$, and $\Omega_{1}=\Omega\left(\mathcal{J}^{-}\right), \Omega_{1}=\Omega\left(\mathcal{J}^{+}\right)$, then

- $\chi^{(1)}$ imposes the "old" small field conditions on $\alpha, \beta$ and $\phi$ in $\left(\Omega\left(\mathcal{J}^{-}\right) \cup \Omega\left(\mathcal{J}^{+}\right)\right) \backslash$ $\left(\Omega\left(\mathcal{J}^{-}\right) \cap \Omega\left(\mathcal{J}^{+}\right)\right)$

- $\chi_{\mathfrak{A}}^{(2)}$ imposes the "new" small and large field conditions on $\alpha$ and $\beta$ in the region $\left(\Omega\left(\mathcal{J}^{-}\right) \cap \Omega\left(\mathcal{J}^{+}\right)\right) \backslash \Lambda(\mathcal{J})$, the complement of the "small field region of the first kind" for $\mathcal{J}$ in the previous "small field regions"

- $\chi_{\mathfrak{A}}^{(3)}$ imposes small field conditions on $\phi$ in $\left(\Omega\left(\mathcal{J}^{-}\right) \cap \Omega\left(\mathcal{J}^{+}\right)\right) \backslash \Lambda(\mathcal{J})$ Observe that $\chi_{\mathfrak{A}, \delta}$ does not depend on the values of the fields on the set $\Lambda$.

For the small/large field conditions of the second kind, we use (as in Theorem II.16) the characteristic function $\chi_{\delta}(Y ; \alpha, \beta)$ (defined for $\delta>0$, a subset $Y \subset X$ and fields $\alpha, \beta$ ) which takes the value one if

- $|\alpha(\mathbf{x})|,|\beta(\mathbf{x})| \leq \mathrm{R}(\delta)$ for all $\mathbf{x} \in Y$ and

- $|\nabla \alpha(b)|,|\nabla \beta(b)| \leq \mathrm{R}^{\prime}(\delta)$ for all bonds $b$ on $X$ that have at least one end in $Y$ and - $|\alpha(\mathbf{x})-\beta(\mathbf{x})| \leq \mathrm{r}(\delta)$ for all $\mathbf{x}$ within a distance one of $Y$

and which takes the value zero otherwise.

Definition A.2 Let $\mathfrak{S}$ be a hierarchy in the sense of Definition II.4, and let $\mathcal{J}$ be a decimation interval for $\mathfrak{S}$ of scale $2 t$. Define

$$
\chi_{\mathcal{J}}(\alpha, \phi, \beta)=\chi_{\mathfrak{A}, t}\left(\Omega\left(\mathcal{J}^{-}\right), \Omega\left(\mathcal{J}^{+}\right) ; \alpha, \phi, \beta\right) \quad \chi_{2 t}(\Lambda(\mathcal{J}) \backslash \Omega(\mathcal{J}) ; \alpha, \beta)
$$

where

$$
\mathfrak{A}=\left(\Lambda(\mathcal{J}), P_{\alpha}(\mathcal{J}), P_{\beta}(\mathcal{J}), P_{\alpha}^{\prime}(\mathcal{J}), P_{\beta}^{\prime}(\mathcal{J}), Q(\mathcal{J})\right) \in F_{\delta}\left(\Omega\left(\mathcal{J}^{-}\right) \cap \Omega\left(\mathcal{J}^{+}\right)\right)
$$

The second factor imposes the small field conditions in the difference between the small field regions of "the first and the second" kind for $\mathcal{J}$.

Lemma A.3 Let $\Omega_{1}, \Omega_{2} \subset X$ and $\delta>0$. Then

$$
\begin{aligned}
\chi_{\delta}\left(\Omega_{1} ; \alpha, \phi\right) \chi_{\delta}\left(\Omega_{2} ; \phi, \beta\right) & \sum_{\mathfrak{A} \in F_{\delta}\left(\Omega_{1} \cap \Omega_{2}\right)} \chi_{2 \delta}\left(\Lambda_{\mathfrak{A}} ; \alpha, \beta\right) \chi_{\mathrm{r}(\delta)}\left(\Lambda_{\mathfrak{A}}, \alpha-\phi\right) \chi_{\mathrm{r}(\delta)}\left(\Lambda_{\mathfrak{A}}, \phi-\beta\right) \chi_{\mathfrak{A}, \delta}\left(\Omega_{1}, \Omega_{2} ; \alpha, \phi, \beta\right)
\end{aligned}
$$


Proof: Again set $\Omega_{0}=\Omega_{1} \cap \Omega_{2}$. By definition,

$$
\begin{array}{rll}
\chi_{\delta}\left(\Omega_{1} ; \alpha, \phi\right) \chi_{\delta}\left(\Omega_{2} ; \phi, \beta\right) & \\
=\chi_{\mathrm{R}(\delta)}\left(\Omega_{1}, \alpha\right) \chi_{\mathrm{R}(\delta)}\left(\Omega_{1}, \phi\right) & \chi_{\mathrm{R}(\delta)}\left(\Omega_{2}, \phi\right) \chi_{\mathrm{R}(\delta)}\left(\Omega_{2}, \beta\right) \\
& \chi_{\mathrm{R}^{\prime}(\delta)}\left(\Omega_{1}^{*}, \nabla \alpha\right) \chi_{\mathrm{R}^{\prime}(\delta)}\left(\Omega_{1}^{*}, \nabla \phi\right) & \chi_{\mathrm{R}^{\prime}(\delta)}\left(\Omega_{2}^{*}, \nabla \phi\right) \chi_{\mathrm{R}^{\prime}(\delta)}\left(\Omega_{2}^{*}, \nabla \beta\right) \\
& \chi_{\mathrm{r}(\delta)}\left(\Omega_{1}^{\star}, \alpha-\phi\right) & \chi_{\mathrm{r}(\delta)}\left(\Omega_{2}^{\star}, \phi-\beta\right) \\
= & \chi^{(1)}\left(\Omega_{1}, \Omega_{2} ; \alpha, \phi, \beta\right) \cdot \chi_{\mathrm{R}(\delta)}\left(\Omega_{0}, \alpha\right) & \chi_{\mathrm{R}(\delta)}\left(\Omega_{0}, \beta\right) \chi_{\mathrm{R}^{\prime}(\delta)}\left(\Omega_{0}^{*}, \nabla \alpha\right) \chi_{\mathrm{R}^{\prime}(\delta)}\left(\Omega_{0}^{*}, \nabla \beta\right) \\
& \chi_{\mathrm{R}(\delta)}\left(\Omega_{0}, \phi\right) \chi_{\mathrm{R}^{\prime}(\delta)}\left(\Omega_{0}^{*}, \nabla \phi\right) & \chi_{\mathrm{r}(\delta)}\left(\Omega_{0}^{\star}, \alpha-\phi\right) \chi_{\mathrm{r}(\delta)}\left(\Omega_{0}^{\star}, \phi-\beta\right)
\end{array}
$$

The first factor, $\chi^{(1)}\left(\Omega_{1}, \Omega_{2} ; \alpha, \phi, \beta\right)$, in (A.1) was defined in Definition A.1, and involves only fields at points of $\left(\Omega_{1}^{\star} \cup \Omega_{2}^{\star}\right) \backslash \Omega_{0}$. The next four factors involve only the external fields $\alpha$ and $\beta$ at points $\mathbf{x} \in \Omega_{0}^{\star}$. To introduce the more restrictive small field conditions of scale $2 \delta$, we expand

$$
\begin{aligned}
\chi_{\mathrm{R}(\delta)}\left(\Omega_{0}, \alpha\right) & =\prod_{\mathbf{x} \in \Omega_{0}}\left[\chi_{\mathrm{R}(2 \delta)}(\alpha(\mathbf{x}))+\chi_{\mathrm{R}(2 \delta), \mathrm{R}(\delta)}(\alpha(\mathbf{x}))\right] \\
& =\sum_{P_{\alpha} \subset \Omega_{0}} \chi_{\mathrm{R}(2 \delta)}\left(\Omega_{0} \backslash P_{\alpha}, \alpha\right) \chi_{\mathrm{R}(2 \delta), \mathrm{R}(\delta)}\left(P_{\alpha}, \alpha\right) \\
\chi_{\mathrm{R}(\delta)}\left(\Omega_{0}, \beta\right) & =\sum_{P_{\beta} \subset \Omega_{0}} \chi_{\mathrm{R}(2 \delta)}\left(\Omega_{0} \backslash P_{\beta}, \beta\right) \chi_{\mathrm{R}(2 \delta), \mathrm{R}(\delta)}\left(P_{\beta}, \beta\right) \\
\chi_{\mathrm{R}^{\prime}(\delta)}\left(\Omega_{0}^{*}, \nabla \alpha\right) & =\sum_{P_{\alpha}^{\prime} \subset \Omega_{0}^{*}} \chi_{\mathrm{R}^{\prime}(2 \delta)}\left(\Omega_{0}^{*} \backslash P_{\alpha}^{\prime}, \nabla \alpha\right) \chi_{\mathrm{R}^{\prime}(2 \delta), \mathrm{R}^{\prime}(\delta)}\left(P_{\alpha}^{\prime}, \nabla \alpha\right) \\
\chi_{\mathrm{R}^{\prime}(\delta)}\left(\Omega_{0}^{*}, \nabla \beta\right) & =\sum_{P_{\beta}^{\prime} \subset \Omega_{0}^{*}} \chi_{\mathrm{R}^{\prime}(2 \delta)}\left(\Omega_{0}^{*} \backslash P_{\beta}^{\prime}, \nabla \beta\right) \chi_{\mathrm{R}^{\prime}(2 \delta), \mathrm{R}^{\prime}(\delta)}\left(P_{\beta}^{\prime}, \nabla \beta\right) \\
1 & =\sum_{Q \subset \Omega_{0}^{\star}} \chi_{\mathrm{r}(2 \delta)}\left(\Omega_{0}^{\star} \backslash Q, \alpha-\beta\right) \chi_{\mathrm{r}(2 \delta), \infty}(Q, \alpha-\beta)
\end{aligned}
$$

and get, for the product of the four factors in (A.1) that depend only on the external fields on $\Omega_{0}^{\star}$,

$$
\begin{gathered}
\chi_{\mathrm{R}(\delta)}\left(\Omega_{0}, \alpha\right) \chi_{\mathrm{R}(\delta)}\left(\Omega_{0}, \beta\right) \chi_{\mathrm{R}^{\prime}(\delta)}\left(\Omega_{0}^{*}, \nabla \alpha\right) \chi_{\mathrm{R}^{\prime}(\delta)}\left(\Omega_{0}^{*}, \nabla \beta\right) \\
\sum_{\left(\Lambda, P_{\alpha}, P_{\beta}, P_{\alpha}^{\prime}, P_{\beta}^{\prime}, Q\right) \in F_{\delta}\left(\Omega_{0}\right)} \begin{array}{ll}
\chi_{\mathrm{R}(2 \delta)}\left(\Omega_{0} \backslash P_{\alpha}, \alpha\right) & \chi_{\mathrm{R}(2 \delta), \mathrm{R}(\delta)}\left(P_{\alpha}, \alpha\right) \\
\chi_{\mathrm{R}(2 \delta)}\left(\Omega_{0} \backslash P_{\beta}, \beta\right) & \chi_{\mathrm{R}(2 \delta), \mathrm{R}(\delta)}\left(P_{\beta}, \beta\right) \\
\chi_{\mathrm{R}^{\prime}(2 \delta)}\left(\Omega_{0}^{*} \backslash P_{\alpha}^{\prime}, \nabla \alpha\right) & \chi_{\mathrm{R}^{\prime}(2 \delta), \mathrm{R}^{\prime}(\delta)}\left(P_{\alpha}^{\prime}, \nabla \alpha\right) \\
\chi_{\mathrm{R}^{\prime}(2 \delta)}\left(\Omega_{0}^{*} \backslash P_{\beta}^{\prime}, \nabla \beta\right) & \chi_{\mathrm{R}^{\prime}(2 \delta), \mathrm{R}^{\prime}(\delta)}\left(P_{\beta}^{\prime}, \nabla \beta\right) \\
\chi_{\mathrm{r}(2 \delta)}\left(\Omega_{0}^{\star} \backslash Q, \alpha-\beta\right) & \chi_{\mathrm{r}(2 \delta), \infty}(Q, \alpha-\beta)
\end{array} \\
=\sum_{\mathfrak{A} \in F_{\delta}\left(\Omega_{0}\right)} \chi_{\mathfrak{A}}^{(2)}(\alpha, \beta) \chi_{2 \delta}\left(\Lambda_{\mathfrak{A}} ; \alpha, \beta\right)
\end{gathered}
$$


For each $\mathfrak{A}=\left(\Lambda, P_{\alpha}, P_{\beta}, P_{\alpha}^{\prime}, P_{\beta}^{\prime}, Q\right) \in F_{\delta}\left(\Omega_{0}\right)$ we write the last four factors of (A.1)

$$
\begin{aligned}
\chi_{\mathrm{R}(\delta)}\left(\Omega_{0}, \phi\right) \chi_{\mathrm{R}^{\prime}(\delta)}\left(\Omega_{0}^{*}, \nabla \phi\right) \chi_{\mathrm{r}(\delta)}\left(\Omega_{0}^{\star}, \alpha-\phi\right) \chi_{\mathrm{r}(\delta)}\left(\Omega_{0}^{\star}, \phi-\beta\right) \\
\quad=\chi_{\mathfrak{A}}^{(3)}(\alpha, \phi, \beta) \cdot \chi_{\mathrm{R}(\delta)}(\Lambda, \phi) \chi_{\mathrm{R}^{\prime}(\delta)}\left(\Lambda^{*}, \nabla \phi\right) \chi_{\mathrm{r}(\delta)}\left(\Lambda^{\star}, \alpha-\phi\right) \chi_{\mathrm{r}(\delta)}\left(\Lambda^{\star}, \phi-\beta\right)
\end{aligned}
$$

Inserting (A.2) and (A.3) into (A.1) we get

$$
\begin{array}{r}
\chi_{\delta}\left(\Omega_{1} ; \alpha, \phi\right) \chi_{\delta}\left(\Omega_{2} ; \phi, \beta\right)=\sum_{\mathfrak{A} \in \mathcal{A}} \chi_{2 \delta}\left(\Lambda_{\mathfrak{A}} ; \alpha, \beta\right) \chi_{\mathrm{r}(\delta)}\left(\Lambda_{\mathfrak{A}}^{\star}, \alpha-\phi\right) \chi_{\mathrm{r}(\delta)}\left(\Lambda_{\mathfrak{A}}^{\star}, \phi-\beta\right) \\
\chi_{\mathrm{R}(\delta)}\left(\Lambda_{\mathfrak{A}}, \phi\right) \chi_{\mathrm{R}^{\prime}(\delta)}\left(\Lambda_{\mathfrak{A}}^{*}, \nabla \phi\right) \chi_{\mathfrak{A}, \delta}\left(\Omega_{1}, \Omega_{2} ; \alpha, \phi, \beta\right)
\end{array}
$$

In Lemma A.5 below we show that

$$
\begin{array}{r}
\chi_{2 \delta}(\Lambda ; \alpha, \beta) \chi_{\mathrm{R}(\delta)}(\Lambda, \phi) \chi_{\mathrm{R}^{\prime}(\delta)}\left(\Lambda^{*}, \nabla \phi\right) \chi_{\mathrm{r}(\delta)}\left(\Lambda^{\star}, \alpha-\phi\right) \chi_{\mathrm{r}(\delta)}\left(\Lambda^{\star}, \phi-\beta\right) \\
=\chi_{2 \delta}(\Lambda ; \alpha, \beta) \chi_{\mathrm{r}(\delta)}\left(\Lambda^{\star}, \alpha-\phi\right) \chi_{\mathrm{r}(\delta)}\left(\Lambda^{\star}, \phi-\beta\right)
\end{array}
$$

If we insert this into (A.4) we get

$$
\begin{aligned}
\chi_{\delta}\left(\Omega_{1} ; \alpha, \phi\right) & \chi_{\delta}\left(\Omega_{2} ; \phi, \beta\right) \\
& =\sum_{\mathfrak{A} \in \mathcal{A}} \chi_{2 \delta}\left(\Lambda_{\mathfrak{A}} ; \alpha, \beta\right) \chi_{\mathrm{r}(\delta)}\left(\Lambda_{\mathfrak{A}}^{\star}, \alpha-\phi\right) \chi_{\mathrm{r}(\delta)}\left(\Lambda_{\mathfrak{A}}^{\star}, \phi-\beta\right) \chi_{\mathfrak{A}, \delta}\left(\Omega_{1}, \Omega_{2} ; \alpha, \phi, \beta\right) \\
& =\sum_{\mathfrak{A} \in \mathcal{A}} \chi_{2 \delta}\left(\Lambda_{\mathfrak{A}} ; \alpha, \beta\right) \chi_{\mathrm{r}(\delta)}\left(\Lambda_{\mathfrak{A}}, \alpha-\phi\right) \chi_{\mathrm{r}(\delta)}\left(\Lambda_{\mathfrak{A}}, \phi-\beta\right) \chi_{\mathfrak{A}, \delta}\left(\Omega_{1}, \Omega_{2} ; \alpha, \phi, \beta\right)
\end{aligned}
$$

We were free to drop the factors $\chi_{\mathrm{r}(\delta)}\left(\Lambda_{\mathfrak{A}}^{\star} \backslash \Lambda_{\mathfrak{A}}, \alpha-\phi\right) \chi_{\mathrm{r}(\delta)}\left(\Lambda_{\mathfrak{A}}^{\star} \backslash \Lambda_{\mathfrak{A}}, \phi-\beta\right)$ from the term $\chi_{\mathrm{r}(\delta)}\left(\Lambda_{\mathfrak{A}}^{\star}, \alpha-\phi\right) \chi_{\mathrm{r}(\delta)}\left(\Lambda_{\mathfrak{A}}^{\star}, \phi-\beta\right)$ because they also appear in $\chi_{\mathfrak{A}, \delta}\left(\Omega_{1}, \Omega_{2} ; \alpha, \phi, \beta\right)$.

Lemma A.4 Let $\mathfrak{S}$ be a hierarchy for scale $2 \delta$. Set $\varepsilon=2^{-\operatorname{depth}(\mathfrak{S})}(2 \delta)$.

(a) Let $\mathcal{J}$ be a decimation interval centred on $\tau$. If $\left|\alpha_{\tau}(\mathbf{x})\right|>\mathrm{R}(\varepsilon)$, for some $\mathbf{x} \in X \backslash \Lambda(\mathcal{J})$, then

$$
\prod_{\substack{\text { decimation intervals } \\ \tilde{\mathcal{J}}=\left[\tau_{l}, \tau_{r}\right] \subset \mathcal{J}}} \chi_{\tilde{\mathcal{J}}}\left(\alpha_{\tau_{l}}, \alpha_{\tau_{c}}, \alpha_{\tau_{r}}\right)=0
$$

where $\tau_{c}=\frac{\tau_{l}+\tau_{r}}{2}$.

(b) If $\left|\alpha_{\delta}(\mathbf{x})\right|>\mathrm{R}(\varepsilon)$, for some $\mathbf{x} \in X \backslash \Lambda_{\mathfrak{S}}$, then

$$
\chi_{\mathfrak{A}, \delta}\left(\Omega([0, \delta]), \Omega([\delta, 2 \delta]) ; \alpha, \alpha_{\delta}, \beta\right) \prod_{\substack{\text { decimation intervals } \\ \mathcal{J}=\left[\tau_{l}, \tau_{r}\right] \varsubsetneqq[0,2 \delta]}} \chi_{\tilde{\mathcal{J}}}\left(\alpha_{\tau_{l}}, \alpha_{\tau_{c}}, \alpha_{\tau_{r}}\right)=0
$$

where $\mathfrak{A}=\left(\Lambda_{\mathfrak{S}}, P_{\alpha}([0,2 \delta]), \cdots, Q([0,2 \delta])\right), \tau_{c}=\frac{\tau_{l}+\tau_{r}}{2}$. 
Proof: We prove part (a). In the event that $\mathbf{x} \in\left(\Omega\left(\mathcal{J}^{-}\right) \cup \Omega\left(\mathcal{J}^{+}\right)\right) \backslash \Lambda(\mathcal{J})$, the factor $\chi_{\mathcal{J}}\left(\alpha_{\tau_{l}}, \alpha_{\tau}, \alpha_{\tau_{r}}\right)$ vanishes

o when $\mathbf{x} \in\left(\Omega\left(\mathcal{J}^{-}\right) \cup \Omega\left(\mathcal{J}^{+}\right)\right) \backslash\left(\Omega\left(\mathcal{J}^{-}\right) \cap \Omega\left(\mathcal{J}^{+}\right)\right)$because of the $\left.\chi_{\mathrm{R}(\delta)}\left(\Omega_{1} \cup \Omega_{2}\right) \backslash \Omega_{0}, \phi\right)$ in $\chi^{(1)}\left(\Omega_{1}, \Omega_{2} ; \alpha, \phi, \beta\right)$, with $\Omega_{1}=\Omega\left(\mathcal{J}^{-}\right), \Omega_{2}=\Omega\left(\mathcal{J}^{+}\right), \Omega_{0}=\Omega\left(\mathcal{J}^{-}\right) \cap \Omega\left(\mathcal{J}^{+}\right)$, $\delta=\frac{1}{2}|\mathcal{J}|, \phi=\alpha_{\tau}$

o when $\mathbf{x} \in\left(\Omega\left(\mathcal{J}^{-}\right) \cap \Omega\left(\mathcal{J}^{+}\right)\right) \backslash \Lambda(\mathcal{J})$ because of the factor $\chi_{\mathrm{R}(\delta)}\left(\Omega_{0} \backslash \Lambda, \phi\right)$ in $\chi_{\mathfrak{A}}^{(3)}(\alpha, \phi, \beta)$, with $\Omega_{0}=\Omega\left(\mathcal{J}^{-}\right) \cap \Omega\left(\mathcal{J}^{+}\right), \Lambda=\Lambda(\mathcal{J}), \delta=\frac{1}{2}|\mathcal{J}|, \phi=\alpha_{\tau}$

Observe that, in these two cases $\Lambda(\mathcal{J}) \neq X$, so that we necessarily have $\delta \geq \varepsilon$. In the event that $\mathbf{x} \in X \backslash\left(\Omega\left(\mathcal{J}^{-}\right) \cup \Omega\left(\mathcal{J}^{+}\right)\right)$, there is a unique decimation interval $\tilde{\mathcal{J}}=\left[\tau_{l}, \tau_{r}\right] \subset \mathcal{J}$ having $\tau_{r}=\tau$ with $\mathbf{x} \in \Omega\left(\tilde{\mathcal{J}}^{+}\right) \backslash \Omega(\tilde{\mathcal{J}})$. In this case, the factor $\chi_{\tilde{\mathcal{J}}}\left(\alpha_{\tau_{l}}, \alpha_{\tau_{c}}, \alpha_{\tau_{r}}\right)$ vanishes $\circ$ when $\mathbf{x} \in \Omega\left(\tilde{\mathcal{J}}^{+}\right) \backslash\left(\Omega\left(\tilde{\mathcal{J}}^{-}\right) \cap \Omega\left(\tilde{\mathcal{J}}^{+}\right)\right)$because of the factor $\chi_{\mathrm{R}(\delta)}\left(\Omega_{2} \backslash \Omega_{0}, \beta\right)$ in $\chi^{(1)}\left(\Omega_{1}, \Omega_{2} ; \alpha, \phi, \beta\right)$, with $\Omega_{2}=\Omega\left(\tilde{\mathcal{J}}^{+}\right), \Omega_{0}=\Omega\left(\tilde{\mathcal{J}}^{-}\right) \cap \Omega\left(\tilde{\mathcal{J}}^{+}\right), \delta=\frac{1}{2}|\tilde{\mathcal{J}}|, \beta=\alpha_{\tau}$

$\circ$ when $\mathbf{x} \in\left(\Omega\left(\tilde{\mathcal{J}}^{-}\right) \cap \Omega\left(\tilde{\mathcal{J}}^{+}\right)\right) \backslash \Lambda(\tilde{\mathcal{J}})$ because of the factors $\chi_{\mathrm{R}(2 \delta)}\left(\Omega_{0} \backslash\left(P_{\beta} \cup \Lambda\right), \beta\right)$ and $\chi_{\mathrm{R}(2 \delta), \mathrm{R}(\delta)}\left(P_{\beta}, \beta\right)$ in $\chi_{\mathfrak{A}}^{(2)}(\alpha, \beta)$, with $\Omega_{0}=\Omega\left(\tilde{\mathcal{J}}^{-}\right) \cap \Omega\left(\tilde{\mathcal{J}}^{+}\right), P_{\beta}=P_{\beta}(\tilde{\mathcal{J}}), \Lambda=\Lambda(\tilde{\mathcal{J}})$, $\delta=\frac{1}{2}|\tilde{\mathcal{J}}|, \beta=\alpha_{\tau}$

o when $\mathbf{x} \in \Lambda(\tilde{\mathcal{J}}) \backslash \Omega(\tilde{\mathcal{J}})$ because of the factor $\chi_{2 t}(\Lambda(\mathcal{J}) \backslash \Omega(\mathcal{J}) ; \alpha, \beta)$ in the $\chi_{\mathcal{J}}(\alpha, \phi, \beta)$ of Definition A.2, with $\mathcal{J}$ replaced by $\tilde{\mathcal{J}}, 2 t=|\tilde{\mathcal{J}}|, \beta=\alpha_{\tau}$.

Observe that, in these three cases $\Omega(\tilde{\mathcal{J}}) \neq X$, so that we necessarily have $t, \delta \geq \varepsilon$.

Lemma A.5 Let $\delta>0$ be sufficiently small and $\Lambda \subset \Omega_{0}$. Assume that

$$
\begin{array}{rlrl}
|\alpha|_{\Lambda},|\beta|_{\Lambda} \leq \mathrm{R}(2 \delta) & |\nabla \alpha|_{\Lambda^{*}},|\nabla \beta|_{\Lambda^{*}} & \leq \mathrm{R}^{\prime}(2 \delta) \\
|\alpha-\phi|_{\Lambda^{*}} & \leq \mathrm{r}(\delta) & |\phi-\beta|_{\Lambda^{\star}} & \leq \mathrm{r}(\delta)
\end{array}
$$

Then

$$
|\phi|_{\Lambda} \leq \mathrm{R}(\delta), \quad|\nabla \phi|_{\Lambda^{*}} \leq \mathrm{R}^{\prime}(\delta)
$$

Proof: It follows from our assumptions that

$$
|\nabla \phi-\nabla \alpha|_{\Lambda^{*}} \leq\|\nabla\||\phi-\alpha|_{\Lambda^{\star}} \leq\|\nabla\| \mathrm{r}(\delta)
$$

where $\|\nabla\|$ is the operator norm of the gradient. Consequently, by (F.3.d,e),

$$
|\phi|_{\Lambda} \leq \mathrm{R}(2 \delta)+\mathrm{r}(\delta) \leq \mathrm{R}(\delta)
$$

and

$$
|\nabla \phi|_{\Lambda^{*}} \leq \mathrm{R}^{\prime}(2 \delta)+\|\nabla\| \mathrm{r}(\delta) \leq \mathrm{R}^{\prime}(\delta)
$$




\section{Appendix B: Bounds on Weight Factors}

Lemma B.1 Let $\mathfrak{S}$ be a hierarchy for scale $\delta$ and $\varepsilon=2^{-n} \delta$ with $n \geq \operatorname{depth}(\mathfrak{S})$. Let $\tau \in \varepsilon \mathbb{Z} \cap[0, \delta]$ and $\tau^{\prime} \in \varepsilon \mathbb{Z} \cap(0, \delta)$.

$$
\sup _{\substack{\mathbf{x}, \mathbf{y} \in X \\ \kappa * \tau(\mathbf{y})<\infty}} e^{-\frac{\mathrm{m}}{3} d(\mathbf{x}, \mathbf{y})} \frac{\kappa_{* \tau}(\mathbf{y})}{\kappa_{* \tau}(\mathbf{x})} \leq 2, \sup _{\substack{\mathbf{x}, \mathbf{y} \in X \\ \kappa \tau(\mathbf{y})<\infty}} e^{-\frac{\mathrm{m}}{3} d(\mathbf{x}, \mathbf{y})} \frac{\kappa_{\tau}(\mathbf{y})}{\kappa_{\tau}(\mathbf{x})} \leq 2
$$

(ii) Let $0 \leq k \leq n$ and set

$$
\begin{aligned}
& \mathcal{J}_{* k}=\left\{\begin{array}{cc}
{\left[\tau^{\prime}, \tau^{\prime}+2^{-k} \delta\right]} & \text { if } k \geq \mathfrak{d}\left(\tau^{\prime}\right) \\
\text { the unique decimation interval } & \text { if } k<\mathfrak{d}\left(\tau^{\prime}\right) \\
\text { of length } 2^{-k} \delta \text { that contains } \tau^{\prime} & \text { if }
\end{array}\right. \\
& \mathcal{J}_{k}=\left\{\begin{array}{cc}
{\left[\tau^{\prime}-2^{-k} \delta, \tau^{\prime}\right]} & \text { if } k \geq \mathfrak{d}\left(\tau^{\prime}\right) \\
\text { the unique decimation interval } & \text { if } k<\mathfrak{d}\left(\tau^{\prime}\right)
\end{array}\right.
\end{aligned}
$$

Then

$$
\kappa_{* \tau^{\prime}}(\mathbf{y}) \leq 4 e^{\frac{\mathrm{m}}{3} d\left(\mathbf{y}, \Lambda\left(\mathcal{J}_{* k}\right)\right)} \mathrm{R}\left(2^{-k} \delta\right) \text { and } \kappa_{\tau^{\prime}}(\mathbf{y}) \leq 4 e^{\frac{\mathrm{m}}{3} d\left(\mathbf{y}, \Lambda\left(\mathcal{J}_{k}\right)\right)} \mathrm{R}\left(2^{-k} \delta\right) \quad \text { for all } \mathbf{y} \in \Lambda_{\tau^{\prime}}^{c}
$$

The $\kappa_{* \tau^{\prime}}(\mathbf{y})$ bound also applies when $\tau^{\prime}=0$ if we take $\mathfrak{d}(0)=0$ and $\Lambda_{0}=\emptyset$. The $\kappa_{\tau^{\prime}}(\mathbf{y})$ bound also applies when $\tau^{\prime}=\delta$ if we take $\Lambda_{\delta}=\emptyset$.

Proof: (i) We prove the first inequality. Let $\mathbf{x}, \mathbf{y} \in X$. It suffices to consider the case that $\kappa_{* \tau}(\mathbf{x})$ and $\kappa_{* \tau}(\mathbf{y})$ are both finite. Let $\mathcal{J}=\left[\tau, \tau+2^{k} \varepsilon\right]$ and $\mathcal{J}^{\prime \prime}=\left[\tau, \tau+2^{\ell} \varepsilon\right]$ be the maximal decimation intervals with $\tau$ as left endpoint such that $\mathbf{x} \in \Lambda(\mathcal{J}), \mathbf{y} \in \Lambda\left(\mathcal{J}^{\prime \prime}\right)$. Then $\kappa_{* \tau}(\mathbf{x})=\mathrm{R}\left(2^{k} \varepsilon\right)$ and $\kappa_{* \tau}(\mathbf{y})=\mathrm{R}\left(2^{\ell} \varepsilon\right)$ if $\tau \neq 0$ and $\kappa_{* \tau}(\mathbf{x})=2 \mathrm{R}\left(2^{k} \varepsilon\right)$ and $\kappa_{* \tau}(\mathbf{y})=2 \mathrm{R}\left(2^{\ell} \varepsilon\right)$ if $\tau=0$. If $k \leq \ell+1$ then $\frac{\kappa_{* \tau}(\mathbf{y})}{\kappa_{* \tau}(\mathbf{x})}=\frac{\mathrm{R}\left(2^{\ell} \varepsilon\right)}{\mathrm{R}\left(2^{k} \varepsilon\right)} \leq \frac{\mathrm{R}\left(2^{\ell} \varepsilon\right)}{\mathrm{R}\left(2^{\ell+1} \varepsilon\right)} \leq 2$ by (F.3.a). If $k>\ell+1$, then $\mathbf{x} \in \Lambda(\mathcal{J})$, while $\mathbf{y} \in \Lambda\left(\mathcal{J}^{\prime}\right)^{c}$ for $\mathcal{J}^{\prime}=\left[\tau, \tau+2^{\ell+1} \varepsilon\right] \varsubsetneqq \mathcal{J}$. Hence, in this case, we have $\frac{\kappa_{* \tau}(\mathbf{y})}{\kappa_{* \tau}(\mathbf{x})}=\frac{\mathrm{R}\left(2^{\ell} \varepsilon\right)}{\mathrm{R}\left(2^{k} \varepsilon\right)} \leq e^{\frac{\mathrm{m}}{3} d(\mathbf{x}, \mathbf{y})}$ by (F.5).

(ii) Again, we prove the first inequality. Let $\mathcal{J}^{\prime \prime}=\left[\tau^{\prime}, \tau^{\prime}+t\right]$ be the maximal decimation interval with $\tau^{\prime}$ as left hand end point such that $\mathbf{y} \in \Lambda\left(\mathcal{J}^{\prime \prime}\right)$. We automatically have $t \geq \varepsilon$, since $\Lambda\left(\left[\tau^{\prime}, \tau^{\prime}+\varepsilon\right]\right)=X$, and $t \leq \frac{\delta}{2^{\mathfrak{d}\left(\tau^{\prime}\right)}}$, since no decimation interval having $\tau^{\prime}$ as an endpoint has length longer than $\frac{\delta}{2^{\mathfrak{d}\left(\tau^{\prime}\right)}}$. By definition, $\kappa_{* \tau^{\prime}}(\mathbf{y})=\mathrm{R}(t)$ if $\tau^{\prime} \neq 0$ and $\kappa_{* \tau^{\prime}}(\mathbf{y})=2 \mathrm{R}(t)$ if $\tau^{\prime}=0$. The desired bound is trivial if $2^{-k} \delta \leq 2 t$, so assume that $2^{-k} \delta>2 t$. Set

$$
\mathcal{J}^{\prime}= \begin{cases}{\left[\tau^{\prime}, \tau^{\prime}+2 t\right]} & \text { if } t<\frac{\delta}{2^{\mathfrak{d}\left(\tau^{\prime}\right)}} \\ {\left[\tau^{\prime}-t, \tau^{\prime}+t\right]=\mathcal{J}_{\tau^{\prime}}} & \text { if } t=\frac{\delta}{2^{\mathfrak{o}\left(\tau^{\prime}\right)}}\end{cases}
$$


Then $\mathbf{y} \in \Lambda\left(\mathcal{J}^{\prime}\right)^{c}$ and $\mathcal{J}^{\prime} \varsubsetneqq \mathcal{J}_{* k}$, so that

$$
\kappa_{* \tau^{\prime}}(\mathbf{y}) \leq 2 \mathrm{R}(t) \leq 2 e^{\frac{\mathrm{m}}{3} d(\mathbf{x}, \mathbf{y})} \mathrm{R}\left(2^{-k} \delta\right)
$$

for all $\mathbf{x} \in \Lambda\left(\mathcal{J}_{* k}\right)$, by (F.5). 


\section{Appendix C: Normalization Constants}

In this appendix, we prove

Lemma II.7 There is a unique function $\delta \in(0,1) \rightarrow \mathcal{Z}_{\delta} \in(0,1)$ that obeys

$$
\mathcal{Z}_{2 \delta}=\mathcal{Z}_{\delta}^{2} \int_{|z| \leq \mathrm{r}(\delta)} \frac{d z^{*} \wedge d z}{2 \pi i} e^{-|z|^{2}} \quad \lim _{\delta \rightarrow 0+} \frac{1}{\delta} \log \mathcal{Z}_{\delta}=0
$$

Furthermore,

$$
\left|\ln \mathcal{Z}_{\delta}\right| \leq e^{-\mathrm{r}(\delta)^{2}}
$$

Proof: Define, for all $0<\delta<1$,

$$
i(\delta)=\frac{1}{2 \delta} \log \int_{|z| \leq \mathrm{r}(\delta)} \frac{d z^{*} \wedge d z}{2 \pi i} e^{-|z|^{2}}
$$

and observe that $i(\delta)<0$. The condition relating $\mathcal{Z}_{2 \delta}$ to $\mathcal{Z}_{\delta}$ is equivalent to

$$
\frac{1}{2 \delta} \log \mathcal{Z}_{2 \delta}=\frac{1}{\delta} \log \mathcal{Z}_{\delta}+i(\delta)
$$

Iterating gives

$$
\frac{1}{\delta} \log \mathcal{Z}_{\delta}=\frac{1}{2^{-n} \delta} \log \mathcal{Z}_{2^{-n} \delta}+\sum_{\ell=1}^{n} i\left(2^{-\ell} \delta\right)
$$

Existence and uniqueness will follow from convergence of the series $\sum_{\ell=1}^{\infty} i\left(2^{-\ell} \delta\right)$, which we now prove.

Since

$$
\begin{aligned}
\int_{|z| \leq \mathrm{r}(\delta)} \frac{d z^{*} \wedge d z}{2 \pi i} e^{-|z|^{2}} & =1-\int_{|(x, y)| \geq \mathrm{r}(\delta)} \frac{d x d y}{\pi} e^{-\left(x^{2}+y^{2}\right)}=1-\frac{1}{\pi} \int_{\mathrm{r}(\delta)}^{\infty} d r \int_{0}^{2 \pi} d \theta r e^{-r^{2}}=1-\int_{\mathrm{r}(\delta)^{2}}^{\infty} d s e^{-s} \\
& =1-e^{-\mathrm{r}(\delta)^{2}}
\end{aligned}
$$

and $|\ln (1-x)| \leq \frac{|x|}{1-|x|} \leq 2|x|$ for all $|x| \leq \frac{1}{2}$, we have $|i(\delta)| \leq \frac{1}{\delta} e^{-\mathrm{r}(\delta)^{2}}$. Hence the series

$$
\delta e^{\mathrm{r}(\delta)^{2}} \sum_{\ell=1}^{\infty}\left|i\left(2^{-\ell} \delta\right)\right| \leq \sum_{\ell=1}^{\infty} 2^{\ell} e^{-\left(\mathrm{r}\left(2^{-\ell} \delta\right)^{2}-\mathrm{r}(\delta)^{2}\right)}
$$


By (F.7.d),

$$
\begin{aligned}
\mathrm{r}\left(2^{-\ell} \delta\right)^{2}-\mathrm{r}(\delta)^{2} & =\sum_{p=1}^{\ell}\left(\mathrm{r}\left(2^{-p} \delta\right)^{2}-\mathrm{r}\left(2^{-p+1} \delta\right)^{2}\right) \geq \sum_{p=1}^{\ell} \mathrm{r}\left(2^{-p} \delta\right)\left(\mathrm{r}\left(2^{-p} \delta\right)-\mathrm{r}\left(2^{-p+1} \delta\right)\right) \\
& \geq \sum_{p=1}^{\ell} 3 L\left(2^{-p} \delta\right) \geq 3 \ell
\end{aligned}
$$

so the series

$$
\delta e^{\mathrm{r}(\delta)^{2}} \sum_{\ell=1}^{\infty}\left|i\left(2^{-\ell} \delta\right)\right| \leq \sum_{\ell=1}^{\infty} 2^{\ell} e^{-3 \ell}=\frac{2 / e^{3}}{1-2 / e^{3}} \leq 1
$$

does indeed converge and

$$
\log \mathcal{Z}_{\delta}=\delta \sum_{\ell=1}^{\infty} i\left(2^{-\ell} \delta\right)=\sum_{\ell=1}^{\infty} 2^{\ell-1} \log \int_{|z| \leq \mathrm{r}\left(2^{-\ell} \delta\right)} \frac{d z^{*} \wedge d z}{2 \pi i} e^{-|z|^{2}}
$$

is bounded in absolute value by $e^{-\mathrm{r}(\delta)^{2}}$. 


\section{Appendix D: Bounds on the Propagator}

Throughout this appendix we assume that $\mathfrak{h} \equiv 1$. Then Recall that $X=\mathbb{Z}^{\mathrm{D}} / L \mathbb{Z}^{\mathrm{D}}$. In (II.16), we assumed that the one-particle operator $\mathrm{h}=\nabla^{*} \mathcal{H} \nabla$ where $\mathcal{H}: L^{2}\left(X^{*}\right) \rightarrow$ $L^{2}\left(X^{*}\right)$ is a translation invariant, self-adjoint operator all of whose eigenvalues lie between $c_{\mathcal{H}}>0$ and $C_{\mathcal{H}}>0$ and for which

$$
D_{\mathcal{H}}=\sum_{\substack{\mathbf{x} \in X \\ 1 \leq i, j \leq \mathrm{D}}} e^{6 \mathrm{~m} d(\mathbf{x}, 0)}\left|\mathcal{H}\left(b_{i}(0), b_{j}(\mathbf{x})\right)\right|<\infty
$$

Here, for each $1 \leq i \leq \mathrm{D}$ and $\mathbf{x} \in X, b_{i}(\mathbf{x})=\left(\mathbf{x}, \mathbf{x}+e_{i}\right)$ denotes the bond with base point $\mathbf{x}$ and direction $e_{i}$. Under this hypothesis, the kernel of $\mathrm{h}$ is

$$
\begin{aligned}
\mathrm{h}(\mathbf{x}, \mathbf{y})=\sum_{1 \leq i, j \leq \mathrm{D}}\left[\mathcal{H}\left(b_{i}\left(\mathbf{x}-e_{i}\right), b_{j}\left(\mathbf{y}-e_{j}\right)\right)-\mathcal{H}\left(b_{i}\left(\mathbf{x}-e_{i}\right), b_{j}(\mathbf{y})\right)\right. \\
\left.-\mathcal{H}\left(b_{i}(\mathbf{x}), b_{j}\left(\mathbf{y}-e_{j}\right)\right)+\mathcal{H}\left(b_{i}(\mathbf{x}), b_{j}(\mathbf{y})\right)\right]
\end{aligned}
$$

The norm $\|\mathrm{h}\| \leq \mathcal{N}_{6 \mathrm{~m}}(\mathrm{~h} ; 1,1) \leq 4 e^{12 \mathrm{~m}} D_{\mathcal{H}}$ and the constant $K_{j}=\mathcal{N}_{6 \mathrm{~m}}(\mathrm{~h}-\mu ; 1,1)$ of Lemma III.21 obeys

$$
K_{j} \leq 4 e^{12 \mathrm{~m}} D_{\mathcal{H}}+|\mu|
$$

Furthermore, we have the following bounds.

Lemma D.1 Set $K_{j}^{\prime}=\max \left\{|\mu|+4 \mathrm{D} D_{\mathcal{H}} e^{10 \mathrm{~m}}, 1\right\}$. Let $S \subset X$ and $A: L^{2}(X) \rightarrow L^{2}(X)$. For all $\tau \geq 0$ and $\mathbf{x} \in X$,

$$
|A([1-j(\tau)] S \alpha)(\mathbf{x})| \leq \tau K_{j}^{\prime} e^{K_{j}^{\prime} \tau}\left|\|A \mid\|\left(\left[|\mu|+e^{-5 \operatorname{m} d\left(\mathbf{x}, S^{c}\right)}\right] \max _{\mathbf{y} \in S}|\alpha(\mathbf{y})|+\max _{b \in S^{*}}|\nabla \alpha(b)|\right)\right.
$$

For all $\tau \geq 0$ and $S^{\prime} \subset X$

$$
\sum_{\mathbf{x} \in S^{\prime}}|A([1-j(\tau)] S \alpha)(\mathbf{x})| \leq \tau K_{j}^{\prime} e^{K_{j}^{\prime} \tau}||\left|A \|\left(\left[|\mu|+e^{-5 \operatorname{md}\left(S^{\prime}, S^{c}\right)}\right] \max _{\mathbf{y} \in S}|\alpha(\mathbf{y})|+\max _{b \in S^{*}}|\nabla \alpha(b)|\right)\right| S \mid
$$

Proof: Write

$$
\begin{aligned}
([1-j(\tau)] S \alpha)(\mathbf{y}) & =\left(1-e^{\mu \tau}\right) S \alpha(\mathbf{y})+e^{\mu \tau}\left(\left[1-e^{-\tau \nabla^{*} \mathcal{H} \nabla}\right] S \alpha\right)(\mathbf{y}) \\
& =\left(1-e^{\mu \tau}\right) S \alpha(\mathbf{y})+e^{\mu \tau} \int_{0}^{\tau} d \tau^{\prime}\left(\left[e^{-\tau^{\prime} \nabla^{*} \mathcal{H} \nabla} \nabla^{*} \mathcal{H} \nabla\right] S \alpha\right)(\mathbf{y})
\end{aligned}
$$


As above

$$
\begin{aligned}
& \sum_{\mathbf{z} \in X} e^{5 \mathrm{~m} d(\mathbf{x}, \mathbf{z})}\left|\left(A e^{-\tau^{\prime} \nabla^{*} \mathcal{H} \nabla}\right)(\mathbf{x}, \mathbf{z})\right| \leq e^{4 \tau^{\prime} D_{\mathcal{H}} e^{10 \mathrm{~m}}}\|A\| \mid \\
& \sup _{\mathbf{z} \in X} \sum_{b \in X^{*}} e^{5 \mathrm{~m}[d(\mathbf{z}, b)+1]}\left|\left(\nabla^{*} \mathcal{H}\right)(\mathbf{z}, b)\right| \leq 2 D_{\mathcal{H}} e^{10 \mathrm{~m}}
\end{aligned}
$$

For any bond $b=\left(\mathbf{y}, \mathbf{y}^{\prime}\right)$

$$
\begin{aligned}
\nabla(S \alpha)(b) & =S\left(\mathbf{y}^{\prime}\right) \alpha\left(\mathbf{y}^{\prime}\right)-S(\mathbf{y}) \alpha(\mathbf{y}) \\
& =\left[S\left(\mathbf{y}^{\prime}\right)-S(\mathbf{y})\right] \alpha\left(\mathbf{y}^{\prime}\right)+S(\mathbf{y})\left[\alpha\left(\mathbf{y}^{\prime}\right)-\alpha(\mathbf{y})\right] \\
& =\alpha\left(\mathbf{y}^{\prime}\right)(\nabla S)(b)+S(\mathbf{y})(\nabla \alpha)(b)
\end{aligned}
$$

The second term is nonzero only for $\mathbf{y} \in S$ and hence for $b \in S^{*}$. The first term is bounded in magnitude by $\left|\alpha\left(\mathbf{y}^{\prime}\right)\right|$ and is nonzero only if $b$ connects a point of $S$ to a point of $S^{c}$. In this case, possibly replacing $b$ by $-b$, we can always arrange that $\mathbf{y}^{\prime}$ is in $S$.

The part of $(A[1-j(\tau)] S \alpha)(\mathbf{x})$ in which the last $\nabla$ of (D.2), multiplied on the left by $A$, acts on $\alpha$ is bounded by

$$
e^{\mu \tau} \tau\left\|\left|\|A\| e^{4 \tau D_{\mathcal{H}} e^{10 \mathrm{~m}}} 2 D_{\mathcal{H}} e^{10 \mathrm{~m}} \max _{b \in S^{*}}\right| \nabla \alpha(b)\left|\leq \tau K_{j}^{\prime} e^{K_{j}^{\prime} \tau}\right|\right\| A \|\left|\max _{b \in S^{*}}\right| \nabla \alpha(b) \mid
$$

The part in which the last $\nabla$ of (D.2) acts on the characteristic function is bounded by

$$
\begin{gathered}
e^{\mu \tau} \tau\|A\| \|^{4 \tau D_{\mathcal{H}} e^{10 \mathrm{~m}}} 2 D_{\mathcal{H}} e^{10 \mathrm{~m}} e^{-5 \operatorname{md}\left(\mathbf{x}, S^{c}\right)} \max _{\substack{\mathbf{y}^{\prime} \in S \\
d\left(\mathbf{y}^{\prime}, S^{c}\right) \leq 1}}\left|\alpha\left(\mathbf{y}^{\prime}\right)\right| \\
\leq \tau K_{j}^{\prime} e^{K_{j}^{\prime} \tau}\|A\||| e^{-5 \mathrm{~m} d\left(\mathbf{x}, S^{c}\right)} \max _{\mathbf{y} \in S}|\alpha(\mathbf{y})|
\end{gathered}
$$

The first bound now follows from

$$
\left|1-e^{\mu \tau}\right| \leq|\mu| \tau e^{|\mu| \tau} \leq|\mu| \tau e^{K_{j}^{\prime} \tau}
$$

The proof of the second bound is similar.

Corollary D.2 Let $\mathfrak{c}^{\prime}>0$ and recall, from (III.2), that

$$
j_{\mathfrak{c}^{\prime}}(\tau)(\mathbf{x}, \mathbf{y})= \begin{cases}j(\tau)(\mathbf{x}, \mathbf{y}) & \text { if } d(\mathbf{x}, \mathbf{y}) \leq \mathfrak{c}^{\prime} \\ 0 & \text { otherwise }\end{cases}
$$

For all $\tau \geq 0$ and $\mathbf{x} \in X$,

$$
\left|\left(\left[1-j_{\mathfrak{c}^{\prime}}(\tau)\right] \alpha\right)(\mathbf{x})\right| \leq \tau K_{j}^{\prime} e^{K_{j}^{\prime} \tau}\left(\left[|\mu|+e^{-5 m \mathfrak{c}^{\prime}}\right] \max _{\substack{\mathbf{y} \in X \\(\mathbf{x}, \mathbf{y}) \leq \mathfrak{c}^{\prime}}}|\alpha(\mathbf{y})|+\max _{\substack{b \in X^{*} \\ d(\mathbf{x}, b) \leq \mathfrak{c}^{\prime}}}|\nabla \alpha(b)|\right)
$$

Proof: Just apply the previous lemma with $A$ being the identity operator and $S$ the set of points $\mathbf{y} \in X$ that are within a distance $\mathfrak{c}^{\prime}$ of $\mathbf{x}$. 


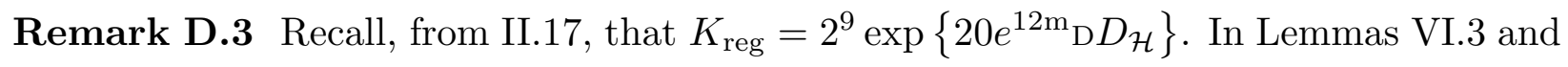
VI.7, we used

$$
4+2 e^{2 K_{j}} \leq 4+2 e^{2} \exp \left\{4 e^{12 \mathrm{~m}} D_{\mathcal{H}}\right\} \leq 2^{5} \exp \left\{4 e^{12 \mathrm{~m}} D_{\mathcal{H}}\right\} \leq K_{\text {reg }}
$$

and

$$
2 K_{j}^{\prime} e^{3 K_{j}+K_{j}^{\prime}} \leq 2 e^{3 K_{j}+2 K_{j}^{\prime}} \leq 2 e^{5} \exp \left\{20 \mathrm{D} e^{12 \mathrm{~m}} D_{\mathcal{H}}\right\} \leq K_{\text {reg }}
$$

Here, we have used that $|\mu| \leq 1$.

Lemma D.4 For all $\alpha \in L^{2}(X)$ and all $0 \leq \varepsilon \leq 1$,

$$
c_{\mathcal{H}} e^{-4 \mathrm{D} C_{\mathcal{H}}} \varepsilon\|\nabla \alpha\|^{2} \leq\left\langle\alpha^{*},\left[1-e^{-\varepsilon \nabla^{*} \mathcal{H} \nabla}\right] \alpha\right\rangle \leq C_{\mathcal{H}} \varepsilon\|\nabla \alpha\|^{2}
$$

Proof: We have

$$
\mathbb{1}-e^{-\varepsilon \nabla^{*} \mathcal{H} \nabla}=-\int_{0}^{1} d t \frac{d}{d t} e^{-\varepsilon t \nabla^{*} \mathcal{H} \nabla}=\int_{0}^{1} d t \varepsilon \nabla^{*} \mathcal{H} \nabla e^{-\varepsilon t \nabla^{*} \mathcal{H} \nabla}
$$

Since $e^{-\varepsilon t \nabla^{*} \mathcal{H} \nabla}$ commutes with $\varepsilon \nabla^{*} \mathcal{H} \nabla$ and all of the eigenvalues of $e^{-\varepsilon t \nabla^{*} \mathcal{H} \nabla}$ lie between $e^{-\varepsilon t C_{\mathcal{H}}\|\nabla\|^{2}} \geq e^{-4 \varepsilon t_{\mathrm{D}} C_{\mathcal{H}}}$ and one

$$
\begin{aligned}
\left\langle\alpha^{*},\left[11-e^{-\varepsilon \nabla^{*} \mathcal{H} \nabla}\right] \alpha\right\rangle & \geq \int_{0}^{1} d t \varepsilon e^{-4 \varepsilon t \mathrm{D} C_{\mathcal{H}}}\left\langle\alpha^{*}, \nabla^{*} \mathcal{H} \nabla \alpha\right\rangle \geq \varepsilon e^{-4 \varepsilon \mathrm{D} C_{\mathcal{H}}}\left\langle\alpha, \nabla^{*} \mathcal{H} \nabla \alpha\right\rangle \\
& \geq c_{\mathcal{H}} \varepsilon e^{-4 \varepsilon \mathrm{D} C_{\mathcal{H}}}\|\nabla \alpha\|^{2}
\end{aligned}
$$

Similarly,

$$
\left\langle\alpha^{*},\left[\mathbb{1}-e^{-\varepsilon \nabla^{*} \mathcal{H} \nabla}\right] \alpha\right\rangle \leq \int_{0}^{1} d t \varepsilon\left\langle\alpha^{*}, \nabla^{*} \mathcal{H} \nabla \alpha\right\rangle \leq C_{\mathcal{H}} \varepsilon\|\nabla \alpha\|^{2}
$$




\section{Appendix E: Bounds on the Background Field}

In this appendix we provide both pointwise and norm bounds on the background field as well as comparisons between the background field and the original field $\alpha_{\tau}$. Throughout subsections E.1-E.4 of the appendix, we fix a hierarchy $\mathfrak{S}$ for scale $\delta$ and write

$$
\gamma_{* \tau}=\left\{\begin{array}{ll}
\alpha^{*} & \text { if } \tau=0 \\
\Gamma_{* \mathfrak{S}}\left(\tau ; \alpha_{*}, \vec{\alpha}_{*}\right) & \text { if } \tau \in(0, \delta) \\
\beta^{*} & \text { if } \tau=\delta
\end{array}\right\} \quad \gamma_{\tau}=\left\{\begin{array}{ll}
\alpha & \text { if } \tau=0 \\
\Gamma_{\mathfrak{S}}(\tau ; \vec{\alpha}, \beta) & \text { if } \tau \in(0, \delta) \\
\beta & \text { if } \tau=\delta
\end{array}\right\}
$$

We also fix an integer $n \geq \operatorname{depth} S$ and set $\varepsilon=2^{-n} \delta$. Recall, from Notation II.2, that, for each decimation point $\tau \in \cap(0, \delta)$,

$\circ$ the decimation index, $\mathfrak{d}(\tau)$, of $\tau$ is determined by $\tau \in \frac{\delta}{2^{\mathfrak{d}(\tau)}} \mathbb{Z} \backslash \frac{2 \delta}{2^{\mathfrak{d}(\tau)}} \mathbb{Z}$. By convention, we also set $\mathfrak{d}(0)=\mathfrak{d}(\delta)=0$ and, if $\tau$ is not a decimation point, $\mathfrak{d}(\tau)=\infty$.

$\circ \mathcal{J}_{\tau}=\left[\tau-\frac{\delta}{2^{\mathfrak{d}(\tau)}}, \tau+\frac{\delta}{2^{\mathfrak{d}(\tau)}}\right], \mathcal{J}_{\tau}^{-}=\left[\tau-\frac{\delta}{2^{\mathfrak{\jmath}(\tau)}}, \tau\right]$ and $\mathcal{J}_{\tau}^{+}=\left[\tau, \tau+\frac{\delta}{2^{\mathfrak{d}(\tau)}}\right]$

$\circ \Lambda_{\tau}=\Lambda\left(\left[\tau-\frac{\delta}{2^{\mathfrak{d}(\tau)}}, \tau+\frac{\delta}{2^{\mathfrak{d}(\tau)}}\right]\right)$. By convention, we also set $\Lambda_{0}=\Lambda_{\delta}=\emptyset$, and, if $\tau$ is not a decimation point, $\Lambda_{\tau}^{c}=\emptyset$.

$\circ$ In this appendix, we use $\varepsilon_{\tau}=\frac{\delta}{2^{\mathfrak{d}(\tau)}}=\frac{1}{2}\left|\mathcal{J}_{\tau}\right|=\left|\mathcal{J}_{\tau}^{-}\right|=\left|\mathcal{J}_{\tau}^{+}\right|$to denote the lattice spacing of the coarsest lattice $\frac{\delta}{2^{k}} \mathbb{Z}$ that contains $\tau$. By convention, we set $\varepsilon_{0}=\varepsilon_{\delta}=\delta$.

In subsection E.5, we fix a hierarchy $\mathfrak{S}$ for scale $2 \delta$. We further assume, throughout this appendix, that the field $\alpha_{\tau}$ is compatible with $\mathfrak{S}$ in the following sense. (In the integral operator $\mathcal{I}_{\mathfrak{S}}$, the field $\alpha_{\tau}$ is compatible with $\mathfrak{S}$.)

Definition E.1 A field configuration $\alpha_{\tau}(\mathbf{x})$ is said to be compatible with the hierarchy $\mathfrak{S}$ if, for each decimation interval $\left[\tau_{-}, \tau_{+}\right]$,

$$
\begin{aligned}
\left|\alpha_{\tau_{-}}(\mathbf{x})\right|,\left|\alpha_{\tau_{+}}(\mathbf{x})\right| & \leq \mathrm{R}\left(\tau_{+}-\tau_{-}\right) & & \text {for all } \mathbf{x} \in \Lambda\left(\left[\tau_{-}, \tau_{+}\right]\right) \\
\left|\nabla \alpha_{\tau_{-}}(b)\right|,\left|\nabla \alpha_{\tau_{+}}(b)\right| & \leq \mathrm{R}^{\prime}\left(\tau_{+}-\tau_{-}\right) & & \text {for all } b \in \Lambda\left(\left[\tau_{-}, \tau_{+}\right]\right)^{*} \\
\left|\alpha_{\tau_{+}}(\mathbf{x})-\alpha_{\tau_{-}}(\mathbf{x})\right| & \leq \mathrm{r}\left(\tau_{+}-\tau_{-}\right) & & \text {for all } \mathbf{x} \in \Lambda\left(\left[\tau_{-}, \tau_{+}\right]\right)^{\star}
\end{aligned}
$$

where we recall that, for each $S \subset X, S^{*}$ is the set of bonds with at least one end in $S$ and $S^{\star}$ is the set of points in $X$ that are connected to some point of $S$ by some bond.

Remark E.2 Compatibility implies

$$
\left|\alpha_{\tau}(\mathbf{x})\right| \leq \min \left\{\kappa_{\tau}(\mathbf{x}), \kappa_{* \tau}(\mathbf{x})\right\} \quad|\alpha(\mathbf{x})| \leq \kappa_{* 0}(\mathbf{x}) \quad|\beta(\mathbf{x})| \leq \kappa_{\delta}(\mathbf{x})
$$

for all $\tau \in \varepsilon \mathbb{Z} \cap(0, \delta)$ and $\mathbf{x} \in X$. 


\section{E.1 Additional Descriptions}

We now rewrite the definition, Definition II.9, of the background field (still with $j(t)$ being interpreted as the $\mathfrak{h}$-operator of (III.1)) in a way that makes more clear which coefficients $\Gamma_{\tau}^{\tau^{\prime}}(\mathfrak{S}), \Gamma_{\tau}^{\tau^{\prime}}(\mathfrak{S})$ are nonzero. The coefficients $\Gamma_{* \tau}^{\tau^{\prime}}(\mathfrak{S})=\Gamma_{* \tau}^{\tau^{\prime}}$ and $\Gamma_{\tau}^{\tau^{\prime}}(\mathfrak{S})=\Gamma_{\tau}^{\tau^{\prime}}$ were defined as follows. For $\tau \in(0, \delta)$,

$\circ$ For $\tau=\tau^{\prime}$,

$$
\Gamma_{* \tau}^{\tau}=\Gamma_{\tau}^{\tau}=\Lambda_{\tau}^{c}
$$

$\circ$ For $\tau \neq \tau^{\prime}, \Gamma_{* \tau}^{\tau^{\prime}}=0$ unless $\tau>\tau^{\prime}$ and $\left[\tau^{\prime}, \tau\right]$ is strictly contained in a decimation interval with $\tau^{\prime}$ as its left endpoint. If $\mathcal{J}$ is the smallest such decimation interval and $\delta^{\prime}$ its length, then

$$
\Gamma_{* \tau}^{\tau^{\prime}}=j\left(\tau-\tau^{\prime}-\frac{\delta^{\prime}}{2}\right) \Lambda(\mathcal{J}) j\left(\frac{\delta^{\prime}}{2}\right) \Lambda_{\tau^{\prime}}^{c}
$$

○ Similarly for $\tau \neq \tau^{\prime}, \Gamma_{\tau}^{\tau^{\prime}}=0$ unless $\tau<\tau^{\prime}$ and $\left[\tau, \tau^{\prime}\right]$ is strictly contained in a decimation interval with $\tau^{\prime}$ as its right endpoint. If $\mathcal{J}$ is the smallest such interval and $\delta^{\prime}$ its length, then

$$
\Gamma_{\tau}^{\tau^{\prime}}=j\left(\tau^{\prime}-\tau-\frac{\delta^{\prime}}{2}\right) \Lambda(\mathcal{J}) j\left(\frac{\delta^{\prime}}{2}\right) \Lambda_{\tau^{\prime}}^{c}
$$

Observe that if $\mathcal{J}$ is a decimation interval, with $\tau^{\prime}$ as right hand endpoint and which contains $\tau$ in its interior, then $\mathfrak{d}(\tau)>\mathfrak{d}\left(\tau^{\prime}\right)$ and $\tau^{\prime}$ is the smallest element of $\varepsilon_{\tau^{\prime}} \mathbb{Z}$ that is above $\tau$. Also observe that if $\mathfrak{d}\left(\tau^{\prime}\right)>n$, then $\Lambda_{\tau^{\prime}}^{c}=\emptyset$ so that $\Gamma_{* \tau}^{\tau^{\prime}}=\Gamma_{\tau}^{\tau^{\prime}}=0$.

Definition E.3 For each $\tau \in(0, \delta)$, set

$$
\begin{aligned}
& \mathcal{T}_{r}(\tau, \delta)=\left\{\tau^{\prime} \in(\tau, \delta] \cap \varepsilon \mathbb{Z} \mid \mathfrak{d}\left(\tau^{\prime}\right)<\mathfrak{d}(\tau), \tau^{\prime}=\min \left\{\tau^{\prime \prime} \in \varepsilon_{\tau^{\prime}} \mathbb{Z} \mid \tau^{\prime \prime}>\tau\right\}\right\} \\
& \mathcal{T}_{l}(\tau, \delta)=\left\{\tau^{\prime} \in[0, \tau) \cap \varepsilon \mathbb{Z} \mid \mathfrak{d}\left(\tau^{\prime}\right)<\mathfrak{d}(\tau), \tau^{\prime}=\max \left\{\tau^{\prime \prime} \in \varepsilon_{\tau^{\prime}} \mathbb{Z} \mid \tau^{\prime \prime}<\tau\right\}\right\}
\end{aligned}
$$

The figure below provides an example. In it, $\varepsilon=1, n=5$ so that $\delta=32 \varepsilon, \tau=14 \varepsilon$ so that $\mathfrak{d}(\tau)=4, \varepsilon_{\tau}=2 \varepsilon, \mathcal{T}_{r}(\tau, \delta)=\{16 \varepsilon, 32 \varepsilon\}$ and $\mathcal{T}_{l}(\tau, \delta)=\{12 \varepsilon, 8 \varepsilon, 0\}$.

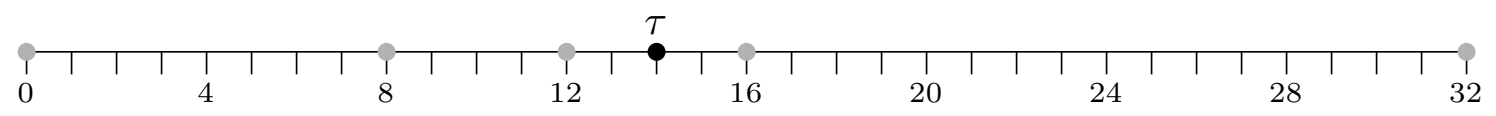

As $\delta \in \mathcal{T}_{r}(\tau, \delta)$ and $0 \in \mathcal{T}_{l}(\tau, \delta)$ both $\mathcal{T}_{r}(\tau, \delta)$ and $\mathcal{T}_{l}(\tau, \delta)$ are always nonempty. When $\tau$ and $\delta$ are clear from the context, we drop them from the notation. Also, for each $\tau^{\prime} \in \mathcal{T}_{r}$, let $\pi\left(\tau^{\prime}\right)$ denote the predecessor element of $\tau^{\prime}$ in $\mathcal{T}_{r}$, which is the largest element of $\mathcal{T}_{r}$ that is strictly smaller than $\tau^{\prime}$. When $\tau^{\prime}$ is the smallest element of $\mathcal{T}_{r}$, set $\pi\left(\tau^{\prime}\right)=\tau$. Similarly, for each $\tau^{\prime} \in \mathcal{T}_{l}$, let $\sigma\left(\tau^{\prime}\right)$ be the successor element of $\tau^{\prime}$, which is the smallest element of $\mathcal{T}_{l}$ that is strictly larger than $\tau^{\prime}$. When $\tau^{\prime}$ is the largest element of $\mathcal{T}_{l}, \sigma\left(\tau^{\prime}\right)=\tau$. 
Lemma E.4 Let $\tau \in \varepsilon \mathbb{Z} \cap(0, \delta)$.

(i)

$$
\begin{gathered}
\gamma_{\tau}=\Lambda_{\tau}^{c} \alpha_{\tau}+\sum_{\tau^{\prime} \in \mathcal{T}_{r}(\tau, \delta)} j\left(\pi\left(\tau^{\prime}\right)-\tau\right) \Lambda_{\pi\left(\tau^{\prime}\right)} j\left(\tau^{\prime}-\pi\left(\tau^{\prime}\right)\right) \Lambda_{\tau^{\prime}}^{c} \alpha_{\tau^{\prime}} \\
\gamma_{* \tau}=\Lambda_{\tau}^{c} \alpha_{\tau}^{*}+\sum_{\tau^{\prime} \in \mathcal{T}_{l}(\tau, \delta)} j\left(\tau-\sigma\left(\tau^{\prime}\right)\right) \Lambda_{\sigma\left(\tau^{\prime}\right)} j\left(\sigma\left(\tau^{\prime}\right)-\tau^{\prime}\right) \Lambda_{\tau^{\prime}}^{c} \alpha_{\tau^{\prime}}^{*}
\end{gathered}
$$

(ii)

$$
\Lambda_{\tau}=\bigcup_{\tau^{\prime} \in \mathcal{T}_{r}} \Lambda_{\pi\left(\tau^{\prime}\right)} \cap \Lambda_{\tau^{\prime}}^{c} \quad \Lambda_{\tau}=\bigcup_{\tau^{\prime} \in \mathcal{T}_{l}} \Lambda_{\sigma\left(\tau^{\prime}\right)} \cap \Lambda_{\tau^{\prime}}^{c}
$$

provides two partitions of $\Lambda_{\tau}$ into disjoint subsets. Let $\tau_{r} \in \mathcal{T}_{r}$ and $\tau_{l} \in \mathcal{T}_{l}$. Then we have $\sigma\left(\tau_{l}\right)-\varepsilon_{\sigma\left(\tau_{l}\right)}=\tau_{l}$ and $\pi\left(\tau_{r}\right)+\varepsilon_{\pi\left(\tau_{r}\right)}=\tau_{r}$. If

$$
\Lambda_{\pi\left(\tau_{r}\right)} \cap \Lambda_{\tau_{r}}^{c} \cap \Lambda_{\sigma\left(\tau_{l}\right)} \cap \Lambda_{\tau_{l}}^{c} \neq \emptyset
$$

then $\left[\tau_{l}, \tau_{r}\right]$ is a decimation interval of length $\min \left\{\varepsilon_{\tau_{l}}, \varepsilon_{\tau_{r}}\right\}$ and

$$
\Lambda_{\pi\left(\tau_{r}\right)} \Lambda_{\tau_{r}}^{c} \Lambda_{\sigma\left(\tau_{l}\right)} \Lambda_{\tau_{l}}^{c}=\Lambda\left(\left[\tau_{l}, \tau_{r}\right]\right) \Lambda_{\tau_{l}}^{c} \Lambda_{\tau_{r}}^{c}
$$

as well.

In the figures below, the partitions of $\Lambda_{\tau}$, stated in Lemma E.4.ii, are illustrated for the example inside Definition E.3.
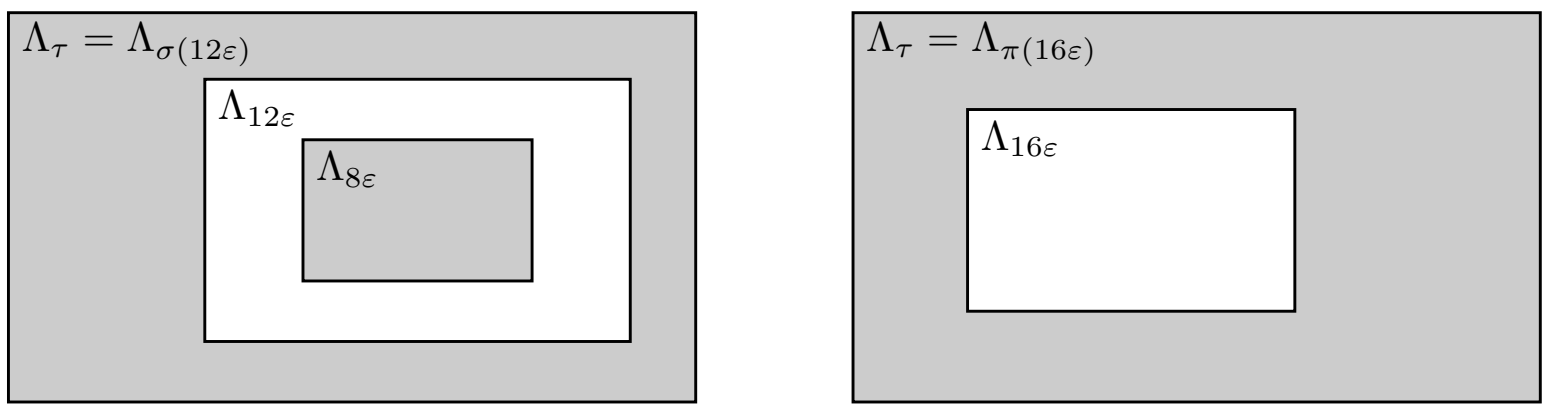

In the proof of Lemma E.4 we need

\section{Remark E.5}

(i) If $\tau^{\prime}, \tilde{\tau}^{\prime} \in \mathcal{T}_{r}(\tau, \delta)$ and $\tau^{\prime}<\tilde{\tau}^{\prime}$ then $\mathfrak{d}\left(\tau^{\prime}\right)>\mathfrak{d}\left(\tilde{\tau}^{\prime}\right)$.

If $\tau^{\prime}, \tilde{\tau}^{\prime} \in \mathcal{T}_{l}(\tau, \delta)$ and $\tau^{\prime}<\tilde{\tau}^{\prime}$ then $\mathfrak{d}\left(\tau^{\prime}\right)<\mathfrak{d}\left(\tilde{\tau}^{\prime}\right)$.

For each $0<\mathfrak{d}<\mathfrak{d}(\tau)$ there is exactly one $\tau^{\prime} \in \mathcal{T}_{l}(\tau, \delta) \cup \mathcal{T}_{r}(\tau, \delta)$ with $\mathfrak{d}\left(\tau^{\prime}\right)=\mathfrak{d}$.

(ii) If $\tau^{\prime}, \tilde{\tau}^{\prime} \in \mathcal{T}_{l}(\tau, \delta) \cup \mathcal{T}_{r}(\tau, \delta)$ and $\mathfrak{d}\left(\tau^{\prime}\right)>\mathfrak{d}\left(\tilde{\tau}^{\prime}\right)$, then

$$
\Lambda_{\tilde{\tau}^{\prime}} \subset \Lambda_{\tau^{\prime}} \subset \Lambda_{\tau}
$$


Proof: (i) Let $\tau^{\prime}<\tilde{\tau}^{\prime}$ both be in $\mathcal{T}_{r}(\tau, \delta)$ and suppose that $\mathfrak{d}\left(\tau^{\prime}\right) \leq \mathfrak{d}\left(\tilde{\tau}^{\prime}\right)$. Then both $\tau^{\prime}$ and $\tilde{\tau}^{\prime}$ are in $\varepsilon_{\tilde{\tau}^{\prime}} \mathbb{Z}$ and $\tilde{\tau}^{\prime}$ cannot be the element of $\varepsilon_{\tilde{\tau}^{\prime}} \mathbb{Z}$ closest to $\tau$, which is a contradiction.

Let $\tau^{\prime} \in \mathcal{T}_{l}(\tau, \delta) \backslash\{0\}$ and $\tilde{\tau}^{\prime} \in \mathcal{T}_{r}(\tau, \delta) \backslash\{\delta\}$ and suppose that $\mathfrak{d}\left(\tau^{\prime}\right)=\mathfrak{d}\left(\tilde{\tau}^{\prime}\right)$. Then $\left[\tau^{\prime}-\varepsilon_{\tau^{\prime}}, \tau^{\prime}+\varepsilon_{\tau^{\prime}}\right]$ and $\left[\tilde{\tau}^{\prime}-\varepsilon_{\tilde{\tau}^{\prime}}, \tilde{\tau}^{\prime}+\varepsilon_{\tilde{\tau}^{\prime}}\right]$ are both decimation intervals of length $2 \varepsilon_{\tau^{\prime}}$ and both contain $\tau$ in their interiors. So they must be identical. That's impossible since $\tau^{\prime}<\tau<\tilde{\tau}^{\prime}$ forces $\tau^{\prime} \neq \tilde{\tau}^{\prime}$.

Let $0 \leq \mathfrak{d}<\mathfrak{d}(\tau)$. There is exactly one decimation interval of length $\frac{\delta}{2^{\mathfrak{d}}}$ that contains $\tau$. Call it $\left[\tau^{\prime}, \tilde{\tau}^{\prime}\right]$. If $\mathfrak{d}>0$, then exactly one of $\tau^{\prime}, \tilde{\tau}^{\prime}$ has decimation index $\mathfrak{d}$. If $\mathfrak{d}=0$, then both of $\tau^{\prime}, \tilde{\tau}^{\prime}$ have decimation index $\mathfrak{d}$. If $\mathfrak{d}\left(\tau^{\prime}\right)=\mathfrak{d}$, then $\tau^{\prime} \in \mathcal{T}_{l}$. If $\mathfrak{d}\left(\tilde{\tau}^{\prime}\right)=\mathfrak{d}$, then $\tilde{\tau}^{\prime} \in \mathcal{T}_{r}$.

(ii) All of $\left[\tau-\varepsilon_{\tau}, \tau+\varepsilon_{\tau}\right]$, $\left[\tau^{\prime}-\varepsilon_{\tau^{\prime}}, \tau^{\prime}+\varepsilon_{\tau^{\prime}}\right]$ and $\left[\tilde{\tau}^{\prime}-\varepsilon_{\tilde{\tau}^{\prime}}, \tilde{\tau}^{\prime}+\varepsilon_{\tilde{\tau}^{\prime}}\right]$ are decimation intervals that contain $\tau$ in their interiors. Since $\mathfrak{d}(\tau)>\mathfrak{d}\left(\tau^{\prime}\right)>\mathfrak{d}\left(\tilde{\tau}^{\prime}\right)$, the first is contained in the second, which, in turn is contained in the third. Consequently,

$$
\Lambda\left(\left[\tilde{\tau}^{\prime}-\varepsilon_{\tilde{\tau}^{\prime}}, \tilde{\tau}^{\prime}+\varepsilon_{\tilde{\tau}^{\prime}}\right]\right) \subset \Lambda\left(\left[\tau^{\prime}-\varepsilon_{\tau^{\prime}}, \tau^{\prime}+\varepsilon_{\tau^{\prime}}\right]\right) \subset \Lambda\left(\left[\tilde{\tau}^{\prime}-\varepsilon_{\tilde{\tau}^{\prime}}, \tilde{\tau}^{\prime}+\varepsilon_{\tilde{\tau}^{\prime}}\right]\right)
$$

(If $\mathfrak{d}\left(\tilde{\tau}^{\prime}\right)=0$, drop consideration of $\left[\tilde{\tau}^{\prime}-\varepsilon_{\tilde{\tau}^{\prime}}, \tilde{\tau}^{\prime}+\varepsilon_{\tilde{\tau}^{\prime}}\right]$ and use that $\Lambda_{\tilde{\tau}^{\prime}}=\emptyset$.)

Proof of Lemma E.4: (i) We give the proof for $\gamma_{\tau}$. We have already observed that $\Gamma_{\tau}^{\tau^{\prime}}$ may be nonzero only for $\tau^{\prime}=\tau$ or $\tau^{\prime} \in \mathcal{T}_{r}$.

Now fix any $\tau^{\prime} \in \mathcal{T}_{r}$. Then $\left[\tau, \tau^{\prime}\right]$ is strictly contained in a decimation interval with $\tau^{\prime}$ as its right endpoint, namely $\left[\tau^{\prime}-\varepsilon_{\tau^{\prime}}, \tau^{\prime}\right]$. Denote by $\mathcal{J}$ the smallest such interval and by $\delta^{\prime}$ its length, so that

$$
\Gamma_{\tau}^{\tau^{\prime}}=j\left(\tau^{\prime}-\tau-\frac{\delta^{\prime}}{2}\right) \Lambda\left(\left[\tau^{\prime}-\delta^{\prime}, \tau^{\prime}\right]\right) j\left(\frac{\delta^{\prime}}{2}\right) \Lambda_{\tau^{\prime}}^{c}
$$

If $\tau^{\prime}-\frac{\delta^{\prime}}{2}=\tau$, then all elements of $\left(\tau, \tau^{\prime}\right)$ have decimation index strictly larger than $\mathfrak{d}(\tau)$ and $\tau^{\prime}$ is the smallest element of $\mathcal{T}_{r}$.

If $\tau^{\prime}-\frac{\delta^{\prime}}{2}>\tau$, then $\tau$ is contained in the interior of the decimation interval $\left[\tau^{\prime}-\delta^{\prime}, \tau^{\prime}-\frac{\delta^{\prime}}{2}\right]$ so that $\tau^{\prime}-\frac{\delta^{\prime}}{2}$ is the smallest element of $\frac{\delta^{\prime}}{2} \mathbb{Z}$ above $\tau$. As $\tau^{\prime}-\frac{\delta^{\prime}}{2}$ has the same decimation index as $\frac{\delta^{\prime}}{2}$, we have $\tau^{\prime}-\frac{\delta^{\prime}}{2} \in \mathcal{T}_{r}$. All elements of $\left(\tau^{\prime}-\frac{\delta^{\prime}}{2}, \tau^{\prime}\right)$ have decimation index strictly larger than that of $\frac{\delta^{\prime}}{2}$, and so cannot be in $\mathcal{T}_{r}$ (because $\tau^{\prime}-\frac{\delta^{\prime}}{2}$ has smaller decimation index and is closer to $\tau)$, we have $\pi\left(\tau^{\prime}\right)=\tau^{\prime}-\frac{\delta^{\prime}}{2}$. In both cases, $\Lambda\left(\left[\tau^{\prime}-\delta^{\prime}, \tau^{\prime}\right]\right)=\Lambda_{\pi\left(\tau^{\prime}\right)}$ so that

$$
\Gamma_{\tau}^{\tau^{\prime}}=j\left(\pi\left(\tau^{\prime}\right)-\tau\right) \Lambda_{\pi\left(\tau^{\prime}\right)} j\left(\tau^{\prime}-\pi\left(\tau^{\prime}\right)\right) \Lambda_{\tau^{\prime}}^{c}
$$

as desired. 
If $\tau^{\prime}-\frac{\delta^{\prime}}{2}<\tau$, then $\left[\tau^{\prime}-\frac{\delta^{\prime}}{2}, \tau^{\prime}\right]$ is a decimation interval that contains $\tau$ in its interior, contradicting the assumption that $\left[\tau^{\prime}-\delta^{\prime}, \tau^{\prime}\right]$ is the shortest such decimation interval.

The proof for $\gamma_{* \tau}$ is similar.

(ii) Observe that $\Lambda_{\tau^{\prime}} \subset \Lambda_{\pi\left(\tau^{\prime}\right)}$ for all $\tau^{\prime} \in \mathcal{T}_{r}$ and $\Lambda_{\tau^{\prime}} \subset \Lambda_{\sigma\left(\tau^{\prime}\right)}$ for all $\tau^{\prime} \in \mathcal{T}_{l}$ and that $\tau^{\prime}=\delta \in \mathcal{T}_{r}$ has $\Lambda_{\delta}^{c}=X$ and $\tau^{\prime}=0 \in \mathcal{T}_{l}$ has $\Lambda_{0}^{c}=X$. Consequently

$$
\Lambda_{\tau}=\bigcup_{\tau^{\prime} \in \mathcal{T}_{r}} \Lambda_{\pi\left(\tau^{\prime}\right)} \cap \Lambda_{\tau^{\prime}}^{c} \quad \Lambda_{\tau}=\bigcup_{\tau^{\prime} \in \mathcal{T}_{l}} \Lambda_{\sigma\left(\tau^{\prime}\right)} \cap \Lambda_{\tau^{\prime}}^{c}
$$

provides two partitions of $\Lambda_{\tau}$ into disjoint subsets.

We have already shown, in part $(\mathrm{i})$, that $\pi\left(\tau_{r}\right)+\frac{\delta}{2^{\mathrm{d}\left(\pi\left(\tau_{r}\right)\right)}}=\tau_{r}$ for all $\tau_{r} \in \mathcal{T}_{r}$.

Now fix any $\tau_{r} \in \mathcal{T}_{r}$ and $\tau_{l} \in \mathcal{T}_{l}$ and assume that

$$
\mathbf{x} \in \Lambda_{\pi\left(\tau_{r}\right)} \cap \Lambda_{\tau_{r}}^{c} \cap \Lambda_{\sigma\left(\tau_{l}\right)} \cap \Lambda_{\tau_{l}}^{c} \neq \emptyset
$$

Write, as in part (i),

$$
\pi\left(\tau_{r}\right)=\tau_{r}-\frac{1}{2} \delta_{r}^{\prime} \quad \sigma\left(\tau_{l}\right)=\tau_{l}+\frac{1}{2} \delta_{l}^{\prime}
$$

Both

$$
\begin{aligned}
{\left[\pi\left(\tau_{r}\right)-\frac{1}{2} \delta_{r}^{\prime}, \pi\left(\tau_{r}\right)+\frac{1}{2} \delta_{r}^{\prime}\right] } & =\left[\tau_{r}-\delta_{r}^{\prime}, \tau_{r}\right] \\
{\left[\sigma\left(\tau_{l}\right)-\frac{1}{2} \delta_{l}^{\prime}, \sigma\left(\tau_{l}\right)+\frac{1}{2} \delta_{l}^{\prime}\right] } & =\left[\tau_{l}, \tau_{l}+\delta_{l}^{\prime}\right]
\end{aligned}
$$

are decimation intervals that contain $\tau$ in their interiors. Hence one must be contained in the other. Say that $\delta_{r}^{\prime} \leq \delta_{l}^{\prime}$ so that the first is contained in the second and $\Lambda_{\sigma\left(\tau_{l}\right)} \subset \Lambda_{\pi\left(\tau_{r}\right)}$. To prove the claim, it suffices to show that $\tau_{l}+\delta_{l}^{\prime}=\tau_{r}$ - i.e. that the right hand ends of the two intervals coincide - since then $\left[\tau_{l}, \tau_{r}\right]$ is a decimation interval and $\Lambda\left(\left[\tau_{l}, \tau_{r}\right]\right)=$ $\Lambda_{\sigma\left(\tau_{l}\right)} \subset \Lambda_{\pi\left(\tau_{r}\right)}$.

Suppose that they do not coincide. That is,

$$
\sigma\left(\tau_{l}\right)-\frac{1}{2} \delta_{l}^{\prime} \leq \pi\left(\tau_{r}\right)-\frac{1}{2} \delta_{r}^{\prime}<\pi\left(\tau_{r}\right)+\frac{1}{2} \delta_{r}^{\prime}<\sigma\left(\tau_{l}\right)+\frac{1}{2} \delta_{l}^{\prime}
$$

or equivalently,

$$
\tau_{l} \leq \tau_{r}-\delta_{r}^{\prime}<\tau<\tau_{r}<\tau_{l}+\delta_{l}^{\prime}
$$

Since $\tau_{r} \in\left(\tau_{l}, \tau_{l}+\delta_{l}^{\prime}\right)$, we have $\mathcal{J}_{\tau_{r}} \subset\left[\tau_{l}, \tau_{l}+\delta_{l}^{\prime}\right]$ leading to the contradiction

$$
\Lambda_{\tau_{r}}^{c} \cap \Lambda_{\sigma\left(\tau_{l}\right)}=\Lambda\left(\mathcal{J}_{\tau_{r}}\right)^{c} \cap \Lambda\left(\left[\tau_{l}, \tau_{l}+\delta_{l}^{\prime}\right]\right)=\emptyset
$$

The argument that the left hand ends of the intervals coincide when $\delta_{r}^{\prime} \geq \delta_{l}^{\prime}$ is similar. 


\section{E.2 Comparison to the case " $j(\tau)=\mathfrak{h} "$}

Recall, from (II.16), that $j(\tau)=e^{-\tau(\mathrm{h}-\mu)}$ with $\mathrm{h}=\nabla^{*} \mathcal{H} \nabla$ where $\mathcal{H}: L^{2}\left(X^{*}\right) \rightarrow$ $L^{2}\left(X^{*}\right)$ is a translation invariant, self-adjoint operator all of whose eigenvalues lie between $c_{\mathcal{H}}>0$ and $C_{\mathcal{H}}>0$ and for which

$$
D_{\mathcal{H}}=\sum_{\substack{\mathbf{x} \in X \\ 1 \leq i, j \leq d}} e^{6 \mathrm{~m} d(\mathbf{x}, 0)}\left|\mathcal{H}\left(b_{i}(0), b_{j}(\mathbf{x})\right)\right|<\infty
$$

Here, for each $1 \leq i \leq d$ and $\mathbf{x} \in X, b_{i}(\mathbf{x})=\left(\mathbf{x}, \mathbf{x}+e_{i}\right)$ denotes the bond with base point $\mathrm{x}$ and direction $e_{i}$.

If $j(\tau)$ were the $j(0)=\mathfrak{h}$, the background field would reduce to

Definition E.6 Define, for each $0<\tau<\delta$,

$$
\begin{aligned}
\hat{\gamma}_{* \tau} & =\Lambda_{\tau}^{c} \alpha_{\tau}^{*}+\sum_{\tau^{\prime} \in \mathcal{T}_{l}(\tau, \delta)} \Lambda_{\sigma\left(\tau^{\prime}\right)} \Lambda_{\tau^{\prime}}^{c} \mathfrak{h} \alpha_{\tau^{\prime}}^{*} \\
\hat{\gamma}_{\tau} & =\Lambda_{\tau}^{c} \alpha_{\tau}+\sum_{\tau^{\prime} \in \mathcal{T}_{r}(\tau, \delta)} \Lambda_{\pi\left(\tau^{\prime}\right)} \Lambda_{\tau^{\prime}}^{c} \mathfrak{h} \alpha_{\tau^{\prime}}
\end{aligned}
$$

We now prove some bounds on the difference between $\gamma_{(*) \tau}$ and $\hat{\gamma}_{(*) \tau}$ and derive some consequences of the bounds.

Proposition E.7 Assume that $\mathfrak{h} \equiv 1$. Let $0<\tau<\delta$. If $\mathbf{x} \in \Lambda_{\tau}^{c}$, then

$$
\left|\gamma_{* \tau}(\mathbf{x})-\hat{\gamma}_{* \tau}(\mathbf{x})\right|,\left|\gamma_{\tau}(\mathbf{x})-\hat{\gamma}_{\tau}(\mathbf{x})\right| \leq \frac{1}{2} e^{-\mathrm{mc}\left(\varepsilon_{\tau}\right)}
$$

If $\mathcal{J}$ is a decimation interval that contains $\tau$ and $\mathbf{x} \in \Lambda(\mathcal{J})$, then

$$
\left|\gamma_{* \tau}(\mathbf{x})-\hat{\gamma}_{* \tau}(\mathbf{x})\right|,\left|\gamma_{\tau}(\mathbf{x})-\hat{\gamma}_{\tau}(\mathbf{x})\right| \leq\left(2 e^{K_{j}}+1\right) \mathrm{r}(|\mathcal{J}|)
$$

The proof of this proposition uses ${ }^{(1)}$

Lemma E.8 Let $0<\tau<\delta$ and $\mathcal{T}_{r}=\left\{\tau_{1}<\tau_{2}<\cdots<\tau_{p}=\delta\right\}$.

(a) If $\mathbf{x} \in \Lambda_{\tau}^{c}$, then

$$
\gamma_{\tau}(\mathbf{x})=\alpha_{\tau}(\mathbf{x})+E_{a}(\tau, \mathbf{x}) \text { with }\left|E_{a}(\tau, \mathbf{x})\right| \leq \frac{1}{2} e^{-\mathrm{mc}\left(\varepsilon_{\tau}\right)}
$$

(1) In Lemma E.8, we do not assume that $\mathfrak{h}$ is identically one. 
If $\mathbf{x} \in \Lambda_{\tau_{\ell-1}} \cap \Lambda_{\tau_{\ell}}^{c}$, for some $1 \leq \ell \leq p$ (with the convention that $\tau_{0}=\tau$ ), then

(b) when $d\left(\mathbf{x}, \Lambda_{\tau_{\ell}}\right) \geq \frac{1}{2} \mathfrak{c}\left(\varepsilon_{\tau_{\ell}}\right)$ (automatic for $\ell=p$ ) and $d\left(\mathbf{x}, \Lambda_{\tau_{\ell-1}}^{c}\right) \geq \frac{1}{2} \mathfrak{c}\left(2 \varepsilon_{\tau_{\ell-1}}\right)$,

$$
\gamma_{\tau}(\mathbf{x})=\mathfrak{h} \alpha_{\tau_{\ell}}(\mathbf{x})+\left(\left[j\left(\tau_{\ell}-\tau\right)-\mathfrak{h}\right] \Lambda_{\tau_{\ell-1}} \Lambda_{\tau_{\ell}}^{c} \alpha_{\tau_{\ell}}\right)(\mathbf{x})+E_{b}(\tau, \mathbf{x}) \text { with }\left|E_{b}(\tau, \mathbf{x})\right| \leq 2 e^{-m \mathfrak{c}\left(\varepsilon_{\tau_{\ell}}\right)}
$$

(c) when $d\left(\mathbf{x}, \Lambda_{\tau_{\ell-1}}^{c}\right) \leq \frac{1}{2} \mathfrak{c}\left(2 \varepsilon_{\tau_{\ell-1}}\right)$

$$
\begin{aligned}
\gamma_{\tau}(\mathbf{x})= & \mathfrak{h} \alpha_{\tau_{\ell}}(\mathbf{x})-\left(j\left(\tau_{\ell-1}-\tau\right) \Lambda_{\tau_{\ell-1}}^{c} \Lambda\left(\left[\tau_{\ell-1}, \tau_{\ell}\right]\right)\left[\alpha_{\tau_{\ell}}-\alpha_{\tau_{\ell-1}}\right]\right)(\mathbf{x}) \\
& +\left(\left\{\left[j\left(\tau_{\ell}-\tau\right)-\mathfrak{h}\right]-j\left(\tau_{\ell-1}-\tau\right) \Lambda_{\tau_{\ell-1}}^{c}\left[j\left(\tau_{\ell}-\tau_{\ell-1}\right)-\mathfrak{h}\right]\right\} \Lambda\left(\left[\tau_{\ell-1}, \tau_{\ell}\right]\right) \Lambda_{\tau_{\ell}}^{c} \alpha_{\tau_{\ell}}\right)(\mathbf{x}) \\
& +E_{c}(\tau, \mathbf{x}) \text { with }\left|E_{c}(\tau, \mathbf{x})\right| \leq 2 e^{-\operatorname{mc}\left(\varepsilon_{\tau_{\ell}}\right)}
\end{aligned}
$$

(d) when $\ell<p$ and $d\left(\mathbf{x}, \Lambda_{\tau_{\ell}}\right) \leq \frac{1}{2} \mathfrak{c}\left(\varepsilon_{\tau_{\ell}}\right)$

$$
\begin{aligned}
\gamma_{\tau}(\mathbf{x}) & =\mathfrak{h} \alpha_{\tau_{\ell}}(\mathbf{x})+\left(\left[j\left(\tau_{\ell}-\tau\right)-\mathfrak{h}\right] \Lambda\left(\left[\tau_{\ell}, \tau_{\ell+1}\right]\right) \Lambda_{\tau_{\ell}}^{c}\left[\alpha_{\tau_{\ell}}-\alpha_{\tau_{\ell+1}}\right]\right)(\mathbf{x}) \\
& +\left(\left\{\left[j\left(\tau_{\ell}-\tau\right)-\mathfrak{h}\right]+j\left(\tau_{\ell}-\tau\right) \Lambda_{\tau_{\ell}}\left[j\left(\tau_{\ell+1}-\tau_{\ell}\right)-\mathfrak{h}\right]\right\} \Lambda\left(\left[\tau_{\ell}, \tau_{\ell+1}\right]\right) \Lambda_{\tau_{\ell+1}}^{c} \alpha_{\tau_{\ell+1}}\right)(\mathbf{x}) \\
& +E_{d}(\tau, \mathbf{x}) \text { with }\left|E_{d}(\tau, \mathbf{x})\right| \leq 2 e^{-\operatorname{mc}\left(\varepsilon_{\tau_{\ell}}\right)}
\end{aligned}
$$

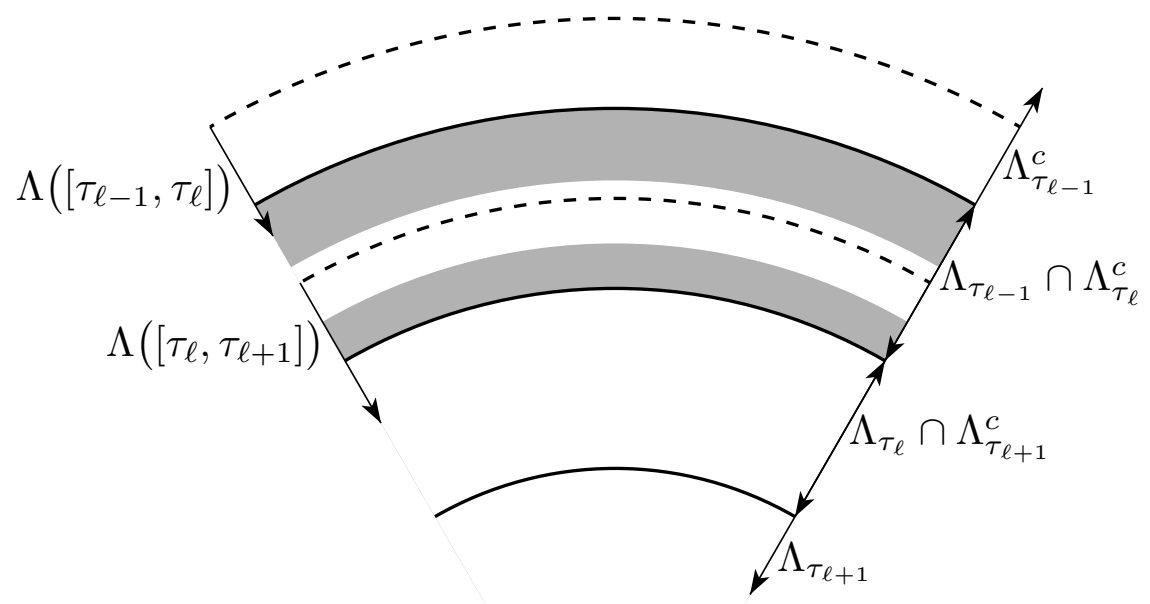

For $\mathbf{x}$ running over $\Lambda=\Lambda([0, \delta])$, we also have that

(e) if $0<\tau<\frac{\delta}{2}$, then

$$
\begin{aligned}
& \gamma_{* \tau}(\mathbf{x})=\left(j(\tau) \alpha^{*}\right)(\mathbf{x})+E_{* e}(\mathbf{x}) \\
& \begin{aligned}
& \gamma_{\tau}(\mathbf{x})=\left(j(\delta-\tau) \Lambda\left(\left[\frac{\delta}{2}, \delta\right]\right) \beta-j\left(\frac{\delta}{2}-\tau\right) \Lambda^{c}\left[j\left(\frac{\delta}{2}\right)-\mathfrak{h}\right] \Lambda\left(\left[\frac{\delta}{2}, \delta\right]\right) \beta\right)(\mathbf{x}) \\
&+\left(j\left(\frac{\delta}{2}-\tau\right) \Lambda^{c} \Lambda\left(\left[\frac{\delta}{2}, \delta\right]\right)\left[\alpha_{\frac{\delta}{2}}-\beta\right]\right)(\mathbf{x})+E_{e}(\mathbf{x}) \\
& \text { with } \sum_{\mathbf{x} \in \Lambda}\left|E_{* e}(\mathbf{x})\right| \leq e^{-\operatorname{mc}(\delta)}\left|\Lambda^{c}\right| \quad \text { and } \quad \sum_{\mathbf{x} \in \Lambda}\left|E_{e}(\mathbf{x})\right| \leq e^{-\operatorname{mc}(\delta)}\left|\Lambda^{c}\right|
\end{aligned}
\end{aligned}
$$


(f) if $\tau=\frac{\delta}{2}$, then

$$
\begin{aligned}
\gamma_{* \tau}(\mathbf{x}) & =\left(j(\tau) \alpha^{*}\right)(\mathbf{x})+E_{* f}(\mathbf{x}) \\
\gamma_{\tau}(\mathbf{x}) & =(j(\delta-\tau) \beta)(\mathbf{x})+E_{f}(\mathbf{x}) \\
\text { with } & \sum_{\mathbf{x} \in \Lambda}\left|E_{* f}(\mathbf{x})\right| \leq e^{-\mathrm{mc}(\delta)}\left|\Lambda^{c}\right| \quad \text { and } \quad \sum_{\mathbf{x} \in \Lambda}\left|E_{f}(\mathbf{x})\right| \leq e^{-\mathrm{mc}(\delta)}\left|\Lambda^{c}\right|
\end{aligned}
$$

(g) if $\frac{\delta}{2}<\tau<\delta$, then

$$
\begin{gathered}
\gamma_{* \tau}(\mathbf{x})=\left(j(\tau) \Lambda\left(\left[0, \frac{\delta}{2}\right]\right) \alpha^{*}-j\left(\tau-\frac{\delta}{2}\right) \Lambda^{c}\left[j\left(\frac{\delta}{2}\right)-\mathfrak{h}\right] \Lambda\left(\left[0, \frac{\delta}{2}\right]\right) \alpha^{*}\right)(\mathbf{x}) \\
+\left(j\left(\tau-\frac{\delta}{2}\right) \Lambda^{c} \Lambda\left(\left[0, \frac{\delta}{2}\right]\right)\left[\alpha_{\frac{\delta}{2}}^{*}-\alpha^{*}\right]\right)(\mathbf{x})+E_{* g}(\mathbf{x}) \\
\gamma_{\tau}(\mathbf{x})=(j(\delta-\tau) \beta)(\mathbf{x})+E_{g}(\mathbf{x}) \\
\text { with } \sum_{\mathbf{x} \in \Lambda}\left|E_{* g}(\mathbf{x})\right| \leq e^{-\mathrm{mc}(\delta)}\left|\Lambda^{c}\right| \quad \text { and } \quad \sum_{\mathbf{x} \in \Lambda}\left|E_{g}(\mathbf{x})\right| \leq e^{-\mathrm{mc}(\delta)}\left|\Lambda^{c}\right|
\end{gathered}
$$

Proof: Recall from Lemma E.4 that

$$
\gamma_{\tau}=\Lambda_{\tau}^{c} \alpha_{\tau}+\sum_{k=1}^{p} j\left(\tau_{k-1}-\tau\right) \Lambda_{\tau_{k-1}} j\left(\tau_{k}-\tau_{k-1}\right) \Lambda_{\tau_{k}}^{c} \alpha_{\tau_{k}}
$$

For each $1 \leq k \leq p$,

$$
\begin{aligned}
& \left|\left(j\left(\tau_{k-1}-\tau\right) \Lambda_{\tau_{k-1}} j\left(\tau_{k}-\tau_{k-1}\right) \Lambda_{\tau_{k}}^{c} \alpha_{\tau_{k}}\right)(\mathbf{x})\right| \\
& \leq N_{0}\left(\chi_{\{\mathbf{x}\}} j\left(\tau_{k-1}-\tau\right) \Lambda_{\tau_{k-1}} j\left(\tau_{k}-\tau_{k-1}\right) \Lambda_{\tau_{k}}^{c} ; 1, \kappa_{\tau_{k}}\right) \\
& \leq 4 \mathrm{R}\left(2 \varepsilon_{\tau_{k^{\prime}}}\right) e^{-\frac{9}{2} \mathrm{~m} \max \left\{d\left(\mathbf{x}, \Lambda_{\tau_{k-1}}\right), d\left(\mathbf{x}, \Lambda_{\tau_{k}}^{c}\right)\right\}}\left\|j\left(\tau_{k-1}-\tau\right)\right\|\|\| j\left(\tau_{k}-\tau_{k-1}\right) \| \\
& \leq 4 e^{K_{j}} \mathrm{R}\left(\varepsilon_{\tau_{k^{\prime}}}\right) e^{-\frac{9}{2} \mathrm{~m} \max \left\{d\left(\mathbf{x}, \Lambda_{\tau_{k-1}}\right), d\left(\mathbf{x}, \Lambda_{\tau_{k}}^{c}\right)\right\}}
\end{aligned}
$$

where $\chi_{\{\mathbf{x}\}}$ is the characteristic function of the set $\{\mathbf{x}\}$ and $k^{\prime}$ is the maximum of $k-1$ and the largest $\ell^{\prime}$ with $\mathrm{x} \in \Lambda_{\tau_{\ell^{\prime}}}$. If $\mathrm{x} \in \Lambda_{\tau}^{c}$, then $k^{\prime}=k-1$. For the second inequality we used Lemma G.5.ii with

○ $L_{1}$ being the single point set $\{\mathbf{x}\}, L_{2}=\Lambda_{\tau_{k-1}}=\Lambda\left(\left[\tau_{k}-2 \varepsilon_{\tau_{k-1}}, \tau_{k}\right]\right), L_{3}=\Lambda_{\tau_{k}}^{c}$,

○ with $d$ replaced by $0, \delta_{1}=0, \delta_{2}=\frac{\mathrm{m}}{3} d, \delta=\frac{9}{2} \mathrm{~m} d, \tilde{d}=5 \mathrm{~m} d$,

$\circ O_{2}=\Lambda_{\tau_{k^{\prime}}}, \kappa=\kappa_{\tau_{k}}$ and $\mathrm{R}=4 \mathrm{R}\left(2 \varepsilon_{\tau_{k^{\prime}}}\right)$. This choice of $\mathrm{R}$ is justified by Lemma B.1.ii.

(a) First consider the case that $\mathbf{x} \in \Lambda_{\tau}^{c}$ (which forces $\tau \in \varepsilon \mathbb{Z}$ ). Then, the $k=1$ term in 
(E.1) vanishes so that

$$
\begin{array}{rlrl}
\left|\gamma_{\tau}(\mathbf{x})-\alpha_{\tau}(\mathbf{x})\right| & =\left|\sum_{k=2}^{p}\left(j\left(\tau_{k-1}-\tau\right) \Lambda_{\tau_{k-1}} j\left(\tau_{k}-\tau_{k-1}\right) \Lambda_{\tau_{k}}^{c} \alpha_{\tau_{k}}\right)(\mathbf{x})\right| \\
& \leq \sum_{k=2}^{p} 4 e^{K_{j}} \mathrm{R}\left(\varepsilon_{\tau_{k-1}}\right) e^{-\frac{9}{2} \mathrm{~m} d\left(\mathbf{x}, \Lambda_{\tau_{k-1}}\right)} & \\
& \leq \sum_{k=2}^{p} 4 e^{K_{j}} \mathrm{R}\left(\varepsilon_{\tau_{k-1}}\right) e^{-\frac{9}{2} \mathrm{mc}\left(2 \varepsilon_{\tau}\right)} \quad & \text { (put } \ell=1 \text { in the figure below) } \\
& \leq \sum_{k=2}^{p} \frac{1}{4} e^{-4 \mathrm{~m} \mathfrak{c}\left(2 \varepsilon_{\tau}\right)} & \text { by (F.6.b) } \\
& \leq \frac{1}{4} e^{-2 \mathrm{~m} \mathfrak{c}\left(2 \varepsilon_{\tau}\right)} \sum_{k=2}^{p} e^{-2 \mathrm{~m} \mathfrak{c}\left(2 \varepsilon_{\tau_{k-1}}\right)} & \\
& \leq \frac{1}{2} e^{-\mathrm{mc}\left(\varepsilon_{\tau}\right)} \quad \text { by (F.4.a,d) }
\end{array}
$$

(b,c,d) Now we consider the case $\mathbf{x} \in \Lambda_{\tau_{\ell-1}} \cap \Lambda_{\tau_{\ell}}^{c}$ with $1 \leq \ell \leq p$. In particular, $\mathbf{x} \in \Lambda_{\tau}$. Set

$$
K= \begin{cases}\{\ell\} & \text { in case }(\mathrm{b}) \\ \{\ell, \ell-1\} & \text { in case }(\mathrm{c}) \\ \{\ell, \ell+1\} & \text { in case }(\mathrm{d})\end{cases}
$$

As in the last paragraph

$$
\left|\gamma_{\tau}(\mathbf{x})-\sum_{k \in K} j\left(\tau_{k-1}-\tau\right) \Lambda_{\tau_{k-1}} j\left(\tau_{k}-\tau_{k-1}\right) \Lambda_{\tau_{k}}^{c} \alpha_{\tau_{k}}\right| \leq e^{-\mathrm{m} \mathfrak{c}\left(\varepsilon_{\tau_{\ell}}\right)}
$$

In case (b), when $d\left(\mathbf{x}, \Lambda_{\tau_{\ell-1}}^{c}\right) \geq \frac{1}{2} \mathfrak{c}\left(2 \varepsilon_{\tau_{\ell-1}}\right)$ and $d\left(\mathbf{x}, \Lambda_{\tau_{\ell}}\right) \geq \frac{1}{2} \mathfrak{c}\left(\varepsilon_{\tau_{\ell}}\right)$, we write the $k=\ell$ term as

$$
\begin{aligned}
& j\left(\tau_{\ell-1}-\tau\right) \Lambda_{\tau_{\ell-1}} j\left(\tau_{\ell}-\tau_{\ell-1}\right) \Lambda_{\tau_{\ell}}^{c} \alpha_{\tau_{\ell}} \\
& =j\left(\tau_{\ell}-\tau\right) \Lambda_{\tau_{\ell}}^{c} \alpha_{\tau_{\ell}}-j\left(\tau_{\ell-1}-\tau\right) \Lambda_{\tau_{\ell-1}}^{c} j\left(\tau_{\ell}-\tau_{\ell-1}\right) \Lambda_{\tau_{\ell}}^{c} \alpha_{\tau_{\ell}} \\
& =\Lambda_{\tau_{\ell-1}} \Lambda_{\tau_{\ell}}^{c} \mathfrak{h} \alpha_{\tau_{\ell}}+\left[j\left(\tau_{\ell}-\tau\right)-\mathfrak{h}\right] \Lambda_{\tau_{\ell-1}} \Lambda_{\tau_{\ell}}^{c} \alpha_{\tau_{\ell}} \\
& \quad \quad+j\left(\tau_{\ell}-\tau\right) \Lambda_{\tau_{\ell-1}}^{c} \alpha_{\tau_{\ell}}-j\left(\tau_{\ell-1}-\tau\right) \Lambda_{\tau_{\ell-1}}^{c} j\left(\tau_{\ell}-\tau_{\ell-1}\right) \Lambda_{\tau_{\ell}}^{c} \alpha_{\tau_{\ell}}
\end{aligned}
$$

and bound, as in (E.2),

$$
\begin{aligned}
\left|\left(j\left(\tau_{\ell-1}-\tau\right) \Lambda_{\tau_{\ell-1}^{c}} j\left(\tau_{\ell}-\tau_{\ell-1}\right) \Lambda_{\tau_{\ell}}^{c} \alpha_{\tau_{\ell}}\right)(\mathbf{x})\right| & \leq 4 e^{K_{j}} \mathrm{R}\left(2 \varepsilon_{\tau_{\ell-1}}\right) e^{-\frac{9}{4} \mathrm{mc}\left(2 \varepsilon_{\tau_{\ell-1}}\right)} \\
& \leq \frac{1}{4} e^{-\mathrm{mc}\left(\varepsilon_{\tau_{\ell-1}}\right)} \\
\left|\left(j\left(\tau_{\ell}-\tau\right) \Lambda_{\tau_{\ell-1}}^{c} \alpha_{\tau_{\ell}}\right)(\mathbf{x})\right| & \leq \frac{1}{4} e^{-\mathrm{mc}\left(\varepsilon_{\tau_{\ell-1}}\right)}
\end{aligned}
$$




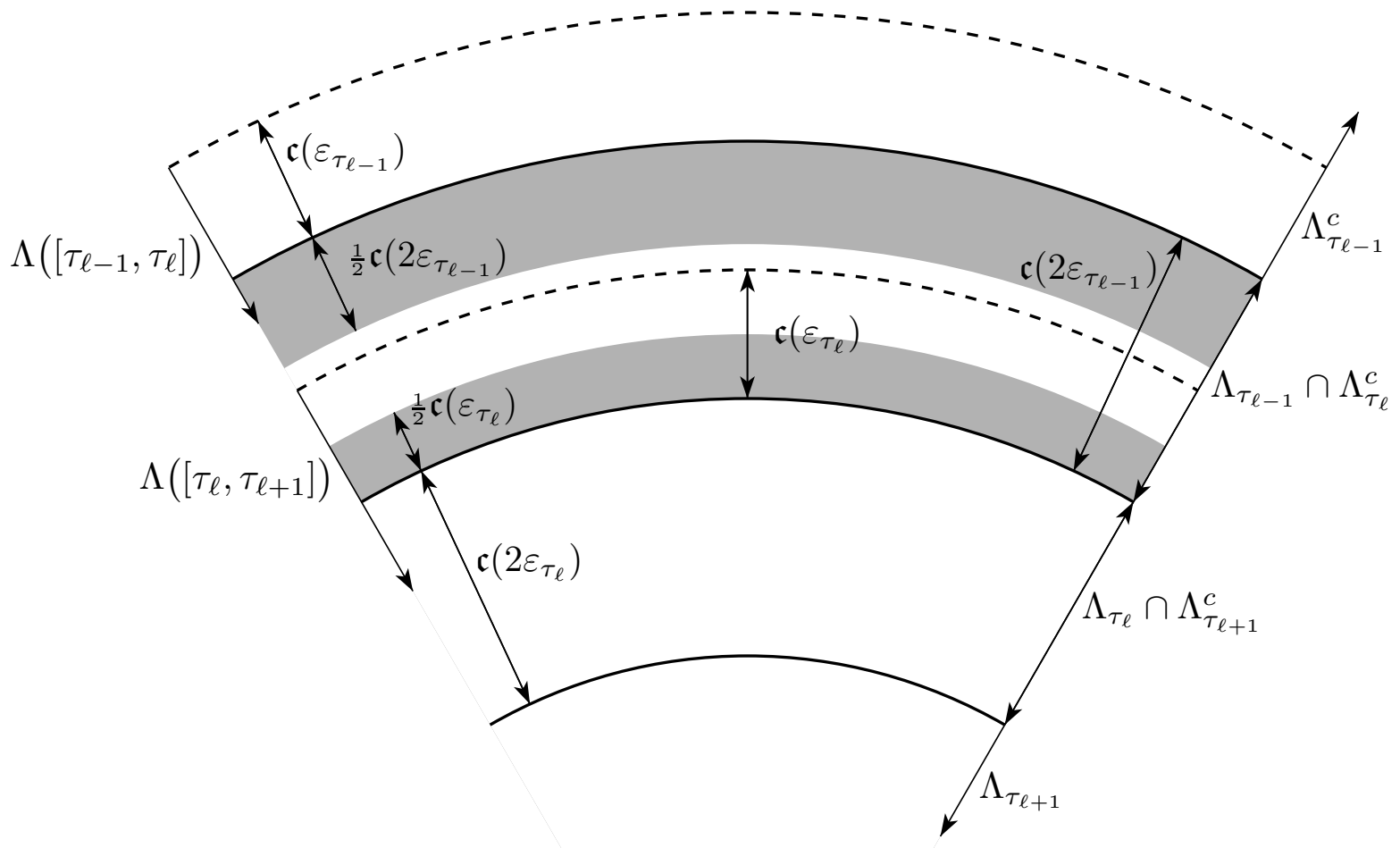

For case $(\mathrm{c})$, when $d\left(\mathbf{x}, \Lambda_{\tau_{\ell-1}}^{c}\right) \leq \frac{1}{2} \mathfrak{c}\left(2 \varepsilon_{\tau_{\ell-1}}\right)$ and hence $d\left(\mathbf{x}, \Lambda_{\tau_{\ell}}\right) \geq \frac{1}{2} \mathfrak{c}\left(2 \varepsilon_{\tau_{\ell-1}}\right)$, we first observe that

$$
\Lambda_{\tau_{\ell}} \Subset \Lambda_{\tau_{\ell-1}}=\Lambda\left(\left[\tau_{\ell-1}-\varepsilon_{\tau_{\ell-1}}, \tau_{\ell}\right]\right) \Subset \Lambda\left(\left[\tau_{\ell-1}, \tau_{\ell}\right]\right)
$$

Here the symbol $A \Subset B$ signifies that $A$ is a proper subset of $B$ unless $B=X$.

So the $k=\ell$ term is

$$
\begin{aligned}
& j\left(\tau_{\ell-1}-\tau\right) \Lambda_{\tau_{\ell-1}} j\left(\tau_{\ell}-\tau_{\ell-1}\right) \Lambda_{\tau_{\ell}}^{c} \alpha_{\tau_{\ell}} \\
& =j\left(\tau_{\ell}-\tau\right) \Lambda\left(\left[\tau_{\ell-1}, \tau_{\ell}\right]\right) \Lambda_{\tau_{\ell}}^{c} \alpha_{\tau_{\ell}}-j\left(\tau_{\ell-1}-\tau\right) \Lambda_{\tau_{\ell-1}}^{c} j\left(\tau_{\ell}-\tau_{\ell-1}\right) \Lambda\left(\left[\tau_{\ell-1}, \tau_{\ell}\right]\right) \Lambda_{\tau_{\ell}}^{c} \alpha_{\tau_{\ell}} \\
& \quad+j\left(\tau_{\ell-1}-\tau\right) \Lambda_{\tau_{\ell-1}} j\left(\tau_{\ell}-\tau_{\ell-1}\right) \Lambda\left(\left[\tau_{\ell-1}, \tau_{\ell}\right]\right)^{c} \alpha_{\tau_{\ell}} \\
& =\Lambda\left(\left[\tau_{\ell-1}, \tau_{\ell}\right]\right) \Lambda_{\tau_{\ell}}^{c} \mathfrak{h} \alpha_{\tau_{\ell}}-j\left(\tau_{\ell-1}-\tau\right) \Lambda_{\tau_{\ell-1}}^{c} \Lambda\left(\left[\tau_{\ell-1}, \tau_{\ell}\right]\right) \Lambda_{\tau_{\ell}}^{c} \alpha_{\tau_{\ell}} \\
& \quad+\left\{\left[j\left(\tau_{\ell}-\tau\right)-\mathfrak{h}\right]-j\left(\tau_{\ell-1}-\tau\right) \Lambda_{\tau_{\ell-1}}^{c}\left[j\left(\tau_{\ell}-\tau_{\ell-1}\right)-\mathfrak{h}\right]\right\} \Lambda\left(\left[\tau_{\ell-1}, \tau_{\ell}\right]\right) \Lambda_{\tau_{\ell}}^{c} \alpha_{\tau_{\ell}} \\
& \quad+j\left(\tau_{\ell-1}-\tau\right) \Lambda_{\tau_{\ell-1}} j\left(\tau_{\ell}-\tau_{\ell-1}\right) \Lambda\left(\left[\tau_{\ell-1}, \tau_{\ell}\right]\right)^{c} \alpha_{\tau_{\ell}}
\end{aligned}
$$


and the $k=\ell-1$ term, which is only present when $\ell \geq 2$, is

$$
\begin{aligned}
& j\left(\tau_{\ell-2}-\tau\right) \Lambda_{\tau_{\ell-2}} j\left(\tau_{\ell-1}-\tau_{\ell-2}\right) \Lambda_{\tau_{\ell-1}}^{c} \alpha_{\tau_{\ell-1}}=j\left(\tau_{\ell-1}-\tau\right) \Lambda_{\tau_{\ell-1}}^{c} \Lambda\left(\left[\tau_{\ell-1}, \tau_{\ell}\right]\right) \alpha_{\tau_{\ell-1}} \\
& \quad-j\left(\tau_{\ell-2}-\tau\right) \Lambda_{\tau_{\ell-2}}^{c} j\left(\tau_{\ell-1}-\tau_{\ell-2}\right) \Lambda_{\tau_{\ell-1}}^{c} \Lambda\left(\left[\tau_{\ell-1}, \tau_{\ell}\right]\right) \alpha_{\tau_{\ell-1}} \\
& \quad+j\left(\tau_{\ell-2}-\tau\right) \Lambda_{\tau_{\ell-2}} j\left(\tau_{\ell-1}-\tau_{\ell-2}\right) \Lambda\left(\left[\tau_{\ell-1}, \tau_{\ell}\right]\right)^{c} \alpha_{\tau_{\ell-1}}
\end{aligned}
$$

As in (E.2),

$$
\begin{aligned}
\left|\left(j\left(\tau_{\ell-1}-\tau\right) \Lambda_{\tau_{\ell-1}} j\left(\tau_{\ell}-\tau_{\ell-1}\right) \Lambda\left(\left[\tau_{\ell-1}, \tau_{\ell}\right]\right)^{c} \alpha_{\tau_{\ell}}\right)(\mathbf{x})\right| & \leq \frac{1}{4} e^{-4 \mathrm{mc}\left(\varepsilon_{\tau_{\ell-1}}\right)} \\
\left|\left(j\left(\tau_{\ell-2}-\tau\right) \Lambda_{\tau_{\ell-2}}^{c} j\left(\tau_{\ell-1}-\tau_{\ell-2}\right) \Lambda_{\tau_{\ell-1}}^{c} \Lambda\left(\left[\tau_{\ell-1}, \tau_{\ell}\right]\right) \alpha_{\tau_{\ell-1}}\right)(\mathbf{x})\right| & \leq \frac{1}{4} e^{-2 \mathrm{mc}\left(\varepsilon_{\tau_{\ell-2}}\right)} \\
\left|\left(j\left(\tau_{\ell-2}-\tau\right) \Lambda_{\tau_{\ell-2}} j\left(\tau_{\ell-1}-\tau_{\ell-2}\right) \Lambda\left(\left[\tau_{\ell-1}, \tau_{\ell}\right]\right)^{c} \alpha_{\tau_{\ell-1}}\right)(\mathbf{x})\right| & \leq \frac{1}{4} e^{-4 \mathrm{mc}\left(\varepsilon_{\tau_{\ell-1}}\right)}
\end{aligned}
$$

Adding the representations of the $k=\ell$ and $k=\ell-1$ terms and using the fact that $\Lambda_{\tau_{\ell-1}}^{c} \Lambda_{\tau_{\ell}}^{c}=\Lambda_{\tau_{\ell-1}}^{c}$, we see that - up to an error of at most $2 e^{-\mathrm{mc}\left(\varepsilon_{\tau_{\ell}}\right)}$

$$
\begin{aligned}
\gamma_{\tau}(\mathbf{x})= & \mathfrak{h} \alpha_{\tau_{\ell}}(\mathbf{x})-\left(j\left(\tau_{\ell-1}-\tau\right) \Lambda_{\tau_{\ell-1}}^{c} \Lambda\left(\left[\tau_{\ell-1}, \tau_{\ell}\right]\right)\left[\alpha_{\tau_{\ell}}-\alpha_{\tau_{\ell-1}}\right]\right)(\mathbf{x}) \\
& +\left(\left\{\left[j\left(\tau_{\ell}-\tau\right)-\mathfrak{h}\right]-j\left(\tau_{\ell-1}-\tau\right) \Lambda_{\tau_{\ell-1}}^{c}\left[j\left(\tau_{\ell}-\tau_{\ell-1}\right)-\mathfrak{h}\right]\right\} \Lambda\left(\left[\tau_{\ell-1}, \tau_{\ell}\right]\right) \Lambda_{\tau_{\ell}}^{c} \alpha_{\tau_{\ell}}\right)(\mathbf{x})
\end{aligned}
$$

(When $\ell=1,\left(j\left(\tau_{\ell-1}-\tau\right) \Lambda_{\tau_{\ell-1}}^{c} \Lambda\left(\left[\tau_{\ell-1}, \tau_{\ell}\right]\right)\right)(\mathbf{x})=0$, since $\left.\mathbf{x} \in \Lambda_{\tau}.\right)$

For case $(\mathrm{d})$, when $\ell<p$ and $d\left(\mathbf{x}, \Lambda_{\tau_{\ell}}\right) \leq \frac{1}{2} \mathfrak{c}\left(\varepsilon_{\tau_{\ell}}\right)$ so that $d\left(\mathbf{x}, \Lambda_{\tau_{\ell-1}}^{c}\right) \geq \frac{1}{2} \mathfrak{c}\left(2 \varepsilon_{\tau_{\ell-1}}\right)$, we first observe that $\mathbf{x} \in \Lambda\left(\left[\tau_{\ell}, \tau_{\ell+1}\right]\right)$ and that

$$
\Lambda_{\tau_{\ell+1}} \Subset \Lambda_{\tau_{\ell}}=\Lambda\left(\left[\tau_{\ell}-\varepsilon_{\tau_{\ell}}, \tau_{\ell+1}\right]\right) \Subset \Lambda\left(\left[\tau_{\ell}, \tau_{\ell+1}\right]\right)
$$

So the $k=\ell$ term is

$$
\begin{gathered}
j\left(\tau_{\ell-1}-\tau\right) \Lambda_{\tau_{\ell-1}} j\left(\tau_{\ell}-\tau_{\ell-1}\right) \Lambda_{\tau_{\ell}}^{c} \alpha_{\tau_{\ell}} \\
=j\left(\tau_{\ell}-\tau\right) \Lambda\left(\left[\tau_{\ell}, \tau_{\ell+1}\right]\right) \Lambda_{\tau_{\ell}}^{c} \alpha_{\tau_{\ell}}+j\left(\tau_{\ell}-\tau\right) \Lambda\left(\left[\tau_{\ell}, \tau_{\ell+1}\right]\right)^{c} \alpha_{\tau_{\ell}} \\
\quad-j\left(\tau_{\ell-1}-\tau\right) \Lambda_{\tau_{\ell-1}}^{c} j\left(\tau_{\ell}-\tau_{\ell-1}\right) \Lambda_{\tau_{\ell}}^{c} \alpha_{\tau_{\ell}}
\end{gathered}
$$

and the $k=\ell+1$ term is

$$
\begin{aligned}
& j\left(\tau_{\ell}-\tau\right) \Lambda_{\tau_{\ell}} j\left(\tau_{\ell+1}-\tau_{\ell}\right) \Lambda_{\tau_{\ell+1}}^{c} \alpha_{\tau_{\ell+1}} \\
& =j\left(\tau_{\ell}-\tau\right) \Lambda_{\tau_{\ell}} \Lambda\left(\left[\tau_{\ell}, \tau_{\ell+1}\right]\right) \Lambda_{\tau_{\ell+1}}^{c} \alpha_{\tau_{\ell+1}} \\
& \quad+j\left(\tau_{\ell}-\tau\right) \Lambda_{\tau_{\ell}}\left(j\left(\tau_{\ell+1}-\tau_{\ell}\right)-\mathfrak{h}\right) \Lambda\left(\left[\tau_{\ell}, \tau_{\ell+1}\right]\right) \Lambda_{\tau_{\ell+1}}^{c} \alpha_{\tau_{\ell+1}} \\
& \quad+j\left(\tau_{\ell}-\tau\right) \Lambda_{\tau_{\ell}} j\left(\tau_{\ell+1}-\tau_{\ell}\right) \Lambda\left(\left[\tau_{\ell}, \tau_{\ell+1}\right]\right)^{c} \alpha_{\tau_{\ell+1}}
\end{aligned}
$$


As in (E.2)

$$
\begin{aligned}
\left|\left(j\left(\tau_{\ell}-\tau\right) \Lambda\left(\left[\tau_{\ell}, \tau_{\ell+1}\right]\right)^{c} \alpha_{\tau_{\ell}}\right)(\mathbf{x})\right| & \leq \frac{1}{4} e^{-2 \mathrm{mc}\left(\varepsilon_{\tau_{\ell}}\right)} \\
\left|\left(j\left(\tau_{\ell-1}-\tau\right) \Lambda_{\tau_{\ell-1}}^{c} j\left(\tau_{\ell}-\tau_{\ell-1}\right) \Lambda_{\tau_{\ell}}^{c} \alpha_{\tau_{\ell}}\right)(\mathbf{x})\right| & \leq \frac{1}{4} e^{-\mathrm{mc}\left(\varepsilon_{\tau_{\ell-1}}\right)} \\
\left|\left(j\left(\tau_{\ell}-\tau\right) \Lambda_{\tau_{\ell}} j\left(\tau_{\ell+1}-\tau_{\ell}\right) \Lambda\left(\left[\tau_{\ell}, \tau_{\ell+1}\right]\right)^{c} \alpha_{\tau_{\ell+1}}\right)(\mathbf{x})\right| & \leq \frac{1}{4} e^{-2 \mathrm{mc}\left(\varepsilon_{\tau_{\ell}}\right)}
\end{aligned}
$$

Adding the representations of the $k=\ell$ and $k=\ell+1$ terms and using the fact that $\Lambda_{\tau_{\ell}}^{c} \Lambda_{\tau_{\ell+1}}^{c}=\Lambda_{\tau_{\ell}}^{c}$, we see that - up to an error at $\mathbf{x}$ of at most $2 e^{-\mathrm{mc}\left(\varepsilon_{\tau_{\ell}}\right)}$

$$
\begin{gathered}
\gamma_{\tau}=j\left(\tau_{\ell}-\tau\right) \Lambda\left(\left[\tau_{\ell}, \tau_{\ell+1}\right]\right) \Lambda_{\tau_{\ell}}^{c} \alpha_{\tau_{\ell}}+j\left(\tau_{\ell}-\tau\right) \Lambda_{\tau_{\ell}} \Lambda\left(\left[\tau_{\ell}, \tau_{\ell+1}\right]\right) \Lambda_{\tau_{\ell+1}}^{c} \alpha_{\tau_{\ell+1}} \\
+j\left(\tau_{\ell}-\tau\right) \Lambda_{\tau_{\ell}}\left(j\left(\tau_{\ell+1}-\tau_{\ell}\right)-\mathfrak{h}\right) \Lambda\left(\left[\tau_{\ell}, \tau_{\ell+1}\right]\right) \Lambda_{\tau_{\ell+1}}^{c} \alpha_{\tau_{\ell+1}} \\
=j\left(\tau_{\ell}-\tau\right)\left[\Lambda\left(\left[\tau_{\ell}, \tau_{\ell+1}\right]\right) \Lambda_{\tau_{\ell+1}}^{c} \alpha_{\tau_{\ell+1}}+\Lambda\left(\left[\tau_{\ell}, \tau_{\ell+1}\right]\right) \Lambda_{\tau_{\ell}}^{c}\left[\alpha_{\tau_{\ell}}-\alpha_{\tau_{\ell+1}}\right]\right] \\
+j\left(\tau_{\ell}-\tau\right) \Lambda_{\tau_{\ell}}\left(j\left(\tau_{\ell+1}-\tau_{\ell}\right)-\mathfrak{h}\right) \Lambda\left(\left[\tau_{\ell}, \tau_{\ell+1}\right]\right) \Lambda_{\tau_{\ell+1}}^{c} \alpha_{\tau_{\ell+1}}
\end{gathered}
$$

Writing, in the first term, $j\left(\tau_{\ell}-\tau\right)=\mathfrak{h}+\left[j\left(\tau_{\ell}-\tau\right)-\mathfrak{h}\right]$ and evaluating at $\mathbf{x}$, we have

$$
\begin{aligned}
\gamma_{\tau}(\mathbf{x})=\mathfrak{h} \alpha_{\tau_{\ell}}(\mathbf{x})+([ & \left.\left.j\left(\tau_{\ell}-\tau\right)-\mathfrak{h}\right] \Lambda\left(\left[\tau_{\ell}, \tau_{\ell+1}\right]\right) \Lambda_{\tau_{\ell+1}}^{c} \alpha_{\tau_{\ell+1}}\right)(\mathbf{x}) \\
& +\left(\left[j\left(\tau_{\ell}-\tau\right)-\mathfrak{h}\right] \Lambda\left(\left[\tau_{\ell}, \tau_{\ell+1}\right]\right) \Lambda_{\tau_{\ell}}^{c}\left[\alpha_{\tau_{\ell}}-\alpha_{\tau_{\ell+1}}\right]\right)(\mathbf{x}) \\
& +\left(j\left(\tau_{\ell}-\tau\right) \Lambda_{\tau_{\ell}}\left[j\left(\tau_{\ell+1}-\tau_{\ell}\right)-\mathfrak{h}\right] \Lambda\left(\left[\tau_{\ell}, \tau_{\ell+1}\right]\right) \Lambda_{\tau_{\ell+1}}^{c} \alpha_{\tau_{\ell+1}}\right)(\mathbf{x})
\end{aligned}
$$

up to an error of at most $2 e^{-\mathrm{mc}\left(\varepsilon_{\tau_{\ell}}\right)}$.

(e,f,g) We give the proof for $\gamma_{* \tau}$. Recall from Lemma E.4.i, that, on $\Lambda$,

$$
\gamma_{* \tau}^{*}=\sum_{\tau^{\prime} \in \mathcal{T}_{l}(\tau, \delta)} j\left(\tau-\sigma\left(\tau^{\prime}\right)\right) \Lambda_{\sigma\left(\tau^{\prime}\right)} j\left(\sigma\left(\tau^{\prime}\right)-\tau^{\prime}\right) \Lambda_{\tau^{\prime}}^{c} \alpha_{\tau^{\prime}}
$$

As in (E.2), if $0<\tau<\delta$ (otherwise $\mathcal{T}_{l}(\tau, \delta)$ is empty),

$$
\begin{aligned}
& \sum_{\substack{\mathbf{x} \in \Lambda \\
(\tau, \delta) \backslash\left\{0, \frac{\delta}{2}\right\}}}\left|\left(j\left(\tau-\sigma\left(\tau^{\prime}\right)\right) \Lambda_{\sigma\left(\tau^{\prime}\right)} j\left(\sigma\left(\tau^{\prime}\right)-\tau^{\prime}\right) \Lambda_{\tau^{\prime}}^{c} \alpha_{\tau^{\prime}}\right)(\mathbf{x})\right| \\
& \leq \sum_{\substack{\tau^{\prime} \in \mathcal{T}_{l}(\tau, \delta) \\
\tau^{\prime} \neq 0, \frac{\delta}{2}}} N_{0}\left(\Lambda j\left(\tau-\sigma\left(\tau^{\prime}\right)\right) \Lambda_{\sigma\left(\tau^{\prime}\right)} j\left(\sigma\left(\tau^{\prime}\right)-\tau^{\prime}\right) \Lambda_{\tau^{\prime}}^{c} ; 1, \kappa_{* \tau^{\prime}}\right)\left|\Lambda_{\tau^{\prime}}^{c}\right| \\
& \leq \sum_{\substack{\tau^{\prime} \in \mathcal{T}_{l}(\tau, \delta) \\
\tau^{\prime} \neq 0, \frac{\delta}{2}}} 4 e^{K_{j}} \mathrm{R}(\delta) e^{-\frac{9}{2} \mathrm{~m} d\left(\Lambda, \Lambda_{\tau^{\prime}}^{c}\right)}\left|\Lambda^{c}\right| \\
& \leq \sum_{\tau^{\prime} \in \mathcal{T}_{l}(\tau, \delta) \backslash\{0\}} 4 e^{K_{j}} \mathrm{R}(\delta) e^{-\frac{9}{2} \mathrm{mc}\left(2 \varepsilon_{\tau^{\prime}}\right)}\left|\Lambda^{c}\right| \\
& \leq \sum_{\tau^{\prime} \in \mathcal{T}_{l}(\tau, \delta) \backslash\{0\}} \frac{1}{4} e^{-4 \mathrm{mc}\left(2 \varepsilon_{\tau^{\prime}}\right)}\left|\Lambda^{c}\right| \quad \text { by (F.6.b) } \\
& \leq \frac{1}{4} e^{-\mathrm{mc}(\delta)}\left|\Lambda^{c}\right| \quad \text { by (F.4.d) }
\end{aligned}
$$


Case $0<\tau \leq \frac{\delta}{2}$ : In this case $\tau^{\prime}=\frac{\delta}{2} \notin \mathcal{T}_{l}(\tau, \delta)$ and the $\tau^{\prime}=0 \in \mathcal{T}_{l}(\tau, \delta)$ contribution to $\gamma_{* \tau}^{*}$ is

$$
j(\tau-\sigma(0)) \Lambda_{\sigma(0)} j(\sigma(0)) \alpha=j(\tau) \alpha-j(\tau-\sigma(0)) \Lambda_{\sigma(0)}^{c} j(\sigma(0)) \alpha
$$

For $\tau=\frac{\delta}{2}, \sigma(0)=\tau$ and the second term vanishes on $\Lambda$. For $0<\tau<\frac{\delta}{2}$, by Lemma B.1.i,

$$
\begin{aligned}
& \sum_{\mathbf{x} \in \Lambda}\left|\left(j(\tau-\sigma(0)) \Lambda_{\sigma(0)}^{c} j(\sigma(0)) \alpha\right)(\mathbf{x})\right| \leq \sum_{\substack{\mathbf{x} \in \Lambda, \mathbf{z} \in X \\
\mathbf{y} \in \Lambda_{\sigma(0)}}}|j(\tau-\sigma(0))(\mathbf{x}, \mathbf{y})||j(\sigma(0))(\mathbf{y}, \mathbf{z})| \kappa_{* 0}(\mathbf{z}) \\
& \leq 4 \mathrm{R}(\delta) e^{-\frac{9}{2} \mathrm{~m} d\left(\Lambda, \Lambda_{\sigma(0)}^{c}\right)} \sum_{\substack{\mathbf{x} \in \Lambda, \mathbf{z} \in X \\
\mathbf{y} \in \Lambda_{\sigma(0)}^{c}}}|j(\tau-\sigma(0))(\mathbf{x}, \mathbf{y})| e^{\frac{9}{2} \mathrm{~m} d(\mathbf{x}, \mathbf{y})}|j(\sigma(0))(\mathbf{y}, \mathbf{z})| e^{\frac{1}{3} \mathrm{~m} d(\mathbf{x}, \mathbf{z})} \\
& \leq 4 \mathrm{R}(\delta) e^{-\frac{9}{2} \mathrm{~m} d\left(\Lambda, \Lambda_{\sigma(0)}^{c}\right)}\left|\Lambda_{\sigma(0)}^{c}\right||| j(\tau-\sigma(0))\|\|\|j(\sigma(0))\| \mid \\
& \leq 4 e^{K_{j}} \mathrm{R}(\delta) e^{-\frac{9}{2} \mathrm{mc}(\delta / 2)}\left|\Lambda_{\sigma(0)}^{c}\right| \quad \text { since } \tau<\frac{\delta}{2}, \text { so that } \sigma(0)<\frac{\delta}{2} \text { and } \varepsilon_{\sigma(0)} \leq \frac{\delta}{4} \\
& \leq \frac{1}{4} e^{-4 \mathrm{mc}(\delta)}\left|\Lambda^{c}\right| \quad \text { by (F.6.b) }
\end{aligned}
$$

Case $\frac{\delta}{2}<\tau<\delta$ : In this case $\tau^{\prime}=\frac{\delta}{2} \in \mathcal{T}_{l}(\tau, \delta)$. The $\tau^{\prime}=0$ term is

$$
\begin{aligned}
j(\tau-\sigma(0)) \Lambda_{\sigma(0)} & j(\sigma(0)) \alpha=j\left(\tau-\frac{\delta}{2}\right) \Lambda j\left(\frac{\delta}{2}\right) \alpha \\
=j(\tau) \Lambda & \left(\left[0, \frac{\delta}{2}\right]\right) \alpha-j\left(\tau-\frac{\delta}{2}\right) \Lambda^{c} j\left(\frac{\delta}{2}\right) \Lambda\left(\left[0, \frac{\delta}{2}\right]\right) \alpha+j\left(\tau-\frac{\delta}{2}\right) \Lambda j\left(\frac{\delta}{2}\right) \Lambda\left(\left[0, \frac{\delta}{2}\right]\right)^{c} \alpha \\
=j(\tau) \Lambda & \left(\left[0, \frac{\delta}{2}\right]\right) \alpha-j\left(\tau-\frac{\delta}{2}\right) \Lambda^{c}\left[j\left(\frac{\delta}{2}\right)-\mathfrak{h}\right] \Lambda\left(\left[0, \frac{\delta}{2}\right]\right) \alpha-j\left(\tau-\frac{\delta}{2}\right) \Lambda^{c} \Lambda\left(\left[0, \frac{\delta}{2}\right]\right) \alpha \\
& +j\left(\tau-\frac{\delta}{2}\right) \Lambda j\left(\frac{\delta}{2}\right) \Lambda\left(\left[0, \frac{\delta}{2}\right]\right)^{c} \alpha
\end{aligned}
$$

and the $\tau^{\prime}=\frac{\delta}{2}$ term is

$$
\begin{aligned}
j\left(\tau-\sigma\left(\frac{\delta}{2}\right)\right) \Lambda_{\sigma\left(\frac{\delta}{2}\right)} j\left(\sigma\left(\frac{\delta}{2}\right)-\frac{\delta}{2}\right) \Lambda_{\frac{\delta}{2}}^{c} \alpha_{\frac{\delta}{2}}= & j\left(\tau-\frac{\delta}{2}\right) \Lambda^{c} \Lambda\left(\left[0, \frac{\delta}{2}\right]\right) \alpha_{\frac{\delta}{2}} \\
& -j\left(\tau-\sigma\left(\frac{\delta}{2}\right)\right) \Lambda_{\sigma\left(\frac{\delta}{2}\right)}^{c} j\left(\sigma\left(\frac{\delta}{2}\right)-\frac{\delta}{2}\right) \Lambda^{c} \Lambda\left(\left[0, \frac{\delta}{2}\right]\right) \alpha_{\frac{\delta}{2}} \\
& +j\left(\tau-\sigma\left(\frac{\delta}{2}\right)\right) \Lambda_{\sigma\left(\frac{\delta}{2}\right)} j\left(\sigma\left(\frac{\delta}{2}\right)-\frac{\delta}{2}\right) \Lambda\left(\left[0, \frac{\delta}{2}\right]\right)^{c} \alpha_{\frac{\delta}{2}}
\end{aligned}
$$

As in (E.3),

$$
\begin{array}{r}
\sum_{\mathbf{x} \in \Lambda}\left|\left(j\left(\tau-\frac{\delta}{2}\right) \Lambda j\left(\frac{\delta}{2}\right) \Lambda\left(\left[0, \frac{\delta}{2}\right]\right)^{c} \alpha\right)(\mathbf{x})\right| \leq \frac{1}{4} e^{-4 \mathrm{mc}(\delta / 2)}\left|\Lambda^{c}\right| \\
\sum_{\mathbf{x} \in \Lambda}\left|\left(j\left(\tau-\sigma\left(\frac{\delta}{2}\right)\right) \Lambda_{\sigma\left(\frac{\delta}{2}\right)}^{c} j\left(\sigma\left(\frac{\delta}{2}\right)-\frac{\delta}{2}\right) \Lambda^{c} \Lambda\left(\left[0, \frac{\delta}{2}\right]\right) \alpha_{\frac{\delta}{2}}\right)(\mathbf{x})\right| \leq \frac{1}{8} e^{-4 \mathrm{mc}(\delta / 2)}\left|\Lambda^{c}\right| \\
\sum_{\mathbf{x} \in \Lambda}\left|\left(j\left(\tau-\sigma\left(\frac{\delta}{2}\right)\right) \Lambda_{\sigma\left(\frac{\delta}{2}\right)} j\left(\sigma\left(\frac{\delta}{2}\right)-\frac{\delta}{2}\right) \Lambda\left(\left[0, \frac{\delta}{2}\right]\right)^{c} \alpha_{\frac{\delta}{2}}\right)(\mathbf{x})\right| \leq \frac{1}{8} e^{-4 \mathrm{mc}(\delta / 2)}\left|\Lambda^{c}\right|
\end{array}
$$


Proof of Proposition E.7: $\quad$ For $\mathbf{x} \in \Lambda_{\tau}^{c}, \hat{\gamma}_{\tau}(\mathbf{x})=\alpha_{\tau}(\mathbf{x})$ and $\hat{\gamma}_{* \tau}=\alpha_{\tau}(\mathbf{x})^{*}$ and the desired bound follows immediately from Lemma E.8 and its analog for $\gamma_{* \tau}$. For the rest of the proof we restrict to $\mathrm{x} \in \Lambda_{\tau}$.

We prove the bound on $\left|\gamma_{\tau}(\mathbf{x})-\hat{\gamma}_{\tau}(\mathbf{x})\right|$. The bound on $\left|\gamma_{* \tau}(\mathbf{x})-\hat{\gamma}_{* \tau}(\mathbf{x})\right|$ is proven similarly. Write $\mathcal{T}_{r}=\left\{\tau_{1}<\tau_{2}<\cdots<\tau_{p}=\delta\right\}$ and fix any $1 \leq \ell \leq p$, any decimation interval $\mathcal{J}$ containing $\tau$ and any $\mathbf{x} \in \Lambda_{\tau_{\ell-1}} \cap \Lambda_{\tau_{\ell}}^{c} \cap \Lambda(\mathcal{J})$. Note that $\Lambda_{\tau_{\ell}}^{c} \cap \Lambda(\mathcal{J})$ is empty unless $|\mathcal{J}| \leq \varepsilon_{\tau_{\ell}}$ and that $2 e^{-\mathrm{mc}\left(\varepsilon_{\tau_{\ell}}\right)} \leq \frac{1}{8} \leq \frac{1}{8} \mathrm{r}(|\mathcal{J}|)$ by (F.4.c).

For case (b) of Lemma E.8, it suffices to bound

$$
\begin{aligned}
& \left|\left(\left[j\left(\tau_{\ell}-\tau\right)-\mathbb{1}\right] \Lambda_{\tau_{\ell-1}} \Lambda_{\tau_{\ell}}^{c} \alpha_{\tau_{\ell}}\right)(\mathbf{x})\right| \\
& \quad \leq\left(\tau_{\ell}-\tau\right) K_{j}^{\prime} e^{K_{j}^{\prime}}\left(\left[|\mu|+e^{-5 \mathrm{~m} d\left(\mathbf{x}, \Lambda_{\tau_{\ell-1}}^{c} \cup \Lambda_{\tau_{\ell}}\right)}\right] \max _{\mathbf{y} \in \Lambda_{\tau_{\ell-1}}}\left|\alpha_{\tau_{\ell}}(\mathbf{y})\right|+\max _{b \in \Lambda_{\tau_{\ell-1}}^{*}}\left|\nabla \alpha_{\tau_{\ell}}(b)\right|\right) \\
& \quad \leq 2 \varepsilon_{\tau_{\ell-1}} K_{j}^{\prime} e^{K_{j}^{\prime}}\left(\left[|\mu|+e^{-\frac{5}{2} \mathrm{mc}\left(\varepsilon_{\tau_{\ell}}\right)}\right] \mathrm{R}\left(2 \varepsilon_{\tau_{\ell-1}}\right)+\mathrm{R}^{\prime}\left(2 \varepsilon_{\tau_{\ell-1}}\right)\right) \\
& \quad \leq \varepsilon_{\tau_{\ell}} K_{j}^{\prime} e^{K_{j}^{\prime}}\left(\left[|\mu|+e^{-\mathrm{mc}}\right] \mathrm{R}\left(\varepsilon_{\tau_{\ell}}\right)+\mathrm{R}^{\prime}\left(\varepsilon_{\tau_{\ell}}\right)\right) \quad \text { by (F.3.a,e), (F.4.a) } \\
& \quad \leq \frac{1}{32} \mathrm{r}\left(\varepsilon_{\tau_{\ell}}\right) \leq \frac{1}{32} \mathrm{r}(|\mathcal{J}|) \quad \text { by (F.6.c), (F.3.b) }
\end{aligned}
$$

In the first inequality, we used Lemma D.1. In the second inequality we used that both $\tau$ and $\tau_{\ell}$ are in $\left[\tau_{\ell-1}-\varepsilon_{\tau_{\ell-1}}, \tau_{\ell-1}+\varepsilon_{\tau_{\ell-1}}\right]$ to bound $\tau_{\ell}-\tau$ by $2 \varepsilon_{\tau_{\ell-1}}$. In the third inequality we repeatedly used $t \mathrm{R}^{(\prime)}(t) \leq 2 t \mathrm{R}^{(\prime)}(2 t)$, which gives $t \mathrm{R}^{(\prime)}(t) \leq\left(2^{\ell} t\right) \mathrm{R}^{(\prime)}\left(2^{\ell} t\right)$ for all $\ell \in \mathbb{N}$.

For case (c), we use the bounds

$$
\begin{array}{r}
\left|\left(\left[j\left(\tau_{\ell}-\tau\right)-\mathbb{1}\right] \Lambda\left(\left[\tau_{\ell-1}, \tau_{\ell}\right]\right) \Lambda_{\tau_{\ell}}^{c} \alpha_{\tau_{\ell}}\right)(\mathbf{x})\right| \leq \frac{1}{16} \mathrm{r}(|\mathcal{J}|) \\
\left|\left(j\left(\tau_{\ell-1}-\tau\right) \Lambda_{\tau_{\ell-1}}^{c}\left[j\left(\tau_{\ell}-\tau_{\ell-1}\right)-\mathbb{1}\right] \Lambda\left(\left[\tau_{\ell-1}, \tau_{\ell}\right]\right) \Lambda_{\tau_{\ell}}^{c} \alpha_{\tau_{\ell}}\right)(\mathbf{x})\right| \leq \frac{1}{32} \mathrm{r}(|\mathcal{J}|)
\end{array}
$$

which are proven as above, together with

$$
\begin{aligned}
& \left|\left(j\left(\tau_{\ell-1}-\tau\right) \Lambda_{\tau_{\ell-1}}^{c} \Lambda\left(\left[\tau_{\ell-1}, \tau_{\ell}\right]\right)\left[\alpha_{\tau_{\ell}}-\alpha_{\tau_{\ell-1}}\right]\right)(\mathbf{x})\right| \\
& \leq e^{-\mathrm{m} d\left(\Lambda(\mathcal{J}), \Lambda_{\tau_{\ell-1}}^{c}\right)}\left\|j\left(\tau_{\ell-1}-\tau\right)\right\|\left|\sup _{\mathbf{y} \in \Lambda_{\tau_{\ell-1}^{c}}^{c} \cap \Lambda\left(\left[\tau_{\ell-1}, \tau_{\ell}\right]\right)}\right| \alpha_{\tau_{\ell}}(\mathbf{y})-\alpha_{\tau_{\ell-1}}(\mathbf{y}) \mid \\
& \leq e^{-\mathrm{m} d\left(\Lambda(\mathcal{J}), \Lambda_{\tau_{\ell-1}}^{c}\right)} e^{K_{j}} \mathrm{r}\left(\varepsilon_{\tau_{\ell-1}}\right) \\
& \leq e^{K_{j}} \begin{cases}\mathrm{r}\left(\varepsilon_{\tau_{\ell-1}}\right) \leq 2 \mathrm{r}(|\mathcal{J}|) & \text { if }|\mathcal{J}| \leq 2 \varepsilon_{\tau_{\ell-1}} \\
e^{-\operatorname{mc}\left(2 \varepsilon_{\tau_{\ell-1}}\right)} \mathrm{r}\left(\varepsilon_{\tau_{\ell-1}}\right) \leq 2 \varepsilon_{\tau_{\ell-1}} \mathfrak{v r}\left(\varepsilon_{\tau_{\ell-1}}\right) \leq 1 \leq \mathrm{r}(|\mathcal{J}|) & \text { if }|\mathcal{J}|>2 \varepsilon_{\tau_{\ell-1}}\end{cases}
\end{aligned}
$$

by (F.3.b), (F.4.c) and (F.6.a). For case (d) of Lemma E.8, it suffices to bound

$$
\begin{array}{r}
\left|\left(\left[j\left(\tau_{\ell}-\tau\right)-\mathbb{1}\right] \Lambda\left(\left[\tau_{\ell}, \tau_{\ell+1}\right]\right) \Lambda_{\tau_{\ell}}^{c}\left[\alpha_{\tau_{\ell}}-\alpha_{\tau_{\ell+1}}\right]\right)(\mathbf{x})\right| \\
\left|\left(\left[j\left(\tau_{\ell}-\tau\right)-\mathbb{1}\right] \Lambda\left(\left[\tau_{\ell}, \tau_{\ell+1}\right]\right) \Lambda_{\tau_{\ell+1}}^{c} \alpha_{\tau_{\ell+1}}\right)(\mathbf{x})\right| \leq \frac{1}{32} \mathrm{r}\left(\varepsilon_{\tau_{\ell}}\right) \\
\left.\mid\left(j\left(\varepsilon_{\ell}\right) \tau\right) \Lambda_{\tau_{\ell}}\left[j\left(\tau_{\ell+1}-\tau_{\ell}\right)-\mathbb{1}\right] \Lambda\left(\left[\tau_{\ell}, \tau_{\ell+1}\right]\right) \Lambda_{\tau_{\ell+1}}^{c} \alpha_{\tau_{\ell+1}}\right)(\mathbf{x}) \mid \leq \frac{1}{32} \mathrm{r}\left(\varepsilon_{\tau_{\ell}}\right)
\end{array}
$$

since, as we have observed, $|\mathcal{J}| \leq \varepsilon_{\tau_{\ell}}$. These bounds are proven as above. 
Corollary E.9 Assume that $\mathfrak{h} \equiv 1$.

(a) Let $0<\tau<\delta$. If $\mathbf{x} \in \Lambda_{\tau}^{c}$, then

$$
\left|\gamma_{* \tau}(\mathbf{x})-\gamma_{\tau}(\mathbf{x})^{*}\right| \leq e^{-\mathrm{mc}\left(\varepsilon_{\tau}\right)}
$$

Let $\mathcal{J}$ be a decimation interval of that contains $\tau$. If $\mathbf{x} \in \Lambda(\mathcal{J})$, then

$$
\left|\gamma_{* \tau}(\mathbf{x})-\gamma_{\tau}(\mathbf{x})^{*}\right| \leq\left(4 e^{K_{j}}+3\right) \operatorname{r}(|\mathcal{J}|)
$$

(b) Let $0<\tau<\delta$. If $\tau \in 2 \varepsilon \mathbb{Z}$ and $\mathbf{x} \in \Lambda(\mathcal{J})$, then

$$
\begin{aligned}
& \left|\gamma_{* \tau}(\mathbf{x})-\gamma_{\tau \pm \varepsilon}(\mathbf{x})^{*}\right| \leq\left(4 e^{K_{j}}+3\right) \mathrm{r}(|\mathcal{J}|) \\
& \left|\gamma_{\tau}(\mathbf{x})-\gamma_{* \tau \pm \varepsilon}(\mathbf{x})^{*}\right| \leq\left(4 e^{K_{j}}+3\right) \mathrm{r}(|\mathcal{J}|)
\end{aligned}
$$

If $\tau \pm \varepsilon \in\{0, \delta\}$ and $\mathbf{x} \in \Lambda(\mathcal{J})$, then

$$
\begin{aligned}
& \left|\gamma_{* \tau}(\mathbf{x})-\gamma_{\tau \pm \varepsilon}(\mathbf{x})^{*}\right| \leq 2\left(e^{K_{j}}+1\right) \mathrm{r}(|\mathcal{J}|) \\
& \left|\gamma_{\tau}(\mathbf{x})-\gamma_{* \tau \pm \varepsilon}(\mathbf{x})^{*}\right| \leq 2\left(e^{K_{j}}+1\right) \mathrm{r}(|\mathcal{J}|)
\end{aligned}
$$

(c) Let $0 \leq \tau<\delta$ and set

$$
\tilde{\gamma}_{\tau, \varepsilon}= \begin{cases}j(\tau) \alpha-j(\delta-\tau-\varepsilon) \Lambda\left(\left[\frac{\delta}{2}, \delta\right]\right) \beta & \text { if } 0 \leq \tau<\frac{\delta}{2}-\varepsilon \\ j(\tau) \alpha-j(\delta-\tau-\varepsilon) \beta & \text { if } \tau=\frac{\delta}{2}-\varepsilon, \frac{\delta}{2} \\ j(\tau) \Lambda\left(\left[0, \frac{\delta}{2}\right]\right) \alpha-j(\delta-\tau-\varepsilon) \beta & \text { if } \frac{\delta}{2}<\tau<\delta\end{cases}
$$

and

$$
\tilde{\tilde{\gamma}}_{\tau, \varepsilon}=j(\tau) \tilde{\Omega} \alpha-j(\delta-\tau-\varepsilon) \tilde{\Omega} \beta
$$

where $\tilde{\Omega}$ the set of all point in $X$ that are within a distance $\mathfrak{c}(\delta)$ of $\Omega([0, \delta])$. Then

$$
\sum_{\mathbf{x} \in \Lambda}\left|\gamma_{* \tau}(\mathbf{x})^{*}-\gamma_{\tau+\varepsilon}(\mathbf{x})-\tilde{\gamma}_{\tau, \varepsilon}(\mathbf{x})\right| \leq 3 e^{K_{j}} \mathrm{r}(\delta)\left|\Lambda^{c}\right|
$$

and

$$
\sum_{\mathbf{x} \in \Lambda} e^{-4 \mathrm{~m} d(\mathbf{x}, \Omega)}\left|\tilde{\gamma}_{\tau, \varepsilon}(\mathbf{x})-\tilde{\tilde{\gamma}}_{\tau, \varepsilon}(\mathbf{x})\right| \leq \frac{1}{8} e^{-3 \mathrm{mc}(\delta)}\left|\tilde{\Omega}^{c}\right|
$$


Proof: (a) The result for $\mathbf{x} \in \Lambda_{\tau}^{c}$ follows immediately from Proposition E.7, so we restrict to $\mathbf{x} \in \Lambda_{\tau}$ for the rest of the proof.

To prove the bound on $\left|\gamma_{* \tau}(\mathbf{x})-\gamma_{\tau}(\mathbf{x})^{*}\right|$ it suffices to prove that $\left|\hat{\gamma}_{* \tau}(\mathbf{x})-\hat{\gamma}_{\tau}(\mathbf{x})^{*}\right| \leq$ $\mathrm{r}(|\mathcal{J}|)$, which we now proceed to do. By Definition E.6 and Lemma E.4.ii, for $\mathbf{x} \in \Lambda(\mathcal{J}) \subset$ $\Lambda_{\tau}$

$$
\hat{\gamma}_{* \tau}(\mathbf{x})-\hat{\gamma}_{\tau}(\mathbf{x})^{*}=\sum_{\substack{\tau_{r} \in \mathcal{T}_{r}(\tau, \delta) \\ \tau_{l} \in \mathcal{T}_{l}(\tau, \delta)}} \Lambda_{\pi\left(\tau_{r}\right)}(\mathbf{x}) \Lambda_{\tau_{r}}^{c}(\mathbf{x}) \Lambda_{\sigma\left(\tau_{l}\right)}(\mathbf{x}) \Lambda_{\tau_{l}}^{c}(\mathbf{x})\left[\alpha_{\tau_{l}}(\mathbf{x})^{*}-\alpha_{\tau_{r}}(\mathbf{x})^{*}\right]
$$

and furthermore, since $\bigcup_{\substack{\tau_{r} \in \mathcal{T}_{r} \\ \tau_{l} \in \mathcal{T}_{l}}} \Lambda_{\pi\left(\tau_{r}\right)} \cap \Lambda_{\tau_{r}}^{c} \cap \Lambda_{\sigma\left(\tau_{l}\right)} \cap \Lambda_{\tau_{l}}^{c}$ is a partition of $\Lambda_{\tau}$ into disjoint subsets, at most one term in this sum is nonzero and it is

$$
\Lambda\left(\left[\tau_{l}, \tau_{r}\right]\right)(\mathbf{x}) \Lambda_{\tau_{l}}^{c}(\mathbf{x}) \Lambda_{\tau_{r}}^{c}(\mathbf{x})\left[\alpha_{\tau_{l}}(\mathbf{x})^{*}-\alpha_{\tau_{r}}(\mathbf{x})^{*}\right]
$$

This term is bounded by $\mathrm{r}\left(\tau_{r}-\tau_{l}\right)$. If $|\mathcal{J}|>\tau_{r}-\tau_{l}$, then at least one of $\tau_{l}, \tau_{r}$ must be in the interior of $\mathcal{J}$. If, for example, $\tau_{l}$ is in the interior of $\mathcal{J}$, then $\left[\tau_{l}-\varepsilon_{\tau_{l}}, \tau_{l}+\varepsilon_{\tau_{l}}\right] \subset \mathcal{J}$ so that $\Lambda(\mathcal{J}) \subset \Lambda_{\tau_{l}}$ and our one potentially nonzero term is in fact zero for all $\mathbf{x} \in \Lambda(\mathcal{J})$. Hence the one possibly nonzero term is bounded by $\mathrm{r}(|\mathcal{J}|)$.

(b) To prove the bound on $\left|\gamma_{* \tau}(\mathbf{x})-\gamma_{\tau \pm \varepsilon}(\mathbf{x})^{*}\right|$ in the case that $\tau \in 2 \varepsilon \mathbb{Z}$, we prove that $\left|\hat{\gamma}_{* \tau}(\mathbf{x})-\hat{\gamma}_{\tau \pm \varepsilon}(\mathbf{x})^{*}\right| \leq \mathrm{r}(|\mathcal{J}|)$. Again, by Definition E.6 and Lemma E.4.ii, for $\mathbf{x} \in \Lambda(\mathcal{J}) \subset$ $\Lambda_{\tau} \subset \Lambda_{\tau \pm \varepsilon}$,

$$
\hat{\gamma}_{* \tau}(\mathbf{x})-\hat{\gamma}_{\tau \pm \varepsilon}(\mathbf{x})^{*}=\sum_{\substack{\tau_{r} \in \mathcal{T}_{r}(\tau \pm \varepsilon, \delta) \\ \tau_{l} \in \mathcal{T}_{l}(\tau, \delta)}} \Lambda_{\pi\left(\tau_{r}\right)}(\mathbf{x}) \Lambda_{\tau_{r}}^{c}(\mathbf{x}) \Lambda_{\sigma\left(\tau_{l}\right)}(\mathbf{x}) \Lambda_{\tau_{l}}^{c}(\mathbf{x})\left[\alpha_{\tau_{l}}(\mathbf{x})^{*}-\alpha_{\tau_{r}}(\mathbf{x})^{*}\right]
$$

○ If $\tau_{r} \in \mathcal{T}_{r}(\tau \pm \varepsilon, \delta)$ happens to have $\varepsilon_{\tau_{r}}<\varepsilon_{\tau}$, then, $\Lambda_{\tau_{r}}$ has $\tau$ as an end point and is properly contained in $\left[\tau-\varepsilon_{\tau}, \tau+\varepsilon_{\tau}\right]$. Hence $\Lambda_{\tau} \subset \Lambda_{\tau_{r}}$ and $\Lambda_{\tau_{r}}^{c} \Lambda_{\sigma\left(\tau_{l}\right)}=0$ for all $\tau_{l} \in \mathcal{T}_{l}(\tau, \delta)$

○ No $\tau_{r} \in \mathcal{T}_{r}(\tau+\varepsilon, \delta)$ can have $\varepsilon_{\tau_{r}}=\varepsilon_{\tau}$, because the first element of $\varepsilon_{\tau_{r}} \mathbb{Z}=\varepsilon_{\tau} \mathbb{Z}$ above $\tau+\varepsilon$ is $\tau+\varepsilon_{\tau}$, which does not satisfy $\varepsilon_{\tau+\varepsilon_{\tau}}=\varepsilon_{\tau}$.

○ If $\tau_{r} \in \mathcal{T}_{r}(\tau-\varepsilon, \delta)$ happens to have $\varepsilon_{\tau_{r}}=\varepsilon_{\tau}$, then $\tau_{r}=\tau$ and $\Lambda_{\tau_{r}}^{c} \Lambda_{\sigma\left(\tau_{l}\right)}=\Lambda_{\tau}^{c} \Lambda_{\sigma\left(\tau_{l}\right)}=0$ for all $\tau_{l} \in \mathcal{T}_{l}(\tau, \delta)$.

○ If $\tau_{r} \in \mathcal{T}_{r}(\tau \pm \varepsilon, \delta)$ happens to have $\varepsilon_{\tau_{r}}>\varepsilon_{\tau}$, then, because $\tau \pm \varepsilon$ is a nearest neighbour of $\tau$ and is in $\varepsilon \mathbb{Z} \backslash 2 \varepsilon \mathbb{Z}$, it is necessary that $\tau_{r}>\tau$ and indeed $\tau_{r}$ is the smallest element of $\varepsilon_{\tau_{r}} \mathbb{Z}$ above $\tau$ too so that $\tau_{r} \in \mathcal{T}_{r}(\tau, \delta)$ too. Conversely, every $\tau_{r} \in \mathcal{T}_{r}(\tau, \delta)$ is also in $\mathcal{T}_{r}(\tau \pm \varepsilon, \delta)$. Denote by $\pi^{\prime}\left(\tau_{r}\right)$ and $\pi\left(\tau_{r}\right)$ the predecessor elements of $\tau_{r}$ in $\mathcal{T}_{r}(\tau \pm \varepsilon, \delta)$ and $\mathcal{T}_{r}(\tau, \delta)$ respectively. If $\varepsilon_{\pi^{\prime}\left(\tau_{r}\right)} \geq \varepsilon_{\pi\left(\tau_{r}\right)}$, then $\pi^{\prime}\left(\tau_{r}\right)=\pi\left(\tau_{r}\right)$. If $\varepsilon_{\pi^{\prime}\left(\tau_{r}\right)}<\varepsilon_{\pi\left(\tau_{r}\right)}$, then $\pi\left(\tau_{r}\right)=\tau$ and $\Lambda_{\pi^{\prime}\left(\tau_{r}\right)}(\mathbf{x})=\Lambda_{\pi\left(\tau_{r}\right)}(\mathbf{x})=1$. 
Hence, once again,

$$
\begin{aligned}
\hat{\gamma}_{* \tau}(\mathbf{x})-\hat{\gamma}_{\tau \pm \varepsilon}(\mathbf{x})^{*} & =\sum_{\substack{\tau_{r} \in \mathcal{T}_{r}(\tau, \delta) \\
\tau_{l} \in \mathcal{T}_{l}(\tau, \delta)}} \Lambda_{\pi\left(\tau_{r}\right)}(\mathbf{x}) \Lambda_{\tau_{r}}^{c}(\mathbf{x}) \Lambda_{\sigma\left(\tau_{l}\right)}(\mathbf{x}) \Lambda_{\tau_{l}}^{c}(\mathbf{x})\left[\alpha_{\tau_{l}}(\mathbf{x})^{*}-\alpha_{\tau_{r}}(\mathbf{x})^{*}\right] \\
& =\hat{\gamma}_{* \tau}(\mathbf{x})-\hat{\gamma}_{\tau}(\mathbf{x})^{*}
\end{aligned}
$$

and the desired result follows from $\left|\hat{\gamma}_{* \tau}(\mathbf{x})-\hat{\gamma}_{\tau}(\mathbf{x})^{*}\right| \leq \mathrm{r}(|\mathcal{J}|)$.

Finally, we prove the bound on $\left|\gamma_{* \tau}(\mathbf{x})-\gamma_{\tau \pm \varepsilon}(\mathbf{x})^{*}\right|$ in the case that $\tau \pm \varepsilon \in\{0, \delta\}$. In this case, either $\tau=\varepsilon$ so that $\gamma_{\tau \pm \varepsilon}(\mathbf{x})^{*}=\alpha_{0}(\mathbf{x})^{*} \equiv \alpha(\mathbf{x})^{*}$ or $\tau=\delta-\varepsilon$ so that $\gamma_{\tau \pm \varepsilon}(\mathbf{x})^{*}=\alpha_{\delta}(\mathbf{x})^{*} \equiv \beta(\mathbf{x})^{*}$. We prove that $\left|\hat{\gamma}_{* \tau}(\mathbf{x})-\alpha_{\tau \pm \varepsilon}(\mathbf{x})^{*}\right| \leq \operatorname{r}(|\mathcal{J}|)$. Again, by Definition E.6 and Lemma E.4.ii, for $\mathbf{x} \in \Lambda(\mathcal{J}) \subset \Lambda_{\tau}$,

$$
\hat{\gamma}_{* \tau}(\mathbf{x})-\hat{\gamma}_{\tau \pm \varepsilon}(\mathbf{x})^{*}=\sum_{\tau_{l} \in \mathcal{T}_{l}(\tau, \delta)} \Lambda_{\sigma\left(\tau_{l}\right)}(\mathbf{x}) \Lambda_{\tau_{l}}^{c}(\mathbf{x})\left[\alpha_{\tau_{l}}(\mathbf{x})^{*}-\alpha_{\tau \pm \varepsilon}(\mathbf{x})^{*}\right]
$$

In the case that $\tau=\varepsilon, \mathcal{T}_{l}(\tau, \delta)$ contains exactly one element, namely $\tau_{l}=0=\tau \pm \varepsilon$, and the right hand side is exactly zero. In the case that $\tau=\delta-\varepsilon, \mathcal{T}_{l}(\tau, \delta)=\left\{\delta-2^{j} \varepsilon \mid 1 \leq j \leq n\right\}$ so that

$$
\begin{aligned}
\hat{\gamma}_{* \tau}(\mathbf{x})-\hat{\gamma}_{\tau \pm \varepsilon}(\mathbf{x})^{*} & =\sum_{j=1}^{n} \Lambda_{\delta-2^{j-1} \varepsilon}(\mathbf{x}) \Lambda_{\delta-2^{j} \varepsilon}^{c}(\mathbf{x})\left[\alpha_{\delta-2^{j} \varepsilon}(\mathbf{x})^{*}-\alpha_{\delta}(\mathbf{x})^{*}\right] \\
& =\sum_{j=1}^{n} \Lambda\left(\left[\delta-2^{j} \varepsilon, \delta\right]\right)(\mathbf{x}) \Lambda_{\delta-2^{j} \varepsilon}^{c}(\mathbf{x})\left[\alpha_{\delta-2^{j} \varepsilon}(\mathbf{x})^{*}-\alpha_{\delta}(\mathbf{x})^{*}\right]
\end{aligned}
$$

Since $\bigcup_{j=1}^{n} \Lambda_{\delta-2^{j-1}{ }_{\varepsilon}} \Lambda_{\delta-2^{j} \varepsilon}^{c}$ is a partition of $\Lambda_{\delta-\varepsilon}=\Lambda_{\tau}$ into disjoint subsets, at most one term in this sum is nonzero. If this term is the $j^{\text {th }}$, it is bounded by $\operatorname{r}\left(2^{j} \varepsilon\right)$. If $|\mathcal{J}|>2^{j} \varepsilon$, then $\Lambda(\mathcal{J}) \subset \Lambda_{\delta-2^{j} \varepsilon}$ and our one potentially nonzero term is in fact zero for all $\mathbf{x} \in \Lambda(\mathcal{J})$. Hence the one possibly nonzero term is bounded by $\mathrm{r}(|\mathcal{J}|)$.

(c, first bound) It suffices to combine parts (e), (f) and (g) of Lemma E.8 with the bounds

$$
\begin{aligned}
\sum_{\mathbf{x} \in \Lambda} \mid\left(j\left(\frac{\delta}{2}-\tau-\varepsilon\right)\right. & \left.\Lambda^{c} \Lambda\left(\left[\frac{\delta}{2}, \delta\right]\right)\left[\alpha_{\frac{\delta}{2}}-\beta\right]\right)(\mathbf{x}) \mid \\
& \leq \sum_{\substack{\mathbf{x} \in \Lambda \\
\mathbf{y} \in \Lambda^{c} \Lambda\left(\left[\frac{\delta}{2}, \delta\right]\right)}}\left|j\left(\frac{\delta}{2}-\tau-\varepsilon\right)(\mathbf{x}, \mathbf{y})\right|\left|\left[\alpha_{\frac{\delta}{2}}-\beta\right](\mathbf{y})\right| \\
& \leq \sum_{\substack{\mathbf{x} \in \Lambda \\
\mathbf{y} \in \Lambda^{c} \Lambda\left(\left[\frac{\delta}{2}, \delta\right]\right)}}\left|j\left(\frac{\delta}{2}-\tau-\varepsilon\right)(\mathbf{x}, \mathbf{y})\right| \mathrm{r}\left(\frac{\delta}{2}\right) \\
& \leq\left\|j\left(\frac{\delta}{2}-\tau-\varepsilon\right)\right\||| \Lambda^{c}\left|\mathrm{r}\left(\frac{\delta}{2}\right) \leq 2 e^{K_{j}} \mathrm{r}(\delta)\right| \Lambda^{c} \mid
\end{aligned}
$$


and, using Lemma D.1,

$$
\begin{aligned}
& \sum_{\mathbf{x} \in \Lambda}\left|\left(j\left(\frac{\delta}{2}-\tau-\varepsilon\right) \Lambda^{c}\left[j\left(\frac{\delta}{2}\right)-\mathbb{1}\right] \Lambda\left(\left[\frac{\delta}{2}, \delta\right]\right) \beta\right)(\mathbf{x})\right| \\
& \leq \sum_{\substack{\mathbf{x} \in \Lambda \\
\mathbf{y} \in \Lambda^{c}}}\left|j\left(\frac{\delta}{2}-\tau-\varepsilon\right)(\mathbf{x}, \mathbf{y})\right|\left|\left\{\left[j\left(\frac{\delta}{2}\right)-\mathbb{1}\right] \Lambda\left(\left[\frac{\delta}{2}, \delta\right]\right) \beta\right\}(\mathbf{y})\right| \\
& \leq \sum_{\substack{\mathbf{x} \in \Lambda \\
\mathbf{y} \in \Lambda^{c}}}\left|j\left(\frac{\delta}{2}-\tau-\varepsilon\right)(\mathbf{x}, \mathbf{y})\right| e^{5 \mathrm{~m} d(\mathbf{x}, \mathbf{y})} \frac{\delta}{2} K_{j}^{\prime} e^{K_{j}^{\prime} \frac{\delta}{2}} \\
& e^{-5 \operatorname{md} d(\Lambda, \mathbf{y})}\left(\left[|\mu|+e^{-5 \operatorname{md} d\left(\mathbf{y}, \Lambda\left(\left[\frac{\delta}{2}, \delta\right]\right)^{c}\right)}\right] \max _{\mathbf{z} \in \Lambda\left(\left[\frac{\delta}{2}, \delta\right]\right)}|\beta(\mathbf{z})|+\max _{b \in \Lambda\left(\left[\frac{\delta}{2}, \delta\right]\right)^{*}}|\nabla \beta(b)|\right) \\
& \leq\left\|j\left(\frac{\delta}{2}-\tau-\varepsilon\right)\right\||| \Lambda^{c} \mid \frac{\delta}{2} e^{2 K_{j}^{\prime}}\left(\left[|\mu|+e^{-5 \operatorname{m} d\left(\Lambda, \Lambda\left(\left[\frac{\delta}{2}, \delta\right]\right)^{c}\right)}\right] \mathrm{R}\left(\frac{\delta}{2}\right)+\mathrm{R}^{\prime}\left(\frac{\delta}{2}\right)\right) \\
& \leq \frac{\delta}{2} e^{K_{j}} e^{2 K_{j}^{\prime}}\left(\left[|\mu|+e^{-5 \mathrm{mc}(\delta / 2)}\right] \mathrm{R}\left(\frac{\delta}{2}\right)+\mathrm{R}^{\prime}\left(\frac{\delta}{2}\right)\right)\left|\Lambda^{c}\right| \\
& \leq \frac{1}{32} \mathrm{r}\left(\frac{\delta}{2}\right)\left|\Lambda^{c}\right| \leq \frac{1}{16} \mathrm{r}(\delta)\left|\Lambda^{c}\right| \quad \text { by (F.4.a), (F.6.c), (F.3.b) }
\end{aligned}
$$

when $0 \leq \tau<\frac{\delta}{2}-\varepsilon$ and the similar bounds

$$
\begin{gathered}
\sum_{\mathbf{x} \in \Lambda}\left|\left(j\left(\tau-\frac{\delta}{2}\right) \Lambda^{c} \Lambda\left(\left[0, \frac{\delta}{2}\right]\right)\left[\alpha_{\frac{\delta}{2}}-\alpha\right]\right)(\mathbf{x})\right| \leq 2 e^{K_{j}} \mathrm{r}(\delta)\left|\Lambda^{c}\right| \\
\sum_{\mathbf{x} \in \Lambda}\left|\left(j\left(\tau-\frac{\delta}{2}\right) \Lambda^{c}\left[j\left(\frac{\delta}{2}\right)-\mathbb{1}\right] \Lambda\left(\left[0, \frac{\delta}{2}\right]\right) \alpha\right)(\mathbf{x})\right| \leq \frac{1}{16} \mathrm{r}(\delta)\left|\Lambda^{c}\right|
\end{gathered}
$$

when $\frac{\delta}{2}<\tau<\delta$. We have used the compatibility assumption of Definition E.1. Since $\left(2 e^{K_{j}}+2 e^{-\operatorname{mc}(\delta)}+\frac{1}{16}\right) \mathrm{r}(\delta)\left|\Lambda^{c}\right| \leq 3 e^{K_{j}} \mathrm{r}(\delta)\left|\Lambda^{c}\right|$, by (F.4.c), the desired results follow.

(c, second bound) We have

$$
\tilde{\gamma}_{\tau, \varepsilon}-\tilde{\tilde{\gamma}}_{\tau, \varepsilon}= \begin{cases}j(\tau) \tilde{\Omega}^{c} \alpha-j(\delta-\tau-\varepsilon) \tilde{\Omega}^{c} \Lambda\left(\left[\frac{\delta}{2}, \delta\right]\right) \beta & \text { if } 0 \leq \tau<\frac{\delta}{2}-\varepsilon \\ j(\tau) \tilde{\Omega}^{c} \alpha-j(\delta-\tau-\varepsilon) \tilde{\Omega}^{c} \beta & \text { if } \tau=\frac{\delta}{2}-\varepsilon, \frac{\delta}{2} \\ j(\tau) \tilde{\Omega}^{c} \Lambda\left(\left[0, \frac{\delta}{2}\right]\right) \alpha-j(\delta-\tau-\varepsilon) \tilde{\Omega}^{c} \beta & \text { if } \frac{\delta}{2}<\tau<\delta\end{cases}
$$

All terms are bounded in the same way. For example, by Lemma B.1.i and Definition II.13,

$$
\begin{aligned}
\sum_{\mathbf{x} \in \Lambda} e^{-4 \mathrm{~m} d(\mathbf{x}, \Omega)}\left|\left(j(\tau) \tilde{\Omega}^{c} \alpha\right)(\mathbf{x})\right| & \leq \sum_{\substack{\mathbf{x} \in \Lambda \\
\mathbf{y} \in \tilde{\Omega}^{c}}} e^{-4 \mathrm{~m} d(\mathbf{x}, \Omega)}|j(\tau)(\mathbf{x}, \mathbf{y})| \kappa_{* 0}(\mathbf{y}) \\
& \leq 4 \mathrm{R}(\delta) \sum_{\substack{\mathbf{x} \in \Lambda \\
\mathbf{y} \in \tilde{\Omega}^{c}}}|j(\tau)(\mathbf{x}, \mathbf{y})| e^{\frac{\mathrm{m}}{3} d(\mathbf{x}, \mathbf{y})} e^{4 \operatorname{m} d(\mathbf{x}, \mathbf{y})} e^{-4 \mathrm{~m} d(\mathbf{y}, \Omega)} \\
& \leq 4\left|\left\|j(\tau)\left|\| e^{-4 \mathrm{~m} d\left(\Omega, \tilde{\Omega}^{c}\right)} \mathrm{R}(\delta)\right| \tilde{\Omega}^{c} \mid\right.\right. \\
& \leq 4 e^{K_{j}} e^{-4 \mathrm{~m} \mathfrak{c}(\delta)} \mathrm{R}(\delta)\left|\tilde{\Omega}^{c}\right| \\
& \leq \frac{1}{16} e^{-3 \operatorname{mc}(\delta)}\left|\tilde{\Omega}^{c}\right| \quad \text { by }(\mathrm{F} .6 . \mathrm{b})
\end{aligned}
$$


Lemma E.10 Assume that $\mathfrak{h} \equiv 1$.

(i) Let $t \in \varepsilon \mathbb{Z} \cap(0, \delta)$ and $\mathbf{x} \in \Lambda_{t}^{c}$ with

$$
d\left(\mathbf{x}, \Lambda_{t}\right) \geq \mathfrak{c}\left(\varepsilon_{t}\right) \quad d\left(\mathbf{x}, \Lambda\left(\left[t-\varepsilon_{t}, t\right]\right)^{c}\right) \geq \mathfrak{c}\left(\varepsilon_{t}\right) \quad d\left(\mathbf{x}, \Lambda\left(\left[t, t+\varepsilon_{t}\right]\right)^{c}\right) \geq \mathfrak{c}\left(\varepsilon_{t}\right)
$$

(If $t \notin 2 \varepsilon \mathbb{Z}$, then $\Lambda\left(\left[t-\varepsilon_{t}, t\right]\right)^{c}=\Lambda\left(\left[t-\varepsilon_{t}, t\right]\right)^{c}=\emptyset$ and the conditions $d\left(\mathbf{x}, \Lambda\left(\left[t-\varepsilon_{t}, t\right]\right)^{c}\right) \geq$ $\mathfrak{c}\left(\varepsilon_{t}\right)$ and $d\left(\mathbf{x}, \Lambda\left(\left[t, t+\varepsilon_{t}\right]\right)^{c}\right) \geq \mathfrak{c}\left(\varepsilon_{t}\right)$ are vacuous. $)$ Then

$$
\begin{aligned}
\left|\gamma_{\tau}(\mathbf{x})-\left(j\left(t+\varepsilon_{t}-\tau\right) B_{\mathbf{x}, \varepsilon_{t}} \Lambda_{t+\varepsilon_{t}}^{c} \alpha_{t+\varepsilon_{t}}\right)(\mathbf{x})\right| \leq e^{-\mathrm{mc}\left(\varepsilon_{t}\right)} & \text { if } t<\tau \leq t+\varepsilon_{t} \\
\left|\gamma_{\tau}(\mathbf{x})-\left(j(t-\tau) B_{\mathbf{x}, \varepsilon_{t}} \Lambda_{t}^{c} \alpha_{t}\right)(\mathbf{x})\right| \leq e^{-\mathrm{mc}\left(\varepsilon_{t}\right)} & \text { if } t-\varepsilon_{t}<\tau \leq t \\
\left|\gamma_{* \tau}(\mathbf{x})-\left(j(\tau-t) B_{\mathbf{x}, \varepsilon_{t}} \Lambda_{t}^{c} \alpha_{t}^{*}\right)(\mathbf{x})\right| \leq e^{-\mathrm{mc}\left(\varepsilon_{t}\right)} & \text { if } t \leq \tau<t+\varepsilon_{t} \\
\left|\gamma_{* \tau}(\mathbf{x})-\left(j\left(\tau-t+\varepsilon_{t}\right) B_{\mathbf{x}, \varepsilon_{t}} \Lambda_{t-\varepsilon_{t}}^{c} \alpha_{t-\varepsilon_{t}}^{*}\right)(\mathbf{x})\right| \leq e^{-\mathrm{mc}\left(\varepsilon_{t}\right)} & \text { if } t-\varepsilon_{t} \leq \tau<t
\end{aligned}
$$

for any set

$$
B_{\mathbf{x}, \varepsilon_{t}} \supset\left\{\mathbf{y} \in X \mid d(\mathbf{x}, \mathbf{y}) \leq \mathfrak{c}\left(\varepsilon_{t}\right)\right\}
$$

(ii) Let $\mathbf{x} \in X$ obey $d\left(\mathbf{x}, \Lambda([0, \delta])^{c}\right) \geq \mathfrak{c}(\delta)$. Then

$$
\begin{aligned}
\left|\gamma_{\tau}(\mathbf{x})-\left(j(\delta-\tau) B_{\mathbf{x}, \delta} \beta\right)(\mathbf{x})\right| \leq e^{-\operatorname{mc}(\delta)} & \text { if } 0<\tau<\delta \\
\left|\gamma_{* \tau}(\mathbf{x})-\left(j(\tau) B_{\mathbf{x}, \delta} \alpha^{*}\right)(\mathbf{x})\right| \leq e^{-\operatorname{mc}(\delta)} & \text { if } 0<\tau<\delta
\end{aligned}
$$

for any set

$$
B_{\mathbf{x}, \delta} \supset\{\mathbf{y} \in T \mid d(\mathbf{x}, \mathbf{y}) \leq \mathfrak{c}(\delta)\}
$$

Proof: (i) We prove the first bound. The proofs of the other three bounds are very similar. Fix any $\tau \in \varepsilon \mathbb{Z}$ obeying $t<\tau \leq t+\varepsilon_{t}$. By Definition II.9, $\gamma_{\tau}(\mathbf{x})=\sum_{\tau^{\prime} \geq \tau}\left(\Gamma_{\tau}^{\tau^{\prime}} \alpha_{\tau^{\prime}}\right)(\mathbf{x})$. Fix any $\tau \leq \tau^{\prime} \leq \delta$ with $\Gamma_{\tau}^{\tau^{\prime}} \neq 0$. By Lemma E.4.i, $\tau^{\prime} \in\{\tau\} \cup \mathcal{T}_{r}(\tau, \delta)$. Set

$L_{x}=\left\{\mathbf{x} \in X \mid d\left(\mathbf{x}, \Lambda_{t}\right) \geq \mathfrak{c}\left(\varepsilon_{t}\right), d\left(\mathbf{x}, \Lambda\left(\left[t-\varepsilon_{t}, t\right]\right)^{c}\right) \geq \mathfrak{c}\left(\varepsilon_{t}\right), d\left(\mathbf{x}, \Lambda\left(\left[t, t+\varepsilon_{t}\right]\right)^{c}\right) \geq \mathfrak{c}\left(\varepsilon_{t}\right)\right\}$

when $t \in 2 \varepsilon \mathbb{Z}$ and $L_{x}=\left\{\mathbf{x} \in X \mid d\left(\mathbf{x}, \Lambda_{t}\right) \geq \mathfrak{c}(\varepsilon)\right\}$ when $t \notin 2 \varepsilon \mathbb{Z}$.

Case 1: $\tau \leq \tau^{\prime}<t+\varepsilon_{t}$. In this case $\varepsilon_{\tau^{\prime}}<\varepsilon_{t}$, (as is true for all times in $\left(t, t+\varepsilon_{t}\right)$ ) so that any $\mathbf{x} \in L_{x}$ obeys

$$
\mathbf{x} \in \Lambda\left(\left[t, t+\varepsilon_{t}\right]\right) \subset \Lambda_{\tau^{\prime}}
$$

By our rules for constructing small field sets, either $\Lambda_{\tau^{\prime}}^{c}=\emptyset$, or $\Lambda\left(\left[t, t+\varepsilon_{t}\right]\right)=\Lambda_{\tau^{\prime}}$ (in which case $\varepsilon_{\tau^{\prime}}=\frac{1}{2} \varepsilon_{t}$ and the distance from $\mathbf{x}$ to $\Lambda_{\tau^{\prime}}^{c}$ is at least $\mathfrak{c}\left(\varepsilon_{t}\right)=\mathfrak{c}\left(2 \varepsilon_{\tau^{\prime}}\right)$ ) or 
$d\left(\Lambda\left(\left[t, t+\varepsilon_{t}\right]\right), \Lambda_{\tau^{\prime}}^{c}\right) \geq \mathfrak{c}\left(2 \varepsilon_{\tau^{\prime}}\right)$. So the distance from $L_{x}$ to $\Lambda_{\tau^{\prime}}^{c}$ is at least $\mathfrak{c}\left(2 \varepsilon_{\tau^{\prime}}\right)$, unless $\Lambda_{\tau^{\prime}}^{c} \equiv 0$. Therefore, for $\tau=\tau^{\prime},\left(\Gamma_{\tau}^{\tau^{\prime}} \alpha_{\tau^{\prime}}\right)(\mathbf{x})=0$ and for $\tau<\tau^{\prime}$,

$$
\begin{aligned}
\left|\left(\Gamma_{\tau}^{\tau^{\prime}} \alpha_{\tau^{\prime}}\right)(\mathbf{x})\right| & =\left|\left(j\left(\pi\left(\tau^{\prime}\right)-\tau\right) \Lambda_{\pi\left(\tau^{\prime}\right)} j\left(\tau^{\prime}-\pi\left(\tau^{\prime}\right)\right) \Lambda_{\tau^{\prime}}^{c} \alpha_{\tau^{\prime}}\right)(\mathbf{x})\right| \\
& \leq N_{0}\left(L_{x} j\left(\pi\left(\tau^{\prime}\right)-\tau\right) \Lambda_{\pi\left(\tau^{\prime}\right)} j\left(\tau^{\prime}-\pi\left(\tau^{\prime}\right)\right) \Lambda_{\tau^{\prime}}^{c} ; 1, \kappa_{\tau^{\prime}}\right) \\
& \leq 4 \mathrm{R}\left(\varepsilon_{t}\right) e^{-4 \mathrm{mc}\left(2 \varepsilon_{\tau^{\prime}}\right)} e^{K_{j}\left(\tau^{\prime}-\tau\right)}
\end{aligned}
$$

by Lemma G.5.ii with $\kappa=\kappa_{\tau^{\prime}}, \mathrm{R}=4 \mathrm{R}\left(\varepsilon_{t}\right), L_{1}=L_{x} \subset \Lambda\left(\left[t, t+\varepsilon_{t}\right]\right)=O_{2}, L_{2}=\Lambda_{\pi\left(\tau^{\prime}\right)}$ and $L_{3}=\Lambda_{\tau^{\prime}}^{c}$. For this and all other applications of the lemma in this proof, $d$ is replaced by $0, \delta_{1}=0, \delta_{2}=\frac{\mathrm{m}}{3} d, \delta=4 \mathrm{~m} d$ and $\tilde{d}=5 \mathrm{~m} d$.

Case 2: $\tau^{\prime}>t+\varepsilon_{t}$. In this case $2 \varepsilon_{t}<2 \varepsilon_{\pi\left(\tau^{\prime}\right)} \leq \varepsilon_{\tau^{\prime}}$ ( since all times $t^{\prime} \in\left(\tau^{\prime}-2 \varepsilon_{\pi\left(\tau^{\prime}\right)}, \tau^{\prime}\right)$, including $t+\varepsilon_{t}$ have $\left.\varepsilon_{t^{\prime}}<2 \varepsilon_{\pi\left(\tau^{\prime}\right)}\right)$. Now $t-\varepsilon_{t}$ is the element of $2 \varepsilon_{t} \mathbb{Z}$ below $t+\varepsilon_{t}$ that is nearest $t+\varepsilon_{t}$. As $\tau^{\prime}-2 \varepsilon_{\pi\left(\tau^{\prime}\right)}$ is an element of $2 \varepsilon_{t} \mathbb{Z}$ that is below $t+\varepsilon_{t}$, we have $\tau^{\prime}-2 \varepsilon_{\pi\left(\tau^{\prime}\right)} \leq t-\varepsilon_{t}<t+\varepsilon_{t}<\tau^{\prime}$. Hence $\Lambda_{\pi\left(\tau^{\prime}\right)}=\Lambda\left(\left[\tau^{\prime}-2 \varepsilon_{\pi\left(\tau^{\prime}\right)}, \tau^{\prime}\right]\right) \subset \Lambda_{t}$. So the distance from $L_{x}$ to $\Lambda_{\pi\left(\tau^{\prime}\right)}$ is at least $\mathfrak{c}\left(\varepsilon_{t}\right)$. Therefore,

$$
\begin{aligned}
\left|\left(\Gamma_{\tau}^{\tau^{\prime}} \alpha_{\tau^{\prime}}\right)(\mathbf{x})\right| & =\left|\left(j\left(\pi\left(\tau^{\prime}\right)-\tau\right) \Lambda_{\pi\left(\tau^{\prime}\right)} j\left(\tau^{\prime}-\pi\left(\tau^{\prime}\right)\right) \Lambda_{\tau^{\prime}}^{c} \alpha_{\tau^{\prime}}\right)(\mathbf{x})\right| \\
& \leq N_{0}\left(L_{x} j\left(\pi\left(\tau^{\prime}\right)-\tau\right) \Lambda_{\pi\left(\tau^{\prime}\right)} j\left(\tau^{\prime}-\pi\left(\tau^{\prime}\right)\right) \Lambda_{\tau^{\prime}}^{c} ; 1, \kappa_{\tau^{\prime}}\right) \\
& \leq 4 \mathrm{R}\left(2 \varepsilon_{\pi\left(\tau^{\prime}\right)}\right) e^{-4 \operatorname{mc}\left(\varepsilon_{t}\right)} e^{K_{j}\left(\tau^{\prime}-\tau\right)}
\end{aligned}
$$

by Lemma G.5.ii with $\mathrm{R}=4 \mathrm{R}\left(2 \varepsilon_{\pi\left(\tau^{\prime}\right)}\right), L_{1}=L_{x}, O_{2}=L_{2}=\Lambda_{\pi\left(\tau^{\prime}\right)}$ and $L_{3}=\Lambda_{\tau^{\prime}}^{c}$.

Case 3: $\tau^{\prime}=t+\varepsilon_{t}$. If $\tau=t+\varepsilon_{t}$ too, then

$$
\left(\Gamma_{\tau}^{\tau^{\prime}} \alpha_{\tau^{\prime}}\right)(\mathbf{x})=\left(\Lambda_{\tau^{\prime}}^{c} \alpha_{\tau^{\prime}}\right)(\mathbf{x})=\left(j\left(t+\varepsilon_{t}-\tau\right) B_{\mathbf{x}, \varepsilon_{t}} \Lambda_{t+\varepsilon_{t}}^{c} \alpha_{t+\varepsilon_{t}}\right)(\mathbf{x})
$$

In general $\varepsilon_{\tau^{\prime}} \geq 2 \varepsilon_{\pi\left(\tau^{\prime}\right)}$ and $\varepsilon_{\tau^{\prime}} \geq 2 \varepsilon_{t}$. Furthermore, since $t=\tau^{\prime}-\varepsilon_{t}<\tau<\tau^{\prime}$, we have $2 \varepsilon_{\pi\left(\tau^{\prime}\right)} \leq \varepsilon_{\tau^{\prime}}$. Hence $\Lambda\left(\left[t, t+\varepsilon_{t}\right]\right) \subset \Lambda\left(\left[\tau^{\prime}-2 \varepsilon_{\pi\left(\tau^{\prime}\right)}, \tau^{\prime}\right]\right)=\Lambda_{\pi\left(\tau^{\prime}\right)}$. So the distance from $\mathbf{x}$ to $\Lambda_{\pi\left(\tau^{\prime}\right)}^{c}$ is at least $\mathfrak{c}\left(\varepsilon_{t}\right)$. As well, by definition, the distance from $\mathbf{x}$ to $B_{\mathbf{x}, \varepsilon_{t}}^{c}$ is at least $\mathfrak{c}\left(\varepsilon_{t}\right)$. Therefore, setting $L_{1}=\left\{\mathbf{y} \in X \mid d\left(\mathbf{y}, \Lambda\left(\left[t, t+\varepsilon_{t}\right]\right)^{c}\right) \geq \mathfrak{c}\left(\varepsilon_{t}\right), d\left(\mathbf{y}, B_{\mathbf{x}, \varepsilon_{t}}^{c}\right) \geq \mathfrak{c}\left(\varepsilon_{t}\right)\right\}$,

$$
\begin{aligned}
& \left|\left(\Gamma_{\tau}^{\tau^{\prime}} \alpha_{\tau^{\prime}}\right)(\mathbf{x})-\left(j\left(t+\varepsilon_{t}-\tau\right) B_{\mathbf{x}, \varepsilon_{t}} \Lambda_{t+\varepsilon_{t}}^{c} \alpha_{t+\varepsilon_{t}}\right)(\mathbf{x})\right| \\
& =\left|\left(\left\{j\left(\pi\left(\tau^{\prime}\right)-\tau\right) \Lambda_{\pi\left(\tau^{\prime}\right)} j\left(\tau^{\prime}-\pi\left(\tau^{\prime}\right)\right)-j\left(\tau^{\prime}-\tau\right) B_{\mathbf{x}, \varepsilon_{t}}\right\} \Lambda_{\tau^{\prime}}^{c} \alpha_{\tau^{\prime}}\right)(\mathbf{x})\right| \\
& =\left|\left(\left\{j\left(\tau^{\prime}-\tau\right) B_{\mathbf{x}, \varepsilon_{t}}^{c}-j\left(\pi\left(\tau^{\prime}\right)-\tau\right) \Lambda_{\pi\left(\tau^{\prime}\right)}^{c} j\left(\tau^{\prime}-\pi\left(\tau^{\prime}\right)\right)\right\} \Lambda_{\tau^{\prime}}^{c} \alpha_{\tau^{\prime}}\right)(\mathbf{x})\right| \\
& \leq N_{0}\left(L_{1} j\left(\tau^{\prime}-\tau\right) B_{\mathbf{x}, \varepsilon_{t}}^{c} \Lambda_{\tau^{\prime}}^{c} ; 1, \kappa_{\tau^{\prime}}\right) \\
& \quad \quad+N_{0}\left(L_{1} j\left(\pi\left(\tau^{\prime}\right)-\tau\right) \Lambda_{\pi\left(\tau^{\prime}\right)}^{c} j\left(\tau^{\prime}-\pi\left(\tau^{\prime}\right)\right) \Lambda_{\tau^{\prime}}^{c} ; 1, \kappa_{\tau^{\prime}}\right) \\
& \leq 8 \mathrm{R}\left(\varepsilon_{t}\right) e^{-4 \operatorname{mc}\left(\varepsilon_{t}\right)} e^{K_{j}\left(\tau^{\prime}-\tau\right)}
\end{aligned}
$$


by Lemma G.5.ii with $\mathrm{R}=4 \mathrm{R}\left(\varepsilon_{t}\right), L_{3}=\Lambda_{\tau^{\prime}}^{c}$,

$$
O_{2}=\Lambda\left(\left[\tau^{\prime}-\varepsilon_{t}, \tau^{\prime}\right]\right)=\Lambda\left(\left[t, t+\varepsilon_{t}\right]\right) \supset L_{1}
$$

and $L_{2}$ being $B_{\mathbf{x}, \varepsilon_{t}}^{c}$ for the first bound and $\Lambda_{\pi\left(\tau^{\prime}\right)}^{c}$ for the second bound.

We have now shown that $\left|\gamma_{\tau}(\mathbf{x})-\left(j\left(t+\varepsilon_{t}-\tau\right) B_{\mathbf{x}, \varepsilon_{t}} \Lambda_{t+\varepsilon_{t}}^{c} \alpha_{t+\varepsilon_{t}}\right)(\mathbf{x})\right|$ is bounded by

$$
\begin{aligned}
& \sum_{\tau^{\prime} \in\{\tau\} \cup \mathcal{T}_{r}(\tau, \delta)} 8 \mathrm{R}\left(\varepsilon_{t}\right) e^{-4 \mathrm{mc}\left(\min \left\{\varepsilon_{t}, 2 \varepsilon_{\tau^{\prime}}\right\}\right)} e^{K_{j}} \leq \sum_{\tau^{\prime} \in\{\tau\} \cup \mathcal{T}_{r}(\tau, \delta)} \frac{1}{2} e^{-\frac{15}{4} \mathrm{mc}\left(\min \left\{\varepsilon_{t}, 2 \varepsilon_{\tau^{\prime}}\right\}\right)} \\
& \leq e^{-\operatorname{mc}\left(\varepsilon_{t}\right)} \sum_{\tau^{\prime} \in\{\tau\} \cup \mathcal{T}_{r}(\tau, \delta)} e^{-\frac{11}{8} \mathrm{mc}\left(\varepsilon_{\tau^{\prime}}\right)} \leq e^{-\mathrm{mc}\left(\varepsilon_{t}\right)} \sum_{\mathfrak{d}^{\prime}=0}^{n} e^{-\frac{11}{8} \mathrm{mc}\left(2^{\mathfrak{o}^{\prime}} \varepsilon\right)} \\
& \leq e^{-\mathrm{m} \mathfrak{c}\left(\varepsilon_{t}\right)}
\end{aligned}
$$

by (F.6.b) and (F.4.a,d).

(ii) We prove the first bound. The proof of the second bound is similar. Fix any $\tau \in \varepsilon \mathbb{Z}$ obeying $0<\tau<\delta$. By definition $\gamma_{\tau}(\mathbf{x})=\sum_{\tau^{\prime} \geq \tau}\left(\Gamma_{\tau}^{\tau^{\prime}} \alpha_{\tau^{\prime}}\right)(\mathbf{x})$. Fix any $\tau \leq \tau^{\prime} \leq \delta$ with $\Gamma_{\tau}^{\tau^{\prime}} \neq 0$. By Lemma E.4.i, $\tau^{\prime} \in\{\tau\} \cup \mathcal{T}_{r}(\tau, \delta)$. Set

$$
L_{x}=\left\{\mathbf{x} \in X \mid d\left(\mathbf{x}, \Lambda([0, \delta])^{c}\right) \geq \mathfrak{c}(\delta)\right\}
$$

Case 1: $\tau \leq \tau^{\prime}<\delta$. By our rules for constructing small field sets, either $\Lambda_{\tau^{\prime}}^{c}=\emptyset$, or $\Lambda([0, \delta])=\Lambda_{\tau^{\prime}}\left(\right.$ in which case $\left.\varepsilon_{\tau^{\prime}}=\frac{\delta}{2}\right)$ or $d\left(\Lambda([0, \delta]), \Lambda_{\tau^{\prime}}^{c}\right) \geq \mathfrak{c}\left(2 \varepsilon_{\tau^{\prime}}\right)$. Consequently, if $\Lambda_{\tau^{\prime}}^{c} \neq$ $\emptyset$, the distance from $L_{x}$ to $\Lambda_{\tau^{\prime}}^{c}$ is at least $\mathfrak{c}\left(2 \varepsilon_{\tau^{\prime}}\right)$. Therefore, for $\tau=\tau^{\prime},\left(\Gamma_{\tau}^{\tau^{\prime}} \alpha_{\tau^{\prime}}\right)(\mathbf{x})=0$ and for $\tau<\tau^{\prime}$,

$$
\begin{aligned}
\left|\left(\Gamma_{\tau}^{\tau^{\prime}} \alpha_{\tau^{\prime}}\right)(\mathbf{x})\right| & =\left|\left(j\left(\pi\left(\tau^{\prime}\right)-\tau\right) \Lambda_{\pi\left(\tau^{\prime}\right)} j\left(\tau^{\prime}-\pi\left(\tau^{\prime}\right)\right) \Lambda_{\tau^{\prime}}^{c} \alpha_{\tau^{\prime}}\right)(\mathbf{x})\right| \\
& \leq N_{0}\left(L_{x} j\left(\pi\left(\tau^{\prime}\right)-\tau\right) \Lambda_{\pi\left(\tau^{\prime}\right)} j\left(\tau^{\prime}-\pi\left(\tau^{\prime}\right)\right) \Lambda_{\tau^{\prime}}^{c} ; 1, \kappa_{\tau^{\prime}}\right) \\
& \leq 4 \mathrm{R}(\delta) e^{-4 \operatorname{mc}\left(2 \varepsilon_{\tau^{\prime}}\right)} e^{K_{j}\left(\tau^{\prime}-\tau\right)}
\end{aligned}
$$

by Lemma G.5.ii with $\kappa=\kappa_{\tau^{\prime}}, \mathrm{R}=4 \mathrm{R}(\delta), O_{2}=\Lambda([0, \delta]) \supset L_{x}=L_{1}, L_{2}=\Lambda_{\pi\left(\tau^{\prime}\right)}$ and $L_{3}=\Lambda_{\tau^{\prime}}^{c}$.

Case 2: $\tau^{\prime}=\delta$. As $\Lambda([0, \delta]) \subset \Lambda\left(\left[\delta-2 \varepsilon_{\pi\left(\tau^{\prime}\right)}, \delta\right]\right)=\Lambda_{\pi\left(\tau^{\prime}\right)}$ the distance from $L_{x}$ to $\Lambda_{\pi\left(\tau^{\prime}\right)}^{c}$ is at least $\mathfrak{c}(\delta)$. We are assuming that the distance from $\mathbf{x}$ to $B_{\mathbf{x}, \delta}^{c}$ is also at least $\mathfrak{c}(\delta)$ so 
that, setting $L_{1}=\left\{\mathbf{y} \in L_{x} \mid d\left(\mathbf{y}, B_{\mathbf{x}, \delta}^{c}\right) \geq \mathfrak{c}(\delta)\right\}$, we have

$$
\begin{aligned}
& \left|\left(\Gamma_{\tau}^{\delta} \beta\right)(\mathbf{x})-\left(j(\delta-\tau) B_{\mathbf{x}, \delta} \beta\right)(\mathbf{x})\right| \\
& =\left|\left(\left\{j\left(\pi\left(\tau^{\prime}\right)-\tau\right) \Lambda_{\pi\left(\tau^{\prime}\right)} j\left(\tau^{\prime}-\pi\left(\tau^{\prime}\right)\right)-j(\delta-\tau) B_{\mathbf{x}, \delta}\right\} \beta\right)(\mathbf{x})\right| \\
& =\left|\left(\left\{j(\delta-\tau) B_{\mathbf{x}, \delta}^{c}-j\left(\pi\left(\tau^{\prime}\right)-\tau\right) \Lambda_{\pi\left(\tau^{\prime}\right)}^{c} j\left(\tau^{\prime}-\pi\left(\tau^{\prime}\right)\right)\right\} \beta\right\}(\mathbf{x})\right| \\
& \leq N_{0}\left(L_{1} j(\delta-\tau) B_{\mathbf{x}, \delta}^{c} ; 1, \kappa_{\delta}\right) \\
& \quad \quad+N_{0}\left(L_{1} j\left(\pi\left(\tau^{\prime}\right)-\tau\right) \Lambda_{\pi\left(\tau^{\prime}\right)}^{c} j\left(\tau^{\prime}-\pi\left(\tau^{\prime}\right)\right) ; 1, \kappa_{\delta}\right) \\
& \leq 8 \operatorname{R}(\delta) e^{-4 \mathrm{mc}(\delta)} e^{K_{j}(\delta-\tau)}
\end{aligned}
$$

by Lemma G.5.ii with $\mathrm{R}=4 \mathrm{R}(\delta), L_{3}=X$

$$
O_{2}=\Lambda([0, \delta]) \supset L_{1}
$$

and $L_{2}$ being $B_{\mathbf{x}, \delta}^{c}$ for the first bound and $\Lambda_{\pi\left(\tau^{\prime}\right)}^{c}$ for the second bound.

The desired bound now follows from (E.4) with $\varepsilon_{t}=\delta$.

\section{E.3 The Size of the Background Field}

In this subsection, we show that the background fields $\gamma_{* \tau}(\mathbf{x}), \gamma_{\tau}(\mathbf{x})$ obey roughly the same bounds as the large and small field conditions impose on the integration variables $\alpha_{\tau}(\mathbf{x})$.

Proposition E.11 Assume that $\mathfrak{h} \equiv 1$.

(i) Let $\tau \in \varepsilon \mathbb{Z} \cap(0, \delta)$ and $\mathcal{J}$ be a decimation interval that contains $\tau$ in its interior. If $\mathbf{x} \in \Lambda(\mathcal{J})$, then

$$
\left|\gamma_{* \tau}(\mathbf{x})\right|,\left|\gamma_{\tau}(\mathbf{x})\right| \leq 40 e^{K_{j}} \mathrm{R}(|\mathcal{J}|)
$$

(ii) For all $\tau \in \varepsilon \mathbb{Z} \cap(0, \delta)$ and $\mathbf{x} \in \Lambda_{\tau}^{c}$

$$
\left|\gamma_{* \tau}(\mathbf{x})-\alpha_{\tau}(\mathbf{x})^{*}\right|,\left|\gamma_{\tau}(\mathbf{x})-\alpha_{\tau}(\mathbf{x})\right| \leq \frac{1}{2} e^{-\mathrm{m} \mathfrak{c}\left(\varepsilon_{\tau}\right)}
$$

Proof: (i) The proof follows Lemma E.13, below.

(ii) follows immediately from Proposition E.7. 
Lemma E.12 Assume that $\mathfrak{h} \equiv 1$. Let $\left[t_{-}, t_{+}\right]$be a decimation interval in $[0, \delta]$ with centre $t=\frac{1}{2}\left(t_{+}+t_{-}\right)$. Recall that the sets $\tilde{P}_{\alpha}^{\prime}(\mathcal{J}), \tilde{P}_{\beta}^{\prime}(\mathcal{J}), \tilde{Q}(\mathcal{J})$ were defined in Proposition III.36 and that the sets $\tilde{P}_{\alpha}(\mathcal{J}), \tilde{P}_{\beta}(\mathcal{J})$ were defined in Proposition III.3\%.

(i) If $b \in \tilde{P}_{\alpha}^{\prime}\left(\left[t_{-}, t_{+}\right]\right)$, then

$$
\left|\nabla \gamma_{* \tau}(b)\right| \geq \frac{1}{2} \mathrm{R}^{\prime}\left(t_{+}-t_{-}\right) \quad \text { for all } \quad t_{-} \leq \tau<t
$$

(ii) If $b \in \tilde{P}_{\beta}^{\prime}\left(\left[t_{-}, t_{+}\right]\right)$, then

$$
\left|\nabla \gamma_{\tau}(b)\right| \geq \frac{1}{2} \mathrm{R}^{\prime}\left(t_{+}-t_{-}\right) \quad \text { for all } \quad t<\tau \leq t_{+}
$$

(iii) If $\mathbf{x} \in \tilde{Q}\left(\left[t_{-}, t_{+}\right]\right)$, then

$$
\left|\gamma_{* t}(\mathbf{x})^{*}-\gamma_{t+\varepsilon}(\mathbf{x})\right|+\left|\gamma_{* t-\varepsilon}(\mathbf{x})^{*}-\gamma_{t}(\mathbf{x})\right| \geq \frac{1}{4} \mathrm{r}\left(t_{+}-t_{-}\right)
$$

(iv) If $\mathbf{x} \in \tilde{P}_{\alpha}\left(\left[t_{-}, t_{+}\right]\right)$, then

$$
\left|\gamma_{* \tau}(\mathbf{x})\right| \geq \frac{1}{2} \mathrm{R}\left(t_{+}-t_{-}\right) \quad \text { for all } \quad t_{-} \leq \tau<t
$$

(v) If $\mathbf{x} \in \tilde{P}_{\beta}\left(\left[t_{-}, t_{+}\right]\right)$, then

$$
\left|\gamma_{\tau}(\mathbf{x})\right| \geq \frac{1}{2} \mathrm{R}\left(t_{+}-t_{-}\right) \quad \text { for all } \quad t<\tau \leq t_{+}
$$

Proof: $\quad$ Set $\mathfrak{c}_{ \pm}=\mathfrak{c}\left(t-t_{-}\right)=\mathfrak{c}\left(t_{+}-t\right)$ and

$$
J_{\tau, \pm}(\mathbf{x}, \mathbf{y})=j_{\mathfrak{c}_{ \pm}}(\tau)(\mathbf{x}, \mathbf{y})= \begin{cases}j(\tau)(\mathbf{x}, \mathbf{y}) & \text { if } d(\mathbf{x}, \mathbf{y}) \leq \mathfrak{c}_{ \pm} \\ 0 & \text { otherwise }\end{cases}
$$

Recall that $j(\tau)=e^{\tau \mu} e^{-\tau \nabla^{*} \mathcal{H} \nabla}$ with $\mathcal{H}$ obeying (II.16).

(i), (ii) We give the proof for part (ii). The proof of part (i) is similar. Let $t<\tau \leq t_{+}$. As $b \in \tilde{P}_{\beta}^{\prime}\left(\left[t_{-}, t_{+}\right]\right)$, both end points $\mathbf{x} \in b$ satisfy the hypotheses,

$$
d\left(\mathbf{x}, \Lambda\left(\left[t_{-}, t_{+}\right]\right)\right), d\left(\mathbf{x}, \Lambda\left(\left[t_{-}, t\right]\right)^{c}\right), d\left(\mathbf{x}, \Lambda\left(\left[t, t_{+}\right]\right)^{c}\right)>\mathfrak{c}_{ \pm}
$$

of Lemma E.10.i. (Again, when $t_{+}-t_{-} \leq 2 \varepsilon, \Lambda\left(\left[t_{-}, t\right]\right)=\Lambda\left(\left[t, t_{+}\right]=X\right.$ and the conditions $d\left(\mathbf{x}, \Lambda\left(\left[t_{-}, t\right]\right)^{c}\right), d\left(\mathbf{x}, \Lambda\left(\left[t, t_{+}\right]\right)^{c}\right)>\mathfrak{c}_{ \pm}$are vacuous. $)$Furthermore, all points $\mathbf{y}$ within a 
distance $\mathfrak{c}_{ \pm}$of either end point $\mathbf{x} \in b$, are in $\Lambda_{t}^{c} \subset \Lambda_{t_{+}}^{c}$. Hence, by Lemma E.10.i, with $B_{\mathbf{x}, \varepsilon_{t}}=\left\{\mathbf{y} \in X \mid d(\mathbf{x}, \mathbf{y}) \leq \mathfrak{c}_{ \pm}\right\}$,

$$
\left|\nabla\left(\gamma_{\tau}-\left(J_{t_{+}-\tau, \pm} \alpha_{t_{+}}\right)\right)(b)\right| \leq 2 \max _{\mathbf{x} \in b}\left|\gamma_{\tau}(\mathbf{x})-\left(J_{t_{+}-\tau, \pm} \alpha_{t_{+}}\right)(\mathbf{x})\right| \leq 2 e^{-\mathbf{m c}_{ \pm}}
$$

Write $b=\left(\mathbf{x}, \mathbf{x}+e_{i}\right)=b_{i}(\mathbf{x})$. As $J_{t_{+}-\tau, \pm}$ is translation invariant

$$
\begin{aligned}
\nabla\left(\left(J_{t_{+}-\tau, \pm} \alpha_{t_{+}}\right)\right)(b) & =\left(J_{t_{+}-\tau, \pm} \alpha_{t_{+}}\right)\left(\mathbf{x}+e_{i}\right)-\left(J_{t_{+}-\tau, \pm} \alpha_{t_{+}}\right)(\mathbf{x}) \\
& =\sum_{\mathbf{y}} J_{t_{+}-\tau, \pm}\left(\mathbf{x}+e_{i}, \mathbf{y}\right) \alpha_{t_{+}}(\mathbf{y})-\sum_{\mathbf{y}} J_{t_{+}-\tau, \pm}(\mathbf{x}, \mathbf{y}) \alpha_{t_{+}}(\mathbf{y}) \\
& =\sum_{\mathbf{y}} J_{t_{+}-\tau, \pm}\left(\mathbf{x}, \mathbf{y}-e_{i}\right) \alpha_{t_{+}}(\mathbf{y})-\sum_{\mathbf{y}} J_{t_{+}-\tau, \pm}(\mathbf{x}, \mathbf{y}) \alpha_{t_{+}}(\mathbf{y}) \\
& =\sum_{\mathbf{y}} J_{t_{+}-\tau, \pm}(\mathbf{x}, \mathbf{y}) \alpha_{t_{+}}\left(\mathbf{y}+e_{i}\right)-\sum_{\mathbf{y}} J_{t_{+}-\tau, \pm}(\mathbf{x}, \mathbf{y}) \alpha_{t_{+}}(\mathbf{y}) \\
& =\sum_{\mathbf{y}} J_{t_{+}-\tau, \pm}(\mathbf{x}, \mathbf{y})\left(\nabla \alpha_{t_{+}}\right)\left(b_{i}(\mathbf{y})\right)
\end{aligned}
$$

Since $b \in \tilde{P}_{\beta}^{\prime}\left(\left[t_{-}, t_{+}\right]\right)$, we have $\left|\nabla \alpha_{t_{+}}(b)\right|>\mathrm{R}^{\prime}\left(t_{+}-t_{-}\right)$and

$$
\begin{aligned}
\left|\nabla \gamma_{\tau}(b)\right| & =\left|\left(J_{t_{+}-\tau, \pm} \nabla \alpha_{t_{+}}\right)(b)+\nabla\left(\gamma_{\tau}-J_{t_{+}-\tau, \pm} \alpha_{t_{+}}\right)(b)\right| \\
& \geq\left|\left(J_{t_{+}-\tau, \pm} \nabla \alpha_{t_{+}}\right)(b)\right|-2 e^{-\mathrm{mc}_{ \pm}} \\
& \geq\left|\nabla \alpha_{t_{+}}(b)\right|-\left|\left(\left(\mathbb{1}-J_{t_{+}-\tau, \pm}\right) \nabla \alpha_{t_{+}}\right)(b)\right|-2 e^{-\mathrm{mc}_{ \pm}} \\
& \geq \mathrm{R}^{\prime}\left(t_{+}-t_{-}\right)-\left|\left(\left(\mathbb{1}-J_{t_{+}-\tau, \pm}\right) \nabla \alpha_{t_{+}}\right)(b)\right|-2 e^{-\mathrm{mc_{ \pm }}}
\end{aligned}
$$

where we are using $\left(J_{t_{+}-\tau, \pm} \nabla \alpha_{t_{+}}\right)$(b) to refer to the last line of (E.5). By Lemma III.21.ii,

$$
\begin{aligned}
\left|\left(\left(\mathbb{1}-J_{t_{+}-\tau, \pm}\right) \nabla \alpha_{t_{+}}\right)(b)\right| & \leq\left\|\mathbb{1}-j\left(t_{+}-\tau\right)\right\| \mid \sup \left\{\left|\nabla \alpha_{t_{+}}\left(b_{i}(\mathbf{y})\right)\right| \mid d(\mathbf{x}, \mathbf{y}) \leq \mathfrak{c}_{ \pm}\right\} \\
& \leq\left(t_{+}-t\right) K_{j} e^{K_{j}\left(t_{+}-t\right)} \sup \left\{\left|\nabla \alpha_{t_{+}}\left(b_{i}(\mathbf{y})\right)\right| \mid d(\mathbf{x}, \mathbf{y}) \leq \mathfrak{c}_{ \pm}\right\}
\end{aligned}
$$

For all $\mathbf{y}$ with $d(\mathbf{x}, \mathbf{y}) \leq \mathfrak{c}_{ \pm}, b_{i}(\mathbf{y})$ is necessarily in $\Lambda\left(\left[t, t_{+}\right]\right)^{*}$. Hence,

$$
\left|\nabla \alpha_{t_{+}}\left(b_{i}(\mathbf{y})\right)\right| \leq \mathrm{R}^{\prime}\left(t_{+}-t\right)
$$

so that

$$
\left|\left(\left(\mathbb{1}-J_{t_{+}-\tau, \pm}\right) \nabla \alpha_{t_{+}}\right)(b)\right| \leq K_{j} e^{K_{j}\left(t_{+}-t\right)}\left(t_{+}-t\right) \mathrm{R}^{\prime}\left(t_{+}-t\right)
$$

and

$$
\left|\nabla \gamma_{\tau}(b)\right| \geq \mathrm{R}^{\prime}\left(t_{+}-t_{-}\right)-K_{j} e^{K_{j}\left(t_{+}-t\right)}\left(t_{+}-t\right) \mathrm{R}^{\prime}\left(t_{+}-t\right)-2 e^{-\mathrm{mc}_{ \pm}} \geq \frac{1}{2} \mathrm{R}^{\prime}\left(t_{+}-t_{-}\right)
$$


by (F.3.e), (F.4.c) and Hypothesis F.7.i.

(iii) Since $\mathbf{x} \in \tilde{Q}\left(\left[t_{-}, t_{+}\right]\right)$, we have $\left|\alpha_{t_{+}}(\mathbf{x})-\alpha_{t_{-}}(\mathbf{x})\right|>\mathrm{r}\left(t_{+}-t_{-}\right)$. Hence at least one of $\left|\alpha_{t_{+}}(\mathbf{x})-\alpha_{t}(\mathbf{x})\right|,\left|\alpha_{t}(\mathbf{x})-\alpha_{t_{-}}(\mathbf{x})\right|$ must be at least $\frac{1}{2} \mathrm{r}\left(t_{+}-t_{-}\right)$. We prove that in the former case $\left|\gamma_{* t}(\mathbf{x})^{*}-\gamma_{t+\varepsilon}(\mathbf{x})\right| \geq \frac{1}{4} \mathrm{r}\left(t_{+}-t_{-}\right)$. The proof that in the latter case $\left|\gamma_{* t-\varepsilon}(\mathbf{x})^{*}-\gamma_{t}(\mathbf{x})\right| \geq \frac{1}{4} \mathrm{r}\left(t_{+}-t_{-}\right)$is similar. So assume that $\left|\alpha_{t_{+}}(\mathbf{x})-\alpha_{t}(\mathbf{x})\right| \geq \frac{1}{2} \mathrm{r}\left(t_{+}-t_{-}\right)$. As in the proof of part (ii), using the third and first bounds of Lemma E.10.i,

$$
\begin{aligned}
&\left|\gamma_{* t}(\mathbf{x})^{*}-\gamma_{t+\varepsilon}(\mathbf{x})\right|=\mid \alpha_{t}(\mathbf{x})-\alpha_{t_{+}}(\mathbf{x})+\left(\gamma_{* t}^{*}-\alpha_{t}\right)(\mathbf{x})+\left(\mathbb{1}-J_{t_{+}-t-\varepsilon, \pm}\right) \alpha_{t_{+}}(\mathbf{x}) \\
&-\left(\gamma_{t+\varepsilon}-J_{t_{+}-t-\varepsilon, \pm} \alpha_{t_{+}}\right)(\mathbf{x}) \mid \\
& \geq \frac{1}{2} \mathrm{r}\left(t_{+}-t_{-}\right)-\left|\left(\mathbb{1}-J_{t_{+}-t-\varepsilon, \pm}\right) \alpha_{t_{+}}(\mathbf{x})\right|-2 e^{-\mathrm{mc}_{ \pm}}
\end{aligned}
$$

since $\Lambda_{t_{+}} \subset \Lambda_{t}$ so that $d\left(\mathbf{x}, \Lambda_{t}\right)>\mathfrak{c}_{ \pm}$implies that $d\left(\mathbf{x}, \Lambda_{t_{+}}\right)>\mathfrak{c}_{ \pm}$too. By Lemma D.1 with $S=\left\{\mathbf{y} \in X \mid d(\mathbf{x}, \mathbf{y}) \leq \mathfrak{c}_{ \pm}\right\}$

$\left|\left(\mathbb{1}-J_{t_{+}-t-\varepsilon, \pm}\right) \alpha_{t_{+}}(\mathbf{x})\right| \leq\left(t_{+}-t\right) K_{j}^{\prime} e^{K_{j}^{\prime}\left(t_{+}-t\right)}\left(\left[|\mu|+e^{-5 m c_{ \pm}}\right] \max _{\mathbf{y} \in S}\left|\alpha_{t_{+}}(\mathbf{y})\right|+\max _{b \in S^{*}}\left|\nabla \alpha_{t_{+}}(b)\right|\right)$

Again, any $\mathbf{y}$ within a distance $\mathfrak{c}_{ \pm}$of $\mathbf{x} \in \tilde{Q}\left(\left[t_{-}, t_{+}\right]\right)$is necessarily in $\Lambda\left(\left[t, t_{+}\right]\right)$. Hence

$$
\max _{\mathbf{y} \in S}\left|\alpha_{t_{+}}(\mathbf{y})\right| \leq \mathrm{R}\left(t_{+}-t\right) \quad \max _{b \in S^{*}}\left|\nabla \alpha_{t_{+}}(b)\right| \leq \mathrm{R}^{\prime}\left(t_{+}-t\right)
$$

so that

$$
\begin{aligned}
\left|\left(\mathbb{1}-J_{t_{+}-t-\varepsilon, p}\right) \alpha_{\tau_{+}}(\mathrm{x})\right| & \leq K_{j}^{\prime} e^{K_{j}^{\prime}\left(t_{+}-t\right)}\left(t_{+}-t\right)\left(|\mu| \mathrm{R}\left(t_{+}-t\right)+e^{-5 \mathrm{mc}_{ \pm}} \mathrm{R}\left(t_{+}-t\right)+\mathrm{R}^{\prime}\left(t_{+}-t\right)\right) \\
& \leq \frac{1}{32} \mathrm{r}\left(t_{+}-t\right) \quad \text { by (F.6.c) and (F.4.a) }
\end{aligned}
$$

and

$$
\begin{aligned}
\left|\gamma_{* t}(\mathbf{x})^{*}-\gamma_{t+\varepsilon}(\mathbf{x})\right| & \geq \frac{1}{2} \mathrm{r}\left(t_{+}-t_{-}\right)-\frac{1}{32} \mathrm{r}\left(t_{+}-t\right)-2 e^{-\mathrm{mc}_{ \pm}} \\
& \geq \frac{1}{4} \mathrm{r}\left(t_{+}-t_{-}\right) \quad \text { by (F.3.b) and (F.4.c) }
\end{aligned}
$$

(iv, $\mathrm{v})$ We give the proof for part (v). The proof of part (iv) is similar. Let $t<\tau \leq t_{+}$. As $\mathbf{x} \in \tilde{P}_{\beta}\left(\left[t_{-}, t_{+}\right]\right)$, it satisfies the hypotheses

$$
d\left(\mathbf{x}, \Lambda\left(\left[t_{-}, t_{+}\right]\right)\right), d\left(\mathbf{x}, \Lambda\left(\left[t_{-}, t\right]\right)^{c}\right), d\left(\mathbf{x}, \Lambda\left(\left[t, t_{+}\right]\right)^{c}\right)>\mathfrak{c}_{ \pm}
$$

of Lemma E.10.i. (Again, when $t_{+}-t_{-} \leq 2 \varepsilon, \Lambda\left(\left[t_{-}, t\right]\right)=\Lambda\left(\left[t, t_{+}\right]=X\right.$ and the conditions $d\left(\mathbf{x}, \Lambda\left(\left[t_{-}, t\right]\right)^{c}\right), d\left(\mathbf{x}, \Lambda\left(\left[t, t_{+}\right]\right)^{c}\right)>\mathfrak{c}_{ \pm}$are vacuous. $)$As well, all points $\mathbf{y}$ within a distance $\mathfrak{c}_{ \pm}$of $\mathbf{x}$, are in $\Lambda_{t}^{c} \subset \Lambda_{t_{+}}^{c}$. Hence, by Lemma E.10.i, with $B_{\mathbf{x}, \varepsilon_{t}}=\left\{\mathbf{y} \in X \mid d(\mathbf{x}, \mathbf{y}) \leq \mathfrak{c}_{ \pm}\right\}$,

$$
\left|\gamma_{\tau}(\mathbf{x})-\left(J_{t_{+}-\tau, \pm} \alpha_{t_{+}}\right)(\mathbf{x})\right| \leq e^{-\mathrm{mc}_{ \pm}}
$$


Since $\mathbf{x} \in \tilde{P}_{\beta}\left(\left[t_{-}, t_{+}\right]\right)$, we have $\left|\alpha_{t_{+}}(\mathbf{x})\right|>\mathrm{R}\left(t_{+}-t_{-}\right)$and

$$
\begin{aligned}
\left|\gamma_{\tau}(\mathbf{x})\right| & =\left|\left(J_{t_{+}-\tau, \pm} \alpha_{t_{+}}\right)(\mathbf{x})+\left(\gamma_{\tau}-J_{t_{+}-\tau, \pm} \alpha_{t_{+}}\right)(\mathbf{x})\right| \\
& \geq\left|\left(J_{t_{+}-\tau, \pm} \alpha_{t_{+}}\right)(\mathbf{x})\right|-e^{-\mathrm{mc}_{ \pm}} \\
& \geq\left|\alpha_{t_{+}}(\mathbf{x})\right|-\left|\left(\left(\mathbb{1}-J_{t_{+}-\tau, \pm}\right) \alpha_{t_{+}}\right)(\mathbf{x})\right|-e^{-\mathbf{m c}_{ \pm}} \\
& \geq \mathrm{R}\left(t_{+}-t_{-}\right)-\left|\left(\left(\mathbb{1}-J_{t_{+}-\tau, \pm}\right) \alpha_{t_{+}}\right)(\mathbf{x})\right|-e^{-\mathbf{m c}_{ \pm}}
\end{aligned}
$$

By Corollary D.2,

$$
\begin{aligned}
& \left|\left(\left(\mathbb{1}-J_{t_{+}-\tau, \pm}\right) \alpha_{t_{+}}\right)(\mathbf{x})\right| \\
& \quad \leq\left(t_{+}-t\right) K_{j}^{\prime} e^{K_{j}^{\prime}\left(t_{+}-t\right)}\left(\left[|\mu|+e^{-5 \mathbf{m c}_{ \pm}}\right] \max _{\substack{\mathbf{y} \in X \\
d(\mathbf{x}, \mathbf{y}) \leq \mathfrak{c}_{ \pm}}}\left|\alpha_{t_{+}}(\mathbf{y})\right|+\max _{\substack{b \in X^{*} \\
d(\mathbf{x}, b) \leq \mathfrak{c}_{ \pm}}}\left|\nabla \alpha_{t_{+}}(b)\right|\right)
\end{aligned}
$$

All $\mathbf{y} \in X$ with $d(\mathbf{x}, \mathbf{y}) \leq \mathfrak{c}_{ \pm}$, are necessarily in $\Lambda\left(\left[t, t_{+}\right]\right)$and all bonds $b$ with $d(\mathbf{x}, b) \leq \mathfrak{c}_{ \pm}$, are necessarily in $\Lambda\left(\left[t, t_{+}\right]\right)^{*}$. Hence,

$$
\left|\alpha_{t_{+}}(\mathbf{y})\right| \leq \mathrm{R}\left(t_{+}-t\right) \quad \text { and } \quad\left|\nabla \alpha_{t_{+}}(b)\right| \leq \mathrm{R}^{\prime}\left(t_{+}-t\right)
$$

so that, by (F.4.a), (F.6.c) and (F.3.b),

$$
\begin{aligned}
\left|\left(\left(\mathbb{1}-J_{t_{+}-\tau, \pm}\right) \alpha_{t_{+}}\right)(\mathbf{x})\right| & \leq K_{j}^{\prime} e^{K_{j}^{\prime}\left(t_{+}-t\right)} \varepsilon\left(t_{+}-t\right)\left(\left[|\mu|+e^{-5 \mathrm{mc}_{ \pm}}\right] \mathrm{R}\left(t_{+}-t\right)+\mathrm{R}^{\prime}\left(t_{+}-t\right)\right) \\
& \leq \frac{1}{16} \mathrm{r}\left(t_{+}-t_{-}\right)
\end{aligned}
$$

and, by (F.3.d) and (F.4.c),

$$
\left|\gamma_{\tau}(b)\right| \geq \mathrm{R}\left(t_{+}-t_{-}\right)-\frac{1}{16} \mathrm{r}\left(t_{+}-t_{-}\right)-e^{-\mathrm{mc}_{ \pm}} \geq \frac{1}{2} \mathrm{R}\left(t_{+}-t_{-}\right)
$$

Lemma E.13 Let $\tau \in \varepsilon \mathbb{Z} \cap(0, \delta)$ and $\mathcal{J}$ be a decimation interval that contains $\tau$ in its interior. Then

$$
\begin{array}{r}
\sum_{\tau^{\prime} \in[0, \delta)} \mathcal{N}_{4 \mathrm{~m}}\left(\Gamma_{* \tau}^{\tau^{\prime}} ; e^{\frac{\mathrm{m}}{2} d(\mathbf{x}, \Lambda(\mathcal{J}))}, \kappa_{* \tau^{\prime}}\right) \leq \begin{cases}40 e^{K_{j} \tau} \mathrm{R}(|\mathcal{J}|) & \text { if } \mathcal{J} \varsubsetneqq[0, \delta] \\
16 e^{K_{j} \tau} \mathrm{R}(\delta) & \text { if } \mathcal{J}=[0, \delta]\end{cases} \\
\sum_{\tau^{\prime} \in(0, \delta]} \mathcal{N}_{4 \mathrm{~m}}\left(\Gamma_{\tau}^{\tau^{\prime}} ; e^{\frac{\mathrm{m}}{2} d(\mathbf{x}, \Lambda(\mathcal{J}))}, \kappa_{\tau^{\prime}}\right) \leq \begin{cases}40 e^{K_{j}(\delta-\tau)} \mathrm{R}(|\mathcal{J}|) & \text { if } \mathcal{J} \varsubsetneqq[0, \delta] \\
16 e^{K_{j}(\delta-\tau)} \mathrm{R}(\delta) & \text { if } \mathcal{J}=[0, \delta]\end{cases}
\end{array}
$$


Proof: We prove the first bound. The proof of the second is virtually identical. Write $\mathcal{J}=\left[\tau_{-}, \tau_{+}\right]$and let $\tau_{+}-\tau_{-}=\frac{1}{2^{p}} \delta$. We first consider the case that $\tau^{\prime} \in \mathcal{T}_{l}(\tau, \delta)$ with $\mathfrak{d}\left(\sigma\left(\tau^{\prime}\right)\right)>p$. We claim that $\mathcal{J}$ is exactly the decimation interval $\mathcal{J}_{* p}$ of Lemma B.1.

○ If $\mathfrak{d}\left(\tau^{\prime}\right)>p$, then, since $\tau^{\prime}$ is the largest element of $\varepsilon_{\tau^{\prime}} \mathbb{Z}$ below $\tau$ and $\tau_{-}$is an element of $\varepsilon_{\tau^{\prime}} \mathbb{Z}$ below $\tau$, we have $\tau_{-}<\tau^{\prime}<\tau<\tau_{+}$so that $\mathcal{J}$ the unique decimation interval of length $\frac{1}{2^{p}} \delta$ that contains $\tau^{\prime}$.

○ If $\mathfrak{d}\left(\tau^{\prime}\right) \leq p<\mathfrak{d}\left(\sigma\left(\tau^{\prime}\right)\right)$, then $\tau_{-}<\sigma\left(\tau^{\prime}\right)$, since $\sigma\left(\tau^{\prime}\right)$ is the largest element of $\frac{\delta}{2^{\mathfrak{o}\left(\sigma\left(\tau^{\prime}\right)\right)} \mathbb{Z}}$ below $\tau$. And $\tau_{-} \geq \tau^{\prime}$, since $\tau_{-}$is the largest element of $\frac{\delta}{2^{p}} \mathbb{Z}$ below $\tau$. And it is impossible to have $\tau^{\prime}<\tau_{-}<\sigma\left(\tau^{\prime}\right)$ since then $\tau_{-}$would be in $\mathcal{T}_{l}(\tau, \delta)$ and $\sigma\left(\tau^{\prime}\right)$ would not be the successor of $\tau^{\prime}$. So $\tau_{-}=\tau^{\prime}$ and $\mathcal{J}=\left[\tau^{\prime}, \tau^{\prime}+\frac{\delta}{2^{p}}\right]$.

Consequently,

$$
\begin{aligned}
& \mathcal{N}_{4 \mathrm{~m}}\left(\Gamma_{* \tau}^{\tau^{\prime}} ; e^{\frac{\mathrm{m}}{2} d(\mathbf{x}, \Lambda(\mathcal{J}))}, \kappa_{* \tau^{\prime}}\right)=\mathcal{N}_{4 \mathrm{~m}}\left(j\left(\tau-\sigma\left(\tau^{\prime}\right)\right) \Lambda_{\sigma\left(\tau^{\prime}\right)} j\left(\sigma\left(\tau^{\prime}\right)-\tau^{\prime}\right) \Lambda_{\tau^{\prime}}^{c} ; e^{\frac{\mathrm{m}}{2} d(\mathbf{x}, \Lambda(\mathcal{J}))}, \kappa_{* \tau^{\prime}}\right) \\
& \leq 4 \mathrm{R}(|\mathcal{J}|) e^{-\frac{\mathrm{m}}{6} d\left(\Lambda(\mathcal{J}), \Lambda_{\tau^{\prime}}^{c}\right)} \mathcal{N}_{5 \mathrm{~m}}\left(j\left(\tau-\sigma\left(\tau^{\prime}\right)\right) ; 1,1\right) \mathcal{N}_{5 \mathrm{~m}}\left(j\left(\sigma\left(\tau^{\prime}\right)-\tau^{\prime}\right) ; 1,1\right) \\
& \leq 4 \mathrm{R}(|\mathcal{J}|) e^{-\frac{\mathrm{m}}{6} d\left(\Lambda(\mathcal{J}), \Lambda_{\tau^{\prime}}^{c}\right)}\left\|j\left(\tau-\sigma\left(\tau^{\prime}\right)\right)\right\|\|\| j\left(\sigma\left(\tau^{\prime}\right)-\tau^{\prime}\right) \| \mid \\
& \leq 4 e^{K_{j}\left(\tau-\tau^{\prime}\right)} \mathrm{R}(|\mathcal{J}|) \begin{cases}e^{-\frac{\mathrm{m}}{6} \mathfrak{c}\left(\left|\mathcal{J}_{\tau^{\prime}}\right|\right)} & \text { if }\left|\mathcal{J}_{\tau^{\prime}}\right|<|\mathcal{J}| \\
1 & \text { otherwise }\end{cases}
\end{aligned}
$$

For the first inequality we used Lemma G.5.i.c with $d$ replaced by $4 \mathrm{~m} d$,

$$
\begin{aligned}
L_{1} & =X, L_{2}=\Lambda_{\sigma\left(\tau^{\prime}\right)}, \quad L_{3}=\Lambda_{\tau^{\prime}}^{c}, \quad O_{1}=O_{2}=\Lambda(\mathcal{J}) \\
\delta_{1} & =\frac{\mathrm{m}}{2} d, \quad \delta_{2}=\frac{\mathrm{m}}{3} d, \delta=\frac{\mathrm{m}}{6} d, \tilde{d}=5 \mathrm{~m} d, \quad \kappa=\kappa_{* \tau^{\prime}}, \mathrm{R}=4 \mathrm{R}(|\mathcal{J}|)
\end{aligned}
$$

and $D_{O} \geq \delta\left(L_{3}, O_{1}\right)=\frac{1}{6} \operatorname{m} d\left(\Lambda(\mathcal{J}), \Lambda_{\tau^{\prime}}^{c}\right)$. (The hypothesis that $\kappa(\mathbf{x})=\kappa_{* \tau^{\prime}}(\mathbf{x}) \leq$ $R e^{\delta_{2}\left(\mathbf{x}, O_{2}\right)}=4 \mathrm{R}(|\mathcal{J}|) e^{\frac{m}{3} d(\mathbf{x}, \Lambda(\mathcal{J}))}$ for all $\mathbf{x} \in X$ is fulfilled by Lemma B.1.ii, since, as we observed above, $\mathcal{J}$ is the decimation interval $\mathcal{J}_{* p}$ of that Lemma). The last inequality follows from Lemma III.21.i.

We next consider the case that $\tau^{\prime} \in \mathcal{T}_{l}(\tau, \delta)$ with $p \geq \mathfrak{d}\left(\sigma\left(\tau^{\prime}\right)\right)$. In this case $\Lambda_{\sigma\left(\tau^{\prime}\right)} \subset$ $\Lambda(\mathcal{J})$ and

$$
\begin{aligned}
\mathcal{N}_{4 \mathrm{~m}}\left(\Gamma_{* \tau}^{\tau^{\prime}} ; e^{\frac{\mathrm{m}}{2} d(\mathbf{x}, \Lambda(\mathcal{J}))}\right. & \left., \kappa_{* \tau^{\prime}}\right) \leq \mathcal{N}_{4 \mathrm{~m}}\left(j\left(\tau-\sigma\left(\tau^{\prime}\right)\right) \Lambda_{\sigma\left(\tau^{\prime}\right)} j\left(\sigma\left(\tau^{\prime}\right)-\tau^{\prime}\right) \Lambda_{\tau^{\prime}}^{c} ; 1, \kappa_{* \tau^{\prime}}\right) \\
& \leq 4 \mathrm{R}\left(\left|\mathcal{J}_{\sigma\left(\tau^{\prime}\right)}\right|\right) \mathcal{N}_{5 \mathrm{~m}}\left(j\left(\tau-\sigma\left(\tau^{\prime}\right)\right) ; 1,1\right) \mathcal{N}_{5 \mathrm{~m}}\left(j\left(\sigma\left(\tau^{\prime}\right)-\tau^{\prime}\right) ; 1,1\right) \\
& \leq 4 \mathrm{R}\left(\left|\mathcal{J}_{\sigma\left(\tau^{\prime}\right)}\right|\right)\left\|j\left(\tau-\sigma\left(\tau^{\prime}\right)\right)\right\|\|\| j\left(\sigma\left(\tau^{\prime}\right)-\tau^{\prime}\right) \| \mid \\
& \leq 4 e^{K_{j}\left(\tau-\tau^{\prime}\right)} \mathrm{R}\left(\left|\mathcal{J}_{\sigma\left(\tau^{\prime}\right)}\right|\right)
\end{aligned}
$$

by Lemma G.5.ii with $d$ replaced by $4 \mathrm{~m} d$,

$$
\begin{aligned}
L_{1} & =X, L_{2}=\Lambda_{\sigma\left(\tau^{\prime}\right)}, \quad L_{3}=\Lambda_{\tau^{\prime}}^{c}, \quad O_{2}=\Lambda_{\sigma\left(\tau^{\prime}\right)} \\
\delta & =\delta_{1}=0, \quad \delta_{2}=\frac{\mathrm{m}}{3} d, \tilde{d}=5 \mathrm{~m} d, \quad \kappa=\kappa_{* \tau^{\prime}}, \mathrm{R}=4 \mathrm{R}\left(\left|\mathcal{J}_{\sigma\left(\tau^{\prime}\right)}\right|\right)
\end{aligned}
$$


Finally, as $\Gamma_{* \tau}^{\tau}=\Lambda_{\tau}^{c}$,

$$
\begin{aligned}
\mathcal{N}_{4 \mathrm{~m}}\left(\Gamma_{* \tau}^{\tau} ; e^{\mathrm{m} / 2 d(\mathbf{x}, \Lambda(\mathcal{J}))}, \kappa_{* \tau}\right) & =\mathcal{N}_{4 \mathrm{~m}}\left(\Lambda_{\tau}^{c} ; 1, \kappa_{* \tau} e^{-\mathrm{m} / 2 d(\mathbf{x}, \Lambda(\mathcal{J}))}\right) \leq 4 \mathrm{R}(|\mathcal{J}|) \mathcal{N}_{4 \mathrm{~m}}\left(\Lambda_{\tau}^{c} ; 1,1\right) \\
& =4 \mathrm{R}(|\mathcal{J}|)
\end{aligned}
$$

Again we used Lemma B.1.ii.

Using (F.4.d) to sum (E.6) over $\tau^{\prime} \in \mathcal{T}_{l}(\tau, \delta)$ with $\mathfrak{d}\left(\tau^{\prime}\right)>p+1$ (so that $\left|\mathcal{J}_{\tau^{\prime}}\right|<|\mathcal{J}|$ ), using (E.6) up to twice more for the cases $\mathfrak{d}\left(\tau^{\prime}\right)=p+1$ (so that $\left|\mathcal{J}_{\tau^{\prime}}\right|=|\mathcal{J}|$ ), and $\mathfrak{d}\left(\tau^{\prime}\right) \leq p<\mathfrak{d}\left(\sigma\left(\tau^{\prime}\right)\right)$, using (F.3.c) to sum (E.7) over $\tau^{\prime} \in \mathcal{T}_{l}(\tau, \delta)$ with $\mathfrak{d}\left(\sigma\left(\tau^{\prime}\right)\right) \leq p$ and finally adding (E.8) gives the bound for $\mathcal{J} \varsubsetneqq[0, \delta]$. When $\mathcal{J}=[0, \delta]$, the case $\mathfrak{d}\left(\sigma\left(\tau^{\prime}\right)\right) \leq p$ is absent.

Proof of Proposition E.11.i: For all $\mathrm{x} \in \Lambda(\mathcal{J})$

$$
\left|\gamma_{* \tau}(\mathbf{x})\right| \leq\left|\sum_{\tau^{\prime} \leq \tau}\left(\Gamma_{* \tau}^{\tau^{\prime}} \alpha_{\tau^{\prime}}\right)(\mathbf{x})^{*}\right| \leq \sum_{\tau^{\prime} \leq \tau} \mathcal{N}_{0}\left(\Gamma_{* \tau}^{\tau^{\prime}} ; e^{\frac{\mathbf{m}}{2} d(\mathbf{x}, \Lambda(\mathcal{J}))}, \kappa_{* \tau^{\prime}}\right)
$$

by Remark E.2 and the assumption that $d(\mathbf{x}, \Lambda(\mathcal{J}))=0$. Now just apply Lemma E.13. The proof of the bound on $\gamma_{\tau}(\mathbf{x})$ is similar.

\section{E.4 Comparison of $\gamma_{\tau+\varepsilon}$ and $j(\varepsilon) \gamma_{\tau}$}

\section{Lemma E.14}

(a) Let $\tau, \tau^{\prime} \in(0, \delta]$ and $t>0$. If $[\tau-t, \tau) \cap \varepsilon \mathbb{Z}=\emptyset$, then

$$
\Gamma_{\tau-t}^{\tau^{\prime}}=j(t) \Gamma_{\tau}^{\tau^{\prime}} \quad \Gamma(\tau-t ; \vec{\alpha}, \beta)=j(t) \Gamma(\tau ; \vec{\alpha}, \beta)
$$

(b) Let $\tau, \tau^{\prime} \in[0, \delta)$ and $t>0$. If $(\tau, \tau+t] \cap \varepsilon \mathbb{Z}=\emptyset$, then

$$
\Gamma_{* \tau+t}^{\tau^{\prime}}=j(t) \Gamma_{* \tau}^{\tau^{\prime}} \quad \Gamma\left(\tau+t ; \alpha_{*}, \vec{\alpha}_{*}\right)=j(t) \Gamma\left(\tau ; \alpha_{*}, \vec{\alpha}\right)
$$

Proof: We prove part (a). It suffices to prove the $\tau^{\prime}$ formula, since the other one follows from it. Since $\Lambda_{\tau^{\prime}}^{c}=\emptyset$ for all $\tau^{\prime}$ with $\mathfrak{d}\left(\tau^{\prime}\right)>n$, it suffices to consider $\tau^{\prime} \in \varepsilon \mathbb{Z} \cap(0, \delta]$. If $\tau>\tau^{\prime}$, then $\tau-t>\tau^{\prime}$ too, so that $\Gamma_{\tau-t}^{\tau^{\prime}}=\Gamma_{\tau}^{\tau^{\prime}}=0$. So it suffices to consider $\tau \leq \tau^{\prime}$.

Case $\tau^{\prime}-\tau \geq \varepsilon$ : Let $\left[\tau^{\prime}-\frac{\delta}{2^{d}}, \tau^{\prime}\right]$ be the shortest decimation interval with $\tau^{\prime}$ as right hand end point that properly contains $\left[\tau, \tau^{\prime}\right]$. (If no such decimation interval exists, then 
$\Gamma_{\tau-t}^{\tau^{\prime}}=\Gamma_{\tau}^{\tau^{\prime}}=0$.) Since $\tau^{\prime}-\tau \geq \varepsilon$, we have $d \leq n$ and $\tau^{\prime}-\frac{\delta}{2^{d}} \in \varepsilon \mathbb{Z}$. Hence $\left[\tau^{\prime}-\frac{\delta}{2^{d}}, \tau^{\prime}\right]$ also properly contains $\left[\tau-t, \tau^{\prime}\right]$ and

$$
\begin{aligned}
\Gamma_{\tau}^{\tau^{\prime}} & =j\left(\tau^{\prime}-\tau-\frac{\delta}{2^{d+1}}\right) \Lambda\left(\left[\tau^{\prime}-\frac{\delta}{2^{d}}, \tau^{\prime}\right]\right) j\left(\frac{\delta}{2^{d+1}}\right) \Lambda_{\tau^{\prime}}^{c} \\
\Gamma_{\tau-t}^{\tau^{\prime}} & =j\left(\tau^{\prime}-\tau+t-\frac{\delta}{2^{d+1}}\right) \Lambda\left(\left[\tau^{\prime}-\frac{\delta}{2^{d}}, \tau^{\prime}\right]\right) j\left(\frac{\delta}{2^{d+1}}\right) \Lambda_{\tau^{\prime}}^{c} \\
& =j(t) \Gamma_{\tau}^{\tau^{\prime}}
\end{aligned}
$$

Case $0 \leq \tau^{\prime}-\tau<\varepsilon$ : In this case $\tau^{\prime}-\tau+t<\varepsilon$ too, since otherwise $\tau^{\prime}-\varepsilon$ would be an element of $\varepsilon \mathbb{Z}$ in $[\tau-t, \tau)$. Let $\mathcal{J}_{\tau}$ and $\mathcal{J}_{\tau-t}$ be the shortest decimation intervals with $\tau^{\prime}$ as right hand end point that properly contain $\left[\tau, \tau^{\prime}\right]$ and $\left[\tau-t, \tau^{\prime}\right]$, respectively. Both are contained in $\left[\tau^{\prime}-\varepsilon, \tau\right]$. Hence $\Lambda\left(\mathcal{J}_{\tau}\right)=\Lambda\left(\mathcal{J}_{\tau-t}\right)=X$ and

$$
\begin{array}{rlrl}
\Gamma_{\tau}^{\tau^{\prime}} & =j\left(\tau^{\prime}-\tau-\frac{1}{2}\left|\mathcal{J}_{\tau}\right|\right) \Lambda\left(\mathcal{J}_{\tau}\right) j\left(\frac{1}{2}\left|\mathcal{J}_{\tau}\right|\right) \Lambda_{\tau^{\prime}}^{c} & =j\left(\tau^{\prime}-\tau\right) \Lambda_{\tau}^{c} \\
\Gamma_{\tau-t}^{\tau^{\prime}} & =j\left(\tau^{\prime}-\tau+t-\frac{1}{2}\left|\mathcal{J}_{\tau-t}\right|\right) \Lambda\left(\mathcal{J}_{\tau-t}\right) j\left(\frac{1}{2}\left|\mathcal{J}_{\tau-t}\right|\right) \Lambda_{\tau^{\prime}}^{c}=j\left(\tau^{\prime}-\tau+t\right) \Lambda_{\tau^{\prime}}^{c} \\
& =j(t) \Gamma_{\tau}^{\tau^{\prime}}
\end{array}
$$

\section{Lemma E.15}

(i) Let $\tau \in \varepsilon \mathbb{Z} \cap(0, \delta)$. If $\mathfrak{d}(\tau)=n$ (that is, $\tau \in \varepsilon \mathbb{Z} \backslash 2 \varepsilon \mathbb{Z}$ ), then

$$
\Lambda_{\tau}\left[\gamma_{* \tau}-j(\varepsilon) \gamma_{* \tau-\varepsilon}\right]=\Lambda_{\tau}\left[\gamma_{\tau}-j(\varepsilon) \gamma_{\tau+\varepsilon}\right]=0
$$

If $0<\mathfrak{d}(\tau)<n$ (that is, $\tau \in 2 \varepsilon \mathbb{Z}$ ), then

$$
\begin{array}{r}
\sum_{\tau^{\prime} \in[0, \delta)} \mathcal{N}_{3 \mathrm{~m}}\left(\Lambda_{\tau}\left[\Gamma_{* \tau}^{\tau^{\prime}}-j(\varepsilon) \Gamma_{* \tau-\varepsilon}^{\tau^{\prime}}\right] ; 1, \kappa_{* \tau^{\prime}}\right) \leq e^{-\mathrm{mc}\left(\varepsilon_{\tau}\right)} \\
\sum_{\tau^{\prime} \in(0, \delta]} \mathcal{N}_{3 \mathrm{~m}}\left(\Lambda_{\tau}\left[\Gamma_{\tau}^{\tau^{\prime}}-j(\varepsilon) \Gamma_{\tau+\varepsilon}^{\tau^{\prime}}\right] ; 1, \kappa_{\tau^{\prime}}\right) \leq e^{-\mathrm{mc}\left(\varepsilon_{\tau}\right)}
\end{array}
$$

(ii) Let $O \subset \Omega_{\mathfrak{S}}=\Omega([0, \delta]), r>0$ and define the weight factor

$$
\lambda(\mathbf{x})= \begin{cases}r & \text { if } \mathbf{x} \in O \\ \infty & \text { if } \mathbf{x} \notin O\end{cases}
$$

Then

$$
\begin{gathered}
\sum_{\tau \in(0, \delta]} \mathcal{N}_{2 \mathrm{~m}}\left(\left[\Gamma_{* \tau}^{0}-j(\varepsilon) \Gamma_{* \tau-\varepsilon}^{0}\right] O ; e^{-\mathrm{m} d(\mathbf{x}, O)}, \lambda\right) \leq 4 e^{K_{j}} r e^{-2 \mathrm{~m} d\left(O, \Omega_{\mathscr{S}}^{c}\right)} \\
\sum_{\tau \in[0, \delta)} \mathcal{N}_{2 \mathrm{~m}}\left(\left[\Gamma_{\tau}^{\delta}-j(\varepsilon) \Gamma_{\tau+\varepsilon}^{\delta}\right] O ; e^{-\mathrm{m} d(\mathbf{x}, O)}, \lambda\right) \leq 4 e^{K_{j}} r e^{-2 \mathrm{~m} d\left(O, \Omega_{\mathscr{S}}^{c}\right)}
\end{gathered}
$$

Here we set $\Gamma_{* \delta}^{0}=\Gamma_{0}^{\delta}=j(\delta)$ and $\Gamma_{* 0}^{0}=\Gamma_{\delta}^{\delta}=\mathbb{1}$. 
Proof: (i) The vanishing when $\mathfrak{d}(\tau)=n$ is proven in Lemma E.16, below. Now assume that $0<\mathfrak{d}(\tau)<n$ and write $\varepsilon_{\tau}=2^{\mathfrak{d}} \varepsilon$. (That is, $\mathfrak{d}=n-\mathfrak{d}(\tau)$.) The same Lemma gives

$$
\begin{gathered}
\sum_{\tau^{\prime} \in[0, \delta)} \mathcal{N}_{3 \mathrm{~m}}\left(\Lambda_{\tau}\left[\Gamma_{* \tau}^{\tau^{\prime}}-j(\varepsilon) \Gamma_{* \tau-\varepsilon}^{\tau^{\prime}}\right] ; 1, \kappa_{* \tau^{\prime}}\right) \\
=\mathcal{N}_{3 \mathrm{~m}}\left(\Lambda_{\tau} j\left(\frac{1}{2} \varepsilon_{\tau}\right) \Lambda\left(\left[\tau-\varepsilon_{\tau}, \tau\right]\right)^{c} j\left(\frac{1}{2} \varepsilon_{\tau}\right) \Lambda_{\tau-\varepsilon_{\tau}}^{c} ; 1, \kappa_{* \tau-\varepsilon_{\tau}}\right) \\
+\sum_{\ell=1}^{\mathfrak{d}-1} \mathcal{N}_{3 \mathrm{~m}}\left(\Lambda_{\tau} j\left(2^{\ell-1} \varepsilon\right) \Lambda\left(\left[\tau-2^{\ell} \varepsilon, \tau\right]\right) j\left(2^{\ell-1} \varepsilon\right) \Lambda_{\tau-2^{\ell} \varepsilon}^{c} ; 1, \kappa_{* \tau-2^{\ell} \varepsilon}\right) \\
\quad+\mathcal{N}_{3 \mathrm{~m}}\left(\Lambda_{\tau} j(\varepsilon) \Lambda_{\tau-\varepsilon}^{c} ; 1, \kappa_{* \tau-\varepsilon}\right)
\end{gathered}
$$

For each $1 \leq \ell \leq \mathfrak{d}-1$, we apply Lemma G.5.i.a, with $d$ replaced by $3 \mathrm{~m} d$,

$$
\begin{aligned}
& L_{1}=\Lambda_{\tau}, L_{2}=\Lambda\left(\left[\tau-2^{\ell} \varepsilon, \tau\right]\right), L_{3}=\Lambda_{\tau-2^{\ell} \varepsilon}^{c}, \quad O_{1}=X, O_{2}=\Lambda_{\tau} \\
& \delta_{1}=0, \delta_{2}=\frac{\mathrm{m}}{3} d, \delta=\frac{3 \mathrm{~m}}{2} d, \tilde{d}=5 \mathrm{~m} d, \quad \kappa=\kappa_{* \tau-2^{\ell} \varepsilon}, \quad \mathrm{R}=4 \mathrm{R}\left(2 \varepsilon_{\tau}\right)
\end{aligned}
$$

and $D_{L} \geq \frac{3}{2} \mathrm{~m} d\left(\Lambda_{\tau}, \Lambda_{\tau-2^{\ell} \varepsilon}^{c}\right) \geq \frac{3}{2} \mathrm{mc}\left(2^{\ell+1} \varepsilon\right)$, to get

$$
\begin{aligned}
\mathcal{N}_{3 \mathrm{~m}}\left(\Lambda_{\tau} j\right. & \left.\left(2^{\ell-1} \varepsilon\right) \Lambda\left(\left[\tau-\varepsilon_{\ell}, \tau\right]\right) j\left(2^{\ell-1} \varepsilon\right) \Lambda_{\tau-\varepsilon_{\ell}}^{c} ; 1, \kappa_{* \tau-\varepsilon_{\ell}}\right) \\
& \leq 4 \mathrm{R}\left(2 \varepsilon_{\tau}\right) e^{-\frac{3}{2} \mathrm{~m} d\left(\Lambda_{\tau}, \Lambda_{\tau-\varepsilon_{\ell}}^{c}\right)}\left\|j\left(2^{\ell-1} \varepsilon\right)\right\| \mid\left\|j\left(2^{\ell-1} \varepsilon\right)\right\| \\
& \leq 4 e^{K_{j} 2^{\ell} \varepsilon} \mathrm{R}\left(2 \varepsilon_{\tau}\right) e^{-\frac{3}{2} \mathrm{~m} \mathfrak{c}\left(2^{\ell+1} \varepsilon\right)} \\
& \leq \frac{1}{4} e^{-\frac{5}{4} \mathrm{~m} \mathfrak{c}\left(2^{\ell+1} \varepsilon\right)}
\end{aligned}
$$

by (F.6.b).

For the last term, we again apply Lemma G.5.i.a, this time with

$$
\begin{aligned}
& L_{1}=\Lambda_{\tau}, L_{2}=L_{3}=\Lambda_{\tau-\varepsilon}^{c}, \quad O_{1}=X, O_{2}=\Lambda_{\tau} \\
& \delta_{1}=0, \delta_{2}=\frac{\mathrm{m}}{3} d, \delta=\frac{3 \mathrm{~m}}{2} d, \tilde{d}=5 \mathrm{~m} d, \quad \kappa=\kappa_{* \tau-\varepsilon}, \quad \mathrm{R}=4 \mathrm{R}\left(2 \varepsilon_{\tau}\right)
\end{aligned}
$$

and $D_{L} \geq \frac{3}{2} \mathrm{~m} d\left(\Lambda_{\tau}, \Lambda_{\tau-\varepsilon}^{c}\right) \geq \frac{3}{2} \mathrm{mc}(2 \varepsilon)$, to get

$$
\mathcal{N}_{3 \mathrm{~m}}\left(\Lambda_{\tau} j(\varepsilon) \Lambda_{\tau-\varepsilon}^{c} ; 1, \kappa_{* \tau-\varepsilon}\right) \leq 4 \mathrm{R}\left(2 \varepsilon_{\tau}\right) e^{K_{j} \varepsilon} e^{-\frac{3}{2} \mathrm{mc}(2 \varepsilon)} \leq \frac{1}{4} e^{-\frac{5}{4} \mathrm{mc}(2 \varepsilon)}
$$

For the first term, the same Lemma gives

$$
\begin{aligned}
\mathcal{N}_{3 \mathrm{~m}}\left(\Lambda_{\tau} j\right. & \left.\left(\frac{1}{2} \varepsilon_{\tau}\right) \Lambda\left(\left[\tau-\varepsilon_{\tau}, \tau\right]\right)^{c} j\left(\frac{1}{2} \varepsilon_{\tau}\right) \Lambda_{\tau-\varepsilon_{\tau}}^{c} ; 1, \kappa_{* \tau-\varepsilon_{\tau}}\right) \\
& \leq 4 \mathrm{R}\left(2 \varepsilon_{\tau}\right) e^{-\frac{3}{2} \mathrm{~m} d\left(\Lambda_{\tau}, \Lambda\left(\left[\tau-\varepsilon_{\tau}, \tau\right]\right)^{c}\right)}\left\|j\left(\frac{1}{2} \varepsilon_{\tau}\right)\right\| \mid\left\|j\left(\frac{1}{2} \varepsilon_{\tau}\right)\right\| \\
& \leq 4 e^{K_{j} \varepsilon_{\tau}} \mathrm{R}\left(2 \varepsilon_{\tau}\right) e^{-\frac{3}{2} \mathrm{~m} \mathfrak{c}\left(\varepsilon_{\tau}\right)} \\
& \leq \frac{1}{4} e^{-\frac{5}{4} \mathrm{~m} \mathfrak{c}\left(\varepsilon_{\tau}\right)}
\end{aligned}
$$


Using (F.4.d) to bound the sum over $1<\ell<\mathfrak{d}-1$ and adding the bounds on the first and last terms gives the desired bound for $\Lambda_{\tau}\left[\Gamma_{* \tau}^{\tau^{\prime}}-j(\varepsilon) \Gamma_{* \tau-\varepsilon}^{\tau^{\prime}}\right]$. The proof of the other bound is similar.

(ii) We prove the first bound. If $\tau \neq 2^{\ell} \varepsilon$ for all $\ell \in\{0, \cdots, n\}$, then $\Gamma_{* \tau}^{0}-j(\varepsilon) \Gamma_{* \tau-\varepsilon}^{0}=0$ by Remark III.5.iv. If $\tau=2^{\ell} \varepsilon$ for some $\ell \in\{1, \cdots, n-1\}$, then

$$
\begin{aligned}
\Gamma_{* \tau}^{0}-j(\varepsilon) \Gamma_{* \tau-\varepsilon}^{0} & =\Lambda\left(\left[0,2^{\ell+1} \varepsilon\right]\right) j\left(2^{\ell} \varepsilon\right)-j\left(2^{\ell-1} \varepsilon\right) \Lambda\left(\left[0,2^{\ell} \varepsilon\right]\right) j\left(2^{\ell-1} \varepsilon\right) \\
& =-\Lambda\left(\left[0,2^{\ell+1} \varepsilon\right]\right)^{c} j\left(2^{\ell} \varepsilon\right)+j\left(2^{\ell-1} \varepsilon\right) \Lambda\left(\left[0,2^{\ell} \varepsilon\right]\right)^{c} j\left(2^{\ell-1} \varepsilon\right)
\end{aligned}
$$

Therefore,

$$
\begin{aligned}
& \mathcal{N}_{2 \mathrm{~m}}\left(\left[\Gamma_{* \tau}^{0}-j(\varepsilon) \Gamma_{* \tau-\varepsilon}^{0}\right] O ; e^{-\mathrm{m} d(\mathbf{x}, O)}, \lambda\right) \\
& \leq \mathcal{N}_{2 \mathrm{~m}}\left(\Lambda\left(\left[0,2^{\ell+1} \varepsilon\right]\right)^{c} j\left(2^{\ell} \varepsilon\right) O ; e^{-\mathrm{m} d(\mathbf{x}, O)}, \lambda\right) \\
& \quad+\mathcal{N}_{2 \mathrm{~m}}\left(j\left(2^{\ell-1} \varepsilon\right) \Lambda\left(\left[0,2^{\ell} \varepsilon\right]\right)^{c} j\left(2^{\ell-1} \varepsilon\right) O ; e^{-\mathrm{m} d(\mathbf{x}, O)}, \lambda\right) \\
& \leq r e^{-2 \mathrm{~m} d\left(O, \Lambda\left(\left[0,2^{\ell+1} \varepsilon\right]\right)^{c}\right)}\left\|j\left(2^{\ell} \varepsilon\right)\right\|+r e^{-2 \mathrm{~m} d\left(O, \Lambda\left(\left[0,2^{\ell} \varepsilon\right]\right)^{c}\right)}\left\|j\left(2^{\ell-1} \varepsilon\right)\right\|^{2} \\
& \leq 2 r e^{K_{j} 2^{\ell} \varepsilon} \begin{cases}e^{-2 \mathrm{~m}\left[d\left(O, \Omega_{\mathfrak{S}}^{c}\right)+\mathfrak{c}\left(2^{\ell+1} \varepsilon\right)\right]} & \text { if } 1 \leq \ell \leq n-2 \\
e^{-2 \mathrm{~m} d\left(O, \Omega_{\mathfrak{S}}^{c}\right)} & \text { if } \ell=n-1\end{cases}
\end{aligned}
$$

In the second inequality we applied Lemma G.5.ii with $d$ replaced by $2 \mathrm{~m} d$,

$$
L_{1}=X, L_{3}=O_{1}=O_{2}=O, \quad \delta_{1}=\mathrm{m} d, \delta_{2}=0, \delta=2 \mathrm{~m} d, \tilde{d}=5 \mathrm{~m} d, \quad \kappa=\lambda, \mathrm{R}=r
$$

and

$$
L_{2}= \begin{cases}\Lambda\left(\left[0,2^{\ell+1} \varepsilon\right]\right)^{c} & \text { for the first summand } \\ \Lambda\left(\left[0,2^{\ell} \varepsilon\right]\right)^{c} & \text { for the second summand }\end{cases}
$$

Similarly, for $\tau=\varepsilon$,

$$
\begin{aligned}
\mathcal{N}_{2 \mathrm{~m}}\left(\left[\Gamma_{* \varepsilon}^{0}-j(\varepsilon) \Gamma_{* 0}^{0}\right] O ; e^{-\mathrm{m} d(\mathbf{x}, O)}, \lambda\right) & =\mathcal{N}_{2 \mathrm{~m}}\left(\Lambda([0,2 \varepsilon])^{c} j(\varepsilon) O ; e^{-\mathrm{m} d(\mathbf{x}, O)}, \lambda\right) \\
& \leq r e^{-2 \mathrm{~m}\left[d\left(O, \Omega_{\mathfrak{S}}^{c}\right)+\mathfrak{c}(2 \varepsilon)\right]} e^{K_{j} \varepsilon}
\end{aligned}
$$

and, for $\tau=\delta$,

$$
\begin{aligned}
\mathcal{N}_{2 \mathrm{~m}}\left(\left[\Gamma_{* \delta}^{0}-j(\varepsilon) \Gamma_{* \delta-\varepsilon}^{0}\right] O ; e^{-\mathrm{m} d(\mathbf{x}, O)}, \lambda\right) & =\mathcal{N}_{2 \mathrm{~m}}\left(j\left(\frac{\delta}{2}\right) \Lambda_{\mathfrak{S}}^{c} j\left(\frac{\delta}{2}\right) O ; e^{-\mathrm{m} d(\mathbf{x}, O)}, \lambda\right) \\
& \leq r e^{-2 \mathrm{~m} d\left(O, \Omega_{\mathfrak{S}}^{c}\right)} e^{K_{j} \delta}
\end{aligned}
$$

Summing up the last three bounds, using (F.4.d), gives

$$
\begin{aligned}
& \sum_{\tau \in(0, \delta]} \mathcal{N}_{2 \mathrm{~m}}\left(\left[\Gamma_{* \tau}^{0}-j(\varepsilon) \Gamma_{* \tau-\varepsilon}^{0}\right] O ;\right.\left.e^{-\mathrm{m} d(\mathbf{x}, O)}, \lambda\right) \\
& \leq e^{K_{j}} r e^{-2 \mathrm{~m} d\left(O, \Omega_{\mathfrak{S}}^{c}\right)}\left[\sum_{\ell=0}^{n-2} 2 e^{-2 \mathrm{~m} \mathfrak{c}\left(2^{\ell+1} \varepsilon\right)}+2+1\right] \\
& \leq e^{K_{j}} r e^{-2 \mathrm{~m} d\left(O, \Omega_{\mathfrak{S}}^{c}\right)}\left[\sum_{\ell=0}^{n-2} \frac{1}{2^{\ell+1} \varepsilon} e^{-2 \mathrm{mc}\left(2^{\ell+1} \varepsilon\right)}+2+1\right] \\
& \leq 4 e^{K_{j}} r e^{-2 \mathrm{~m} d\left(O, \Omega_{\mathfrak{S}}^{c}\right)}
\end{aligned}
$$


which is the desired result.

Lemma E.16 Let $\tau \in \varepsilon \mathbb{Z} \cap(0, \delta)$. Recall that $\varepsilon=2^{-n} \delta$ with the integer $n \geq$ depthS .

(i) If $\mathfrak{d}(\tau)=n$ (that is, $\tau \in \varepsilon \mathbb{Z} \backslash 2 \varepsilon \mathbb{Z})$, then $\Lambda_{\tau}\left(\gamma_{\tau}-j(\varepsilon) \gamma_{\tau+\varepsilon}\right)=0$.

If $0<\mathfrak{d}(\tau)<n$ (that is, $\tau \in 2 \varepsilon \mathbb{Z})$, then

$$
\begin{gathered}
\Lambda_{\tau}\left(\gamma_{\tau}-j(\varepsilon) \gamma_{\tau+\varepsilon}\right)=\Lambda_{\tau}\left\{j\left(\frac{1}{2} \varepsilon_{\tau}\right) \Lambda\left(\left[\tau, \tau+\varepsilon_{\tau}\right]\right)^{c} j\left(\frac{1}{2} \varepsilon_{\tau}\right) \Lambda_{\tau+\varepsilon_{\tau}}^{c} \alpha_{\tau+\varepsilon_{\tau}}\right. \\
-\sum_{\ell=1}^{n-\mathfrak{d}(\tau)-1}\left(2^{\ell-1} \varepsilon\right) \Lambda\left(\left[\tau, \tau+2^{\ell} \varepsilon\right]\right) j\left(2^{\ell-1} \varepsilon\right) \Lambda_{\tau+2^{\ell} \varepsilon}^{c} \alpha_{\tau+2^{\ell} \varepsilon} \\
\left.-j(\varepsilon) \Lambda_{\tau+\varepsilon}^{c} \alpha_{\tau+\varepsilon}\right\}
\end{gathered}
$$

where, if $\tau=\delta-\varepsilon_{\tau}$, then $\alpha_{\tau+\varepsilon_{\tau}}=\beta$.

(ii) If $\mathfrak{d}(\tau)=n$ (that is, $\tau \in \varepsilon \mathbb{Z} \backslash 2 \varepsilon \mathbb{Z})$, then $\Lambda_{\tau}\left(\gamma_{* \tau}-j(\varepsilon) \gamma_{* \tau-\varepsilon}\right)=0$.

If $0<\mathfrak{d}(\tau)<n$ (that is, $\tau \in 2 \varepsilon \mathbb{Z})$, then

$$
\begin{aligned}
\Lambda_{\tau}\left(\gamma_{* \tau}-j(\varepsilon) \gamma_{* \tau-\varepsilon}\right)=\Lambda_{\tau}\left\{j\left(\frac{1}{2} \varepsilon_{\tau}\right)\right. & \Lambda\left(\left[\tau-\varepsilon_{\tau}, \tau\right]\right)^{c} j\left(\frac{1}{2} \varepsilon_{\tau}\right) \Lambda_{\tau-\varepsilon_{\tau}}^{c} \alpha_{\tau-\varepsilon_{\tau}}^{*} \\
& -\sum_{\ell=1}^{n-\mathfrak{d}(\tau)-1} j\left(2^{\ell-1} \varepsilon\right) \Lambda\left(\left[\tau-2^{\ell} \varepsilon, \tau\right]\right) j\left(2^{\ell-1} \varepsilon\right) \Lambda_{\tau-2^{\ell} \varepsilon}^{c} \alpha_{\tau-2^{\ell} \varepsilon}^{*} \\
& \left.-j(\varepsilon) \Lambda_{\tau-\varepsilon}^{c} \alpha_{\tau-\varepsilon}^{*}\right\}
\end{aligned}
$$

where, if $\tau=\varepsilon_{\tau}$, then $\alpha_{* \tau-\varepsilon_{\tau}}=\alpha^{*}$.

Proof: We give the proof for part (ii). The proof of part (i) is similar. For $\tau \in(\varepsilon, \delta)$ and $\tau^{\prime} \in[0, \delta)$, directly from Definition II.9,

$$
\Lambda_{\tau} \Gamma_{* \tau}^{\tau^{\prime}}=\left\{\begin{array}{rr}
0 & \text { if } \tau \notin\left(\tau^{\prime}, \tau^{\prime}+\varepsilon_{\tau^{\prime}}\right) \\
\Lambda_{\tau} j\left(\tau-\tau^{\prime}-2^{m-1} \varepsilon\right) \Lambda\left(\left[\tau^{\prime}, \tau^{\prime}+2^{m} \varepsilon\right]\right) j\left(2^{m-1} \varepsilon\right) \Lambda_{\tau^{\prime}}^{c} & \text { if } \tau \in\left[\tau^{\prime}+2^{m-1} \varepsilon, \tau^{\prime}+2^{m} \varepsilon\right) \\
& \text { with } m \geq 1,2^{m} \varepsilon \leq \varepsilon_{\tau^{\prime}}
\end{array}\right.
$$

(so that, in particular, $\Lambda_{\tau} \Gamma_{* \tau}^{\tau^{\prime}} \neq 0$ only for $\varepsilon_{\tau}<\varepsilon_{\tau^{\prime}}$ ) and

$$
j(\varepsilon) \Gamma_{* \tau-\varepsilon}^{\tau^{\prime}}=\left\{\begin{array}{lr}
0 & \text { if } \tau \notin\left(\tau^{\prime}, \tau^{\prime}+\varepsilon_{\tau^{\prime}}\right] \\
j(\varepsilon) \Lambda_{\tau^{\prime}}^{c} & \text { if } \tau=\tau^{\prime}+\varepsilon \\
j\left(\tau-\tau^{\prime}-2^{m^{\prime}-1} \varepsilon\right) \Lambda\left(\left[\tau^{\prime}, \tau^{\prime}+2^{m^{\prime}} \varepsilon\right]\right) j\left(2^{m^{\prime}-1} \varepsilon\right) \Lambda_{\tau^{\prime}}^{c} & \text { if } \tau \in\left(\tau^{\prime}+2^{m^{\prime}-1} \varepsilon, \tau^{\prime}+2^{m^{\prime}} \varepsilon\right] \\
& \text { with } m^{\prime} \geq 1,2^{m^{\prime}} \varepsilon \leq \varepsilon_{\tau^{\prime}}
\end{array}\right.
$$

(so that, in particular, $j(\varepsilon) \Gamma_{* \tau-\varepsilon}^{\tau^{\prime}} \neq 0$ only for $\varepsilon_{\tau}<\varepsilon_{\tau^{\prime}}$ or $\tau=\tau^{\prime}+\varepsilon_{\tau^{\prime}}$ ). 
○ If $\tau=\tau^{\prime}+\varepsilon$ with $\tau^{\prime} \in \varepsilon \mathbb{Z} \backslash 2 \varepsilon \mathbb{Z}$ so that $\varepsilon_{\tau^{\prime}}=\varepsilon$, then

$$
\Lambda_{\tau}\left\{\Gamma_{* \tau}^{\tau^{\prime}}-j(\varepsilon) \Gamma_{* \tau-\varepsilon}^{\tau^{\prime}}\right\}=-\Lambda_{\tau} j(\varepsilon) \Lambda_{\tau^{\prime}}^{c}
$$

This gives the last term in the statement, for the case $\tau \in 2 \varepsilon \mathbb{Z}$.

$\circ$ If $\tau=\tau^{\prime}+\varepsilon$ with $\varepsilon_{\tau^{\prime}}>\varepsilon$, then $m=1$ and

$$
\Lambda_{\tau}\left\{\Gamma_{* \tau}^{\tau^{\prime}}-j(\varepsilon) \Gamma_{* \tau-\varepsilon}^{\tau^{\prime}}\right\}=\Lambda_{\tau} \Lambda\left(\left[\tau^{\prime}, \tau^{\prime}+2 \varepsilon\right]\right) j(\varepsilon) \Lambda_{\tau^{\prime}}^{c}-\Lambda_{\tau} j(\varepsilon) \Lambda_{\tau^{\prime}}^{c}=0
$$

since $\Lambda_{\tau}=\Lambda\left(\left[\tau^{\prime}, \tau^{\prime}+2 \varepsilon\right]\right)$. This (together with the last $\circ$ ) gives the $\mathfrak{d}(\tau)=n$ case in the statement.

○ If $\tau=\tau^{\prime}+2^{k} \varepsilon$ for some $k>0$ with $2^{k} \varepsilon<\varepsilon_{\tau^{\prime}}$, then $m=k+1$ and $m^{\prime}=k$. As $\varepsilon_{\tau}=2^{k} \varepsilon$, we have $\Lambda_{\tau}=\Lambda\left(\left[\tau^{\prime}, \tau^{\prime}+2^{k+1} \varepsilon\right]\right)$ and

$$
\begin{aligned}
\Lambda_{\tau}\left\{\Gamma_{* \tau}^{\tau^{\prime}}\right. & \left.-j(\varepsilon) \Gamma_{* \tau-\varepsilon}^{\tau^{\prime}}\right\} \\
& =\Lambda\left(\left[\tau^{\prime}, \tau^{\prime}+2^{k+1} \varepsilon\right]\right)\left\{j\left(2^{k} \varepsilon\right)-j\left(2^{k-1} \varepsilon\right) \Lambda\left(\left[\tau^{\prime}, \tau^{\prime}+2^{k} \varepsilon\right]\right) j\left(2^{k-1} \varepsilon\right)\right\} \Lambda_{\tau^{\prime}}^{c} \\
& =\Lambda_{\tau} j\left(2^{k-1} \varepsilon\right) \Lambda\left(\left[\tau^{\prime}, \tau^{\prime}+2^{k} \varepsilon\right]\right)^{c} j\left(2^{k-1} \varepsilon\right) \Lambda_{\tau^{\prime}}^{c}
\end{aligned}
$$

In this case $\varepsilon<2^{k} \varepsilon=\varepsilon_{\tau}$. This gives the first term in the statement, for the case $\tau \in 2 \varepsilon \mathbb{Z}$.

○ If $\tau=\tau^{\prime}+\varepsilon_{\tau^{\prime}}$, with $\varepsilon_{\tau^{\prime}}>\varepsilon$, then $\Gamma_{* \tau}^{\tau^{\prime}}=0$ and $2^{m^{\prime}} \varepsilon=\varepsilon_{\tau^{\prime}}$ so that

$$
\Lambda_{\tau}\left\{\Gamma_{* \tau}^{\tau^{\prime}}-j(\varepsilon) \Gamma_{* \tau-\varepsilon}^{\tau^{\prime}}\right\}=-\Lambda_{\tau} j\left(\frac{1}{2} \varepsilon_{\tau^{\prime}}\right) \Lambda\left(\left[\tau^{\prime}, \tau^{\prime}+\varepsilon_{\tau^{\prime}}\right]\right) j\left(\frac{1}{2} \varepsilon_{\tau^{\prime}}\right) \Lambda_{\tau^{\prime}}^{c}
$$

In this case $\varepsilon_{\tau}>\varepsilon_{\tau^{\prime}}$. This gives the $\ell^{\text {th }}$ term in the statement, with $\ell$ determined by $2^{\ell} \varepsilon=\varepsilon_{\tau^{\prime}}$, for the case $\tau \in 2 \varepsilon \mathbb{Z}$.

○ If $\tau \in\left(\tau^{\prime}, \tau^{\prime}+\varepsilon_{\tau^{\prime}}\right)$ but $\tau \neq \tau^{\prime}+2^{k} \varepsilon$ for all $k \geq 0$ with $2^{k} \varepsilon \leq \varepsilon_{\tau^{\prime}}$, then $m=m^{\prime} \geq 1$ with $2^{m} \varepsilon=2^{m^{\prime}} \varepsilon \leq \varepsilon_{\tau^{\prime}}$ and $\Gamma_{* \tau}^{\tau^{\prime}}=j(\varepsilon) \Gamma_{* \tau-\varepsilon}^{\tau^{\prime}}$.

Finally, we consider $\tau=\varepsilon$. Then

$$
\gamma_{* \varepsilon}=\Gamma_{* \varepsilon}^{\varepsilon} \alpha_{\varepsilon}^{*}+\Gamma_{* \varepsilon}^{0} \alpha^{*}=\Lambda_{\varepsilon}^{c} \alpha_{\varepsilon}^{*}+\Lambda([0,2 \varepsilon]) j(\varepsilon) \alpha^{*}
$$

so that

$$
\Lambda_{\varepsilon} \gamma_{* \varepsilon}=\Lambda([0,2 \varepsilon]) j(\varepsilon) \alpha^{*} \quad \Lambda_{\varepsilon} j(\varepsilon) \gamma_{* 0}=\Lambda([0,2 \varepsilon]) j(\varepsilon) \alpha^{*}
$$

and

$$
\Lambda_{\tau}\left(\gamma_{* \tau}-j(\varepsilon) \gamma_{* \tau-\varepsilon}\right)=0
$$


Lemma E.17 Assume that $\mathfrak{h} \equiv 1$. Then

$$
\begin{aligned}
\sum_{\tau \in(0, \delta)}\left|\left\langle\gamma_{* \tau}-\gamma_{\tau}^{*}, \Lambda_{\tau}\left(\gamma_{\tau}-j(\varepsilon) \gamma_{\tau+\varepsilon}\right)\right\rangle\right| & \leq \frac{1}{4} \sum_{\tau^{\prime} \in(0, \delta)} e^{-\mathrm{mc}\left(\varepsilon_{\tau^{\prime}}\right)}\left|\Lambda_{\tau^{\prime}}^{c}\right| \\
\sum_{\tau \in(0, \delta)}\left|\left\langle\gamma_{\tau}-\gamma_{* \tau}^{*}, \Lambda_{\tau}\left(\gamma_{* \tau}-j(\varepsilon) \gamma_{* \tau-\varepsilon}\right)\right\rangle\right| & \leq \frac{1}{4} \sum_{\tau^{\prime} \in(0, \delta)} e^{-\mathrm{mc}\left(\varepsilon_{\tau^{\prime}}\right)}\left|\Lambda_{\tau^{\prime}}^{c}\right|
\end{aligned}
$$

Proof: We prove the first bound. Write $\varepsilon_{\tau}=2^{\mathfrak{d}} \varepsilon$. (That is, $\mathfrak{d}=n-\mathfrak{d}(\tau)$.) By Lemma E.16.i, for $\mathfrak{d}>0$,

$$
\begin{gathered}
\Lambda_{\tau}\left(\gamma_{\tau}-j(\varepsilon) \gamma_{\tau+\varepsilon}\right)=\Lambda_{\tau}\left\{j\left(\frac{1}{2} \varepsilon_{\tau}\right) \Lambda\left(\left[\tau, \tau+\varepsilon_{\tau}\right]\right)^{c} j\left(\frac{1}{2} \varepsilon_{\tau}\right) \Lambda_{\tau+\varepsilon_{\tau}}^{c} \alpha_{\tau+\varepsilon_{\tau}}\right. \\
-\sum_{\ell=1}^{\mathfrak{d}-1} j\left(2^{\ell-1} \varepsilon\right) \Lambda\left(\left[\tau, \tau+2^{\ell} \varepsilon\right]\right) j\left(2^{\ell-1} \varepsilon\right) \Lambda_{\tau+2^{\ell} \varepsilon}^{c} \alpha_{\tau+2^{\ell} \varepsilon} \\
\left.-j(\varepsilon) \Lambda_{\tau+\varepsilon}^{c} \alpha_{\tau+\varepsilon}\right\}
\end{gathered}
$$

and, if $\mathfrak{d}=0$, then $\Lambda_{\tau}\left(\gamma_{\tau}-j(\varepsilon) \gamma_{\tau+\varepsilon}\right)=0$.

For most terms that result from inserting this into the left hand side of the first claim, we shall use the bound

$$
\begin{aligned}
\left|\left\langle\gamma_{* \tau}-\gamma_{\tau}^{*}, \Lambda_{\tau} A \Lambda_{\tau^{\prime}}^{c} \alpha_{\tau^{\prime}}\right\rangle\right| & \leq \sup _{\mathbf{y} \in \Lambda_{\tau}}\left|\gamma_{* \tau}(\mathbf{y})-\gamma_{\tau}(\mathbf{y})^{*}\right| N_{0}\left(\Lambda_{\tau} A \Lambda_{\tau^{\prime}}^{c} ; 1, \kappa_{\tau^{\prime}}\right)\left|\Lambda_{\tau^{\prime}}^{c}\right| \\
& \leq 80 e^{K_{j}} \mathrm{R}\left(2 \varepsilon_{\tau}\right) N_{0}\left(\Lambda_{\tau} A \Lambda_{\tau^{\prime}}^{c} ; 1, \kappa_{\tau^{\prime}}\right)\left|\Lambda_{\tau^{\prime}}^{c}\right|
\end{aligned}
$$

by Proposition E.11.i. Using this bound, we have, for each $\tau \in(0, \delta)$ with $\tau \in 2 \varepsilon \mathbb{Z}$ and each $1 \leq \ell \leq \mathfrak{d}-1$,

$$
\begin{aligned}
\mid\left\langle\gamma_{* \tau}\right. & \left.-\gamma_{\tau}^{*}, \Lambda_{\tau} j\left(2^{\ell-1} \varepsilon\right) \Lambda\left(\left[\tau, \tau+2^{\ell} \varepsilon\right]\right) j\left(2^{\ell-1} \varepsilon\right) \Lambda_{\tau+2^{\ell} \varepsilon}^{c} \alpha_{\tau+2^{\ell} \varepsilon}\right\rangle \mid \\
& \leq 80 e^{K_{j}} \mathrm{R}\left(2 \varepsilon_{\tau}\right) N_{0}\left(\Lambda_{\tau} j\left(2^{\ell-1} \varepsilon\right) \Lambda\left(\left[\tau, \tau+2^{\ell} \varepsilon\right]\right) j\left(2^{\ell-1} \varepsilon\right) \Lambda_{\tau+2^{\ell} \varepsilon}^{c} ; 1, \kappa_{\tau+2^{\ell} \varepsilon}\right)\left|\Lambda_{\tau+2^{\ell} \varepsilon}^{c}\right| \\
& \leq 320 e^{K_{j}} \mathrm{R}\left(2 \varepsilon_{\tau}\right)^{2} e^{K_{j} 2^{\ell} \varepsilon} e^{-4 \mathrm{~m} d\left(\Lambda_{\tau}, \Lambda_{\tau+2^{\ell} \varepsilon}^{c}\right)}\left|\Lambda_{\tau+2^{\ell} \varepsilon}^{c}\right| \\
& \leq 320 \mathrm{R}\left(\varepsilon_{\tau}\right)^{2} e^{2 K_{j}} e^{-4 \mathrm{mc}\left(2^{\ell+1} \varepsilon\right)}\left|\Lambda_{\tau+2^{\ell} \varepsilon}^{c}\right| \\
& \leq \frac{1}{8} e^{-\mathrm{mc}\left(2^{\ell} \varepsilon\right)}\left|\Lambda_{\tau+2^{\ell} \varepsilon}^{c}\right| \quad \text { by (F.4.a) and (F.6.b) }
\end{aligned}
$$

by Lemma G.5.ii with $d$ replaced by $0, \delta_{1}=0, \delta_{2}=\frac{\mathrm{m}}{3} d, \delta=4 \mathrm{~m} d, \tilde{\delta}=5 \mathrm{~m} d, \kappa=\kappa_{\tau+2^{\ell} \varepsilon}$, $\mathrm{R}=4 \mathrm{R}\left(2 \varepsilon_{\tau}\right), O_{2}=\Lambda_{\tau}=L_{1}, L_{2}=\Lambda\left(\left[\tau, \tau+2^{\ell} \varepsilon\right]\right)$ and $L_{3}=\Lambda_{\tau+2^{\ell} \varepsilon}^{c}$.

We also have, for each $\tau \in 2 \varepsilon \mathbb{Z} \cap(0, \delta)$,

$$
\begin{aligned}
\mid\left\langle\gamma_{* \tau}-\right. & \left.\gamma_{\tau}^{*}, \Lambda_{\tau} j(\varepsilon) \Lambda_{\tau+\varepsilon}^{c} \alpha_{\tau+\varepsilon}\right\rangle \mid \\
& \leq 80 e^{K_{j}} \mathrm{R}\left(2 \varepsilon_{\tau}\right) N_{0}\left(\Lambda_{\tau} j(\varepsilon) \Lambda_{\tau+\varepsilon}^{c} ; 1, \kappa_{\tau+\varepsilon}\right)\left|\Lambda_{\tau+\varepsilon}^{c}\right| \\
& \leq 320 e^{K_{j}} \mathrm{R}\left(2 \varepsilon_{\tau}\right)^{2} e^{K_{j} \varepsilon} e^{-4 \mathrm{mc}(2 \varepsilon)}\left|\Lambda_{\tau+\varepsilon}^{c}\right| \\
& \leq \frac{1}{8} e^{-\mathrm{mc}(\varepsilon)}\left|\Lambda_{\tau+\varepsilon}^{c}\right|
\end{aligned}
$$


by Lemma G.5.ii with $d$ replaced by $0, \delta_{1}=0, \delta_{2}=\frac{\mathrm{m}}{3} d, \delta=4 \mathrm{~m} d, \tilde{\delta}=5 \mathrm{~m} d, \kappa=\kappa_{\tau+\varepsilon}$, $\mathrm{R}=4 \mathrm{R}\left(2 \varepsilon_{\tau}\right), O_{2}=\Lambda_{\tau}=L_{1}=L_{2}$ and $L_{3}=\Lambda_{\tau+\varepsilon}^{c}$, followed by (F.4.a) and (F.6.b).

We still have the " $\Lambda\left(\left[\tau, \tau+\varepsilon_{\tau}\right]\right)^{c}=\Lambda_{\tau+\frac{1}{2} \varepsilon_{\tau}}^{c}$ " terms to deal with. For these, we use

$$
\begin{aligned}
& \left|\left\langle\gamma_{* \tau}-\gamma_{\tau}^{*}, \Lambda_{\tau} j\left(\frac{1}{2} \varepsilon_{\tau}\right) \Lambda_{\tau+\frac{1}{2} \varepsilon_{\tau}}^{c} j\left(\frac{1}{2} \varepsilon_{\tau}\right) \Lambda_{\tau+\varepsilon_{\tau}}^{c} \alpha_{\tau+\varepsilon_{\tau}}\right\rangle\right| \\
& \quad \leq \sum_{\mathbf{x}, \mathbf{y}, \mathbf{z} \in X}\left|\gamma_{* \tau}(\mathbf{x})-\gamma_{\tau}(\mathbf{x})^{*}\right| \Lambda_{\tau}(\mathbf{x}) j\left(\frac{1}{2} \varepsilon_{\tau}\right)(\mathbf{x}, \mathbf{y}) \Lambda_{\tau+\frac{1}{2} \varepsilon_{\tau}}^{c}(\mathbf{y}) j\left(\frac{1}{2} \varepsilon_{\tau}\right)(\mathbf{y}, \mathbf{z}) \Lambda_{\tau+\varepsilon_{\tau}}^{c}(\mathbf{z})\left|\alpha_{\tau+\varepsilon_{\tau}}(\mathbf{z})\right| \\
& \leq \sum_{\mathbf{x}, \mathbf{y}, \mathbf{z} \in X} 320 e^{K_{j}} \mathrm{R}\left(\varepsilon_{\tau}\right)^{2} e^{\frac{\mathrm{m}}{3} d(\mathbf{x}, \mathbf{z})} \Lambda_{\tau}(\mathbf{x}) j\left(\frac{1}{2} \varepsilon_{\tau}\right)(\mathbf{x}, \mathbf{y}) \Lambda_{\tau+\frac{1}{2} \varepsilon_{\tau}}^{c}(\mathbf{y}) j\left(\frac{1}{2} \varepsilon_{\tau}\right)(\mathbf{y}, \mathbf{z}) \Lambda_{\tau+\varepsilon_{\tau}}^{c}(\mathbf{z})
\end{aligned}
$$

by Proposition E.11.i, and the fact, from Lemma B.1.ii, that

$$
\left|\alpha_{\tau+\varepsilon_{\tau}}(\mathbf{z})\right| \leq \kappa_{\tau+\varepsilon_{\tau}}(\mathbf{z}) \leq 4 \mathrm{R}\left(\varepsilon_{\tau}\right) e^{\frac{\mathrm{m}}{3} d\left(\mathbf{z}, \Lambda\left(\left[\tau, \tau+\varepsilon_{\tau}\right]\right)\right.} \leq 4 \mathrm{R}\left(\varepsilon_{\tau}\right) e^{\frac{\mathrm{m}}{3} d(\mathbf{x}, \mathbf{z})}
$$

when $\mathbf{x} \in \Lambda_{\tau} \subset \Lambda\left(\left[\tau, \tau+\varepsilon_{\tau}\right]\right)$. For all $\mathbf{x}, \mathbf{y}, \mathbf{z}$ for which the summand does not vanish,

$$
e^{\frac{\mathrm{m}}{3} d(\mathbf{x}, \mathbf{z})} \leq e^{-4 \mathrm{~m} d\left(\Lambda_{\tau}, \Lambda_{\tau+\frac{1}{2} \varepsilon_{\tau}}^{c}\right)} e^{4 \mathrm{~m} d(\mathbf{x}, \mathbf{y})} e^{\frac{\mathrm{m}}{3} d(\mathbf{x}, \mathbf{z})} \leq e^{-4 \mathrm{~m} d\left(\Lambda_{\tau}, \Lambda_{\tau+\frac{1}{2} \varepsilon_{\tau}}^{c}\right)} e^{5 \mathrm{~m} d(\mathbf{x}, \mathbf{y})} e^{5 \mathrm{~m} d(\mathbf{y}, \mathbf{z})}
$$

and we have

$$
\begin{aligned}
\left|\left\langle\gamma_{* \tau}-\gamma_{\tau}^{*}, \Lambda_{\tau} j\left(\frac{1}{2} \varepsilon_{\tau}\right) \Lambda_{\tau+\frac{1}{2} \varepsilon_{\tau}}^{c} j\left(\frac{1}{2} \varepsilon_{\tau}\right) \Lambda_{\tau+\varepsilon_{\tau}}^{c} \alpha_{\tau+\varepsilon_{\tau}}\right\rangle\right| & \\
& \leq \sum_{\mathbf{y} \in X} 320 e^{K_{j}} \mathrm{R}\left(\varepsilon_{\tau}\right)^{2} e^{-4 \mathrm{~m} d\left(\Lambda_{\tau}, \Lambda_{\tau+\frac{1}{2} \varepsilon_{\tau}}^{c}\right)}|| j\left(\frac{1}{2} \varepsilon_{\tau}\right)\left\|\Lambda_{\tau+\frac{1}{2} \varepsilon_{\tau}}^{c}(\mathbf{y})\right\| j\left(\frac{1}{2} \varepsilon_{\tau}\right) \| \mid \\
& \leq 320 e^{K_{j}} \mathrm{R}\left(\varepsilon_{\tau}\right)^{2} e^{K_{j} \varepsilon_{\tau}} e^{-4 \mathrm{mc}\left(\varepsilon_{\tau}\right)}\left|\Lambda_{\tau+\frac{1}{2} \varepsilon_{\tau}}^{c}\right| \\
& \leq \frac{1}{8} e^{-\mathrm{mc}\left(\frac{1}{2} \varepsilon_{\tau}\right)}\left|\Lambda_{\tau+\frac{1}{2} \varepsilon_{\tau}}^{c}\right|
\end{aligned}
$$

by (F.4.a) and (F.6.b). All together

$$
\begin{aligned}
& \sum_{\tau \in(0, \delta)}\left|\left\langle\gamma_{* \tau}-\gamma_{\tau}^{*}, \Lambda_{\tau}\left(\gamma_{\tau}-j(\varepsilon) \gamma_{\tau+\varepsilon}\right)\right\rangle\right| \\
& \leq \sum_{\tau \in 2 \varepsilon \mathbb{Z} \cap(0, \delta)}\left\{\frac{1}{8} e^{-\mathrm{mc}\left(\frac{1}{2} \varepsilon_{\tau}\right)}\left|\Lambda_{\tau+\frac{1}{2} \varepsilon_{\tau}}^{c}\right|+\sum_{\ell=0}^{n-\mathfrak{o}(\tau)-1} \frac{1}{8} e^{-\mathrm{mc}\left(2^{\ell} \varepsilon\right)}\left|\Lambda_{\tau+2^{\ell} \varepsilon}^{c}\right|\right\} \\
& \leq \sum_{\tau^{\prime} \in(0, \delta)} \frac{1}{8} e^{-\mathrm{mc}\left(\varepsilon_{\tau^{\prime}}\right)}\left|\Lambda_{\tau^{\prime}}^{c}\right|\left[\#\left\{\tau \in(0, \delta) \mid \tau+\frac{1}{2} \varepsilon_{\tau}=\tau^{\prime}\right\}\right. \\
& \left.+\#\left\{(\tau, \ell) \mid \tau \in(0, \delta), 0 \leq \ell \leq n-\mathfrak{d}(\tau)-1, \tau+2^{\ell} \varepsilon=\tau^{\prime}\right\}\right]
\end{aligned}
$$

Here, we have used that $\tau+2^{\ell} \varepsilon \in(0, \delta)$ for all $\tau \in(0, \delta)$ and $\ell<n-\mathfrak{d}(\tau)$. On the other hand, given any $\tau^{\prime} \in(0, \delta)$ there is at most one $\tau \in(0, \delta)$ with $\tau+\frac{1}{2} \varepsilon_{\tau}=\tau^{\prime}$ 
(because it is necessary that $\frac{1}{2} \varepsilon_{\tau}=\varepsilon_{\tau^{\prime}}$ ), and there is at most one pair $(\tau, \ell)$ with $\tau \in(0, \delta)$, $0 \leq \ell \leq n-\mathfrak{d}(\tau)-1$ and $\tau+2^{\ell} \varepsilon=\tau^{\prime}$ (because it is necessary that $2^{\ell} \varepsilon=\varepsilon_{\tau^{\prime}}$ ). So we end up with

$$
\sum_{\tau \in(0, \delta)}\left|\left\langle\gamma_{* \tau}-\gamma_{\tau}^{*}, \Lambda_{\tau}\left(\gamma_{\tau}-j(\varepsilon) \gamma_{\tau+\varepsilon}\right)\right\rangle\right| \leq \sum_{\tau^{\prime} \in(0, \delta)} \frac{1}{4} e^{-m \mathfrak{c}\left(\varepsilon_{\tau^{\prime}}\right)}\left|\Lambda_{\tau^{\prime}}^{c}\right|
$$

\section{E.5 Error Terms in the Recursive Construction}

Now assume that $\mathfrak{S}$ is a hierarchy for scale $2 \delta$, preceded by hierarchies $\left(\mathfrak{S}_{1}, \mathfrak{S}_{2}\right)$ for scale $\delta$. Let $\varepsilon=2^{-n} \delta$ with $n \geq \max \left\{\operatorname{depth}\left(\mathfrak{S}_{1}\right), \operatorname{depth}\left(\mathfrak{S}_{2}\right)\right\}$. For simplicity we again write $\Lambda=\Lambda_{\mathfrak{S}}$. As in (V.19) we set

$$
\begin{gathered}
\partial_{\mathfrak{c}} \Gamma_{* \tau}= \begin{cases}0 & \text { if } \tau \in[0, \delta) \text { or } \tau=2 \delta \\
\Lambda j_{\delta} & \text { if } \tau=\delta \\
\partial \Gamma_{* \tau}+\Gamma_{* \tau-\delta}^{0}\left(\mathfrak{S}_{2}\right) \Lambda j_{\delta} & \text { if } \tau \in(\delta, 2 \delta)\end{cases} \\
\partial_{\mathfrak{c}} \Gamma_{\tau}= \begin{cases}\partial \Gamma_{\tau}+\Gamma_{* \tau}^{\delta}\left(\mathfrak{S}_{1}\right) \Lambda j_{\delta} & \text { if } \tau \in(0, \delta) \\
\Lambda j_{\delta} & \text { if } \tau=\delta \\
0 & \text { if } \tau \in(\delta, 2 \delta] \text { or } \tau=0\end{cases}
\end{gathered}
$$

with $j_{\delta}=j(\delta)-j_{\mathfrak{c}}(\delta)$ and, as in Proposition III.6,

$$
\begin{aligned}
& \partial \Gamma_{* \tau}=j\left(\tau-\delta-2^{m-1} \varepsilon\right) \Lambda\left(\left[\delta, \delta+2^{m} \varepsilon\right]\right)^{c} j\left(2^{m-1} \varepsilon\right) \Lambda j(\delta) \quad \text { for } \tau \in\left[\delta+2^{m-1} \varepsilon, \delta+2^{m} \varepsilon\right) \\
& \partial \Gamma_{\tau}=j\left(\delta-2^{m-1} \varepsilon-\tau\right) \Lambda\left(\left[\delta-2^{m} \varepsilon, \delta\right]\right)^{c} j\left(2^{m-1} \varepsilon\right) \Lambda j(\delta) \quad \text { for } \tau \in\left(\delta-2^{m} \varepsilon, \delta-2^{m-1} \varepsilon\right]
\end{aligned}
$$

with $1 \leq m \leq n$.

\section{Lemma E.18}

$$
\begin{array}{r}
\mathcal{N}_{2 \mathrm{~m}}\left(\partial_{\mathfrak{c}} \Gamma_{* \tau} ; e^{-\frac{3}{2} \mathrm{~m} d(\mathbf{x}, \Lambda)}, \kappa_{* 0}\right) \leq 4 e^{2 K_{j}} \mathrm{R}(2 \delta)\left(\delta e^{-\mathrm{m} \mathfrak{c}}+e^{-\mathrm{m} \mathfrak{c}(\delta)}\right) \\
\mathcal{N}_{2 \mathrm{~m}}\left(\partial_{\mathfrak{c}} \Gamma_{\tau} ; e^{-\frac{3}{2} \mathrm{~m} d(\mathbf{x}, \Lambda)}, \kappa_{2 \delta}\right) \leq 4 e^{2 K_{j}} \mathrm{R}(2 \delta)\left(\delta e^{-\mathrm{m} \mathfrak{c}}+e^{-\mathrm{m} \mathfrak{c}(\delta)}\right)
\end{array}
$$

Proof: We prove the first bound.

In the case $\tau=\delta$ we use Remark G.4.i, Lemma B.1.i and Lemma III.21.iii to see that

$$
\begin{aligned}
\mathcal{N}_{2 \mathrm{~m}}\left(\partial_{\mathfrak{c}} \Gamma_{* \delta} ; e^{-\frac{3}{2} \mathrm{~m} d(\mathbf{x}, \Lambda)}, \kappa_{* 0}\right) & =\mathcal{N}_{2 \mathrm{~m}}\left(\Lambda j_{\delta} ; e^{-\frac{3}{2} \mathrm{~m} d(\mathbf{x}, \Lambda)}, \kappa_{* 0}\right)=\mathcal{N}_{2 \mathrm{~m}}\left(\Lambda j_{\delta} ; 1, \kappa_{* 0}\right) \\
& \leq\left\|j_{\delta}\right\| 2 \mathrm{R}(2 \delta) \sup _{\mathbf{x}, \mathbf{y} \in X}\left(e^{\left.-\operatorname{md} d(\mathbf{x}, \mathbf{y}) \frac{\kappa_{* 0}(\mathbf{y})}{\kappa_{* 0}(\mathbf{x})}\right)}\right. \\
& \leq 4 \delta K_{j} \mathrm{R}(2 \delta) e^{K_{j} \delta} e^{-\mathrm{m} \mathfrak{c}}
\end{aligned}
$$


Now let $\tau \in(\delta, 2 \delta)$. There is a unique $1 \leq m \leq n$ such that $\tau \in\left[\delta+2^{m-1} \varepsilon, \delta+2^{m} \varepsilon\right)$. We estimate $\mathcal{N}_{2 \mathrm{~m}}\left(\partial \Gamma_{* \tau} ; e^{-\frac{3}{2} \mathrm{~m} d(\mathbf{x}, \Lambda)}, \kappa_{* 0}\right)$ and $\mathcal{N}_{2 \mathrm{~m}}\left(\Gamma_{* \tau-\delta}^{0}\left(\mathfrak{S}_{2}\right) \Lambda j_{\delta} ; e^{-\frac{3}{2} \mathrm{~m} d(\mathbf{x}, \Lambda)}, \kappa_{* 0}\right)$ separately.

By $[\mathrm{BFKT} 4,(\mathrm{IV} .1)]$

$$
\begin{aligned}
\mathcal{N}_{2 \mathrm{~m}}\left(\partial \Gamma_{* \tau} ; e^{-\frac{3}{2} \mathrm{~m} d(\mathbf{x}, \Lambda)}, \kappa_{* 0}\right) & \\
= & \mathcal{N}_{2 \mathrm{~m}}\left(j\left(\tau-\delta-2^{m-1} \varepsilon\right) \Lambda\left(\left[\delta, \delta+2^{m} \varepsilon\right]\right)^{c} j\left(2^{m-1} \varepsilon\right) \Lambda j(\delta) ; e^{-\frac{3}{2} \mathrm{~m} d(\mathbf{x}, \Lambda)}, \kappa_{* 0}\right) \\
\leq & \mathcal{N}_{2 \mathrm{~m}}\left(j\left(\tau-\delta-2^{m-1} \varepsilon\right) ; e^{-\frac{3}{2} \mathrm{~m} d(\mathbf{x}, \Lambda)}, e^{-\frac{3}{2} \mathrm{~m} d(\mathbf{x}, \Lambda)}\right) \\
& \quad \mathcal{N}_{2 \mathrm{~m}}\left(\Lambda\left(\left[\delta, \delta+2^{m} \varepsilon\right]\right)^{c} j\left(2^{m-1} \varepsilon\right) \Lambda j(\delta) ; e^{-\frac{3}{2} \mathrm{~m} d(\mathbf{x}, \Lambda)}, \kappa_{* 0}\right)
\end{aligned}
$$

Using Remark G.4.i, we bound the first factor on the right hand side of (E.9) by

$$
\begin{aligned}
\mathcal{N}_{2 \mathrm{~m}}(j(\tau & \left.\left.-\delta-2^{m-1} \varepsilon\right) ; e^{-\frac{3}{2} \mathrm{~m} d(\mathbf{x}, \Lambda)}, e^{-\frac{3}{2} \mathrm{~m} d(\mathbf{x}, \Lambda)}\right) \\
& \leq\left\|j\left(\tau-\delta-2^{m-1} \varepsilon\right)\right\| \sup _{\mathbf{x}, \mathbf{y} \in X} e^{-\frac{3}{2} \mathrm{~m} d(\mathbf{x}, \mathbf{y})} \frac{e^{-\frac{3}{2} \mathrm{~m} d(\mathbf{y}, \Lambda)}}{e^{-\frac{3}{2} \mathrm{~m} d(\mathbf{x}, \Lambda)}} \\
& \leq e^{K_{j}\left(\tau-\delta-2^{m-1} \varepsilon\right)}
\end{aligned}
$$

The second factor on the right hand side of (E.9) is bounded by

$$
\begin{aligned}
\mathcal{N}_{2 \mathrm{~m}}\left(\Lambda\left(\left[\delta, \delta+2^{m} \varepsilon\right]\right)^{c}\right. & \left.j\left(2^{m-1} \varepsilon\right) \Lambda j(\delta) ; e^{-\frac{3}{2} \mathrm{~m} d(\mathbf{x}, \Lambda)}, \kappa_{* 0}\right) \\
\leq & 4 \mathrm{R}(2 \delta) e^{-\operatorname{m} d\left(\Lambda, \Lambda\left(\left[\delta, \delta+2^{m} \varepsilon\right]\right)^{c}\right)}\left\|j\left(2^{m-1} \varepsilon\right)\right\|\|\| j(\delta) \| \\
\leq & 4 e^{K_{j}\left(\delta+2^{m-1} \varepsilon\right)} \mathrm{R}(2 \delta) e^{-\operatorname{mc}\left(2^{m} \varepsilon\right)}
\end{aligned}
$$

Here we used Lemma G.5.ii with $d$ replaced by $2 \mathrm{~m} d$ and

$$
\begin{aligned}
& L_{1}=\Lambda\left(\left[\delta, \delta+2^{m} \varepsilon\right]\right)^{c}, L_{2}=\Lambda, L_{3}=X, \quad O_{1}=O_{2}=\Lambda \\
& \delta_{1}=\frac{3 \mathrm{~m}}{2} d, \delta_{2}=\frac{\mathrm{m}}{3} d, \delta=\mathrm{m} d, \tilde{d}=5 \mathrm{~m} d, \quad \kappa=\kappa_{* 0}, \mathrm{R}=4 \mathrm{R}(2 \delta)
\end{aligned}
$$

Putting the last two estimates together we get

$$
\mathcal{N}_{2 \mathrm{~m}}\left(\partial \Gamma_{* \tau} ; e^{-\frac{3}{2} \mathrm{~m} d(\mathbf{x}, \Lambda)}, \kappa_{* 0}\right) \leq 4 e^{2 K_{j} \delta} \mathrm{R}(2 \delta) e^{-\mathrm{m} \mathfrak{c}\left(2^{m} \varepsilon\right)} \leq 4 e^{K_{j}} \mathrm{R}(2 \delta) e^{-\mathrm{m} \mathfrak{c}(\delta)}
$$

Similarly

$$
\begin{aligned}
\mathcal{N}_{2 \mathrm{~m}}\left(\Gamma_{* \tau-\delta}^{0}\right. & \left.\left(\mathfrak{S}_{2}\right) \Lambda j_{\delta} ; e^{-\frac{3}{2} \mathrm{~m} d(\mathbf{x}, \Lambda)}, \kappa_{* 0}\right) \\
= & \mathcal{N}_{2 \mathrm{~m}}\left(j\left(\tau-\delta-2^{m-1} \varepsilon\right) \Lambda\left(\left[\delta, \delta+2^{m} \varepsilon\right]\right) j\left(2^{m-1} \varepsilon\right) \Lambda j_{\delta} ; e^{-\frac{3}{2} \mathrm{~m} d(\mathbf{x}, \Lambda)}, \kappa_{* 0}\right) \\
\leq & \mathcal{N}_{2 \mathrm{~m}}\left(j\left(\tau-\delta-2^{m-1} \varepsilon\right) ; e^{-\frac{3}{2} \mathrm{~m} d(\mathbf{x}, \Lambda)}, e^{-\frac{3}{2} \mathrm{~m} d(\mathbf{x}, \Lambda)}\right) \\
& \quad \mathcal{N}_{2 \mathrm{~m}}\left(\Lambda\left(\left[\delta, \delta+2^{m} \varepsilon\right]\right) j\left(2^{m-1} \varepsilon\right) \Lambda j_{\delta} ; e^{-\frac{3}{2} \mathrm{~m} d(\mathbf{x}, \Lambda)}, \kappa_{* 0}\right) \\
\leq & e^{K_{j}\left(\tau-\delta-2^{m-1} \varepsilon\right)} 4 \mathrm{R}(2 \delta)\left\|j\left(2^{m-1} \varepsilon\right)\right\|\|\|_{\delta} \| \leq 4 e^{K_{j} \tau} \mathrm{R}(2 \delta) \delta K_{j} e^{-\mathrm{m} \mathfrak{c}} \\
\leq & 4 K_{j} e^{K_{j}} \mathrm{R}(2 \delta) \delta e^{-\mathrm{m} \mathfrak{c}}
\end{aligned}
$$




\section{Remark E.19}

(a) Let $\tau \in(0, \delta]$ and $t>0$. If $[\tau-t, \tau) \cap \varepsilon \mathbb{Z}=\emptyset$, then $\partial \Gamma_{\tau-t}=j(t) \partial \Gamma_{\tau}$.

(b) Let $\tau \in[0, \delta)$ and $t>0$. If $(\tau, \tau+t] \cap \varepsilon \mathbb{Z}=\emptyset$, then $\partial \Gamma_{* \tau+t}=j(t) \partial \Gamma_{* \tau}$.

Proof: $\quad$ For all $\tau \in(0, \delta]$

$$
\partial \Gamma_{\tau} \beta=\Gamma_{\mathfrak{S}}(\tau ; \vec{\alpha}, \beta)-\Gamma_{\mathfrak{S}_{1}}\left(\tau ; \vec{\alpha}_{l}, \Lambda_{\mathfrak{S} j} j(\delta) \beta+\Lambda_{\mathfrak{S}}^{c} \alpha_{\delta}\right)
$$

Apply Lemma E.14.

Lemma E.20 Write $\partial_{j} \Gamma_{* \tau}=\partial_{\mathfrak{c}} \Gamma_{* \tau}-j(\varepsilon) \partial_{\mathfrak{c}} \Gamma_{* \tau-\varepsilon}$.

(i) $\quad \partial_{j} \Gamma_{* \tau}=0 \quad$ if $\tau \in(0,2 \delta] \backslash\left\{\delta, \delta+\varepsilon, \delta+\varepsilon_{1}, \cdots, \delta+\frac{\delta}{2}, 2 \delta\right\}$

Furthermore

$$
\begin{aligned}
& \partial_{j} \Gamma_{* \delta}=\Lambda j_{\delta} \\
& \partial_{j} \Gamma_{* \delta+\varepsilon}=\Lambda([\delta, \delta+2 \varepsilon])^{c} j(\varepsilon) \Lambda j_{\mathfrak{c}}(\delta) \\
& \partial_{j} \Gamma_{* \delta+2^{\ell} \varepsilon}=\Lambda\left(\left[\delta, \delta+2^{\ell+1} \varepsilon\right]\right)^{c} j\left(2^{\ell} \varepsilon\right) \Lambda j_{\mathfrak{c}}(\delta)-j\left(2^{\ell-1} \varepsilon\right) \Lambda\left(\left[\delta, \delta+2^{\ell} \varepsilon\right]\right)^{c} j\left(2^{\ell-1} \varepsilon\right) \Lambda j_{\mathfrak{c}}(\delta) \\
& \partial_{j} \Gamma_{* 2 \delta}=-j(\delta) \Lambda j_{\delta}-j\left(\frac{\delta}{2}\right) \Lambda([\delta, 2 \delta])^{c} j\left(\frac{\delta}{2}\right) \Lambda j_{\mathfrak{c}}(\delta) \\
& \text { for } \ell=1, \cdots, n-1
\end{aligned}
$$

(ii)

$\sum_{\tau \in(\delta, 2 \delta)} \mathcal{N}_{3 \mathrm{~m}}\left(\partial_{j} \Gamma_{* \tau} ; e^{-\frac{\mathrm{m}}{2} d(\mathbf{x}, \Lambda)}, \kappa_{* 0}\right)+\mathcal{N}_{3 \mathrm{~m}}\left(\partial_{j} \Gamma_{* 2 \delta}+j(\delta) \Lambda j_{\delta} ; e^{-\frac{\mathrm{m}}{2} d(\mathbf{x}, \Lambda)}, \kappa_{* 0}\right) \leq e^{-\frac{1}{2} \mathrm{~m} \mathfrak{c}(\delta)}$

Proof: (i) If $\tau \in(\delta, 2 \delta)$ and $m=\min \left\{m^{\prime} \mid \tau \in\left(\delta, \delta+2^{m^{\prime}} \varepsilon\right)\right\}$ then, by construction

$$
\begin{aligned}
\partial_{\mathfrak{c}} \Gamma_{* \tau}= & \partial \Gamma_{* \tau}+\Gamma_{* \tau-\delta}^{0}\left(\mathfrak{S}_{2}\right) \Lambda j_{\delta} \\
= & j\left(\tau-\delta-2^{m-1} \varepsilon\right) \Lambda\left(\left[\delta, \delta+2^{m} \varepsilon\right]\right)^{c} j\left(2^{m-1} \varepsilon\right) \Lambda j(\delta) \\
& +j\left(\tau-\delta-2^{m-1} \varepsilon\right) \Lambda\left(\left[\delta, \delta+2^{m} \varepsilon\right]\right) j\left(2^{m-1} \varepsilon\right) \Lambda j_{\delta} \\
= & j(\tau-\delta) \Lambda j(\delta)-j\left(\tau-\delta-2^{m-1} \varepsilon\right) \Lambda\left(\left[\delta, \delta+2^{m} \varepsilon\right]\right) j\left(2^{m-1} \varepsilon\right) \Lambda j(\delta) \\
& \quad+j\left(\tau-\delta-2^{m-1} \varepsilon\right) \Lambda\left(\left[\delta, \delta+2^{m} \varepsilon\right]\right) j\left(2^{m-1} \varepsilon\right) \Lambda j_{\delta} \\
= & j(\tau-\delta) \Lambda j(\delta)-j\left(\tau-\delta-2^{m-1} \varepsilon\right) \Lambda\left(\left[\delta, \delta+2^{m} \varepsilon\right]\right) j\left(2^{m-1} \varepsilon\right) \Lambda j_{\mathfrak{c}}(\delta)
\end{aligned}
$$


Consequently, if $\tau \in(\delta, 2 \delta)$ is not of the form $\delta+2^{\ell} \varepsilon$ for any $\ell=0, \cdots, n$, then $\partial_{\mathfrak{c}} \Gamma_{* \tau}-$ $j(\varepsilon) \partial_{\mathfrak{c}} \Gamma_{* \tau-\varepsilon}=0$. If $\tau=\delta+2^{\ell} \varepsilon$ for some $\ell=1, \cdots, n-1$, then

$$
\begin{aligned}
\partial_{\mathfrak{c}} \Gamma_{* \tau}-j(\varepsilon) \partial_{\mathfrak{c}} \Gamma_{* \tau-\varepsilon}= & -\Lambda\left(\left[\delta, \delta+2^{\ell+1} \varepsilon\right]\right) j\left(2^{\ell} \varepsilon\right) \Lambda j_{\mathfrak{c}}(\delta) \\
& \quad+j\left(2^{\ell-1} \varepsilon\right) \Lambda\left(\left[\delta, \delta+2^{\ell} \varepsilon\right]\right) j\left(2^{\ell-1} \varepsilon\right) \Lambda j_{\mathfrak{c}}(\delta) \\
=\Lambda([\delta, \delta & \left.\left.+2^{\ell+1} \varepsilon\right]\right)^{c} j\left(2^{\ell} \varepsilon\right) \Lambda j_{\mathfrak{c}}(\delta) \\
& \quad-j\left(2^{\ell-1} \varepsilon\right) \Lambda\left(\left[\delta, \delta+2^{\ell} \varepsilon\right]\right)^{c} j\left(2^{\ell-1} \varepsilon\right) \Lambda j_{\mathfrak{c}}(\delta)
\end{aligned}
$$

If $\tau \in(0, \delta)$, both $\partial_{\mathfrak{c}} \Gamma_{* \tau}$ and $j(\varepsilon) \partial_{\mathfrak{c}} \Gamma_{* \tau-\varepsilon}$ are zero.

For $\tau=\delta$, we have $\partial_{\mathfrak{c}} \Gamma_{* \delta}-j(\varepsilon) \partial_{\mathfrak{c}} \Gamma_{* \delta-\varepsilon}=\Lambda j_{\delta}$.

For $\tau=\delta+\varepsilon$,

$$
\begin{aligned}
\partial_{\mathfrak{c}} \Gamma_{* \delta+\varepsilon}-j(\varepsilon) \partial_{\mathfrak{c}} \Gamma_{* \delta} & =j(\varepsilon) \Lambda j(\delta)-\Lambda([\delta, \delta+2 \varepsilon]) j(\varepsilon) \Lambda j_{\mathfrak{c}}(\delta)-j(\varepsilon) \Lambda j_{\delta} \\
& =\Lambda([\delta, \delta+2 \varepsilon])^{c} j(\varepsilon) \Lambda j_{\mathfrak{c}}(\delta)
\end{aligned}
$$

For $\tau=2 \delta$,

$$
\begin{aligned}
\partial_{\mathfrak{c}} \Gamma_{* 2 \delta}-j(\varepsilon) \partial_{\mathfrak{c}} \Gamma_{* 2 \delta-\varepsilon} & =-j(\delta) \Lambda j(\delta)+j\left(\frac{\delta}{2}\right) \Lambda([\delta, 2 \delta]) j\left(\frac{\delta}{2}\right) \Lambda j_{\mathfrak{c}}(\delta) \\
& =-j(\delta) \Lambda j_{\delta}-j\left(\frac{\delta}{2}\right) \Lambda([\delta, 2 \delta])^{c} j\left(\frac{\delta}{2}\right) \Lambda j_{\mathfrak{c}}(\delta)
\end{aligned}
$$

(ii) By part (i),

$$
\begin{aligned}
\sum_{\tau \in(\delta, 2 \delta)} \mathcal{N}_{3 \mathrm{~m}}\left(\partial_{j} \Gamma_{* \tau}\right. & \left.; e^{-\frac{1}{2} \mathrm{~m} d(\mathbf{x}, \Lambda)}, \kappa_{* 0}\right)+\mathcal{N}_{3 \mathrm{~m}}\left(\partial_{j} \Gamma_{* 2 \delta}+j(\delta) \Lambda j_{\delta} ; e^{-\frac{1}{2} \mathrm{~m} d(\mathbf{x}, \Lambda)}, \kappa_{* 0}\right) \\
\leq & \sum_{\ell=0}^{n-1} \mathcal{N}_{3 \mathrm{~m}}\left(\Lambda\left(\left[\delta, \delta+2^{\ell+1} \varepsilon\right]\right)^{c} j\left(2^{\ell} \varepsilon\right) \Lambda j_{\mathfrak{c}}(\delta) ; e^{-\frac{1}{2} \mathrm{~m} d(\mathbf{x}, \Lambda)}, \kappa_{* 0}\right) \\
& +\sum_{\ell=1}^{n} \mathcal{N}_{3 \mathrm{~m}}\left(j\left(2^{\ell-1} \varepsilon\right) \Lambda\left(\left[\delta, \delta+2^{\ell} \varepsilon\right]\right)^{c} j\left(2^{\ell-1} \varepsilon\right) \Lambda j_{\mathfrak{c}}(\delta) ; e^{-\frac{1}{2} \mathrm{~m} d(\mathbf{x}, \Lambda)}, \kappa_{* 0}\right)
\end{aligned}
$$

To bound the terms of the first sum on the right hand side of (E.10), we use Lemma G.5.ii with $d$ replaced by $3 \mathrm{~m} d$ and

$$
\begin{aligned}
L_{1} & =\Lambda\left(\left[\delta, \delta+2^{\ell+1} \varepsilon\right]\right)^{c}, L_{2}=\Lambda, L_{3}=X, \quad O_{1}=O_{2}=\Lambda \\
\delta_{1} & =\frac{\mathrm{m}}{2} d, \delta_{2}=\frac{\mathrm{m}}{3} d, \delta=\mathrm{m} d, \tilde{d}=5 \mathrm{~m} d, \quad \kappa=\kappa_{* 0}, \mathrm{R}=4 \mathrm{R}(2 \delta)
\end{aligned}
$$

to get

$$
\begin{aligned}
\mathcal{N}_{3 \mathrm{~m}}\left(\Lambda\left(\left[\delta, \delta+2^{\ell+1} \varepsilon\right]\right)^{c} j\left(2^{\ell} \varepsilon\right) \Lambda j_{\mathfrak{c}}(\delta)\right. & \left.; e^{-\frac{1}{2} \mathrm{~m} d(\mathbf{x}, \Lambda)}, \kappa_{* 0}\right) \\
& \leq 4 \mathrm{R}(2 \delta) e^{-\mathrm{m} d\left(\Lambda, \Lambda\left(\left[\delta, \delta+2^{\ell+1} \varepsilon\right]\right)^{c}\right)}\left\|j\left(2^{\ell} \varepsilon\right)\right\|\|\| j_{\mathfrak{c}}(\delta) \| \\
& \leq 4 e^{K_{j}\left(\delta+2^{\ell} \varepsilon\right)} \mathrm{R}(2 \delta) e^{-\mathrm{mc}\left(2^{\ell+1} \varepsilon\right)}
\end{aligned}
$$


To bound the terms of the second sum on the right hand side of (E.10), we use [BFKT4,(IV.1)] to see that

$$
\begin{aligned}
\mathcal{N}_{3 \mathrm{~m}}\left(j\left(2^{\ell-1} \varepsilon\right) \Lambda\left(\left[\delta, \delta+2^{\ell} \varepsilon\right]\right)^{c} j\left(2^{\ell-1} \varepsilon\right) \Lambda j_{\mathfrak{c}}(\delta) ; e^{-\frac{1}{2} \mathrm{~m} d(\mathbf{x}, \Lambda)}, \kappa_{* 0}\right) \\
\leq \mathcal{N}_{3 \mathrm{~m}}\left(j\left(2^{\ell-1} \varepsilon\right) ; e^{-\frac{1}{2} \mathrm{~m} d(\mathbf{x}, \Lambda)}, e^{-\frac{1}{2} \mathrm{~m} d(\mathbf{x}, \Lambda)}\right) \\
\mathcal{N}_{3 \mathrm{~m}}\left(\Lambda\left(\left[\delta, \delta+2^{\ell} \varepsilon\right]\right)^{c} j\left(2^{\ell-1} \varepsilon\right) \Lambda j_{\mathfrak{c}}(\delta) ; e^{-\frac{1}{2} \mathrm{~m} d(\mathbf{x}, \Lambda)}, \kappa_{* 0}\right)
\end{aligned}
$$

Remark G.4.i gives the bound

$$
\begin{aligned}
\mathcal{N}_{3 \mathrm{~m}}\left(j\left(2^{\ell-1} \varepsilon\right) ; e^{-\frac{1}{2} \mathrm{~m} d(\mathbf{x}, \Lambda)}, e^{-\frac{1}{2} \mathrm{~m} d(\mathbf{x}, \Lambda)}\right) & \leq\left\|j\left(2^{\ell-1} \varepsilon\right)\right\| \sup _{\mathbf{x}, \mathbf{y} \in X} e^{-\frac{1}{2} \mathrm{~m} d(\mathbf{x}, \mathbf{y})} \frac{e^{-\frac{1}{2} \mathrm{~m} d(\mathbf{y}, \Lambda)}}{e^{-\frac{1}{2} \mathrm{~m} d(\mathbf{x}, \Lambda)}} \\
& \leq e^{K_{j} 2^{\ell-1} \varepsilon}
\end{aligned}
$$

for the first factor on the right hand side. (E.11), with $\ell$ replaced by $\ell-1$, shows that the second factor is bounded by $4 e^{K_{j}\left(\delta+2^{\ell-1} \varepsilon\right)} \mathrm{R}(2 \delta) e^{-\operatorname{mc}\left(2^{\ell} \varepsilon\right)}$. Consequently

$$
\begin{aligned}
\mathcal{N}_{3 \mathrm{~m}}\left(j\left(2^{\ell-1} \varepsilon\right) \Lambda\left(\left[\delta, \delta+2^{\ell} \varepsilon\right]\right)^{c} j\left(2^{\ell-1} \varepsilon\right) \Lambda j_{\mathfrak{c}}(\delta)\right. & \left.; e^{-\frac{1}{2} \mathrm{~m} d(\mathbf{x}, \Lambda)}, \kappa_{* 0}\right) \\
& \leq 4 e^{K_{j}\left(\delta+2^{\ell} \varepsilon\right)} \mathrm{R}(2 \delta) e^{-\mathrm{mc}\left(2^{\ell} \varepsilon\right)}
\end{aligned}
$$

Inserting (E.11) and (E.12),

$$
\text { (E.10) } \leq 4 e^{K_{j} 2 \delta} \mathrm{R}(2 \delta)\left\{\sum_{\ell=0}^{n-1} e^{-\mathrm{mc}\left(2^{\ell+1} \varepsilon\right)}+\sum_{\ell=1}^{n} e^{-\mathrm{mc}\left(2^{\ell} \varepsilon\right)}\right\} \leq e^{-\frac{1}{2} \mathrm{mc}(\delta)}
$$

by (F.4.d) and (F.6.b). 


\section{Appendix F: Properties of the Various Constants}

The model under consideration is determined by the kinetic energy $\mathrm{h}=\nabla^{*} \mathcal{H} \nabla$, the two-body potential $2 v(\mathbf{x}, \mathbf{y})$, the temperature $T>0$, and the chemical potential $\mu$. We are assuming that both $\mathcal{H}$ and $v$ are exponentially decaying. That is, there is a "mass" $\mathrm{m}>0$ such that

$$
\begin{aligned}
D_{\mathcal{H}} & =\sum_{\substack{\mathbf{x} \in X \\
1 \leq i, j \leq d}} e^{6 \mathrm{~m} d(\mathbf{x}, 0)}\left|\mathcal{H}\left(b_{i}(0), b_{j}(\mathbf{x})\right)\right| \\
\|v\| \| & =\sup _{\mathbf{x} \in X} \sum_{\mathbf{y} \in X} e^{5 \mathrm{~m} d(\mathbf{x}, \mathbf{y})}|v(\mathbf{x}, \mathbf{y})|
\end{aligned}
$$

are finite. See (II.16) and (II.5). We have also assumed that there are constants $0<$ $c_{\mathcal{H}}<C_{\mathcal{H}}$ such that all of the eigenvalues of $\mathcal{H}$ lie between $c_{\mathcal{H}}$ and $C_{\mathcal{H}}$. In Lemmas III.21 and D.1, we introduced constants $K_{j}, K_{j}^{\prime}$ (depending only on $\mathcal{D}_{\mathcal{H}}, \mathrm{m}$ and $\mu$ ) for bounds on the semigroup $j(t)=e^{-t(\mathrm{~h}-\mu)}$. Our bounds are uniform for two-body potentials lying in the annulus $\frac{1}{4} \mathfrak{v} \leq\|\| v \| \leq \frac{1}{2} \mathfrak{v}$ and for which the lowest eigenvalue obeys $v_{1} \geq c_{v}\|v\|$. See Hypothesis II.14. The constant $\mathfrak{v}>0$ must be sufficiently small and the constant $c_{v} \in(0,1)$ is arbitrary. The chemical potential $\mu$ is required to obey $|\mu| \leq \max \left\{K_{\mu} \mathfrak{v}^{e_{\mu}}, 1\right\}$ with strictly positive $K_{\mu}$ and $\frac{1}{2}<e_{\mu} \leq 1$.

For the bounds and the construction of the large field/small field decomposition, we introduced, in (II.17)-(II.20), the cutoff functions

$$
\begin{aligned}
& \mathrm{r}(t)=\left(\frac{1}{t \mathfrak{v}}\right)^{e_{\mathrm{r}}} \quad \mathrm{R}(t)=\left(\frac{1}{t \mathfrak{v}}\right)^{e_{\mathrm{R}}} \mathrm{r}(t) \quad \mathrm{R}^{\prime}(t)=\left(\frac{1}{t}\right)^{e_{\mathrm{R}^{\prime}}} \mathrm{r}(t) \\
& \mathfrak{c}=\log ^{2} \frac{1}{\mathfrak{v}} \quad \mathfrak{c}(t)=\log ^{2} \frac{1}{t \mathfrak{v}} \quad \ell(t)=\left(\frac{1}{t \mathfrak{v}}\right)^{e_{\ell}}
\end{aligned}
$$

with strictly positive exponents $e_{\mathrm{r}}, e_{\mathrm{R}}, e_{\mathrm{R}^{\prime}}$ and $e_{\ell}$ that obey

$$
\begin{array}{rlc}
3 e_{\mathrm{R}}+4 e_{\mathrm{r}}<1 & 1 \leq 4 e_{\mathrm{R}}+2 e_{\mathrm{r}} & 2\left(e_{\mathrm{R}}+e_{\mathrm{r}}\right)<e_{\mu} \leq 1 \\
e_{\mathrm{R}^{\prime}}+e_{\mathrm{r}}<1 & \frac{1}{2} \leq e_{\mathrm{R}^{\prime}} & e_{\ell}<2 e_{\mathrm{r}}
\end{array}
$$

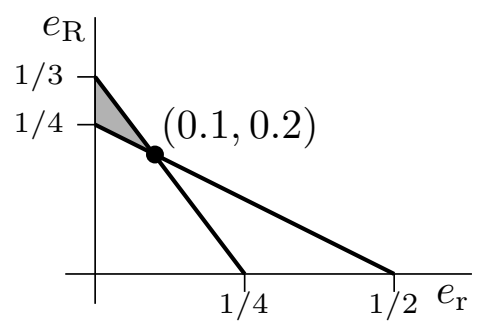

Our main results Theorems II.16 and II.18 apply when $\mathfrak{v}$ and the time interval length $\theta$ are sufficiently small. The precise restrictions are determined by a number of technical condtions that are specified in Hypothesis F.7 at the end of this appendix. 
Clearly

$$
\begin{gathered}
\mathrm{R}(2 t) \leq \mathrm{R}(t) \leq 2 \mathrm{R}(2 t) \\
\mathrm{r}(2 t) \leq \mathrm{r}(t) \leq 2 \mathrm{r}(2 t) \\
\sum_{n=1}^{\infty} R\left(2^{n} t\right) \leq 6 \mathrm{R}(t)
\end{gathered}
$$

Lemma F.1 There is a constant $t_{\max }>0$, depending only on $e_{\mathrm{r}}, e_{\mathrm{R}}$ and $e_{\mathrm{R}^{\prime}}$ such that, for all $0<t \leq t_{\max }$ and $0<\mathfrak{v} \leq 1$,

$$
\begin{aligned}
\mathrm{R}(2 t)+\mathrm{r}(t) & <\mathrm{R}(t) \\
\mathrm{R}^{\prime}(2 t)+\|\nabla\| \mathrm{r}(t) \leq \mathrm{R}^{\prime}(t) & \leq 2 \mathrm{R}^{\prime}(2 t) \\
\frac{\mathrm{r}(t)}{\mathrm{R}(t)} & \leq \frac{1}{2^{10}}
\end{aligned}
$$

Proof: These all follow directly from the definitions (F.1) and conditions (F.2).

Lemma F.2 There is a constant $0<\mathfrak{v}_{\max }<1$, depending only on $\mathrm{m}$, such that, for all $0<t<\frac{1}{2}$ and $0<\mathfrak{v} \leq \mathfrak{v}_{\max }$

$$
\begin{aligned}
& \mathfrak{c}<\mathfrak{c}(2 t)<\mathfrak{c}(t) \leq 2 \mathfrak{c}(2 t) \\
& e^{-\mathrm{m} \mathfrak{c}} \leq \mathfrak{v} \\
& e^{-\frac{1}{4} \mathrm{~m} \mathfrak{c}(t)} \leq \min \left\{t \mathfrak{v}, \frac{1}{32}\right\} \\
& \sum_{k=0}^{\infty} \frac{1}{2^{-k} t} e^{-\frac{1}{8} m \mathfrak{c}\left(2^{-k} t\right)} \leq 1
\end{aligned}
$$

Proof: Parts (F.4.a-c) are obvious. For (F.4.d),

$$
\begin{aligned}
\sum_{k=0}^{\infty} \frac{1}{2^{-k} t} e^{-\frac{1}{8} \mathrm{mc}\left(2^{-k} t\right)} & =\sum_{k=0}^{\infty} e^{\log \frac{2^{k}}{t}-\frac{1}{8} \mathrm{~m} \log ^{2} \frac{2^{k}}{t \mathfrak{v}}} \leq \sum_{k=0}^{\infty} e^{\log \frac{2^{k}}{t}-2 \log \frac{2^{k}}{t \mathfrak{v}}} \leq \sum_{k=0}^{\infty} e^{-\log \frac{2^{k}}{t \mathfrak{v}}}=\sum_{k=0}^{\infty} \frac{t \mathfrak{v}}{2^{k}} \\
& =2 t \mathfrak{v}
\end{aligned}
$$

For the first inequality we used $\frac{1}{8} \mathrm{~m} \log \frac{2}{\mathfrak{v}} \geq 2$ and for the second inequality we used $\mathfrak{v} \leq 1$. 
Lemma F.3 There are constants $t_{\max }>0$ and $0<\mathfrak{v}_{\max }<1$, depending only on $\mathrm{m}$, such that the following is true for all $0<\delta \leq t_{\max }$ and $0<\mathfrak{v} \leq \mathfrak{v}_{\max }$. Let $\mathfrak{S}$ be a hierarchy for scale $\delta$ and let $\mathcal{J}^{\prime} \varsubsetneqq \mathcal{J}$ be decimation intervals for $\mathfrak{S}$, with lengths $t^{\prime}$ and $t$, respectively. For all $\mathbf{x} \in \Lambda(\mathcal{J})$ and $\mathbf{y} \in \Lambda\left(\mathcal{J}^{\prime}\right)^{c}$ we have

$$
\mathrm{R}\left(\frac{t^{\prime}}{2}\right) \leq e^{\frac{\mathrm{m}}{3} d(\mathbf{x}, \mathbf{y})} \mathrm{R}(t)
$$

Proof: Recall from (F.3.a) that $\mathrm{R}(t) \leq 2 \mathrm{R}(2 t)$. So

$$
\mathrm{R}\left(\frac{t^{\prime}}{2}\right) \leq \frac{2 t}{t^{\prime}} \mathrm{R}(t)
$$

and, by Definition II.4,

$$
d(\mathbf{x}, \mathbf{y}) \geq \mathfrak{c}\left(t^{\prime}\right)=\log ^{2} \frac{1}{t^{\prime} \mathfrak{v}}=\left(\log \frac{2 t}{t^{\prime}}+\log \frac{1}{2 t \mathfrak{v}}\right)^{2} \geq 2 \log \frac{1}{2 t \mathfrak{v}} \log \frac{2 t}{t^{\prime}} \geq \frac{3}{\mathrm{~m}} \log \frac{2 t}{t^{\prime}}
$$

assuming $2 \log \frac{1}{2 t \mathfrak{v}} \geq \frac{3}{\mathrm{~m}}$.

The next Proposition involves the constant $K_{d}=\sup _{\mathbf{y} \in X} \sum_{\mathbf{x} \in X} e^{-d(\mathbf{x}, \mathbf{y})}>1$, which is a characteristic quantity of the spatial lattice $X$ alone and was defined in the proof of Lemma III.42.

Lemma F.4 There are constants $t_{\max }>0$ and $\mathfrak{v}_{\max }>0$ (depending only on $e_{\mathrm{R}}, e_{\mathrm{R}^{\prime}}, e_{\mathrm{r}}$, $e_{\ell}, K_{d}, K_{\mu}, K_{j}^{\prime}$ and $\left.K_{j}\right)$, such that

$$
\begin{aligned}
2^{48} K_{d} e^{6 K_{j}} t \mathfrak{v} \mathrm{r}(t) \mathrm{R}(t)^{3} & <\frac{1}{40} \\
96 e^{K_{j}} \mathrm{R}(t) e^{-\frac{1}{4} \mathrm{~m} \mathfrak{c}(t)} & \leq 1 \\
e^{2 K_{j}^{\prime}+K_{j}} t\left\{\mathrm{R}^{\prime}(t)+e^{-\mathrm{mc}} \mathrm{R}(t)+|\mu| \mathrm{R}(t)\right\} & \leq \frac{1}{32} \mathrm{r}(t) \\
19+4 \mathrm{D}+10 \log \mathrm{R}(t) & \leq \frac{1}{4} \ell(2 t)
\end{aligned}
$$

for all $0<t \leq t_{\max }$ and $0<\mathfrak{v} \leq \mathfrak{v}_{\max }$.

Proof: For (F.6.a), just recall that $t \mathfrak{v} r(t) \mathrm{R}(t)^{3}=(t \mathfrak{v})^{1-3 e_{\mathrm{R}}-4 e_{\mathrm{r}}}$ and $3 e_{\mathrm{R}}+4 e_{r}<1$.

By (F.4.c), $\mathrm{R}(t) e^{-\frac{1}{4} \mathrm{~m} \mathfrak{c}(t)} \leq t \mathfrak{v} \mathrm{R}(t)=(t \mathfrak{v})^{1-e_{\mathrm{R}}-e_{\mathrm{r}}}$ tends to zero as $t \mathfrak{v} \rightarrow 0$ and (F.6.b) follows.

Since (F.4.b)

$$
\begin{array}{rl}
e^{2 K_{j}^{\prime}+K_{j}} & t\left\{\mathrm{R}^{\prime}(t)+e^{-\mathrm{mc}} \mathrm{R}(t)+|\mu| \mathrm{R}(t)\right\} \\
\leq e^{2 K_{j}^{\prime}+K_{j}}\left\{t^{1-e_{\mathrm{R}^{\prime}}}+(t \mathfrak{v})^{1-e_{\mathrm{R}}}+K_{\mu} t^{1-e_{\mathrm{R}}} \mathfrak{v}^{e_{\mu}-e_{\mathrm{R}}}\right\} \mathrm{r}(t) \leq \frac{1}{32} \mathrm{r}(t)
\end{array}
$$

and the constraint (F.6.c) is satisfied if $t$ is small enough.

The inequality (F.6.d) is satisfied since the ratio between $\log \mathrm{R}(t)=\left(e_{\mathrm{R}}+e_{\mathrm{r}}\right) \log \frac{1}{t \mathfrak{v}}$ and $\ell(2 t)=\left(\frac{1}{2 t \mathfrak{v}}\right)^{e_{\ell}}$ converges to zero as $t \mathfrak{v}$ tends to zero. 
As in the proof of Proposition III.36, set $K_{L 2}=\frac{1}{32} \min \left\{c_{\mathcal{H}} e^{-4 \mathrm{D} C_{\mathcal{H}}}, \frac{1}{32}\right\}$.

Lemma F.5 There are constants ${ }^{(1)} C_{L}$ and $t_{\max }$ such that, setting $L(t)=C_{L} r(t)^{2}$,

$$
\begin{aligned}
\frac{1}{2} K_{L 2} t \mathrm{R}^{\prime}(t)^{2} & \geq L(t) \\
K_{L 2} \mathrm{r}(t)^{2} & \geq L(t) \\
\frac{c_{v}}{512} t \mathfrak{v} \mathrm{R}(t)^{4} & \geq L(t) \\
6 e^{2 K_{j}^{\prime}}\left(t\left[|\mu|+e^{-5 \mathrm{mc}}\right] \mathrm{R}(2 t)+t \mathrm{R}^{\prime}(t)\right) & \leq \frac{L(t)}{\mathrm{r}(t)} \leq \frac{1}{3}(\mathrm{r}(t)-\mathrm{r}(2 t)) \\
2^{11} \sum_{p=q}^{n} \ell\left(2^{p} \varepsilon\right)\left(\sum_{k=q-1}^{p-1} \mathfrak{c}\left(2^{k} \varepsilon\right)\right)^{3} & \leq L\left(2^{q} \varepsilon\right)
\end{aligned}
$$

for all $0<t \leq t_{\max }, 0<\mathfrak{v} \leq \mathfrak{v}_{\max }$ (with the $\mathfrak{v}_{\max }$ of Lemma F.2) and all $\varepsilon>0$ and $n \in \mathbb{N}$ with $2^{n} \varepsilon \leq t_{\max }$.

Proof: Using (F.4.b), the constraint (F.7.d) will be satisfied if

$$
6 e^{2 K_{j}^{\prime}}\left(t^{1-e_{\mathrm{R}}}\left[K_{\mu} \mathfrak{v}^{e_{\mu}-e_{\mathrm{R}}}+\mathfrak{v}^{5-e_{\mathrm{R}}}\right]+t^{1-e_{\mathrm{R}^{\prime}}}\right) \mathrm{r}(t) \leq \frac{L(t)}{\mathrm{r}(t)}=C_{L} \mathrm{r}(t) \leq \frac{1}{3}\left(1-\frac{1}{2^{e_{\mathrm{r}}}}\right) \mathrm{r}(t)
$$

As long as $\mathfrak{v}$ is smaller than the $\mathfrak{v}_{\max }$ of Lemma F.2 and $t$ is small enough, we may choose $L(t)$ to be a small constant times $\mathrm{r}(t)^{2}$. We may also choose this constant to be smaller than $\frac{1}{2} K_{L 2}$ and $\frac{c_{v}}{512}$. Then constraints (F.7.a,b,c) are also satisfied.

Observe that, for any $0<2 t \mathfrak{v} \leq 1$ and real $b \geq 1$,

$$
\begin{aligned}
\left(\log \frac{1}{t \mathfrak{v}}\right)^{b+1} & =\left(\log 2+\log \frac{1}{2 t \mathfrak{v}}\right)^{b+1} \geq\left(\log \frac{1}{2 t \mathfrak{v}}\right)^{b+1}+(b+1)(\log 2)\left(\log \frac{1}{2 t \mathfrak{v}}\right)^{b} \\
& \geq\left(\log \frac{1}{2 t \mathfrak{v}}\right)^{b+1}+\left(\log \frac{1}{2 t \mathfrak{v}}\right)^{b}
\end{aligned}
$$

Iterating, we have, for all $\varepsilon>0$ and $0 \leq m<n$ with $0<2^{n} \varepsilon \mathfrak{v} \leq 1$ and any real $b \geq 1$,

$$
\left(\log \frac{1}{2^{m} \varepsilon \mathfrak{v}}\right)^{b+1} \geq\left(\log \frac{1}{2^{n} \varepsilon \mathfrak{v}}\right)^{b+1}+\sum_{j=m+1}^{n}\left(\log \frac{1}{2^{j} \varepsilon \mathfrak{v}}\right)^{b} \Longrightarrow \sum_{j=m}^{n}\left(\log \frac{1}{2^{j} \varepsilon \mathfrak{v}}\right)^{b} \leq 2\left(\log \frac{1}{2^{m} \varepsilon \mathfrak{v}}\right)^{b+1}
$$

and hence

$$
\begin{aligned}
2^{11} \sum_{p=q}^{n} \ell\left(2^{p} \varepsilon\right)\left(\sum_{k=q-1}^{p-1} \mathfrak{c}\left(2^{k} \varepsilon\right)\right)^{3} & \leq 2^{14} \sum_{p=q}^{n} \ell\left(2^{p} \varepsilon\right)\left(\log \frac{1}{2^{q-1} \varepsilon \mathfrak{v}}\right)^{9} \\
& \leq 2^{23}\left(\log \frac{1}{2^{q} \varepsilon \mathfrak{v}}\right)^{9} \sum_{p=q}^{n} \ell\left(2^{p} \varepsilon\right)
\end{aligned}
$$

(1) The constant $C_{L}$ depends only on $c_{\mathcal{H}}, C_{\mathcal{H}}, c_{v}, e_{\mathrm{R}^{\prime}}$ and $e_{\mathrm{r}}$. The constant $t_{\text {max }}$ depends only on $C_{L}$, $K_{j}^{\prime}, K_{\mu}, e_{\mu}, e_{\mathrm{R}}, e_{\mathrm{r}}, e_{\ell}$ 
Since, for any $e_{\ell}>0$,

$$
\sum_{p=q}^{n}\left(\frac{1}{2^{p} \varepsilon \mathfrak{v}}\right)^{e_{\ell}}=\left(\frac{1}{\varepsilon \mathfrak{v}}\right)^{e_{\ell}} \sum_{p=q}^{n} \frac{1}{2^{p e_{\ell}}}=\frac{1}{1-2^{-e_{\ell}}}\left(\frac{1}{2^{q} \varepsilon \mathfrak{v}}\right)^{e_{\ell}}
$$

the left hand side of (F.7.e)

$$
2^{11} \sum_{p=q}^{n} \ell\left(2^{p} \varepsilon\right)\left(\sum_{k=q-1}^{p-1} \mathfrak{c}\left(2^{k} \varepsilon\right)\right)^{3} \leq \frac{2^{23}}{1-2^{-e_{\ell}}}\left(\log \frac{1}{2^{q^{\varepsilon} \mathfrak{v}}}\right)^{9}\left(\frac{1}{2^{q} \varepsilon \mathfrak{v}}\right)^{e_{\ell}}
$$

is indeed smaller that $L\left(2^{q} \varepsilon\right)=C_{L}\left(\frac{1}{2^{q} \varepsilon \mathfrak{v}}\right)^{2 e_{\mathrm{r}}}$ if $e_{\ell}<2 e_{\mathrm{r}}$ and $2^{n} \varepsilon \mathfrak{v}$ is small enough.

During the construction, we introduced the auxiliary constants

○ $K_{R}=2^{12} K_{j}^{2}$ and $K_{E}=2^{23}$ in Theorem III.24,

○ $K_{D}=2^{35} e^{6 K_{j}}$ and $K_{L}=2^{48} e^{6 K_{j}}$ in Theorem III.26,

- $K_{\Delta}=2^{40} e^{10 K_{j}}$ in Theorem III.27 and

○ $K_{Q}=2^{9} e^{2 K_{j}}$ and $K_{\mathcal{V}}=2^{25} e^{6 K_{j}}$ in Propositions V.12 and V.13, respectively.

Lemma F.6 There is a constant $t_{\max }>0$ (depending only on $e_{\mathrm{R}}, e_{\mathrm{r}}$ and $K_{j}$ ), such that, for all $0<t \leq t_{\max }$ and $0<\mathfrak{v} \leq 1$, we have

$$
\begin{aligned}
& {\left[\frac{e^{t K_{j}}}{2^{1-2 e_{\mathrm{r}}}}+\frac{K_{j}^{2} e^{2 K_{j} t}}{K_{R}}(2 t \mathfrak{v})^{2 e_{\mathrm{r}}}+\left(\frac{\sqrt{K_{R}}}{2^{1-e_{\mathrm{R}}-2 e_{\mathrm{r}}}} \frac{t e^{-\mathrm{m} \mathfrak{c}}}{(t \mathfrak{v})^{e} \mathrm{R}^{+2 e_{\mathrm{r}}}}+\frac{2^{5+e_{\mathrm{r}}} K_{j} e^{K_{j} t}}{\sqrt{K_{R}}}\right)^{2}\right] \leq 1} \\
& \frac{e^{2 t K_{j}}}{2^{1-\left(2 e_{\mathrm{R}}+4 e_{\mathrm{r}}\right)}}+\frac{16 K_{j} e^{8 K_{j} t} e^{-\mathrm{m} \mathfrak{c}}}{K_{E} \mathrm{r}(2 t)^{2} \mathrm{R}(2 t)^{2} \mathfrak{v}}+\frac{1}{K_{E}} 2^{51}(2 t \mathfrak{v}) \mathrm{r}(2 t) \mathrm{R}(2 t)^{3}+\frac{2^{10} e^{-\frac{1}{2} \mathrm{~m} \mathfrak{c}}}{K_{E} \mathfrak{v} \mathrm{R}(2 t)^{2}}+\frac{2^{20}}{K_{E}} \leq 1 \\
& 2^{-2 e_{\mathrm{r}}} e^{2 K_{j}}+2^{28}(t \mathfrak{v}) \mathrm{r}(t) \mathrm{R}(t)^{3} \leq 1
\end{aligned}
$$

Proof: (a) Since $e_{\mathrm{r}}<\frac{1}{10}$,

$$
\frac{e^{t K_{j}}}{2^{1-2 e_{\mathrm{r}}}}+\left(\frac{2^{5+e_{\mathrm{r}}} K_{j} e^{K_{j} t}}{\sqrt{K_{R}}}\right)^{2} \leq\left(\frac{1}{2^{1-2 e_{\mathrm{r}}}}+\frac{1}{2^{2-2 e_{\mathrm{r}}}}\right) e^{2 K_{j} t} \leq \frac{7}{8} e^{2 K_{j} t}
$$

By (F.4.b), all remaining contributions may be made arbitrarily small by choosing $t_{\max }$ small enough.

(b) Since $1-\left(2 e_{\mathrm{R}}+4 e_{\mathrm{r}}\right)=1-\left(3 e_{\mathrm{R}}+4 e_{\mathrm{r}}\right)+e_{\mathrm{R}}>e_{\mathrm{R}} \geq \frac{1}{5}$, by (II.17), we have

$$
\frac{e^{2 t K_{j}}}{2^{1-\left(2 e_{\mathrm{R}}+4 e_{\mathrm{r}}\right)}}+\frac{1}{K_{E}} 2^{51}(2 t \mathfrak{v}) \mathrm{r}(2 t) \mathrm{R}(2 t)^{3}+\frac{2^{20}}{K_{E}} \leq 1-\frac{1}{2^{8}}
$$

by (F.6.a), if $t$ is small enough. Using (F.4.b) and (II.17), the remaining two terms can be made arbitrarily small.

(c) is obvious. 
The precise smallness assumptions on $\theta$ and $\mathfrak{v}$ in Theorems II.16 and II.18 are

\section{Hypothesis F.7}

(i) $\theta$ is smaller than $\frac{c_{v}}{2^{48} e^{6 K_{j}}\left(1+K_{\mu}^{3}\right)},\left(\frac{1-2^{-2\left(-3 e_{\mathrm{R}}-4 e_{\mathrm{r}}\right)}}{2^{17} e^{10 K_{j}}}\right)^{\frac{1}{1-2 e_{\mathrm{R}}-4 e_{\mathrm{r}}}}$ and each of the $t_{\max }$ 's of Lemmas F.1 to F.6.

(ii) $\mathfrak{v}$ is smaller than $\left(\frac{1}{2^{12} e^{2 K_{j}}}\right)^{\frac{1}{1-2 e_{\mathrm{R}}-4 e_{\mathrm{r}}}}$ and each of the $\mathfrak{v}_{\max }$ 's of Lemmas F.1 to F.5.

These are also all of the restrictions that we put on the constants $\Theta$ and $\mathfrak{v}_{0}$ of Theorems III.24, III.26 and Proposition III.29. 


\section{Appendix G: Changes of Variables and Estimates of Operator Norms}

The basic change of variables formula for the operator norms of Definition III.18 is Proposition III.19. It, and consequences of it, are proven in [BFKT4, §IV]. For the purposes of our construction, we need variants of these results for special situations, namely that the operators implementing the change of variables have restricted ranges or that the functions to which the change of variables is applied are polynomials. The first is treated in Proposition G.1 and the second in Lemma G.2 and Corollary G.3. Also, in Remark G.4 and Lemma G.5, we develop tools to bound the operator norms of Definition III.18.

We work in the same abstract environment as in $[\mathrm{BFKT} 4, \S \mathrm{IV}]$. We are given weight factors $\kappa_{1}, \cdots, \kappa_{s}$ on an abstract metric space $X$ with metric $d$. We consider analytic functions $f\left(\alpha_{1}, \cdots, \alpha_{s} ; \mathfrak{h}\right)$ of the complex fields $\alpha_{1}, \cdots, \alpha_{s}$ and the additional "history" field $\mathfrak{h}$. Denote by $w$ the weight system with metric $d$ that associates the weight factor $\kappa_{j}$ to the field $\alpha_{j}$, and the constant weight factor 1 to the history field $\mathfrak{h}$ (see Definition III.12).

Proposition G.1 Let $\Gamma_{j}, 1 \leq j \leq r$, be $\mathfrak{h}$-operators on $\mathbb{C}^{X}$. Let $\Lambda \subset X$ and, for each $1 \leq j \leq r, \Lambda_{j}$ be either $\Lambda$ or $\Lambda^{c}$. Set

$$
\tilde{f}\left(\alpha_{1}, \cdots, \alpha_{s-1}, \beta_{1}, \cdots, \beta_{r} ; \mathfrak{h}\right)=f\left(\alpha_{1}, \cdots, \alpha_{s-1}, \sum_{j=1}^{r} \Lambda_{j} \Gamma_{j} \beta_{j} ; \mathfrak{h}\right)
$$

Furthermore let $\tilde{\kappa}_{i}, 1 \leq i \leq s-1$ and $\lambda_{j}, 1 \leq j \leq r$ be weight factors. Let $\tilde{w}$ be the weight system with metric $d$ that associates the weight factor $\tilde{\kappa}_{i}$ to the field $\alpha_{i}$, for $1 \leq i \leq s-1$, the weight factor $\lambda_{j}$ to the field $\beta_{j}$, for $1 \leq j \leq r$, and the constant weight factor 1 to the history field $\mathfrak{h}$. Assume that $\tilde{\kappa}_{i}(\mathbf{x}) \leq \kappa_{i}(\mathbf{x})$ for all $1 \leq i \leq s-1$ and $\mathbf{x} \in X$ and that there is a $\nu>1$ such that

$$
\sum_{\substack{1 \leq j \leq r \\ \Lambda_{j}=\Lambda}} N_{d}\left(\Lambda_{j} \Gamma_{j} ; \kappa_{s}, \lambda_{j}\right) \leq \frac{1}{\nu} \quad \text { and } \quad \sum_{\substack{1 \leq j \leq r \\ \Lambda_{j}=\Lambda^{c}}} N_{d}\left(\Lambda_{j} \Gamma_{j} ; \kappa_{s}, \lambda_{j}\right) \leq \frac{1}{\nu}
$$

Then

$$
\|\tilde{f}\|_{\tilde{w}} \leq C_{\nu}\|f\|_{w} \quad \text { with } C_{\nu}= \begin{cases}\frac{4 \nu}{(e \ln \nu)^{2}} & \text { if } \nu \leq e \\ \frac{4}{\nu} & \text { if } e \leq \nu<4 \\ 1 & \text { if } \nu \geq 4\end{cases}
$$


Proof: We introduce auxiliary fields $\left\{\beta_{j}^{\prime}\right\}_{1 \leq j \leq r}$ and $\left\{\gamma_{1}, \gamma_{2}\right\}$ and define

$$
\begin{aligned}
f^{\prime}\left(\alpha_{1}, \cdots, \alpha_{s-1}, \gamma_{1}, \gamma_{2} ; \mathfrak{h}\right) & =f\left(\alpha_{1}, \cdots, \alpha_{s-1}, \Lambda \gamma_{1}+\Lambda^{c} \gamma_{2} ; \mathfrak{h}\right) \\
f^{\prime \prime}\left(\alpha_{1}, \cdots, \alpha_{s-1}, \beta_{1}^{\prime}, \cdots, \beta_{r}^{\prime} ; \mathfrak{h}\right) & =f^{\prime}\left(\alpha_{1}, \cdots, \alpha_{s-1}, \sum_{\substack{1 \leq j \leq r \\
\Lambda_{j}=\Lambda}} \beta_{j}^{\prime}, \sum_{\substack{1 \leq j \leq r \\
\Lambda_{j}=\Lambda}} \beta_{j}^{\prime} ; \mathfrak{h}\right)
\end{aligned}
$$

Then

$$
\tilde{f}\left(\alpha_{1}, \cdots, \alpha_{s-1}, \beta_{1}, \cdots, \beta_{r} ; \mathfrak{h}\right)=f^{\prime \prime}\left(\alpha_{1}, \cdots, \alpha_{s-1}, \Lambda_{1} \Gamma_{1} \beta_{1}, \cdots, \Lambda_{r} \Gamma_{r} \beta_{r} ; \mathfrak{h}\right)
$$

Set, for each $1 \leq j \leq r, t_{j}=N_{d}\left(\Lambda_{j} \Gamma_{j} ; \kappa_{s}, \lambda_{j}\right)$ and introduce the auxiliary weight system $w^{\prime}$ with metric $d$ that associates the weight factor one to the history field,

○ the weight factor $\kappa_{i}$ to the field $\alpha_{i}$, for each $1 \leq i \leq s-1$,

$\circ$ the weight factor $\lambda_{j}^{\prime}(\mathbf{x})=t_{j} \kappa_{s}(\mathbf{x})$ to the field $\beta_{j}^{\prime}$, for each $1 \leq j \leq r$, and

o the weight factors $\kappa_{1}^{\prime}(\mathbf{x})=\sum_{\substack{1 \leq j \leq r \\ \Lambda_{j}=\Lambda}} t_{j} \kappa_{s}(\mathbf{x})$ and $\kappa_{2}^{\prime}(\mathbf{x})=\sum_{\substack{1 \leq j \leq r \\ \Lambda_{j}=\Lambda^{c}}} t_{j} \kappa_{s}(\mathbf{x})$, respectively, to

the fields $\gamma_{1}$ and $\gamma_{2}$.

By [BFKT4, Proposition IV.4],

$$
\left\|\tilde{f}\left(\alpha_{1}, \cdots, \alpha_{s-1}, \beta_{1}, \cdots, \beta_{r} ; \mathfrak{h}\right)\right\|_{\tilde{w}} \leq\left\|f^{\prime \prime}\left(\alpha_{1}, \cdots, \alpha_{s-1}, \beta_{1}^{\prime}, \cdots, \beta_{r}^{\prime} ; \mathfrak{h}\right)\right\|_{w^{\prime}}
$$

since

$$
N_{d}\left(\Lambda_{j} \Gamma_{j} ; \lambda_{j}^{\prime}, \lambda_{j}\right)=N_{d}\left(\Lambda_{j} \Gamma_{j} ; t_{j} \kappa_{s}, \lambda_{j}\right)=\frac{1}{t_{j}} N_{d}\left(\Lambda_{j} \Gamma_{j} ; \kappa_{s}, \lambda_{j}\right)=1
$$

By [BFKT4, Lemma IV.5.i], applied twice,

$$
\left\|f^{\prime \prime}\left(\alpha_{1}, \cdots, \alpha_{s-1}, \beta_{1}^{\prime}, \cdots, \beta_{r}^{\prime} ; \mathfrak{h}\right)\right\|_{w^{\prime}} \leq\left\|f^{\prime}\left(\alpha_{1}, \cdots, \alpha_{s-1}, \gamma_{1}, \gamma_{2} ; \mathfrak{h}\right)\right\|_{w^{\prime}}
$$

since,

$$
\sum_{\substack{1 \leq j \leq r \\ \Lambda_{j}=\Lambda}} \sup _{\mathbf{x}} \frac{\lambda_{j}^{\prime}(\mathbf{x})}{\kappa_{1}^{\prime}(\mathbf{x})}=\sum_{\substack{1 \leq j \leq r \\ \Lambda_{j}=\Lambda^{c}}} \sup _{\mathbf{x}} \frac{\lambda_{j}^{\prime}(\mathbf{x})}{\kappa_{2}^{\prime}(\mathbf{x})}=1
$$

By part (iii) of [BFKT4, Lemma IV.5], with $r$ replaced by 2 ,

$$
\left\|f^{\prime}\left(\alpha_{1}, \cdots, \alpha_{s-1}, \gamma_{1}, \gamma_{2} ; \mathfrak{h}\right)\right\|_{w^{\prime}} \leq C_{\nu}\left\|f\left(\alpha_{1}, \cdots, \alpha_{s} ; \mathfrak{h}\right)\right\|_{w}
$$

since $C_{\nu}=C_{\nu, 2}$ and

$$
\sup _{\mathbf{x}} \frac{\kappa_{1}^{\prime}(\mathbf{x})}{\kappa_{s}(\mathbf{x})}=\sum_{\substack{1 \leq j \leq r \\ \Lambda_{j}=\Lambda}} t_{j} \leq \frac{1}{\nu} \quad \text { and } \quad \sup _{\mathbf{x}} \frac{\kappa_{1}^{\prime}(\mathbf{x})}{\kappa_{s}(\mathbf{x})}=\sum_{\substack{1 \leq j \leq r \\ \Lambda_{j}=\Lambda^{c}}} t_{j} \leq \frac{1}{\nu}
$$


In $\S \mathrm{V}$, we use a refinement of [BFKT4, Proposition IV.4] for functions of low degree in the fields that exploits exponential decay of operators away from a given subset $L$ of $X$. This estimate is contained in

Lemma G.2 Let $A_{1}, \cdots, A_{s}$ be $\mathfrak{h}$-operators on $\mathbb{C}^{X}$ and let $\kappa_{1}^{\prime}, \cdots \kappa_{s}^{\prime}$ be weight factors. Furthermore, let $f\left(\alpha_{1}, \cdots, \alpha_{s} ; \mathfrak{h}\right)$ be a polynomial in the fields $\alpha_{1}, \cdots, \alpha_{s}$ and set

$$
f^{\prime}\left(\alpha_{1}, \cdots, \alpha_{s} ; \mathfrak{h}\right)=f\left(A_{1} \alpha_{1}, \cdots, A_{s} \alpha_{s} ; \mathfrak{h}\right)
$$

(a) Assume that $f\left(\alpha_{1}, \cdots, \alpha_{s} ; \mathfrak{h}\right)$ is homogeneous of degree $d_{j}$ in $\alpha_{j}$ for each $1 \leq j \leq s$. Then

$$
\left\|f^{\prime}\right\|_{\tilde{w}} \leq\|f\|_{w} \prod_{j=1}^{s} N_{d}\left(A_{j} ; \kappa_{j}, \kappa_{j}^{\prime}\right)^{d_{j}}
$$

where $\tilde{w}$ is the weight system with metric d that associates the weight factor $\kappa_{j}^{\prime}$ to the field $\alpha_{j}$ and the constant weight factor 1 to the history field $\mathfrak{h}$.

(b) Let $t_{1}, \cdots, t_{s} \in \mathbb{R}$ with $t_{1}+\cdots+t_{s} \leq 0$. Also, Let $d^{\prime}$ and $\delta$ be metrics that obey

$$
d \geq d^{\prime}+\left(\sum_{\substack{i=1 \\ t_{i} \geq 0}}^{s} t_{i}\right) \delta
$$

Denote by $w^{\prime}$ the weight system with metric $d^{\prime}$ that associates the weight factor $\kappa_{j}^{\prime}$ to the field $\alpha_{j}$ and the constant weight factor 1 to the history field $\mathfrak{h}$.

Assume that $f\left(\alpha_{1}, \cdots, \alpha_{s} ; \mathfrak{h}\right)$ is homogenous of degree one in each of the fields $\alpha_{1}, \cdots, \alpha_{s}$. Then, for any subset $L$ of $X$,

$$
\left\|f^{\prime}\right\|_{w^{\prime}} \leq\|f\|_{w} \prod_{j=1}^{s} N_{d^{\prime}}\left(A_{j} ; \kappa_{j} e^{t_{j} \delta(\mathbf{x}, L)}, \kappa_{j}^{\prime}\right)
$$

Proof: (a) follows from [BFKT4, Proposition IV.4] by scaling.

(b) Denote by $w_{\text {aux }}$ the weight system with metric $d^{\prime}$ that associates the weight factor $\kappa_{j} e^{t_{j} \delta(\mathbf{x}, L)}$ to the field $\alpha_{j}$. We claim that

$$
\|f\|_{w_{\text {aux }}} \leq\|f\|_{w}
$$

The claim follows from (G.1), since, by part (a),

$$
\left\|f^{\prime}\right\|_{w^{\prime}} \leq\|f\|_{w_{\text {aux }}} \prod_{j=1}^{s} N_{d^{\prime}}\left(A_{j} ; \kappa_{j} e^{t_{j} \delta(\mathbf{x}, L)}, \kappa_{j}^{\prime}\right)
$$


To prove (G.1) we may assume that there is $r \geq 0$ such that $t_{1}, \cdots, t_{r} \geq 0$ and $t_{r+1}, \cdots, t_{s} \leq 0$. By hypothesis, $\quad \sum_{i=1}^{r} t_{i} \leq \sum_{j=r+1}^{s}\left(-t_{j}\right)$. Therefore there are $a_{i j} \geq 0$, $i=1, \cdots, r, j=r+1, \cdots, s$ such that

$$
\begin{gathered}
t_{i}=\sum_{j=r+1}^{s} a_{i j} \quad \text { for } i=1, \cdots, r \\
\left(-t_{j}\right) \geq \sum_{i=1}^{r} a_{i j} \quad \text { for } j=r+1, \cdots, s
\end{gathered}
$$

Now fix $\mathbf{x}_{1}, \cdots, \mathbf{x}_{s} \in X, \ell \geq 0$ and $\overrightarrow{\mathbf{z}} \in X^{\ell}$. Let $T$ be a tree whose set of vertices contains $\mathbf{x}_{1}, \cdots, \mathbf{x}_{s}, \mathbf{z}_{1}, \cdots, \mathbf{z}_{\ell}$. Denote by $\operatorname{length}_{\delta}(T)$ the length of $T$ with respect to the metric $\delta$. For each $1 \leq i, j \leq s$, the points $\mathbf{x}_{i}$ and $\mathbf{x}_{j}$ can be connected by a path in $T$. Therefore

$$
\delta\left(\mathbf{x}_{i}, L\right) \leq \delta\left(\mathbf{x}_{j}, L\right)+\operatorname{length}_{\delta}(T)
$$

Consequently

$$
\begin{aligned}
\sum_{i=1}^{s} t_{i} \delta\left(\mathbf{x}_{i}, L\right) & =\sum_{i=1}^{r} \sum_{j=r+1}^{s} a_{i j} \delta\left(\mathbf{x}_{i}, L\right)+\sum_{i=r+1}^{s} t_{i} \delta\left(\mathbf{x}_{i}, L\right) \\
& \leq \sum_{i=1}^{r} \sum_{j=r+1}^{s} a_{i j} \delta\left(\mathbf{x}_{j}, L\right)+\sum_{i=1}^{r} \sum_{j=r+1}^{s} a_{i j} \operatorname{length}_{\delta}(T)+\sum_{j=r+1}^{s} t_{j} \delta\left(\mathbf{x}_{j}, L\right) \\
& =\sum_{j=r+1}^{s}\left(t_{j}+\sum_{i=1}^{r} a_{i j}\right) \delta\left(\mathbf{x}_{j}, L\right)+\sum_{i=1}^{r} t_{i} \operatorname{length}_{\delta}(T) \\
& \leq\left(\sum_{i=1}^{r} t_{i}\right) \operatorname{length}_{\delta}(T)
\end{aligned}
$$

by (G.2). Therefore

$$
\operatorname{length}_{d^{\prime}}(T)+\sum_{i=1}^{s} t_{i} \delta\left(\mathbf{x}_{i}, L\right) \leq \operatorname{length}_{d}(T)
$$

This holds for any tree $T$, whose set of vertices contains $\mathbf{x}_{1}, \cdots, \mathbf{x}_{s}, \mathbf{z}_{1}, \cdots, \mathbf{z}_{\ell}$, so that

$$
\tau_{d^{\prime}}\left(\mathbf{x}_{1}, \cdots, \mathbf{x}_{s}, \overrightarrow{\mathbf{z}}\right)+\sum_{i=1}^{s} t_{i} \delta\left(\mathbf{x}_{i}, L\right) \leq \tau_{d}\left(\mathbf{x}_{1}, \cdots, \mathbf{x}_{s}, \overrightarrow{\mathbf{z}}\right)
$$

If we expand

$$
f\left(\alpha_{1}, \cdots, \alpha_{s} ; \mathfrak{h}\right)=\sum_{\ell \geq 0} \sum_{\substack{\mathbf{x}_{1}, \cdots, \mathbf{x}_{\ell} \in X \\ \mathbf{z} \in X^{\ell}}} a_{\ell}\left(\mathbf{x}_{1}, \cdots, \mathbf{x}_{s} ; \overrightarrow{\mathbf{z}}\right) \alpha_{1}\left(\mathbf{x}_{1}\right) \cdots \alpha_{s}\left(\mathbf{x}_{s}\right) \mathfrak{h}\left(\mathbf{z}_{1}\right) \cdots \mathfrak{h}\left(\mathbf{z}_{\ell}\right)
$$

and apply Definition III.12, we get (G.1) 
Corollary G.3 Let $h\left(\gamma_{1}, \cdots, \gamma_{r} ; \mathfrak{h}\right)$ be a multilinear form in the fields $\gamma_{1}, \cdots, \gamma_{r}$. Furthermore, let

$$
\Gamma_{i}^{j}, \tilde{\Gamma}_{i}^{j}, A_{j} \text { and } \tilde{A}_{j}
$$

$(i=1, \cdots, r ; j=1, \cdots, s)$ be $\mathfrak{h}$-operators on $\mathbb{C}^{X}$. Set

$$
\begin{aligned}
f_{1}\left(\alpha_{1}, \cdots, \alpha_{s} ; \mathfrak{h}\right) & =h\left(\sum_{j=1}^{s} \Gamma_{1}^{j} \alpha_{j}, \cdots, \sum_{j=1}^{s} \Gamma_{r}^{j} \alpha_{j} ; \mathfrak{h}\right) \\
f_{2}\left(\alpha_{1}, \cdots, \alpha_{s} ; \mathfrak{h}\right) & =h\left(\sum_{j=1}^{s} \Gamma_{1}^{j} \alpha_{j}, \cdots, \sum_{j=1}^{s} \Gamma_{r}^{j} \alpha_{j} ; \mathfrak{h}\right)-h\left(\sum_{j=1}^{s} \tilde{\Gamma}_{1}^{j} \alpha_{j}, \cdots, \sum_{j=1}^{s} \tilde{\Gamma}_{r}^{j} \alpha_{j} ; \mathfrak{h}\right) \\
f_{3}\left(\alpha_{1}, \cdots, \alpha_{s} ; \mathfrak{h}\right) & =h\left(\sum_{j=1}^{s} A_{1} \Gamma_{1}^{j} \alpha_{j}, \cdots, \sum_{j=1}^{s} A_{r} \Gamma_{r}^{j} \alpha_{j} ; \mathfrak{h}\right)-h\left(\sum_{j=1}^{s} \tilde{A}_{1} \Gamma_{1}^{j} \alpha_{j}, \cdots, \sum_{j=1}^{s} \tilde{A}_{r} \Gamma_{r}^{j} \alpha_{j} ; \mathfrak{h}\right) \\
& -h\left(\sum_{j=1}^{s} A_{1} \tilde{\Gamma}_{1}^{j} \alpha_{j}, \cdots, \sum_{j=1}^{s} A_{r} \tilde{\Gamma}_{r}^{j} \alpha_{j} ; \mathfrak{h}\right)+h\left(\sum_{j=1}^{s} \tilde{A}_{1} \tilde{\Gamma}_{1}^{j} \alpha_{j}, \cdots, \sum_{j=1}^{s} \tilde{A}_{r} \tilde{\Gamma}_{r}^{j} \alpha_{j} ; \mathfrak{h}\right)
\end{aligned}
$$

Let $\lambda_{1}, \cdots, \lambda_{r}$ be weight factors. Let $w^{\prime}$ be the weight system with some metric $d^{\prime}$ that associates the weight factor $\kappa_{j}$ to the field $\alpha_{j}$; and let $w_{\lambda}$ be the weight system with metric $d$ that associates the weight factor $\lambda_{i}$ to the field $\gamma_{i}$. Let $\delta$ either be 0 or a metric which obeys

$$
d \geq d^{\prime}+(r-1) \delta
$$

and let $L \subset X$. Then

$$
\left\|f_{1}\right\|_{w^{\prime}} \leq\|h\|_{w_{\lambda}} \prod_{i=1}^{r}\left(\sum_{j=1}^{s} N_{d^{\prime}}\left(\Gamma_{i}^{j} ; \lambda_{i} e^{t_{i} \delta(\mathbf{x}, L)}, \kappa_{j}\right)\right)
$$

where each $t_{i}, 1 \leq i \leq r$, is either 1 or $-(r-1)$ and at least one of them is $-(r-1)$

where

$$
\left\|f_{2}\right\|_{w^{\prime}} \leq r\|h\|_{w_{\lambda}} \sigma_{\delta} \sigma^{r-1}
$$

$$
\begin{aligned}
\sigma & =\max _{i=1, \cdots, r} \max \left\{\sum_{j=1}^{s} N_{d^{\prime}}\left(\Gamma_{i}^{j} ; \lambda_{i} e^{\delta(\mathbf{x}, L)}, \kappa_{j}\right), \sum_{j=1}^{s} N_{d^{\prime}}\left(\tilde{\Gamma}_{i}^{j} ; \lambda_{i} e^{\delta(\mathbf{x}, L)}, \kappa_{j}\right)\right\} \\
\sigma_{\delta} & =\max _{i=1, \cdots, r} \sum_{j=1}^{s} \frac{\kappa_{j}}{\lambda_{i}}\left\|\Gamma_{i}^{j}-\tilde{\Gamma}_{i}^{j}\right\| \|
\end{aligned}
$$

(iii)

$$
\left\|f_{3}\right\|_{w^{\prime}} \leq r^{2}\|h\|_{w_{\lambda}} \sigma_{\delta} a_{\delta}(\sigma a)^{r-1}
$$

where

$$
\begin{aligned}
a & =\max _{\substack{i=1, \cdots, \cdots, t=1,-(r-1)}} \max \left\{N_{d^{\prime}}\left(A_{i} ; \lambda_{i} e^{t \delta(\mathbf{x}, L)}, \lambda_{i} e^{t \delta(\mathbf{x}, L)}\right), N_{d^{\prime}}\left(\tilde{A}_{i} ; \lambda_{i} e^{t \delta(\mathbf{x}, L)}, \lambda_{i} e^{t \delta(\mathbf{x}, L)}\right)\right\} \\
a_{\delta} & =\max _{\substack{i=1, \cdots, r, r \\
t=1,-(r-1)}} N_{d^{\prime}}\left(A_{i}-\tilde{A}_{i} ; \lambda_{i} e^{t \delta(\mathbf{x}, L)}, \lambda_{i} e^{t \delta(\mathbf{x}, L)}\right)
\end{aligned}
$$


Proof: (i) It suffices to prove that, for each choice of $1 \leq j_{1}, \cdots, j_{r} \leq s$, the $\|\cdot\|_{w^{\prime}}$ norm of $h\left(\Gamma_{1}^{j_{1}} \alpha_{j_{1}}, \cdots, \Gamma_{r}^{j_{r}} \alpha_{j_{r}} ; \mathfrak{h}\right)$ is bounded by $\|h\|_{w_{\lambda}} \prod_{i=1}^{r} N_{d^{\prime}}\left(\Gamma_{i}^{j_{i}} ; \lambda_{i} e^{t_{i} \delta(\mathbf{x}, L)}, \kappa_{j_{i}}\right)$. This follows from Lemma G.2.b and [BFKT4, Lemma IV.5.ii] (when two or more of the $j_{i}$ 's happen to be the same).

(ii) Write the telescoping sum

$$
\begin{gathered}
f_{2}\left(\alpha_{1}, \cdots, \alpha_{s}\right)=h\left(\sum_{j=1}^{s}\left(\Gamma_{1}^{j}-\tilde{\Gamma}_{1}^{j}\right) \alpha_{j}, \sum_{j=1}^{s} \Gamma_{2}^{j} \alpha_{j}, \cdots, \sum_{j=1}^{s} \Gamma_{r}^{j} \alpha_{j}\right) \\
+h\left(\sum_{j=1}^{s} \tilde{\Gamma}_{1}^{j} \alpha_{j}, \sum_{j=1}^{s}\left(\Gamma_{2}^{j}-\tilde{\Gamma}_{2}^{j}\right) \alpha_{j}, \cdots, \sum_{j=1}^{s} \Gamma_{r}^{j} \alpha_{j}\right)+\cdots \\
\cdots+h\left(\sum_{j=1}^{s} \tilde{\Gamma}_{1}^{j} \alpha_{j}, \sum_{j=1}^{s} \tilde{\Gamma}_{2}^{j} \alpha_{j}, \cdots, \sum_{j=1}^{s}\left(\tilde{\Gamma}_{r}^{j}-\tilde{\Gamma}_{r}^{j}\right) \alpha_{j}\right)
\end{gathered}
$$

and apply part (i) to each of the summands. For term number $i_{0}$, which contains $\Gamma_{i_{0}}^{j}-\tilde{\Gamma}_{i_{0}}^{j}$, choose $t_{i_{0}}=-(r-1)$ and $t_{i}=1$ for all $i \neq i_{0}$. (We have suppressed the argument $\mathfrak{h}$ and will do so for the rest of the proof.)

(iii) Write the telescoping sum

$$
\begin{aligned}
& f_{3}\left(\alpha_{1}, \cdots, \alpha_{s}\right) \\
& =h\left(\sum_{j=1}^{s} A_{1}\left(\Gamma_{1}^{j}-\tilde{\Gamma}_{1}^{j}\right) \alpha_{j}, \cdots, \sum_{j=1}^{s} A_{r} \Gamma_{r}^{j} \alpha_{j}\right)-h\left(\sum_{j=1}^{s} \tilde{A}_{1}\left(\Gamma_{1}^{j}-\tilde{\Gamma}_{1}^{j}\right) \alpha_{j}, \cdots, \sum_{j=1}^{s} \tilde{A}_{r} \Gamma_{r}^{j} \alpha_{j}\right) \\
& \quad+\cdots+ \\
& \quad+h\left(\sum_{j=1}^{s} A_{1} \tilde{\Gamma}_{1}^{j} \alpha_{j}, \cdots, \sum_{j=1}^{s} A_{r}\left(\tilde{\Gamma}_{r}^{j}-\tilde{\Gamma}_{r}^{j}\right) \alpha_{j}\right)-h\left(\sum_{j=1}^{s} \tilde{A}_{1} \tilde{\Gamma}_{1}^{j} \alpha_{j}, \cdots, \sum_{j=1}^{s} \tilde{A}_{r}\left(\tilde{\Gamma}_{r}^{j}-\tilde{\Gamma}_{r}^{j}\right) \alpha_{j}\right)
\end{aligned}
$$

We claim that the $\|\cdot\|_{w}$ norm of each of the $r$ lines is bounded by $r\|h\|_{w_{\lambda}} \sigma_{\delta} a_{\delta}(\sigma a)^{r-1}$. We prove this for the first line. The proof for the other lines is similar. We again write a telescoping sum

$$
\begin{aligned}
h\left(\sum_{j=1}^{s} A_{1}\left(\Gamma_{1}^{j}-\tilde{\Gamma}_{1}^{j}\right) \alpha_{j}, \cdots, \sum_{j=1}^{s} A_{r} \Gamma_{r}^{j} \alpha_{j}\right)-h\left(\sum_{j=1}^{s} \tilde{A}_{1}\left(\Gamma_{1}^{j}-\tilde{\Gamma}_{1}^{j}\right) \alpha_{j}, \cdots, \sum_{j=1}^{s} \tilde{A}_{r} \Gamma_{r}^{j} \alpha_{j}\right) \\
=h\left(\sum_{j=1}^{s}\left(A_{1}-\tilde{A}_{1}\right)\left(\Gamma_{1}^{j}-\tilde{\Gamma}_{1}^{j}\right) \alpha_{j}, \sum_{j=1}^{s} A_{2} \Gamma_{2}^{j} \alpha_{j}, \cdots, \sum_{j=1}^{s} A_{r} \Gamma_{r}^{j} \alpha_{j}\right) \\
+h\left(\sum_{j=1}^{s} \tilde{A}_{1}\left(\Gamma_{1}^{j}-\tilde{\Gamma}_{1}^{j}\right) \alpha_{j}, \sum_{j=1}^{s}\left(A_{2}-\tilde{A}_{2}\right) \Gamma_{2}^{j} \alpha_{j}, \cdots, \sum_{j=1}^{s} A_{r} \Gamma_{r}^{j} \alpha_{j}\right) \\
+\cdots+ \\
+h\left(\sum_{j=1}^{s} \tilde{A}_{1}\left(\Gamma_{1}^{j}-\tilde{\Gamma}_{1}^{j}\right) \alpha_{j}, \sum_{j=1}^{s} \tilde{A}_{2} \Gamma_{2}^{j} \alpha_{j}, \cdots, \sum_{j=1}^{s}\left(A_{r}-\tilde{A}_{r}\right) \Gamma_{r}^{j} \alpha_{j}\right)
\end{aligned}
$$


By the first bound, the $\|\cdot\|_{w}$ norm of the first term is bounded by

$$
\begin{aligned}
& \|h\|_{w_{\lambda}}\left(\sum_{j=1}^{s} N_{d^{\prime}}\left(\left(A_{1}-\tilde{A}_{1}\right)\left(\Gamma_{1}^{j}-\tilde{\Gamma}_{1}^{j}\right) ; \lambda_{1} e^{-(r-1) \delta(\mathbf{x}, L)}, \kappa_{j}\right)\right) \prod_{i=2}^{r}\left(\sum_{j=1}^{s} N_{d^{\prime}}\left(A_{i} \Gamma_{i}^{j} ; \lambda_{i} e^{\delta(\mathbf{x}, L)}, \kappa_{j}\right)\right) \\
& \leq\|h\|_{w_{\lambda}} N_{d^{\prime}}\left(A_{1}-\tilde{A}_{1} ; \lambda_{1} e^{-(r-1) \delta(\mathbf{x}, L)}, \lambda_{1} e^{-(r-1) \delta(\mathbf{x}, L)}\right)\left(\sum_{j=1}^{s} N_{d^{\prime}}\left(\Gamma_{1}^{j}-\tilde{\Gamma}_{1}^{j} ; \lambda_{1} e^{-(r-1) \delta(\mathbf{x}, L)}, \kappa_{j}\right)\right) \\
& \qquad \prod_{i=2}^{r}\left(N_{d^{\prime}}\left(A_{i} ; \lambda_{i} e^{\delta(\mathbf{x}, L)}, \lambda_{i} e^{\delta(\mathbf{x}, L)}\right) \sum_{j=1}^{s} N_{d^{\prime}}\left(\Gamma_{i}^{j} ; \lambda_{i} e^{\delta(\mathbf{x}, L)}, \kappa_{j}\right)\right) \\
& \leq\|h\|_{w_{\lambda}}\left(a_{\delta} \sum_{j=1}^{s} N_{d^{\prime}}\left(\Gamma_{1}^{j}-\tilde{\Gamma}_{1}^{j} ; \lambda_{1} e^{-(r-1) \delta(\mathbf{x}, L)}, \kappa_{j}\right)\right) \prod_{i=2}^{r}\left(\sum_{j=1}^{s} a N_{d^{\prime}}\left(\Gamma_{i}^{j} ; \lambda_{i} e^{\delta(\mathbf{x}, L)}, \kappa_{j}\right)\right) \\
& \leq\|h\|_{w_{\lambda}} \sigma_{\delta} a_{\delta}(\sigma a)^{r-1}
\end{aligned}
$$

by [BFKT4, Remark IV.3.ii]. The norm of the second term is bounded by

$$
\begin{gathered}
\|h\|_{w_{\lambda}}\left(\sum_{j=1}^{s} N_{d^{\prime}}\left(\tilde{A}_{1}\left(\Gamma_{1}^{j}-\tilde{\Gamma}_{1}^{j}\right) ; \lambda_{1} e^{-(r-1) \delta(\mathbf{x}, L)}, \kappa_{j}\right)\right)\left(\sum_{j=1}^{s} N_{d^{\prime}}\left(\left(A_{2}-\tilde{A}_{2}\right) \Gamma_{2}^{j} ; \lambda_{2} e^{\delta(\mathbf{x}, L)}, \kappa_{j}\right)\right) \\
\prod_{i=3}^{r}\left(\sum_{j=1}^{s} N_{d^{\prime}}\left(A_{i} \Gamma_{i}^{j} ; \lambda_{i} e^{\delta(\mathbf{x}, L)}, \kappa_{j}\right)\right) \\
\leq\|h\|_{w_{\lambda}}\left(\sigma_{\delta} a\right)\left(\sigma a_{\delta}\right)(\sigma a)^{r-2}
\end{gathered}
$$

Similarly, one bounds the norms of each of the other $r-2$ terms by $\|h\|_{w_{\lambda}} \sigma_{\delta} a_{\delta}(\sigma a)^{r-1}$.

In order to apply Proposition III.19, [BFKT4, Corollary IV.6] or Lemma G.2 we need techniques to estimate operator norms. They are given in Remark G.4 and Lemma G.5, below.

Remark G.4 Let $A$ be an $\mathfrak{h}$-linear map from $\mathbb{C}^{X}$ to $\mathbb{C}^{X}$. Let $d_{1}, d_{2}$ be metrics with $d_{1}-d_{2} \geq d$. Furthermore let $\kappa, \kappa^{\prime}$ be weight factors.

(i)

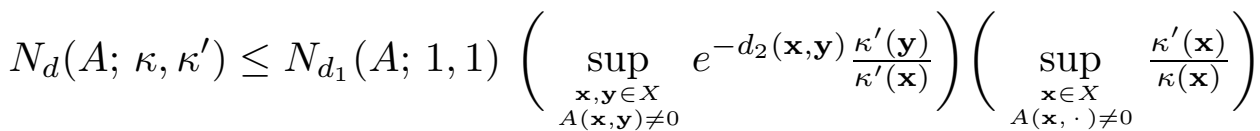

$$
\begin{aligned}
& N_{d}\left(A ; \kappa, \kappa^{\prime}\right) \leq N_{d_{1}}(A ; 1,1)\left(\sup _{\substack{\mathbf{x}, \mathbf{y} \in X \\
A(\mathbf{x}, \mathbf{y}) \neq 0}} e^{-d_{2}(\mathbf{x}, \mathbf{y})} \frac{\kappa(\mathbf{y})}{\kappa(\mathbf{x})}\right)\left(\sup _{\substack{\mathbf{y} \in X \\
A(,, \mathbf{y}) \neq 0}} \frac{\kappa^{\prime}(\mathbf{y})}{\kappa(\mathbf{y})}\right)
\end{aligned}
$$

(ii) Let $\mathfrak{c}>0$ and define the $\mathfrak{h}$-linear operator $A_{\mathfrak{c}}$ by

$$
A_{\mathfrak{c}}(\mathbf{x} ; \overrightarrow{\mathbf{z}} ; \mathbf{y})= \begin{cases}A(\mathbf{x} ; \overrightarrow{\mathbf{z}} ; \mathbf{y}) & \text { if } d_{2}(\mathbf{x}, \mathbf{y}) \leq \mathfrak{c} \\ 0 & \text { if } d_{2}(\mathbf{x}, \mathbf{y})>\mathfrak{c}\end{cases}
$$


Then

$$
N_{d}\left(A_{\mathfrak{c}}-A ; \kappa, \kappa^{\prime}\right) \leq e^{-\mathfrak{c}} N_{d_{1}}\left(A ; \kappa, \kappa^{\prime}\right)
$$

(iii) Denote

$$
K=\sup _{\substack{\mathbf{x}, \mathbf{y} \in X \\ A(\mathbf{x} ; \cdot ; \mathbf{y}) \neq 0}} \frac{\kappa^{\prime}(\mathbf{y})}{\kappa(\mathbf{x})} e^{-d_{2}(\mathbf{x}, \mathbf{y})}
$$

Then

$$
N_{d}\left(A ; \kappa, \kappa^{\prime}\right) \leq K N_{d_{1}}(A ; 1,1)
$$

(iv) Let $J$ be a linear operator on $\mathbb{C}^{X}$. Then

$$
\begin{aligned}
N_{d}(\operatorname{exph}(J) ; \kappa, \kappa) & \leq e^{N_{d}(J ; \kappa, \kappa)} \\
N_{d}(\operatorname{exph}(J)-\mathfrak{h} ; \kappa, \kappa) & \leq N_{d}(J ; \kappa, \kappa) e^{N_{d}(J ; \kappa, \kappa)}
\end{aligned}
$$

Proof: To prove parts (i) and (ii), observe that, for all $\mathbf{x}, \mathbf{y} \in X$ and all $\overrightarrow{\mathbf{z}} \in X^{(1)}$

$$
e^{\tau_{d}(\operatorname{supp}(\mathbf{x}, \overrightarrow{\mathbf{z}}, \mathbf{y}))}|A(\mathbf{x} ; \overrightarrow{\mathbf{z}} ; \mathbf{y})| \frac{\kappa^{\prime}(\mathbf{y})}{\kappa(\mathbf{x})} \leq e^{-d_{2}(\mathbf{x}, \mathbf{y})} e^{\tau_{d_{1}}(\operatorname{supp}(\mathbf{x}, \overrightarrow{\mathbf{z}}, \mathbf{y}))}|A(\mathbf{x} ; \overrightarrow{\mathbf{z}} ; \mathbf{y})| \frac{\kappa^{\prime}(\mathbf{y})}{\kappa(\mathbf{x})}
$$

This immediately gives part (ii). The two inequalities of part (i) follow by writing

$$
\frac{\kappa^{\prime}(\mathbf{y})}{\kappa(\mathbf{x})}=\frac{\kappa^{\prime}(\mathbf{y})}{\kappa^{\prime}(\mathbf{x})} \quad \frac{\kappa^{\prime}(\mathbf{x})}{\kappa(\mathbf{x})}=\frac{\kappa(\mathbf{y})}{\kappa(\mathbf{x})} \quad \frac{\kappa^{\prime}(\mathbf{y})}{\kappa(\mathbf{y})}
$$

Similarly, part (iii) follows from

$$
\begin{aligned}
e^{\tau_{d}(\operatorname{supp}(\mathbf{x}, \overrightarrow{\mathbf{z}}, \mathbf{y}))}|A(\mathbf{x} ; \overrightarrow{\mathbf{z}} ; \mathbf{y})| \frac{\kappa^{\prime}(\mathbf{y})}{\kappa(\mathbf{x})} & \leq K e^{d_{2}(\mathbf{x}, \mathbf{y})} e^{\tau_{d}(\operatorname{supp}(\mathbf{x}, \overrightarrow{\mathbf{z}}, \mathbf{y}))}|A(\mathbf{x} ; \overrightarrow{\mathbf{z}} ; \mathbf{y})| \\
& \leq K e^{\tau_{d_{1}}(\operatorname{supp}(\mathbf{x}, \overrightarrow{\mathbf{z}}, \mathbf{y}))}|A(\mathbf{x} ; \overrightarrow{\mathbf{z}} ; \mathbf{y})|
\end{aligned}
$$

Part (iv) follows from the expansion

$$
\operatorname{exph}(J)=e^{\bar{J}}=\mathfrak{h}+\sum_{\ell=1}^{\infty} \frac{1}{\ell !} \bar{J}^{\ell}
$$

and [BFKT4, (IV.1)]. 
In Appendix E, we use a more sophisticated

\section{Lemma G.5 Let}

○ $L_{1}, L_{2}, L_{3}, O_{1}, O_{2} \subset X$

- $A_{1}, A_{2}$ be $\mathfrak{h}$-linear operators on $\mathbb{C}^{X}$

- $\delta_{1}, \delta_{2}, \delta, \tilde{d}$ metrics on $X$

$\circ \mathrm{R}>0$ and $\kappa$ a weight factor such that $\kappa(\mathbf{x}) \leq \mathrm{R} e^{\delta_{2}\left(\mathbf{x}, O_{2}\right)}$ for all $\mathbf{x} \in L_{3}$. Set

$$
\begin{aligned}
& D_{L}=\max \left\{\delta\left(L_{1}, L_{2}\right), \delta\left(L_{2}, L_{3}\right), \delta\left(L_{3}, L_{1}\right)\right\} \\
& D_{O}=\max \left\{D_{L}, \delta\left(L_{1}, O_{1}\right), \delta\left(L_{2}, O_{1}\right), \delta\left(L_{3}, O_{1}\right)\right\}
\end{aligned}
$$

(i) Assume that $d+\delta_{2}+\delta \leq \tilde{d}$. If

(a) $L_{j} \subset O_{2}$ for at least one $j \in\{1,2,3\}$ and $D=D_{L}$

or if

(b) $L_{j} \subset O_{2}$ for at least one $j \in\{1,2,3\}, \delta_{1} \geq \delta$ and $D=D_{O}$ or if

(c) $O_{1} \subset O_{2}, \delta_{1} \geq \delta+\delta_{2}$ and $D=D_{O}$

then

$$
N_{d}\left(L_{1} A_{1} L_{2} A_{2} L_{3} ; e^{\delta_{1}\left(\mathbf{x}, O_{1}\right)}, \kappa\right) \leq \mathrm{R} e^{-D} N_{\tilde{d}}\left(L_{1} A_{1} L_{2} ; 1,1\right) N_{\tilde{d}}\left(L_{2} A_{2} L_{3} ; 1,1\right)
$$

(ii) Assume that $d+\delta_{1}+\delta_{2}+\delta \leq \tilde{d}$ and that $L_{i} \subset O_{1}, L_{j} \subset O_{2}$ for some $1 \leq i, j \leq 3$. Then

$$
N_{d}\left(L_{1} A_{1} L_{2} A_{2} L_{3} ; e^{-\delta_{1}\left(\mathbf{x}, O_{1}\right)}, \kappa\right) \leq \mathrm{R} e^{-D_{L}} N_{\tilde{d}}\left(L_{1} A_{1} L_{2} ; 1,1\right) N_{\tilde{d}}\left(L_{2} A_{2} L_{3} ; 1,1\right)
$$

Proof: We may assume that $\mathrm{R}=1$ and that $\kappa(\mathbf{x})=e^{\delta_{2}\left(\mathbf{x}, O_{2}\right)}$. We write the kernel of the operator $L_{1} A_{1} L_{2} A_{2} L_{3}$ as

$$
\left(L_{1} A_{1} L_{2} A_{2} L_{3}\right)(\mathbf{x} ; \overrightarrow{\mathbf{z}} ; \mathbf{y})=\sum_{\substack{\mathbf{u} \in L_{2} \\ \overrightarrow{\mathbf{z}}_{1}, \mathbf{z}_{\mathbf{2}} \in X(1) \\ \mathbf{z}_{1}(\mathbf{u}) \circ \overrightarrow{\mathbf{z}}_{2}=\overrightarrow{\mathbf{z}}}}\left(L_{1} A_{1} L_{2}\right)\left(\mathbf{x} ; \overrightarrow{\mathbf{z}}_{1} ; \mathbf{u}\right)\left(L_{2} A_{2} L_{3}\right)\left(\mathbf{u} ; \overrightarrow{\mathbf{z}}_{2} ; \mathbf{y}\right)
$$

(i) Fix $\mathbf{x} \in L_{1}, \mathbf{u} \in L_{2}, \mathbf{y} \in L_{3}, \overrightarrow{\mathbf{z}}_{1}, \overrightarrow{\mathbf{z}}_{2} \in X^{(1)}$. Then

$$
\begin{array}{r}
-\delta_{1}\left(\mathbf{x}, O_{1}\right)+\tau_{d}\left(\operatorname{supp}\left(\mathbf{x} ; \overrightarrow{\mathbf{z}}_{1} \circ \mathbf{u} \circ \overrightarrow{\mathbf{z}}_{2} ; \mathbf{y}\right)\right)+\delta_{2}\left(\mathbf{y}, O_{2}\right) \\
\leq \tau_{d}\left(\operatorname{supp}\left(\mathbf{x} ; \overrightarrow{\mathbf{z}}_{1} ; \mathbf{u}\right)\right)+\tau_{d}\left(\operatorname{supp}\left(\mathbf{u} ; \overrightarrow{\mathbf{z}}_{2} ; \mathbf{y}\right)\right)+\delta(\mathbf{x}, \mathbf{u})+\delta(\mathbf{u}, \mathbf{y})+\delta_{2}\left(\mathbf{y}, O_{2}\right) \\
-\delta_{1}\left(\mathbf{x}, O_{1}\right)-\delta(\mathbf{x}, \mathbf{u})-\delta(\mathbf{u}, \mathbf{y}) \\
\leq \tau_{\tilde{d}}\left(\operatorname{supp}\left(\mathbf{x} ; \overrightarrow{\mathbf{z}}_{1} ; \mathbf{u}\right)\right)+\tau_{\tilde{d}}\left(\operatorname{supp}\left(\mathbf{u} ; \overrightarrow{\mathbf{z}}_{2} ; \mathbf{y}\right)\right)-\delta_{2}(\mathbf{x}, \mathbf{u})-\delta_{2}(\mathbf{u}, \mathbf{y})+\delta_{2}\left(\mathbf{y}, O_{2}\right) \\
-\delta_{1}\left(\mathbf{x}, O_{1}\right)-\delta(\mathbf{x}, \mathbf{u})-\delta(\mathbf{u}, \mathbf{y})
\end{array}
$$


As $\mathbf{x} \in L_{1}, \mathbf{u} \in L_{2}, \mathbf{y} \in L_{3}$

$$
\begin{aligned}
\delta(\mathbf{x}, \mathbf{u})+\delta(\mathbf{u}, \mathbf{y}) & \geq D_{L} \\
\delta\left(\mathbf{x}, O_{1}\right)+\delta(\mathbf{x}, \mathbf{u})+\delta(\mathbf{u}, \mathbf{y}) & \geq D_{O}
\end{aligned}
$$

If $L_{j} \subset O_{2}$ for at least one $j \in\{1,2,3\}$ then

$$
-\delta_{2}(\mathbf{x}, \mathbf{u})-\delta_{2}(\mathbf{u}, \mathbf{y})+\delta_{2}\left(\mathbf{y}, O_{2}\right) \leq 0
$$

Therefore

$$
-\delta_{2}(\mathbf{x}, \mathbf{u})-\delta_{2}(\mathbf{u}, \mathbf{y})+\delta_{2}\left(\mathbf{y}, O_{2}\right)-\delta_{1}\left(\mathbf{x}, O_{1}\right)-\delta(\mathbf{x}, \mathbf{u})-\delta(\mathbf{u}, \mathbf{y}) \leq \begin{cases}-D_{L} & \text { in case a }) \\ -D_{O} & \text { in case b) }\end{cases}
$$

In case $\mathrm{c}), \delta_{1}\left(\mathbf{x}, O_{1}\right) \geq \delta_{2}\left(\mathbf{x}, O_{2}\right)+\delta\left(\mathbf{x}, O_{1}\right)$ so that

$$
\begin{aligned}
& -\delta_{2}(\mathbf{x}, \mathbf{u})-\delta_{2}(\mathbf{u}, \mathbf{y})+\delta_{2}\left(\mathbf{y}, O_{2}\right)-\delta_{1}\left(\mathbf{x}, O_{1}\right)-\delta(\mathbf{x}, \mathbf{u})-\delta(\mathbf{u}, \mathbf{y}) \\
& \quad \leq-\delta_{2}(\mathbf{x}, \mathbf{u})-\delta_{2}(\mathbf{u}, \mathbf{y})+\delta_{2}\left(\mathbf{y}, O_{2}\right)-\delta_{2}\left(\mathbf{x}, O_{2}\right)-\delta\left(\mathbf{x}, O_{1}\right)-\delta(\mathbf{x}, \mathbf{u})-\delta(\mathbf{u}, \mathbf{y}) \\
& \quad \leq-D_{O}
\end{aligned}
$$

Consequently, in all three cases

$$
\begin{aligned}
-\delta_{1}\left(\mathbf{x}, O_{1}\right)+\tau_{d}\left(\operatorname{supp}\left(\mathbf{x} ; \overrightarrow{\mathbf{z}}_{1} \circ \mathbf{u} \circ \overrightarrow{\mathbf{z}}_{2} ; \mathbf{y}\right)\right)+\delta_{2}\left(\mathbf{y}, O_{2}\right) \\
\leq-D+\tau_{\tilde{d}}\left(\operatorname{supp}\left(\mathbf{x} ; \overrightarrow{\mathbf{z}}_{1} ; \mathbf{u}\right)\right)+\tau_{\tilde{d}}\left(\operatorname{supp}\left(\mathbf{u} ; \overrightarrow{\mathbf{z}}_{2} ; \mathbf{y}\right)\right)
\end{aligned}
$$

so that

$$
\begin{aligned}
& e^{-\delta_{1}\left(\mathbf{x}, O_{1}\right)} e^{\tau_{d}\left(\operatorname{supp}\left(\mathbf{x} ; \overrightarrow{\mathbf{z}}_{1} \circ \mathbf{u} \circ \overrightarrow{\mathbf{z}}_{2} ; \mathbf{y}\right)\right)}\left|\left(L_{1} A_{1} L_{2}\right)\left(\mathbf{x} ; \overrightarrow{\mathbf{z}}_{1} ; \mathbf{u}\right)\left(L_{2} A_{2} L_{3}\right)\left(\mathbf{u} ; \overrightarrow{\mathbf{z}}_{2} ; \mathbf{y}\right)\right| \kappa(\mathbf{y}) \\
& \quad \leq e^{-D} e^{\tau_{\tilde{d}}\left(\operatorname{supp}\left(\mathbf{x} ; \overrightarrow{\mathbf{z}}_{1} ; \mathbf{u}\right)\right)}\left|\left(L_{1} A_{1} L_{2}\right)\left(\mathbf{x} ; \overrightarrow{\mathbf{z}}_{1} ; \mathbf{u}\right)\right| e^{\tau_{\tilde{d}}\left(\operatorname{supp}\left(\mathbf{u} ; \overrightarrow{\mathbf{z}}_{2} ; \mathbf{y}\right)\right)}\left|\left(L_{2} A_{2} L_{3}\right)\left(\mathbf{u} ; \overrightarrow{\mathbf{z}}_{2} ; \mathbf{y}\right)\right|
\end{aligned}
$$

If we define the auxiliary $\mathfrak{h}$-linear operators $\tilde{A}_{1}$ and $\tilde{A}_{2}$ by

$$
\tilde{A}_{1}(\mathbf{x} ; \overrightarrow{\mathbf{z}} ; \mathbf{y})=e^{\tau_{\tilde{d}}(\operatorname{supp}(\mathbf{x} ; \overrightarrow{\mathbf{z}} ; \mathbf{y}))}\left|A_{1}(\mathbf{x} ; \overrightarrow{\mathbf{z}} ; \mathbf{y})\right| \quad \tilde{A}_{2}(\mathbf{x} ; \overrightarrow{\mathbf{z}} ; \mathbf{y})=e^{\tau_{\tilde{d}}(\operatorname{supp}(\mathbf{x} ; \overrightarrow{\mathbf{z}} ; \mathbf{y}))}\left|A_{2}(\mathbf{x} ; \overrightarrow{\mathbf{z}} ; \mathbf{y})\right|
$$

we now have, by [BFKT4, (IV.1)],

$$
\begin{aligned}
N_{d}\left(L_{1} A_{1} L_{2} A_{2} L_{3} ; e^{\delta_{1}\left(\mathbf{x}, O_{1}\right)}, \kappa\right) & \leq \mathrm{R} e^{-D} N_{0}\left(L_{1} \tilde{A}_{1} L_{2} \tilde{A}_{2} L_{3} ; 1,1\right) \\
& \leq \mathrm{R} e^{-D} N_{0}\left(L_{1} \tilde{A}_{1} L_{2} ; 1,1\right) N_{0}\left(L_{2} \tilde{A}_{2} L_{3} ; 1,1\right) \\
& =\mathrm{R} e^{-D} N_{\tilde{d}}\left(L_{1} A_{1} L_{2} ; 1,1\right) N_{\tilde{d}}\left(L_{2} A_{2} L_{3} ; 1,1\right)
\end{aligned}
$$

(ii) As in part (i), fix $\mathbf{x} \in L_{1}, \mathbf{u} \in L_{2}, \mathbf{y} \in L_{3}, \overrightarrow{\mathbf{z}}_{1}, \overrightarrow{\mathbf{z}}_{2} \in X^{(1)}$ and bound

$$
\begin{aligned}
\delta_{1}\left(\mathbf{x}, O_{1}\right)+\tau_{d}\left(\operatorname{supp}\left(\mathbf{x} ; \overrightarrow{\mathbf{z}}_{1} \circ \mathbf{u} \circ \overrightarrow{\mathbf{z}}_{2} ; \mathbf{y}\right)\right)+\delta_{2}\left(\mathbf{y}, O_{2}\right) \\
\leq \tau_{d}\left(\operatorname{supp}\left(\mathbf{x} ; \overrightarrow{\mathbf{z}}_{1} ; \mathbf{u}\right)\right)+\tau_{d}\left(\operatorname{supp}\left(\mathbf{u} ; \overrightarrow{\mathbf{z}}_{2} ; \mathbf{y}\right)\right)+\delta(\mathbf{x}, \mathbf{u})+\delta(\mathbf{u}, \mathbf{y})+\delta_{1}\left(\mathbf{x}, O_{1}\right) \\
\quad+\delta_{2}\left(\mathbf{y}, O_{2}\right)-\delta(\mathbf{x}, \mathbf{u})-\delta(\mathbf{u}, \mathbf{y}) \\
\leq \tau_{\tilde{d}}\left(\operatorname{supp}\left(\mathbf{x} ; \overrightarrow{\mathbf{z}}_{1} ; \mathbf{u}\right)\right)+\tau_{\tilde{d}}\left(\operatorname{supp}\left(\mathbf{u} ; \overrightarrow{\mathbf{z}}_{2} ; \mathbf{y}\right)\right)-\left(\delta_{1}(\mathbf{x}, \mathbf{u})+\delta_{1}(\mathbf{u}, \mathbf{y})-\delta_{1}\left(\mathbf{x}, O_{1}\right)\right) \\
\quad-\left(\delta_{2}(\mathbf{x}, \mathbf{u})+\delta_{2}(\mathbf{u}, \mathbf{y})-\delta_{2}\left(\mathbf{y}, O_{2}\right)\right)-(\delta(\mathbf{x}, \mathbf{u})+\delta(\mathbf{u}, \mathbf{y})) \\
\leq-D_{L}+\tau_{\tilde{d}}\left(\operatorname{supp}\left(\mathbf{x} ; \overrightarrow{\mathbf{z}}_{1} ; \mathbf{u}\right)\right)+\tau_{\tilde{d}}\left(\operatorname{supp}\left(\mathbf{u} ; \overrightarrow{\mathbf{z}}_{2} ; \mathbf{y}\right)\right)
\end{aligned}
$$




\section{Appendix H: Symbol Table}

\begin{tabular}{|c|c|c|}
\hline Notation & Definition & Comments \\
\hline$\nabla$ & $(\nabla f)((\mathbf{x}, \mathbf{y}))=f(\mathbf{y})-f(\mathbf{x})$ & discrete gradient \\
\hline$\|v\|$ & $\sup _{\mathbf{x} \in X} \sum_{\mathbf{y} \in X} e^{5 \mathrm{~m} d(\mathbf{x}, \mathbf{y})}|v(\mathbf{x}, \mathbf{y})|$ & (II.5) \\
\hline$|\|A \mid\|$ & $\mathcal{N}_{5 \mathrm{~m}}(A ; 1,1)$ & Definition III.20 \\
\hline$\chi_{\theta}(\Omega ; \alpha, \beta)$ & Theorem II.16 & small field conditions \\
\hline $\mathfrak{S}$ & Definition II.4 & hierarchy \\
\hline$d \mu_{\Omega, \mathrm{r}}\left(z^{*}, z\right)$ & $\prod_{\mathbf{x} \in \Omega} \frac{d z(\mathbf{x})^{*} \wedge d z(\mathbf{x})}{2 \pi \imath} e^{-z(\mathbf{x})^{*} z(\mathbf{x})} \chi(|z(\mathbf{x})|<r)$ & before (II.1) \\
\hline $\mathcal{D}_{\Omega ; 0}\left(\varepsilon ; \alpha_{*}, \beta\right)$ & before (II.2) & part of effective action \\
\hline $\mathcal{D}_{\Omega ; \theta}\left(\alpha_{*}, \beta\right)$ & $\lim _{m \rightarrow \infty} \mathcal{D}_{\Omega ; m}\left(2^{-m} \theta ; \alpha_{*}, \beta\right)$ & $(\mathrm{II} .2)$ \\
\hline$d(\mathbf{x}, \mathbf{y})$ & standard metric on $\mathrm{X}$ & \\
\hline $\mathfrak{d}(\tau)$ & Notation II.2.iii & decimation index of $\tau$ \\
\hline $\operatorname{exph}(J)$ & $e^{\mathfrak{h} J \mathfrak{h} h}$ & Definition III.4.iii \\
\hline$\Gamma_{* \mathfrak{S}}, \Gamma_{\mathfrak{S}}$ & Definition II.9 & background field \\
\hline$\Gamma_{* \tau}^{\tau^{\prime}}(\mathfrak{S}), \Gamma_{\tau}^{\tau^{\prime}}(\mathfrak{S})$ & Definition II.9 & coefficients \\
\hline $\mathrm{h}$ & $\nabla^{*} \mathcal{H} \nabla$ & kinetic energy \\
\hline $\mathcal{H}$ & kernel in kinetic energy & see beginning of Appendix D \\
\hline$\overline{I_{\theta}\left(\alpha^{*}, \beta\right)}$ & $\lim _{m \rightarrow \infty} I_{m}\left(2^{-m} \theta ; \alpha^{*}, \beta\right)$ & Theorem II.16 \\
\hline$I_{n}\left(\varepsilon ; \alpha^{*}, \beta\right)$ & (I.3), (I.10) & effective density \\
\hline $\mathcal{I}_{\left(\mathfrak{S} ; \alpha^{*}, \beta\right)}$ & Definition II.8.ii & large field integral operator \\
\hline$\overline{\mathcal{I}_{\left(\mathcal{J} ; \alpha^{*}, \beta\right)}}$ & Definition II.8.i & large field integral operator \\
\hline $\mathcal{J}_{\tau}$ & Notation II.2.iii & decimation interval centred on $\tau$ \\
\hline $\mathcal{J}_{\tau}^{-}$ & Notation II.2.iii & left half of $\mathcal{J}_{\tau}$ \\
\hline $\mathcal{J}_{\tau}^{+}$ & Notation II.2.iii & right half of $\mathcal{J}_{\tau}$ \\
\hline$j(t)$ & $\operatorname{exph}(-t(\mathrm{~h}-\mu))$ & (III.1) \\
\hline$j_{\mathfrak{c}}(\tau)(\mathbf{x}, \mathbf{y})$ & $j(\tau)(\mathbf{x}, \mathbf{y}) \cdot \begin{cases}1 & \text { if } d(\mathbf{x}, \mathbf{y}) \leq \mathfrak{c} \\
0 & \text { if } d(\mathbf{x}, \mathbf{y})>\mathfrak{c}\end{cases}$ & (III.2) \\
\hline
\end{tabular}




\begin{tabular}{|c|c|c|}
\hline$K_{d}$ & $\sup _{\mathbf{y} \in X} \sum_{\mathbf{x} \in X} e^{-d(\mathbf{x}, \mathbf{y})}$ & in proof of Lemma III.42 \\
\hline$K_{D}$ & $2^{35} e^{6 K_{j}}$ & Theorem III.26 \\
\hline$K_{\Delta}$ & $2^{40} e^{10 K_{j}}$ & Theorem III.27 \\
\hline$K_{E}$ & $2^{23}$ & Theorem III.24 \\
\hline$K_{L}$ & $2^{48} e^{6 K_{j}}$ & Theorem III.26 \\
\hline$K_{L 2}$ & $\frac{1}{32} \min \left\{c_{\mathcal{H}} e^{-4 \mathrm{D} C_{\mathcal{H}}}, \frac{1}{32}\right\}$ & in proof of Proposition III.36 \\
\hline$K_{Q}$ & $2^{9} e^{2 K_{j}}$ & Proposition V.12 \\
\hline$K_{R}$ & $2^{12} K_{j}^{2}$ & Theorem III.24 \\
\hline$K_{\mathcal{V}}$ & $2^{25} e^{6 K_{j}}$ & Proposition V.13 \\
\hline$\Lambda(\mathcal{J})$ & Definitions II.4, A.1, §V.1 & small field set \\
\hline$N_{\delta}\left(A ; \kappa, \kappa^{\prime}\right)$ & Definition III.18 & weighted $L^{1}-L^{\infty}$ operator norm \\
\hline$\Omega(\mathcal{J})$ & Definitions II.4, §V.3 & small field set \\
\hline$P_{\alpha}(\mathcal{J}), P_{\beta}(\mathcal{J})$ & Definitions II.4, A.1, $\S \mathrm{V} .1$ & large field sets \\
\hline$P_{\alpha}^{\prime}(\mathcal{J}), P_{\beta}^{\prime}(\mathcal{J})$ & Definitions II.4, A.1, §V.1 & large field sets \\
\hline$Q(\mathcal{J})$ & Definitions II.4, A.1, §V.1 & large field set \\
\hline$Q_{\varepsilon, \delta}\left(\alpha_{*}, \beta ; \vec{\gamma}_{*}, \vec{\gamma}\right)$ & (II.10) & dominant quadratic part \\
\hline$Q_{\mathfrak{S}}\left(\alpha_{*}, \beta ; \vec{\alpha}_{*}, \vec{\alpha}\right)$ & $Q_{\varepsilon, \delta}\left(\alpha_{*}, \beta ; \Gamma_{* \mathfrak{S}}\left(\cdot ; \alpha_{*}, \vec{\alpha}_{*}\right), \Gamma_{\mathfrak{S}}(\cdot ; \vec{\alpha}, \beta)\right)$ & $($ II.9), (III.3) \\
\hline$R(\mathcal{J})$ & Definitions II.4, $\S \mathrm{V} .3$ & Stokes' large field sets \\
\hline $\mathfrak{R}_{\Omega ; t}$ & before (II.1) & renormalization group operator \\
\hline$V_{\Omega, \delta}\left(\varepsilon ; \alpha_{*}, \beta\right)$ & (II.1) & principal interaction \\
\hline$V_{\Omega ; \theta}\left(\alpha_{*}, \beta\right)$ & $\lim _{m \rightarrow \infty} V_{\Omega ; \theta}\left(2^{-m} \theta ; \alpha_{*}, \beta\right)$ & $(\mathrm{II} .4)$ \\
\hline $\mathcal{V}_{\mathfrak{S}}\left(\alpha_{*}, \beta ; \vec{\alpha}_{*}, \vec{\alpha}\right)$ & $(\mathrm{II} .11)$ & dominant quartic part \\
\hline$v(\mathbf{x}, \mathbf{y})$ & two-body potential & see Hypothesis II.14 \\
\hline$X$ & $\mathbb{Z}^{\mathrm{D}} / L \mathbb{Z}^{\mathrm{D}}$ & space \\
\hline$Y^{*}$ & bonds with at least one end in $Y$ & just before Notation II.2 \\
\hline$Y^{\star}$ & points within distance one of $Y$ & just before Notation II.2 \\
\hline $\mathcal{Z}_{\delta}$ & Lemma II.7 & normalization constant \\
\hline
\end{tabular}




\section{References}

[BFKT1] Tadeusz Balaban, Joel Feldman, Horst Knörrer and Eugene Trubowitz, A Functional Integral Representation for Many Boson Systems. I: The Partition Function, Annales Henri Poincaré 9 (2008), 1229-1273.

[BFKT2] Tadeusz Balaban, Joel Feldman, Horst Knörrer and Eugene Trubowitz, A Functional Integral Representation for Many Boson Systems. II: Correlation Functions, Annales Henri Poincaré, 9 (2008), 1275-1307.

[BFKT3] Tadeusz Balaban, Joel Feldman, Horst Knörrer and Eugene Trubowitz, Power Series Representations for Bosonic Effective Actions, Journal of Statistical Physics, 134 (2009), 839-857.

[BFKT4] Tadeusz Balaban, Joel Feldman, Horst Knörrer and Eugene Trubowitz, Power Series Representations for Complex Bosonic Effective Actions, preprint.

[BFKT5] Tadeusz Balaban, Joel Feldman, Horst Knörrer and Eugene Trubowitz, Power Series Representations for Complex Bosonic Effective Actions. II: A Small Field Renormalization Group Flow, preprint.

[BFI] David Brydges and Paul Federbush, The Cluster Expansion in Statistical Physics, Commun. Math. Phys. 49 (1976), 233-246.

[BFII] David Brydges and Paul Federbush, The Cluster Expansion for Potentials with Exponential Fall-off, Commun. Math. Phys. 53 (1977), 19-30.

[Br] David C. Brydges, A short course on cluster expansions, in Phénomènes critiques, systèmes aléatoires, théories de jauge (Les Houches, 1984), 129-183, North-Holland, Amsterdam, 1986.

[C] Camillo Cammarota, Decay of Correlations for Infinite Range Interactions in Unbounded Spin Systems, Commun. Math. Phys. 85 (1982), 517-528.

[E] Robert E. Edwards, Functional analysis: theory and applications, Dover (1995).

[G1] Jean Ginibre, Reduced density matrices of quantum gases. I. Limit of infinite volume. J. Mathematical Phys. 6 (1965) 238-251.

[G2] Jean Ginibre, Some applications of functional integration in Statistical Mechanics, in Statistical Mechanics and Quantum Field Theory, Proceedings of the Les Houches Summer School of Theoretical Physics, edited by C. DeWitt and R. Stora (Gordon and Breach, New York, 1971), 327.

[NO] John W. Negele and Henri Orland, Quantum Many-Particle Systems, Addison-Wesley (1988). 